\title{
Enantioselective Insertion of Alkynyl Carbenes into Si-H Bonds: An Efficient Access to Chiral Propargylsilanes and Allenylsilanes
}

\author{
Liang-Liang Yang, Jing Ouyang, Hui-Na Zou, Shou-Fei Zhu* and Qi-Lin Zhou* \\ State Key Laboratory and Institute of Elemento-Organic Chemistry, College of Chemistry, Nankai \\ University, Tianjin 300071, China \\ ${ }^{*}$ Correspondence to: sfzhu@nankai.edu.cn (S.-F.Z.); qlzhou@nankai.edu.cn (Q.-L.Z.).
}

\section{Table of Contents}

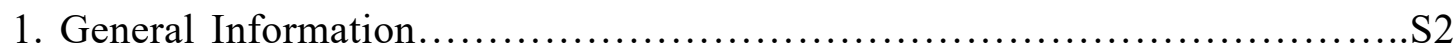

2. Condition Optimization of Enantioselective $\mathrm{Si}-\mathrm{H}$ Bond Insertion................S3

3. Condition Optimization of Isomerization of Propargylsilanes to

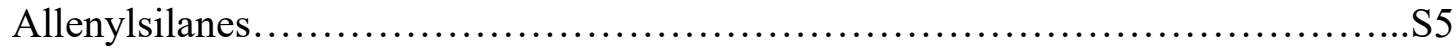

4. Preparation and Analytical Data of Hydrazones.............................S6

5. Typical Procedure for $\mathrm{Si}-\mathrm{H}$ Bond Insertion Reaction and Analytical Data of Insertion

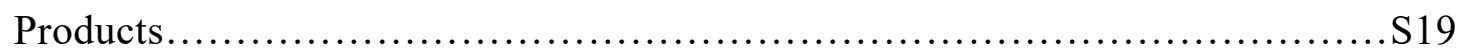

6. Transformations of $\mathrm{Si}-\mathrm{H}$ Bond Insertion Products.................................................S37

7. Typical Procedure for Isomerization of Propargylsilanes to Allenylsilanes and

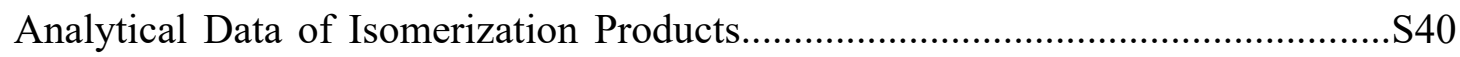

8. Kinetic Isotopic Effect Experiment for $\mathrm{Si}-\mathrm{H}$ Insertion Reaction........................S47

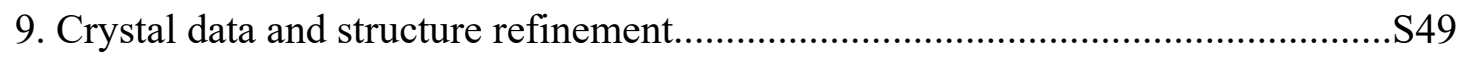

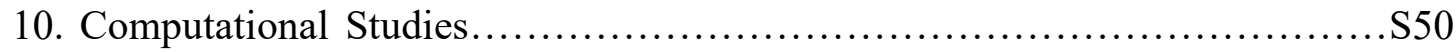

11. NMR Spectra for New Compounds.....................................S60

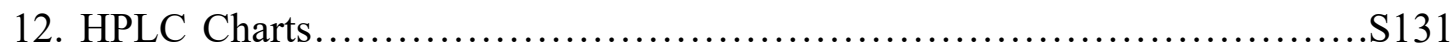

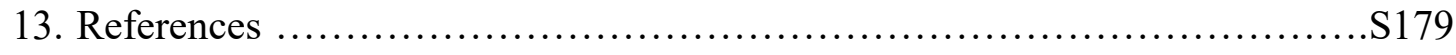




\section{General Information}

All reactions and manipulations were performed using standard Schlenk techniques. All solvents were purified and dried using standard procedures. ${ }^{1}$ All chiral carboxylate dirhodium complexes were purchased from Strem and TCI. All chiral phosphate dirhodium complexes were prepared according to the literature. ${ }^{2}$ All alkynyl ketones were prepared according to the literature. ${ }^{3}$ Dimethylphenylsilane (2a), triethylsilane (2b) and methyldiphenylsilane (2c) were purchased from Aldrich, Alfa, or TCI chemical company and deuterio dimethylphenyl silane (2a-d $\mathbf{1})$ was prepared according to the literature. ${ }^{4}$

NMR spectra were recorded with a Bruker AV 400 spectrometer at $400 \mathrm{MHz}\left({ }^{1} \mathrm{H}\right.$ NMR), $101 \mathrm{MHz}\left({ }^{13} \mathrm{C} \mathrm{NMR}\right), 128 \mathrm{MHz}\left({ }^{11} \mathrm{~B} \mathrm{NMR}\right)$ and $162 \mathrm{MHz}\left({ }^{31} \mathrm{P} \mathrm{NMR}\right)$. Chemical shifts ( $\delta$ values) were reported in ppm down field from internal $\mathrm{Me}_{4} \mathrm{Si}\left({ }^{1} \mathrm{H}\right.$ and ${ }^{13} \mathrm{C}$ NMR). High Resolution Mass Spectra (HRMS) were recorded on an IonSpec FT-ICR mass spectrometer with Electron Spray Ionization (ESI) resource and Waters GCT Premier mass spectrometer. Melting points were measured on a RY-I apparatus and uncorrected. Enantioselectivities were recorded on Agilent HPLC, using a chiral stationary phase column (Daicel Co. CHIRALPAK, CHIRALCEL, Phenomenex). The chiral HPLC methods were calibrated with the corresponding racemic mixtures. 


\section{Condition Optimization of Enantioselective Si-H Bond Insertion}

Scheme S1. Evaluation of sulfonyl groups of hydrazone

2,
entry
1

Reaction conditions: $\mathbf{C 1} / \mathbf{1} / \mathbf{2 a}=0.002: 0.2: 0.6(\mathrm{mmol}), 0.6 \mathrm{mmol} \mathrm{LiO}^{t} \mathrm{Bu}, 3 \mathrm{~mL} \mathrm{CH}_{2} \mathrm{Cl}_{2}$, $0{ }^{\circ} \mathrm{C}, 12 \mathrm{~h}$. Isolated yields were given.

Scheme S2. Evaluation of chiral dirhodium catalysts

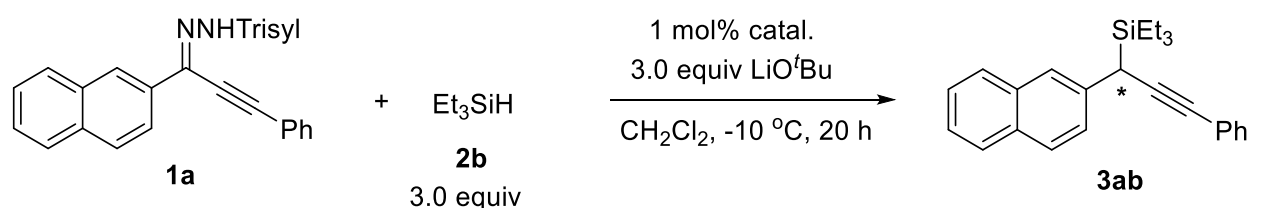<smiles>COP(Oc1ccc2ccccc2c1-c1cccc2ccccc12)Oc1cccc2ccccc12</smiles>

$\mathrm{Rh}_{2}(R-\mathrm{BINP})_{4}$

$48 \%$ yield $54 \%$ ee<smiles>C[R18](C)(C)N1C(=O)c2c(Cl)c(Cl)c(Cl)c(Cl)c2C1=O</smiles>

$$
\mathrm{Rh}_{2}(\text { S-CIPTTL })_{4}
$$

$84 \%$ yield $86 \%$ ee

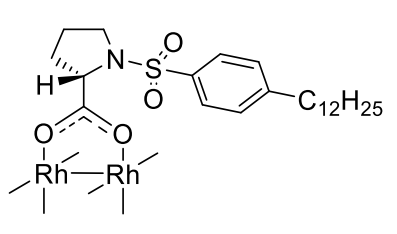

$\mathrm{Rh}_{2}(S-\mathrm{DOSP})_{4}$

$67 \%$ yield $0 \%$ ee

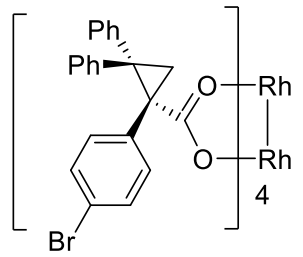

$\mathrm{Rh}_{2}(R-\mathrm{BTPCP})_{4}$ $81 \%$ yield $30 \%$ ee

Reaction conditions: $\mathbf{C} / \mathbf{1 a} / \mathbf{2 b}=0.002: 0.2: 0.6(\mathrm{mmol}), 0.6 \mathrm{mmol} \mathrm{LiO}^{t} \mathrm{Bu}, 3 \mathrm{~mL} \mathrm{CH}_{2} \mathrm{Cl}_{2}$, $-10{ }^{\circ} \mathrm{C}, 20 \mathrm{~h}$. Isolated yields were given. The ee values were determined by HPLC. 
Scheme S3. Evaluation of chiral spiro phosphate dirhodium catalysts using $1 \mathbf{j}$ as the substrate<smiles>CC(C)(C)C#CC(=N)c1ccc2ccccc2c1</smiles>

$1 \mathrm{j}$

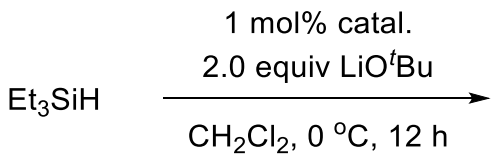

2b

2.0 equiv<smiles>CCSC(C#CC(C)(C)C)c1ccc2ccccc2c1</smiles>

3jb

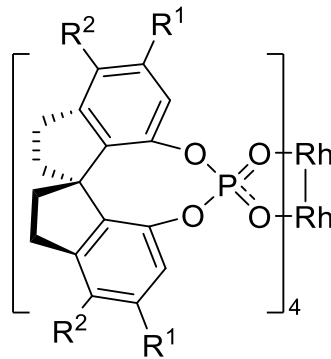

\begin{tabular}{|c|c|c|}
\hline C1 $R^{1}=H$ & $\mathrm{R}^{2}=\mathrm{H}$ & $83 \%$ yield \\
\hline C2 $\mathrm{R}^{1}=\mathrm{C}_{6} \mathrm{H}_{5}$ & $\mathrm{R}^{2}=\mathrm{H}$ & $87 \%$ yield \\
\hline C3 $R^{1}=2-N p$ & $\mathrm{R}^{2}=\mathrm{H}$ & $89 \%$ yield \\
\hline C4 $\mathrm{R}^{1}=4-\left(4-{ }^{t} \mathrm{BuC}_{6} \mathrm{H}_{4}\right) \mathrm{C}_{6} \mathrm{H}_{4}$ & $\mathrm{R}^{2}=\mathrm{H}$ & $89 \%$ yield \\
\hline C5 $\mathrm{R}^{1}=\mathrm{H}$ & $\mathrm{R}^{2}=\mathrm{Br}$ & $57 \%$ yield \\
\hline C6 $R^{1}=H$ & $\mathrm{R}^{2}=\mathrm{C}_{6} \mathrm{H}_{5}$ & $89 \%$ yield \\
\hline
\end{tabular}

Reaction conditions: $\mathbf{C} / \mathbf{1} \mathbf{j} / \mathbf{2} \mathbf{b}=0.002: 0.2: 0.4(\mathrm{mmol}), 0.4 \mathrm{mmol} \mathrm{LiO}^{t} \mathrm{Bu}, 3 \mathrm{~mL} \mathrm{CH}_{2} \mathrm{Cl}_{2}$, $0{ }^{\circ} \mathrm{C}, 12 \mathrm{~h}$. Isolated yields were given. The ee values were determined by HPLC. 


\section{Condition Optimization of Isomerization of Propargylsilanes to}

\section{Allenylsilanes}

Table S1. Catalytic isomerization of propargylsilanes to allenylsilanes<smiles></smiles>

$3 \mathrm{jc}(98 \%$ ee $)$

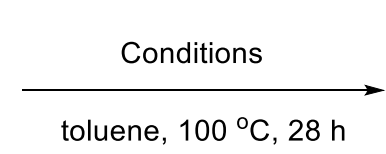

toluene, $100{ }^{\circ} \mathrm{C}, 28 \mathrm{~h}$

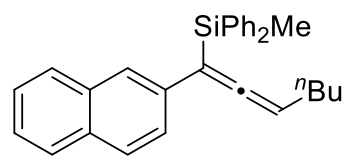

$7 a$

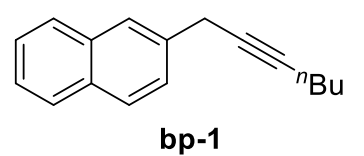<smiles>CC(C)(C)C=C=Cc1ccc2ccccc2c1</smiles>

bp-2

\begin{tabular}{|c|c|c|c|c|}
\hline entry & conditions & yield $(\%)$ & ee $(\%)$ & es $(\%)$ \\
\hline $1^{a}$ & $5 \mathrm{~mol} \%\left(\mathrm{Ph}_{3} \mathrm{P}\right)_{2} \mathrm{Pt} \cdot \mathrm{O}_{2}$ & 84 & 96 & 98 \\
\hline 2 & $5 \mathrm{~mol}_{0} \mathrm{Pt}\left(\mathrm{PPh}_{3}\right)_{4}$ & trace & NA & NA \\
\hline $3^{a}$ & $5 \mathrm{~mol} \% \mathrm{Pt}\left(\mathrm{PPh}_{3}\right)_{4}$, air & 64 & 96 & 98 \\
\hline 4 & $5 \mathrm{~mol}_{0} \mathrm{PPh}_{3}$ & NR & NA & NA \\
\hline 5 & $5 \mathrm{~mol}_{0} \mathrm{OPPh}_{3}$ & NR & NA & NA \\
\hline 6 & $5 \mathrm{~mol} \% \mathrm{Pt} / \mathrm{C}$ & NR & NA & NA \\
\hline $7^{a}$ & $5 \mathrm{~mol} \%\left(\mathrm{Ph}_{3} \mathrm{P}\right)_{2} \mathrm{Pt} \cdot \mathrm{O}_{2}, \mathrm{DMF}, 80^{\circ} \mathrm{C}, 3 \mathrm{~h}$ & trace & NA & NA \\
\hline $8^{a}$ & $20 \mathrm{~mol} \% \mathrm{DBU}, \mathrm{THF}, \mathrm{rt}, 24 \mathrm{~h}$ & $<10 \%{ }^{c}$ & NA & NA \\
\hline 9 & $20 \mathrm{~mol} \%{ }^{i} \operatorname{Pr}_{2} \mathrm{NEt}, \mathrm{THF}, \mathrm{rt}, 24 \mathrm{~h}$ & NR & NA & NA \\
\hline 10 & $20 \mathrm{~mol}^{\circ} \% \mathrm{LiO}^{t} \mathrm{Bu}, \mathrm{THF}, \mathrm{rt}, 24 \mathrm{~h}$ & NR & NA & NA \\
\hline $11^{b}$ & $20 \mathrm{~mol} \% \mathrm{NaO}{ }^{t} \mathrm{Bu}, \mathrm{THF}, \mathrm{rt}, 24 \mathrm{~h}$ & $\operatorname{trace}^{c}$ & NA & NA \\
\hline
\end{tabular}

Reaction conditions: $3 \mathbf{j c} /\left(\mathrm{Ph}_{3} \mathrm{P}\right)_{2} \mathrm{Pt} \cdot \mathrm{O}_{2}=0.05: 0.0025(\mathrm{mmol}), 1 \mathrm{~mL}$ toluene, $100{ }^{\circ} \mathrm{C}$, $28 \mathrm{~h}$. Isolated yields were given. The ee values were determined by HPLC. NR $=$ no reaction. NA $=$ not analyzed. ${ }^{a}>95 \%$ Conv. ${ }^{b} 65 \%$ Conv. ${ }^{c}$ The main products are bp-1 and bp-2. 


\section{Preparation and Analytical Data of Substrates}

\section{Synthesis of alkynyl sulfonylhydrazones}

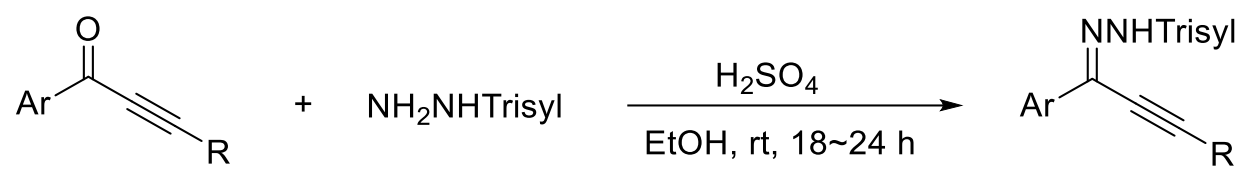

All alkynyl sulfonylhydrazones were prepared according to the literature. ${ }^{3}$

A $100 \mathrm{~mL}$ Schlenk bottle equipped with a magnetic stir bar was charged with 2,4,6triisopropylphenylsulfonyl hydrazone ( $\mathrm{NH}_{2} \mathrm{NHTrisyl}, 1.1$ equiv) and anhydrous ethanol $(4 \mathrm{~mL} / \mathrm{mmol})$. Concentrated $\mathrm{H}_{2} \mathrm{SO}_{4}(0.2 \mathrm{~mL})$ was added dropwise into the bottle at $0{ }^{\circ} \mathrm{C}$ over 5 minutes. After stirring at $0{ }^{\circ} \mathrm{C}$ for additional $10 \mathrm{~min}$, the solution became clear, and alkynyl ketone (1.0 equiv) in ethanol solution $(3-5 \mathrm{~mL})$ was injected into the Schlenk bottle. The pale-yellow slurry was stirred at room temperature for 18-24 hours. The solid was isolated by suction filtration and washed with cold water and enthanol to give white product. If the product is difficult to precipitate, the crude product was purified by column chromatography to afford the alkynyl hydrazone.

\section{Analytical data of alkynyl sulfonylhydrazones}

2,4,6-triisopropyl-N'-(1-(naphthalen-2-yl)-3-phenylprop-2-yn-1ylidene)benzenesulfonohydrazide (1a)<smiles>N=C(C#Cc1ccccc1)c1ccc2ccccc2c1</smiles>

$84 \%$ yield $(3.6 \mathrm{~g})$, white solid, m.p. $=180-182^{\circ} \mathrm{C}$.

$\underline{{ }^{1} \mathrm{H}} \underline{\mathrm{NMR}} \underline{(400} \underline{\mathrm{MHz}}, \underline{\left.\mathrm{CDCl}_{3}\right)}$

$\delta 8.87(\mathrm{~s}, 1 \mathrm{H}), 8.28(\mathrm{~s}, 1 \mathrm{H}), 8.02(\mathrm{dd}, J=8.6,1.7 \mathrm{~Hz}, 1 \mathrm{H}), 7.88-7.77(\mathrm{~m}, 3 \mathrm{H})$, $7.69-7.62$ (m, 2H), $7.55-7.43$ (m, 5H), 7.18 (s, 2H), 4.36 (hept, $J=6.7 \mathrm{~Hz}$, 2H), 2.88 (hept, $J=6.8 \mathrm{~Hz}, 1 \mathrm{H}), 1.35(\mathrm{~d}, J=6.7 \mathrm{~Hz}, 12 \mathrm{H}), 1.23(\mathrm{~d}, J=6.8 \mathrm{~Hz}$, $6 \mathrm{H})$

${ }^{13} \mathrm{C} \underline{\mathrm{NMR}} \underline{(101} \underline{\mathrm{MHz}}, \underline{\left.\mathrm{CDCl}_{3}\right)}$

$\delta 153.5,151.2,134.9,134.1,132.8,132.1,131.6$ (2C signal), 130.4, 128.8, $128.5,128.0,127.7,127.4,127.0,126.5,123.9,122.9,120.3,104.6,77.20$, $34.1,30.2,24.9,23.5$.

$\underline{\operatorname{HRMS}} \underline{(\mathrm{ESI})}$

Calcd for $\mathrm{C}_{34} \mathrm{H}_{36} \mathrm{~N}_{2} \mathrm{O}_{2} \mathrm{~S}[\mathrm{M}+\mathrm{H}]^{+}$: 537.2570, found: 537.2565 . 
N'-(1,3-diphenylprop-2-yn-1-ylidene)-2,4,6-triisopropylbenzenesulfonohydrazide (1b)<smiles>CCCCCC/N=C(\C#Cc1ccccc1)c1ccccc1</smiles>

$77 \%$ yield $(1.5 \mathrm{~g})$, white solid, m.p. $=159-161^{\circ} \mathrm{C}$.

$\underline{{ }^{1} \mathrm{H}} \underline{\mathrm{NMR}} \underline{(400} \underline{\mathrm{MHz}}, \underline{\left.\mathrm{CDCl}_{3}\right)}$

$\delta 8.79(\mathrm{~s}, 1 \mathrm{H}), 7.91-7.84(\mathrm{~m}, 2 \mathrm{H}), 7.65-7.58(\mathrm{~m}, 2 \mathrm{H}), 7.52-7.40(\mathrm{~m}, 3 \mathrm{H})$,

$7.38-7.30$ (m, 3H), 7.17 (s, 2H), 4.31 (hept, $J=6.8 \mathrm{~Hz}, 2 \mathrm{H}$ ), 2.88 (hept, $J=$

$7.1 \mathrm{~Hz}, 1 \mathrm{H}), 1.31(\mathrm{~d}, J=6.8 \mathrm{~Hz}, 12 \mathrm{H}), 1.24(\mathrm{~d}, J=6.9 \mathrm{~Hz}, 6 \mathrm{H})$.

${ }^{13} \mathrm{C} \underline{\mathrm{NMR}} \underline{(101} \underline{\mathrm{MHz}}, \underline{\left.\mathrm{CDCl}_{3}\right)}$

$\delta 153.5,151.2,134.8,134.0,132.1,131.5,130.4,130.0,128.8,128.3,126.5$,

$123.9,120.3,104.5,77.2,34.2,30.1,24.8,23.5$.

$\underline{\mathrm{HRMS}} \underline{(\mathrm{ESI})}$

Calcd for $\mathrm{C}_{30} \mathrm{H}_{34} \mathrm{~N}_{2} \mathrm{O}_{2} \mathrm{~S}[\mathrm{M}+\mathrm{H}]^{+}$: 487.2414, found: 487.2416 .

N'-(1-(2-chlorophenyl)-3-phenylprop-2-yn-1-ylidene)-2,4,6-

triisopropylbenzenesulfonohydrazide (1c)<smiles>N=C(C#Cc1ccccc1)c1ccccc1Cl</smiles>

$56 \%$ yield $(2.3 \mathrm{~g})$, white solid, m.p. $=128-129^{\circ} \mathrm{C}$.

$\underline{{ }^{1} \mathrm{H}} \underline{\mathrm{NMR}} \underline{(400} \underline{\mathrm{MHz}}, \underline{\left.\mathrm{CDCl}_{3}\right)}$

$\delta 8.94(\mathrm{~s}, 1 \mathrm{H}), 7.59-7.52(\mathrm{~m}, 2 \mathrm{H}), 7.50-7.37$ (m, 4H), $7.35(\mathrm{dd}, J=7.8,1.5$

$\mathrm{Hz}, 1 \mathrm{H}), 7.30-7.19$ (m, 2H), 7.18 (s, 2H), 4.26 (hept, $J=6.7 \mathrm{~Hz}, 2 \mathrm{H}), 2.90$

(hept, $J=6.9 \mathrm{~Hz}, 1 \mathrm{H}), 1.26-1.24(\mathrm{~m}, 18 \mathrm{H})$.

${ }^{13} \mathrm{C} \underline{\mathrm{NMR}}\left(101 \mathrm{MHz}, \underline{\left.\mathrm{CDCl}_{3}\right)}\right.$

$\delta 153.6,151.2,133.4,133.0,132.9,132.0,131.3,130.7,130.4,130.3(2 \mathrm{C}$

$\underline{\text { HRMS }} \underline{(\mathrm{ESI})}$

signal), 128.7, 126.6, 123.8, 120.5, 105.1, 78.2, 34.2, 30.0, 24.7, 23.5.

Calcd for $\mathrm{C}_{30} \mathrm{H}_{33} \mathrm{ClN}_{2} \mathrm{O}_{2} \mathrm{~S}[\mathrm{M}+\mathrm{H}]^{+}:$521.2024, found: 521.2032 .

N'-(1-(3-chlorophenyl)-3-phenylprop-2-yn-1-ylidene)-2,4,6-

triisopropylbenzenesulfonohydrazide (1d)

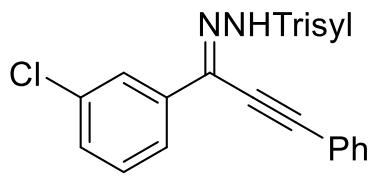

$72 \%$ yield $(1.9 \mathrm{~g})$, white solid, m.p. $=177-178^{\circ} \mathrm{C}$. 


\section{$\underline{{ }^{1} \mathrm{H}} \underline{\mathrm{NMR}} \underline{(400} \underline{\mathrm{MHz}}, \underline{\left.\mathrm{CDCl}_{3}\right)}$}

$\delta 8.81(\mathrm{~s}, 1 \mathrm{H}), 7.86(\mathrm{t}, J=1.8 \mathrm{~Hz}, 1 \mathrm{H}), 7.73(\mathrm{dt}, J=7.4,1.6 \mathrm{~Hz}, 1 \mathrm{H}), 7.63-$

$7.58(\mathrm{~m}, 2 \mathrm{H}), 7.53-7.41(\mathrm{~m}, 3 \mathrm{H}), 7.36-7.24(\mathrm{~m}, 2 \mathrm{H}), 7.18$ (s, 2H), 4.29 (hept, $J=6.7 \mathrm{~Hz}, 2 \mathrm{H}), 2.90$ (hept, $J=6.9 \mathrm{~Hz}, 1 \mathrm{H}), 1.32$ (d, $J=6.7 \mathrm{~Hz}, 12 \mathrm{H}), 1.24$

$(\mathrm{d}, J=6.9 \mathrm{~Hz}, 6 \mathrm{H})$.

${ }^{13} \mathrm{C} \underline{\mathrm{NMR}} \underline{(101} \underline{\mathrm{MHz}}, \underline{\left.\mathrm{CDCl}_{3}\right)}$

$\delta 153.7,151.2,135.8,134.5,133.3,132.1,131.4,130.6,129.9,129.6,128.8$,

$126.3,124.8,123.9,120.0,105.0,76.7,34.2,30.2,24.8,23.5$.

$\underline{\mathrm{HRMS}} \underline{(\mathrm{ESI})}$

Calcd for $\mathrm{C}_{30} \mathrm{H}_{33} \mathrm{ClN}_{2} \mathrm{O}_{2} \mathrm{~S}[\mathrm{M}+\mathrm{H}]^{+}$: 521.2024, found: 521.2026.

N'-(1-(4-chlorophenyl)-3-phenylprop-2-yn-1-ylidene)-2,4,6-

triisopropylbenzenesulfonohydrazide (1e)<smiles>N=C(C#CCNCCc1ccccc1)c1ccc(Cl)cc1</smiles>

$66 \%$ yield $(1.7 \mathrm{~g})$, white solid, m.p. $=177-178^{\circ} \mathrm{C}$.

${ }^{1} \mathrm{H} \underline{\mathrm{NMR}} \underline{(400} \underline{\mathrm{MHz}}, \underline{\left.\mathrm{CDCl}_{3}\right)}$

$\delta 8.78(\mathrm{~s}, 1 \mathrm{H}), 7.79(\mathrm{~d}, J=8.7 \mathrm{~Hz}, 2 \mathrm{H}), 7.63-7.55(\mathrm{~m}, 2 \mathrm{H}), 7.51-7.44(\mathrm{~m}$, $3 \mathrm{H}), 7.32$ (d, $J=8.6 \mathrm{~Hz}, 2 \mathrm{H}), 7.18$ (s, 2H), 4.29 (hept, $J=6.7 \mathrm{~Hz}, 2 \mathrm{H}), 2.89$ (hept, $J=6.9 \mathrm{~Hz}, 1 \mathrm{H}), 1.30(\mathrm{~d}, J=6.8 \mathrm{~Hz}, 12 \mathrm{H}), 1.24(\mathrm{~d}, J=6.9 \mathrm{~Hz}, 6 \mathrm{H})$.

${ }^{13} \mathrm{C} \underline{\mathrm{NMR}} \underline{(101} \underline{\mathrm{MHz}}, \underline{\left.\mathrm{CDCl}_{3}\right)}$

$\delta 153.6,151.1,133.6,133.1,132.1,131.4,130.8,130.5,128.8,128.5,127.7$, $123.9,120.0,104.9,76.8,34.2,30.1,24.8,23.5$.

$\underline{\text { HRMS }} \underline{(E S I)}$

Calcd for $\mathrm{C}_{30} \mathrm{H}_{33} \mathrm{ClN}_{2} \mathrm{O}_{2} \mathrm{~S}[\mathrm{M}+\mathrm{H}]^{+}:$521.2024, found: 521.2020 .

\section{2,4,6-triisopropyl-N'-(1-(4-methoxyphenyl)-3-phenylprop-2-yn-1-} ylidene)benzenesulfonohydrazide (1f)<smiles>COc1ccc(C(=N)C#Cc2ccccc2)cc1</smiles>

$74 \%$ yield $(1.9 \mathrm{~g})$, white solid, m.p. $=163-164{ }^{\circ} \mathrm{C}$.

$\underline{{ }^{1} \mathrm{HMR}} \underline{\mathrm{NM00}} \underline{\mathrm{MHz}}, \underline{\left.\mathrm{CDCl}_{3}\right)}$

$\delta 8.68(\mathrm{~s}, 1 \mathrm{H}), 7.81(\mathrm{~d}, J=8.9 \mathrm{~Hz}, 2 \mathrm{H}), 7.63-7.55(\mathrm{~m}, 2 \mathrm{H}), 7.52-7.39(\mathrm{~m}$, 3H), 7.17 (s, 2H), 6.87 (d, $J=8.9 \mathrm{~Hz}, 2 \mathrm{H}), 4.31$ (hept, $J=6.8 \mathrm{~Hz}, 2 \mathrm{H}), 3.81$ 
(s, 3H), 2.88 (hept, $J=6.9 \mathrm{~Hz}, 1 \mathrm{H}), 1.31$ (d, $J=6.8 \mathrm{~Hz}, 12 \mathrm{H}), 1.24(\mathrm{~d}, J=7.0$ $\mathrm{Hz}, 6 \mathrm{H})$.

$\underline{{ }^{13} \mathrm{C}} \underline{\mathrm{NMR}} \underline{(101} \underline{\mathrm{MHz}}, \underline{\left.\mathrm{CDCl}_{3}\right)}$

$\delta 161.1,153.4,151.1,134.8,132.0,131.6,130.3,128.7,128.0,126.8,123.8$, $\underline{\mathrm{HRMS}} \underline{(\mathrm{ESI})}$

$120.3,113.7,104.1,77.3,55.3,34.1,30.1,24.8,23.5$.

Calcd for $\mathrm{C}_{31} \mathrm{H}_{36} \mathrm{~N}_{2} \mathrm{O}_{3} \mathrm{~S}[\mathrm{M}+\mathrm{H}]^{+}:$517.2519, found: 517.2523.

\section{N'-(1-(4-cyanophenyl)-3-phenylprop-2-yn-1-ylidene)-2,4,6-}

triisopropylbenzenesulfonohydrazide (1g)

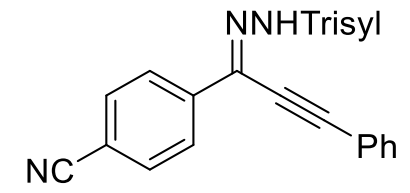

$70 \%$ yield $(0.9 \mathrm{~g})$, white solid, m.p. $=196-198^{\circ} \mathrm{C}$.

$\underline{{ }^{1} \mathrm{H}} \underline{\mathrm{NMR}} \underline{(400} \underline{\mathrm{MHz}}, \underline{\left.\mathrm{CDCl}_{3}\right)}$

$\delta 8.95(\mathrm{~s}, 1 \mathrm{H}), 7.96(\mathrm{~d}, J=8.2 \mathrm{~Hz}, 2 \mathrm{H}), 7.70-7.57(\mathrm{~m}, 4 \mathrm{H}), 7.57-7.41(\mathrm{~m}$,

3H), 7.19 (s, 2H), 4.28 (hept, $J=6.7 \mathrm{~Hz}, 2 \mathrm{H}), 2.90$ (hept, $J=6.9 \mathrm{~Hz}, 1 \mathrm{H}), 1.31$

$(\mathrm{d}, J=6.7 \mathrm{~Hz}, 12 \mathrm{H}), 1.25(\mathrm{~d}, J=6.9 \mathrm{~Hz}, 6 \mathrm{H})$.

${ }^{13} \mathrm{C} \underline{\mathrm{NMR}} \underline{(101} \underline{\mathrm{MHz}}, \underline{\left.\mathrm{CDCl}_{3}\right)}$

$\delta 153.9,151.2,138.2,132.5,132.2,132.1,131.2,130.8,128.9,126.9,124.0$,

$119.8,118.4,113.1,105.7,76.4,34.2,30.1,24.8,23.5$.

$\underline{\mathrm{HRMS}} \underline{(\mathrm{ESI})}$

Calcd for $\mathrm{C}_{31} \mathrm{H}_{33} \mathrm{~N}_{3} \mathrm{O}_{2} \mathrm{~S}[\mathrm{M}+\mathrm{H}]^{+}:$512.2366, found:512.2371.

N'-(3-(4-cyanophenyl)-1-(naphthalen-2-yl)prop-2-yn-1-ylidene)-2,4,6triisopropylbenzenesulfonohydrazide $(1 \mathrm{~h})$<smiles>N#Cc1ccc(C#CC(=N)c2ccc3ccccc3c2)cc1</smiles>

$79 \%$ yield $(1.1 \mathrm{~g})$, yellow solid, m.p. $=187-189^{\circ} \mathrm{C}$.

$\underline{{ }^{1} \mathrm{H}} \underline{\mathrm{NMR}} \underline{(400} \underline{\mathrm{MHz}}, \underline{\left.\mathrm{CDCl}_{3}\right)}$

$\delta 9.07(\mathrm{~s}, 1 \mathrm{H}), 8.22(\mathrm{~s}, 1 \mathrm{H}), 7.98(\mathrm{dd}, J=8.7,1.7 \mathrm{~Hz}, 1 \mathrm{H}), 7.87-7.67(\mathrm{~m}, 7 \mathrm{H})$,

7.50 (p, $J=5.8,5.3 \mathrm{~Hz}, 2 \mathrm{H}), 7.19$ (s, 2H), 4.36 (hept, $J=6.7 \mathrm{~Hz}, 2 \mathrm{H}), 2.90$

(hept, $J=6.8 \mathrm{~Hz}, 1 \mathrm{H}), 1.34(\mathrm{~d}, J=6.7 \mathrm{~Hz}, 12 \mathrm{H}), 1.24(\mathrm{~d}, J=6.8 \mathrm{~Hz}, 6 \mathrm{H})$.

${ }^{13} \mathrm{C} \underline{\mathrm{NMR}} \underline{(101} \underline{\mathrm{MHz}}, \underline{\left.\mathrm{CDCl}_{3}\right)}$ 
$\delta 153.8,151.3,134.1,133.7,132.8,132.7,132.3,131.3$ (2C signal), 128.5 (2C signal), 128.2, 127.7, 127.2, 126.7, 125.1, 124.0, 122.7, 117.9, 113.7, 101.7, $80.6,34.2,30.1,24.8,23.5$.

\section{$\underline{\mathrm{HRMS}} \underline{(\mathrm{ESI})}$}

Calcd for $\mathrm{C}_{35} \mathrm{H}_{35} \mathrm{~N}_{3} \mathrm{O}_{2} \mathrm{~S}[\mathrm{M}+\mathrm{H}]^{+}:$562.2523, found: 562.2520 .

2,4,6-triisopropyl-N'-(1-(naphthalen-2-yl)-3-(thiophen-2-yl)prop-2-yn-1ylidene)benzenesulfonohydrazide (1i)<smiles>N=C(C#Cc1cccs1)c1ccc2ccccc2c1</smiles>

$56 \%$ yield $(0.6 \mathrm{~g})$, white solid, m.p. $=168-170{ }^{\circ} \mathrm{C}$.

$\underline{{ }^{1} \mathrm{H}} \underline{\mathrm{NMR}} \underline{(400} \underline{\mathrm{MHz}}, \underline{\left.\mathrm{CDCl}_{3}\right)}$

$\delta 8.79(\mathrm{~s}, 1 \mathrm{H}), 8.24(\mathrm{~s}, 1 \mathrm{H}), 7.99(\mathrm{dd}, J=8.7,1.6 \mathrm{~Hz}, 1 \mathrm{H}), 7.88-7.74(\mathrm{~m}, 3 \mathrm{H})$,

$7.56-7.44(\mathrm{~m}, 4 \mathrm{H}), 7.22-7.09(\mathrm{~m}, 3 \mathrm{H}), 4.35$ (hept, $J=6.7 \mathrm{~Hz}, 2 \mathrm{H}), 2.88$

(hept, $J=6.9 \mathrm{~Hz}, 1 \mathrm{H}), 1.34(\mathrm{~d}, J=6.7 \mathrm{~Hz}, 12 \mathrm{H}), 1.23(\mathrm{~d}, J=6.9 \mathrm{~Hz}, 6 \mathrm{H})$.

${ }^{13} \mathrm{C} \underline{\mathrm{NMR}} \underline{(101} \underline{\mathrm{MHz}}, \underline{\left.\mathrm{CDCl}_{3}\right)}$

$\delta 153.5,151.2,134.8,134.7,134.1,132.8,131.5$ (2C signal), 130.4, 128.6, 128.1, 127.7 (2C signal), 127.3, 127.1, 126.5, 123.9, 122.9, 119.8, 97.8, 81.0, $34.2,30.2,24.9,23.5$.

$\underline{\mathrm{HRMS}} \underline{(\mathrm{ESI})}$

Calcd for $\mathrm{C}_{32} \mathrm{H}_{34} \mathrm{~N}_{2} \mathrm{O}_{2} \mathrm{~S}_{2}[\mathrm{M}+\mathrm{H}]^{+}:$543.2134, found: 543.2141.

2,4,6-triisopropyl-N'-(1-(naphthalen-2-yl)hept-2-yn-1-

ylidene)benzenesulfonohydrazide (1j)<smiles>CC(C)(C)C#CC(=N)c1ccc2ccccc2c1</smiles>

$74 \%$ yield $(1.9 \mathrm{~g})$, white solid, m.p. $=168-170{ }^{\circ} \mathrm{C}$.

${ }^{1} \mathrm{H} \underline{\mathrm{NMR}} \underline{(400} \underline{\mathrm{MHz}}, \underline{\left.\mathrm{CDCl}_{3}\right)}$

$\delta 8.75(\mathrm{~s}, 1 \mathrm{H}), 8.20(\mathrm{~s}, 1 \mathrm{H}), 7.97(\mathrm{~d}, J=8.7 \mathrm{~Hz}, 1 \mathrm{H}), 7.85-7.71(\mathrm{~m}, 3 \mathrm{H}), 7.51$

- 7.43 (m, 2H), 7.17 (s, 2H), 4.33 (hept, $J=6.8 \mathrm{~Hz}, 2 \mathrm{H}$ ), 2.87 (hept, $J=6.9$

$\mathrm{Hz}, 1 \mathrm{H}), 2.65$ (t, $J=7.1 \mathrm{~Hz}, 2 \mathrm{H}), 1.74-1.70(\mathrm{~m}, 2 \mathrm{H}), 1.60-1.50(\mathrm{~m}, 2 \mathrm{H})$,

$1.34(\mathrm{~d}, J=6.8 \mathrm{~Hz}, 12 \mathrm{H}), 1.23(\mathrm{~d}, J=6.9 \mathrm{~Hz}, 6 \mathrm{H}), 1.02(\mathrm{t}, J=7.3 \mathrm{~Hz}, 3 \mathrm{H})$.

${ }^{13} \mathrm{C} \underline{\mathrm{NMR}} \underline{(101} \underline{\mathrm{MHz}}, \underline{\left.\mathrm{CDCl}_{3}\right)}$

$\delta 153.4,151.1,135.5,134.0,132.8,131.9,131.7,128.5,127.8,127.7,127.3$, $126.9,126.4,123.9,122.9,107.5,70.0,34.1,30.3,30.1,24.9,23.5,22.1,19.4$, 13.5. 
$\underline{\operatorname{HRMS}} \underline{(\mathrm{ESI})}$

Calcd for $\mathrm{C}_{32} \mathrm{H}_{40} \mathrm{~N}_{2} \mathrm{O}_{2} \mathrm{~S}[\mathrm{M}+\mathrm{H}]^{+}:$517.2883, found: 517.2891.

\section{2,4,6-triisopropyl-N'-(1-(naphthalen-2-yl)but-2-yn-1-}

ylidene)benzenesulfonohydrazide $(1 \mathrm{k})$<smiles>CC#CC(=N)c1ccc2ccccc2c1</smiles>

$50 \%$ yield $(0.6 \mathrm{~g})$, white solid, m.p. $=199-200{ }^{\circ} \mathrm{C}$.

$\underline{{ }^{1} \mathrm{H}} \underline{\mathrm{NMR}} \underline{(400} \underline{\mathrm{MHz}}, \underline{\left.\mathrm{CDCl}_{3}\right)}$

$\delta 8.77(\mathrm{~s}, 1 \mathrm{H}), 8.19(\mathrm{~s}, 1 \mathrm{H}), 7.96(\mathrm{~d}, J=8.7 \mathrm{~Hz}, 1 \mathrm{H}), 7.87-7.69(\mathrm{~m}, 3 \mathrm{H}), 7.53$

- 7.42 (m, 2H), 7.17 (s, 2H), 4.33 (hept, $J=6.7 \mathrm{~Hz}, 2 \mathrm{H}$ ), 2.87 (hept, $6.6 \mathrm{~Hz}$,

$1 \mathrm{H}), 2.30(\mathrm{~s}, 3 \mathrm{H}), 1.33(\mathrm{~d}, J=6.7 \mathrm{~Hz}, 12 \mathrm{H}), 1.23(\mathrm{~d}, J=6.6 \mathrm{~Hz}, 6 \mathrm{H})$.

${ }^{13} \mathrm{C} \underline{\mathrm{NMR}} \underline{(101} \underline{\mathrm{MHz}}, \underline{\left.\mathrm{CDCl}_{3}\right)}$

$\delta 153.4,151.2,135.5,134.0,132.8,131.8,131.6,128.5,127.8,127.7,127.3$,

$126.9,126.4,123.9,122.9,102.9,69.4,34.1,30.1,24.8,23.5,4.8$.

$\underline{\operatorname{HRMS}} \underline{(\mathrm{ESI})}$

Calcd for $\mathrm{C}_{29} \mathrm{H}_{34} \mathrm{~N}_{2} \mathrm{O}_{2} \mathrm{~S}[\mathrm{M}+\mathrm{H}]^{+}: 475.2414$, found: 475.2422 .

2,4,6-triisopropyl-N'-(1-(naphthalen-2-yl)-6-phenylhex-2-yn-1-

ylidene)benzenesulfonohydrazide (11)<smiles>N=C(C#Cc1ccccc1)c1ccc2ccccc2c1</smiles>

$47 \%$ yield $(0.7 \mathrm{~g})$, white solid, m.p. $=108-109^{\circ} \mathrm{C}$.

$\underline{{ }^{1} \mathrm{H}} \underline{\mathrm{NMR}} \underline{(400} \underline{\mathrm{MHz}}, \underline{\left.\mathrm{CDCl}_{3}\right)}$

$\delta 8.75(\mathrm{~s}, 1 \mathrm{H}), 8.21(\mathrm{~s}, 1 \mathrm{H}), 7.97(\mathrm{dd}, J=8.7,1.7 \mathrm{~Hz}, 1 \mathrm{H}), 7.85-7.72(\mathrm{~m}, 3 \mathrm{H})$,

$7.50-7.42(\mathrm{~m}, 2 \mathrm{H}), 7.36-7.30(\mathrm{~m}, 2 \mathrm{H}), 7.27-7.21(\mathrm{~m}, 3 \mathrm{H}), 7.17(\mathrm{~s}, 2 \mathrm{H})$,

4.34 (hept, $J=6.8 \mathrm{~Hz}, 2 \mathrm{H}), 2.93-2.80(\mathrm{~m}, 3 \mathrm{H}), 2.64(\mathrm{t}, J=7.1 \mathrm{~Hz}, 2 \mathrm{H}), 2.07$

$(\mathrm{p}, J=7.2 \mathrm{~Hz}, 2 \mathrm{H}), 1.33(\mathrm{~d}, J=6.8 \mathrm{~Hz}, 12 \mathrm{H}), 1.22(\mathrm{~d}, J=6.9 \mathrm{~Hz}, 6 \mathrm{H})$.

${ }^{13} \mathrm{C} \underline{\mathrm{NMR}} \underline{(101} \underline{\mathrm{MHz}}, \underline{\left.\mathrm{CDCl}_{3}\right)}$

$\delta 153.4,151.2,140.6,135.3,134.0,132.8,131.9,131.6,128.6,128.5(2 \mathrm{C}$ signal), 127.9, 127.7, 127.3, 126.9, 126.5, 126.3, 123.9, 122.9, 106.9, 70.4, $34.9,34.1,30.1,29.8,24.9,23.5,19.0$.

$\underline{\text { HRMS }} \underline{(E S I)}$

Calcd for $\mathrm{C}_{37} \mathrm{H}_{42} \mathrm{~N}_{2} \mathrm{O}_{2} \mathrm{~S}[\mathrm{M}+\mathrm{H}]^{+}: 579.3040$, found: 579.3049 . 


\section{N'-(3-cyclopropyl-1-(naphthalen-2-yl)prop-2-yn-1-ylidene)-2,4,6-}

\section{triisopropylbenzenesulfonohydrazide (1m)}<smiles>N=C(C#CC1CC1)c1ccc2ccccc2c1</smiles>

$55 \%$ yield $(0.65 \mathrm{~g})$, white solid, m.p. $=159-161{ }^{\circ} \mathrm{C}$.

$\underline{{ }^{1} \mathrm{H}} \underline{\mathrm{NMR}} \underline{(400} \underline{\mathrm{MHz}}, \underline{\left.\mathrm{CDCl}_{3}\right)}$

$\delta 8.74(\mathrm{~s}, 1 \mathrm{H}), 8.17(\mathrm{~s}, 1 \mathrm{H}), 7.96(\mathrm{dd}, J=8.7,1.8 \mathrm{~Hz}, 1 \mathrm{H}), 7.87-7.70(\mathrm{~m}, 3 \mathrm{H})$,

7.48 (dd, $J=6.3,3.3 \mathrm{~Hz}, 2 \mathrm{H}), 7.19$ (s, 2H), 4.36 (hept, $J=6.8 \mathrm{~Hz}, 2 \mathrm{H}), 2.89$ (hept, $J=6.9 \mathrm{~Hz}, 1 \mathrm{H}), 1.70-1.62(\mathrm{~m}, 1 \mathrm{H}), 1.36(\mathrm{~d}, J=6.8 \mathrm{~Hz}, 12 \mathrm{H}), 1.24$ (d, $J=6.9 \mathrm{~Hz}, 6 \mathrm{H}), 1.16-1.09$ (m, 2H), $1.05-0.98$ (m, 2H).

${ }^{13} \mathrm{C} \underline{\mathrm{NMR}} \underline{(101} \underline{\mathrm{MHz}}, \underline{\left.\mathrm{CDCl}_{3}\right)}$

$\delta 153.4,151.1,135.5,134.0,132.8,131.9,131.6,128.5,127.8,127.6,127.2$, $126.9,126.4,123.8,122.9,110.7,65.0,34.1,30.1,29.7,24.9,23.5,9.8,0.3$.

$\underline{\text { HRMS }} \underline{(E S I)}$

Calcd for $\mathrm{C}_{31} \mathrm{H}_{36} \mathrm{~N}_{2} \mathrm{O}_{2} \mathrm{~S}$ [M $\left.+\mathrm{H}\right]^{+}:$501.2570, found: 501.2575 .

N'-(3-cyclohexyl-1-(naphthalen-2-yl)prop-2-yn-1-ylidene)-2,4,6-

triisopropylbenzenesulfonohydrazide (1n)<smiles>N=C(C#CC1CCCCC1)c1ccc2ccccc2c1</smiles>

$50 \%$ yield $(0.35 \mathrm{~g})$, white solid, m.p. $=140-143{ }^{\circ} \mathrm{C}$.

$\underline{{ }^{1} \mathrm{H}} \underline{\mathrm{NMR}} \underline{(400} \underline{\mathrm{MHz}}, \underline{\left.\mathrm{CDCl}_{3}\right)}$

$\delta 8.70(\mathrm{~s}, 1 \mathrm{H}), 8.20(\mathrm{~s}, 1 \mathrm{H}), 7.97(\mathrm{~d}, J=8.8 \mathrm{~Hz}, 1 \mathrm{H}), 7.85-7.77(\mathrm{~m}, 2 \mathrm{H}), 7.75$ $(\mathrm{d}, J=8.7 \mathrm{~Hz}, 1 \mathrm{H}), 7.50-7.43(\mathrm{~m}, 2 \mathrm{H}), 7.17$ (s, 2H), 4.33 (hept, $J=6.7 \mathrm{~Hz}$, 2H), $2.93-2.79(\mathrm{~m}, 2 \mathrm{H}), 2.06-1.95(\mathrm{~m}, 2 \mathrm{H}), 1.88-1.75(\mathrm{~m}, 2 \mathrm{H}), 1.74-$ $1.56(\mathrm{~m}, 3 \mathrm{H}), 1.53-1.41(\mathrm{~m}, 3 \mathrm{H}), 1.33(\mathrm{~d}, J=6.7 \mathrm{~Hz}, 12 \mathrm{H}), 1.22(\mathrm{~d}, J=6.9$ $\mathrm{Hz}, 6 \mathrm{H})$.

${ }^{13} \mathrm{C} \underline{\mathrm{NMR}} \underline{(101} \underline{\mathrm{MHz}}, \underline{\left.\mathrm{CDCl}_{3}\right)}$

$\delta 153.4,151.1,135.5,134.0,132.8,131.9,131.7,128.5,127.8,127.7,127.3$, $126.9,126.4,123.9,123.0,111.4,69.9,34.1,32.2$, 30.2, 29.9, 25.6, 24.9, 24.7, 23.5.

\section{$\underline{\text { HRMS }} \underline{(\mathrm{ESI})}$}

Calcd for $\mathrm{C}_{34} \mathrm{H}_{42} \mathrm{~N}_{2} \mathrm{O}_{2} \mathrm{~S}[\mathrm{M}+\mathrm{H}]^{+}$: 543.3040, found: 543.3049 . 
2,4,6-triisopropyl-N'-(1-(naphthalen-2-yl)-3-(trimethylsilyl)prop-2-yn-1ylidene)benzenesulfonohydrazide (10)<smiles>CC#CC(=N)c1ccc2ccccc2c1</smiles>

$81 \%$ yield $(0.43 \mathrm{~g})$, white solid, m.p. $=146-147^{\circ} \mathrm{C}$.

$\underline{{ }^{1} \mathrm{H}} \underline{\mathrm{NMR}} \underline{(400} \underline{\mathrm{MHz}}, \underline{\left.\mathrm{CDCl}_{3}\right)}$

$\delta 8.81(\mathrm{~s}, 1 \mathrm{H}), 8.19(\mathrm{~s}, 1 \mathrm{H}), 7.95(\mathrm{~d}, J=8.6 \mathrm{~Hz}, 1 \mathrm{H}), 7.86-7.72(\mathrm{~m}, 3 \mathrm{H}), 7.56$

- 7.44 (m, 2H), 7.17 (s, 2H), 4.33 (hept, $J=6.7 \mathrm{~Hz}, 2 \mathrm{H}$ ), 2.87 (hept, $J=6.9$

$\mathrm{Hz}, 1 \mathrm{H}), 1.34$ (d, $J=6.7 \mathrm{~Hz}, 12 \mathrm{H}), 1.22$ (d, $J=6.9 \mathrm{~Hz}, 6 \mathrm{H}), 0.39$ (s, 9H).

${ }^{13} \mathrm{C} \underline{\mathrm{NMR}} \underline{(101} \underline{\mathrm{MHz}}, \underline{\left.\mathrm{CDCl}_{3}\right)}$

$\delta 153.5,151.1,134.6,134.0,132.8,131.6,131.2,128.5,128.0,127.7,127.3$,

$127.0,126.5,123.9,122.8,112.7,92.1,34.1,30.1,24.9,23.5,-0.3$.

$\underline{\mathrm{HRMS}} \underline{\underline{(\mathrm{ESI})}}$

Calcd for $\mathrm{C}_{31} \mathrm{H}_{40} \mathrm{~N}_{2} \mathrm{O}_{2} \mathrm{SSi}[\mathrm{M}+\mathrm{H}]^{+}:$533.2653, found: 533.2650.

N'-(3-(cyclohex-1-en-1-yl)-1-(naphthalen-2-yl)prop-2-yn-1-ylidene)-2,4,6triisopropylbenzenesulfonohydrazide (1p)<smiles>N=C(C#CC1=CCCCC1)c1ccc2ccccc2c1</smiles>

$74 \%$ yield $(1.0 \mathrm{~g})$, white solid, m.p. $=152-154{ }^{\circ} \mathrm{C}$.

$\underline{{ }^{1} \mathrm{H}} \underline{\mathrm{NMR}} \underline{(400} \underline{\mathrm{MHz}}, \underline{\left.\mathrm{CDCl}_{3}\right)}$

$\delta 8.70(\mathrm{~s}, 1 \mathrm{H}), 8.20(\mathrm{~s}, 1 \mathrm{H}), 7.98(\mathrm{~d}, J=8.6 \mathrm{~Hz}, 1 \mathrm{H}), 7.86-7.70(\mathrm{~m}, 3 \mathrm{H}), 7.50$

$-7.41(\mathrm{~m}, 2 \mathrm{H}), 7.17(\mathrm{~s}, 2 \mathrm{H}), 6.51-6.48(\mathrm{~m}, 1 \mathrm{H}), 4.33$ (hept, $J=6.7 \mathrm{~Hz}, 2 \mathrm{H}$ ),

2.87 (hept, $J=7.0 \mathrm{~Hz}, 1 \mathrm{H}), 2.36-2.29$ (m, 2H), $2.26-2.24$ (m, 2H), $1.81-$

$1.64(\mathrm{~m}, 4 \mathrm{H}), 1.34(\mathrm{~d}, J=6.7 \mathrm{~Hz}, 12 \mathrm{H}), 1.23(\mathrm{~d}, J=6.9 \mathrm{~Hz}, 6 \mathrm{H})$.

${ }^{13} \mathrm{C} \underline{\mathrm{NMR}}\left(101 \mathrm{MHz}, \underline{\left.\mathrm{CDCl}_{3}\right)}\right.$

$\delta 153.4,151.1,140.3,135.5,134.0,132.8,131.8,131.7,128.5,127.9,127.7$, $127.3,126.9,126.4,123.9,123.0,119.1,106.9,75.0,34.1,30.2,28.9,26.0$, $24.9,23.5,22.0,21.2$.

$\underline{\mathrm{HRMS}} \underline{(\mathrm{ESI})}$

Calcd for $\mathrm{C}_{34} \mathrm{H}_{40} \mathrm{~N}_{2} \mathrm{O}_{2} \mathrm{~S}[\mathrm{M}+\mathrm{H}]^{+}:$541.2883, found: 541.2880. 
2,4,6-triisopropyl-N'-(1-(naphthalen-2-yl)undeca-2,4-diyn-1-

ylidene)benzenesulfonohydrazide (1q)<smiles>N=C(C#CC=[In])c1ccc2ccccc2c1</smiles>

$65 \%$ yield $(0.6 \mathrm{~g})$, white solid, m.p. $=91-93{ }^{\circ} \mathrm{C}$.

$\underline{{ }^{1} \mathrm{H}} \underline{\mathrm{NMR}} \underline{(400} \underline{\mathrm{MHz}}, \underline{\left.\mathrm{CDCl}_{3}\right)}$

$\delta 8.86(\mathrm{~s}, 1 \mathrm{H}), 8.17(\mathrm{~d}, J=1.7 \mathrm{~Hz}, 1 \mathrm{H}), 7.93(\mathrm{dd}, J=8.7,1.8 \mathrm{~Hz}, 1 \mathrm{H}), 7.89$ -

$7.69(\mathrm{~m}, 3 \mathrm{H}), 7.53-7.41(\mathrm{~m}, 2 \mathrm{H}), 7.17$ (s, 2H), 4.32 (hept, $J=6.7 \mathrm{~Hz}, 2 \mathrm{H})$,

2.88 (hept, $J=6.9 \mathrm{~Hz}, 1 \mathrm{H}), 2.48(\mathrm{t}, J=7.0 \mathrm{~Hz}, 2 \mathrm{H}), 1.66$ (p, $J=7.1 \mathrm{~Hz}, 2 \mathrm{H})$,

$1.52-1.48(\mathrm{~m}, 3 \mathrm{H}), 1.38-1.32(\mathrm{~m}, 15 \mathrm{H}), 1.23(\mathrm{~d}, J=6.9 \mathrm{~Hz}, 6 \mathrm{H}), 0.93(\mathrm{t}, J$

$=6.7 \mathrm{~Hz}, 3 \mathrm{H})$.

${ }^{13} \mathrm{C} \underline{\mathrm{NMR}} \underline{(101} \underline{\mathrm{MHz}}, \underline{\left.\mathrm{CDCl}_{3}\right)}$

$\delta 153.6,151.2,134.1,134.0,132.8,131.4$ (2C signal) 128.5, 128.0, 127.7, $127.4,127.1,126.5,123.9,122.7,92.3,89.2,63.6,62.3,34.2,31.2,30.1,28.6$, $27.8,24.8,23.5,22.5,19.8,14.0$.

$\underline{\text { HRMS }} \underline{\text { (ESI) }}$

Calcd for $\mathrm{C}_{36} \mathrm{H}_{44} \mathrm{~N}_{2} \mathrm{O}_{2} \mathrm{~S}[\mathrm{M}+\mathrm{H}]^{+}:$569.3196, found: 569.3193 .

N'-(6-chloro-1-(naphthalen-2-yl)hex-2-yn-1-ylidene)-2,4,6-

triisopropylbenzenesulfonohydrazide (1r)<smiles>N=C(C#CCl)c1ccc2ccccc2c1</smiles>

$55 \%$ yield $(0.65 \mathrm{~g})$, white solid, m.p. $=143-145{ }^{\circ} \mathrm{C}$.

$\underline{{ }^{1} \mathrm{H}} \underline{\mathrm{NMR}} \underline{(400} \underline{\mathrm{MHz}}, \underline{\left.\mathrm{CDCl}_{3}\right)}$

$\delta 8.77(\mathrm{~s}, 1 \mathrm{H}), 8.26-8.15(\mathrm{~m}, 1 \mathrm{H}), 7.95(\mathrm{dd}, J=8.7,1.8 \mathrm{~Hz}, 1 \mathrm{H}), 7.86-7.70$

(m, 3H), $7.53-7.40(\mathrm{~m}, 2 \mathrm{H}), 7.17(\mathrm{~s}, 2 \mathrm{H}), 4.34$ (hept, $J=6.7 \mathrm{~Hz}, 2 \mathrm{H}), 3.75$ (t, $J=6.1 \mathrm{~Hz}, 2 \mathrm{H}), 2.89-2.85(\mathrm{~m}, 3 \mathrm{H}), 2.18(\mathrm{p}, J=6.6 \mathrm{~Hz}, 2 \mathrm{H}), 1.34(\mathrm{~d}, J=6.8$

$\mathrm{Hz}, 12 \mathrm{H}), 1.23(\mathrm{~d}, J=6.9 \mathrm{~Hz}, 6 \mathrm{H})$.

${ }^{13} \mathrm{C} \underline{\mathrm{NMR}} \underline{(101} \underline{\mathrm{MHz}}, \underline{\left.\mathrm{CDCl}_{3}\right)}$

$\delta 153.5,151.2,135.0,134.0,132.8,131.7,131.6,128.5,127.9,127.7,127.3$, 127.0, 126.5, 123.9, 122.8, 105.0, 70.8, 43.3, 34.1, 30.6, 30.1, 24.9, 23.5, 17.2. $\underline{\mathrm{HRMS}} \underline{(\mathrm{ESI})}$

Calcd for $\mathrm{C}_{31} \mathrm{H}_{37} \mathrm{ClN}_{2} \mathrm{O}_{2} \mathrm{~S}[\mathrm{M}+\mathrm{H}]^{+}$: 537.2337, found: 537.2337. 
N'-(8-(benzyloxy)-1-(naphthalen-2-yl)oct-2-yn-1-ylidene)-2,4,6-

triisopropylbenzenesulfonohydrazide (1s)<smiles>N=C(C#CCOc1ccccc1)c1ccc2ccccc2c1</smiles>

$47 \%$ yield $(0.66 \mathrm{~g})$, white solid, m.p. $=116-118^{\circ} \mathrm{C}$.

$\underline{{ }^{1} \mathrm{H}} \underline{\mathrm{NMR}} \underline{(400} \underline{\mathrm{MHz}}, \underline{\left.\mathrm{CDCl}_{3}\right)}$

$\delta 8.74(\mathrm{~s}, 1 \mathrm{H}), 8.19(\mathrm{~s}, 1 \mathrm{H}), 7.96(\mathrm{dd}, J=8.8,1.7 \mathrm{~Hz}, 1 \mathrm{H}), 7.84-7.76(\mathrm{~m}, 2 \mathrm{H})$,

$7.74(\mathrm{~d}, J=8.8 \mathrm{~Hz}, 1 \mathrm{H}), 7.51-7.42(\mathrm{~m}, 2 \mathrm{H}), 7.38-7.29(\mathrm{~m}, 4 \mathrm{H}), 7.28-7.22$

(m, 1H), 7.17 (s, 2H), 4.52 (s, 2H), 4.33 (hept, $J=6.7 \mathrm{~Hz}, 2 \mathrm{H}), 3.53$ (t, $J=6.2$

$\mathrm{Hz}, 2 \mathrm{H}), 2.87$ (hept, $J=6.9 \mathrm{~Hz}, 1 \mathrm{H}), 2.65$ (t, $J=7.0 \mathrm{~Hz}, 2 \mathrm{H}), 1.82-1.68$ (m,

$4 \mathrm{H}), 1.67-1.60(\mathrm{~m}, 2 \mathrm{H}), 1.33(\mathrm{~d}, J=6.8 \mathrm{~Hz}, 12 \mathrm{H}), 1.22(\mathrm{~d}, J=6.9 \mathrm{~Hz}, 6 \mathrm{H})$.

${ }^{13} \mathrm{C} \underline{\mathrm{NMR}} \underline{(101} \underline{\mathrm{MHz}}, \underline{\left.\mathrm{CDCl}_{3}\right)}$

$\delta 153.4,151.1,138.4,135.5,134.0,132.8,131.8,131.6,128.5,128.3,127.8$, 127.6 (2C signal), 127.5, 127.3, 126.9, 126.4, 123.8, 122.9, 107.3, 73.0, 70.1, 70.0, 34.1, 30.1, 29.2, 28.1, 25.8, 24.9, 23.5, 19.7.

$\underline{\operatorname{HRMS}} \underline{(\mathrm{ESI})}$

Calcd for $\mathrm{C}_{40} \mathrm{H}_{48} \mathrm{~N}_{2} \mathrm{O}_{3} \mathrm{~S}[\mathrm{M}+\mathrm{H}]^{+}: 637.3458$, found: 637.3467 .

N'-(6-(1H-indol-1-yl)-1-(naphthalen-2-yl)hex-2-yn-1-ylidene)-2,4,6triisopropylbenzenesulfonohydrazide (1t)<smiles>N=C(C#CNn1ccc2ccccc21)c1ccc2ccccc2c1</smiles>

$45 \%$ yield $(0.65 \mathrm{~g})$, pale solid, m.p. $=144-146{ }^{\circ} \mathrm{C}$.

$\left.\underline{{ }^{1} \mathrm{H}} \underline{\mathrm{NMR}} \underline{(400} \underline{\mathrm{MHz}}, \underline{\mathrm{CDCl}_{3}}\right)$

$\delta 8.77(\mathrm{~s}, 1 \mathrm{H}), 8.18(\mathrm{~s}, 1 \mathrm{H}), 7.99-7.93(\mathrm{~m}, 1 \mathrm{H}), 7.85-7.74(\mathrm{~m}, 3 \mathrm{H}), 7.65(\mathrm{~d}$, $J=7.8 \mathrm{~Hz}, 1 \mathrm{H}), 7.52-7.44$ (m, 2H), 7.38 (d, $J=8.2 \mathrm{~Hz}, 1 \mathrm{H}), 7.24-7.17$ (m, $3 \mathrm{H}), 7.16-7.09(\mathrm{~m}, 2 \mathrm{H}), 6.54(\mathrm{~d}, J=3.2 \mathrm{~Hz}, 1 \mathrm{H}), 4.42-4.28(\mathrm{~m}, 4 \mathrm{H}), 2.88$ (hept, $J=6.9 \mathrm{~Hz}, 1 \mathrm{H}), 2.58(\mathrm{t}, J=7.0 \mathrm{~Hz}, 2 \mathrm{H}), 2.26$ (p, $J=6.8 \mathrm{~Hz}, 2 \mathrm{H}), 1.33$ $(\mathrm{d}, J=6.7 \mathrm{~Hz}, 12 \mathrm{H}), 1.23(\mathrm{~d}, J=6.9 \mathrm{~Hz}, 6 \mathrm{H})$.

\section{$\underline{{ }^{13} \mathrm{C}} \underline{\mathrm{NMR}} \underline{(101} \underline{\mathrm{MHz}}, \underline{\left.\mathrm{CDCl}_{3}\right)}$}

$\delta 153.5,151.2,135.9,135.0,134.0,132.8,131.7,131.5,128.7,128.5,128.0$, 127.7 (2C signal), 127.2, 127.0, 126.5, 123.9, 122.8, 121.8, 121.2, 119.6, 109.1, $\underline{\mathrm{HRMS}} \underline{(\mathrm{ESI})}$ $105.5,101.8,70.8,44.9,34.1,30.1,28.8,24.9,23.5,17.2$.

Calcd for $\mathrm{C}_{39} \mathrm{H}_{43} \mathrm{~N}_{3} \mathrm{O}_{2} \mathrm{~S}[\mathrm{M}+\mathrm{H}]^{+}:$618.3149, found: 618.3160 . 
2,4,6-triisopropyl-N'-(1-(4-nitrophenyl)hept-2-yn-1ylidene)benzenesulfonohydrazide (1u)<smiles>CC(C)(C#CC(=N)c1ccc([N+](=O)[O-])cc1)C(=N)O</smiles>

$62 \%$ yield $(0.75 \mathrm{~g})$, yellow solid, m.p. $=149-151^{\circ} \mathrm{C}$.

$\underline{{ }^{1} \mathrm{H}} \underline{\mathrm{NMR}} \underline{(400} \underline{\mathrm{MHz}}, \underline{\left.\mathrm{CDCl}_{3}\right)}$

$\delta 8.91(\mathrm{~s}, 1 \mathrm{H}), 8.18(\mathrm{~d}, J=8.8 \mathrm{~Hz}, 2 \mathrm{H}), 7.94(\mathrm{~d}, J=8.8 \mathrm{~Hz}, 2 \mathrm{H}), 7.19(\mathrm{~s}, 2 \mathrm{H})$, 4.28 (hept, $J=6.8 \mathrm{~Hz}, 2 \mathrm{H}$ ), 2.90 (hept, $J=6.7 \mathrm{~Hz}, 1 \mathrm{H}$ ), 2.63 (t, $J=7.1 \mathrm{~Hz}$, 2H), 1.69 (p, $J=7.2 \mathrm{~Hz}, 2 \mathrm{H}), 1.58-1.44(\mathrm{~m}, 2 \mathrm{H}), 1.31(\mathrm{~d}, J=6.8 \mathrm{~Hz}, 12 \mathrm{H})$, $1.24(\mathrm{~d}, J=6.9 \mathrm{~Hz}, 6 \mathrm{H}), 0.99(\mathrm{t}, J=7.3 \mathrm{~Hz}, 3 \mathrm{H})$.

${ }^{13} \mathrm{C} \underline{\mathrm{NMR}}\left(101 \mathrm{MHz}, \underline{\left.\mathrm{CDCl}_{3}\right)}\right.$

$\delta 153.8,151.2,148.2,140.1,132.6,131.3,127.1,124.0,123.5,109.1,69.3$, $34.1,30.1,30.1,24.8,23.4,22.1,19.4,13.4$.

$\underline{\text { HRMS }} \underline{(E S I)}$

Calcd for $\mathrm{C}_{28} \mathrm{H}_{37} \mathrm{~N}_{3} \mathrm{O}_{4} \mathrm{~S}[\mathrm{M}+\mathrm{H}]^{+}$: 512.2578, found: 512.2573 .

N'-(1-(3,4-dichlorophenyl)hept-2-yn-1-ylidene)-2,4,6triisopropylbenzenesulfonohydrazide (1v)<smiles>CC(C)(Br)C#CC(=N)c1ccc(Cl)c(Cl)c1</smiles>

$67 \%$ yield $(0.75 \mathrm{~g})$, white solid, m.p. $=146-148^{\circ} \mathrm{C}$.

$\underline{{ }^{1} \mathrm{H}} \underline{\mathrm{NMR}} \underline{(400} \underline{\mathrm{MHz}}, \underline{\left.\mathrm{CDCl}_{3}\right)}$

$\delta 8.69(\mathrm{~s}, 1 \mathrm{H}), 7.87(\mathrm{~d}, J=2.1 \mathrm{~Hz}, 1 \mathrm{H}), 7.59(\mathrm{dd}, J=8.4,2.1 \mathrm{~Hz}, 1 \mathrm{H}), 7.38(\mathrm{~d}$, $J=8.4 \mathrm{~Hz}, 1 \mathrm{H}$ ), 7.17 (s, 2H), 4.26 (hept, $J=6.8 \mathrm{~Hz}, 2 \mathrm{H}$ ), 2.89 (hept, $J=6.9$ $\mathrm{Hz}, 1 \mathrm{H}), 2.60$ (t, $J=7.1 \mathrm{~Hz}, 2 \mathrm{H}), 1.74-1.61(\mathrm{~m}, 2 \mathrm{H}), 1.58-1.44(\mathrm{~m}, 2 \mathrm{H})$, $1.31(\mathrm{~d}, J=6.8 \mathrm{~Hz}, 12 \mathrm{H}), 1.24(\mathrm{~d}, J=6.9 \mathrm{~Hz}, 6 \mathrm{H}), 0.99(\mathrm{t}, J=7.3 \mathrm{~Hz}, 3 \mathrm{H})$.

${ }^{13} \mathrm{C} \underline{\mathrm{NMR}} \underline{(101} \underline{\mathrm{MHz}}, \underline{\left.\mathrm{CDCl}_{3}\right)}$

$\delta 153.6,151.2,134.3,133.7,132.9,132.7,131.4,130.2,128.0,125.6,123.9$, 108.4, 69.3, 34.2, 30.2, 30.1, 24.8, 23.5, 22.1, 19.4, 13.5.

$\underline{\mathrm{HRMS}} \underline{(\mathrm{ESI})}$

Calcd for $\mathrm{C}_{28} \mathrm{H}_{36} \mathrm{Cl}_{2} \mathrm{~N}_{2} \mathrm{O}_{2} \mathrm{~S}[\mathrm{M}+\mathrm{H}]^{+}:$535.1947, found: 535.1942 . 
N'-(1-(3,4-dimethylphenyl)hept-2-yn-1-ylidene)-2,4,6-

triisopropylbenzenesulfonohydrazide (1w)

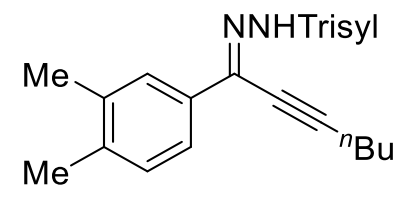

$70 \%$ yield $(1.5 \mathrm{~g})$, white solid, m.p. $=100-101^{\circ} \mathrm{C}$.

${ }^{1} \mathrm{H} \underline{\mathrm{NMR}} \underline{(400} \underline{\mathrm{MHz}}, \underline{\left.\mathrm{CDCl}_{3}\right)}$

$\delta 8.65(\mathrm{~s}, 1 \mathrm{H}), 7.60(\mathrm{~s}, 1 \mathrm{H}), 7.51(\mathrm{dd}, J=7.9,1.9 \mathrm{~Hz}, 1 \mathrm{H}), 7.15(\mathrm{~s}, 2 \mathrm{H}), 7.07$

$(\mathrm{d}, J=7.9 \mathrm{~Hz}, 1 \mathrm{H}), 4.31$ (hept, $J=6.8 \mathrm{~Hz}, 2 \mathrm{H}), 2.87$ (hept, $J=6.9 \mathrm{~Hz}, 1 \mathrm{H}$ ),

$2.58(\mathrm{t}, J=7.1 \mathrm{~Hz}, 2 \mathrm{H}), 2.22(\mathrm{~s}, 6 \mathrm{H}), 1.72-1.62(\mathrm{~m}, 2 \mathrm{H}), 1.57-1.45(\mathrm{~m}, 2 \mathrm{H})$,

$1.31(\mathrm{~d}, J=6.7 \mathrm{~Hz}, 12 \mathrm{H}), 1.23(\mathrm{~d}, J=6.9 \mathrm{~Hz}, 6 \mathrm{H}), 0.97(\mathrm{t}, J=7.3 \mathrm{~Hz}, 3 \mathrm{H})$.

${ }^{13} \mathrm{C} \underline{\mathrm{NMR}} \underline{(101} \underline{\mathrm{MHz}}, \underline{\left.\mathrm{CDCl}_{3}\right)}$

$\delta 153.2,151.1,138.7,136.4,135.7,131.9,131.7,129.4,127.2,124.3,123.7$,

107.0, 70.1, 34.1, 30.2, 30.0, 24.8 (2C signal), 23.5, 22.0, 19.6, 19.3, 13.4.

\section{$\underline{\text { HRMS }} \underline{\underline{(E S I)}}$}

Calcd for $\mathrm{C}_{30} \mathrm{H}_{42} \mathrm{~N}_{2} \mathrm{O}_{2} \mathrm{~S}[\mathrm{M}+\mathrm{H}]^{+}$: 495.3040, found: 495.3045 .

(Z)-N'-(1-(benzofuran-5-yl)hept-2-yn-1-ylidene)-2,4,6-

triisopropylbenzenesulfonohydrazide (1x)<smiles>CC(C)(C#CC(=N)c1ccc2occc2c1)C(=N)O</smiles>

$68 \%$ yield $(1.35 \mathrm{~g})$, white solid, m.p. $=135-136^{\circ} \mathrm{C}$.

$\underline{{ }^{1} \mathrm{H}} \underline{\mathrm{NMR}} \underline{(400} \underline{\mathrm{MHz}}, \underline{\left.\mathrm{CDCl}_{3}\right)}$

$\delta 8.64(\mathrm{~s}, 1 \mathrm{H}), 8.03(\mathrm{~s}, 1 \mathrm{H}), 7.81(\mathrm{dd}, J=8.8,2.0 \mathrm{~Hz}, 1 \mathrm{H}), 7.61(\mathrm{~d}, J=2.0 \mathrm{~Hz}$, 1H), 7.42 (d, $J=8.8 \mathrm{~Hz}, 1 \mathrm{H}), 7.16$ (s, 2H), 6.74 (s, 1H), 4.31 (hept, $J=6.8 \mathrm{~Hz}$, 2H), 2.88 (hept, $J=6.9 \mathrm{~Hz}, 1 \mathrm{H}), 2.61$ (t, $J=7.1 \mathrm{~Hz}, 2 \mathrm{H}), 1.75-1.64(\mathrm{~m}, 2 \mathrm{H})$, $1.59-1.49$ (m, 2H), $1.32(\mathrm{~d}, J=6.7 \mathrm{~Hz}, 12 \mathrm{H}), 1.23(\mathrm{~d}, J=6.9 \mathrm{~Hz}, 6 \mathrm{H}), 1.00$ (t, $J=7.3 \mathrm{~Hz}, 3 \mathrm{H})$.

${ }^{13} \mathrm{C} \underline{\mathrm{NMR}} \underline{(101} \underline{\mathrm{MHz}}, \underline{\left.\mathrm{CDCl}_{3}\right)}$

$\delta 155.8,153.3,151.1,145.8,135.7,131.7,129.5,127.3,123.8,122.9,120.0$, $111.1,107.3,106.8,70.2,34.1,30.3,30.1,24.8,23.5,22.1,19.3,13.5$.

$\underline{\operatorname{HRMS}} \underline{(\mathrm{ESI})}$

Calcd for $\mathrm{C}_{30} \mathrm{H}_{38} \mathrm{~N}_{2} \mathrm{O}_{3} \mathrm{~S}[\mathrm{M}+\mathrm{H}]^{+}$: 507.2676, found: 507.2678. 
(Z)-2,4,6-triisopropyl-N'-(4-phenylbut-3-yn-2-ylidene)benzenesulfonohydrazide (1y)

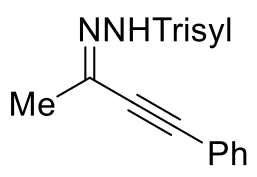

$60 \%$ yield $(1.0 \mathrm{~g})$, white solid, m.p. $=125-127^{\circ} \mathrm{C}$.

$\underline{{ }^{1} \mathrm{H}} \underline{\mathrm{NMR}} \underline{(400} \underline{\mathrm{MHz}}, \underline{\left.\mathrm{CDCl}_{3}\right)}$

$\delta 8.39$ (s, 1H), $7.53-7.48(\mathrm{~m}, 2 \mathrm{H}), 7.47-7.37(\mathrm{~m}, 3 \mathrm{H}), 7.18(\mathrm{~s}, 2 \mathrm{H}), 4.23$ (hept, $J=6.7 \mathrm{~Hz}, 2 \mathrm{H}), 2.91$ (hept, $J=6.9 \mathrm{~Hz}, 1 \mathrm{H}$ ), 2.13 (s, 3H), 1.27 (t, $J=$ $6.8 \mathrm{~Hz}, 18 \mathrm{H})$.

$\underline{{ }^{13} \mathrm{C}} \underline{\mathrm{NMR}} \underline{(101} \underline{\mathrm{MHz}}, \underline{\left.\mathrm{CDCl}_{3}\right)}$

$\delta 153.3,151.2,134.0,132.0,131.5,130.2,128.7,123.8,120.3,102.1,79.0$, $34.1,30.0,24.8,23.5,22.8$.

$\underline{\text { HRMS }} \underline{(\mathrm{ESI})}$

Calcd for $\mathrm{C}_{25} \mathrm{H}_{32} \mathrm{~N}_{2} \mathrm{O}_{2} \mathrm{~S}[\mathrm{M}+\mathrm{H}]^{+}$: 425.2257, found: 425.2250 . 


\section{Typical Procedure for Si-H Bond Insertion Reaction and Analytical Data of Insertion Products}

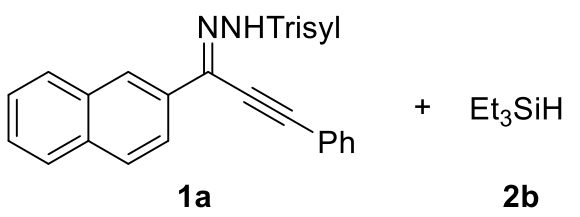
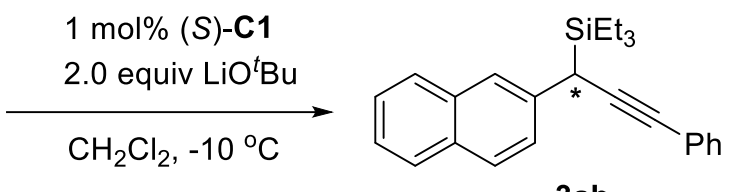

Alkynyl sulfonylhydrazone 1a (107.2 mg, $0.2 \mathrm{mmol}), \mathrm{LiO}^{t} \mathrm{Bu}(32.0 \mathrm{mg}, 0.4 \mathrm{mmol})$ and $\mathbf{C 1}$ (3.0 mg, $0.002 \mathrm{mmol}$ ) were charged into an oven-dried Schlenk tube under argon atmosphere. After the mixture was cooled to $-10^{\circ} \mathrm{C}$, triethyl silane $\mathbf{2 b}(46.5 \mathrm{mg}$, $0.4 \mathrm{mmol}$ ) and $3 \mathrm{~mL}$ dry DCM were injected into the Schlenk tube by syringe. At this temperature, the reaction was kept stirring for $20 \mathrm{~h}$, TLC indicated that the sulfonylhydrazone was consumed completely. The reaction mixture was concentrated and purified by a flash chromatography on silica gel (eluting with petroleum ether/DCM $=20: 1, \mathrm{v} / \mathrm{v})$ to give product $\mathbf{3 a b}$ as a colorless oil $(65.5 \mathrm{mg}, 92 \%$ yield $)$. Note: the compound is unstable when it is exposed to the air. Therefore, the workup of reaction should be operated quickly.

(R)-dimethyl(1-(naphthalen-2-yl)-3-phenylprop-2-yn-1-yl)(phenyl)silane (3aa)<smiles>CC[SeH]C(C#Cc1ccccc1)c1ccc2ccccc2c1</smiles>

Colorless oil, $85 \%$ yield $(64.0 \mathrm{mg}), 90 \%$ ee. HPLC condition: Chiralcel AS-3 column, $n$-hexane $/ i-\mathrm{PrOH}=99: 1$, flow rate $=1.0 \mathrm{~mL} / \mathrm{min}, 230 \mathrm{~nm} \mathrm{UV}$ detector, $t_{\mathrm{R}}=4.13 \mathrm{~min}$ (major) and $t_{\mathrm{R}}=4.53 \mathrm{~min}$ (minor). $[\alpha]_{\mathrm{D}}^{25}=-24.3\left(c 1.0, \mathrm{CHCl}_{3}\right)$.

$\underline{{ }^{1} \mathrm{H}} \underline{\mathrm{NMR}} \underline{(400} \underline{\mathrm{MHz}}, \underline{\left.\mathrm{CDCl}_{3}\right)}$

$\delta 7.64(\mathrm{~d}, J=7.7 \mathrm{~Hz}, 1 \mathrm{H}), 7.55(\mathrm{t}, J=9.1 \mathrm{~Hz}, 2 \mathrm{H}), 7.40(\mathrm{~s}, 1 \mathrm{H}), 7.36-7.23$

(m, 7H), $7.21-7.13(\mathrm{~m}, 5 \mathrm{H}), 7.05(\mathrm{dt}, J=8.6,1.7 \mathrm{~Hz}, 1 \mathrm{H}), 3.57(\mathrm{~s}, 1 \mathrm{H}), 0.34$ $(\mathrm{s}, 3 \mathrm{H}), 0.24(\mathrm{~s}, 3 \mathrm{H})$.

$\underline{{ }^{13} \mathrm{C}} \underline{\mathrm{NMR}} \underline{(101} \underline{\mathrm{MHz}}, \underline{\left.\mathrm{CDCl}_{3}\right)}$

$\delta 136.3,135.8,134.4,133.5,131.7,131.5,129.6,128.2,127.6$ (2C signal), 127.5 (2C signal), 127.4, 126.5, 125.8, 125.2, 124.9, 124.3, 90.0, 83.9, 30.2,$4.8,-4.9$.

$\underline{\operatorname{HRMS}} \underline{(\mathrm{EI})}$

Calcd for $\mathrm{C}_{27} \mathrm{H}_{24} \mathrm{Si}[\mathrm{M}]^{+}: 376.1647$, found: 376.1641 . 
(-)-triethyl(1-(naphthalen-2-yl)-3-phenylprop-2-yn-1-yl)silane (3ab)<smiles>CCCC(C#Cc1ccccc1)c1ccc2ccccc2c1</smiles>

Colorless oil, 92\% yield (65.5 mg), 91\% ee. HPLC condition: Chiralcel AD-3 column, $n$-hexane $/ i-\mathrm{PrOH}=99.3: 0.7$, flow rate $=0.8 \mathrm{~mL} / \mathrm{min}, 230 \mathrm{~nm} \mathrm{UV}$ detector, $t \mathrm{R}=4.64$ $\min \left(\right.$ minor) and $t_{\mathrm{R}}=5.08 \min$ (major). $[\alpha]_{\mathrm{D}}^{25}=-13.6\left(\mathrm{c} 1.0, \mathrm{CHCl}_{3}\right)$.

$\underline{{ }^{1} \mathrm{H}} \underline{\mathrm{NMR}} \underline{(400} \underline{\mathrm{MHz}}, \underline{\left.\mathrm{CDCl}_{3}\right)}$

$\delta 7.82-7.69(\mathrm{~m}, 4 \mathrm{H}), 7.63(\mathrm{dd}, J=7.4,1.5 \mathrm{~Hz}, 1 \mathrm{H}), 7.51-7.50(\mathrm{~m}, 2 \mathrm{H}), 7.48$ $-7.35(\mathrm{~m}, 4 \mathrm{H}), 7.34-7.32(\mathrm{~m}, 1 \mathrm{H}), 3.47(\mathrm{~s}, 1 \mathrm{H}), 1.07$ (t, $J=8.0 \mathrm{~Hz}, 9 \mathrm{H}), 0.69$

$-0.67(\mathrm{~m}, 6 \mathrm{H})$.

${ }^{13} \mathrm{C} \underline{\mathrm{NMR}} \underline{(101} \underline{\mathrm{MHz}}, \underline{\left.\mathrm{CDCl}_{3}\right)}$

$\delta 137.3,133.6,131.6,131.5,128.2,127.7,127.6,127.4$ (2C signal), 126.3, $125.9,124.9,124.8,124.5,90.4,83.1,27.0,7.4,2.4$.

$\underline{\operatorname{HRMS}} \underline{(\mathrm{EI})}$

Calcd for $\mathrm{C}_{25} \mathrm{H}_{28} \mathrm{Si}[\mathrm{M}]^{+}: 356.1960$, found: 356.1957 .

(-)-methyl(1-(naphthalen-2-yl)-3-phenylprop-2-yn-1-yl)diphenylsilane (3ac)<smiles></smiles>

Colorless oil, 84\% yield (73.6 mg), 93\% ee. HPLC condition: Chiralcel AD-3 column, $n$-hexane $/ i$-PrOH $=95: 5$, flow rate $=1.0 \mathrm{~mL} / \mathrm{min}, 254 \mathrm{~nm} \mathrm{UV}$ detector, $t_{\mathrm{R}}=4.04 \mathrm{~min}$ (minor) and $t_{\mathrm{R}}=5.80 \mathrm{~min}$ (major). $[\alpha]_{\mathrm{D}}^{26}=-12.4\left(\mathrm{c} 0.5, \mathrm{CHCl}_{3}\right)$.

$\underline{{ }^{1} \mathrm{H}} \underline{\mathrm{NMR}} \underline{(400} \underline{\mathrm{MHz}}, \underline{\left.\mathrm{CDCl}_{3}\right)}$

$\delta 7.69(\mathrm{~d}, J=7.4 \mathrm{~Hz}, 1 \mathrm{H}), 7.64-7.54(\mathrm{~m}, 4 \mathrm{H}), 7.52-7.44(\mathrm{~m}, 3 \mathrm{H}), 7.39-$ $7.28(\mathrm{~m}, 8 \mathrm{H}), 7.26-7.16(\mathrm{~m}, 5 \mathrm{H}), 7.12(\mathrm{~d}, J=8.3 \mathrm{~Hz}, 1 \mathrm{H}), 4.07$ (s, 1H), 0.59 $(\mathrm{s}, 3 \mathrm{H})$.

\section{$\underline{{ }^{13} \mathrm{C}} \underline{\mathrm{NMR}} \underline{(101} \underline{\mathrm{MHz}}, \underline{\left.\mathrm{CDCl}_{3}\right)}$}

$\delta 135.7,135.4,135.1,134.3,134.0,133.3,131.7,131.5,129.7$ (2C signal), 128.1, 127.7, 127.6, 127.5 (2C signal), 127.4 (2C signal), 126.7, 125.9, 125.8, $125.0,124.2,90.0,84.6,28.9,-5.7$.

$\underline{\operatorname{HRMS}} \underline{(\mathrm{EI})}$

Calcd for $\mathrm{C}_{32} \mathrm{H}_{26} \mathrm{Si}[\mathrm{M}]^{+}$: 438.1804, found: 438.1797 . 


\section{(-)-(1,3-diphenylprop-2-yn-1-yl)triethylsilane (3bb)}<smiles>CCC(C#CC#Cc1ccccc1)c1ccccc1</smiles>

Colorless oil, $87 \%$ yield (53.3 mg), 87\% ee. HPLC condition: Chiralcel OJ-3 column, $n$-hexane $/ i-\mathrm{PrOH}=99: 1$, flow rate $=1.0 \mathrm{~mL} / \mathrm{min}, 230 \mathrm{~nm} \mathrm{UV}$ detector, $t \mathrm{R}=3.83 \mathrm{~min}$ (major) and $t_{\mathrm{R}}=6.06 \mathrm{~min}$ (minor). $[\alpha]_{\mathrm{D}}^{27}=-18.0\left(\mathrm{c} 1.0, \mathrm{CHCl}_{3}\right.$ ).

${ }^{1} \mathrm{H} \underline{\mathrm{NMR}} \underline{(400} \underline{\mathrm{MHz}}, \underline{\left.\mathrm{CDCl}_{3}\right)}$

$\delta 7.43(\mathrm{dd}, J=7.9,1.8 \mathrm{~Hz}, 2 \mathrm{H}), 7.33-7.24(\mathrm{~m}, 7 \mathrm{H}), 7.17-7.10(\mathrm{~m}, 1 \mathrm{H}), 3.47$ $(\mathrm{s}, 1 \mathrm{H}), 0.97(\mathrm{t}, J=8.0 \mathrm{~Hz}, 9 \mathrm{H}), 0.75-0.60(\mathrm{~m}, 6 \mathrm{H})$.

$\underline{{ }^{13} \mathrm{C}} \underline{\mathrm{NMR}} \underline{(101} \underline{\mathrm{MHz}}, \underline{\left.\mathrm{CDCl}_{3}\right)}$

$\delta 139.5,131.4,128.2,128.2,127.3,127.1,125.1,124.5,90.5,82.9,26.7,7.4$, 2.3 .

$\underline{\operatorname{HRMS}} \underline{(\mathrm{EI})}$

Calcd for $\mathrm{C}_{21} \mathrm{H}_{26} \mathrm{Si}[\mathrm{M}]^{+}$: 306.1804, found: 306.1799 .

(-)-(1-(2-chlorophenyl)-3-phenylprop-2-yn-1-yl)triethylsilane (3cb)<smiles>CCC(C#Cc1ccccc1)c1ccccc1Cl</smiles>

Colorless oil, $81 \%$ yield (55.0 mg), 82\% ee. HPLC condition: Phenomenex, Cellulose3 column, $\mathrm{MeOH}$, flow rate $=1.0 \mathrm{~mL} / \mathrm{min}, 230 \mathrm{~nm} \mathrm{UV}$ detector, $t \mathrm{R}=4.26 \mathrm{~min}$ (major) and $t_{\mathrm{R}}=5.36 \mathrm{~min}$ (minor). $[\alpha]_{\mathrm{D}}^{26}=-40.2\left(\mathrm{c} 1.0, \mathrm{CHCl}_{3}\right)$.

$\underline{{ }^{1} \mathrm{H}} \underline{\mathrm{NMR}} \underline{(400} \underline{\mathrm{MHz}}, \underline{\left.\mathrm{CDCl}_{3}\right)}$

$\delta 7.59(\mathrm{~d}, J=8.0 \mathrm{~Hz}, 1 \mathrm{H}), 7.45-7.40(\mathrm{~m}, 2 \mathrm{H}), 7.33-7.22(\mathrm{~m}, 5 \mathrm{H}), 7.08(\mathrm{t}, J$ $=7.6 \mathrm{~Hz}, 1 \mathrm{H}), 4.10(\mathrm{~s}, 1 \mathrm{H}), 0.96(\mathrm{t}, J=7.7 \mathrm{~Hz}, 9 \mathrm{H}), 0.73(\mathrm{q}, J=7.8 \mathrm{~Hz}, 6 \mathrm{H})$.

$\underline{{ }^{13} \mathrm{C}} \underline{\mathrm{NMR}} \underline{(101} \underline{\mathrm{MHz}}, \underline{\left.\mathrm{CDCl}_{3}\right)}$

$\delta 138.2,131.7,131.5,129.3$ (2C signal), 128.2, 127.4, 126.9, 126.3, 124.3, $90.5,82.2,23.6,7.3,2.5$.

$\underline{\operatorname{HRMS}} \underline{(\mathrm{EI})}$

Calcd for $\mathrm{C}_{21} \mathrm{H}_{25} \mathrm{ClSi}[\mathrm{M}]^{+}: 340.1414$, found: 340.1417 . 
(-)-(1-(3-chlorophenyl)-3-phenylprop-2-yn-1-yl)triethylsilane (3db)<smiles>CCC(C#CC#Cc1ccccc1)c1cccc(Cl)c1</smiles>

Colorless oil, 85\% yield (57.8 mg), 95\% ee. HPLC condition: Chiralpak AD-3 column, $n$-hexane $/ i$-PrOH $=99: 1$, flow rate $=1.0 \mathrm{~mL} / \mathrm{min}, 230 \mathrm{~nm} \mathrm{UV}$ detector, $t_{\mathrm{R}}=3.29 \mathrm{~min}$ (minor) and $t_{\mathrm{R}}=3.61$ min (major). $[\alpha]_{\mathrm{D}}^{27}=-10.0\left(\mathrm{c} 1.0, \mathrm{CHCl}_{3}\right)$.

$\underline{{ }^{1} \mathrm{H}} \underline{\mathrm{NMR}} \underline{(400} \underline{\mathrm{MHz}}, \underline{\left.\mathrm{CDCl}_{3}\right)}$

$\delta 7.47-7.41(\mathrm{~m}, 2 \mathrm{H}), 7.34-7.26(\mathrm{~m}, 4 \mathrm{H}), 7.23-7.10(\mathrm{~m}, 3 \mathrm{H}), 3.45(\mathrm{~s}, 1 \mathrm{H})$, $0.97(\mathrm{t}, J=7.9 \mathrm{~Hz}, 9 \mathrm{H}), 0.74-0.60(\mathrm{~m}, 6 \mathrm{H})$.

${ }^{13} \mathrm{C} \underline{\mathrm{NMR}} \underline{(101} \underline{\mathrm{MHz}}, \underline{\left.\mathrm{CDCl}_{3}\right)}$

$\delta 141.9,134.2,131.5,129.4,128.2,127.5,127.0,125.3,125.2,124.2,89.5$, $83.5,26.7,7.3,2.3$.

$\underline{\operatorname{HRMS}} \underline{(\mathrm{EI})}$

Calcd for $\mathrm{C}_{21} \mathrm{H}_{25} \mathrm{ClSi}[\mathrm{M}]^{+}: 340.1414$, found: 340.1418 .

(-)-triethyl(1-(4-methoxyphenyl)-3-phenylprop-2-yn-1-yl)silane (3eb)<smiles></smiles>

Colorless oil, 64\% yield (43.0 mg), 87\% ee. HPLC condition: Chiralcel OJ-3 column, $n$-hexane $/ i-\mathrm{PrOH}=99: 1$, flow rate $=1.0 \mathrm{~mL} / \mathrm{min}, 230 \mathrm{~nm} \mathrm{UV}$ detector, $t_{\mathrm{R}}=4.68 \mathrm{~min}$ (major) and $t_{\mathrm{R}}=5.73 \mathrm{~min}$ (minor). $[\alpha]_{\mathrm{D}}^{27}=-12.2\left(\mathrm{c} 1.0, \mathrm{CHCl}_{3}\right)$.

$\underline{{ }^{1} \mathrm{H}} \underline{\mathrm{NMR}} \underline{(400} \underline{\mathrm{MHz}}, \underline{\left.\mathrm{CDCl}_{3}\right)}$

$\delta 7.46-7.39$ (m, 2H), 7.33 - 7.24 (m, 3H), 7.23 - 7.15 (m, 2H), $6.89-6.79$ (m, 2H), $3.78(\mathrm{~s}, 3 \mathrm{H}), 3.41(\mathrm{~s}, 1 \mathrm{H}), 0.97$ (t, $J=7.9 \mathrm{~Hz}, 9 \mathrm{H}), 0.70-0.60$ (m, $6 \mathrm{H})$.

${ }^{13} \mathrm{C} \underline{\mathrm{NMR}} \underline{(101} \underline{\mathrm{MHz}}, \underline{\left.\mathrm{CDCl}_{3}\right)}$

$\delta 157.3,131.4,131.4,128.2,127.9,127.2,124.6,113.7,90.9,82.7,55.2,25.5$, 7.4, 2.3.

$\underline{\operatorname{HRMS}} \underline{(\mathrm{EI})}$

Calcd for $\mathrm{C}_{22} \mathrm{H}_{28} \mathrm{OSi}[\mathrm{M}]^{+}: 336.1909$, found: 336.1903 . 
<smiles>CC[SiH2]C(C#CC#Cc1ccccc1)c1ccc(Cl)cc1</smiles>

Colorless oil, 80\% yield (50.0 mg), 93\% ee. HPLC condition: Chiralpak IA-3 column, $n$-hexane $/ i$-PrOH $=99.5: 0.5$, flow rate $=0.5 \mathrm{~mL} / \mathrm{min}, 230 \mathrm{~nm} \mathrm{UV}$ detector, $t_{\mathrm{R}}=7.46$ $\min \left(\right.$ minor) and $t_{\mathrm{R}}=7.74 \min$ (major). $[\alpha]_{\mathrm{D}}^{28}=-15.0\left(\mathrm{c} 1.0, \mathrm{CHCl}_{3}\right)$.

$\underline{{ }^{1} \mathrm{H}} \underline{\mathrm{NMR}} \underline{(400} \underline{\mathrm{MHz}}, \underline{\left.\mathrm{CDCl}_{3}\right)}$

$\delta 7.45-7.39(\mathrm{~m}, 2 \mathrm{H}), 7.32-7.20(\mathrm{~m}, 7 \mathrm{H}), 3.45(\mathrm{~s}, 1 \mathrm{H}), 0.97(\mathrm{t}, J=7.9 \mathrm{~Hz}$, $9 \mathrm{H}), 0.75-0.60(\mathrm{~m}, 6 \mathrm{H})$.

${ }^{13} \mathrm{C} \underline{\mathrm{NMR}} \underline{(101} \underline{\mathrm{MHz}}, \underline{\left.\mathrm{CDCl}_{3}\right)}$

$\delta 138.2,131.4,130.7,128.3$ (2C signal), 128.2, 127.5, 124.3, 89.8, 83.3, 26.3, $7.4,2.3$.

$\underline{\operatorname{HRMS}} \underline{(\mathrm{EI})}$

Calcd for $\mathrm{C}_{21} \mathrm{H}_{25} \mathrm{ClSi}\left[\mathrm{M}-\mathrm{C}_{2} \mathrm{H}_{5}\right]^{+}: 311.1023$, found: 311.1015 .

(-)-4-(3-phenyl-1-(triethylsilyl)prop-2-yn-1-yl)benzonitrile (3gb)<smiles>CC[SiH2]C(C#CC#Cc1ccccc1)c1ccc(C#N)cc1</smiles>

Colorless oil, 89\% yield (58.9 mg), 96\% ee. HPLC condition: Chiralpak AD-3 column, $n$-hexane $/ i$-PrOH $=95: 5$, flow rate $=1.0 \mathrm{~mL} / \mathrm{min}, 230 \mathrm{~nm} \mathrm{UV}$ detector, $t_{\mathrm{R}}=4.54 \mathrm{~min}$ (minor) and $t_{\mathrm{R}}=5.86 \mathrm{~min}$ (major). $[\alpha]_{\mathrm{D}}^{25}=-23.6\left(\mathrm{c} 1.0, \mathrm{CHCl}_{3}\right)$.

$\underline{{ }^{1} \mathrm{H}} \underline{\mathrm{NMR}} \underline{(400} \underline{\mathrm{MHz}}, \underline{\left.\mathrm{CDCl}_{3}\right)}$

$\delta 7.57(\mathrm{~d}, J=8.0 \mathrm{~Hz}, 2 \mathrm{H}), 7.47-7.35(\mathrm{~m}, 4 \mathrm{H}), 7.34-7.24(\mathrm{~m}, 3 \mathrm{H}), 3.56(\mathrm{~s}$, $1 \mathrm{H}), 0.97(\mathrm{t}, J=7.9 \mathrm{~Hz}, 9 \mathrm{H}), 0.72-0.60(\mathrm{~m}, 6 \mathrm{H})$.

${ }^{13} \mathrm{C} \underline{\mathrm{NMR}} \underline{(101} \underline{\mathrm{MHz}}, \underline{\left.\mathrm{CDCl}_{3}\right)}$

$\delta 146.0,132.0,131.4,128.2,127.7,127.6,123.8,119.1,108.7,88.5,84.0,27.8$, 7.2, 2.3.

$\underline{\mathrm{HRMS}} \underline{(\mathrm{EI})}$

Calcd for $\mathrm{C}_{22} \mathrm{H}_{25} \mathrm{NSi}[\mathrm{M}]^{+}:$331.1756, found: 331.1749 . 
(-)-4-(3-(naphthalen-2-yl)-3-(triethylsilyl)prop-1-yn-1-yl)benzonitrile (3hb)<smiles>CCC(C#Cc1ccc(C#N)cc1)c1ccc2ccccc2c1</smiles>

Colorless oil, 85\% yield (64.8 mg), 78\% ee. HPLC condition: Chiralpak AD-3 column, $n$-hexane $/ i$-PrOH $=95: 5$, flow rate $=1.0 \mathrm{~mL} / \mathrm{min}, 230 \mathrm{~nm} \mathrm{UV}$ detector, $t_{\mathrm{R}}=6.01 \mathrm{~min}$ (minor) and $t_{\mathrm{R}}=7.77 \mathrm{~min}$ (major). $[\alpha] \mathrm{D}^{26}=-26.0\left(\mathrm{c} 1.0, \mathrm{CHCl}_{3}\right)$.

$\underline{{ }^{1} \mathrm{H}} \underline{\mathrm{NMR}} \underline{(400} \underline{\mathrm{MHz}}, \underline{\left.\mathrm{CDCl}_{3}\right)}$

$\delta 7.90-7.70$ (m, 3H), 7.68 (s, 1H), 7.58 (d, $J=8.3 \mathrm{~Hz}, 2 \mathrm{H}), 7.51(\mathrm{~d}, J=8.3$

$\mathrm{Hz}, 2 \mathrm{H}), 7.48-7.36(\mathrm{~m}, 3 \mathrm{H}), 3.67$ (s, 1H), 0.98 (t, $J=7.9 \mathrm{~Hz}, 9 \mathrm{H}), 0.75-0.60$ $(\mathrm{m}, 6 \mathrm{H})$.

\section{${ }^{13} \mathrm{C} \underline{\mathrm{NMR}} \underline{(101} \underline{\mathrm{MHz}}, \underline{\left.\mathrm{CDCl}_{3}\right)}$}

$\delta 136.4,133.6,132.0,132.0,131.8,129.5,128.0,127.7,127.4,126.2,126.1$, $125.2,124.9,118.8,110.6,96.2,82.0,27.3,7.4,2.5$.

$\underline{\mathrm{HRMS}} \underline{(\mathrm{EI})}$

Calcd for $\mathrm{C}_{26} \mathrm{H}_{27} \mathrm{NSi}[\mathrm{M}]^{+}: 381.1913$, found: 381.1911 .

(-)-triethyl(1-(naphthalen-2-yl)-3-(thiophen-2-yl)prop-2-yn-1-yl)silane (3ib)<smiles>CCSC(C#Cc1cccs1)c1ccc2ccccc2c1</smiles>

Colorless oil, 78\% yield (56.5 mg), 87\% ee. HPLC condition: Chiralpak AD-3 column, $n$-hexane $/ i$-PrOH $=99.5: 0.5$, flow rate $=0.5 \mathrm{~mL} / \mathrm{min}, 230 \mathrm{~nm}$ UV detector, $t_{\mathrm{R}}=7.90$ $\min$ (minor) and $t_{\mathrm{R}}=8.91 \mathrm{~min}$ (major). $[\alpha]_{\mathrm{D}}^{23}=-0.1\left(\mathrm{c} 1.0, \mathrm{CHCl}_{3}\right)$.

$\underline{{ }^{1} \mathrm{H}} \underline{\mathrm{NMR}} \underline{(400} \underline{\mathrm{MHz}}, \underline{\left.\mathrm{CDCl}_{3}\right)}$

$\delta 7.80-7.70(\mathrm{~m}, 3 \mathrm{H}), 7.69(\mathrm{~s}, 1 \mathrm{H}), 7.47-7.35(\mathrm{~m}, 3 \mathrm{H}), 7.20-7.13(\mathrm{~m}, 2 \mathrm{H})$, $6.95(\mathrm{dd}, J=5.1,3.7 \mathrm{~Hz}, 1 \mathrm{H}), 3.66(\mathrm{~s}, 1 \mathrm{H}), 0.98$ (t, $J=7.9 \mathrm{~Hz}, 9 \mathrm{H}), 0.75-$ $0.60(\mathrm{~m}, 6 \mathrm{H})$.

${ }^{13} \mathrm{C} \underline{\mathrm{NMR}} \underline{(101} \underline{\mathrm{MHz}}, \underline{\left.\mathrm{CDCl}_{3}\right)}$

$\delta 136.8,133.6,131.7,130.8,127.8,127.6,127.4,126.8,126.2,126.0,125.9$, $124.9,124.8,124.7,94.6,76.1,27.3,7.4,2.4$.

$\underline{\operatorname{HRMS}} \underline{(\mathrm{EI})}$

Calcd for $\mathrm{C}_{23} \mathrm{H}_{26} \mathrm{SSi}[\mathrm{M}]^{+}: 362.1524$, found: 362.1517 . 


\section{(-)-triethyl(1-(naphthalen-2-yl)hept-2-yn-1-yl)silane (3jb)}<smiles>CCC(C#CC(C)(C)C)c1ccc2ccccc2c1</smiles>

Colorless oil, $87 \%$ yield $(58.5 \mathrm{mg}), 97 \%$ ee. HPLC condition: Chiralcel OJ-3 column, $n$-hexane $/ i$-PrOH $=99.5: 0.5$, flow rate $=0.5 \mathrm{~mL} / \mathrm{min}, 230 \mathrm{~nm} \mathrm{UV}$ detector, $t_{\mathrm{R}}=7.40$ $\min$ (major) and $t_{\mathrm{R}}=8.70 \mathrm{~min}$ (minor). $[\alpha]_{\mathrm{D}^{26}}=-7.0$ (c 1.0, $\mathrm{CHCl}_{3}$ ).

${ }^{1} \mathrm{H} \underline{\mathrm{NMR}} \underline{(400} \underline{\mathrm{MHz}}, \underline{\left.\mathrm{CDCl}_{3}\right)}$

$\delta 7.81-7.69$ (m, 3H), 7.65 (s, 1H), $7.47-7.33$ (m, 3H), 3.39 (s, 1H), $2.35-$ $2.25(\mathrm{~m}, 2 \mathrm{H}), 1.60-1.44(\mathrm{~m}, 4 \mathrm{H}), 0.94(\mathrm{t}, J=7.8 \mathrm{~Hz}, 12 \mathrm{H}), 0.73-0.54(\mathrm{~m}$, $6 \mathrm{H})$.

${ }^{13} \mathrm{C} \underline{\mathrm{NMR}} \underline{(101} \underline{\mathrm{MHz}}, \underline{\left.\mathrm{CDCl}_{3}\right)}$

$\delta$ 138.2, 133.6, 131.6, 127.5 (2C signal), 127.3, 126.4, 125.8, 124.7 (2C signal), $82.8,79.7,31.4,26.2,22.0,18.8,13.6,7.4,2.3$.

$\underline{\operatorname{HRMS}} \underline{(\mathrm{EI})}$

Calcd for $\mathrm{C}_{23} \mathrm{H}_{32} \mathrm{Si}[\mathrm{M}]^{+}$: 336.2273 , found: 336.2268 .

(-)-methyl(1-(naphthalen-2-yl)hept-2-yn-1-yl)diphenylsilane (3jc)<smiles></smiles>

Colorless oil, 83\% yield (69.4 mg), 98\% ee. HPLC condition: Chiralpak AD-3 column, $n$-hexane $/ i-\mathrm{PrOH}=95: 5$, flow rate $=1.0 \mathrm{~mL} / \mathrm{min}, 230 \mathrm{~nm} \mathrm{UV}$ detector, $t_{\mathrm{R}}=3.57 \mathrm{~min}$ (minor) and $t_{\mathrm{R}}=9.71 \mathrm{~min}$ (major). $[\alpha] \mathrm{D}^{24}=-22.4$ (c 1.0, $\mathrm{CHCl}_{3}$ ).

$\underline{{ }^{1} \mathrm{H}} \underline{\mathrm{NMR}} \underline{(400} \underline{\mathrm{MHz}}, \underline{\left.\mathrm{CDCl}_{3}\right)}$

$\delta 7.76-7.69(\mathrm{~m}, 1 \mathrm{H}), 7.64-7.53(\mathrm{~m}, 4 \mathrm{H}), 7.46-7.31(\mathrm{~m}, 9 \mathrm{H}), 7.29-7.22$

(m, 2H), $7.05(\mathrm{dd}, J=8.5,1.9 \mathrm{~Hz}, 1 \mathrm{H}), 3.84(\mathrm{~s}, 1 \mathrm{H}), 2.27-2.21(\mathrm{~m}, 2 \mathrm{H}), 1.43$

(dt, $J=14.0,6.5 \mathrm{~Hz}, 2 \mathrm{H}), 1.34(\mathrm{dt}, J=14.9,7.1 \mathrm{~Hz}, 2 \mathrm{H}), 0.86(\mathrm{t}, J=7.2 \mathrm{~Hz}$, $3 \mathrm{H}), 0.54(\mathrm{~s}, 3 \mathrm{H})$.

$\underline{{ }^{13} \mathrm{C}} \underline{\mathrm{NMR}} \underline{(101} \underline{\mathrm{MHz}}, \underline{\left.\mathrm{CDCl}_{3}\right)}$

$\delta$ 136.6, 135.4, 135.2, 134.7, 134.4, 133.4, 131.7, 129.5 (2C signal), 127.5 (3C signal),127.4, 127.1, 126.9, 125.8, 125.7, 124.8, 84.6, 79.4, 31.1, 28.1, 21.9, $18.8,13.6,-5.8$.

$\underline{{ }^{29} \mathrm{Si}} \underline{\mathrm{NMR}} \underline{(79} \underline{\mathrm{MHz}}, \underline{\left.\mathrm{CDCl}_{3}\right)}$

$\delta-1.9$. 


\section{$\underline{\operatorname{HRMS}} \underline{(\mathrm{EI})}$}

Calcd for $\mathrm{C}_{30} \mathrm{H}_{30} \mathrm{Si}[\mathrm{M}]^{+}$: 418.2117, found: 418.2111 .

(-)-triethyl(1-(naphthalen-2-yl)but-2-yn-1-yl)silane (3kb)<smiles>CC#CC(=CC)c1ccc2ccccc2c1</smiles>

Colorless oil, 75\% yield (44.1 mg), 96\% ee. HPLC condition: Chiralpak AD-3 column, $n$-hexane $/ i-\mathrm{PrOH}=95: 5$, flow rate $=1.0 \mathrm{~mL} / \mathrm{min}, 230 \mathrm{~nm} \mathrm{UV}$ detector, $t_{\mathrm{R}}=3.21 \mathrm{~min}$ (minor) and $t_{\mathrm{R}}=3.87$ min (major). $[\alpha]_{\mathrm{D}^{23}}=-1.8\left(\mathrm{c} 1.0, \mathrm{CHCl}_{3}\right.$ ).

$\underline{{ }^{1} \mathrm{H}} \underline{\mathrm{NMR}} \underline{(400} \underline{\mathrm{MHz}}, \underline{\left.\mathrm{CDCl}_{3}\right)}$

$\delta 7.74(\mathrm{dd}, J=17.4,8.5 \mathrm{~Hz}, 3 \mathrm{H}), 7.65(\mathrm{~s}, 1 \mathrm{H}), 7.45-7.32(\mathrm{~m}, 3 \mathrm{H}), 3.36(\mathrm{~s}$, $1 \mathrm{H}), 1.92(\mathrm{~d}, J=2.6 \mathrm{~Hz}, 3 \mathrm{H}), 0.94(\mathrm{t}, J=7.9 \mathrm{~Hz}, 9 \mathrm{H}), 0.70-0.54(\mathrm{~m}, 6 \mathrm{H})$.

$\underline{{ }^{13} \mathrm{C}} \underline{\mathrm{NMR}} \underline{(101} \underline{\mathrm{MHz}}, \underline{\left.\mathrm{CDCl}_{3}\right)}$

$\delta 138.1,133.6,131.6,127.5$ (2C signal), 127.3, 126.4, 125.8, 124.7 (2C signal), $78.9,77.9,26.3,7.4,3.8,2.3$

\section{$\underline{\operatorname{HRMS}} \underline{(\mathrm{EI})}$}

Calcd for $\mathrm{C}_{20} \mathrm{H}_{26} \mathrm{Si}[\mathrm{M}]^{+}: 294.1804$, found: 294.1800 .

\section{(R)-methyl(1-(naphthalen-2-yl)but-2-yn-1-yl)diphenylsilane (3kc)}<smiles>CC#CC([SnH2])c1ccc2ccccc2c1</smiles>

Colorless oil, 70\% yield (52.7 mg), 96\% ee. HPLC condition: Chiralpak AD-3 column, $n$-hexane $/ i$-PrOH $=95: 5$, flow rate $=1.0 \mathrm{~mL} / \mathrm{min}, 230 \mathrm{~nm} \mathrm{UV}$ detector, $t_{\mathrm{R}}=3.48 \mathrm{~min}$ (minor) and $t_{\mathrm{R}}=12.71 \mathrm{~min}$ (major). $[\alpha]_{\mathrm{D}}{ }^{24}=-15.2$ (c 1.0, $\mathrm{CHCl}_{3}$ ).

$\underline{{ }^{1} \mathrm{H}} \underline{\mathrm{NMR}} \underline{(400} \underline{\mathrm{MHz}}, \underline{\left.\mathrm{CDCl}_{3}\right)}$

$\delta 7.73-7.68(\mathrm{~m}, 1 \mathrm{H}), 7.62-7.54(\mathrm{~m}, 4 \mathrm{H}), 7.44-7.40(\mathrm{~m}, 3 \mathrm{H}), 7.39-7.30$ $(\mathrm{m}, 6 \mathrm{H}), 7.27-7.21(\mathrm{~m}, 2 \mathrm{H}), 7.05(\mathrm{dd}, J=8.5,1.9 \mathrm{~Hz}, 1 \mathrm{H}), 3.81(\mathrm{q}, J=2.7$ $\mathrm{Hz}, 1 \mathrm{H}), 1.84(\mathrm{~d}, J=2.7 \mathrm{~Hz}, 3 \mathrm{H}), 0.54(\mathrm{~s}, 3 \mathrm{H})$.

\section{${ }^{13} \mathrm{C} \underline{\mathrm{NMR}} \underline{(101} \underline{\mathrm{MHz}}, \underline{\left.\mathrm{CDCl}_{3}\right)}$}

$\delta 136.5,135.4,135.1,134.7,134.5,133.3,131.7,129.5$ (2C signal), 127.6, 127.5, 127.4 (2C signal), 127.2, 126.8, 125.8, 125.7, 124.8, 79.6, 78.6, 28.1, $3.9,-5.8$

\section{$\underline{\operatorname{HRMS}} \underline{(\mathrm{EI})}$}


Calcd for $\mathrm{C}_{27} \mathrm{H}_{24} \mathrm{Si}[\mathrm{M}]^{+}$: 376.1647 , found: 376.1639 .

\section{Deterination of the configuration of $3 \mathrm{kc}$}

The silane 3kc was hydrogenated to $\mathbf{3 k c - H}$ by the same method as that for silane 6 (see below).

\section{(S)-methyl(1-(naphthalen-2-yl)butyl)diphenylsilane (3kc-H)}<smiles>CCCCC(CCC)c1ccc2ccccc2c1</smiles>

Colorless oil, 83\% yield (62.0 mg), 94\% ee. HPLC condition: Phenomenex, Cellulose3 column, $5 \mathrm{um}, \mathrm{MeOH}$, flow rate $=1.0 \mathrm{~mL} / \mathrm{min}, 230 \mathrm{~nm} \mathrm{UV}$ detector, $t_{\mathrm{R}}=7.57 \mathrm{~min}$ (major) and $t_{\mathrm{R}}=9.06 \mathrm{~min}$ (minor). $[\alpha]_{\mathrm{D}^{24}}=-52.6$ (c 1.0, $\mathrm{CHCl}_{3}$ ).

$\underline{{ }^{1} \mathrm{H}} \underline{\mathrm{NMR}} \underline{(400} \underline{\mathrm{MHz}}, \underline{\left.\mathrm{CDCl}_{3}\right)}$

$\delta 7.78-7.70(\mathrm{~m}, 1 \mathrm{H}), 7.65-7.58(\mathrm{~m}, 2 \mathrm{H}), 7.56-7.48(\mathrm{~m}, 2 \mathrm{H}), 7.45-7.29$ (m, 9H), $7.28-7.21(\mathrm{~m}, 2 \mathrm{H}), 7.02(\mathrm{dd}, J=8.4,1.8 \mathrm{~Hz}, 1 \mathrm{H}), 2.85$ (dd, $J=11.7$, $3.8 \mathrm{~Hz}, 1 \mathrm{H}), 1.99-1.80(\mathrm{~m}, 2 \mathrm{H}), 1.35-1.28(\mathrm{~m}, 1 \mathrm{H}), 1.20-1.10(\mathrm{~m}, 1 \mathrm{H})$, $0.80(\mathrm{t}, J=7.3 \mathrm{~Hz}, 3 \mathrm{H}), 0.41(\mathrm{~s}, 3 \mathrm{H})$.

\section{${ }^{13} \mathrm{C} \underline{\mathrm{NMR}} \underline{(101} \underline{\mathrm{MHz}}, \underline{\left.\mathrm{CDCl}_{3}\right)}$}

$\delta 140.2,136.2,135.4,135.2,134.9,133.5,131.5,129.3,129.1,127.8,127.7$, 127.6, 127.5, 127.2 (2C signal), 126.3, 125.6, 124.5, 34.9, 32.2, 22.2, 13.7, 5.1 .

$\underline{\operatorname{HRMS}} \underline{(\mathrm{EI})}$

Calcd for $\mathrm{C}_{27} \mathrm{H}_{28} \mathrm{Si}[\mathrm{M}]^{+}: 380.1960$, found: 380.1953 .

The silane $\mathbf{3 k c - H}$ was oxidized to alcohol $\mathbf{3 k c - o l},{ }^{2}$ the absolute configuration of alcohol 3kc-ol was determined by comparing its optical rotation with that in the literature.

\section{(S)-1-(naphthalen-2-yl)butan-1-ol (3kc-ol)}<smiles>CCCC(O)c1ccc2ccccc2c1</smiles> 
White solid, 63\% yield (20 mg), 91\% ee. HPLC condition: Chiralpak IB-3 column, $n$ hexane $/ i-\mathrm{PrOH}=95: 5$, flow rate $=1.0 \mathrm{~mL} / \mathrm{min}, 210 \mathrm{~nm} \mathrm{UV}$ detector, $t_{\mathrm{R}}=9.90 \mathrm{~min}$ (major) and $t_{\mathrm{R}}=10.87 \mathrm{~min}$ (minor). $[\alpha]_{\mathrm{D}}{ }^{26}=-47.0\left(\mathrm{c} 0.5, \mathrm{CHCl}_{3}\right) .\left(\right.$ lit. $[\alpha]_{\mathrm{D}}{ }^{24}=-32.2$ (c $\left.0.05, \mathrm{CHCl}_{3}\right), 76 \%$ ee $\left.(S)\right){ }^{5}$

\section{$\underline{{ }^{1} \mathrm{H}} \underline{\mathrm{NMR}} \underline{(400} \underline{\mathrm{MHz}}, \underline{\left.\mathrm{CDCl}_{3}\right)}$}

$\delta 7.90-7.73(\mathrm{~m}, 4 \mathrm{H}), 7.53-7.42(\mathrm{~m}, 3 \mathrm{H}), 4.92-4.81(\mathrm{~m}, 1 \mathrm{H}), 1.97-1.74$ $(\mathrm{m}, 3 \mathrm{H}), 1.51-1.29(\mathrm{~m}, 2 \mathrm{H}), 0.95(\mathrm{t}, J=7.4 \mathrm{~Hz}, 3 \mathrm{H})$.

\section{(-)-triethyl(1-(naphthalen-2-yl)-6-phenylhex-2-yn-1-yl)silane (3lb)}<smiles>CCSC(C#CC=Cc1ccccc1)c1ccc2ccccc2c1</smiles>

Colorless oil, 81\% yield (64.5 mg), 95\% ee. HPLC condition: Chiralpak AD-3 column, $n$-hexane $/ i-\mathrm{PrOH}=95: 5$, flow rate $=1.0 \mathrm{~mL} / \mathrm{min}, 230 \mathrm{~nm} \mathrm{UV}$ detector, $t_{\mathrm{R}}=3.27 \mathrm{~min}$ (minor) and $t_{\mathrm{R}}=3.85 \mathrm{~min}$ (major). $[\alpha]_{\mathrm{D}}{ }^{27}=-0.1$ (c 1.0, $\left.\mathrm{CHCl}_{3}\right)$.

$\underline{{ }^{1} \mathrm{H}} \underline{\mathrm{NMR}} \underline{(400} \underline{\mathrm{MHz}}, \underline{\left.\mathrm{CDCl}_{3}\right)}$

$\delta 7.80-7.71(\mathrm{~m}, 3 \mathrm{H}), 7.68(\mathrm{~s}, 1 \mathrm{H}), 7.46-7.34(\mathrm{~m}, 3 \mathrm{H}), 7.32-7.25(\mathrm{~m}, 2 \mathrm{H})$,

$7.25-7.16(\mathrm{~m}, 3 \mathrm{H}), 3.42(\mathrm{~s}, 1 \mathrm{H}), 2.80(\mathrm{t}, J=7.7 \mathrm{~Hz}, 2 \mathrm{H}), 2.37-2.27(\mathrm{~m}, 2 \mathrm{H})$,

$1.95-1.79(\mathrm{~m}, 2 \mathrm{H}), 0.95(\mathrm{t}, J=7.9 \mathrm{~Hz}, 10 \mathrm{H}), 0.73-0.60(\mathrm{~m}, 6 \mathrm{H})$.

${ }^{13} \mathrm{C} \underline{\mathrm{NMR}} \underline{(101} \underline{\mathrm{MHz}}, \underline{\left.\mathrm{CDCl}_{3}\right)}$

$\delta 141.9,138.1,133.6,131.6,128.5,128.3,127.5$ (2C signal), 127.3, 126.4,

125.8 (2C signal), 124.7(2C signal), 82.3, 80.4, 35.0, 31.1, 26.3, 18.7, 7.4, 2.4 .

$\underline{\operatorname{HRMS}} \underline{(\mathrm{EI})}$

Calcd for $\mathrm{C}_{28} \mathrm{H}_{34} \mathrm{Si}[\mathrm{M}]^{+}: 398.2430$, found: 398.2425 .

(-)-(3-cyclopropyl-1-(naphthalen-2-yl)prop-2-yn-1-yl)triethylsilane (3mb)<smiles></smiles>

Colorless oil, 63\% yield (40.3 mg), 97\% ee. HPLC condition: Chiralpak AD-3 column, $n$-hexane $/ i-\mathrm{PrOH}=95: 5$, flow rate $=1.0 \mathrm{~mL} / \mathrm{min}, 230 \mathrm{~nm} \mathrm{UV}$ detector, $t_{\mathrm{R}}=3.27 \mathrm{~min}$ (minor) and $t_{\mathrm{R}}=4.04 \mathrm{~min}$ (major). $[\alpha]_{\mathrm{D}}{ }^{26}=-0.1$ (c 1.0, $\mathrm{CHCl}_{3}$ ).

$\underline{{ }^{1} \mathrm{H}} \underline{\mathrm{NMR}} \underline{(400} \underline{\mathrm{MHz}}, \underline{\left.\mathrm{CDCl}_{3}\right)}$ 
$\delta 7.76(\mathrm{~d}, J=8.0 \mathrm{~Hz}, 2 \mathrm{H}), 7.72(\mathrm{~d}, J=8.5 \mathrm{~Hz}, 1 \mathrm{H}), 7.63(\mathrm{~s}, 1 \mathrm{H}), 7.45-7.31$ (m, 3H), $3.35(\mathrm{~s}, 1 \mathrm{H}), 1.33$ (ddt, $J=8.1,5.2,2.5 \mathrm{~Hz}, 1 \mathrm{H}), 0.93$ (t, $J=7.9 \mathrm{~Hz}$, $9 \mathrm{H}), 0.79-0.73(\mathrm{~m}, 2 \mathrm{H}), 0.70-0.68(\mathrm{~m}, 2 \mathrm{H}), 0.65-0.55(\mathrm{~m}, 6 \mathrm{H})$.

$\underline{{ }^{13} \mathrm{C}} \underline{\mathrm{NMR}} \underline{(101} \underline{\mathrm{MHz}}, \underline{\left.\mathrm{CDCl}_{3}\right)}$

$\delta$ 138.1, 133.6, 131.6, 127.5 (2C signal), 127.3, 126.3, 125.8, 124.7, 124.6, $86.0,75.2,26.2,8.2,8.1,7.4,2.3,0.0$.

$\underline{\operatorname{HRMS}} \underline{(\mathrm{EI})}$

Calcd for $\mathrm{C}_{22} \mathrm{H}_{28} \mathrm{Si}[\mathrm{M}]^{+}: 320.1960$, found: 320.1953 .

(-)-(3-cyclopropyl-1-(naphthalen-2-yl)prop-2-yn-1-yl)(methyl)diphenylsilane (3mc)<smiles></smiles>

Colorless oil, 65\% yield (52.3 mg), 98\% ee. HPLC condition: Chiralpak AD-3 column, $n$-hexane $/ i-\mathrm{PrOH}=90: 10$, flow rate $=1.0 \mathrm{~mL} / \mathrm{min}, 230 \mathrm{~nm} \mathrm{UV}$ detector, $t_{\mathrm{R}}=3.66 \mathrm{~min}$ (minor) and $t_{\mathrm{R}}=15.79 \mathrm{~min}$ (major). $[\alpha]_{\mathrm{D}}{ }^{24}=-16.2\left(\mathrm{c} 1.0, \mathrm{CHCl}_{3}\right)$.

$\underline{{ }^{1} \mathrm{H}} \underline{\mathrm{NMR}} \underline{(400} \underline{\mathrm{MHz}}, \underline{\left.\mathrm{CDCl}_{3}\right)}$

$\delta 7.75-7.69$ (m, 1H), $7.64-7.58(\mathrm{~m}, 1 \mathrm{H}), 7.58-7.53(\mathrm{~m}, 3 \mathrm{H}), 7.46-7.38$ (m, 4H), $7.38-7.31(\mathrm{~m}, 5 \mathrm{H}), 7.28-7.24(\mathrm{~m}, 2 \mathrm{H}), 7.04(\mathrm{dd}, J=8.5,1.8 \mathrm{~Hz}$, 1H), $3.80(\mathrm{~s}, 1 \mathrm{H}), 1.29-1.21(\mathrm{~m}, 1 \mathrm{H}), 0.71-0.62(\mathrm{~m}, 2 \mathrm{H}), 0.55-0.47(\mathrm{~m}$, $5 \mathrm{H})$.

\section{$\underline{{ }^{13} \mathrm{C}} \underline{\mathrm{NMR}} \underline{(101} \underline{\mathrm{MHz}}, \underline{\left.\mathrm{CDCl}_{3}\right)}$}

$\delta 136.4,135.4,135.1,134.6,134.4,133.3,131.7,129.5$ (2C signal), 127.6, 127.5, 127.4 (2C signal), 127.2, 126.8, 125.7 (2C signal), 124.8, 87.8, 74.8, $28.1,8.1,8.0,-0.0,-5.8$.

\section{$\underline{\operatorname{HRMS}} \underline{(\mathrm{EI})}$}

Calcd for $\mathrm{C}_{29} \mathrm{H}_{26} \mathrm{Si}[\mathrm{M}]^{+}$: 402.1804, found: 402.1799 .

\section{(-)-(3-cyclohexyl-1-(naphthalen-2-yl)prop-2-yn-1-yl)triethylsilane (3nb)}<smiles>CCSC(C#CC1CCCCC1)c1ccc2ccccc2c1</smiles>

Colorless oil, 80\% yield (58.0 mg), 96\% ee. HPLC condition: Chiralpak AD-3 column, 
$n$-hexane $/ i-\mathrm{PrOH}=95: 5$, flow rate $=1.0 \mathrm{~mL} / \mathrm{min}, 230 \mathrm{~nm} \mathrm{UV}$ detector, $t_{\mathrm{R}}=3.06 \mathrm{~min}$ (minor) and $t_{\mathrm{R}}=3.38 \mathrm{~min}$ (major). $[\alpha]_{\mathrm{D}}{ }^{24}=-0.1$ (c $1.0, \mathrm{CHCl}_{3}$ ).

$\underline{{ }^{1} \mathrm{H}} \underline{\mathrm{NMR}} \underline{(400} \underline{\mathrm{MHz}}, \underline{\left.\mathrm{CDCl}_{3}\right)}$

$\delta 7.80-7.70(\mathrm{~m}, 3 \mathrm{H}), 7.66(\mathrm{~s}, 1 \mathrm{H}), 7.45-7.33(\mathrm{~m}, 3 \mathrm{H}), 3.40(\mathrm{~s}, 1 \mathrm{H}), 2.56-$

$2.43(\mathrm{~m}, 1 \mathrm{H}), 1.92-1.81(\mathrm{~m}, 2 \mathrm{H}), 1.79-1.71(\mathrm{~m}, 2 \mathrm{H}), 1.57-1.46(\mathrm{~m}, 3 \mathrm{H})$, $1.39-1.30(\mathrm{~m}, 3 \mathrm{H}), 0.95(\mathrm{t}, J=7.8 \mathrm{~Hz}, 9 \mathrm{H}), 0.72-0.62(\mathrm{~m}, 6 \mathrm{H})$.

${ }^{13} \mathrm{C} \underline{\mathrm{NMR}} \underline{(101} \underline{\mathrm{MHz}}, \underline{\left.\mathrm{CDCl}_{3}\right)}$

$\delta 138.3,133.6,131.6,127.5,127.4,127.3,126.4,125.8,124.7$ (2C signal), $87.4,79.6,33.3,29.5,26.2,26.0,25.0,7.4,2.3$.

$\underline{\operatorname{HRMS}} \underline{(\mathrm{EI})}$

Calcd for $\mathrm{C}_{25} \mathrm{H}_{34} \mathrm{Si}[\mathrm{M}]^{+}: 362.2430$, found: 362.2427 .

(-)-triethyl(1-(naphthalen-2-yl)-3-(trimethylsilyl)prop-2-yn-1-yl)silane (3ob)<smiles>CC#CC(=CC)c1ccc2ccccc2c1</smiles>

Colorless oil, 87\% yield (61.3 mg), 97\% ee. HPLC condition: Phenomenex, Cellulose3 column, $\mathrm{MeOH}$, flow rate $=1.0 \mathrm{~mL} / \mathrm{min}, 230 \mathrm{~nm} \mathrm{UV}$ detector, $t_{\mathrm{R}}=3.79 \mathrm{~min}$ (minor) and $t_{\mathrm{R}}=8.50 \mathrm{~min}$ (major). $[\alpha]_{\mathrm{D}}{ }^{26}=-5.4\left(\mathrm{c} 1.0, \mathrm{CHCl}_{3}\right)$.

$\underline{{ }^{1} \mathrm{H}} \underline{\mathrm{NMR}} \underline{(400} \underline{\mathrm{MHz}}, \underline{\left.\mathrm{CDCl}_{3}\right)}$

$\delta 7.84-7.77(\mathrm{~m}, 3 \mathrm{H}), 7.69(\mathrm{~s}, 1 \mathrm{H}), 7.51-7.37(\mathrm{~m}, 3 \mathrm{H}), 3.53(\mathrm{~s}, 1 \mathrm{H}), 1.01(\mathrm{t}$, $J=7.9 \mathrm{~Hz}, 9 \mathrm{H}), 0.75-0.64(\mathrm{~m}, 6 \mathrm{H}), 0.27(\mathrm{~s}, 9 \mathrm{H})$.

${ }^{13} \mathrm{C} \underline{\mathrm{NMR}} \underline{(101} \underline{\mathrm{MHz}}, \underline{\left.\mathrm{CDCl}_{3}\right)}$

$\delta 136.9,133.6,131.6,127.7,127.5,127.3,126.2,125.9,124.9,124.8,107.3$, $87.2,27.7,7.4,2.3,0.2$.

$\underline{\operatorname{HRMS}} \underline{(\mathrm{EI})}$

Calcd for $\mathrm{C}_{22} \mathrm{H}_{32} \mathrm{Si}_{2}[\mathrm{M}]^{+}:$352.2043, found: 352.2034 .

(-)-(3-(cyclohex-1-en-1-yl)-1-(naphthalen-2-yl)prop-2-yn-1-yl)triethylsilane (3pb)<smiles></smiles>

Colorless oil, 95\% yield (68.4 mg), 94\% ee. HPLC condition: Chiralpak AD-3 column, $n$-hexane $/ i-\mathrm{PrOH}=99: 1$, flow rate $=1.0 \mathrm{~mL} / \mathrm{min}, 230 \mathrm{~nm} \mathrm{UV}$ detector, $t \mathrm{R}=3.50 \mathrm{~min}$ 
(minor) and $t_{\mathrm{R}}=4.03 \mathrm{~min}$ (major). $[\alpha]_{\mathrm{D}}{ }^{24}=-0.4$ (c 1.0, $\mathrm{CHCl}_{3}$ ).

$\underline{{ }^{1} \mathrm{H}} \underline{\mathrm{NMR}} \underline{(400} \underline{\mathrm{MHz}}, \underline{\left.\mathrm{CDCl}_{3}\right)}$

$\delta 7.79-7.20(\mathrm{~m}, 3 \mathrm{H}), 7.65(\mathrm{~s}, 1 \mathrm{H}), 7.45-7.34(\mathrm{~m}, 3 \mathrm{H}), 6.06(\mathrm{tt}, J=4.0,1.8$

$\mathrm{Hz}, 1 \mathrm{H}), 3.53(\mathrm{~s}, 1 \mathrm{H}), 2.23-2.16(\mathrm{~m}, 2 \mathrm{H}), 2.13-2.06(\mathrm{~m}, 2 \mathrm{H}), 1.70-1.57$

(m, 4H), $0.95(\mathrm{t}, J=7.9 \mathrm{~Hz}, 9 \mathrm{H}), 0.70-0.59(\mathrm{~m}, 6 \mathrm{H})$.

$\left.{ }^{13} \mathrm{C} \underline{\mathrm{NMR}} \underline{(101} \underline{\mathrm{MHz}}, \underline{\mathrm{CDCl}_{3}}\right)$

$\delta 137.7,133.6,132.7,131.6,127.6,127.5,127.3,126.4,125.8,124.8,124.7$,

$121.5,87.1,85.0,29.8,26.8,25.6,22.5,21.7,7.4,2.4$.

$\underline{\operatorname{HRMS}} \underline{(\mathrm{EI})}$

Calcd for $\mathrm{C}_{25} \mathrm{H}_{32} \mathrm{Si}[\mathrm{M}]^{+}: 360.2273$, found: 360.2268 .

(-)-(3-(cyclohex-1-en-1-yl)-1-(naphthalen-2-yl)prop-2-yn-1-

yl)(methyl)diphenylsilane (3pc)<smiles>CCCCC(C#CC1=CCCCC1)c1ccc2ccccc2c1</smiles>

Colorless oil, 85\% yield (75.2 mg), 99\% ee. HPLC condition: Chiralpak AD-3 column, $n$-hexane $/ i$-PrOH $=95: 5$, flow rate $=1.0 \mathrm{~mL} / \mathrm{min}, 230 \mathrm{~nm} \mathrm{UV}$ detector, $t_{\mathrm{R}}=3.94 \mathrm{~min}$ (minor) and $t_{\mathrm{R}}=8.02 \mathrm{~min}$ (major). $[\alpha]_{\mathrm{D}}^{30}=-1.6\left(\mathrm{c} 1.0, \mathrm{CHCl}_{3}\right)$.

$\underline{{ }^{1} \mathrm{H}} \underline{\mathrm{NMR}} \underline{(400} \underline{\mathrm{MHz}}, \underline{\left.\mathrm{CDCl}_{3}\right)}$

$\delta 7.76-7.71(\mathrm{~m}, 1 \mathrm{H}), 7.64-7.54(\mathrm{~m}, 4 \mathrm{H}), 7.49-7.44(\mathrm{~m}, 2 \mathrm{H}), 7.42-7.32$

(m, 7H), $7.29-7.24(\mathrm{~m}, 2 \mathrm{H}), 7.06(\mathrm{dd}, J=8.5,1.9 \mathrm{~Hz}, 1 \mathrm{H}), 6.00-5.94(\mathrm{~m}$,

$1 \mathrm{H}), 3.97(\mathrm{~s}, 1 \mathrm{H}), 2.11-2.02(\mathrm{~m}, 4 \mathrm{H}), 1.66-1.53(\mathrm{~m}, 4 \mathrm{H}), 0.56(\mathrm{~s}, 3 \mathrm{H})$.

$\underline{{ }^{13} \mathrm{C}} \underline{\mathrm{NMR}} \underline{(101} \underline{\mathrm{MHz}}, \underline{\left.\mathrm{CDCl}_{3}\right)}$

$\delta 136.2,135.5,135.2,134.6,134.3,133.4,133.1,131.7,129.6$ (2C signal), 127.7, 127.6, 127.5 (2C signal), 127.3, 126.9, 125.9, 125.7, 124.9, 121.3, 86.7, $86.5,29.5,28.8,25.6,22.5,21.7,-5.7$.

$\underline{\text { HRMS }} \underline{\text { (EI) }}$

Calcd for $\mathrm{C}_{32} \mathrm{H}_{30} \mathrm{Si}[\mathrm{M}]^{+}:$442.2117, found: 442.2108 .

(-)-triethyl(1-(naphthalen-2-yl)undeca-2,4-diyn-1-yl)silane (3qb)<smiles>CCCCCCCC=C(C#CC#CCCCCCCCC)c1ccc2ccccc2c1</smiles> 
Colorless oil, $85 \%$ yield $(66.0 \mathrm{mg}),>99 \%$ ee. HPLC condition: Phenomenex, Cellulose3 column, $\mathrm{MeOH}$, flow rate $=1.0 \mathrm{~mL} / \mathrm{min}, 230 \mathrm{~nm} \mathrm{UV}$ detector, $t_{\mathrm{R}}=7.67 \mathrm{~min}$ (minor) and $t_{\mathrm{R}}=11.16 \mathrm{~min}$ (major). $[\alpha]_{\mathrm{D}}{ }^{24}=+1.8\left(\mathrm{c} 1.0, \mathrm{CHCl}_{3}\right)$.

$\underline{{ }^{1} \mathrm{H}} \underline{\mathrm{NMR}} \underline{(400} \underline{\mathrm{MHz}}, \underline{\left.\mathrm{CDCl}_{3}\right)}$

$\delta 7.80-7.71(\mathrm{~m}, 3 \mathrm{H}), 7.63(\mathrm{~s}, 1 \mathrm{H}), 7.48-7.38(\mathrm{~m}, 2 \mathrm{H}), 7.34-7.29(\mathrm{~m}, 1 \mathrm{H})$, 3.49 (s, 1H), 2.29 (t, $J=7.1 \mathrm{~Hz}, 2 \mathrm{H}), 1.55$ (p, $J=7.2 \mathrm{~Hz}, 2 \mathrm{H}), 1.40$ (dd, $J=$ $10.9,4.7 \mathrm{~Hz}, 2 \mathrm{H}), 1.35-1.25(\mathrm{~m}, 4 \mathrm{H}), 0.98-0.86(\mathrm{~m}, 12 \mathrm{H}), 0.75-0.60$ (m, $6 \mathrm{H})$.

${ }^{13} \mathrm{C} \underline{\mathrm{NMR}} \underline{(101} \underline{\mathrm{MHz}}, \underline{\left.\mathrm{CDCl}_{3}\right)}$

$\delta 136.3,133.6,131.7,127.8,127.6,127.4,126.1,126.0,125.0,124.8,78.3$, $77.3,67.8,65.8,31.3,28.6,28.4,27.0,22.5,19.4,14.0,7.3,2.4$.

$\underline{\operatorname{HRMS}} \underline{(\mathrm{EI})}$

Calcd for $\mathrm{C}_{27} \mathrm{H}_{36} \mathrm{Si}[\mathrm{M}]^{+}$: 388.2586 , found: 388.2582 .

(-)-(6-chloro-1-(naphthalen-2-yl)hex-2-yn-1-yl)triethylsilane (3rb)<smiles></smiles>

Colorless oil, 72\% yield (51.3 mg), 95\% ee. HPLC condition: Chiralcel OJ-3 column, $n$-hexane $/ i$-PrOH $=95: 5$, flow rate $=1.0 \mathrm{~mL} / \mathrm{min}, 230 \mathrm{~nm} \mathrm{UV}$ detector, $t_{\mathrm{R}}=4.27 \mathrm{~min}$ (major) and $t_{\mathrm{R}}=5.56 \mathrm{~min}$ (minor). $[\alpha]_{\mathrm{D}}^{24}=0.0\left(\mathrm{c} 1.0, \mathrm{CHCl}_{3}\right)$.

$\underline{{ }^{1} \mathrm{H}} \underline{\mathrm{NMR}} \underline{(400} \underline{\mathrm{MHz}}, \underline{\left.\mathrm{CDCl}_{3}\right)}$

$\delta 7.81-7.69(\mathrm{~m}, 3 \mathrm{H}), 7.67-7.60(\mathrm{~m}, 1 \mathrm{H}), 7.47-7.30(\mathrm{~m}, 3 \mathrm{H}), 3.71(\mathrm{t}, J=$ $6.4 \mathrm{~Hz}, 2 \mathrm{H}), 3.39$ (t, $J=2.7 \mathrm{~Hz}, 1 \mathrm{H}), 2.49$ (td, $J=6.8,2.7 \mathrm{~Hz}, 2 \mathrm{H}), 2.00$ (p, $J$ $=6.6 \mathrm{~Hz}, 2 \mathrm{H}), 0.94(\mathrm{t}, J=7.9 \mathrm{~Hz}, 9 \mathrm{H}), 0.67-0.57(\mathrm{~m}, 6 \mathrm{H})$.

${ }^{13} \mathrm{C} \underline{\mathrm{NMR}} \underline{(101} \underline{\mathrm{MHz}}, \underline{\left.\mathrm{CDCl}_{3}\right)}$

$\delta 137.8,133.6,131.6,127.6,127.5,127.3,126.3,125.9,124.8,124.7,81.1$, $80.5,43.9,31.9,26.2,16.6,7.4,2.3$.

$\underline{\operatorname{HRMS}} \underline{(\mathrm{EI})}$

Calcd for $\mathrm{C}_{22} \mathrm{H}_{29} \mathrm{ClSi}[\mathrm{M}]^{+}: 356.1727$, found: 356.1716 .

(-)-(8-(benzyloxy)-1-(naphthalen-2-yl)oct-2-yn-1-yl)triethylsilane (3sb)<smiles>CCSC(C#CC(C)OCc1ccccc1)c1ccc2ccccc2c1</smiles> 
Colorless oil, 83\% yield (75.7 mg), 95\% ee. HPLC condition: Chiralpak AD-3 column, $n$-hexane $/ i-\mathrm{PrOH}=95: 5$, flow rate $=1.0 \mathrm{~mL} / \mathrm{min}, 230 \mathrm{~nm} \mathrm{UV}$ detector, $t_{\mathrm{R}}=3.49 \mathrm{~min}$ (minor) and $t_{\mathrm{R}}=4.10 \mathrm{~min}$ (major). $[\alpha]_{\mathrm{D}}^{23}=-7.4$ (c 1.0, $\mathrm{CHCl}_{3}$ ).

\section{$\underline{{ }^{1} \mathrm{H}} \underline{\mathrm{NMR}} \underline{(400} \underline{\mathrm{MHz}}, \underline{\left.\mathrm{CDCl}_{3}\right)}$}

$\delta 7.79-7.69(\mathrm{~m}, 3 \mathrm{H}), 7.64(\mathrm{~s}, 1 \mathrm{H}), 7.44-7.24(\mathrm{~m}, 8 \mathrm{H}), 4.50(\mathrm{~s}, 2 \mathrm{H}), 3.49(\mathrm{t}$, $J=6.5 \mathrm{~Hz}, 2 \mathrm{H}), 3.38$ (t, $J=2.6 \mathrm{~Hz}, 1 \mathrm{H}), 2.30(\mathrm{dt}, J=6.8,3.4 \mathrm{~Hz}, 2 \mathrm{H}), 1.66$ $(\mathrm{q}, J=6.8 \mathrm{~Hz}, 2 \mathrm{H}), 1.62-1.50(\mathrm{~m}, 4 \mathrm{H}), 0.93(\mathrm{t}, J=7.9 \mathrm{~Hz}, 9 \mathrm{H}), 0.67-0.57$ $(\mathrm{m}, 6 \mathrm{H})$.

\section{${ }^{13} \mathrm{C} \underline{\mathrm{NMR}} \underline{(101} \underline{\mathrm{MHz}}, \underline{\left.\mathrm{CDCl}_{3}\right)}$}

$\delta$ 138.6, 138.1, 133.6, 131.5, 128.3, 127.6 (2C signal), 127.5 (2C signal), 127.4, $127.3,126.4,125.8,124.7,82.6,79.9,72.9,70.4,29.4,29.1,26.2,25.6,19.1$, 7.4, 2.3 .

\section{$\underline{\operatorname{HRMS}} \underline{(\mathrm{EI})}$}

Calcd for $\mathrm{C}_{31} \mathrm{H}_{40} \mathrm{OSi}[\mathrm{M}]^{+}:$456.2848, found: 456.2841 .

\section{(-)-1-(6-(naphthalen-2-yl)-6-(triethylsilyl)hex-4-yn-1-yl)-1H-indole (3tb)}<smiles>C=C(C#CC#CC(CC)n1ccc2ccccc21)c1ccc2ccccc2c1</smiles>

Colorless oil, 65\% yield (56.8 mg), 93\% ee. HPLC condition: Chiralpak AD-3 column, $n$-hexane $/ i$-PrOH $=95: 5$, flow rate $=1.0 \mathrm{~mL} / \mathrm{min}, 230 \mathrm{~nm} \mathrm{UV}$ detector, $t_{\mathrm{R}}=3.74 \mathrm{~min}$ (minor) and $t_{\mathrm{R}}=4.38 \mathrm{~min}$ (major). $[\alpha]_{\mathrm{D}}^{25}=-6.4\left(\mathrm{c} 1.0, \mathrm{CHCl}_{3}\right)$.

$\underline{{ }^{1} \mathrm{H}} \underline{\mathrm{NMR}} \underline{(400} \underline{\mathrm{MHz}}, \underline{\left.\mathrm{CDCl}_{3}\right)}$

$\delta 7.81-7.71(\mathrm{~m}, 3 \mathrm{H}), 7.68(\mathrm{~s}, 1 \mathrm{H}), 7.63(\mathrm{~d}, J=7.8 \mathrm{~Hz}, 1 \mathrm{H}), 7.47-7.34(\mathrm{~m}$, 4H), $7.20-7.06(\mathrm{~m}, 3 \mathrm{H}), 6.49$ (d, $J=3.1 \mathrm{~Hz}, 1 \mathrm{H}), 4.29$ (t, $J=6.8 \mathrm{~Hz}, 2 \mathrm{H})$, 3.44 (s, 1H), 2.29 (td, $J=6.7,2.4 \mathrm{~Hz}, 2 \mathrm{H}), 2.04$ (p, $J=6.8 \mathrm{~Hz}, 2 \mathrm{H}), 0.95$ (t, $J$ $=7.9 \mathrm{~Hz}, 9 \mathrm{H}), 0.71-0.61(\mathrm{~m}, 6 \mathrm{H})$.

${ }^{13} \mathrm{C} \underline{\mathrm{NMR}} \underline{(101} \underline{\mathrm{MHz}}, \underline{\left.\mathrm{CDCl}_{3}\right)}$

$\delta 137.9,136.0,133.6,131.6,128.6,128.0,127.7,127.6,127.3,126.3,125.9$, $124.8,124.7,121.4,120.9,119.3,109.4,101.1,81.4,80.9,45.1,29.7,26.3$, $16.6,7.4,2.4$.

$\underline{\text { HRMS }} \underline{(\mathrm{EI})}$

Calcd for $\mathrm{C}_{30} \mathrm{H}_{35} \mathrm{NSi}[\mathrm{M}]^{+}:$437.2539, found: 437.2535. 
(-)-triethyl(1-(4-nitrophenyl)hept-2-yn-1-yl)silane (3ub)<smiles>CCSC(C#CC(Br)(Br)c1ccc([N+](=O)[O-])cc1)c1ccccc1</smiles>

Performed at $0{ }^{\circ} \mathrm{C}$ for $3 \mathrm{~h}$. Colorless oil, $95 \%$ yield $(62.9 \mathrm{mg}), 80 \%$ ee. HPLC condition: Chiralpak IF+IF-3 column, $n$-hexane $/ i$-PrOH $=99.7: 0.3$, flow rate $=0.3 \mathrm{~mL} / \mathrm{min}, 230$ $\mathrm{nm}$ UV detector, $t_{\mathrm{R}}=28.67 \min \left(\right.$ minor) and $t_{\mathrm{R}}=29.63 \mathrm{~min}$ (major). $[\alpha]_{\mathrm{D}}{ }^{24}=-10.6(\mathrm{c}$ $\left.1.0, \mathrm{CHCl}_{3}\right)$.

$\underline{{ }^{1} \mathrm{H}} \underline{\mathrm{NMR}} \underline{(400} \underline{\mathrm{MHz}}, \underline{\left.\mathrm{CDCl}_{3}\right)}$

$\delta 8.12(\mathrm{~d}, J=8.5 \mathrm{~Hz}, 2 \mathrm{H}), 7.36(\mathrm{~d}, J=8.5 \mathrm{~Hz}, 2 \mathrm{H}), 3.37(\mathrm{~s}, 1 \mathrm{H}), 2.27(\mathrm{dt}, J=$ 7.1, 3.4 Hz, 2H), $1.6-1.4(\mathrm{~m}, 4 \mathrm{H}), 0.94(\mathrm{t}, J=7.8 \mathrm{~Hz}, 12 \mathrm{H}), 0.66-0.56(\mathrm{~m}$, $6 \mathrm{H})$.

${ }^{13} \mathrm{C} \underline{\mathrm{NMR}} \underline{(101} \underline{\mathrm{MHz}}, \underline{\left.\mathrm{CDCl}_{3}\right)}$

$\delta 149.5,145.5,127.4,123.4,84.1,78.1,31.2,27.3,22.0,18.7,13.6,7.2,2.2$. $\underline{\operatorname{HRMS}} \underline{(\mathrm{EI})}$

Calcd for $\mathrm{C}_{19} \mathrm{H}_{29} \mathrm{NO}_{2} \mathrm{Si}[\mathrm{M}]^{+}: 331.1968$, found: 331.1962 .

(-)-(1-(3,4-dichlorophenyl)hept-2-yn-1-yl)triethylsilane (3vb)<smiles>CCSC(C#CCBr)c1ccc(Cl)c(Cl)c1</smiles>

Colorless oil, 85\% yield (60.2 mg), 89\% ee. HPLC condition: Chiralpak AD-3 column, $n$-hexane $/ i-\mathrm{PrOH}=95: 5$, flow rate $=1.0 \mathrm{~mL} / \mathrm{min}, 230 \mathrm{~nm} \mathrm{UV}$ detector, $t_{\mathrm{R}}=2.99 \mathrm{~min}$ (minor) and $t_{\mathrm{R}}=3.26 \mathrm{~min}$ (major). $[\alpha]_{\mathrm{D}^{24}}=-2.2$ (c 1.0, $\mathrm{CHCl}_{3}$ ).

$\underline{{ }^{1} \mathrm{H}} \underline{\mathrm{NMR}} \underline{(400} \underline{\mathrm{MHz}}, \underline{\left.\mathrm{CDCl}_{3}\right)}$

$\delta 7.26-7.20(\mathrm{~m}, 2 \mathrm{H}), 6.95(\mathrm{dd}, J=8.3,2.2 \mathrm{~Hz}, 1 \mathrm{H}), 3.10(\mathrm{t}, J=2.6 \mathrm{~Hz}, 1 \mathrm{H})$, $2.18(\mathrm{td}, J=6.9,2.6 \mathrm{~Hz}, 2 \mathrm{H}), 1.48-1.35(\mathrm{~m}, 4 \mathrm{H}), 0.90-0.80(\mathrm{~m}, 15 \mathrm{H}), 0.57$ $-0.47(\mathrm{~m}, 6 \mathrm{H})$.

$\underline{{ }^{13} \mathrm{C}} \underline{\mathrm{NMR}} \underline{(101} \underline{\mathrm{MHz}}, \underline{\left.\mathrm{CDCl}_{3}\right)}$

$\delta 141.3,132.0,129.8,128.7,128.5,126.4,83.7,78.6,31.3,25.6,22.0,18.7$, 13.6, 7.3, 2.2.

$\underline{\operatorname{HRMS}} \underline{(\mathrm{EI})}$

Calcd for $\mathrm{C}_{19} \mathrm{H}_{28} \mathrm{Cl}_{2} \mathrm{Si}[\mathrm{M}]^{+}: 354.1337$, found: 354.1331 . 


\section{(-)-(1-(3,4-dichlorophenyl)hept-2-yn-1-yl)(methyl)diphenylsilane (3vc)}<smiles>CCCCC(C#CC(C)(C)C)c1ccc(Cl)c(Cl)c1</smiles>

Colorless oil, 84\% yield (73.3 mg), 96\% ee. HPLC condition: Chiralpak AD-3 column, $n$-hexane $/ i-\mathrm{PrOH}=95: 5$, flow rate $=1.0 \mathrm{~mL} / \mathrm{min}, 230 \mathrm{~nm} \mathrm{UV}$ detector, $t_{\mathrm{R}}=3.35 \mathrm{~min}$ (minor) and $t_{\mathrm{R}}=10.29 \min$ (major). $[\alpha]_{\mathrm{D}}{ }^{24}=-16.0\left(\mathrm{c} 1.0, \mathrm{CHCl}_{3}\right)$.

$\underline{{ }^{1} \mathrm{H}} \underline{\mathrm{NMR}} \underline{(400} \underline{\mathrm{MHz}}, \underline{\left.\mathrm{CDCl}_{3}\right)}$

$\delta 7.61-7.54(\mathrm{~m}, 2 \mathrm{H}), 7.44-7.34(\mathrm{~m}, 6 \mathrm{H}), 7.34-7.29(\mathrm{~m}, 2 \mathrm{H}), 7.14(\mathrm{~d}, J=$ $8.3 \mathrm{~Hz}, 1 \mathrm{H}), 7.00(\mathrm{~d}, J=2.2 \mathrm{~Hz}, 1 \mathrm{H}), 6.73(\mathrm{dd}, J=8.3,2.2 \mathrm{~Hz}, 1 \mathrm{H}), 3.61$ (s, $1 \mathrm{H}), 2.19(\mathrm{td}, J=7.0,2.4 \mathrm{~Hz}, 2 \mathrm{H}), 1.47-1.35(\mathrm{~m}, 2 \mathrm{H}), 1.33-1.23(\mathrm{~m}, 2 \mathrm{H})$, $0.84(\mathrm{t}, J=7.3 \mathrm{~Hz}, 3 \mathrm{H}), 0.54(\mathrm{~s}, 3 \mathrm{H})$.

${ }^{13} \mathrm{C} \underline{\mathrm{NMR}} \underline{(101} \underline{\mathrm{MHz}}, \underline{\left.\mathrm{CDCl}_{3}\right)}$

$\delta 139.6,135.2,135.0,133.9,133.7,131.7,129.8,129.5$ (2C signal), 128.8, $127.7,127.7,127.6,127.0,85.4,78.3,31.0,27.5,21.8,18.6,13.6,-6.1$.

$\underline{\operatorname{HRMS}} \underline{(\mathrm{EI})}$

Calcd for $\mathrm{C}_{26} \mathrm{H}_{26} \mathrm{Cl}_{2} \mathrm{Si}[\mathrm{M}]^{+}: 436.1181$, found: 436.1173 .

(+)-(1-(3,4-dimethylphenyl)hept-2-yn-1-yl)(methyl)diphenylsilane (3wc)<smiles>CCC[SiH2]C(C#CC(C)(C)C)c1ccc(C)c(C)c1</smiles>

Colorless oil, 65\% yield (51.5 mg), 94\% ee. HPLC condition: Chiralpak AD-3 column, $n$-hexane $/ i$-PrOH $=95: 5$, flow rate $=1.0 \mathrm{~mL} / \mathrm{min}, 230 \mathrm{~nm} \mathrm{UV}$ detector, $t_{\mathrm{R}}=3.36 \mathrm{~min}$ (minor) and $t_{\mathrm{R}}=13.25 \mathrm{~min}$ (major). $[\alpha]_{\mathrm{D}}{ }^{24}=+13.8$ (c 1.0, $\mathrm{CHCl}_{3}$ ).

$\underline{{ }^{1} \mathrm{H}} \underline{\mathrm{NMR}} \underline{(400} \underline{\mathrm{MHz}}, \underline{\left.\mathrm{CDCl}_{3}\right)}$

$\delta 7.63-7.55(\mathrm{~m}, 2 \mathrm{H}), 7.46-7.41(\mathrm{~m}, 2 \mathrm{H}), 7.40-7.25(\mathrm{~m}, 6 \mathrm{H}), 6.88(\mathrm{~d}, J=$ $7.7 \mathrm{~Hz}, 1 \mathrm{H}), 6.73$ (dd, $J=7.7,2.0 \mathrm{~Hz}, 1 \mathrm{H}), 6.65$ (s, 1H), 3.59 (s, 1H), $2.21-$ $2.13(\mathrm{~m}, 5 \mathrm{H}), 2.06(\mathrm{~s}, 3 \mathrm{H}), 1.45-1.35(\mathrm{~m}, 2 \mathrm{H}), 1.33-1.24(\mathrm{~m}, 2 \mathrm{H}), 0.83(\mathrm{t}, J$ $=7.2 \mathrm{~Hz}, 3 \mathrm{H}), 0.51(\mathrm{~s}, 3 \mathrm{H})$.

${ }^{13} \mathrm{C} \underline{\mathrm{NMR}} \underline{(101} \underline{\mathrm{MHz}}, \underline{\left.\mathrm{CDCl}_{3}\right)}$

$\delta$ 136.0, 135.7, 135.4, 135.2, 135.1, 134.7, 133.1, 129.4, 129.3 (2C signal), 129.1, 127.4 (2C signal), 125.1, 83.9, 79.7, 31.1, 27.1, 21.8, 19.6, 19.2, 18.7, $13.6,-5.9$.

$\underline{\operatorname{HRMS}} \underline{(\mathrm{EI})}$ 
Calcd for $\mathrm{C}_{28} \mathrm{H}_{32} \mathrm{Si}[\mathrm{M}]^{+}$: 396.2273, found: 396.2264 .

(-)-(1-(benzofuran-5-yl)hept-2-yn-1-yl)(methyl)diphenylsilane (3xc)<smiles>CCCCC(C#CC(C)(C)C)c1ccc2occc2c1</smiles>

Colorless oil, 75\% yield (61.2 mg), 96\% ee. HPLC condition: Chiralcel OJ-3 column, $n$-hexane $/ i$-PrOH $=95: 5$, flow rate $=1.0 \mathrm{~mL} / \mathrm{min}, 230 \mathrm{~nm} \mathrm{UV}$ detector, $t_{\mathrm{R}}=4.24 \mathrm{~min}$ (major) and $t_{\mathrm{R}}=5.25 \mathrm{~min}$ (minor). $[\alpha]_{\mathrm{D}}^{24}=-13.4\left(\mathrm{c} 1.0, \mathrm{CHCl}_{3}\right)$.

$\underline{{ }^{1} \mathrm{H}} \underline{\mathrm{NMR}} \underline{(400} \underline{\mathrm{MHz}}, \underline{\left.\mathrm{CDCl}_{3}\right)}$

$\delta 7.61-7.55(\mathrm{~m}, 2 \mathrm{H}), 7.51(\mathrm{~d}, J=2.2 \mathrm{~Hz}, 1 \mathrm{H}), 7.44-7.37(\mathrm{~m}, 3 \mathrm{H}), 7.36-$ $7.30(\mathrm{~m}, 3 \mathrm{H}), 7.28-7.20(\mathrm{~m}, 4 \mathrm{H}), 6.85(\mathrm{dd}, J=8.5,1.9 \mathrm{~Hz}, 1 \mathrm{H}), 6.58(\mathrm{~d}, J=$

$2.1 \mathrm{~Hz}, 1 \mathrm{H}), 3.76(\mathrm{t}, J=2.6 \mathrm{~Hz}, 1 \mathrm{H}), 2.25-2.15(\mathrm{~m}, 2 \mathrm{H}), 1.48-1.38(\mathrm{~m}, 2 \mathrm{H})$, $1.35-1.26(\mathrm{~m}, 2 \mathrm{H}), 0.85(\mathrm{t}, J=7.3 \mathrm{~Hz}, 3 \mathrm{H}), 0.53(\mathrm{~s}, 3 \mathrm{H})$.

${ }^{13} \mathrm{C} \underline{\mathrm{NMR}} \underline{(101} \underline{\mathrm{MHz}}, \underline{\left.\mathrm{CDCl}_{3}\right)}$

$\delta 153.4,144.9,135.5,135.2,135.0,134.6,133.3,129.5,129.5,127.6,127.5$, $127.2,124.5,119.9,110.5,106.5,84.3,80.0,31.2,27.6,21.9,18.8,13.7,-5.8$.

\section{$\underline{\mathrm{HRMS}} \underline{(\mathrm{EI})}$}

Calcd for $\mathrm{C}_{28} \mathrm{H}_{28} \mathrm{OSi}[\mathrm{M}]^{+}$: 408.1909, found: 408.1902 . 


\section{Transformations of Si-H Bond Insertion Products}

\section{Synthesis of allyl silane $4^{6}$}<smiles>CC[SiH2]C(C#CC(C)(C)C)c1ccc2ccccc2c1</smiles>

$3 \mathbf{j b}$

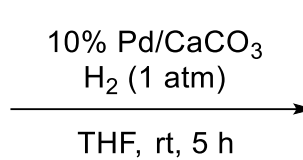

THF, rt, $5 \mathrm{~h}$

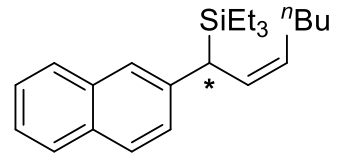

4

To a $25 \mathrm{~mL}$ Schlenk tube added alkynyl silane $\mathbf{3 j b}(89.0 \mathrm{mg}, 0.30 \mathrm{mmol})$ and $\mathrm{Pd} / \mathrm{CaCO}_{3}(5 \% \mathrm{Pd}, 63.0 \mathrm{mg}, 0.003 \mathrm{mmol})$, and filled with $\mathrm{H}_{2}$ gas (1 atm). THF (3 mL) was injected into the Schlenk tube at room temperature, and the reaction mixture was kept stirring for $5 \mathrm{~h}$. TLC indicated that the substrate $\mathbf{3 j b}$ was completely consumed. The solid was filtered off and the solution was concentrated in vacuum. The residue was chromatographied on silica gel (petroleum ether) to give 4 as a colorless oil (66.0 $\mathrm{mg}, 74 \%$ yield, $94 \%$ ee).

HPLC condition: Phenomenex, Cellulose-3 column, $\mathrm{MeOH}$, flow rate $=1.0 \mathrm{~mL} / \mathrm{min}$, $230 \mathrm{~nm}$ UV detector, $t_{\mathrm{R}}=4.82 \mathrm{~min}$ (major) and $t_{\mathrm{R}}=6.15 \mathrm{~min}\left(\right.$ minor). $[\alpha]_{\mathrm{D}}{ }^{25}=+96.4(\mathrm{c}$ $\left.1.0, \mathrm{CHCl}_{3}\right)$.

$\underline{{ }^{1} \mathrm{H}} \underline{\mathrm{NMR}} \underline{(400} \underline{\mathrm{MHz}}, \underline{\left.\mathrm{CDCl}_{3}\right)}$

$\delta 7.78-7.68(\mathrm{~m}, 3 \mathrm{H}), 7.52(\mathrm{~s}, 1 \mathrm{H}), 7.43-7.32(\mathrm{~m}, 2 \mathrm{H}), 7.28(\mathrm{dd}, J=8.5,1.8$ $\mathrm{Hz}, 1 \mathrm{H}), 5.97$ (ddt, $J=12.4,11.0,1.7 \mathrm{~Hz}, 1 \mathrm{H}), 5.47-5.36$ (m, 1H), 3.55 (d, $J$ $=11.6 \mathrm{~Hz}, 1 \mathrm{H}), 2.21-1.99(\mathrm{~m}, 2 \mathrm{H}), 1.38-1.23(\mathrm{~m}, 4 \mathrm{H}), 0.95-0.84(\mathrm{~m}, 12 \mathrm{H})$, $0.56(\mathrm{q}, J=8.1 \mathrm{~Hz}, 6 \mathrm{H})$.

${ }^{13} \mathrm{C} \underline{\mathrm{NMR}} \underline{(101} \underline{\mathrm{MHz}}, \underline{\left.\mathrm{CDCl}_{3}\right)}$

$\delta 141.4,133.8,131.2,128.6,128.5,127.6,127.5,127.2,126.8,125.7,124.5$ (2C signal), 35.0, 31.9, 27.1, 22.4, 14.0, 7.5, 2.4.

$\underline{\operatorname{HRMS}} \underline{(\mathrm{EI})}$

Calcd for $\mathrm{C}_{23} \mathrm{H}_{34} \mathrm{Si}[\mathrm{M}]^{+}: 338.2430$, found: 338.2421 .

\section{Synthesis of allyl silane $5^{7}$}

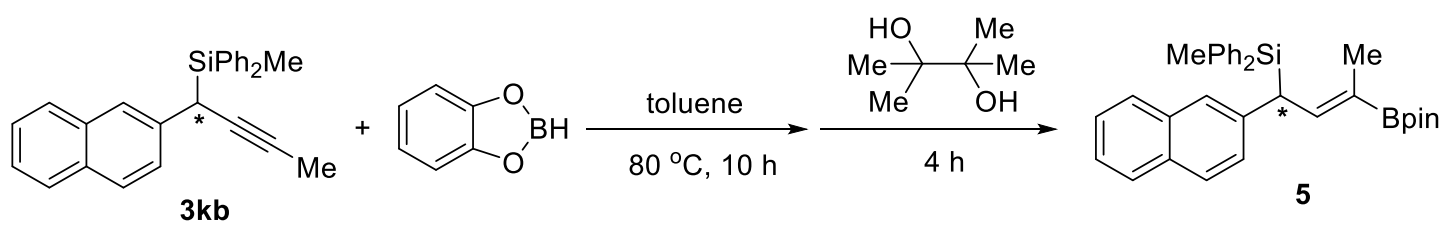

Under argon atmosphere, alkynyl silane 3kb (19.0 mg, $0.05 \mathrm{mmol})$ and catecholborane $(18.0 \mathrm{mg}, 0.15 \mathrm{mmol})$ were charged into $15 \mathrm{~mL}$ Schlenk tube. Dry 
toluene $(1 \mathrm{~mL})$ was injected into the Schlenk tube at room temperature, and the reaction mixture was kept stirring at $80{ }^{\circ} \mathrm{C}$ for $10 \mathrm{~h}$. TLC indicated that the substrate $\mathbf{3 k b}$ was completely consumed. Then the pinacol was added under the argon atmosphere and the reaction mixture was kept stirring at room temperature for 10 hours. The reaction mixture was concentrated and the residue was purified by chromatography on silica gel (petroleum ether) to give $\mathbf{5}$ as a colorless oil (15.0 mg, 60\% yield, 95\% ee).

HPLC condition: Phenomenex, Cellulose-3 column, $\mathrm{MeOH}$, flow rate $=1.0 \mathrm{~mL} / \mathrm{min}$, $230 \mathrm{~nm}$ UV detector, $t_{\mathrm{R}}=4.18 \mathrm{~min}$ (minor) and $t_{\mathrm{R}}=6.99 \min$ (major). $[\alpha]_{\mathrm{D}}{ }^{25}=+35.6$ (c $0.5, \mathrm{CHCl}_{3}$ ).

\section{$\underline{{ }^{1} \mathrm{H}} \underline{\mathrm{NMR}} \underline{(400} \underline{\mathrm{MHz}}, \underline{\left.\mathrm{CDCl}_{3}\right)}$}

$\delta 7.77-7.69(\mathrm{~m}, 1 \mathrm{H}), 7.63-7.55(\mathrm{~m}, 2 \mathrm{H}), 7.52-7.45(\mathrm{~m}, 4 \mathrm{H}), 7.42-7.33$

(m, 5H), $7.32-7.25(\mathrm{~m}, 4 \mathrm{H}), 7.12(\mathrm{dd}, J=8.5,1.9 \mathrm{~Hz}, 1 \mathrm{H}), 6.90-6.86(\mathrm{~m}$, $1 \mathrm{H}), 4.13(\mathrm{~d}, J=11.5 \mathrm{~Hz}, 1 \mathrm{H}), 1.53(\mathrm{~s}, 3 \mathrm{H}), 1.24(\mathrm{~s}, 12 \mathrm{H}), 0.53(\mathrm{~s}, 3 \mathrm{H})$.

$\underline{{ }^{13} \mathrm{C}} \underline{\mathrm{NMR}} \underline{(101} \underline{\mathrm{MHz}}, \underline{\left.\mathrm{CDCl}_{3}\right)}$

$\delta 144.8,139.0,135.4,135.2,135.1,134.8,133.6,131.4,129.3,127.6,127.5$, 127.4 (2C signal), 127.3, 125.8, 125.6, 124.7, 83.0, 39.1, 25.0, 24.5, 14.2, 4.9 .

$\underline{{ }^{11} \mathrm{~B}} \underline{\mathrm{NMR}} \underline{(128} \underline{\mathrm{MHz}}, \underline{\left.\mathrm{CDCl}_{3}\right)}$

$\delta 35.2$.

$\underline{\text { HRMS }} \underline{(E I)}$

Calcd for $\mathrm{C}_{33} \mathrm{H}_{37} \mathrm{BO}_{2} \mathrm{Si}[\mathrm{M}+\mathrm{Na}]^{+}:$527.2554, found: 527.2551.

Synthesis of $(S)$-dimethyl(1-(naphthalen-2-yl)-3-phenylpropyl)(phenyl)silane (6)<smiles>C[I-][C+](C)C(CCc1ccccc1)c1ccc2ccccc2c1</smiles>

Alkynyl silane 3aa (30.0 mg, $0.08 \mathrm{mmol})$ and $\mathrm{Pd} / \mathrm{C}$ (58\% Pd, $1.5 \mathrm{mg}, 0.008 \mathrm{mmol})$ were charged into $25 \mathrm{~mL}$ Schlenk tube, and the tube was filled with $\mathrm{H}_{2}$ gas (1 atm). THF ( $2 \mathrm{~mL}$ ) was injected into the Schlenk tube at room temperature, and the reaction mixture was kept stirring for $10 \mathrm{~h}$. TLC indicated that the substrate 3aa was consumed completely. The solid was filtered off and the solution was concentrated to give compound 6.

Colorless oil, 87\% yield (26.0 mg), 90\% ee. HPLC condition: Chiralpak AD-3 column, $n$-hexane $/ i$-PrOH $=99.5: 0.5$, flow rate $=0.5 \mathrm{~mL} / \mathrm{min}, 230 \mathrm{~nm}$ UV detector, $t_{\mathrm{R}}=6.98$ 
$\min$ (minor) and $t_{\mathrm{R}}=7.34 \min$ (major). $[\alpha]_{\mathrm{D}^{23}}^{23}+1.6\left(\mathrm{c} 0.5, \mathrm{CHCl}_{3}\right)$.

$\underline{{ }^{1} \mathrm{H}} \underline{\mathrm{NMR}} \underline{(400} \underline{\mathrm{MHz}}, \underline{\left.\mathrm{CDCl}_{3}\right)}$

$\delta 7.80(\mathrm{~d}, J=7.7 \mathrm{~Hz}, 1 \mathrm{H}), 7.72(\mathrm{~d}, J=8.3 \mathrm{~Hz}, 2 \mathrm{H}), 7.47-7.27(\mathrm{~m}, 8 \mathrm{H}), 7.26$

$-7.20(\mathrm{~m}, 2 \mathrm{H}), 7.18-7.06(\mathrm{~m}, 2 \mathrm{H}), 7.02$ (d, $J=7.2 \mathrm{~Hz}, 2 \mathrm{H}), 2.66-2.55$ (m,

$1 \mathrm{H}), 2.48-2.31(\mathrm{~m}, 2 \mathrm{H}), 2.26-2.03(\mathrm{~m}, 2 \mathrm{H}), 0.25(\mathrm{~s}, 3 \mathrm{H}), 0.17(\mathrm{~s}, 3 \mathrm{H})$.

${ }^{13} \mathrm{C} \underline{\mathrm{NMR}} \underline{(101} \underline{\mathrm{MHz}}, \underline{\left.\mathrm{CDCl}_{3}\right)}$

$\delta 142.3,140.4,137.3,134.2,133.7,131.5,129.0,128.5,128.2,127.6,127.5$

(3C signal), 127.2, 125.8, 125.7, 125.6, 124.6, 36.2, 35.2, 31.3, -3.8, -5.2.

\section{$\underline{\operatorname{HRMS}} \underline{(\mathrm{EI})}$}

Calcd for $\mathrm{C}_{27} \mathrm{H}_{28} \mathrm{Si}[\mathrm{M}]^{+}: 380.1960$, found: 380.1954 .

\section{Synthesis of (S)-1-(naphthalen-2-yl)-3-phenylpropan-1-ol (6ol)}<smiles>OC(CCc1ccccc1)c1ccc2ccccc2c1</smiles>

Silane 6 was oxidized to alcohol $\mathbf{6 o l},{ }^{2}$ and the absolute configuration of alcohol $\mathbf{6 o l}$ was determined by comparing its optical rotation with that in the literature.

Colorless oil, $30 \%$ yield $(8.0 \mathrm{mg}), 88 \%$ ee. HPLC condition: Chiralcel OD-H column, $n$-hexane $/ i-\mathrm{PrOH}=90: 10$, flow rate $=1.0 \mathrm{~mL} / \mathrm{min}, 230 \mathrm{~nm} \mathrm{UV}$ detector, $t_{\mathrm{R}}=14.63 \mathrm{~min}$ (major) and $t_{\mathrm{R}}=18.48 \mathrm{~min}$ (minor). $[\alpha]_{\mathrm{D}}{ }^{25}=-12.0$ (c 0.15, Ethanol). (lit. $[\alpha]_{\mathrm{D}}{ }^{25}=-12.0$ (c 2.0, Ethanol), $90 \%$ ee $(S)) .^{8}$

\section{$\underline{{ }^{1} \mathrm{H}} \underline{\mathrm{NMR}} \underline{(400} \underline{\mathrm{MHz}}, \underline{\left.\mathrm{CDCl}_{3}\right)}$}

$\delta 7.77-7.84(\mathrm{~m}, 4 \mathrm{H}), 7.44-7.49(\mathrm{~m}, 3 \mathrm{H}), 7.16-7.30(\mathrm{~m}, 5 \mathrm{H}), 4.83-4.86$ $(\mathrm{dd}, \mathrm{J}=5.8 \mathrm{~Hz}, 7.4 \mathrm{~Hz}, 1 \mathrm{H}), 2.65-2.80(\mathrm{~m}, 2 \mathrm{H}), 2.08-2.24(\mathrm{~m}, 2 \mathrm{H}), 2.00$ (s, $1 \mathrm{H}, \mathrm{OH})$. 


\section{Typical Procedure for Isomerization of Propargylsilanes to Allenylsilanes and Analytical Data of Isomerization Products}

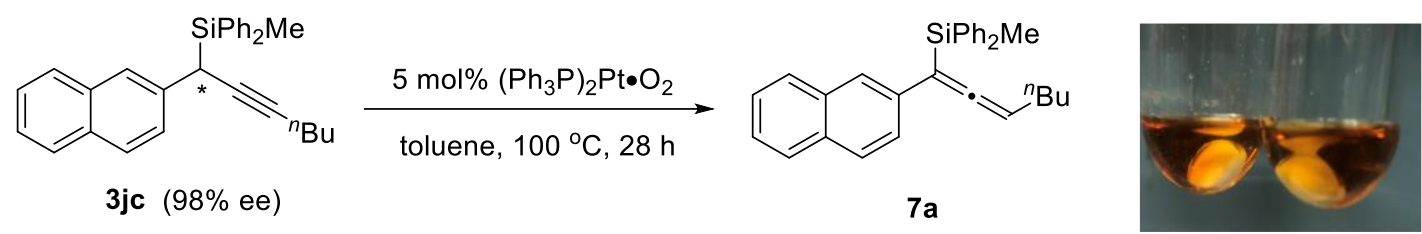

Propargylsilane 3jc $(21.0 \mathrm{mg}, 0.05 \mathrm{mmol})$ and $\left(\mathrm{PPh}_{3}\right)_{2} \mathrm{Pt}^{\circ} \mathrm{O}_{2}{ }^{9}(1.9 \mathrm{mg}, 0.0025$ mmol) were added into a $15 \mathrm{~mL}$ oven-dried tube under argon atmosphere. Dry toluene $(1 \mathrm{~mL})$ was added to the tube by syringe. After the substrate and catalyst were fully mixed, the reaction mixture was stirred at $100{ }^{\circ} \mathrm{C}$ for 28 hours. The reaction mixture was concentrated in vacuum. The residue was purified by column chromatography on silica gel (eluting with petroleum ether/DCM $=20: 1, \mathrm{v} / \mathrm{v}$ ) or thin layer chromatography to give $7 \mathbf{a}$ as a colorless oil.

The complex $\left(\mathrm{Ph}_{3} \mathrm{P}\right)_{2} \mathrm{Pt}^{\cdot} \mathrm{O}_{2}$ is unstable and decompose rapidly to zero-valent platinum and triphenylphosphine oxide $\left(\mathrm{OPPh}_{3}\right)$ above $90{ }^{\circ} \mathrm{C}^{10} .{ }^{1} \mathrm{H}$ and ${ }^{31} \mathrm{P}$ NMR also indicated that the complex $\left(\mathrm{Ph}_{3} \mathrm{P}\right)_{2} \mathrm{Pt}^{\bullet} \mathrm{O}_{2}$ decomposed to triphenylphosphine oxide within the first few minutes in the reaction. The treatment of $\mathbf{3 j} \mathbf{c}$ with $\mathrm{OPPh}_{3}, \mathrm{PPh}_{3}$ and $\mathrm{Pt} / \mathrm{C}$ results no reaction. Based on these observations, we speculate that the active catalyst in the reaction may be platinum nanoparticles.

\section{(-)-methyl(1-(naphthalen-2-yl)hepta-1,2-dien-1-yl)diphenylsilane (7a)}

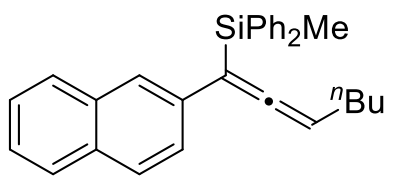

Colorless oil, 84\% yield (17.6 mg), 96\% ee. HPLC condition: Chiralpak AD-3+AD-3 column, $n$-hexane $/ i-\mathrm{PrOH}=99.5: 0.5$, flow rate $=0.5 \mathrm{~mL} / \mathrm{min}, 254 \mathrm{~nm} \mathrm{UV}$ detector, $t_{\mathrm{R}}$ $=14.89 \min$ (minor) and $t_{\mathrm{R}}=15.86 \mathrm{~min}$ (major). $[\alpha]_{\mathrm{D}}{ }^{24}=-54.0\left(\mathrm{c} 0.5, \mathrm{CHCl}_{3}\right)$.

\section{$\underline{{ }^{1} \mathrm{H}} \underline{\mathrm{NMR}} \underline{(400} \underline{\mathrm{MHz}}, \underline{\left.\mathrm{CDCl}_{3}\right)}$}

$\delta 7.76-7.70(\mathrm{~m}, 1 \mathrm{H}), 7.67(\mathrm{~d}, J=8.6 \mathrm{~Hz}, 1 \mathrm{H}), 7.65-7.59(\mathrm{~m}, 4 \mathrm{H}), 7.55(\mathrm{~s}$, 1H), $7.55-7.47(\mathrm{~m}, 2 \mathrm{H}), 7.41-7.31(\mathrm{~m}, 8 \mathrm{H}), 5.19(\mathrm{t}, J=6.8 \mathrm{~Hz}, 1 \mathrm{H}), 2.08-$ $1.95(\mathrm{~m}, 2 \mathrm{H}), 1.34-1.27(\mathrm{~m}, 4 \mathrm{H}), 0.85(\mathrm{t}, J=5.9 \mathrm{~Hz}, 3 \mathrm{H}), 0.76(\mathrm{~s}, 3 \mathrm{H})$.

\section{${ }^{13} \mathrm{C} \underline{\mathrm{NMR}} \underline{(101} \underline{\mathrm{MHz}}, \underline{\left.\mathrm{CDCl}_{3}\right)}$}

$\delta 211.7,136.3$ (2C signal), 135.1 (2C signal), 134.9, 133.4, 132.0, 129.3 (2C signal), 127.8 (3C signal), 127.7, 127.4, 126.6, 126.5, 125.8, 125.3, 97.3, 88.8, $31.6,27.9,22.3,13.9,-2.2$. 
${ }^{29} \mathrm{Si} \underline{\mathrm{NMR}} \underline{(79} \underline{\mathrm{MHz}}, \underline{\left.\mathrm{CDCl}_{3}\right)}$

$\delta-12.5$.

$\underline{\operatorname{HRMS}} \underline{(\mathrm{EI})}$

Calcd for $\mathrm{C}_{30} \mathrm{H}_{30} \mathrm{Si}[\mathrm{M}]^{+}:$418.2117, found: 418.2110 .

(S)-methyl(1-(naphthalen-2-yl)buta-1,2-dien-1-yl)diphenylsilane (7b)<smiles>CC=C=C([SnH2])c1ccc2ccccc2c1</smiles>

Performed at $100{ }^{\circ} \mathrm{C}$ for $30 \mathrm{~h}$. Colorless oil, $73 \%$ yield $(13.7 \mathrm{mg}), 93 \%$ ee. White solid (In general, the allenylsilane $\mathbf{7 b}$ is a viscous liquid that is difficult to cure.), m.p. 92 $94{ }^{\circ} \mathrm{C}$. HPLC condition: Chiralpak AD-3 column, $n$-hexane $/ i-\mathrm{PrOH}=99.5: 0.5$, flow rate $=1.0 \mathrm{~mL} / \mathrm{min}, 230 \mathrm{~nm} \mathrm{UV} \mathrm{detector,} t_{\mathrm{R}}=14.12 \mathrm{~min}$ (minor) and $t_{\mathrm{R}}=14.80 \mathrm{~min}$ (major). $[\alpha] \mathrm{D}^{25}=-36.0$ (c $\left.0.5, \mathrm{CHCl}_{3}\right)$.

$\underline{{ }^{1} \mathrm{H}} \underline{\mathrm{NMR}} \underline{(400} \underline{\mathrm{MHz}}, \underline{\left.\mathrm{CDCl}_{3}\right)}$

$\delta 7.76-7.70(\mathrm{~m}, 1 \mathrm{H}), 7.67(\mathrm{~d}, J=8.6 \mathrm{~Hz}, 1 \mathrm{H}), 7.65-7.58(\mathrm{~m}, 4 \mathrm{H}), 7.57-$ $7.51(\mathrm{~m}, 2 \mathrm{H}), 7.48(\mathrm{dd}, J=8.6,1.8 \mathrm{~Hz}, 1 \mathrm{H}), 7.43-7.31(\mathrm{~m}, 8 \mathrm{H}), 5.14(\mathrm{q}, J=$ $7.1 \mathrm{~Hz}, 1 \mathrm{H}), 1.66(\mathrm{~d}, J=7.1 \mathrm{~Hz}, 3 \mathrm{H}), 0.75(\mathrm{~s}, 3 \mathrm{H})$.

$\underline{{ }^{13} \mathrm{C}} \underline{\mathrm{NMR}} \underline{(101} \underline{\mathrm{MHz}}, \underline{\left.\mathrm{CDCl}_{3}\right)}$

$\delta 212.6,136.3,136.2,135.1$ (2C signal), 134.8, 133.4, 132.0, 129.4, 129.3, 127.8 (3C signal), 127.7, 127.4, 126.7, 126.6, 125.8, 125.3, 96.9, 83.1, 13.2,2.3 .

$\underline{\operatorname{HRMS}} \underline{(\mathrm{EI})}$

Calcd for $\mathrm{C}_{27} \mathrm{H}_{24} \mathrm{Si}[\mathrm{M}]^{+}: 376.1647$, found: 376.1639 .

(-)-(3-cyclopropyl-1-(naphthalen-2-yl)propa-1,2-dien-1-yl)(methyl)diphenylsilane (7c)<smiles>CCCCCC(=CC1CC1)c1ccc2ccccc2c1</smiles>

Performed at $100{ }^{\circ} \mathrm{C}$ for $30 \mathrm{~h}$. Colorless oil, 63\% yield $(12.7 \mathrm{mg}), 86 \%$ ee. HPLC condition: Chiralpak AD-3 column, $n$-hexane $/ i-\mathrm{PrOH}=99: 1$, flow rate $=1.0 \mathrm{~mL} / \mathrm{min}$, $254 \mathrm{~nm} \mathrm{UV} \mathrm{detector,} t_{\mathrm{R}}=3.79 \mathrm{~min}$ (minor) and $t_{\mathrm{R}}=4.28 \mathrm{~min}$ (major). $[\alpha]_{\mathrm{D}}{ }^{25}=-56.8(\mathrm{c}$ $\left.0.5, \mathrm{CHCl}_{3}\right)$.

$\underline{{ }^{1} \mathrm{H}} \underline{\mathrm{NMR}} \underline{(400} \underline{\mathrm{MHz}}, \underline{\left.\mathrm{CDCl}_{3}\right)}$ 
$\delta 7.74-7.65(\mathrm{~m}, 2 \mathrm{H}), 7.64-7.59(\mathrm{~m}, 4 \mathrm{H}), 7.56(\mathrm{~s}, 1 \mathrm{H}), 7.54-7.47(\mathrm{~m}, 2 \mathrm{H})$, $7.40-7.30(\mathrm{~m}, 8 \mathrm{H}), 5.14(\mathrm{~d}, J=6.9 \mathrm{~Hz}, 1 \mathrm{H}), 1.28-1.20(\mathrm{~m}, 1 \mathrm{H}), 0.76(\mathrm{~s}$, $3 \mathrm{H}), 0.67-0.58(\mathrm{~m}, 2 \mathrm{H}), 0.29-0.13(\mathrm{~m}, 2 \mathrm{H})$.

\section{${ }^{13} \mathrm{C} \underline{\mathrm{NMR}} \underline{(101} \underline{\mathrm{MHz}}, \underline{\left.\mathrm{CDCl}_{3}\right)}$}

$\delta 211.8,136.2,136.1,135.1$ (2C signal), 134.6, 133.4, 132.1, 129.4, 127.8 (5C signal), 127.4, 126.6 (2C signal), 125.8, 125.4, 99.4, 92.9, 8.7, 6.7, 6.4, -2.2. $\underline{\operatorname{HRMS}} \underline{(\mathrm{EI})}$

Calcd for $\mathrm{C}_{29} \mathrm{H}_{26} \mathrm{Si}[\mathrm{M}]^{+}:$402.1804, found: 402.1797 .

\section{(-)-(3-(cyclohex-1-en-1-yl)-1-(naphthalen-2-yl)propa-1,2-dien-1-} yl)(methyl)diphenylsilane (7d)<smiles>CCCCCCCCC=CC1=CCCCC1</smiles>

Performed at $100{ }^{\circ} \mathrm{C}$ for $28 \mathrm{~h}$. Colorless oil, $80 \%$ yield $(17.7 \mathrm{mg}), 80 \%$ ee. HPLC condition: Chiralpak IA-3 column, $n$-hexane $/ i-\mathrm{PrOH}=99.2: 0.8$, flow rate $=0.8 \mathrm{~mL} / \mathrm{min}$, $254 \mathrm{~nm} \mathrm{UV} \mathrm{detector,} t_{\mathrm{R}}=8.19 \mathrm{~min}$ (minor) and $t_{\mathrm{R}}=9.66 \mathrm{~min}$ (major). $[\alpha]_{\mathrm{D}}{ }^{25}=-56.8(\mathrm{c}$ $\left.0.5, \mathrm{CHCl}_{3}\right)$.

\section{$\underline{{ }^{1} \mathrm{H}} \underline{\mathrm{NMR}} \underline{(400} \underline{\mathrm{MHz}}, \underline{\left.\mathrm{CDCl}_{3}\right)}$}

$\delta 7.75-7.69(\mathrm{~m}, 1 \mathrm{H}), 7.66(\mathrm{~d}, J=8.6 \mathrm{~Hz}, 1 \mathrm{H}), 7.64-7.59(\mathrm{~m}, 4 \mathrm{H}), 7.56(\mathrm{~s}$, 1H), $7.53-7.47(\mathrm{~m}, 2 \mathrm{H}), 7.41-7.30(\mathrm{~m}, 8 \mathrm{H}), 6.01(\mathrm{~s}, 1 \mathrm{H}), 5.62(\mathrm{~s}, 1 \mathrm{H}), 2.14$ $-2.04(\mathrm{~m}, 3 \mathrm{H}), 2.04-1.92(\mathrm{~m}, 1 \mathrm{H}), 1.68-1.55(\mathrm{~m}, 4 \mathrm{H}), 0.74(\mathrm{~s}, 3 \mathrm{H})$.

$\underline{{ }^{13} \mathrm{C}} \underline{\mathrm{NMR}} \underline{(101} \underline{\mathrm{MHz}}, \underline{\left.\mathrm{CDCl}_{3}\right)}$

$\delta 212.8,136.0$ (2C signal), 135.1 (2C signal), 134.3, 133.4, 132.1, 131.4, 129.4, 127.9 (3C signal), 127.8 (2C signal),127.4, 126.7, 126.6, 125.8, 125.4, 125.0, $100.9,96.1,26.1,25.9,22.6,22.4,-1.8$.

\section{$\underline{\operatorname{HRMS}} \underline{(\mathrm{EI})}$}

Calcd for $\mathrm{C}_{32} \mathrm{H}_{30} \mathrm{Si}[\mathrm{M}]^{+}:$442.2117, found: 442.2108 .

\section{(-)-methyl(1-(naphthalen-2-yl)-3-phenylpropa-1,2-dien-1-yl)diphenylsilane (7e)}<smiles>C/C=C/C(=C\c1ccccc1)/C(=C/c1ccccc1)c1ccc2ccccc2c1</smiles>

Performed at $100{ }^{\circ} \mathrm{C}$ for $36 \mathrm{~h}$. Colorless oil, $86 \%$ yield $(18.8 \mathrm{mg}), 84 \%$ ee. HPLC condition: Chiralpak AD-3 column, $n$-hexane $/ i-\mathrm{PrOH}=95: 5$, flow rate $=1.0 \mathrm{~mL} / \mathrm{min}$, 
$254 \mathrm{~nm} \mathrm{UV} \mathrm{detector,} t_{\mathrm{R}}=3.71 \mathrm{~min}$ (minor) and $t_{\mathrm{R}}=4.16 \mathrm{~min}$ (major). $[\alpha]_{\mathrm{D}}{ }^{26}=-122.8$ (c $\left.0.5, \mathrm{CHCl}_{3}\right)$.

\section{$\underline{{ }^{1} \mathrm{H}} \underline{\mathrm{NMR}} \underline{(400} \underline{\mathrm{MHz}}, \underline{\left.\mathrm{CDCl}_{3}\right)}$}

$\delta 7.74-7.69(\mathrm{~m}, 1 \mathrm{H}), 7.69-7.62(\mathrm{~m}, 6 \mathrm{H}), 7.51(\mathrm{dd}, J=8.5,1.9 \mathrm{~Hz}, 2 \mathrm{H}), 7.41$

- 7.30 (m, 8H), $7.29-7.26(\mathrm{~m}, 4 \mathrm{H}), 7.20-7.14(\mathrm{~m}, 1 \mathrm{H}), 6.29$ (s, 1H), 0.77 (s, $3 \mathrm{H})$.

$\underline{{ }^{13} \mathrm{C}} \underline{\mathrm{NMR}} \underline{(101} \underline{\mathrm{MHz}}, \underline{\left.\mathrm{CDCl}_{3}\right)}$

$\delta 212.9,135.6$ (2C signal), 135.1 (2C signal), 134.1, 133.4, 133.3, 132.3, 129.6

(2C signal), 128.7, 128.0 (2C signal), 127.9 (2C signal), 127.5, 127.1, 126.6

(2C signal), 126.4, 125.9, 125.7, 102.1, 92.8, -2.0.

$\underline{\operatorname{HRMS}} \underline{(\mathrm{EI})}$

Calcd for $\mathrm{C}_{32} \mathrm{H}_{26} \mathrm{Si}[\mathrm{M}]^{+}:$438.1804, found: 438.1797 .

\section{(-)-(1-(3,4-dichlorophenyl)hepta-1,2-dien-1-yl)(methyl)diphenylsilane (7f)}

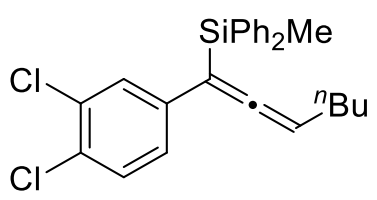

Performed at $100{ }^{\circ} \mathrm{C}$ for $36 \mathrm{~h}$. Colorless oil, $82 \%$ yield $(17.9 \mathrm{mg}), 95 \%$ ee. HPLC condition: Phenomenex, Cellulose-3 column, $3 \mathrm{um}+5 \mathrm{um}, \mathrm{MeOH}$, flow rate $=0.8$ $\mathrm{mL} / \mathrm{min}, 230 \mathrm{~nm} \mathrm{UV}$ detector, $t_{\mathrm{R}}=12.85 \mathrm{~min}$ (major) and $t_{\mathrm{R}}=13.91 \mathrm{~min}$ (minor). $[\alpha]_{\mathrm{D}}{ }^{24}$ $=-58.4\left(\mathrm{c} \mathrm{1.0,} \mathrm{CHCl}_{3}\right)$.

$\underline{{ }^{1} \mathrm{H}} \underline{\mathrm{NMR}} \underline{(400} \underline{\mathrm{MHz}}, \underline{\left.\mathrm{CDCl}_{3}\right)}$

$\delta 7.60-7.51(\mathrm{~m}, 4 \mathrm{H}), 7.43-7.31(\mathrm{~m}, 7 \mathrm{H}), 7.18(\mathrm{~d}, J=8.3 \mathrm{~Hz}, 1 \mathrm{H}), 6.97$ (dd, $J=8.3,2.2 \mathrm{~Hz}, 1 \mathrm{H}), 5.17(\mathrm{t}, J=6.8 \mathrm{~Hz}, 1 \mathrm{H}), 2.06-1.91(\mathrm{~m}, 2 \mathrm{H}), 1.31-1.23$ $(\mathrm{m}, 4 \mathrm{H}), 0.90-0.81(\mathrm{~m}, 3 \mathrm{H}), 0.69(\mathrm{~s}, 3 \mathrm{H})$.

\section{$\underline{{ }^{13} \mathrm{C}} \underline{\mathrm{NMR}} \underline{(101} \underline{\mathrm{MHz}}, \underline{\left.\mathrm{CDCl}_{3}\right)}$}

$\delta 211.4,137.9,135.5,135.4,135.0$ (2C signal), 132.2, 130.0, 129.8, 129.6 (3C signal), 127.9 (2C signal), 127.2, 95.8, 89.2, 31.5, 27.7, 22.3, 13.9, -2.4.

$\underline{\operatorname{HRMS}} \underline{(\mathrm{EI})}$

Calcd for $\mathrm{C}_{26} \mathrm{H}_{26} \mathrm{Cl}_{2} \mathrm{Si}[\mathrm{M}]^{+}: 436.1181$, found: 436.1176 .

(-)-(1-(3,4-dimethylphenyl)hepta-1,2-dien-1-yl)(methyl)diphenylsilane (7g)

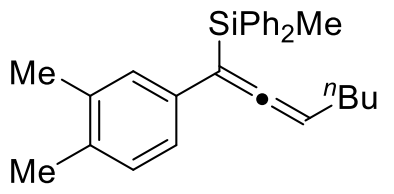


Performed at $100{ }^{\circ} \mathrm{C}$ for $24 \mathrm{~h}$. Colorless oil, 84\% yield (16.6 mg), 93\% ee. HPLC condition: Chiralpak IF-3 column, $n$-hexane $/ i-\mathrm{PrOH}=99.5: 0.5$, flow rate $=0.5 \mathrm{~mL} / \mathrm{min}$, $230 \mathrm{~nm} \mathrm{UV} \mathrm{detector,} t_{\mathrm{R}}=7.53 \mathrm{~min}$ (minor) and $t_{\mathrm{R}}=8.00 \mathrm{~min}$ (major). $[\alpha]_{\mathrm{D}}{ }^{21}=-36.4(\mathrm{c}$ $\left.1.0, \mathrm{CHCl}_{3}\right)$.

\section{$\underline{{ }^{1} \mathrm{H}} \underline{\mathrm{NMR}} \underline{(400} \underline{\mathrm{MHz}}, \underline{\left.\mathrm{CDCl}_{3}\right)}$}

$\delta 7.62-7.53(\mathrm{~m}, 4 \mathrm{H}), 7.40-7.29(\mathrm{~m}, 6 \mathrm{H}), 7.06(\mathrm{~s}, 1 \mathrm{H}), 6.96-6.89(\mathrm{~m}, 2 \mathrm{H})$, $5.07(\mathrm{t}, J=6.8 \mathrm{~Hz}, 1 \mathrm{H}), 2.17(\mathrm{~s}, 3 \mathrm{H}), 2.13(\mathrm{~s}, 3 \mathrm{H}), 1.99-1.89(\mathrm{~m}, 2 \mathrm{H}), 1.32-$ $1.19(\mathrm{~m}, 4 \mathrm{H}), 0.88-0.79(\mathrm{~m}, 3 \mathrm{H}), 0.70(\mathrm{~s}, 3 \mathrm{H})$.

${ }^{13} \mathrm{C} \underline{\mathrm{NMR}} \underline{(101} \underline{\mathrm{MHz}}, \underline{\left.\mathrm{CDCl}_{3}\right)}$

$\delta 210.8,136.5$ (2C signal), 136.3, 135.1 (2C signal), 134.7, 134.4, 129.5, 129.2 (2C signal), 127.7 (3C signal), 125.4, 96.7, 88.3, 31.6, 27.9, 22.3, 19.8, 19.3, $13.9,-2.3$.

$\underline{\operatorname{HRMS}} \underline{(\mathrm{EI})}$

Calcd for $\mathrm{C}_{28} \mathrm{H}_{32} \mathrm{Si}[\mathrm{M}]^{+}:$396.2273, found: 396.2263 .

\section{(-)-(1-(benzofuran-5-yl)hepta-1,2-dien-1-yl)(methyl)diphenylsilane (7h)}

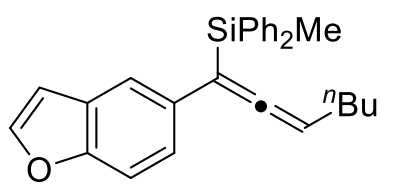

Performed at $100{ }^{\circ} \mathrm{C}$ for $36 \mathrm{~h}$. Colorless oil, $80 \%$ yield $(16.3 \mathrm{mg}), 91 \%$ ee. HPLC condition: Phenomenex, Cellulose-3 column, 3um $+5 \mathrm{um}, \mathrm{MeOH}$, flow rate $=0.8$ $\mathrm{mL} / \mathrm{min}, 230 \mathrm{~nm}$ UV detector, $t_{\mathrm{R}}=13.09 \mathrm{~min}$ (major) and $t_{\mathrm{R}}=13.78 \mathrm{~min}$ (minor). $[\alpha]_{\mathrm{D}}{ }^{24}$ $=-40.0\left(\mathrm{c} 1.0, \mathrm{CHCl}_{3}\right)$.

$\underline{{ }^{1} \mathrm{H}} \underline{\mathrm{NMR}} \underline{(400} \underline{\mathrm{MHz}}, \underline{\left.\mathrm{CDCl}_{3}\right)}$

$\delta 7.64-7.55(\mathrm{~m}, 4 \mathrm{H}), 7.52(\mathrm{~d}, J=2.1 \mathrm{~Hz}, 1 \mathrm{H}), 7.43-7.29(\mathrm{~m}, 8 \mathrm{H}), 7.22(\mathrm{dd}$, $J=8.6,1.8 \mathrm{~Hz}, 1 \mathrm{H}), 6.60(\mathrm{~d}, J=2.2 \mathrm{~Hz}, 1 \mathrm{H}), 5.12(\mathrm{t}, J=6.8 \mathrm{~Hz}, 1 \mathrm{H}), 2.04-$ $1.91(\mathrm{~m}, 2 \mathrm{H}), 1.31-1.22(\mathrm{~m}, 4 \mathrm{H}), 0.89-0.80(\mathrm{~m}, 3 \mathrm{H}), 0.72(\mathrm{~s}, 3 \mathrm{H})$.

${ }^{13} \mathrm{C} \underline{\mathrm{NMR}} \underline{(101} \underline{\mathrm{MHz}}, \underline{\left.\mathrm{CDCl}_{3}\right)}$

$\delta 211.0,153.7,145.0,136.4$ (2C signal), 135.1 (2C signal), 132.2, 129.3, 127.8, 127.5 (3C signal), 124.7, 120.3, 111.0, 106.6, 97.1, 88.3, 31.6, 27.9, 22.3, 13.9, -2.3 .

$\underline{\operatorname{HRMS}} \underline{(\mathrm{EI})}$

Calcd for $\mathrm{C}_{28} \mathrm{H}_{28} \mathrm{OSi}[\mathrm{M}]^{+}:$408.1909, found: 408.1902 . 
<smiles>CCCC=CC(C)(C)C</smiles>

$10 \mathrm{~mol} \%\left(\mathrm{Ph}_{3} \mathrm{P}\right)_{2} \mathrm{Pt}^{\cdot} \cdot \mathrm{O}_{2}$ was used and the reaction was performed at $105{ }^{\circ} \mathrm{C}$ for $48 \mathrm{~h}$. Colorless oil, 50\% yield ( $8.4 \mathrm{mg}$ ), 86\% ee. HPLC condition: Phenomenex, Cellulose-3 column, $\mathrm{MeOH}$, flow rate $=1.0 \mathrm{~mL} / \mathrm{min}, 230 \mathrm{~nm} \mathrm{UV}$ detector, $t_{\mathrm{R}}=4.31 \mathrm{~min}$ (major) and $t_{\mathrm{R}}=4.87 \mathrm{~min}$ (minor). $[\alpha]_{\mathrm{D}}{ }^{26}=-28.8\left(\mathrm{c} 0.5, \mathrm{CHCl}_{3}\right)$.

${ }^{1} \mathrm{H} \underline{\mathrm{NMR}} \underline{(400} \underline{\mathrm{MHz}}, \underline{\left.\mathrm{CDCl}_{3}\right)}$

$\delta 7.81-7.72(\mathrm{~m}, 3 \mathrm{H}), 7.66(\mathrm{~s}, 1 \mathrm{H}), 7.48(\mathrm{dd}, J=8.5,1.8 \mathrm{~Hz}, 1 \mathrm{H}), 7.46-7.36$ $(\mathrm{m}, 2 \mathrm{H}), 5.17(\mathrm{t}, J=6.8 \mathrm{~Hz}, 1 \mathrm{H}), 2.20-2.15(\mathrm{~m}, 2 \mathrm{H}), 1.52-1.43(\mathrm{~m}, 2 \mathrm{H})$, $1.43-1.35(\mathrm{~m}, 2 \mathrm{H}), 1.03-0.94(\mathrm{~m}, 9 \mathrm{H}), 0.91(\mathrm{t}, J=7.2 \mathrm{~Hz}, 3 \mathrm{H}), 0.83-0.74$ $(\mathrm{m}, 6 \mathrm{H})$.

\section{$\underline{{ }^{13} \mathrm{C}} \underline{\mathrm{NMR}} \underline{(101} \underline{\mathrm{MHz}}, \underline{\left.\mathrm{CDCl}_{3}\right)}$}

$\delta 209.9,136.2,133.7,132.0,127.8,127.7,127.5,126.6,125.9,125.4,125.2$, 97.0, 86.8, 31.9, 28.2, 22.4, 13.9, 7.4, 4.1.

\section{$\underline{\operatorname{HRMS}} \underline{(\mathrm{EI})}$}

Calcd for $\mathrm{C}_{23} \mathrm{H}_{32} \mathrm{Si}[\mathrm{M}]^{+}$: 336.2273, found: 336.2267 .

\section{(-)-triethyl(1-(naphthalen-2-yl)-3-phenylpropa-1,2-dien-1-yl)silane (7j)}<smiles>CCCC=CC=Cc1ccc2ccccc2c1</smiles>

Performed at $105{ }^{\circ} \mathrm{C}$ for $48 \mathrm{~h}$. Colorless oil, 64\% yield $(11.4 \mathrm{mg}), 84 \%$ ee. HPLC condition: Chiralpak AD-3 column, $n$-hexane $/ i-\mathrm{PrOH}=99.2: 0.8$, flow rate $=0.8$ $\mathrm{mL} / \mathrm{min}, 254 \mathrm{~nm} \mathrm{UV}$ detector, $t_{\mathrm{R}}=5.12 \mathrm{~min}$ (minor) and $t_{\mathrm{R}}=5.48 \mathrm{~min}$ (major). $[\alpha]_{\mathrm{D}}{ }^{26}$ $=-105\left(\mathrm{c} 0.5, \mathrm{CHCl}_{3}\right)$.

\section{$\underline{{ }^{1} \mathrm{H}} \underline{\mathrm{NMR}} \underline{(400} \underline{\mathrm{MHz}}, \underline{\left.\mathrm{CDCl}_{3}\right)}$}

$\delta 7.82-7.72(\mathrm{~m}, 4 \mathrm{H}), 7.52(\mathrm{dd}, J=8.6,1.7 \mathrm{~Hz}, 1 \mathrm{H}), 7.46-7.39(\mathrm{~m}, 2 \mathrm{H}), 7.36$

$-7.27(\mathrm{~m}, 4 \mathrm{H}), 7.21-7.14(\mathrm{~m}, 1 \mathrm{H}), 6.26(\mathrm{~s}, 1 \mathrm{H}), 1.00(\mathrm{t}, J=7.7 \mathrm{~Hz}, 9 \mathrm{H}), 0.89$

$-0.80(\mathrm{~m}, 6 \mathrm{H})$.

$\underline{{ }^{13} \mathrm{C}} \underline{\mathrm{NMR}} \underline{(101} \underline{\mathrm{MHz}}, \underline{\left.\mathrm{CDCl}_{3}\right)}$

$\delta 210.8,134.8,134.5,133.6,132.3,128.7,128.1,127.8,127.6,126.5,126.4$, $126.3,126.0,125.8,125.6,101.8,91.1,7.5,4.1$.

\section{$\underline{\operatorname{HRMS}} \underline{(\mathrm{EI})}$}


Calcd for $\mathrm{C}_{25} \mathrm{H}_{28} \mathrm{Si}[\mathrm{M}]^{+}: 356.1960$, found: 356.1953 .

(-)-dimethyl(1-(naphthalen-2-yl)-3-phenylpropa-1,2-dien-1-yl)(phenyl)silane (7k)<smiles>CCCCC=Cc1ccc2ccccc2c1</smiles>

Performed at $90{ }^{\circ} \mathrm{C}$ for $48 \mathrm{~h}$. Colorless oil, $47 \%$ yield $(8.9 \mathrm{mg}), 87 \%$ ee. HPLC condition: Chiralpak AD-3 column, $n$-hexane $/ i-\mathrm{PrOH}=99: 1$, flow rate $=1.0 \mathrm{~mL} / \mathrm{min}, 230 \mathrm{~nm} \mathrm{UV}$ detector, $t_{\mathrm{R}}=4.57 \mathrm{~min}$ (minor) and $t_{\mathrm{R}}=5.07 \mathrm{~min}$ (major). $[\alpha]_{\mathrm{D}}{ }^{26}=-74.0\left(\mathrm{c} 0.5, \mathrm{CHCl}_{3}\right)$. $\underline{{ }^{1} \mathrm{H}} \underline{\mathrm{NMR}} \underline{(400} \underline{\mathrm{MHz}}, \underline{\left.\mathrm{CDCl}_{3}\right)}$

$\delta 7.78-7.72(\mathrm{~m}, 1 \mathrm{H}), 7.71-7.66(\mathrm{~m}, 3 \mathrm{H}), 7.64-7.59(\mathrm{~m}, 2 \mathrm{H}), 7.47(\mathrm{dd}, J=$ 8.5, $1.8 \mathrm{~Hz}, 1 \mathrm{H}), 7.41-7.34(\mathrm{~m}, 8 \mathrm{H}), 7.33-7.31(\mathrm{~m}, 1 \mathrm{H}), 7.23-7.17$ (m, $1 \mathrm{H}), 6.37(\mathrm{~s}, 1 \mathrm{H}), 0.56(\mathrm{~s}, 3 \mathrm{H}), 0.54(\mathrm{~s}, 3 \mathrm{H})$.

${ }^{13} \mathrm{C} \underline{\mathrm{NMR}} \underline{(101} \underline{\mathrm{MHz}}, \underline{\left.\mathrm{CDCl}_{3}\right)}$

$\delta 211.4,138.0,134.5,134.0,133.5,133.4$ (2C signal), 132.3, 129.4, 128.8 (2C signal), 128.0, 127.9, 127.5, 126.6 (2C signal), 126.3, 126.0, 125.6, 103.3, 92.2, -1.6 (2C signal).

\section{$\underline{\operatorname{HRMS}} \underline{(\mathrm{EI})}$}

Calcd for $\mathrm{C}_{27} \mathrm{H}_{24} \mathrm{Si}[\mathrm{M}]^{+}: 376.1647$, found: 376.1637 . 


\section{Kinetic Isotopic Effect Experiment for Si-H Insertion Reaction}

\section{Parallel KIE Experiment}

In a $25 \mathrm{ml}$ oven-dried Schlenk tube, alkynyl sulfonylhydrazone 1a (107.2 mg, 0.2 $\mathrm{mmol}), \mathrm{LiO}^{t} \mathrm{Bu}(32.0 \mathrm{mg}, 0.4 \mathrm{mmol}), \mathbf{C 1}(3.0 \mathrm{mg}, 0.002 \mathrm{mmol}$ ) and 1,3,5trimethyloxylbenzene $(16.8 \mathrm{mg}, 0.1 \mathrm{mmol})$ were added. After the sealed tube was evacuated and backfilled with Ar for three times, the mixture was cooled to $-10{ }^{\circ} \mathrm{C}$. At this temperature, dimethylphenyl silane $2 \mathbf{a}$ or $\mathbf{2 a - d _ { 1 }}(54.5 \mathrm{mg}, 0.4 \mathrm{mmol})$ and $3 \mathrm{~mL}$ dry and cooled dichloromethane were injected into the Schlenk tube by syringe. The reaction mixture was stirred at $-10{ }^{\circ} \mathrm{C}$. At the indicated reaction time, $100 \mu \mathrm{L}$ reaction mixture was extracted with a syringe, which was added in $\mathrm{CDCl}_{3}$ (Note: $2 \mu \mathrm{L}$ formic acid was added to quench the reaction) for NMR analysis.

According to the parallel KIE experiment, the initial rate data of the reaction demonstrate that the $\mathrm{kH} / \mathrm{kD}$ almost is 1.0 .

Table S2. Parallel KIE Experiment

\begin{tabular}{|c|c|c|c|c|c|c|}
\hline $\mathrm{t} / \mathrm{min}$ & 0 & 5 & 10 & 20 & 40 & 80 \\
\hline $\mathbf{3 a a} / \%$ & 0 & 5.0 & 7.5 & 13.5 & 21.5 & 36.0 \\
\hline 3aa-d $/ \%$ & 0 & 4.5 & 8.0 & 12.5 & 21.5 & 35.0 \\
\hline
\end{tabular}

${ }^{a}$ Reaction conditions: 1a $(0.2 \mathrm{mmol}), \mathrm{LiO}^{t} \mathrm{Bu}(0.4 \mathrm{mmol}), \mathbf{2 a}(0.4 \mathrm{mmol}), \mathbf{C 1}(0.002$ mmol), $3 \mathrm{~mL} \mathrm{CH}_{2} \mathrm{Cl}_{2},-10{ }^{\circ} \mathrm{C}$. Yields were calculated from ${ }^{1} \mathrm{H}$ NMR spectroscopy with 1,3,5-trimethyloxybenzene as the internal standard.

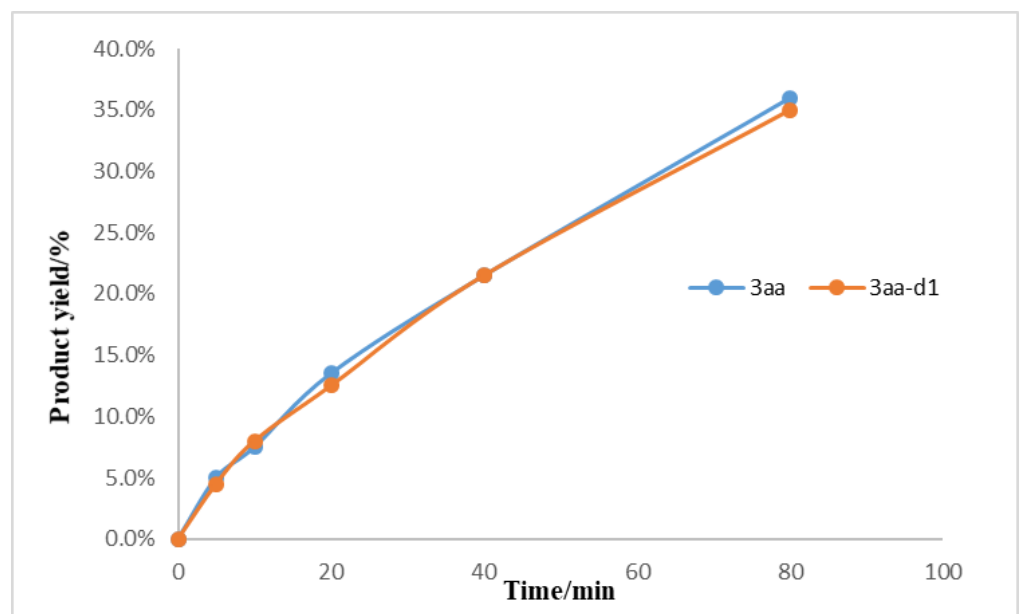




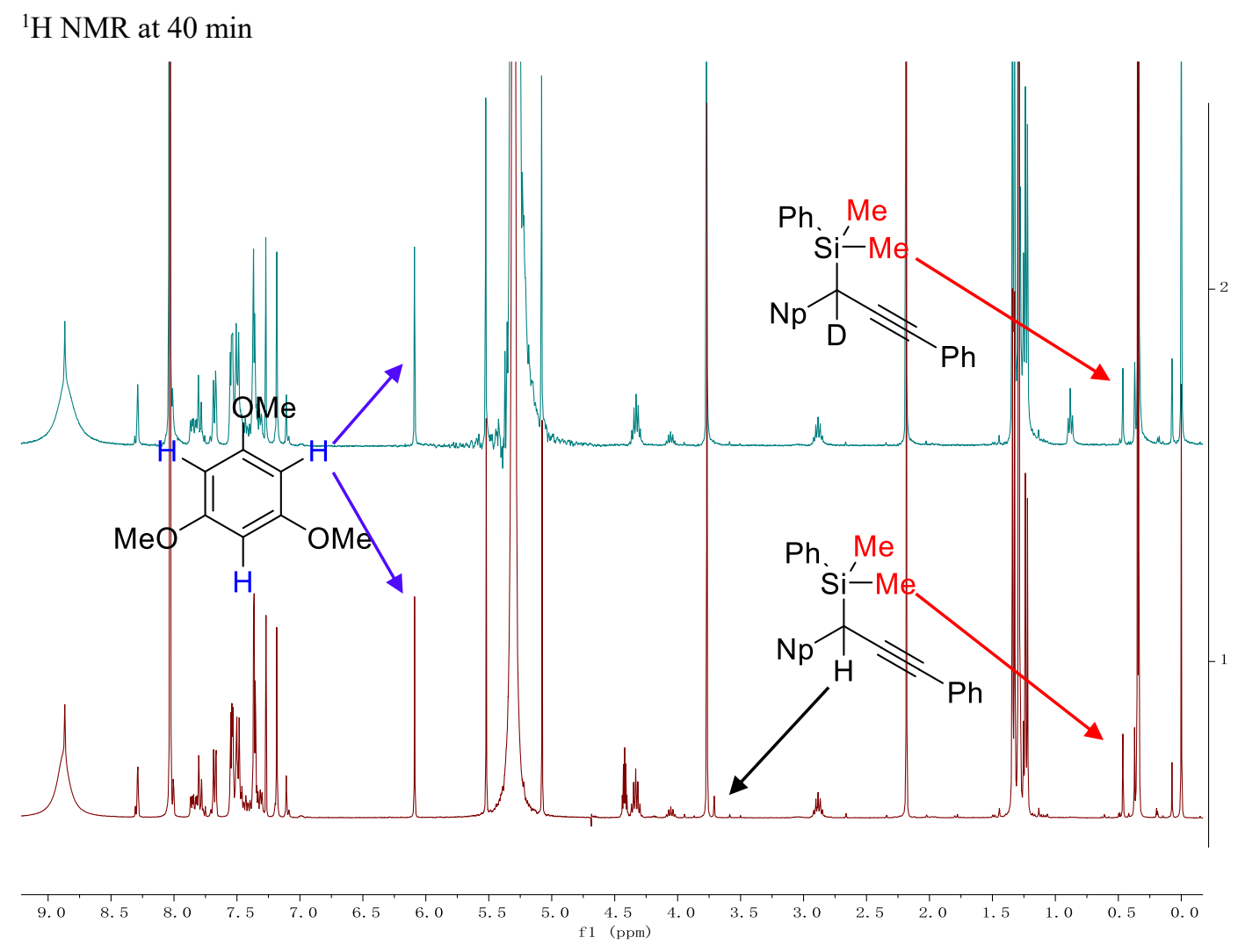




\section{Crystal data and structure refinement}

The crystal structure of compound $(S)-7 \mathbf{b}$ has been deposited at the Cambridge Crystallographic Data Centre (CCDC 2070326). The data is available free of charge at www.ccdc.cam.ac.uk/conts/retrieving.html.<smiles>C#CCC(C)=CC(C)C</smiles>

$(S)-7 \mathbf{b}$

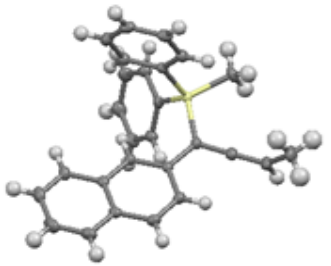

Table S3 Crystal data and structure refinement for $(S)-7 b$.

Identification code

Empirical formula

Formula weight

Temperature/K

Crystal system

Space group

$\mathrm{a} / \AA ̊$

$\mathrm{b} / \AA$

$\mathrm{c} / \AA$

$\alpha /{ }^{\circ}$

$\beta /{ }^{\circ}$

$\gamma^{\circ}$

Volume $/ \AA^{3}$

Z

$\rho_{\text {calc }} / \mathrm{cm}^{3}$

$\mu / \mathrm{mm}^{-1}$

$\mathrm{F}(000)$

Crystal size $/ \mathrm{mm}^{3}$

Radiation r20210315a

$\mathrm{C}_{27} \mathrm{H}_{24} \mathrm{Si}$

376.55

113.15

orthorhombic

$\mathrm{P} 2{ }_{1} 2_{1} 2_{1}$

$8.6331(2)$

$10.3225(3)$

24.0864(6)

90

90

90

2146.46(10)

4

1.165

0.118

800.0

$0.23 \times 0.2 \times 0.19$

$\operatorname{MoK} \alpha(\lambda=0.71073)$

$2 \Theta$ range for data collection $/{ }^{\circ} 4.292$ to 65.668

Index ranges

$-13 \leqslant \mathrm{~h} \leqslant 13,-15 \leqslant \mathrm{k} \leqslant 15,-34 \leqslant 1 \leqslant 36$

Reflections collected

32687

Independent reflections

$7527\left[\mathrm{R}_{\text {int }}=0.0439, \mathrm{R}_{\text {sigma }}=0.0366\right]$

Data/restraints/parameters

$7527 / 0 / 255$

Goodness-of-fit on $\mathrm{F}^{2}$

1.034

Final $\mathrm{R}$ indexes $[\mathrm{I}>=2 \sigma(\mathrm{I})]$

$\mathrm{R}_{1}=0.0397, \mathrm{wR}_{2}=0.0917$

Final $\mathrm{R}$ indexes [all data] $\quad \mathrm{R}_{1}=0.0460, \mathrm{wR}_{2}=0.0952$

Largest diff. peak/hole / e $\AA^{-3} 0.26 /-0.26$

Flack parameter

$-0.05(4)$ 


\section{Computational Studies}

1a, 2a and C1 was used as the model molecular.

Conformational searches were conducted by restraining the position of the rhodium and rhodium-bound atoms. Geometry optimizations and frequency calculations were performed with Gaussian16 $6^{11}$ at the B3LYP-D3/6-31G(d)-LANL2DZ(Rh,I) level of theory before single point energy corrections at the B3LYP-D3/6-311++G(d,p)$\operatorname{SDD}(\mathrm{Rh}, \mathrm{I}) / \mathrm{SMD}(\mathrm{DCM})$ level of theory. Reaction paths were traced by the intrinsic reaction coordinate method for all transition states. All energetics reported throughout the text are in $\mathrm{kcal} / \mathrm{mol}$. Structures were generated using CYLview ${ }^{12}$.

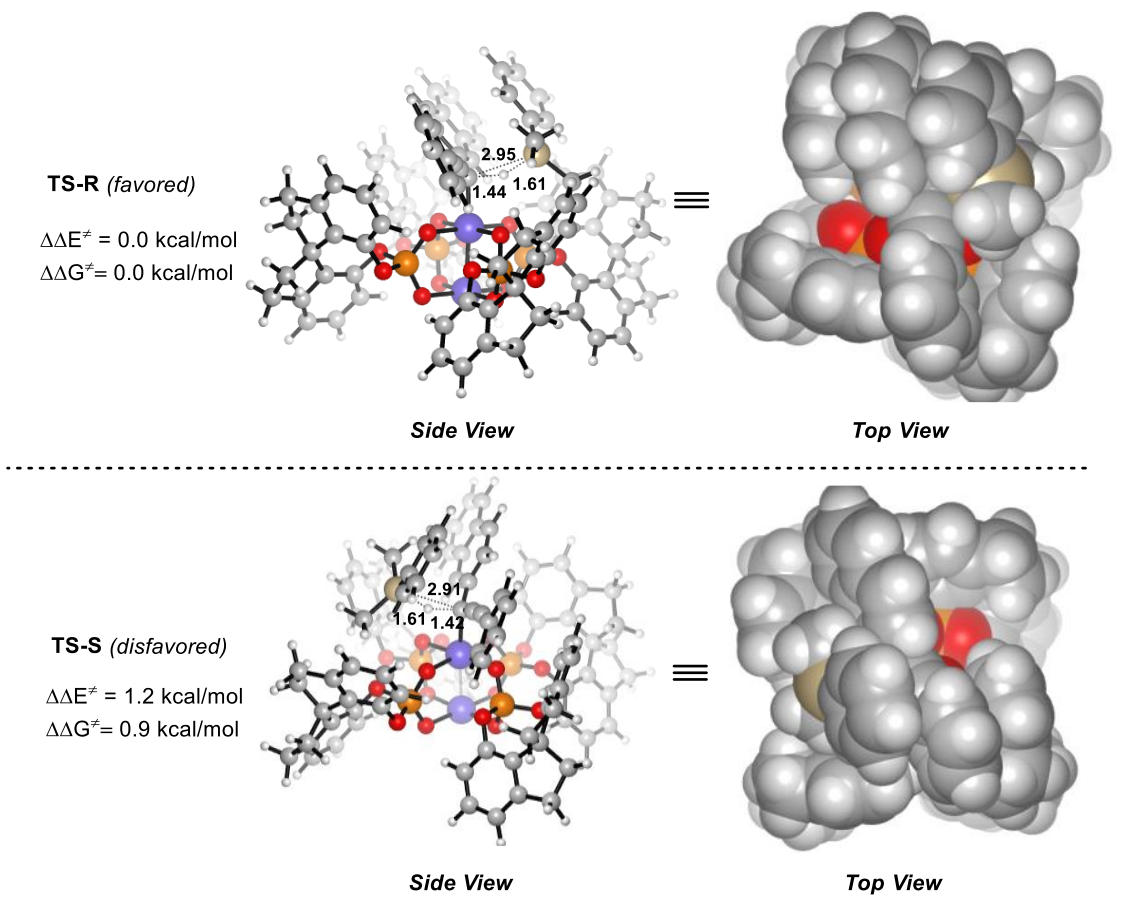

Fig S1. Density functional theory calculations for enantioselectivity. Geometry optimizations and frequency calculations were performed with Gaussian16 at the B3LYP-D3/6-31G(d)LANL2DZ(Rh,I) level of theory before single point energy corrections at the B3LYP-D3/6$311++\mathrm{G}(\mathrm{d}, \mathrm{p})-\mathrm{SDD}(\mathrm{Rh}, \mathrm{I}) / \mathrm{SMD}(\mathrm{DCM})$.

Computed Energies (Hartree)

\begin{tabular}{|l|l|l|l|l|}
\hline Structure & $\begin{array}{l}\text { Electronic } \\
\text { Energy }\end{array}$ & Zero Point Energy & Enthalpy & Free Energy \\
\hline TS-R & -6750.2816 & -6748.7327 & -6748.6338 & -6748.8658 \\
\hline TS-S & -6750.2797 & -6748.7306 & -6748.6317 & -6748.8644 \\
\hline
\end{tabular}

\section{TS-R}

\begin{tabular}{cccc}
$\mathrm{Rh}$ & -0.13847300 & 0.03744500 & 0.12670400 \\
$\mathrm{Rh}$ & -0.38337400 & 0.05260900 & -2.50888700 \\
$\mathrm{P}$ & -2.49764800 & -1.58613600 & -0.93322500 \\
$\mathrm{P}$ & 1.38773700 & -2.19280000 & -1.35157800 \\
& \multicolumn{3}{c}{$\mathrm{S} 50$}
\end{tabular}




\begin{tabular}{|c|c|c|c|}
\hline $\mathrm{P}$ & 1.98348000 & 1.65544700 & -1.39677900 \\
\hline $\mathrm{P}$ & -1.82601900 & 2.31883000 & -1.04905900 \\
\hline $\mathrm{O}$ & -1.59142100 & -1.46215100 & 0.29131600 \\
\hline $\mathrm{O}$ & -1.94223100 & -1.34357400 & -2.32425700 \\
\hline $\mathrm{O}$ & -3.10834400 & -3.09858000 & -0.99966700 \\
\hline $\mathrm{O}$ & -3.80823300 & -0.65982100 & -0.61913600 \\
\hline $\mathrm{O}$ & 1.34760500 & -1.43656000 & -0.02298800 \\
\hline $\mathrm{O}$ & 0.97391600 & -1.52494500 & -2.64619700 \\
\hline $\mathrm{O}$ & 2.89975100 & -2.71135000 & -1.66274700 \\
\hline $\mathrm{O}$ & 0.55653800 & -3.56404900 & -1.05500000 \\
\hline $\mathrm{O}$ & 1.21301000 & 1.41056900 & -2.68280700 \\
\hline $\mathrm{O}$ & 1.28779800 & 1.50355700 & -0.04755800 \\
\hline $\mathrm{O}$ & 2.56249300 & 3.16637200 & -1.51508500 \\
\hline $\mathrm{O}$ & 3.32915800 & 0.73066700 & -1.28331300 \\
\hline $\mathrm{O}$ & -1.73607700 & 1.64746600 & -2.41029000 \\
\hline $\mathrm{O}$ & -1.60696000 & 1.49220800 & 0.21698200 \\
\hline $\mathrm{O}$ & -0.82794100 & 3.60128600 & -0.91718500 \\
\hline $\mathrm{O}$ & -3.31485000 & 2.97086300 & -1.01132400 \\
\hline $\mathrm{C}$ & -3.74072700 & -3.57301100 & 0.15116000 \\
\hline $\mathrm{C}$ & -2.98691900 & -4.28460400 & 1.08417000 \\
\hline $\mathrm{H}$ & -1.94648700 & -4.48738400 & 0.86210000 \\
\hline $\mathrm{C}$ & -3.57451800 & -4.68950800 & 2.28315600 \\
\hline $\mathrm{H}$ & -2.98311400 & -5.23919100 & 3.00935900 \\
\hline $\mathrm{C}$ & -4.89993900 & -4.34812800 & 2.57240400 \\
\hline $\mathrm{H}$ & -5.34103600 & -4.61693100 & 3.52833100 \\
\hline $\mathrm{C}$ & -5.64024600 & -3.64906700 & 1.62339600 \\
\hline $\mathrm{C}$ & -7.06250100 & -3.14056800 & 1.72119900 \\
\hline $\mathrm{H}$ & -7.78429300 & -3.95650200 & 1.57975300 \\
\hline $\mathrm{H}$ & -7.27761300 & -2.69261900 & 2.69739800 \\
\hline $\mathrm{C}$ & -7.13881500 & -2.12577100 & 0.55979900 \\
\hline $\mathrm{H}$ & -8.14785800 & -1.99693800 & 0.15798200 \\
\hline $\mathrm{H}$ & -6.78478800 & -1.14331700 & 0.88896700 \\
\hline $\mathrm{C}$ & -6.14147900 & -2.67215400 & -0.51053400 \\
\hline $\mathrm{C}$ & -5.08284600 & -3.29122800 & 0.38485400 \\
\hline $\mathrm{C}$ & -4.81271500 & -0.66223600 & -1.60343400 \\
\hline $\mathrm{C}$ & -4.74621700 & 0.25946100 & -2.64731400 \\
\hline $\mathrm{H}$ & -3.93371800 & 0.97439900 & -2.67087700 \\
\hline $\mathrm{C}$ & -5.69740500 & 0.20168900 & -3.66708300 \\
\hline $\mathrm{H}$ & -5.64211800 & 0.91683500 & -4.48251100 \\
\hline $\mathrm{C}$ & -6.67894300 & -0.79505800 & -3.67455600 \\
\hline $\mathrm{H}$ & -7.38314500 & -0.86753500 & -4.49923800 \\
\hline $\mathrm{C}$ & -6.71927700 & -1.71141000 & -2.62837800 \\
\hline $\mathrm{C}$ & -7.61624500 & -2.91908400 & -2.45578000 \\
\hline $\mathrm{H}$ & -8.61330100 & -2.62925800 & -2.09562000 \\
\hline
\end{tabular}




\begin{tabular}{|c|c|c|c|}
\hline $\mathrm{H}$ & -7.76442500 & -3.46983000 & -3.39086500 \\
\hline $\mathrm{C}$ & -6.85849500 & -3.74437800 & -1.39250500 \\
\hline $\mathrm{H}$ & -7.50462200 & -4.40276400 & -0.80359100 \\
\hline $\mathrm{H}$ & -6.09634100 & -4.36326300 & -1.87819700 \\
\hline $\mathrm{C}$ & -5.81014100 & -1.62900700 & -1.56174000 \\
\hline $\mathrm{C}$ & 3.58916800 & -3.36341400 & -0.64535800 \\
\hline $\mathrm{C}$ & 4.41229700 & -2.60632600 & 0.18993900 \\
\hline $\mathrm{H}$ & 4.50523300 & -1.54431300 & -0.00958500 \\
\hline $\mathrm{C}$ & 5.06213800 & -3.22468300 & 1.25699800 \\
\hline $\mathrm{H}$ & 5.69786400 & -2.63503000 & 1.91075400 \\
\hline $\mathrm{C}$ & 4.84273500 & -4.58154200 & 1.52810400 \\
\hline $\mathrm{H}$ & 5.30135900 & -5.04827900 & 2.39598800 \\
\hline $\mathrm{C}$ & 4.00714600 & -5.31554400 & 0.69031800 \\
\hline $\mathrm{C}$ & 3.54754700 & -6.75219600 & 0.82763900 \\
\hline $\mathrm{H}$ & 4.33796200 & -7.45359800 & 0.52836600 \\
\hline $\mathrm{H}$ & 3.27340400 & -7.00377800 & 1.85803000 \\
\hline $\mathrm{C}$ & 2.34616300 & -6.81449400 & -0.14329600 \\
\hline $\mathrm{H}$ & 2.15963400 & -7.81543800 & -0.54287800 \\
\hline $\mathrm{H}$ & 1.43731900 & -6.47909800 & 0.36792500 \\
\hline $\mathrm{C}$ & 2.69754500 & -5.78520000 & -1.26390100 \\
\hline $\mathrm{C}$ & 3.41524800 & -4.72701400 & -0.44067100 \\
\hline $\mathrm{C}$ & 0.50690600 & -4.52351700 & -2.07362600 \\
\hline $\mathrm{C}$ & -0.53100600 & -4.46371800 & -3.00258500 \\
\hline $\mathrm{H}$ & -1.27609500 & -3.68273400 & -2.90713600 \\
\hline $\mathrm{C}$ & -0.55539600 & -5.38201500 & -4.05283600 \\
\hline $\mathrm{H}$ & -1.35955000 & -5.33500900 & -4.78116300 \\
\hline $\mathrm{C}$ & 0.46859900 & -6.32391100 & -4.19871500 \\
\hline $\mathrm{H}$ & 0.47141500 & -7.00577500 & -5.04498400 \\
\hline $\mathrm{C}$ & 1.50070500 & -6.35495200 & -3.26576300 \\
\hline $\mathrm{C}$ & 2.74633800 & -7.21406200 & -3.26242000 \\
\hline $\mathrm{H}$ & 2.53285700 & -8.22524700 & -2.88856300 \\
\hline $\mathrm{H}$ & 3.18140800 & -7.32937000 & -4.26088000 \\
\hline $\mathrm{C}$ & 3.67110900 & -6.44683300 & -2.29459100 \\
\hline $\mathrm{H}$ & 4.42257900 & -7.07801700 & -1.81098100 \\
\hline $\mathrm{H}$ & 4.19547900 & -5.65248700 & -2.83671400 \\
\hline $\mathrm{C}$ & 1.51471500 & -5.47637500 & -2.16980600 \\
\hline $\mathrm{C}$ & 4.23104500 & 0.80339800 & -2.35599600 \\
\hline $\mathrm{C}$ & 4.07734700 & -0.08358700 & -3.42156000 \\
\hline $\mathrm{H}$ & 3.27944300 & -0.81466900 & -3.38358000 \\
\hline $\mathrm{C}$ & 4.92309200 & 0.02354900 & -4.52592700 \\
\hline $\mathrm{H}$ & 4.80058500 & -0.66381500 & -5.35787100 \\
\hline $\mathrm{C}$ & 5.88445100 & 1.03760100 & -4.58939800 \\
\hline $\mathrm{H}$ & 6.50581100 & 1.15167000 & -5.47385900 \\
\hline $\mathrm{C}$ & 6.01020800 & 1.92032400 & -3.5212020 \\
\hline
\end{tabular}




\begin{tabular}{|c|c|c|c|}
\hline $\mathrm{C}$ & 6.89819200 & 3.14064900 & -3.40441900 \\
\hline $\mathrm{H}$ & 7.92889200 & 2.86166900 & -3.14420800 \\
\hline $\mathrm{H}$ & 6.94954500 & 3.71296400 & -4.33697100 \\
\hline $\mathrm{C}$ & 6.22778700 & 3.92569700 & -2.25704600 \\
\hline $\mathrm{H}$ & 6.91207800 & 4.58971800 & -1.72055900 \\
\hline $\mathrm{H}$ & 5.40792400 & 4.53376600 & -2.65443900 \\
\hline $\mathrm{C}$ & 5.62334700 & 2.82131800 & -1.33127000 \\
\hline $\mathrm{C}$ & 5.21146100 & 1.79049600 & -2.37250500 \\
\hline $\mathrm{C}$ & 3.27950300 & 3.62230500 & -0.39906800 \\
\hline $\mathrm{C}$ & 2.58037400 & 4.21637600 & 0.6520700 \\
\hline $\mathrm{H}$ & 1.50475700 & 4.32355400 & 0.56737700 \\
\hline $\mathrm{C}$ & 3.27272700 & 4.59901700 & 1.80034600 \\
\hline $\mathrm{H}$ & 2.72816300 & 5.04524300 & 2.6268990 \\
\hline $\mathrm{C}$ & 4.64640000 & 4.35817200 & 1.91612300 \\
\hline $\mathrm{H}$ & 5.17149100 & 4.60645500 & 2.83308600 \\
\hline $\mathrm{C}$ & 5.31885200 & 3.74764800 & 0.86310400 \\
\hline $\mathrm{C}$ & 6.76409200 & 3.30705500 & 0.78644300 \\
\hline $\mathrm{H}$ & 7.42554500 & 4.15145800 & 0.54758100 \\
\hline $\mathrm{H}$ & 7.11708000 & 2.87554400 & 1.72722000 \\
\hline $\mathrm{C}$ & 6.73203600 & 2.28435700 & -0.36881600 \\
\hline $\mathrm{H}$ & 7.69489100 & 2.15650600 & -0.87255900 \\
\hline $\mathrm{H}$ & 6.41684000 & 1.30858400 & 0.01739500 \\
\hline $\mathrm{C}$ & 4.64698300 & 3.39776300 & -0.31993300 \\
\hline $\mathrm{C}$ & -0.93404600 & 4.59420200 & -1.90195600 \\
\hline $\mathrm{C}$ & -0.16297000 & 4.48912600 & -3.05902500 \\
\hline $\mathrm{H}$ & 0.50902100 & 3.64724600 & -3.16845300 \\
\hline $\mathrm{C}$ & -0.30713700 & 5.44882500 & -4.06175000 \\
\hline $\mathrm{H}$ & 0.29057200 & 5.36619900 & -4.96484400 \\
\hline $\mathrm{C}$ & -1.24173100 & 6.48148100 & -3.93190400 \\
\hline $\mathrm{H}$ & -1.38305300 & 7.19982600 & -4.73528600 \\
\hline $\mathrm{C}$ & -2.01021700 & 6.55744600 & -2.77441800 \\
\hline $\mathrm{C}$ & -3.13004800 & 7.51980000 & -2.44213300 \\
\hline $\mathrm{H}$ & -2.73560500 & 8.48889800 & -2.10556500 \\
\hline $\mathrm{H}$ & -3.78104900 & 7.72193500 & -3.29962800 \\
\hline $\mathrm{C}$ & -3.86124000 & 6.78902800 & -1.29632500 \\
\hline $\mathrm{H}$ & -4.40701200 & 7.45883300 & -0.62499300 \\
\hline $\mathrm{H}$ & -4.57708100 & 6.07224600 & -1.71318500 \\
\hline $\mathrm{C}$ & -2.73605000 & 5.99321500 & -0.55665100 \\
\hline $\mathrm{C}$ & -1.84156700 & 5.63294500 & -1.73085900 \\
\hline $\mathrm{C}$ & -3.67658300 & 3.62264200 & 0.17475300 \\
\hline $\mathrm{C}$ & -4.32705900 & 2.88718100 & 1.16418700 \\
\hline $\mathrm{H}$ & -4.55890000 & 1.84680800 & 0.97088700 \\
\hline $\mathrm{C}$ & -4.61579400 & 3.48693800 & 2.38977800 \\
\hline $\mathrm{H}$ & -5.12252500 & 2.91312700 & 3.15804600 \\
\hline
\end{tabular}




\begin{tabular}{|c|c|c|c|}
\hline $\mathrm{C}$ & -4.20368200 & 4.79928600 & 2.64655600 \\
\hline $\mathrm{H}$ & -4.37655200 & 5.24708900 & 3.62192000 \\
\hline $\mathrm{C}$ & -3.54958700 & 5.51483500 & 1.64762000 \\
\hline $\mathrm{C}$ & -2.94883700 & 6.90379300 & 1.71226500 \\
\hline $\mathrm{H}$ & -2.40794400 & 7.08257200 & 2.64811800 \\
\hline $\mathrm{H}$ & -3.72530600 & 7.67800100 & 1.64102500 \\
\hline $\mathrm{C}$ & -2.02503400 & 6.92609900 & 0.47384200 \\
\hline $\mathrm{H}$ & -1.05245800 & 6.48975700 & 0.72731500 \\
\hline $\mathrm{H}$ & -1.85122100 & 7.92994700 & 0.07483400 \\
\hline $\mathrm{C}$ & -3.32310300 & 4.95095400 & 0.38109300 \\
\hline $\mathrm{C}$ & 2.77929500 & -0.75609100 & 6.11943400 \\
\hline $\mathrm{C}$ & 1.68033600 & -1.20551700 & 5.39174800 \\
\hline $\mathrm{C}$ & 1.83585600 & -2.13130000 & 4.34298600 \\
\hline $\mathrm{C}$ & 3.13075100 & -2.60101000 & 4.06075200 \\
\hline $\mathrm{C}$ & 4.23128800 & -2.15693300 & 4.79072200 \\
\hline $\mathrm{C}$ & 4.05858600 & -1.22607100 & 5.81567700 \\
\hline $\mathrm{H}$ & 2.64298700 & -0.02223700 & 6.90820900 \\
\hline $\mathrm{H}$ & 0.69578900 & -0.80913000 & 5.62388800 \\
\hline $\mathrm{H}$ & 3.28678700 & -3.30253100 & 3.24998000 \\
\hline $\mathrm{H}$ & 5.22530700 & -2.52210100 & 4.54819000 \\
\hline $\mathrm{H}$ & 4.91833400 & -0.85369300 & 6.36459600 \\
\hline $\mathrm{Si}$ & 0.40356800 & -2.58661600 & 3.24811200 \\
\hline $\mathrm{H}$ & 0.26733500 & -1.27469800 & 2.33130600 \\
\hline $\mathrm{C}$ & -1.23941600 & -2.68640600 & 4.14179600 \\
\hline $\mathrm{H}$ & -1.38287900 & -1.85128500 & 4.83257700 \\
\hline $\mathrm{H}$ & -2.06395500 & -2.67719100 & 3.42366800 \\
\hline $\mathrm{H}$ & -1.29256600 & -3.62060700 & 4.71527900 \\
\hline $\mathrm{C}$ & 0.74715200 & -4.01321400 & 2.09217800 \\
\hline $\mathrm{H}$ & 0.56668600 & -4.96993800 & 2.59876700 \\
\hline $\mathrm{H}$ & 0.10423400 & -3.94050100 & 1.21379300 \\
\hline $\mathrm{H}$ & 1.77697500 & -3.99595800 & 1.72920800 \\
\hline $\mathrm{C}$ & 0.02882300 & 0.14374900 & 2.19312400 \\
\hline $\mathrm{C}$ & -1.21823400 & 0.32034700 & 2.80819700 \\
\hline $\mathrm{C}$ & -2.34263400 & 0.39011000 & 3.27328500 \\
\hline $\mathrm{C}$ & -3.66831700 & 0.44269000 & 3.76733300 \\
\hline $\mathrm{C}$ & -4.72587900 & -0.09197600 & 3.00233900 \\
\hline $\mathrm{C}$ & -6.03064600 & -0.00685500 & 3.47919900 \\
\hline $\mathrm{C}$ & -6.29834100 & 0.59553000 & 4.71220700 \\
\hline $\mathrm{C}$ & -5.25259700 & 1.11431000 & 5.48040800 \\
\hline $\mathrm{C}$ & -3.94439700 & 1.03832700 & 5.01366200 \\
\hline $\mathrm{H}$ & -4.50270900 & -0.54631000 & 2.04205400 \\
\hline $\mathrm{H}$ & -6.84806700 & -0.40056300 & 2.88533300 \\
\hline $\mathrm{H}$ & -7.32104200 & 0.66114300 & 5.07222700 \\
\hline $\mathrm{H}$ & -5.45931000 & 1.58120100 & 6.4389870 \\
\hline
\end{tabular}




$\begin{array}{lrrr}\mathrm{H} & -3.12357700 & 1.44783100 & 5.59358900 \\ \mathrm{C} & 1.21875500 & 0.76268200 & 2.79243100 \\ \mathrm{C} & 1.09730000 & 1.71476400 & 3.84569000 \\ \mathrm{C} & 2.21312500 & 2.24978200 & 4.44044300 \\ \mathrm{C} & 3.51393900 & 1.83589500 & 4.05124400 \\ \mathrm{C} & 3.64313300 & 0.90804700 & 2.96840600 \\ \mathrm{C} & 2.48349700 & 0.40252600 & 2.34791700 \\ \mathrm{C} & 4.68748200 & 2.28305800 & 4.71007700 \\ \mathrm{C} & 5.92960900 & 1.82918700 & 4.32409700 \\ \mathrm{C} & 6.05808900 & 0.92125000 & 3.24302600 \\ \mathrm{C} & 4.93962600 & 0.48282800 & 2.57411200 \\ \mathrm{H} & 0.10359500 & 2.00629500 & 4.16989700 \\ \mathrm{H} & 2.11205100 & 2.97372500 & 5.24533200 \\ \mathrm{H} & 2.57900100 & -0.30956900 & 1.53658200 \\ \mathrm{H} & 4.58945300 & 2.98556000 & 5.53421300 \\ \mathrm{H} & 6.81942900 & 2.17120300 & 4.84550400 \\ \mathrm{H} & 7.04395200 & 0.57670800 & 2.94390700 \\ \mathrm{H} & 5.02295300 & -0.21032400 & 1.74387000\end{array}$

\section{TS-S}

$\mathrm{Rh}$

$\mathrm{Rh}$

$\mathrm{P}$

$\mathrm{P}$

$\mathrm{P}$

$\mathrm{P}$

$\mathrm{O}$

$\mathrm{O}$

$\mathrm{O}$

$\mathrm{O}$

$\mathrm{O}$

$\mathrm{O}$

$\mathrm{O}$

$\mathrm{O}$

$\mathrm{O}$

$\mathrm{O}$

$\mathrm{O}$

$\mathrm{O}$

$\mathrm{O}$

$\mathrm{O}$

$\mathrm{O}$

$\mathrm{O}$

C

C

$-0.11144000$

0.01995600

0.11512000

$-0.21064600$

0.18107700

$-2.52976600$

2.10228000

1.70506000

$-1.20271000$

$-1.70615100$

2.38154600

$-1.02976700$

$-2.42810600$

$-1.50139600$

$-1.21259400$

1.45995800

$-2.15007500$

$-1.36497600$

1.36360500

1.45739200

0.10705700

1.37967400

1.54520100

$-2.52936000$

2.67167900

3.22627900

$-1.19703800$

3.46229000

0.79096300

$-1.13093000$

$-1.56607600$

1.48346000

0.19901100

$-1.56372200$

1.77965900

$-2.41927300$

$-3.17986500$

3.06649700

$-1.02018600$

$-0.68357900$

3.62532700

$-0.78056900$

$-1.80112400$

$-1.19761000$

$-2.55865200$

$-1.56338900$

$-1.49345000$

0.05043300

$-3.10615600$

$-2.97370000$

$-1.39011600$

$-3.69805000$

$-0.53412100$

$-0.86792700$

1.14279800

$-1.40801800$

$-2.64887400$

1.37379400

$-1.45177300$

$-0.00841400$

0.56278000

$-3.50220100$

$-1.18423100$

2.96724300

$-2.71717800$

$-1.61560400$

3.42061500

3.58513700

$-0.06879800$

2.75763700

4.05250400

1.06608100 


\begin{tabular}{|c|c|c|c|}
\hline $\mathrm{H}$ & 1.67764400 & 4.14382900 & 1.03762400 \\
\hline $\mathrm{C}$ & 3.49375100 & 4.33196100 & 2.21808900 \\
\hline $\mathrm{H}$ & 2.97805300 & 4.68110200 & 3.10798400 \\
\hline $\mathrm{C}$ & 4.87673400 & 4.12147700 & 2.24871800 \\
\hline $\mathrm{H}$ & 5.43759400 & 4.28970400 & 3.16346400 \\
\hline $\mathrm{C}$ & 5.51509500 & 3.64748100 & 1.10721800 \\
\hline $\mathrm{C}$ & 6.96861500 & 3.27515500 & 0.91598800 \\
\hline $\mathrm{H}$ & 7.58380500 & 4.16671100 & 0.73067200 \\
\hline $\mathrm{H}$ & 7.38899900 & 2.76948800 & 1.79062000 \\
\hline $\mathrm{C}$ & 6.91558100 & 2.37128000 & -0.33328200 \\
\hline $\mathrm{H}$ & 7.85154400 & 2.34747300 & -0.89900600 \\
\hline $\mathrm{H}$ & 6.67598500 & 1.34524800 & -0.03660900 \\
\hline $\mathrm{C}$ & 5.72473200 & 2.93028900 & -1.17869400 \\
\hline $\mathrm{C}$ & 4.79571500 & 3.39328200 & -0.07128200 \\
\hline $\mathrm{C}$ & 4.33482500 & 0.92169600 & -2.22608200 \\
\hline $\mathrm{C}$ & 4.16816200 & 0.06905200 & -3.31729300 \\
\hline $\mathrm{H}$ & 3.39624100 & -0.68948600 & -3.27676500 \\
\hline $\mathrm{C}$ & 4.95791000 & 0.25111800 & -4.45302500 \\
\hline $\mathrm{H}$ & 4.82307400 & -0.40909100 & -5.30480300 \\
\hline $\mathrm{C}$ & 5.87179200 & 1.30798600 & -4.52260900 \\
\hline $\mathrm{H}$ & 6.44164800 & 1.48311800 & -5.43143400 \\
\hline $\mathrm{C}$ & 6.01567600 & 2.15222100 & -3.42600600 \\
\hline $\mathrm{C}$ & 6.85732300 & 3.40506100 & -3.30001000 \\
\hline $\mathrm{H}$ & 7.91371800 & 3.16201300 & -3.11928000 \\
\hline $\mathrm{H}$ & 6.82416400 & 4.02341000 & -4.20353500 \\
\hline $\mathrm{C}$ & 6.22903400 & 4.10471200 & -2.07440700 \\
\hline $\mathrm{H}$ & 6.92139000 & 4.76464800 & -1.54259400 \\
\hline $\mathrm{H}$ & 5.36559700 & 4.70069100 & -2.38914600 \\
\hline $\mathrm{C}$ & 5.28184600 & 1.94131500 & -2.24670800 \\
\hline $\mathrm{C}$ & -3.57701000 & 3.64545700 & 0.19415600 \\
\hline $\mathrm{C}$ & -4.28386900 & 2.86068500 & 1.10361500 \\
\hline $\mathrm{H}$ & -4.53811700 & 1.84418500 & 0.82801400 \\
\hline $\mathrm{C}$ & -4.59682200 & 3.37724900 & 2.36064500 \\
\hline $\mathrm{H}$ & -5.14318600 & 2.76416100 & 3.06854600 \\
\hline $\mathrm{C}$ & -4.15688400 & 4.65348400 & 2.72745400 \\
\hline $\mathrm{H}$ & -4.34798300 & 5.03133000 & 3.72857100 \\
\hline $\mathrm{C}$ & -3.45085600 & 5.42105600 & 1.80563100 \\
\hline $\mathrm{C}$ & -2.81608000 & 6.78345400 & 1.99245600 \\
\hline $\mathrm{H}$ & -3.56867000 & 7.58312200 & 1.95197900 \\
\hline $\mathrm{H}$ & -2.30459100 & 6.87785900 & 2.95670400 \\
\hline $\mathrm{C}$ & -1.84807400 & 6.86870900 & 0.79118700 \\
\hline $\mathrm{H}$ & -1.63375900 & 7.89337000 & 0.47274000 \\
\hline $\mathrm{H}$ & -0.89712100 & 6.38728500 & 1.04495800 \\
\hline $\mathrm{C}$ & -2.54520600 & 6.03333700 & -0.32855200 \\
\hline
\end{tabular}




\begin{tabular}{|c|c|c|c|}
\hline $\mathrm{C}$ & -3.19483600 & 4.94420900 & 0.50941600 \\
\hline $\mathrm{C}$ & -0.72881200 & 4.68244100 & -1.70104200 \\
\hline $\mathrm{C}$ & 0.08116700 & 4.63391900 & -2.83503400 \\
\hline $\mathrm{H}$ & 0.73633200 & 3.78362400 & -2.97641900 \\
\hline $\mathrm{C}$ & -0.00259700 & 5.66236300 & -3.77457600 \\
\hline $\mathrm{H}$ & 0.62546200 & 5.62438400 & -4.65993500 \\
\hline $\mathrm{C}$ & -0.91482600 & 6.70957000 & -3.60721800 \\
\hline $\mathrm{H}$ & -1.00782100 & 7.48437500 & -4.36384000 \\
\hline $\mathrm{C}$ & -1.72334200 & 6.72853100 & -2.47488900 \\
\hline $\mathrm{C}$ & -2.82834900 & 7.69811400 & -2.11502000 \\
\hline $\mathrm{H}$ & -2.41998300 & 8.62938800 & -1.69783100 \\
\hline $\mathrm{H}$ & -3.44201500 & 7.97836600 & -2.97807100 \\
\hline $\mathrm{C}$ & -3.62018000 & 6.91205400 & -1.04879500 \\
\hline $\mathrm{H}$ & -4.17102800 & 7.54992800 & -0.35102100 \\
\hline $\mathrm{H}$ & -4.34021200 & 6.24784800 & -1.53906800 \\
\hline $\mathrm{C}$ & -1.61691400 & 5.73090300 & -1.49243600 \\
\hline $\mathrm{C}$ & -4.70371100 & -0.44220900 & -1.84616100 \\
\hline $\mathrm{C}$ & -4.59315700 & 0.51806900 & -2.85062000 \\
\hline $\mathrm{H}$ & -3.74315700 & 1.18850400 & -2.84948800 \\
\hline $\mathrm{C}$ & -5.55560500 & 0.55751300 & -3.86072100 \\
\hline $\mathrm{H}$ & -5.46807200 & 1.30316500 & -4.64538600 \\
\hline $\mathrm{C}$ & -6.59256600 & -0.38066500 & -3.89833000 \\
\hline $\mathrm{H}$ & -7.30782400 & -0.37739900 & -4.71661400 \\
\hline $\mathrm{C}$ & -6.67455200 & -1.33836200 & -2.89234800 \\
\hline $\mathrm{C}$ & -7.63329300 & -2.50304300 & -2.76360200 \\
\hline $\mathrm{H}$ & -8.60746500 & -2.17846500 & -2.37195600 \\
\hline $\mathrm{H}$ & -7.82508300 & -2.99807700 & -3.72158400 \\
\hline $\mathrm{C}$ & -6.90495500 & -3.41891400 & -1.75564700 \\
\hline $\mathrm{H}$ & -7.57571500 & -4.07305500 & -1.18997100 \\
\hline $\mathrm{H}$ & -6.18237000 & -4.04978600 & -2.28451500 \\
\hline $\mathrm{C}$ & -6.12213000 & -2.43070500 & -0.83077900 \\
\hline $\mathrm{C}$ & -5.75232600 & -1.35376700 & -1.83395400 \\
\hline $\mathrm{C}$ & -3.76056800 & -3.49942900 & -0.27256900 \\
\hline $\mathrm{C}$ & -3.03954600 & -4.32538800 & 0.58918700 \\
\hline $\mathrm{H}$ & -2.01628900 & -4.57233000 & 0.33301700 \\
\hline $\mathrm{C}$ & -3.63544100 & -4.78518500 & 1.76395800 \\
\hline $\mathrm{H}$ & -3.07026800 & -5.42539200 & 2.43486200 \\
\hline $\mathrm{C}$ & -4.93061000 & -4.38084900 & 2.10527300 \\
\hline $\mathrm{H}$ & -5.37290000 & -4.69115900 & 3.04794800 \\
\hline $\mathrm{C}$ & -5.63926800 & -3.56667600 & 1.22599900 \\
\hline $\mathrm{C}$ & -7.02477400 & -2.97974500 & 1.39268100 \\
\hline $\mathrm{H}$ & -7.79818900 & -3.74451300 & 1.23964500 \\
\hline $\mathrm{H}$ & -7.17903800 & -2.56690700 & 2.39551300 \\
\hline $\mathrm{C}$ & -7.07907300 & -1.90242300 & 0.28589500 \\
\hline
\end{tabular}




\begin{tabular}{|c|c|c|c|}
\hline $\mathrm{H}$ & -8.08912400 & -1.72111200 & -0.09228800 \\
\hline $\mathrm{H}$ & -6.69463800 & -0.94916500 & 0.66237900 \\
\hline $\mathrm{C}$ & -5.08085300 & -3.15590900 & 0.00424800 \\
\hline $\mathrm{C}$ & 0.55707300 & -4.42890700 & -2.23276700 \\
\hline $\mathrm{C}$ & -0.40208700 & -4.30502700 & -3.23727600 \\
\hline $\mathrm{H}$ & -1.12096400 & -3.49636500 & -3.18069500 \\
\hline $\mathrm{C}$ & -0.38256800 & -5.19839700 & -4.30907400 \\
\hline $\mathrm{H}$ & -1.12566100 & -5.10125300 & -5.09504200 \\
\hline $\mathrm{C}$ & 0.60921400 & -6.18072700 & -4.39933700 \\
\hline $\mathrm{H}$ & 0.64853800 & -6.84463800 & -5.25887400 \\
\hline $\mathrm{C}$ & 1.56421400 & -6.27545800 & -3.39150200 \\
\hline $\mathrm{C}$ & 2.76878200 & -7.18785400 & -3.31282400 \\
\hline $\mathrm{H}$ & 2.48474600 & -8.19508800 & -2.97713500 \\
\hline $\mathrm{H}$ & 3.27330600 & -7.30460800 & -4.27789300 \\
\hline $\mathrm{C}$ & 3.64852700 & -6.47882600 & -2.26220600 \\
\hline $\mathrm{H}$ & 4.33424500 & -7.15036600 & -1.73701000 \\
\hline $\mathrm{H}$ & 4.24512100 & -5.69839400 & -2.74680000 \\
\hline $\mathrm{C}$ & 2.62828200 & -5.79412200 & -1.29328700 \\
\hline $\mathrm{C}$ & 1.52997200 & -5.42112300 & -2.27722700 \\
\hline $\mathrm{C}$ & 3.56813700 & -3.41931600 & -0.57292800 \\
\hline $\mathrm{C}$ & 4.36428300 & -2.71221100 & 0.32804600 \\
\hline $\mathrm{H}$ & 4.51854900 & -1.65474800 & 0.15283400 \\
\hline $\mathrm{C}$ & 4.90617900 & -3.36797000 & 1.43231600 \\
\hline $\mathrm{H}$ & 5.51775400 & -2.80911500 & 2.13494700 \\
\hline $\mathrm{C}$ & 4.60620600 & -4.71589100 & 1.66923800 \\
\hline $\mathrm{H}$ & 4.97374600 & -5.21061300 & 2.56436700 \\
\hline $\mathrm{C}$ & 3.80792800 & -5.40390500 & 0.75877300 \\
\hline $\mathrm{C}$ & 3.28262900 & -6.82244300 & 0.83892300 \\
\hline $\mathrm{H}$ & 2.92173100 & -7.07538800 & 1.84191300 \\
\hline $\mathrm{H}$ & 4.06497600 & -7.55171000 & 0.58896600 \\
\hline $\mathrm{C}$ & 2.15600100 & -6.82500600 & -0.21977800 \\
\hline $\mathrm{H}$ & 1.22442000 & -6.46285800 & 0.22809200 \\
\hline $\mathrm{H}$ & 1.96335000 & -7.81242400 & -0.64922100 \\
\hline $\mathrm{C}$ & 3.32487000 & -4.77722000 & -0.40314800 \\
\hline $\mathrm{C}$ & 2.11783400 & -1.22869600 & 6.46309900 \\
\hline $\mathrm{C}$ & 1.10227600 & -1.62381400 & 5.59349400 \\
\hline $\mathrm{C}$ & 1.39930600 & -2.30393100 & 4.39772000 \\
\hline $\mathrm{C}$ & 2.74921700 & -2.56105300 & 4.09745000 \\
\hline $\mathrm{C}$ & 3.76584800 & -2.16607400 & 4.96339100 \\
\hline $\mathrm{C}$ & 3.45162700 & -1.49990200 & 6.14875100 \\
\hline $\mathrm{H}$ & 1.87080800 & -0.70632000 & 7.38351600 \\
\hline $\mathrm{H}$ & 0.07125700 & -1.38982200 & 5.84343200 \\
\hline $\mathrm{H}$ & 3.01286500 & -3.05676800 & 3.17046900 \\
\hline $\mathrm{H}$ & 4.80337100 & -2.35687900 & 4.70446600 \\
\hline
\end{tabular}




\begin{tabular}{|c|c|c|c|}
\hline $\mathrm{H}$ & 4.24466100 & -1.18130100 & 6.81917600 \\
\hline $\mathrm{Si}$ & 0.07005100 & -2.72834800 & 3.17073300 \\
\hline $\mathrm{H}$ & -0.00222700 & -1.39922400 & 2.26563300 \\
\hline $\mathrm{C}$ & -1.63052800 & -2.85878100 & 3.94590500 \\
\hline $\mathrm{H}$ & -1.84386100 & -2.01928900 & 4.61305100 \\
\hline $\mathrm{H}$ & -2.40601200 & -2.88477600 & 3.17676700 \\
\hline $\mathrm{H}$ & -1.69772400 & -3.78731000 & 4.52775800 \\
\hline $\mathrm{C}$ & 0.51371000 & -4.10717700 & 1.99014400 \\
\hline $\mathrm{H}$ & -0.01798600 & -3.97161000 & 1.04690000 \\
\hline $\mathrm{H}$ & 1.58066200 & -4.10632400 & 1.75634400 \\
\hline $\mathrm{H}$ & 0.24886300 & -5.08033000 & 2.42310000 \\
\hline $\mathrm{C}$ & -0.05200000 & 0.01850500 & 2.20687500 \\
\hline $\mathrm{C}$ & 1.20426400 & 0.40914400 & 2.67818900 \\
\hline $\mathrm{C}$ & 2.34502200 & 0.67067100 & 3.02014400 \\
\hline $\mathrm{C}$ & 3.72317800 & 0.84302500 & 3.28763100 \\
\hline $\mathrm{C}$ & 4.65369800 & 0.42716700 & 2.31467200 \\
\hline $\mathrm{C}$ & 6.01279900 & 0.43676600 & 2.60819000 \\
\hline $\mathrm{C}$ & 6.46262900 & 0.90746200 & 3.84595400 \\
\hline $\mathrm{C}$ & 5.54666400 & 1.37174900 & 4.79404500 \\
\hline $\mathrm{C}$ & 4.18227700 & 1.32995700 & 4.52426500 \\
\hline $\mathrm{H}$ & 4.28033600 & 0.10380400 & 1.35033900 \\
\hline $\mathrm{H}$ & 6.72696600 & 0.08989400 & 1.86747000 \\
\hline $\mathrm{H}$ & 7.52607300 & 0.91803700 & 4.06824900 \\
\hline $\mathrm{H}$ & 5.89767900 & 1.74747600 & 5.75095100 \\
\hline $\mathrm{H}$ & 3.45832300 & 1.64720800 & 5.26684700 \\
\hline $\mathrm{C}$ & -1.12461600 & 0.99847300 & 4.27595100 \\
\hline $\mathrm{C}$ & -1.24689200 & 0.35397000 & 3.00933000 \\
\hline $\mathrm{C}$ & -2.50469200 & -0.01613900 & 2.56218800 \\
\hline $\mathrm{C}$ & -3.65456100 & 0.21033600 & 3.34515000 \\
\hline $\mathrm{C}$ & -3.52941000 & 0.86092700 & 4.61356000 \\
\hline $\mathrm{C}$ & -2.23317800 & 1.24992800 & 5.04858800 \\
\hline $\mathrm{C}$ & -4.93719100 & -0.19644000 & 2.89131900 \\
\hline $\mathrm{C}$ & -6.05770000 & 0.04637300 & 3.65155700 \\
\hline $\mathrm{C}$ & -5.93731300 & 0.69783600 & 4.90605300 \\
\hline $\mathrm{C}$ & -4.70282900 & 1.09178000 & 5.37684400 \\
\hline $\mathrm{H}$ & -0.13516400 & 1.28178500 & 4.61940900 \\
\hline $\mathrm{H}$ & -2.60606700 & -0.51079300 & 1.60683000 \\
\hline $\mathrm{H}$ & -2.13028600 & 1.74897500 & 6.00899200 \\
\hline $\mathrm{H}$ & -5.00134800 & -0.69724900 & 1.93101000 \\
\hline $\mathrm{H}$ & -7.03824000 & -0.25404400 & 3.29343300 \\
\hline $\mathrm{H}$ & -6.82832300 & 0.88612500 & 5.49841100 \\
\hline $\mathrm{H}$ & -4.61418400 & 1.59002400 & 6.33901700 \\
\hline
\end{tabular}




\section{NMR Spectra for New Compounds}

2,4,6-triisopropyl- $N^{\prime}$-(1-(naphthalen-2-yl)-3-phenylprop-2-yn-1ylidene)benzenesulfonohydrazide (1a)

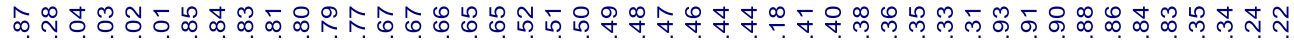

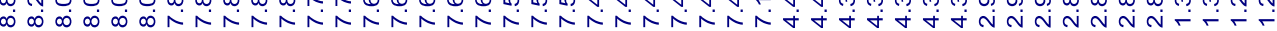<smiles>N=C(C#Cc1ccccc1)c1ccc2ccccc2c1</smiles>

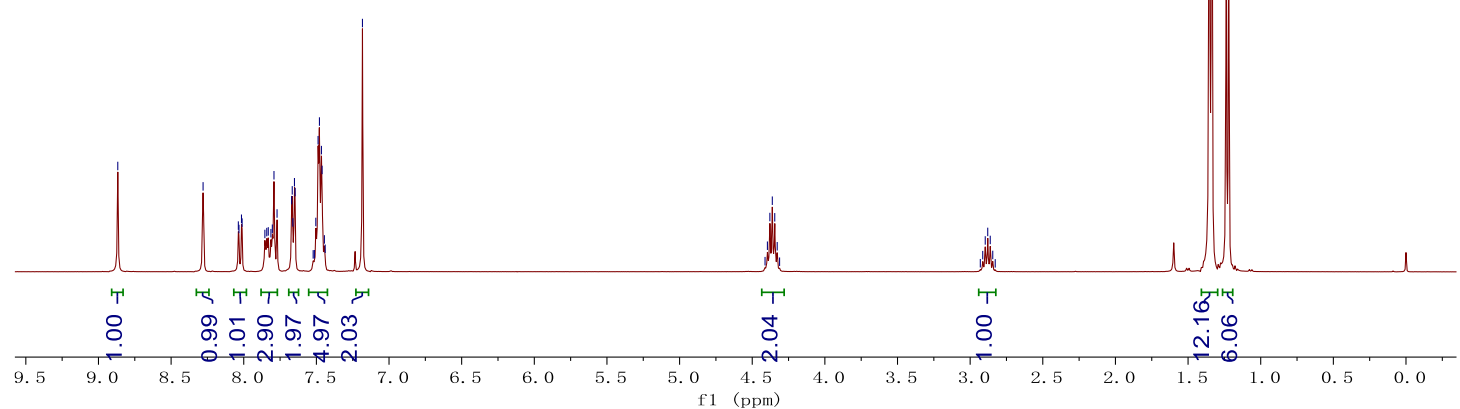

กำ

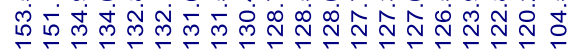

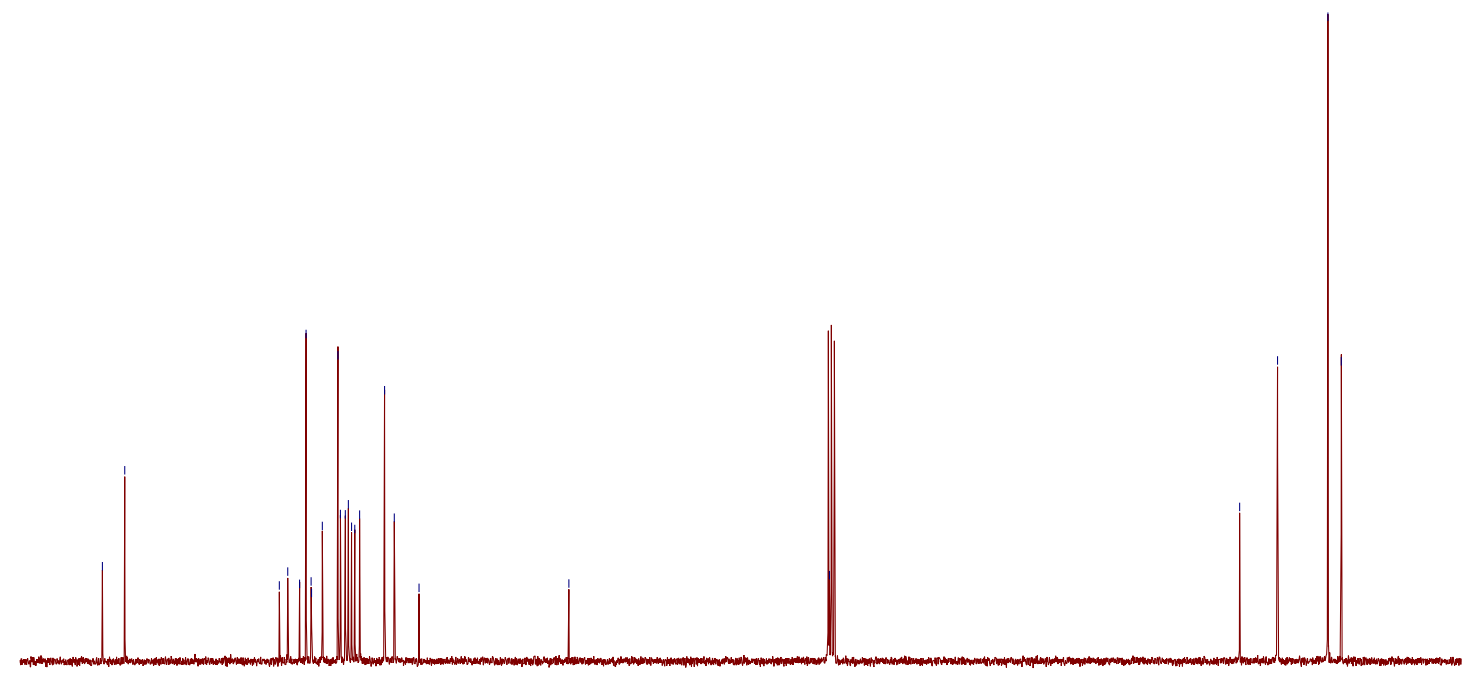

$\begin{array}{lllllllllllllllllllllllllllllllllllllllll}160 & 155 & 150 & 145 & 140 & 135 & 130 & 125 & 120 & 115 & 110 & 105 & 100 & 95 & 90 & 85 & 80 & 75 & 70 & 65 & 60 & 55 & 50 & 45 & 40 & 35 & 30 & 25 & 20 & 15\end{array}$ 
$N^{\prime}$-(1,3-diphenylprop-2-yn-1-ylidene)-2,4,6-triisopropylbenzenesulfonohydrazide (1b)

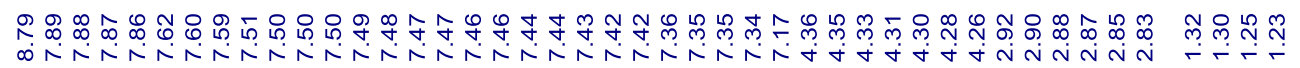

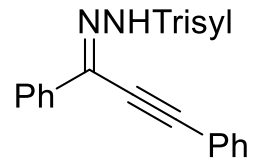

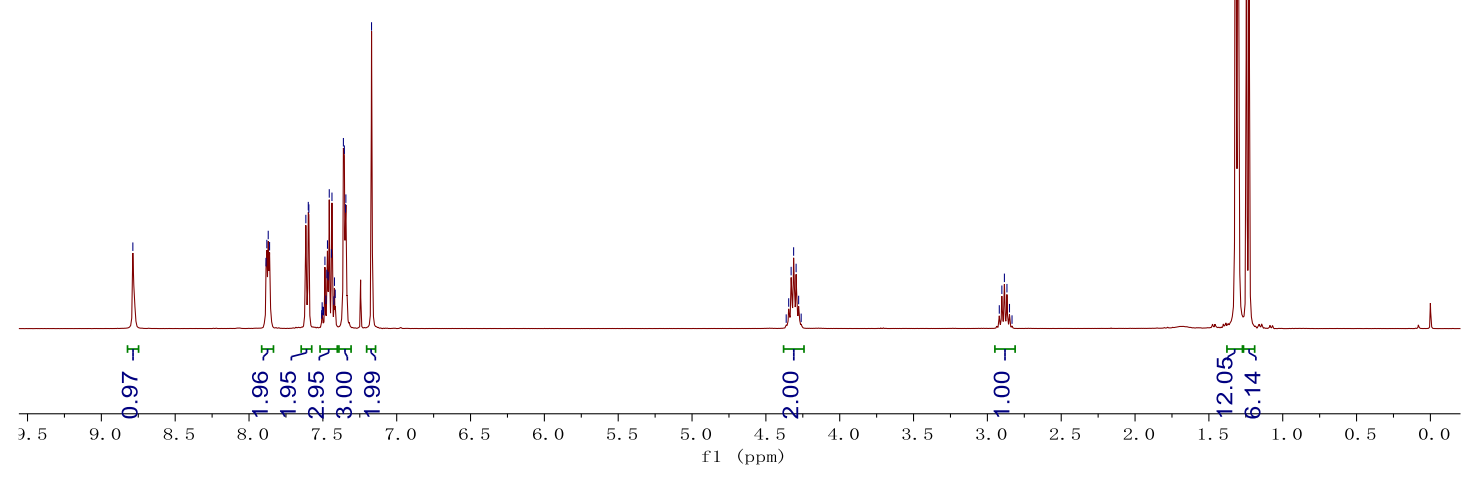

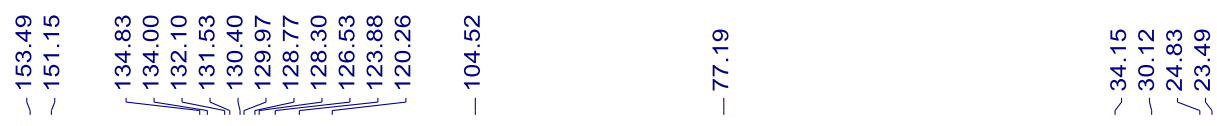

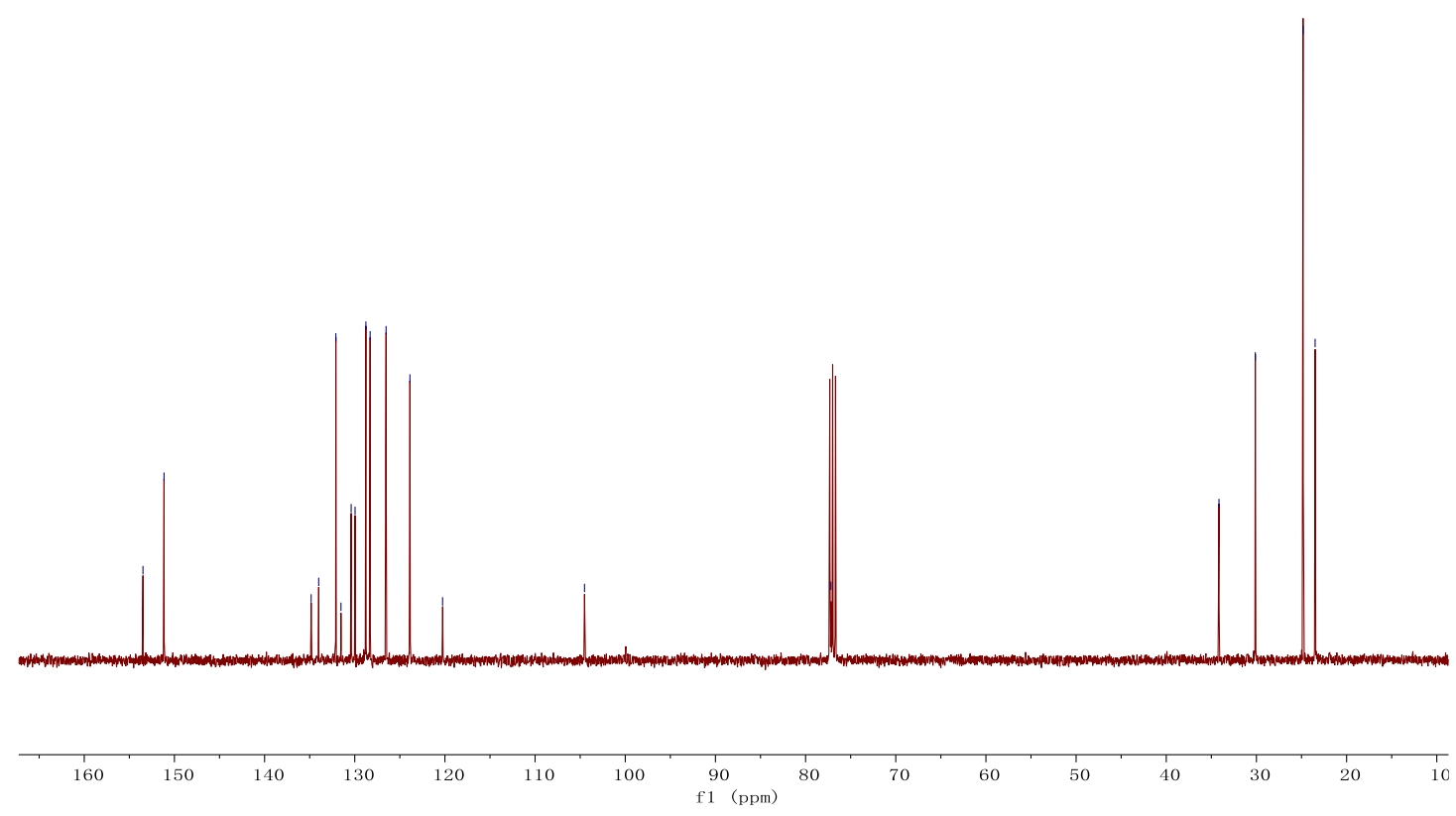


N'-(1-(2-chlorophenyl)-3-phenylprop-2-yn-1-ylidene)-2,4,6triisopropylbenzenesulfonohydrazide (1c)

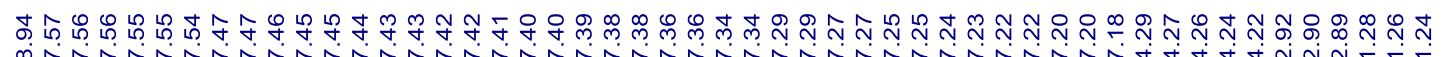

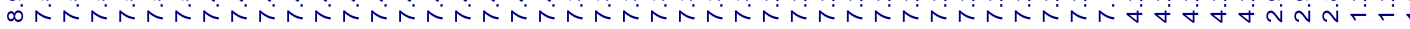<smiles>C=NN=C(C#Cc1ccccc1)c1ccccc1Cl</smiles>

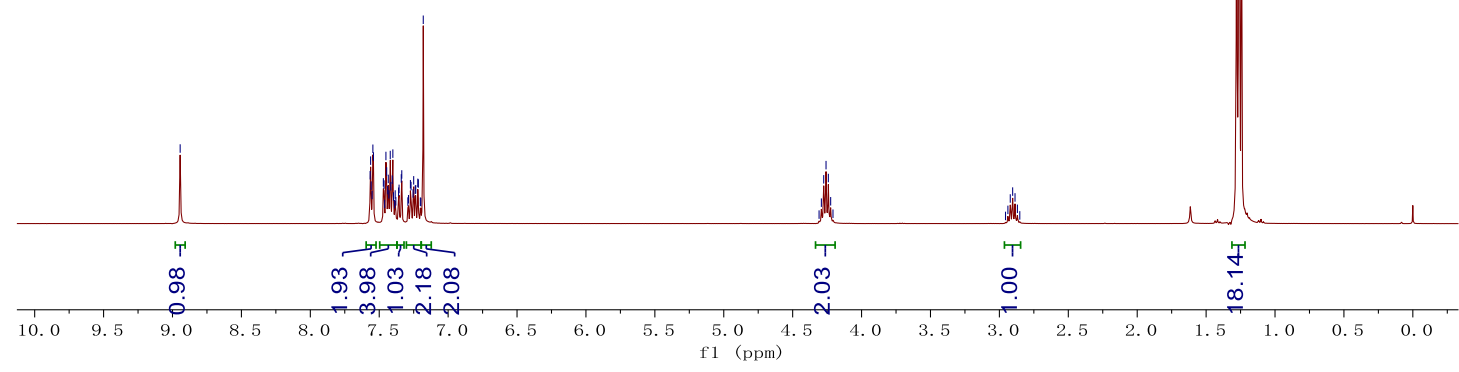

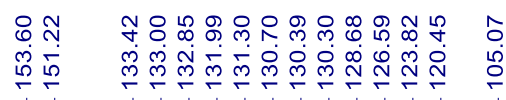

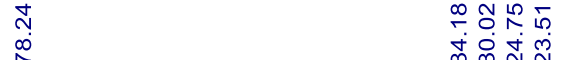

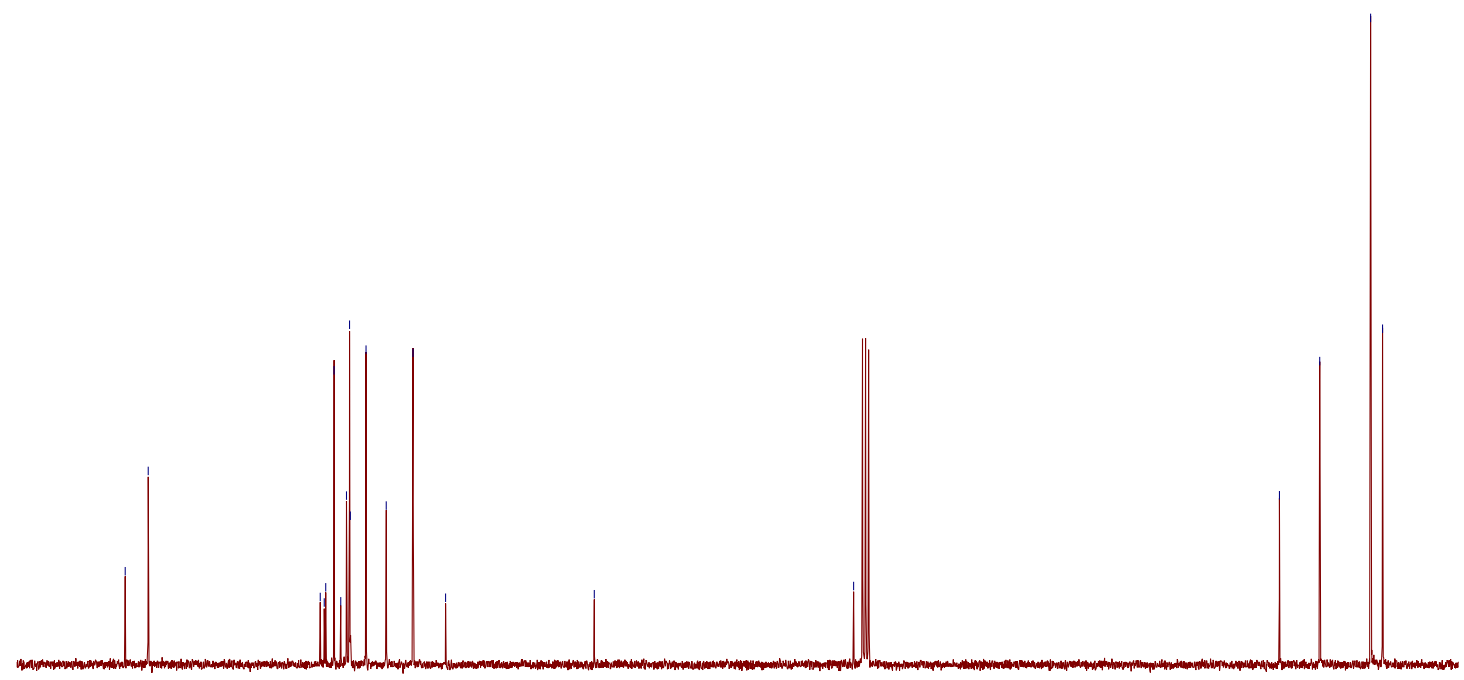

$\begin{array}{llllllllllllllllllllllllllllllllllll}160 & 155 & 150 & 145 & 140 & 135 & 130 & 125 & 120 & 115 & 110 & 105 & 100 & 95 & 90 & 85 & 80 & 75 & 70 & 65 & 60 & 55 & 50 & 45 & 40 & 35 & 30 & 25 & 20 \\ \mathrm{f} 1 & (\mathrm{ppm}) & & & \end{array}$ 
N'-(1-(3-chlorophenyl)-3-phenylprop-2-yn-1-ylidene)-2,4,6triisopropylbenzenesulfonohydrazide (1d)

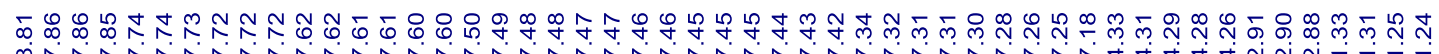

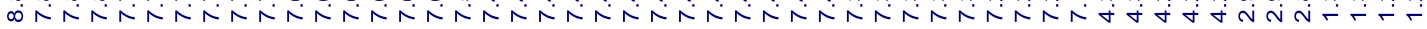<smiles></smiles>

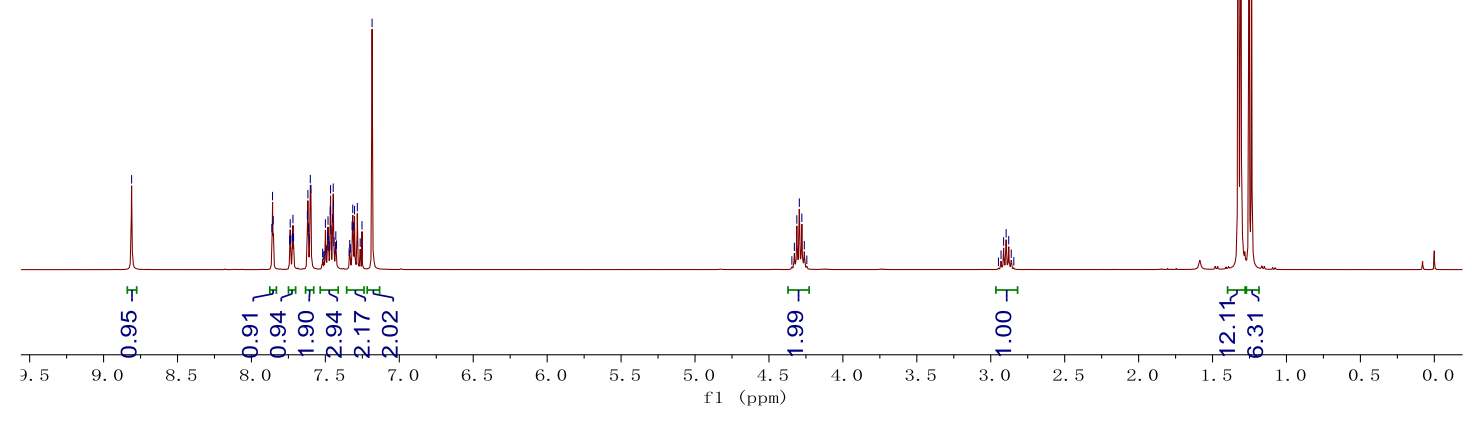

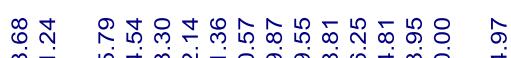

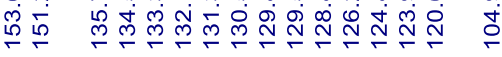

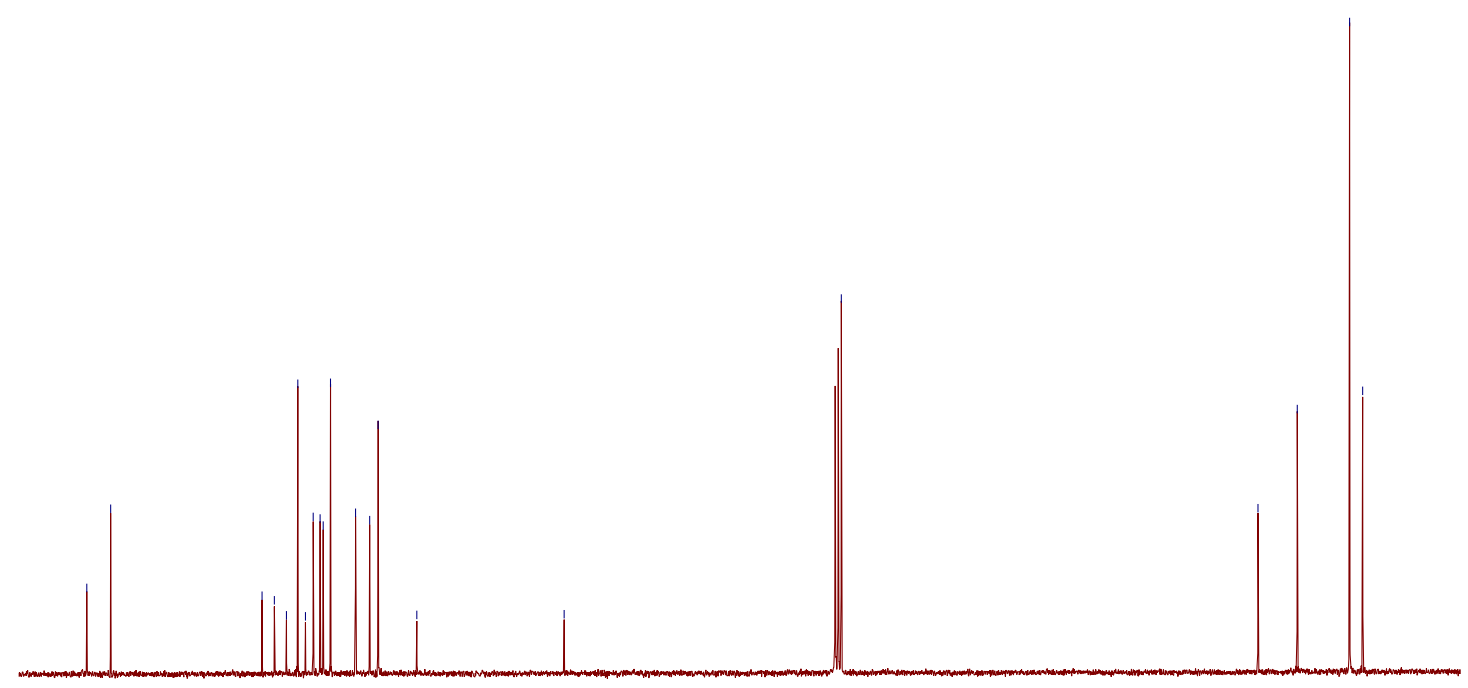

$\begin{array}{lllllllllllllllllllllllllllllllllllll}160 & 155 & 150 & 145 & 140 & 135 & 130 & 125 & 120 & 115 & 110 & 105 & 100 & 95 & 90 & 85 & 80 & 75 & 70 & 65 & 60 & 55 & 50 & 45 & 40 & 35 & 30 & 25 & 20 & 15\end{array}$ 
N'-(1-(4-chlorophenyl)-3-phenylprop-2-yn-1-ylidene)-2,4,6triisopropylbenzenesulfonohydrazide (1e)

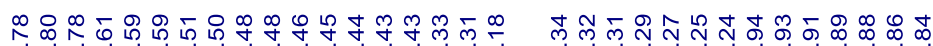

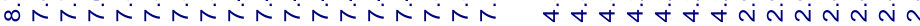

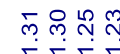<smiles>FC(F)([Mg]N=C(C#Cc1ccccc1)c1ccc(Cl)cc1)c1ccccc1</smiles>

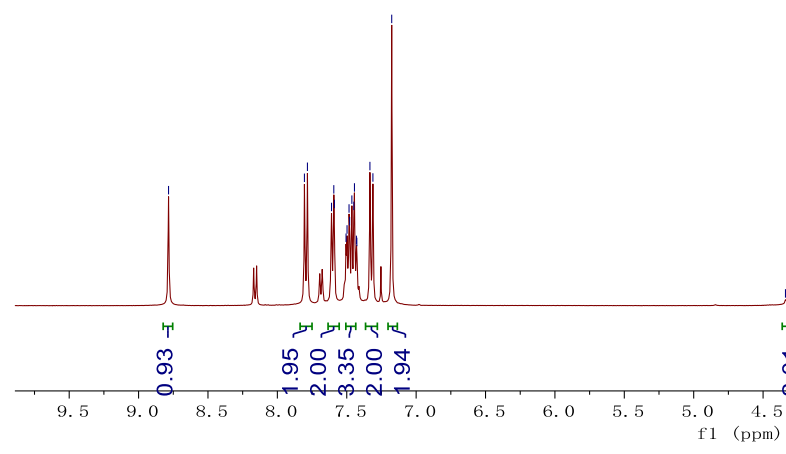

ঢ.

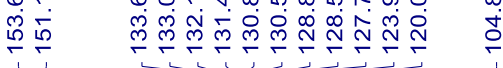

9
$i$
0
1

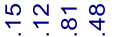

मำ

, $1>$

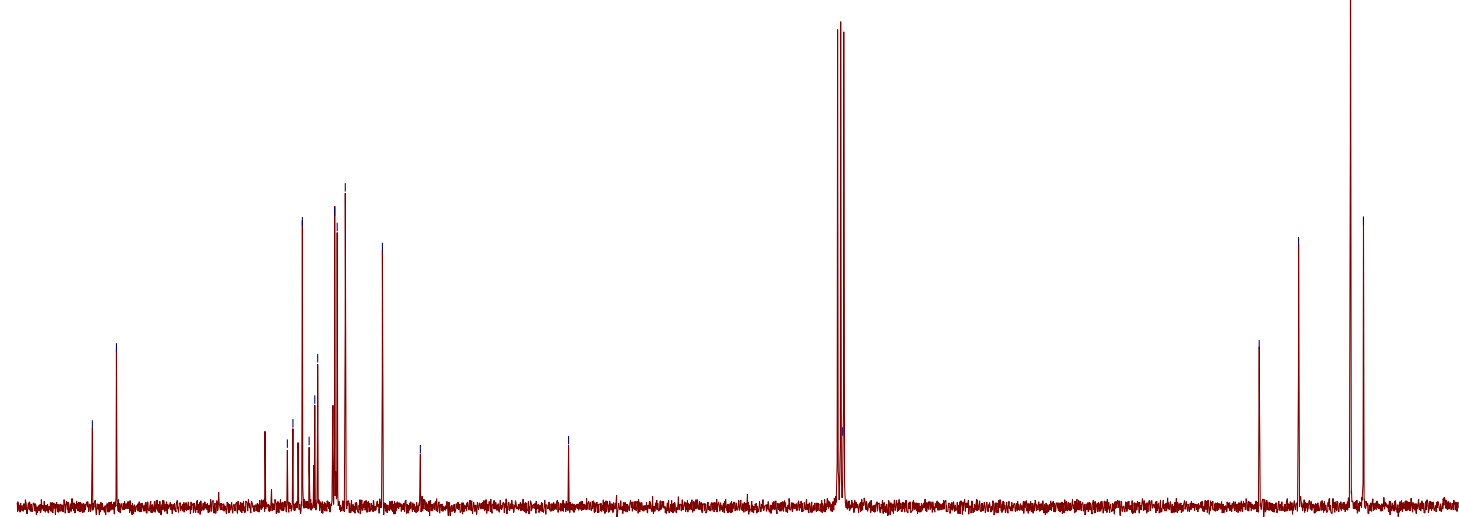

$\begin{array}{lllllllllllllllllllllllllllllllllllllllll}160 & 155 & 150 & 145 & 140 & 135 & 130 & 125 & 120 & 115 & 110 & 105 & 100 & 95 & 90 & 85 & 80 & 75 & 70 & 65 & 60 & 55 & 50 & 45 & 40 & 35 & 30 & 25 & 20 & 15\end{array}$ $\mathrm{f} 1(\mathrm{ppm})$ 
2,4,6-triisopropyl-N'-(1-(4-methoxyphenyl)-3-phenylprop-2-yn-1ylidene)benzenesulfonohydrazide (1f)

œ

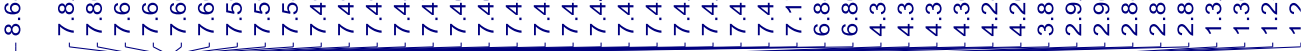
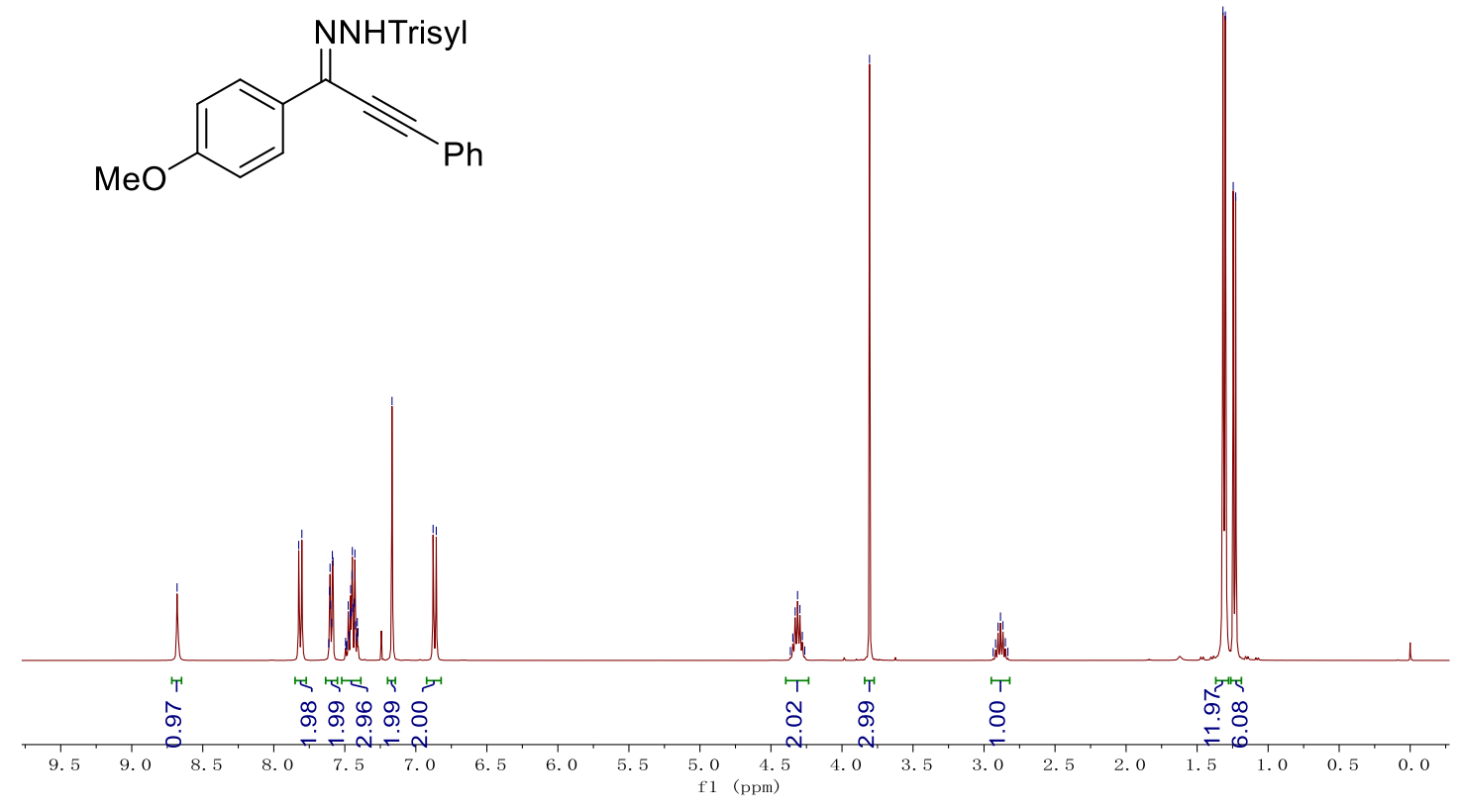

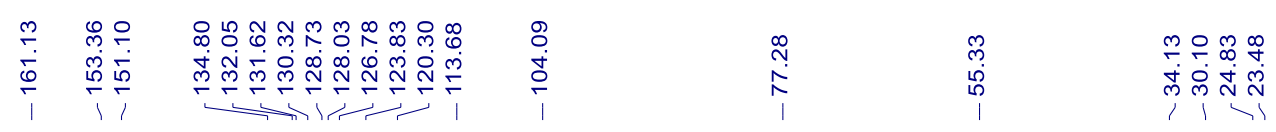

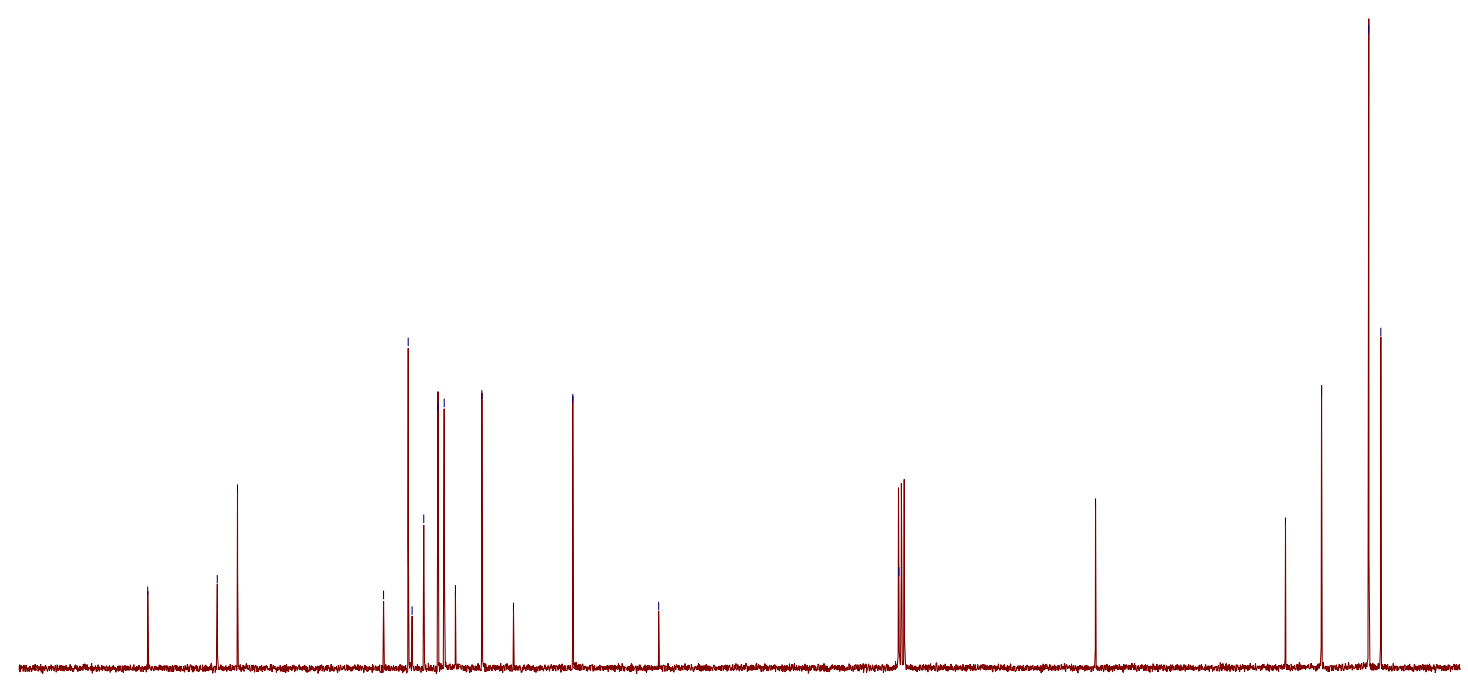

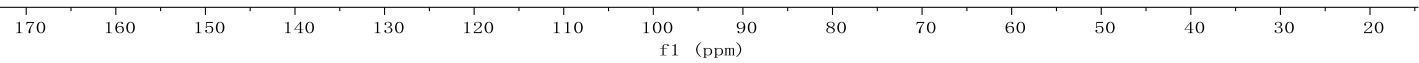


N'-(1-(4-cyanophenyl)-3-phenylprop-2-yn-1-ylidene)-2,4,6triisopropylbenzenesulfonohydrazide (1g)

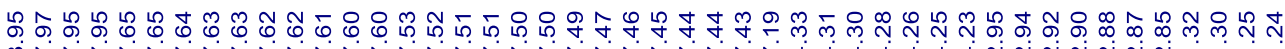

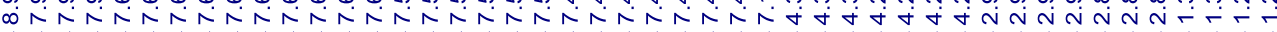<smiles></smiles>

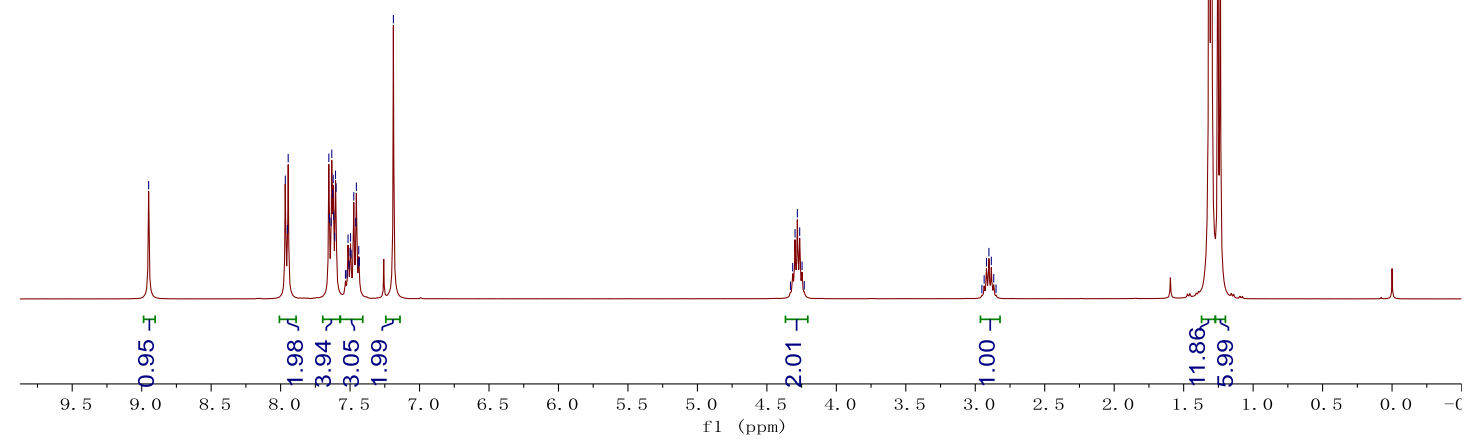

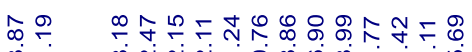

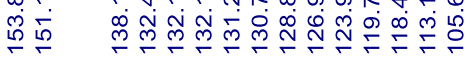

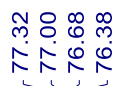

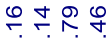

官官突

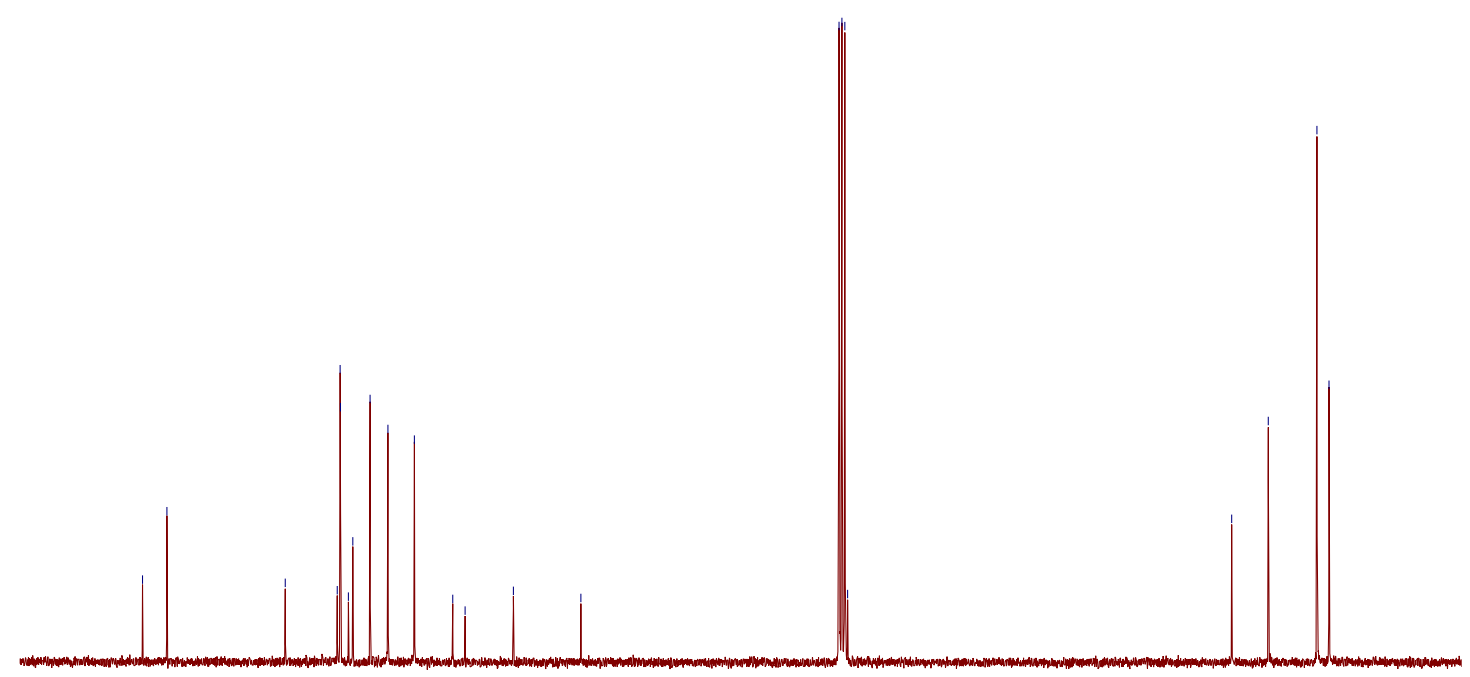


N'-(3-(4-cyanophenyl)-1-(naphthalen-2-yl)prop-2-yn-1-ylidene)-2,4,6triisopropylbenzenesulfonohydrazide (1h)

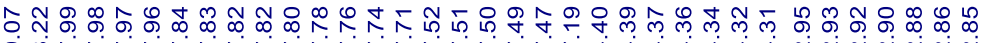

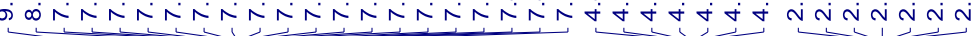

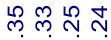<smiles>N#Cc1ccc(C#CC(=N)c2ccc3ccccc3c2)cc1</smiles>

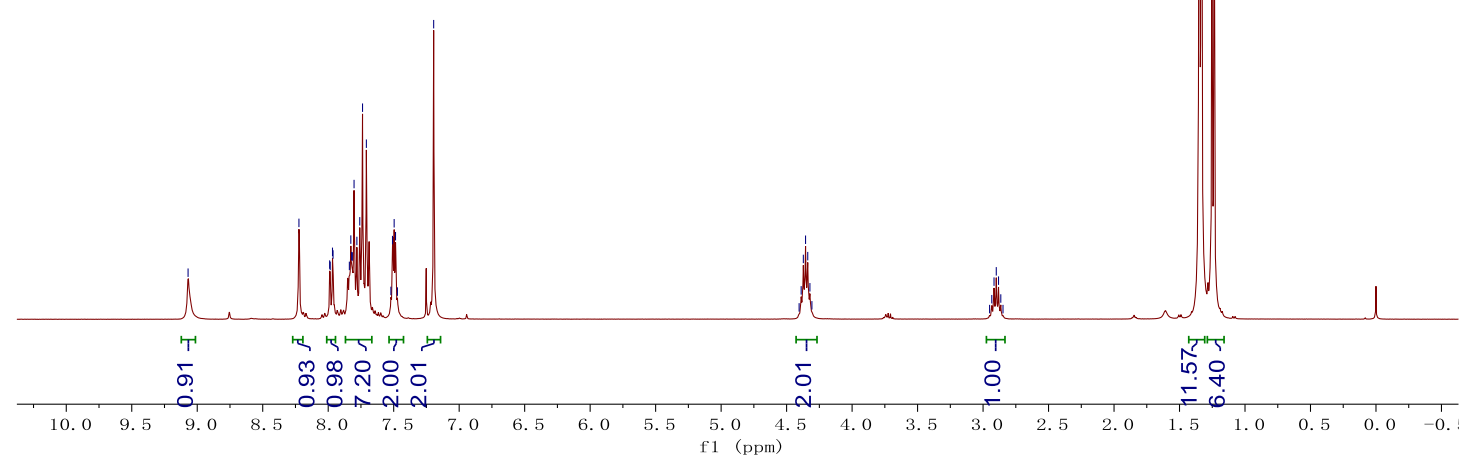

ㅅำ

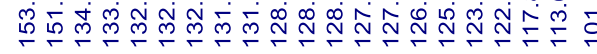

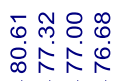

$\stackrel{n}{r} \mp \stackrel{\infty}{\infty} \stackrel{\infty}{\infty} \stackrel{\infty}{\stackrel{n}{n}}$

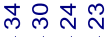

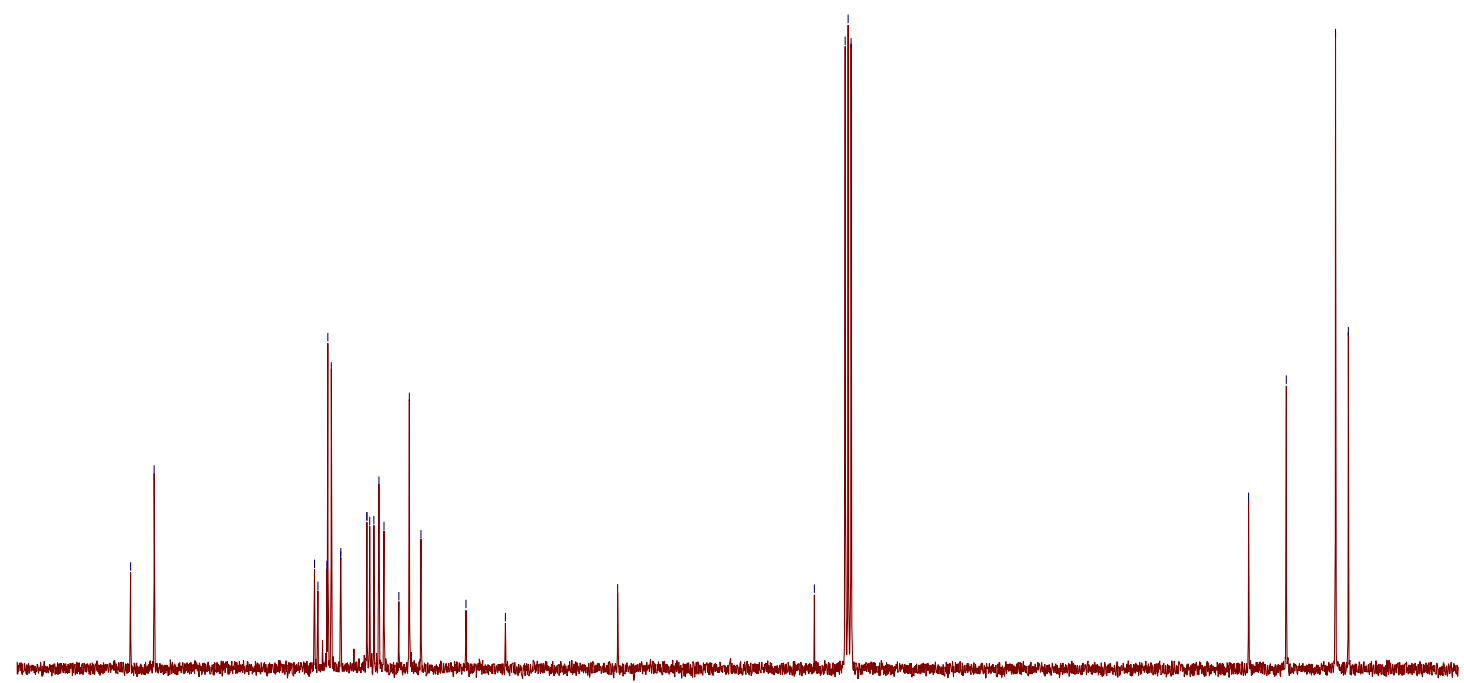

$\begin{array}{llllllllllllllllllllllllllllllllllllll}165 & 160 & 155 & 150 & 145 & 140 & 135 & 130 & 125 & 120 & 115 & 110 & 105 & 100 & 95 & 90 & 85 & 80 & 75 & 70 & 65 & 60 & 55 & 50 & 45 & 40 & 35 & 30 & 25 & 20 & 15\end{array}$ 
2,4,6-triisopropyl-N'-(1-(naphthalen-2-yl)-3-(thiophen-2-yl)prop-2-yn-1ylidene)benzenesulfonohydrazide (1i)

온 舟<smiles>N=C(C#Cc1cccs1)c1ccc2ccccc2c1</smiles>

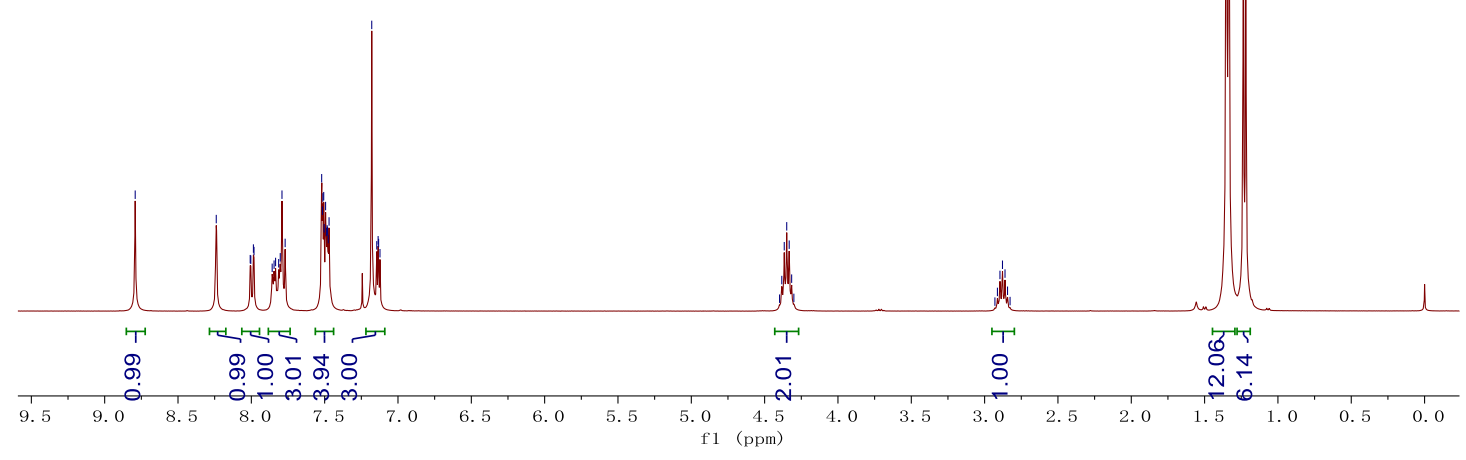

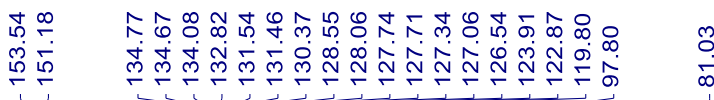

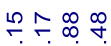

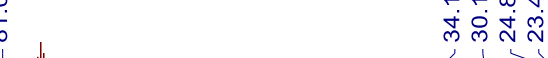

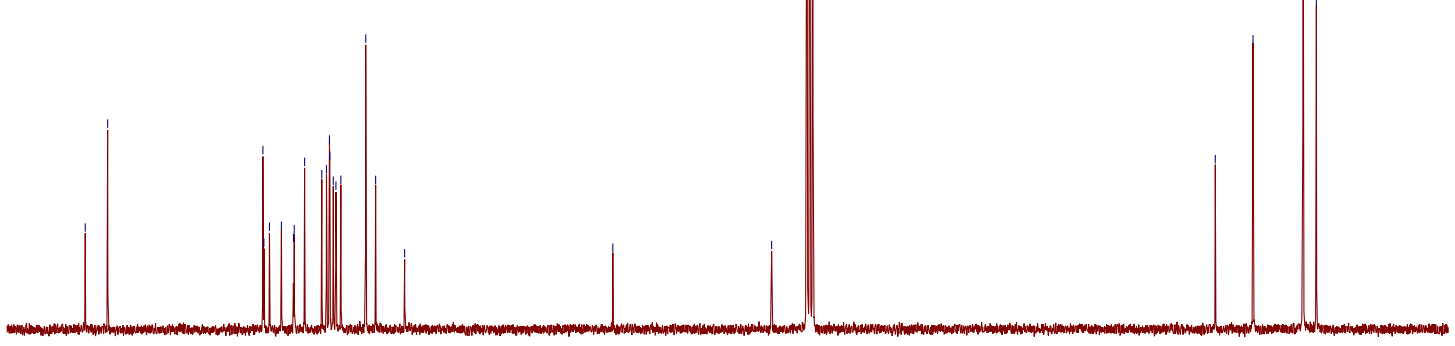

$\begin{array}{llllllllllllllllllllllllllllllllllllllll}160 & 155 & 150 & 145 & 140 & 135 & 130 & 125 & 120 & 115 & 110 & 105 & 100 & 95 & 90 & 85 & 80 & 75 & 70 & 65 & 60 & 55 & 50 & 45 & 40 & 35 & 30 & 25 & 20 & 15 & 1\end{array}$ 
2,4,6-triisopropyl-N'-(1-(naphthalen-2-yl)hept-2-yn-1ylidene)benzenesulfonohydrazide $(\mathbf{1 j})$

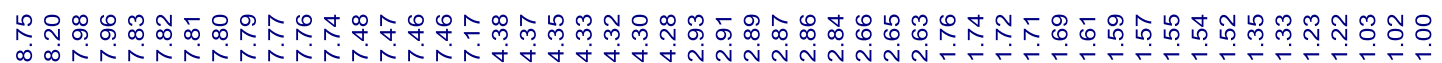<smiles>CCC(C)(C)C#CC(=N)c1ccc2ccccc2c1</smiles>

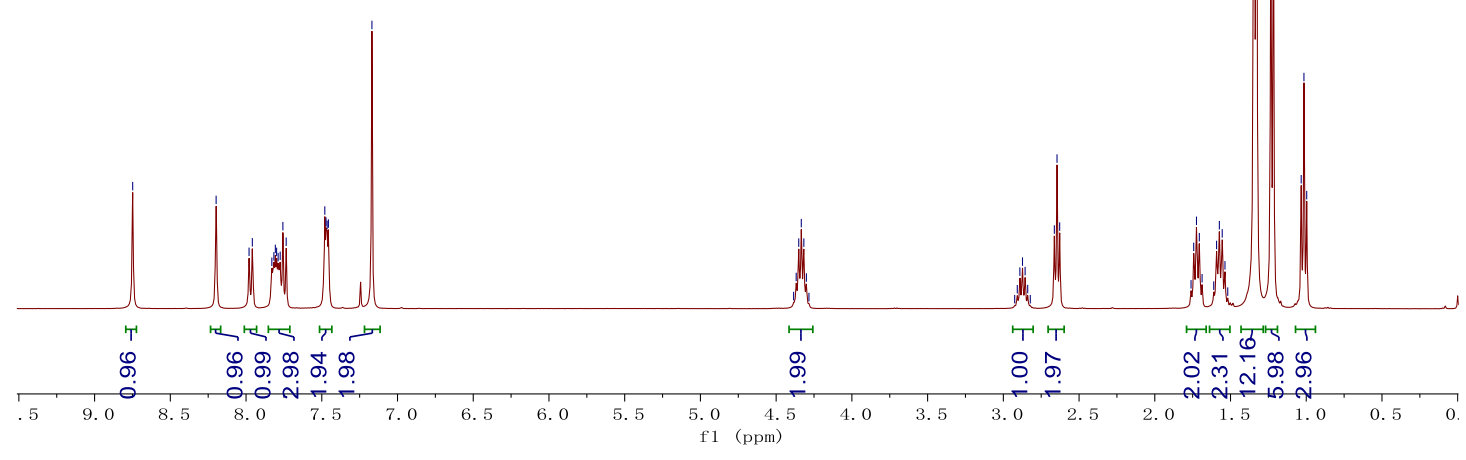

م

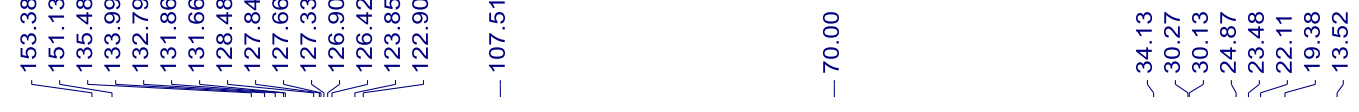

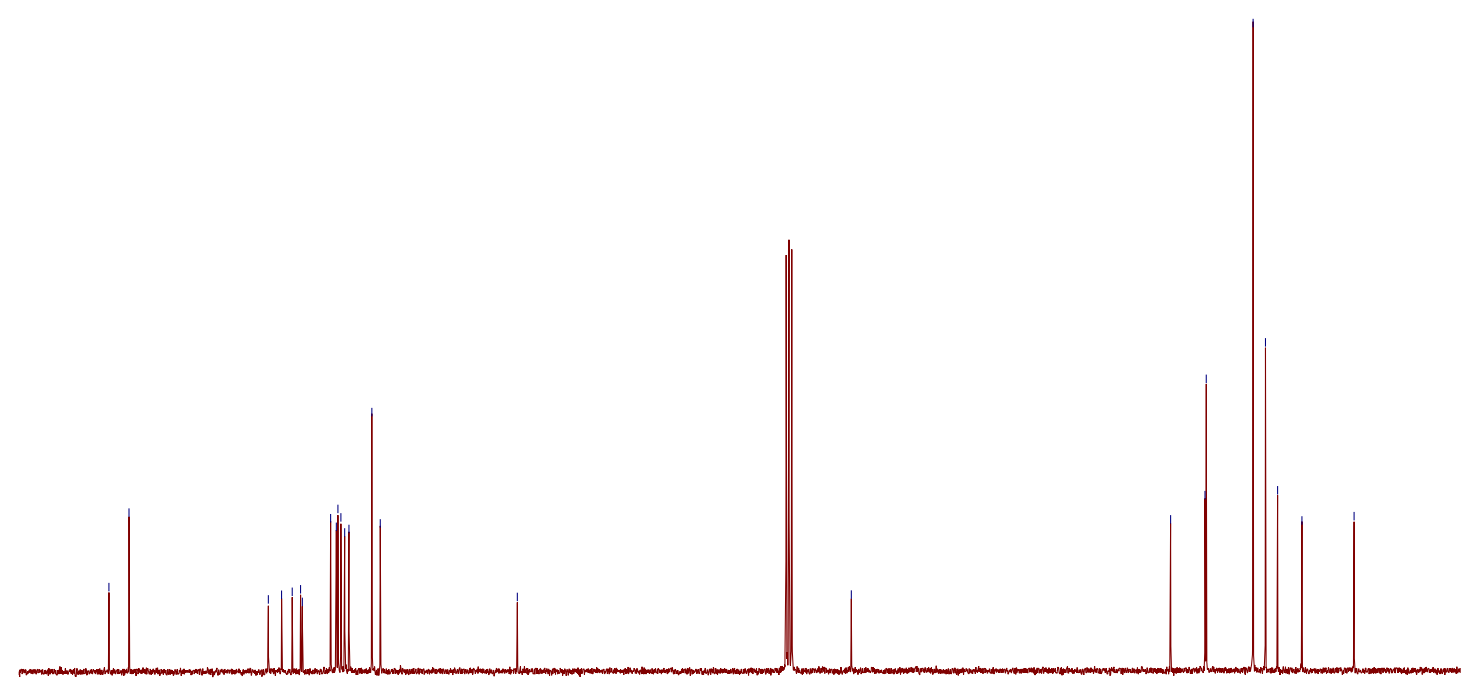

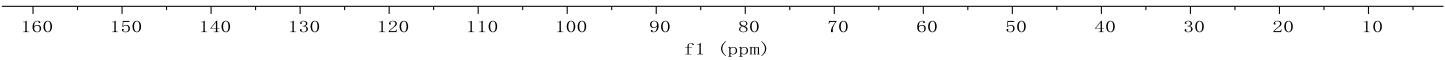


2,4,6-triisopropyl-N'-(1-(naphthalen-2-yl)but-2-yn-1ylidene)benzenesulfonohydrazide (1k)

둗ำ

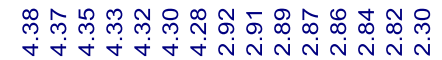

芦

on

$\longrightarrow$ ।<smiles>CC#CC(=N)c1ccc2ccccc2c1</smiles>

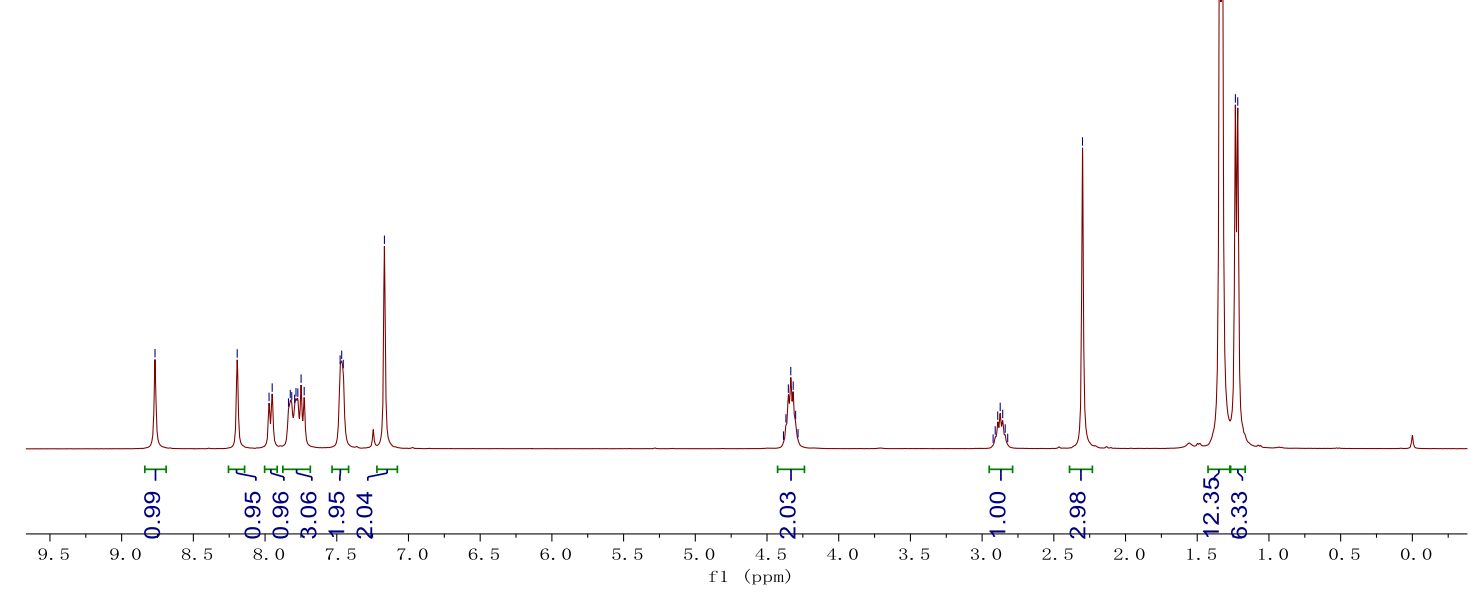

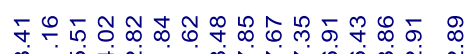

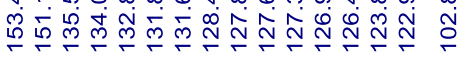

$\hat{m}$
0
0
1

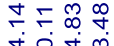

में

$1 / \sqrt{1}$

$\stackrel{\substack{\infty \\ ⿱ 亠 \infty}}{i}$

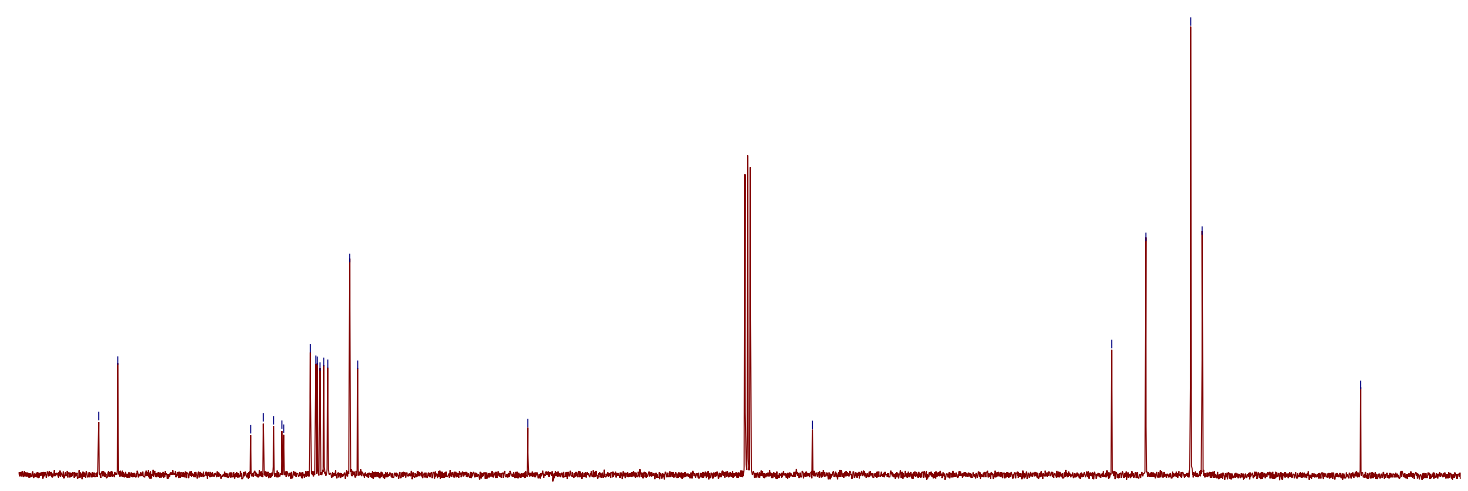


2,4,6-triisopropyl-N'-(1-(naphthalen-2-yl)-6-phenylhex-2-yn-1ylidene)benzenesulfonohydrazide (11)

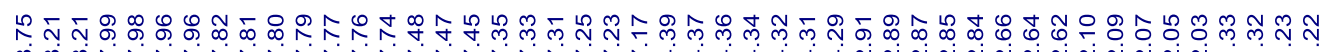
m<smiles>N=C(C#CC(=N)c1ccc2ccccc2c1)c1ccccc1</smiles>

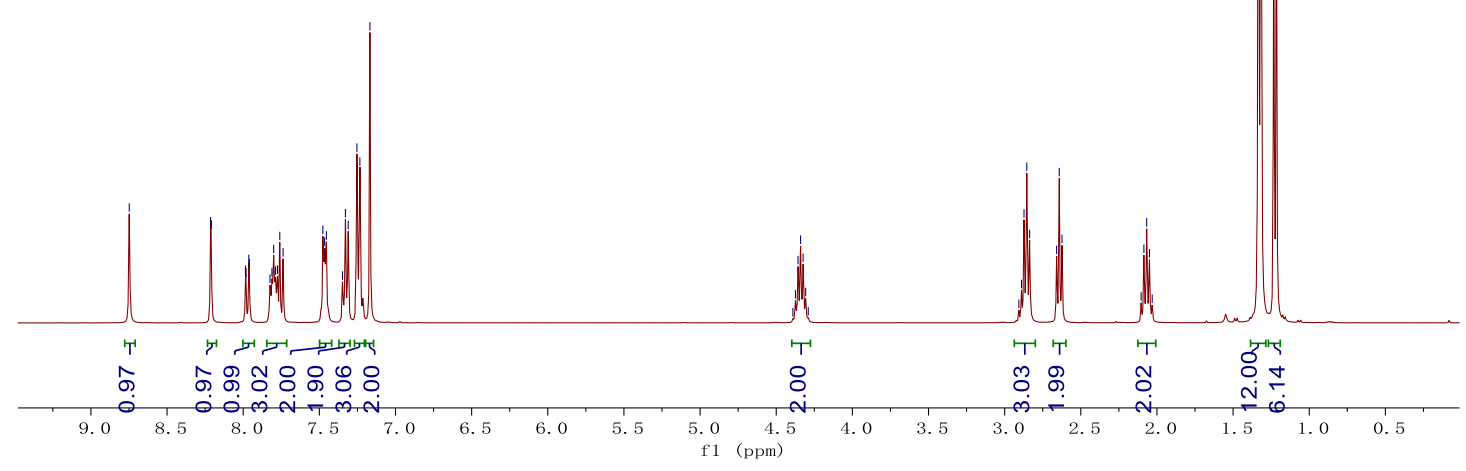

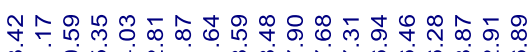

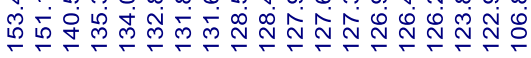

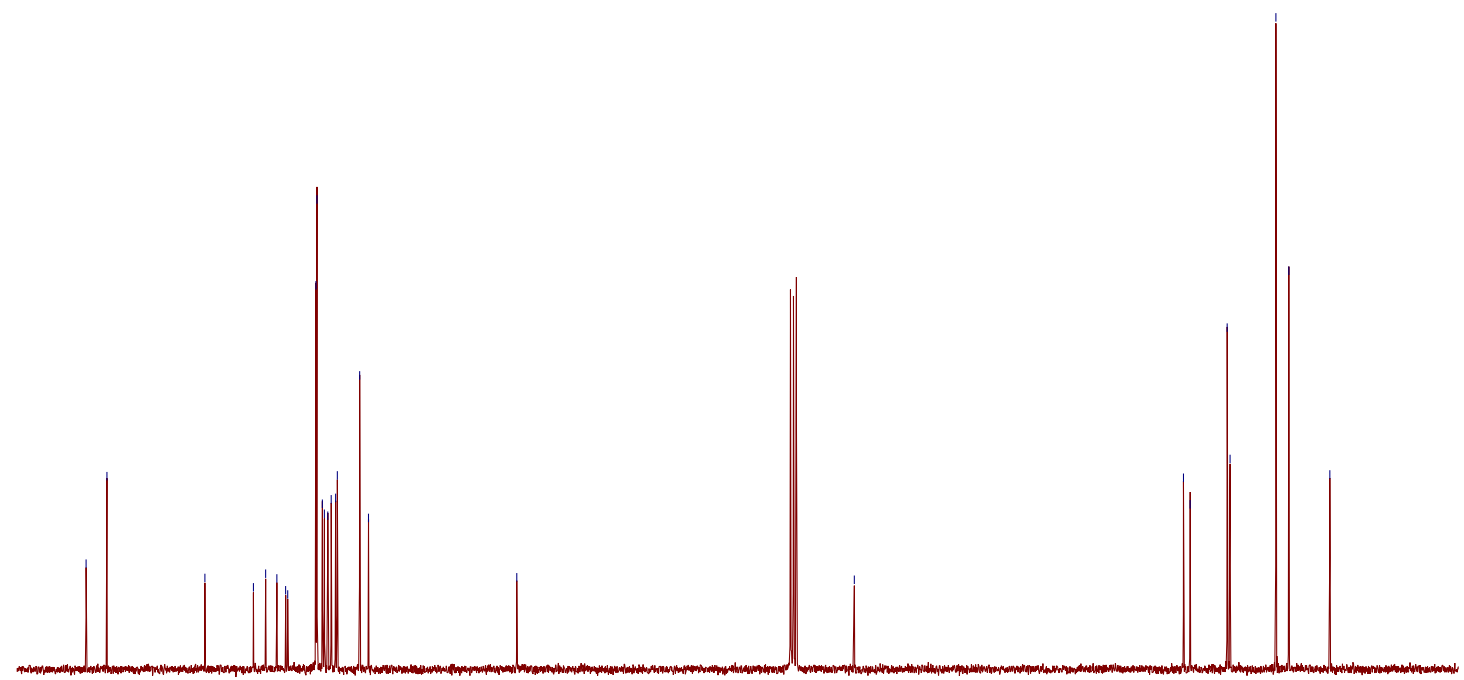

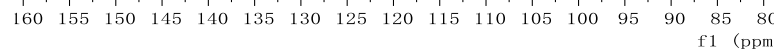


N'-(3-cyclopropyl-1-(naphthalen-2-yl)prop-2-yn-1-ylidene)-2,4,6triisopropylbenzenesulfonohydrazide (1m)

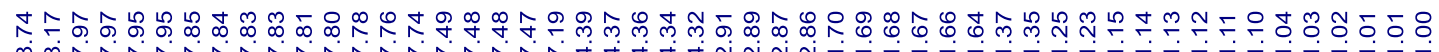

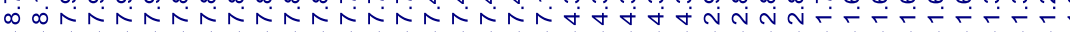<smiles>C(#CC1CC1)c1ccc2ccccc2c1</smiles>

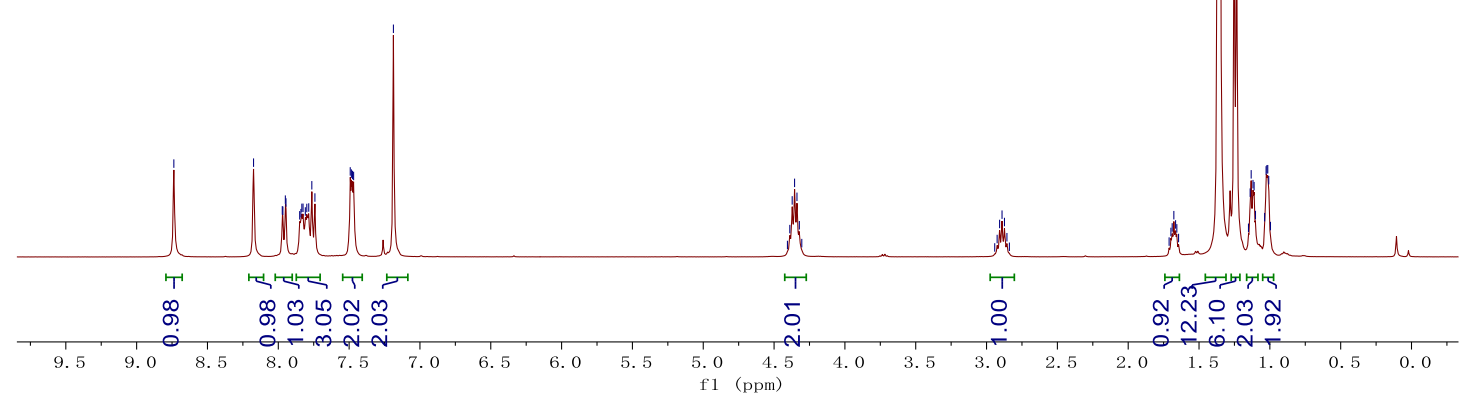

$\int_{\substack{0 \\ 0}}^{\infty}$

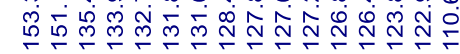

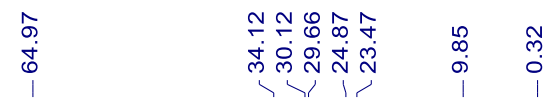

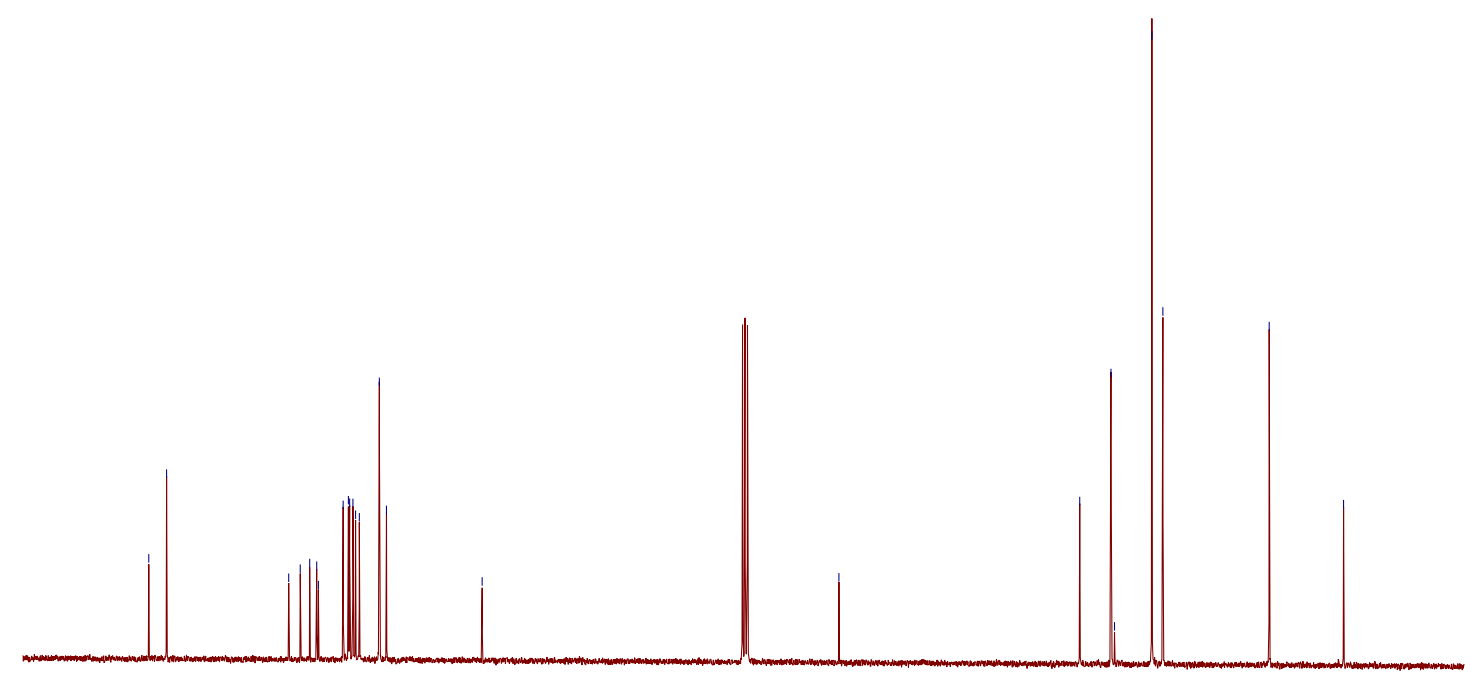

$\begin{array}{lllllllll}160 & 150 & 140 & 130 & 120 & 110 & 100 & 90 & 80 \\ f 1 & & & 1\end{array}$ 
N'-(3-cyclohexyl-1-(naphthalen-2-yl)prop-2-yn-1-ylidene)-2,4,6triisopropylbenzenesulfonohydrazide (1n)

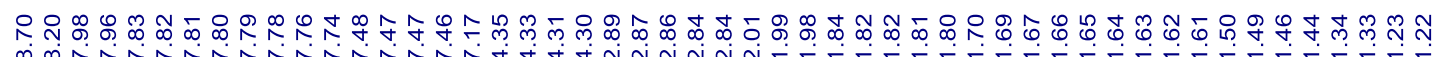

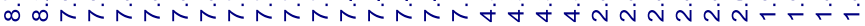<smiles>N=C(C#CC1CCCCC1)c1ccc2ccccc2c1</smiles>

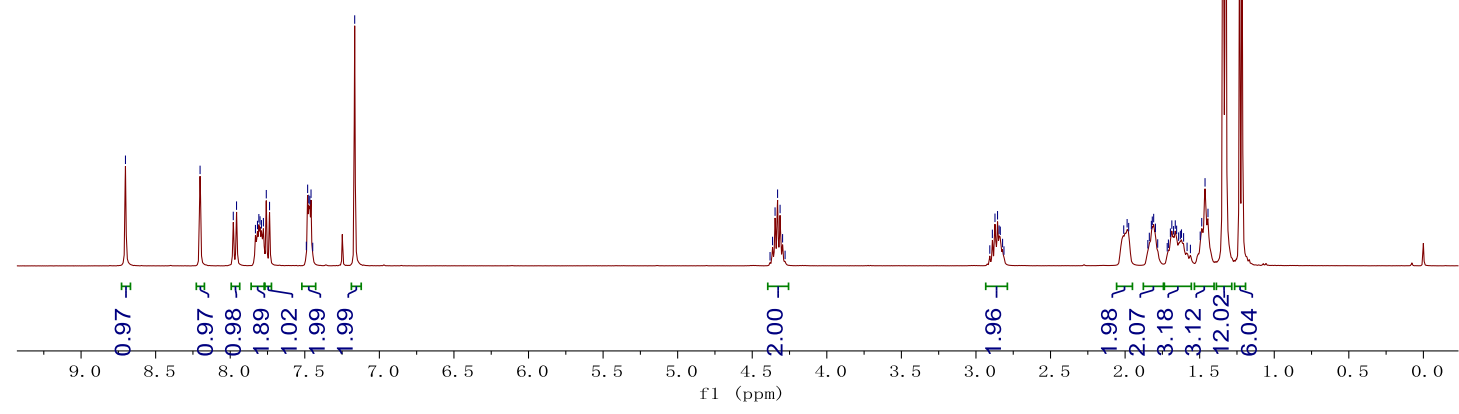

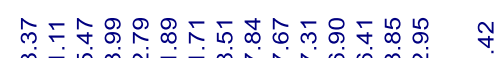

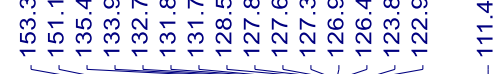

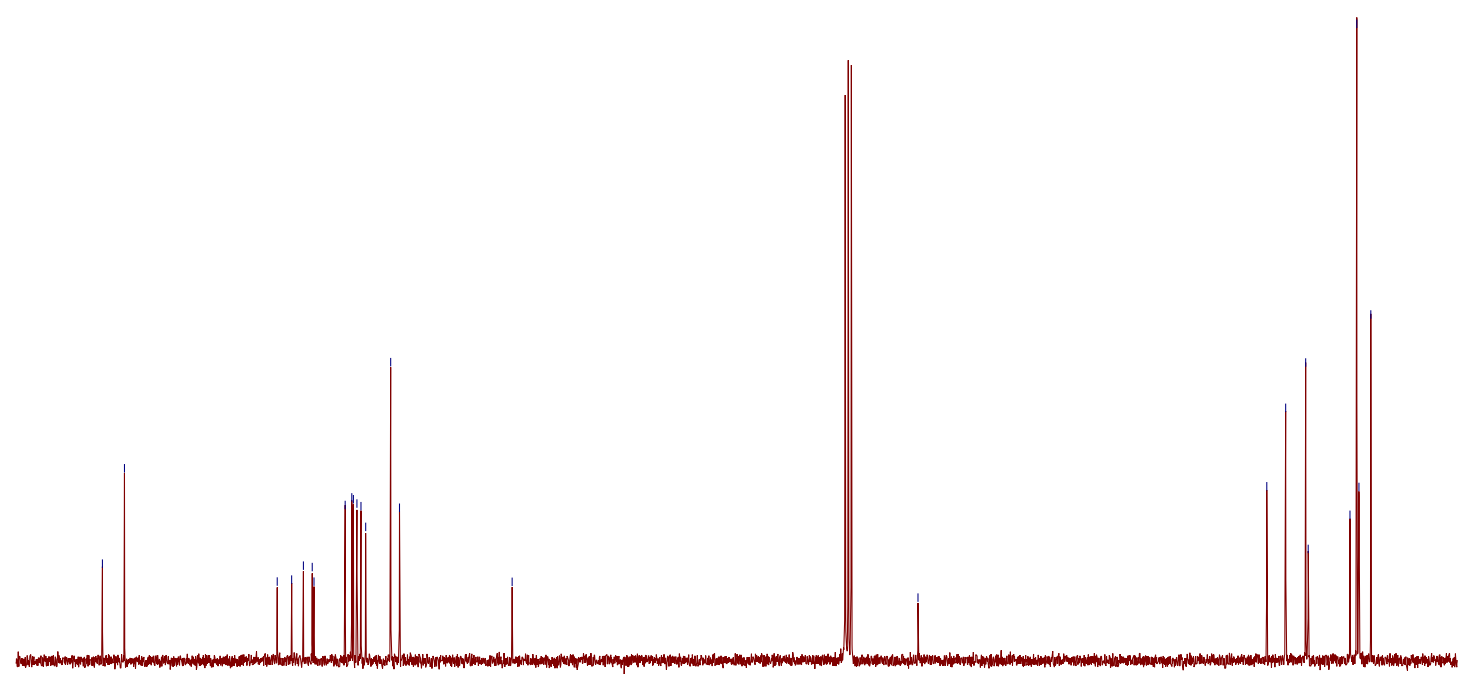

$\begin{array}{lllllllllllllllllllll}160 & 155 & 150 & 145 & 140 & 135 & 130 & 125 & 120 & 115 & 110 & 105 & 100 & 95 & 90 & 85 \\ \text { f1 } & (\mathrm{ppm})\end{array}$ 
2,4,6-triisopropyl-N'-(1-(naphthalen-2-yl)-3-(trimethylsilyl)prop-2-yn-1ylidene)benzenesulfonohydrazide (10)<smiles>CC#CC(=N)c1ccc2ccccc2c1</smiles>

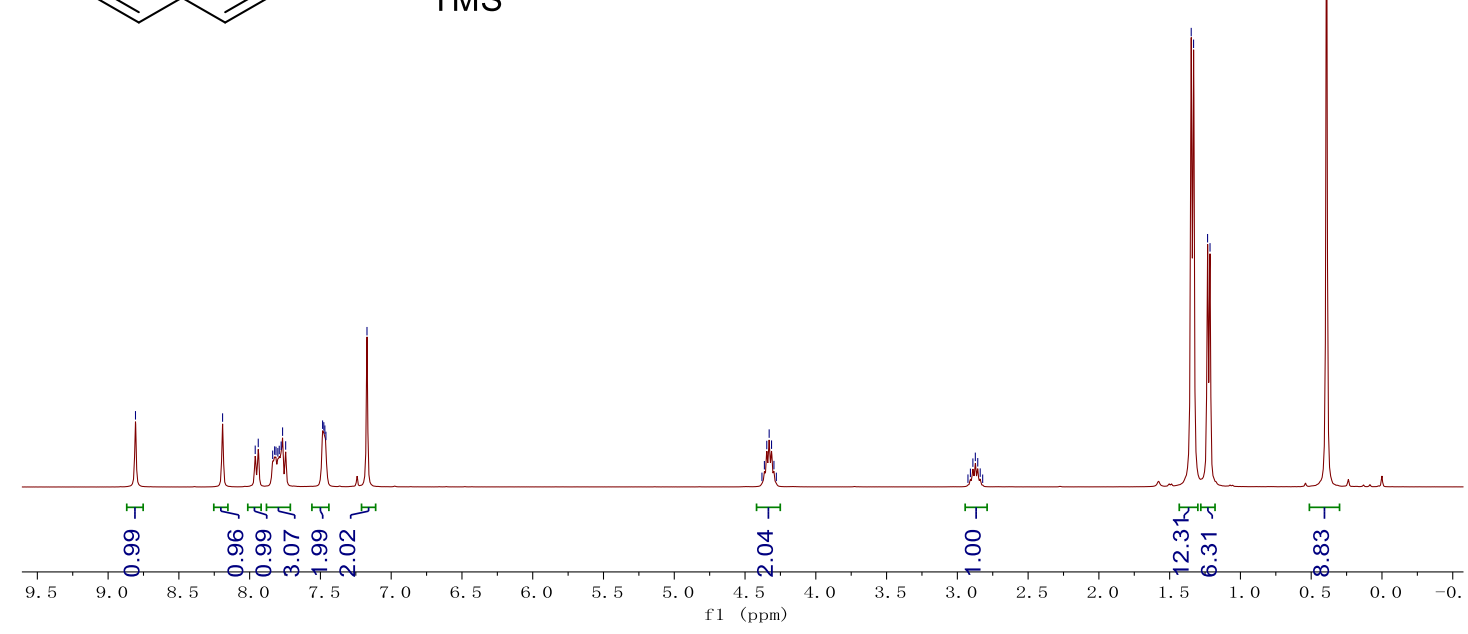

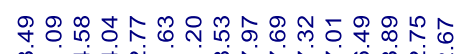

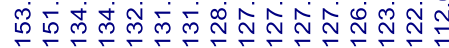

을

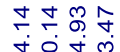

लें

率

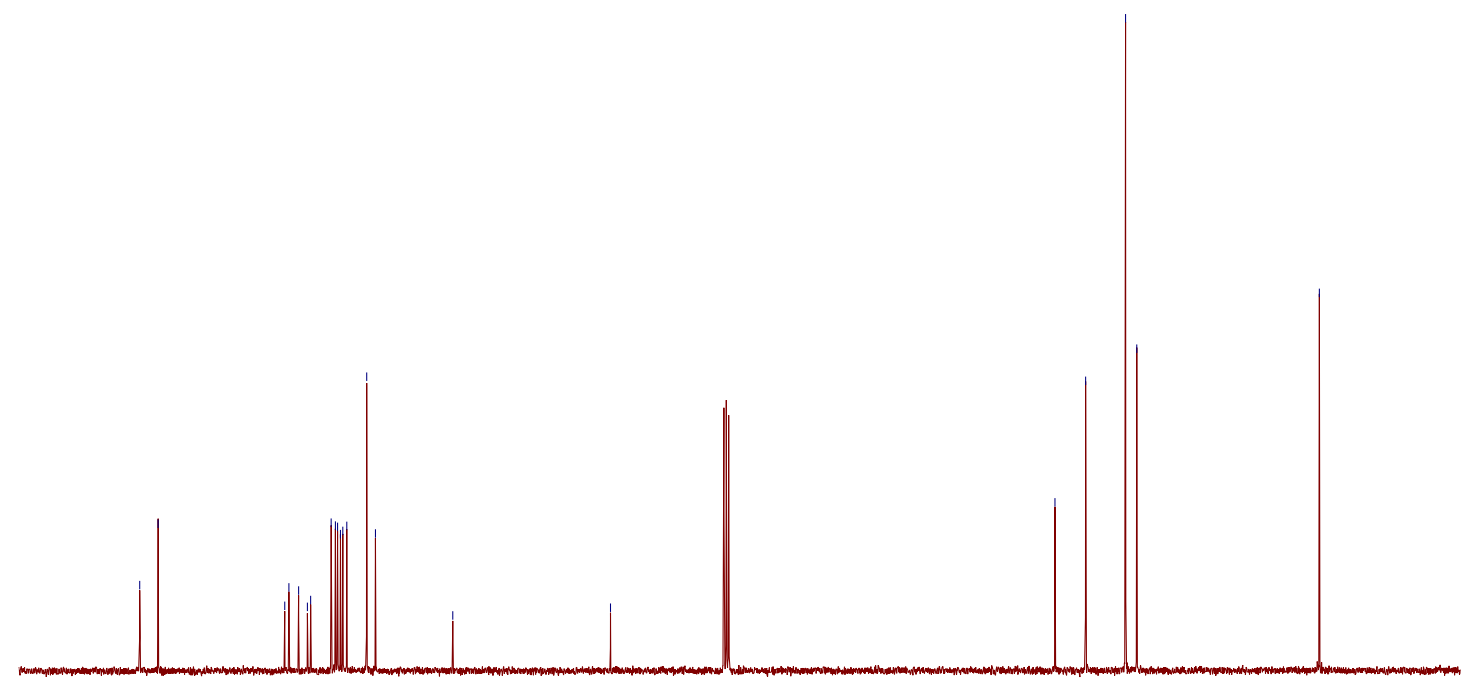

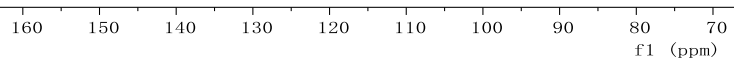


N'-(3-(cyclohex-1-en-1-yl)-1-(naphthalen-2-yl)prop-2-yn-1-ylidene)-2,4,6triisopropylbenzenesulfonohydrazide (1p)

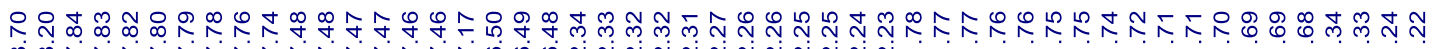

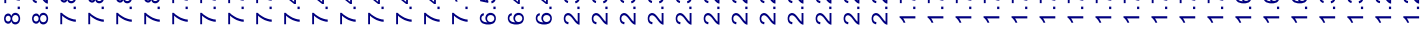<smiles>N=C(C#CC1=CCCCC1)c1ccc2ccccc2c1</smiles>

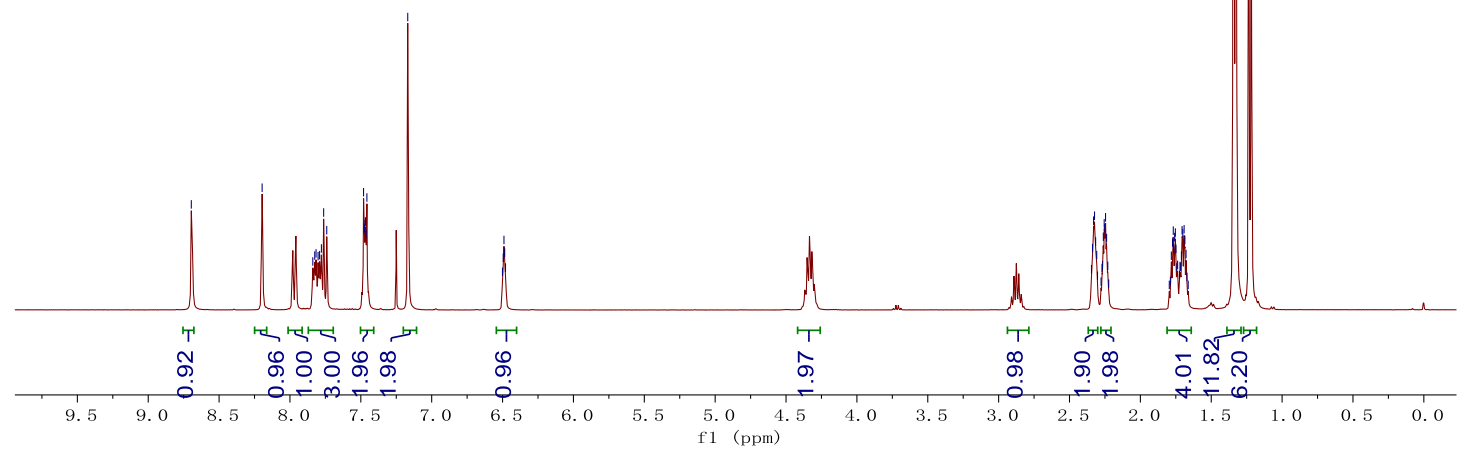

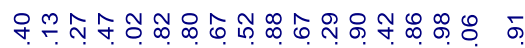

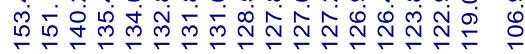

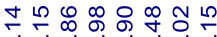

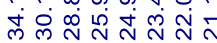

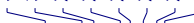

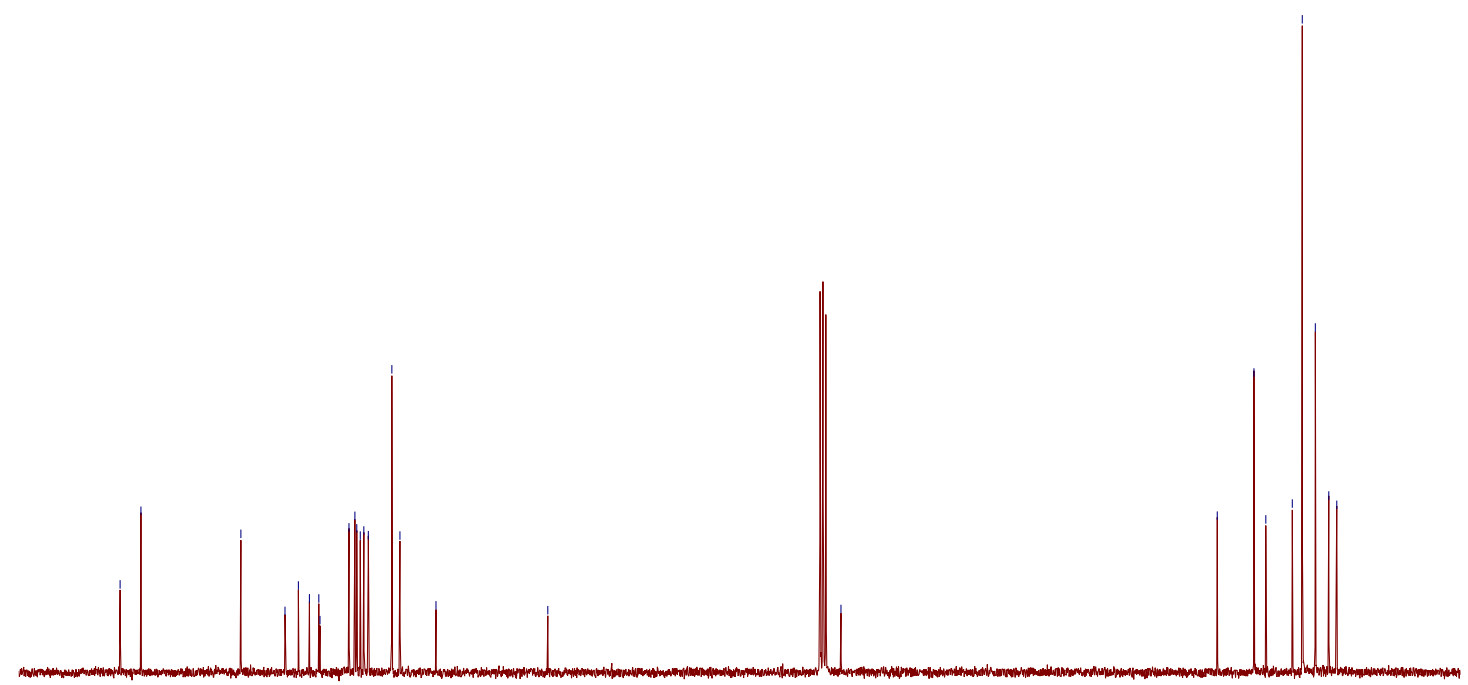

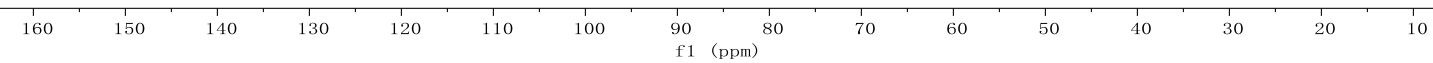


2,4,6-triisopropyl-N'-(1-(naphthalen-2-yl)undeca-2,4-diyn-1ylidene)benzenesulfonohydrazide (1q)

œ

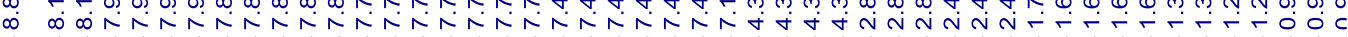<smiles>CC#CC#CC#CC(=N)c1ccc2ccccc2c1</smiles>

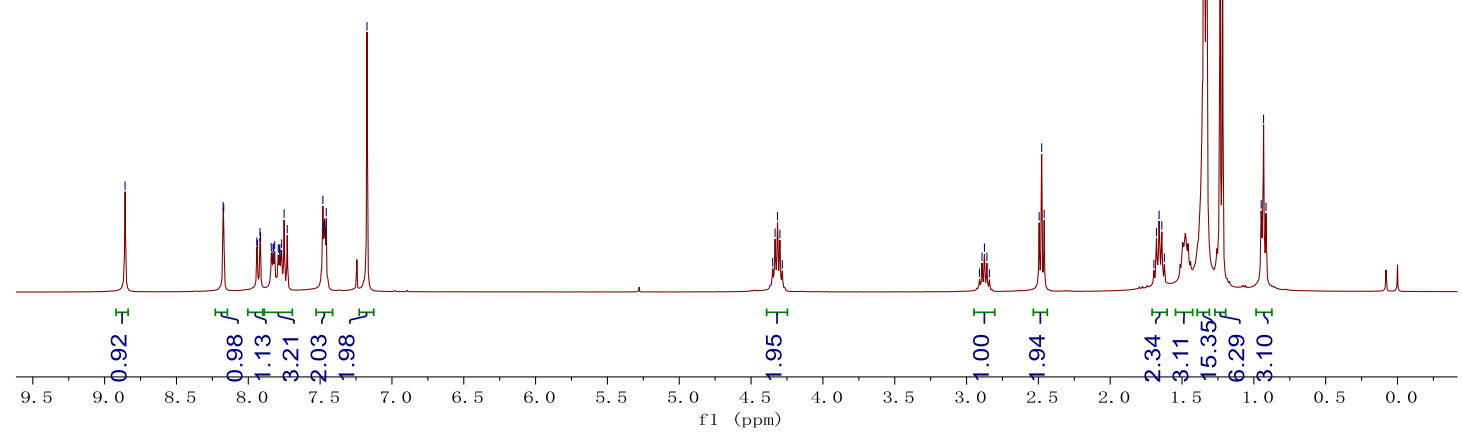

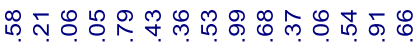

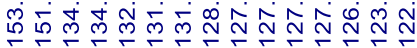

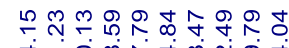

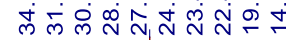

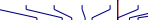

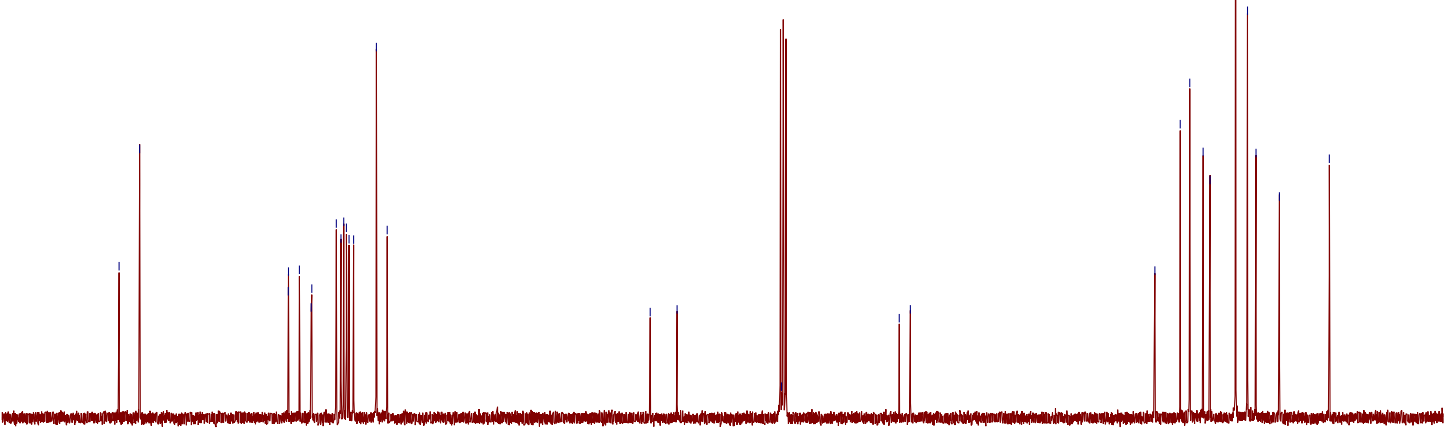

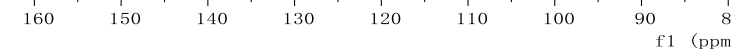


N'-(6-chloro-1-(naphthalen-2-yl)hex-2-yn-1-ylidene)-2,4,6triisopropylbenzenesulfonohydrazide (1r)

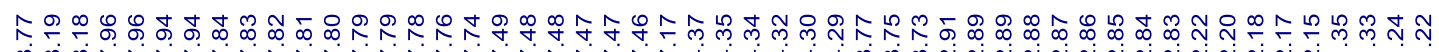

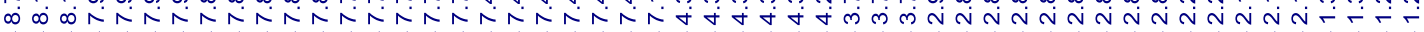<smiles>N=C(C#C[Hg]Cl)c1ccc2ccccc2c1</smiles>

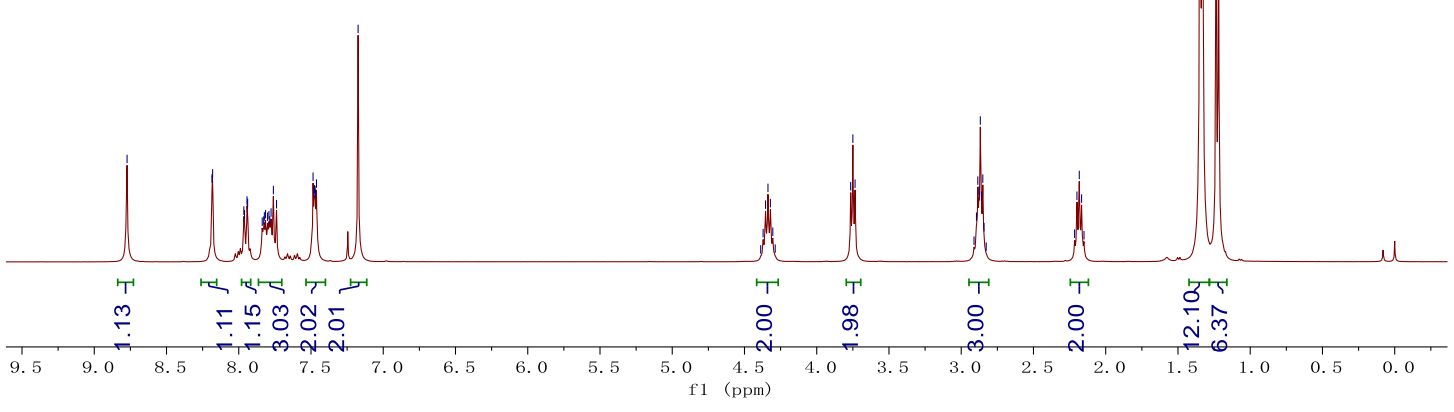

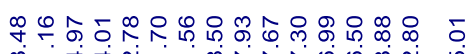

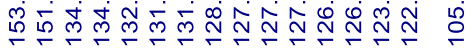

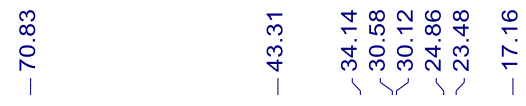

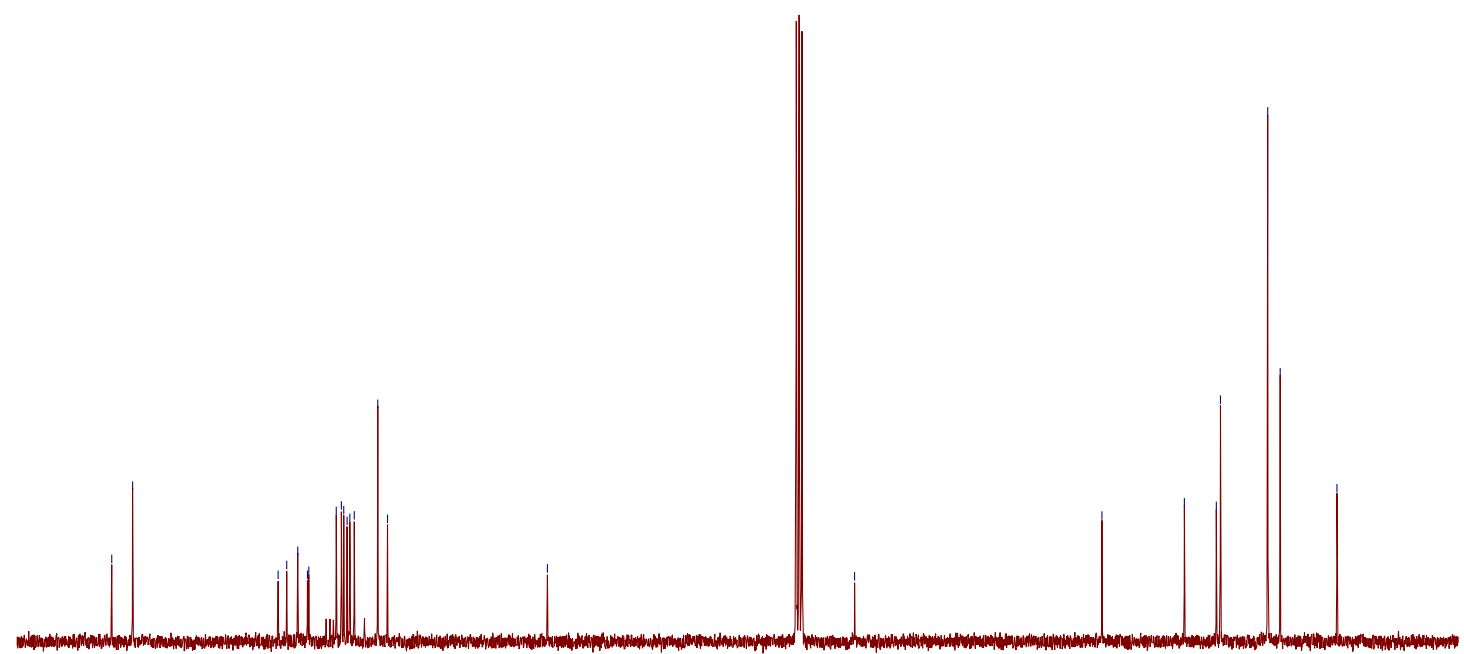

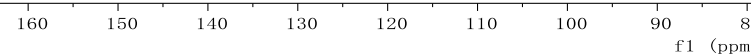


N'-(8-(benzyloxy)-1-(naphthalen-2-yl)oct-2-yn-1-ylidene)-2,4,6triisopropylbenzenesulfonohydrazide (1s)

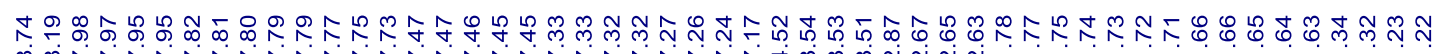

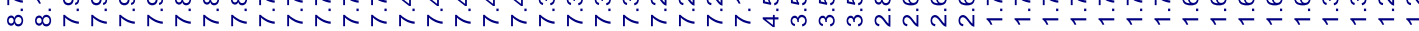<smiles></smiles>

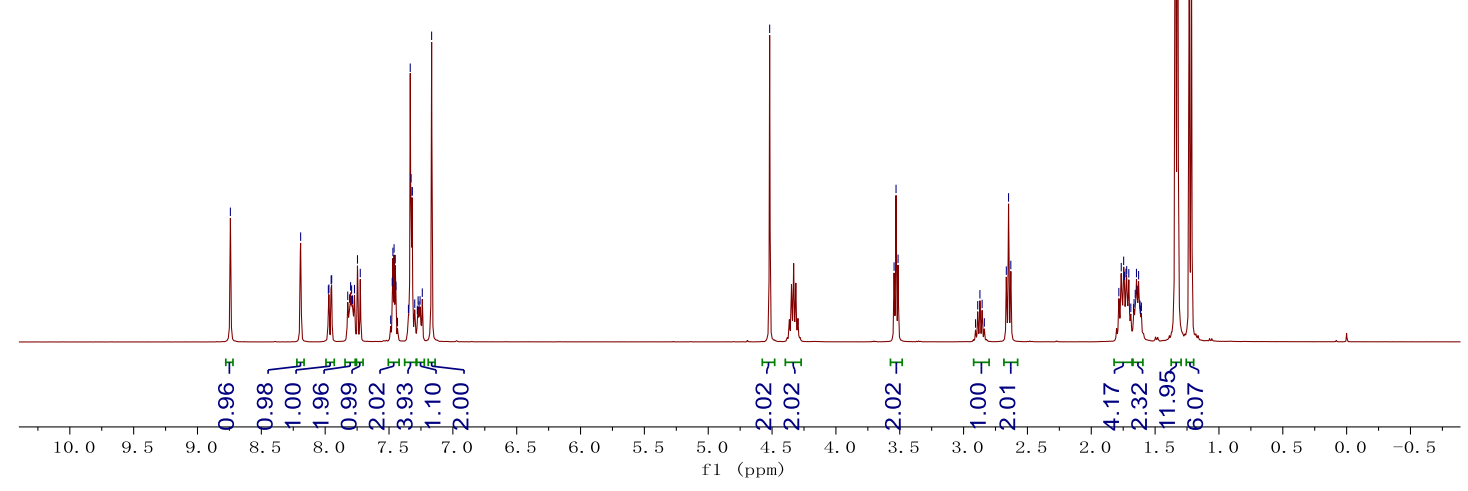

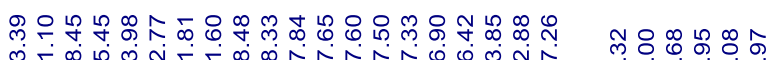

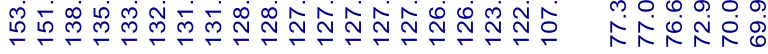

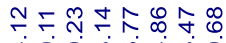
芦官

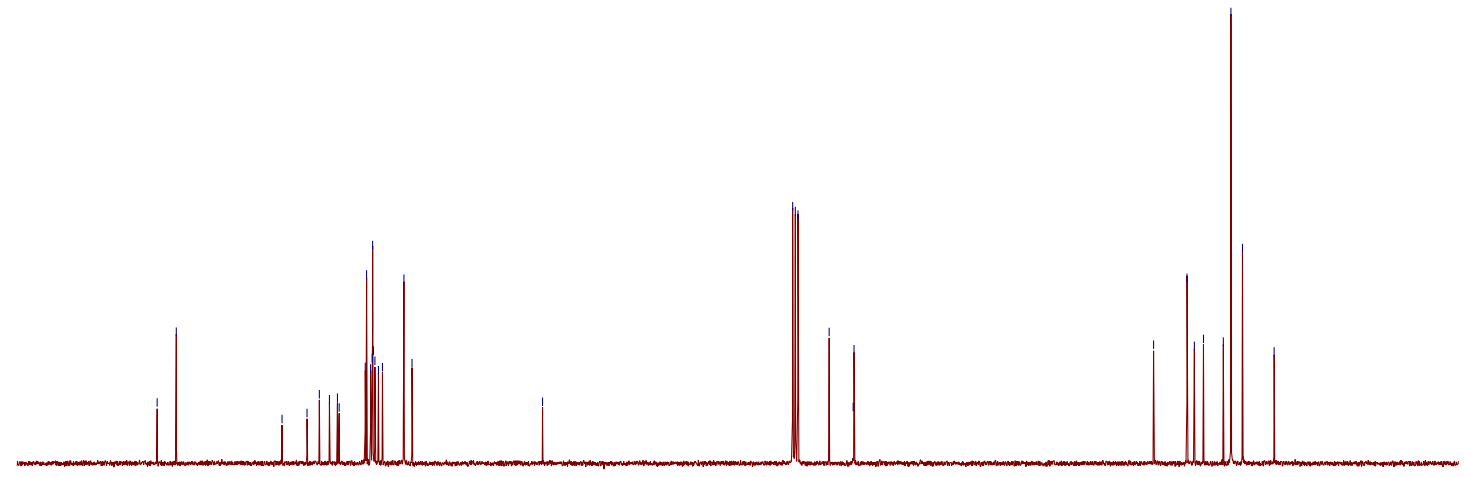

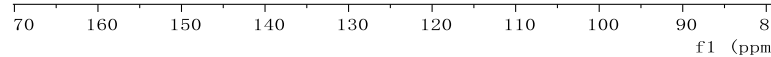


N'-(6-(1H-indol-1-yl)-1-(naphthalen-2-yl)hex-2-yn-1-ylidene)-2,4,6triisopropylbenzenesulfonohydrazide (1t)

Кำ

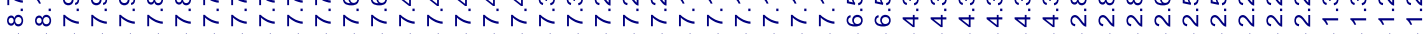<smiles>N=C(C#Cn1ccc2ccccc21)c1ccc2ccccc2c1</smiles>

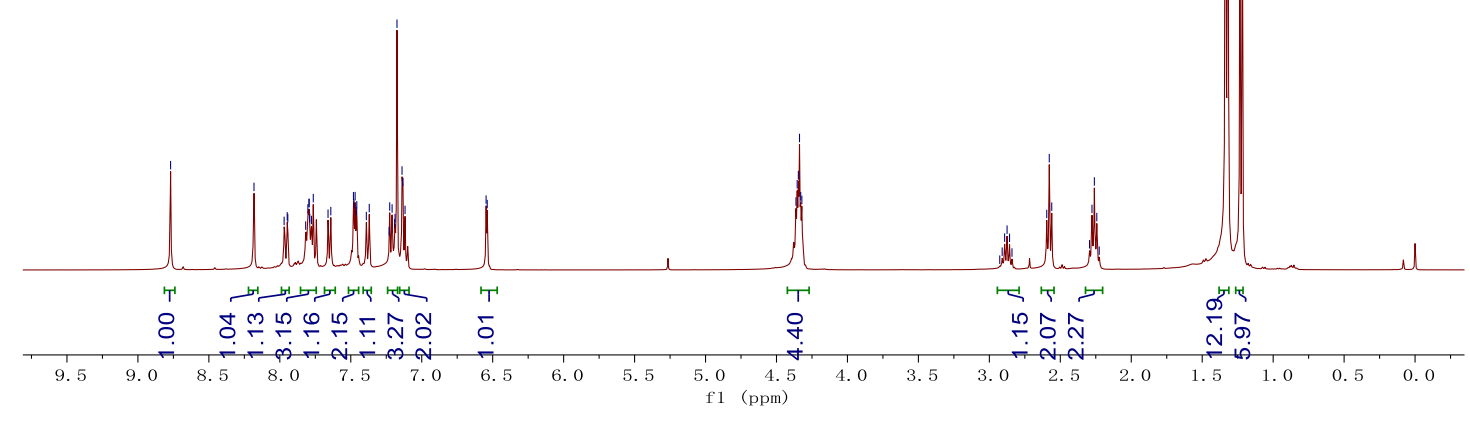

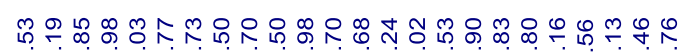

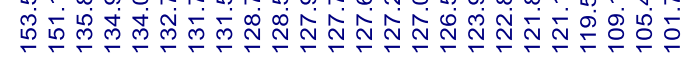

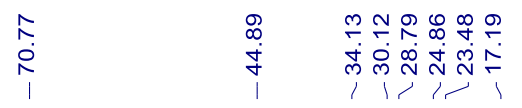

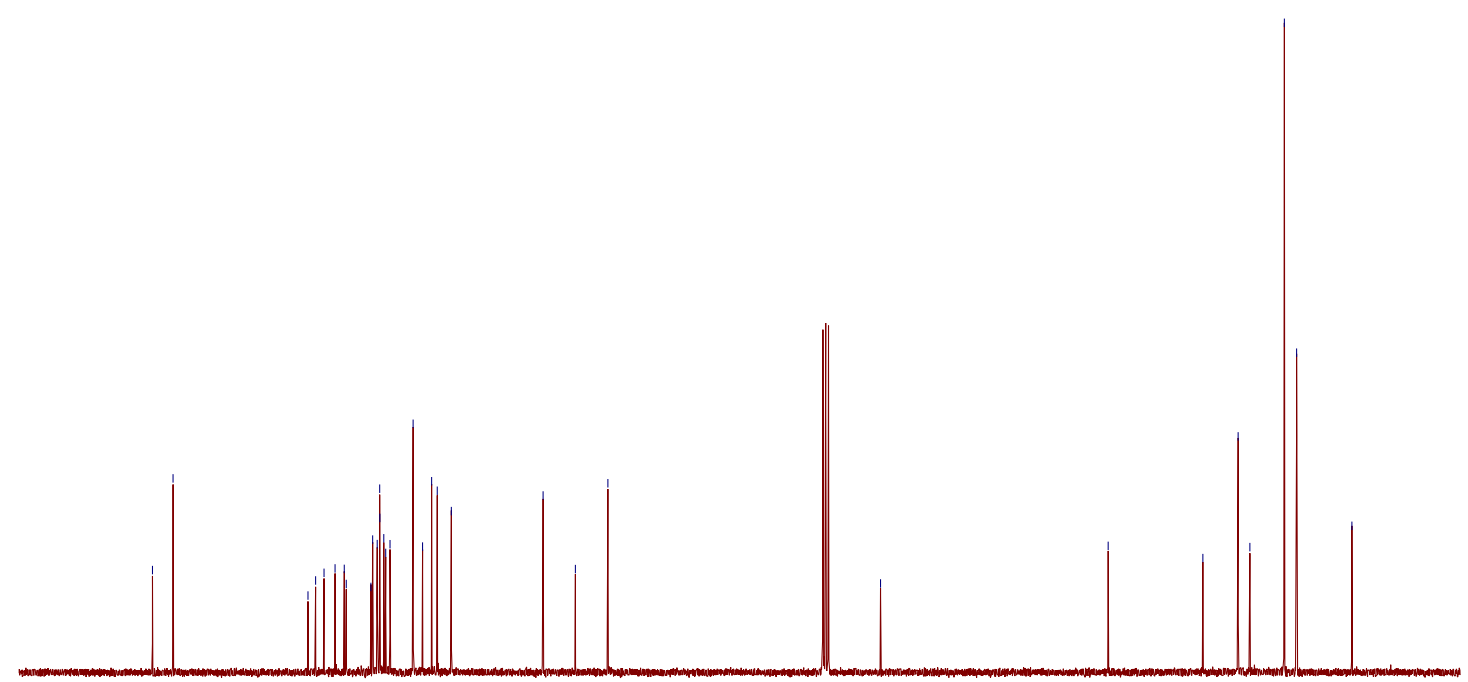

$\begin{array}{rrrrrrrr}160 & 150 & 140 & 130 & 120 & 110 & 100 & 90 \\ & & & & & & & \end{array}$ 
2,4,6-triisopropyl-N'-(1-(4-nitrophenyl)hept-2-yn-1ylidene)benzenesulfonohydrazide (1u)

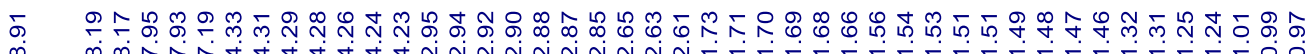

Atatatan<smiles>N=C(C#C[N+](=O)[O-])c1ccc(Br)cc1</smiles>

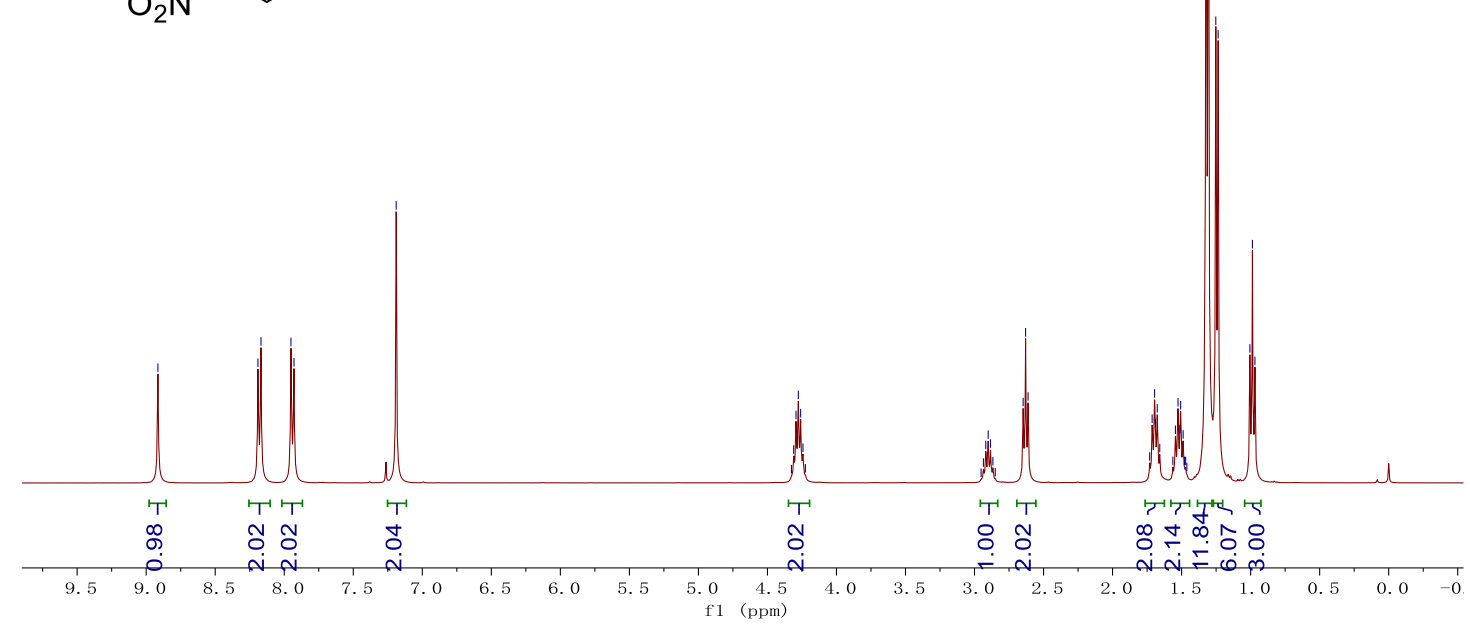

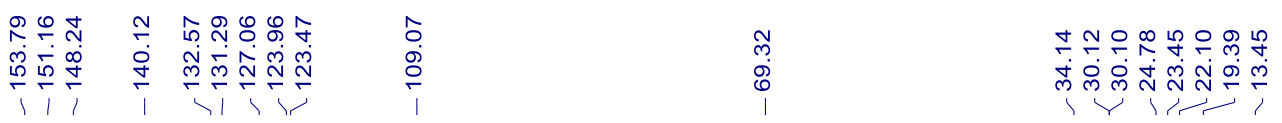
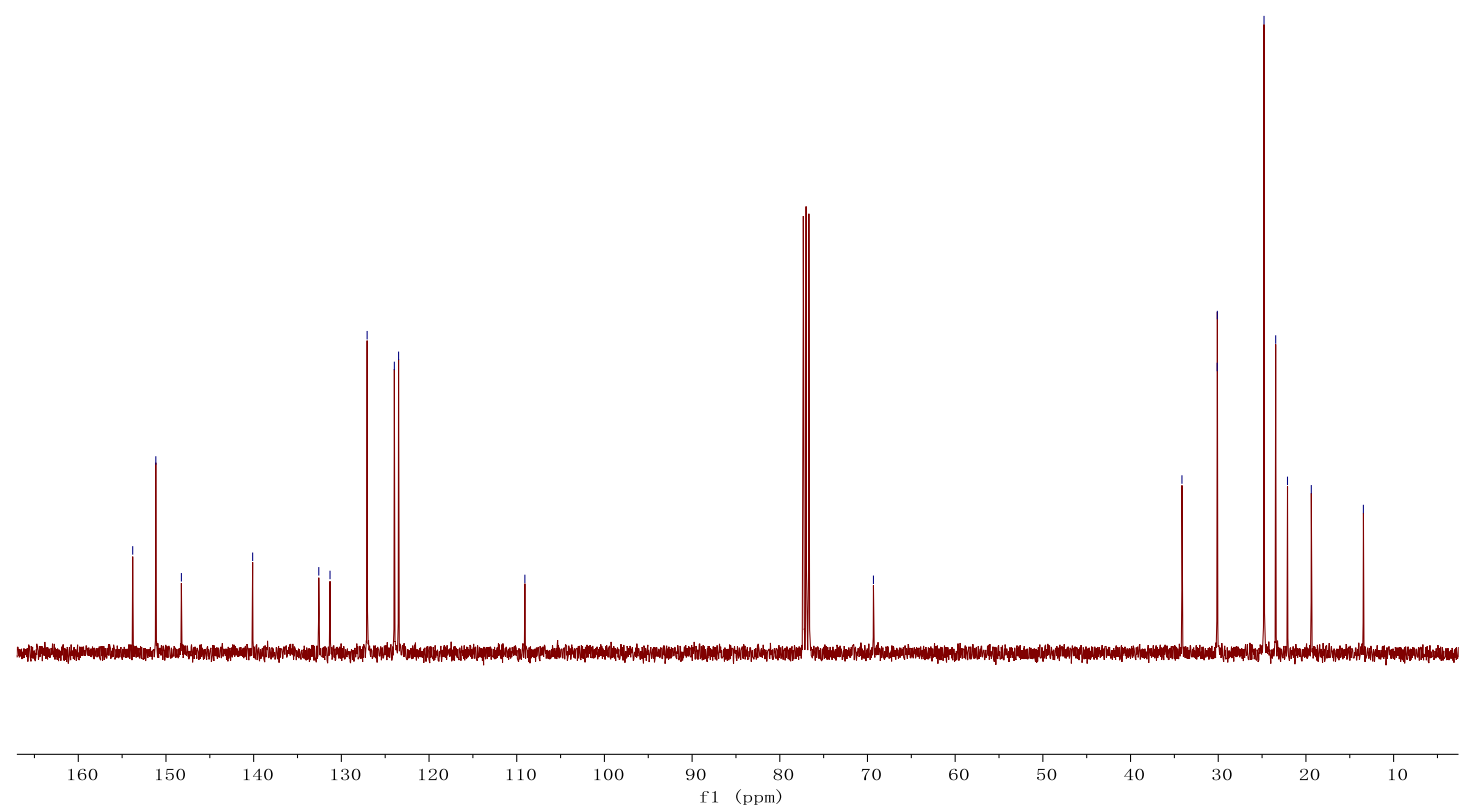
N'-(1-(3,4-dichlorophenyl)hept-2-yn-1-ylidene)-2,4,6triisopropylbenzenesulfonohydrazide (1v)

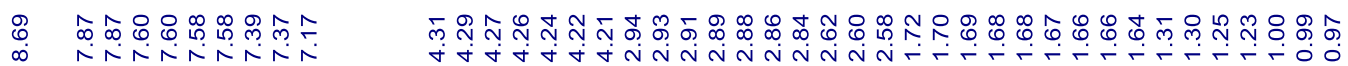<smiles>CC(C)(C)C#CC(=N)c1ccc(Cl)c(Cl)c1</smiles>

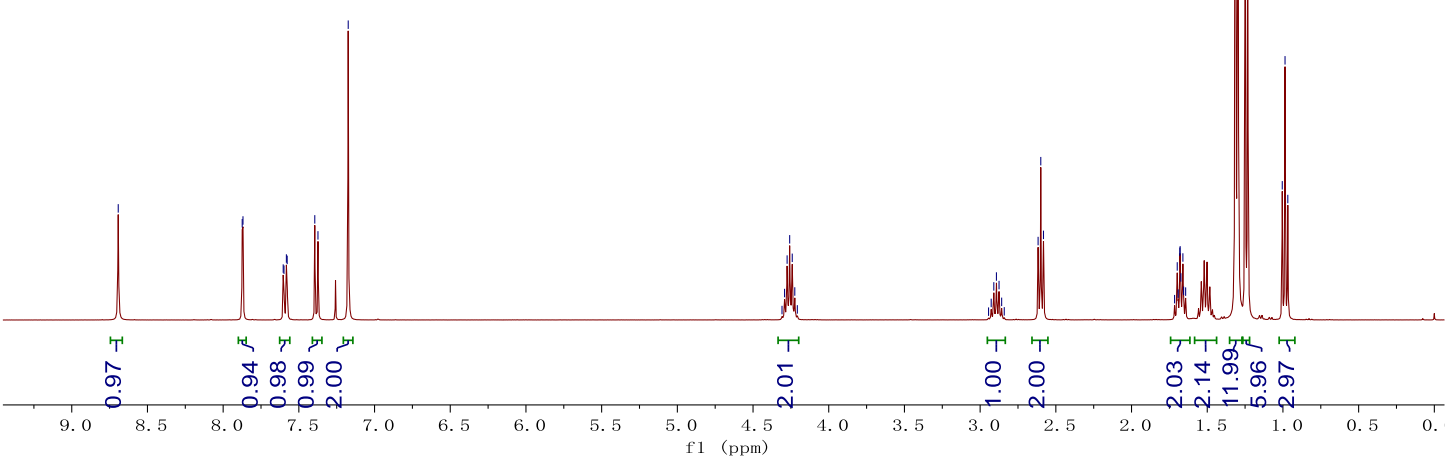

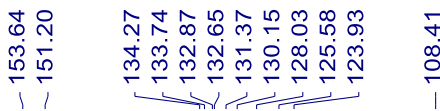

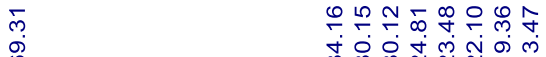

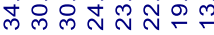

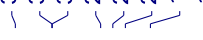

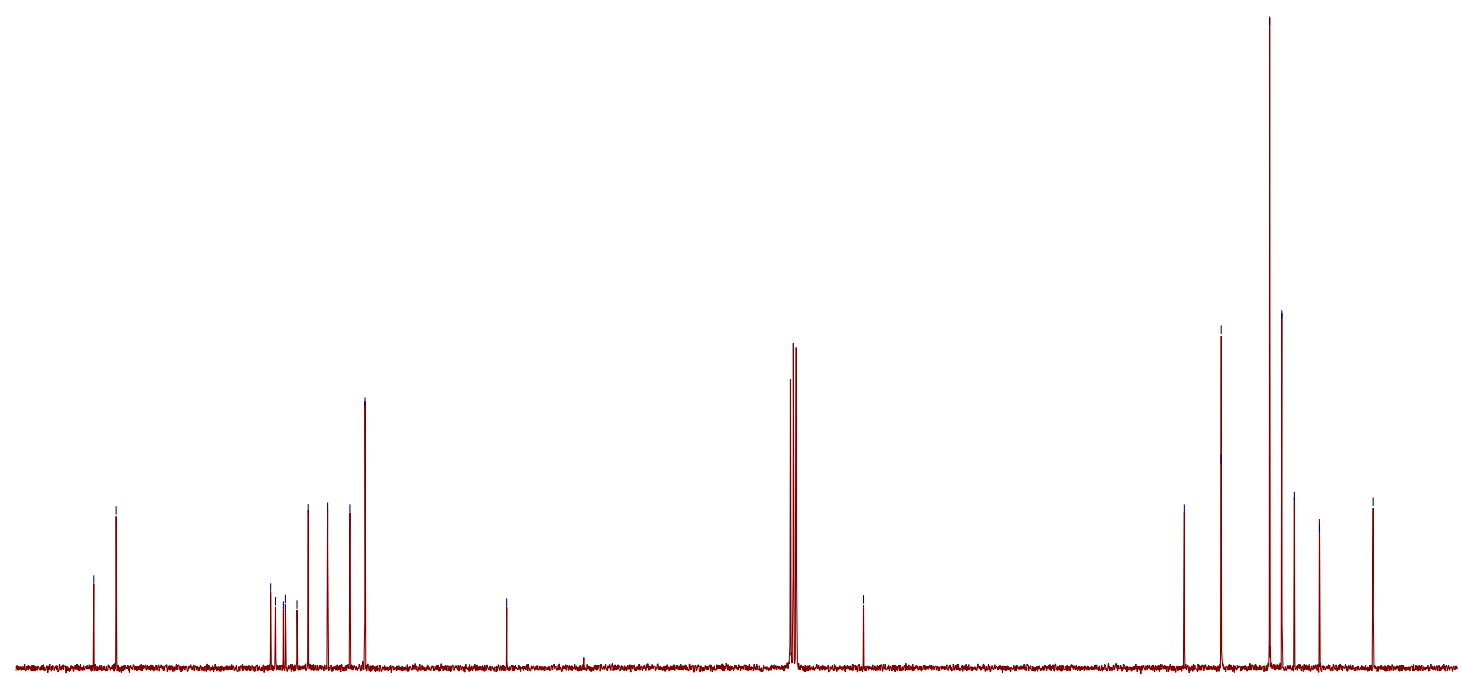

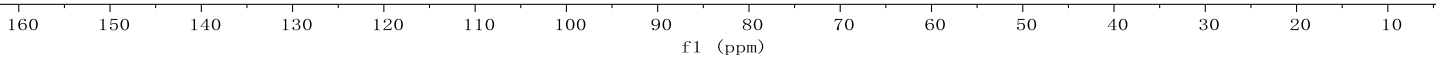


N'-(1-(3,4-dimethylphenyl)hept-2-yn-1-ylidene)-2,4,6triisopropylbenzenesulfonohydrazide (1w)

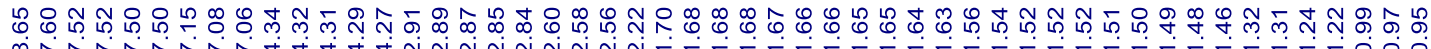

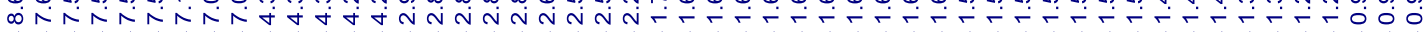<smiles>Cc1ccc(C(=N)C#CC(C)(C)C)cc1C</smiles>

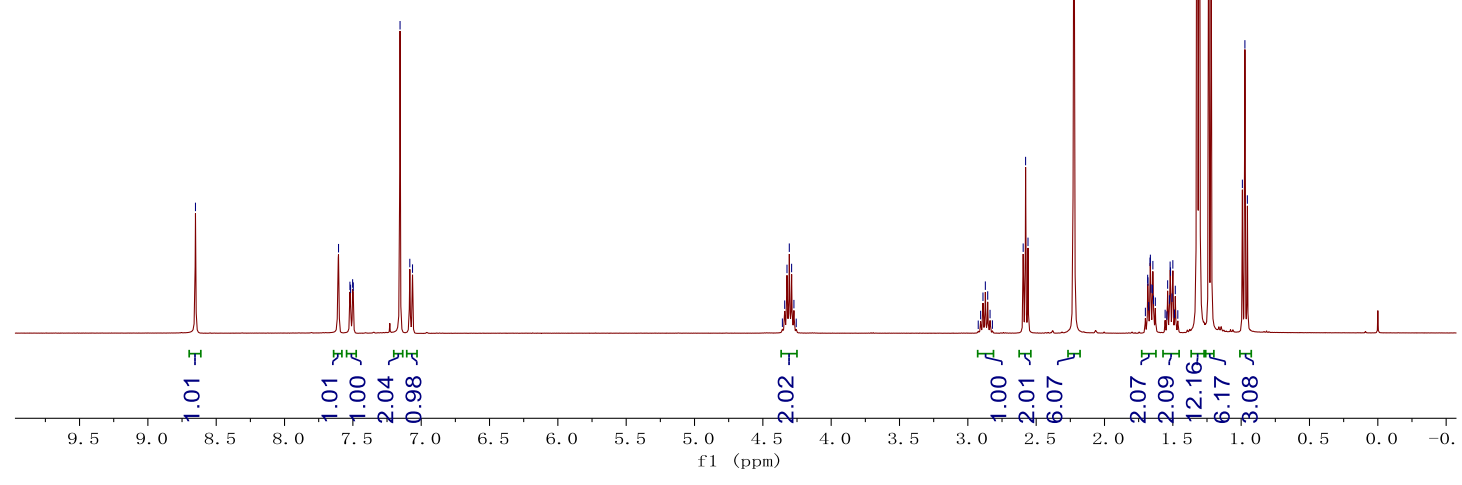

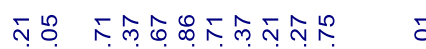

至点

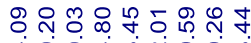

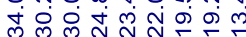

प प प?

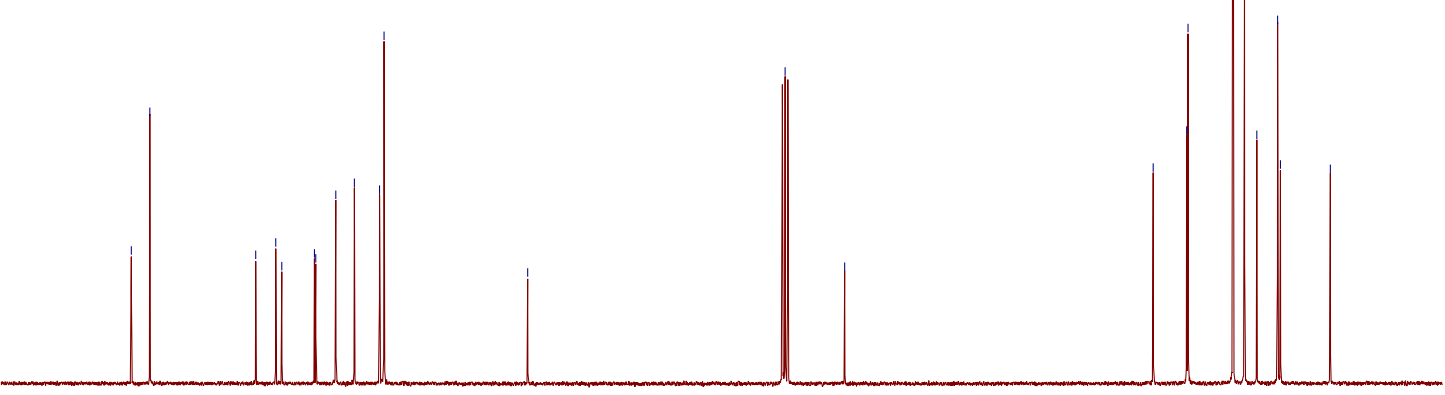

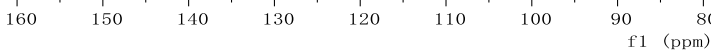


(Z)-N'-(1-(benzofuran-5-yl)hept-2-yn-1-ylidene)-2,4,6-

triisopropylbenzenesulfonohydrazide (1x)

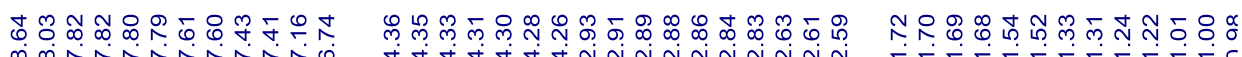<smiles>CCC(C)(C)C#CC(=N)c1ccc2occc2c1</smiles>

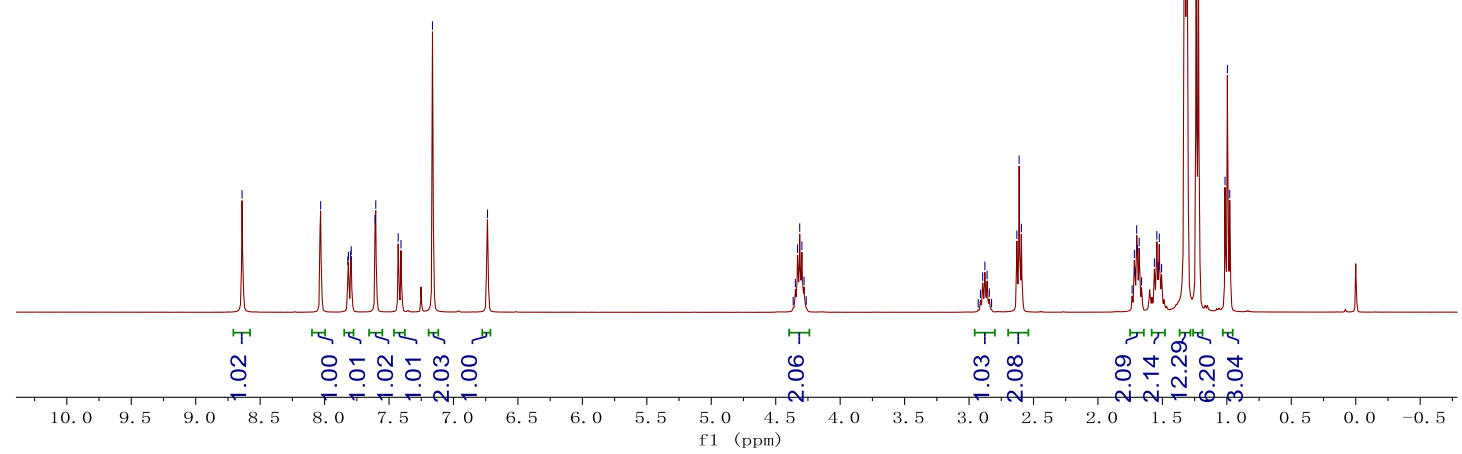

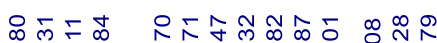

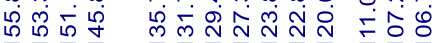

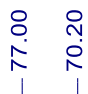

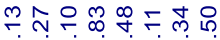

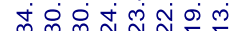

Y

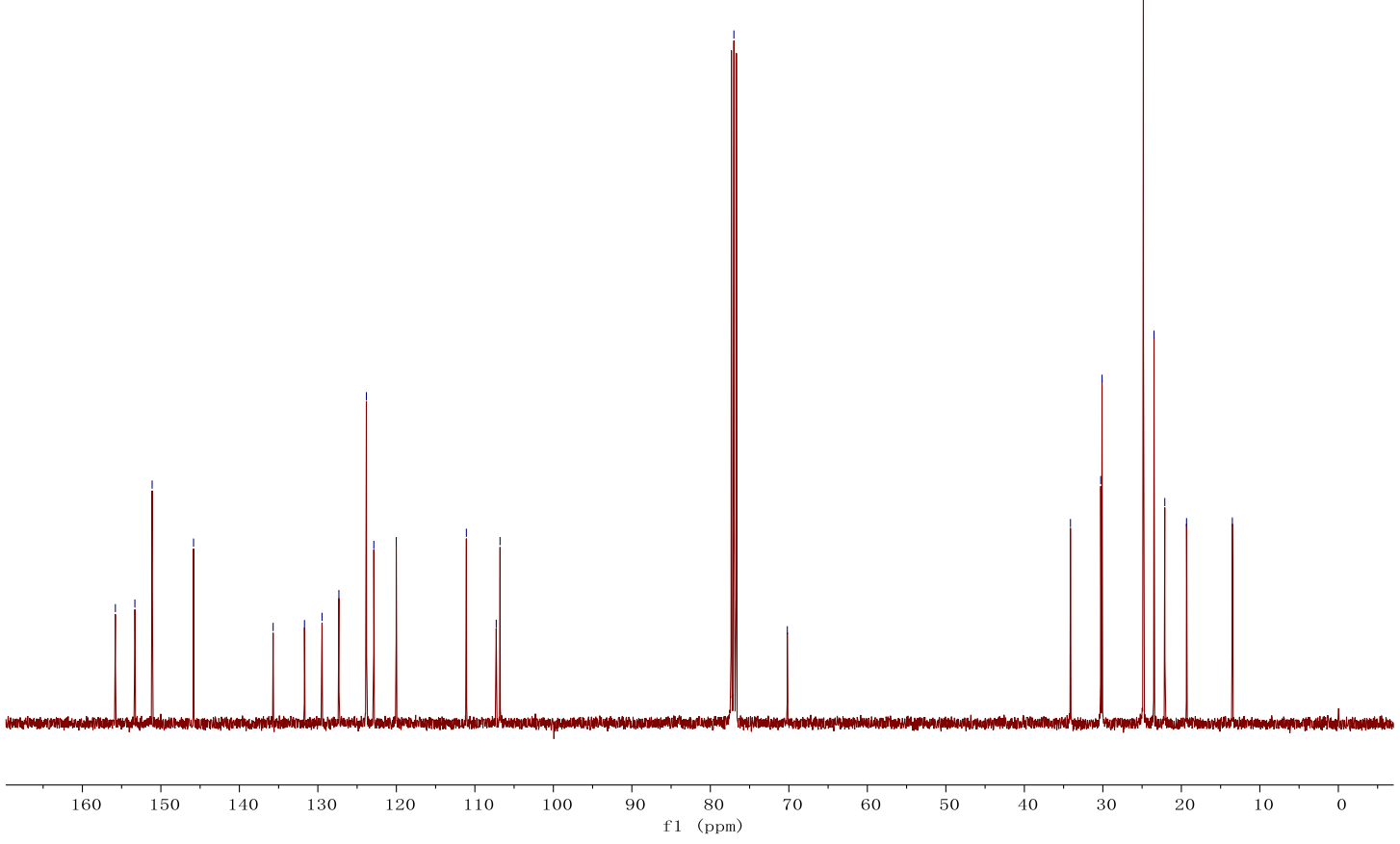


(Z)-2,4,6-triisopropyl-N'-(4-phenylbut-3-yn-2-ylidene)benzenesulfonohydrazide (1y)
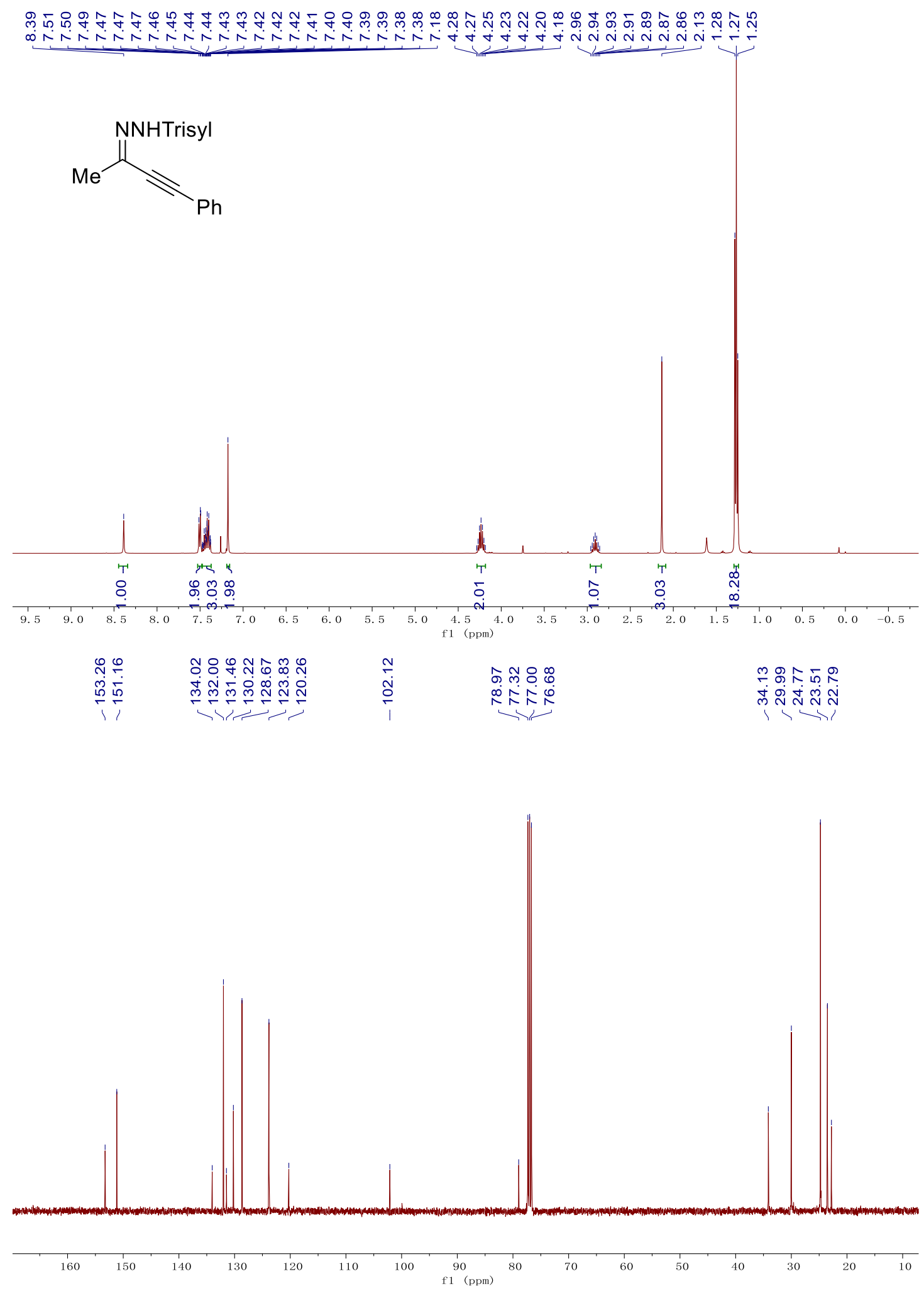
<smiles>CC[SeH]C(C#Cc1ccccc1)c1ccc2ccccc2c1</smiles>

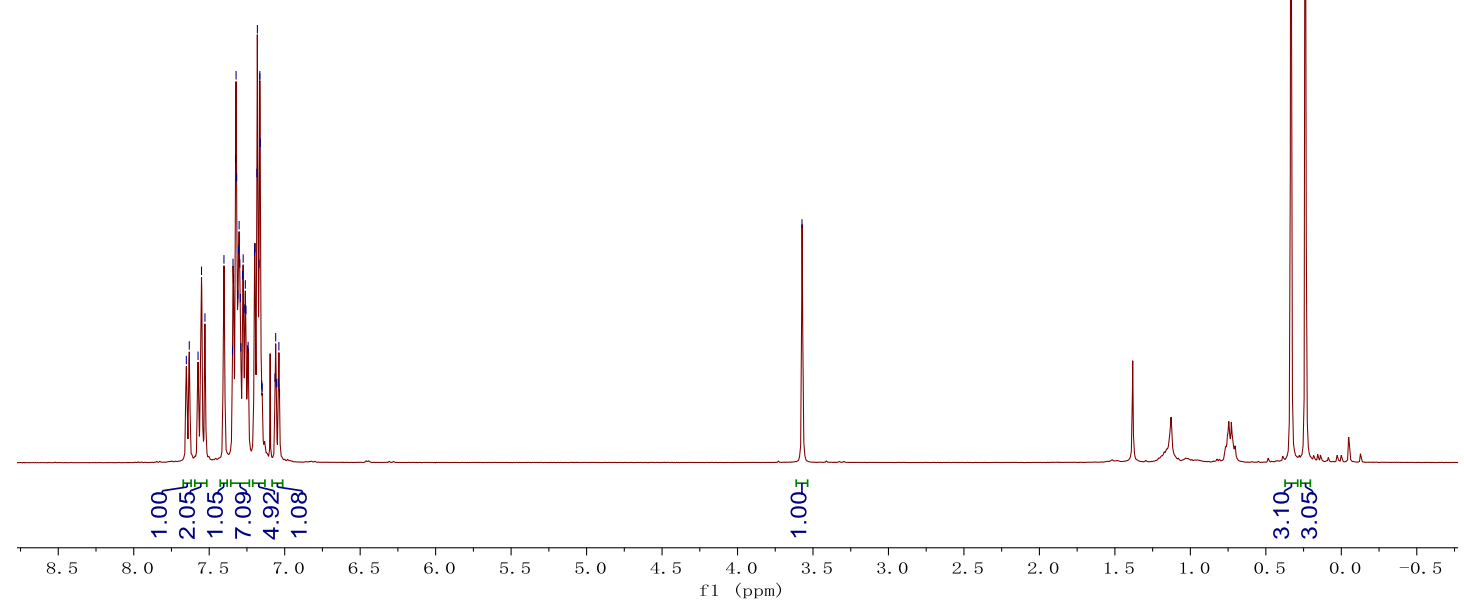

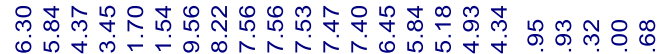

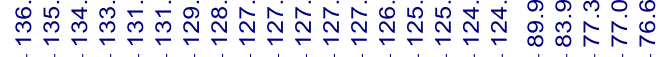

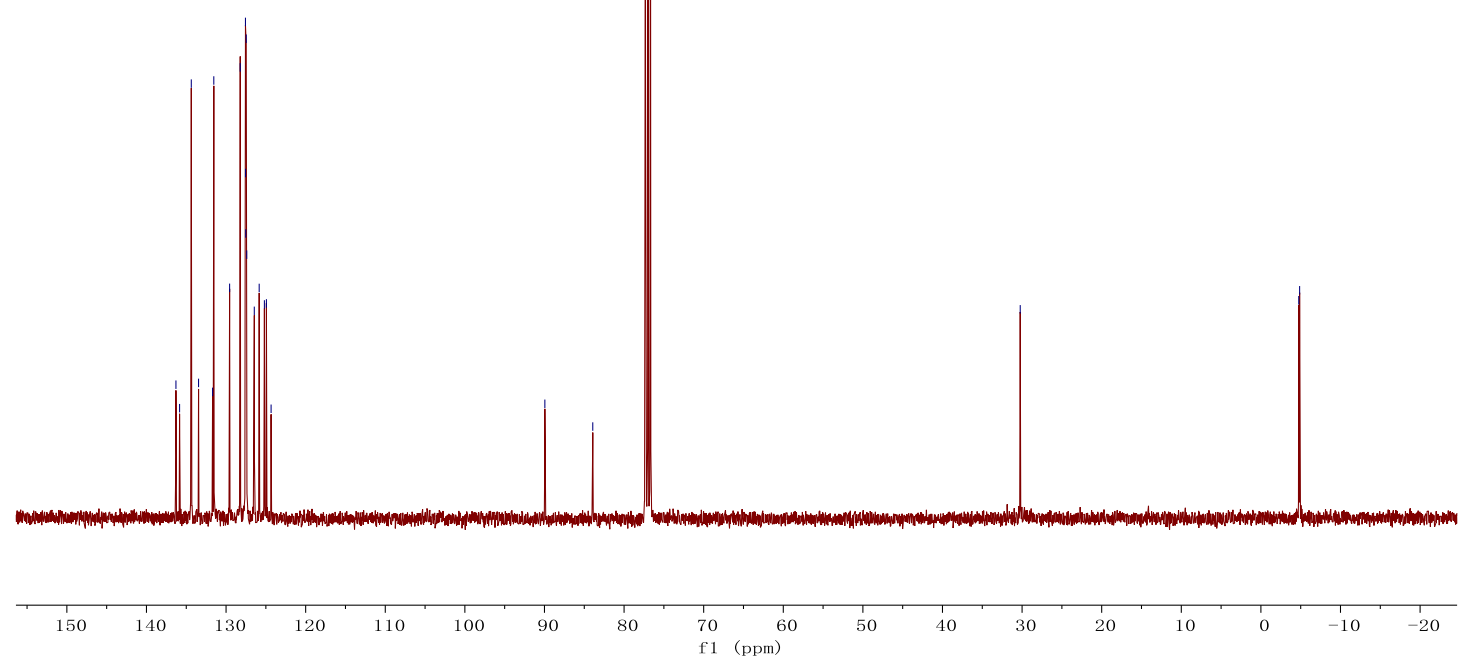


(-)-triethyl(1-(naphthalen-2-yl)-3-phenylprop-2-yn-1-yl)silane (3ab)

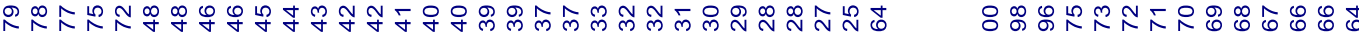
rnkn<smiles>CCC(C#Cc1ccccc1)c1ccc2ccccc2c1</smiles>

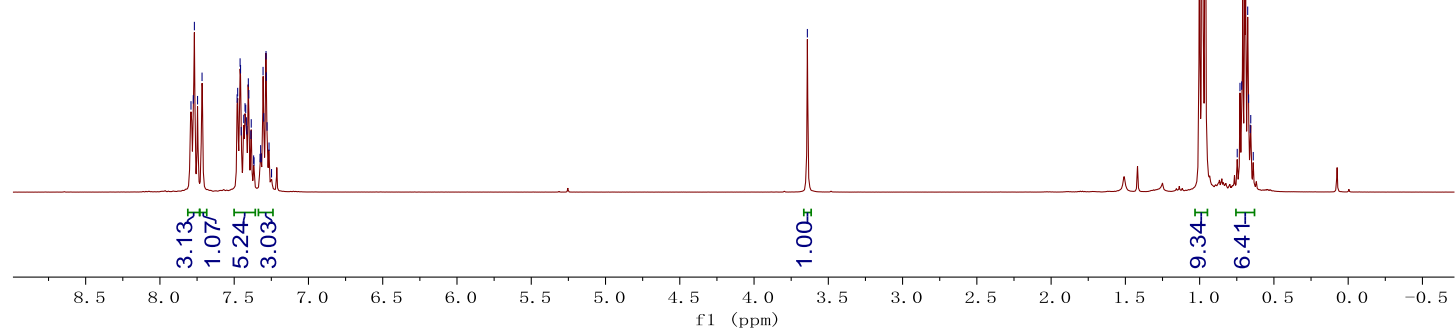

กุ.

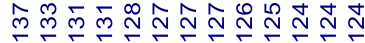

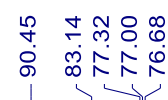

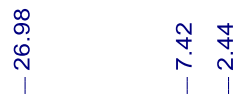
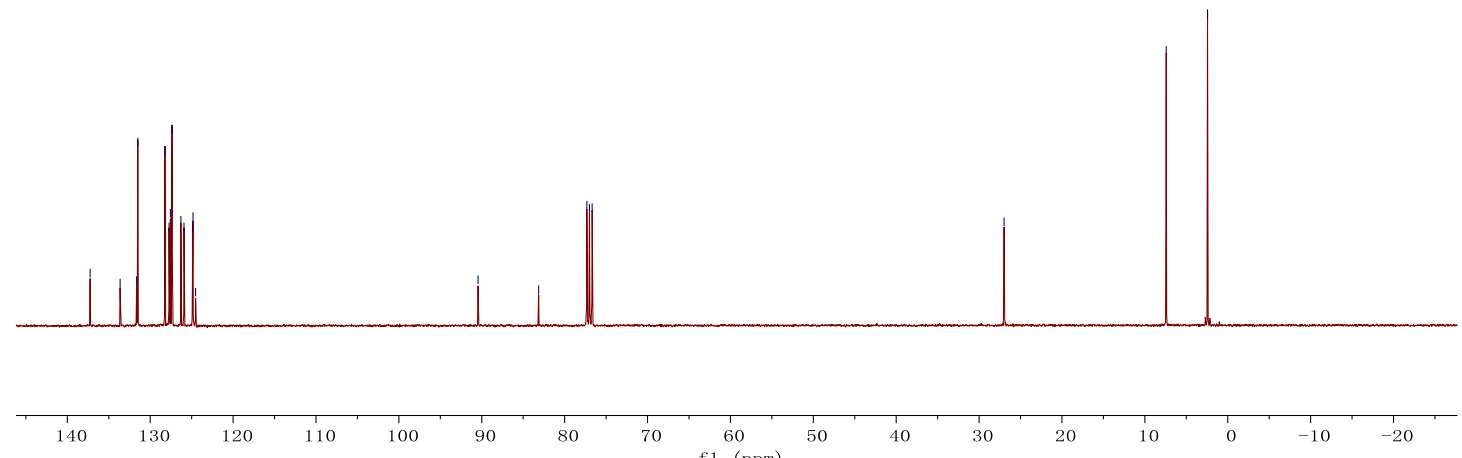
(-)-methyl(1-(naphthalen-2-yl)-3-phenylprop-2-yn-1-yl)diphenylsilane (3ac)

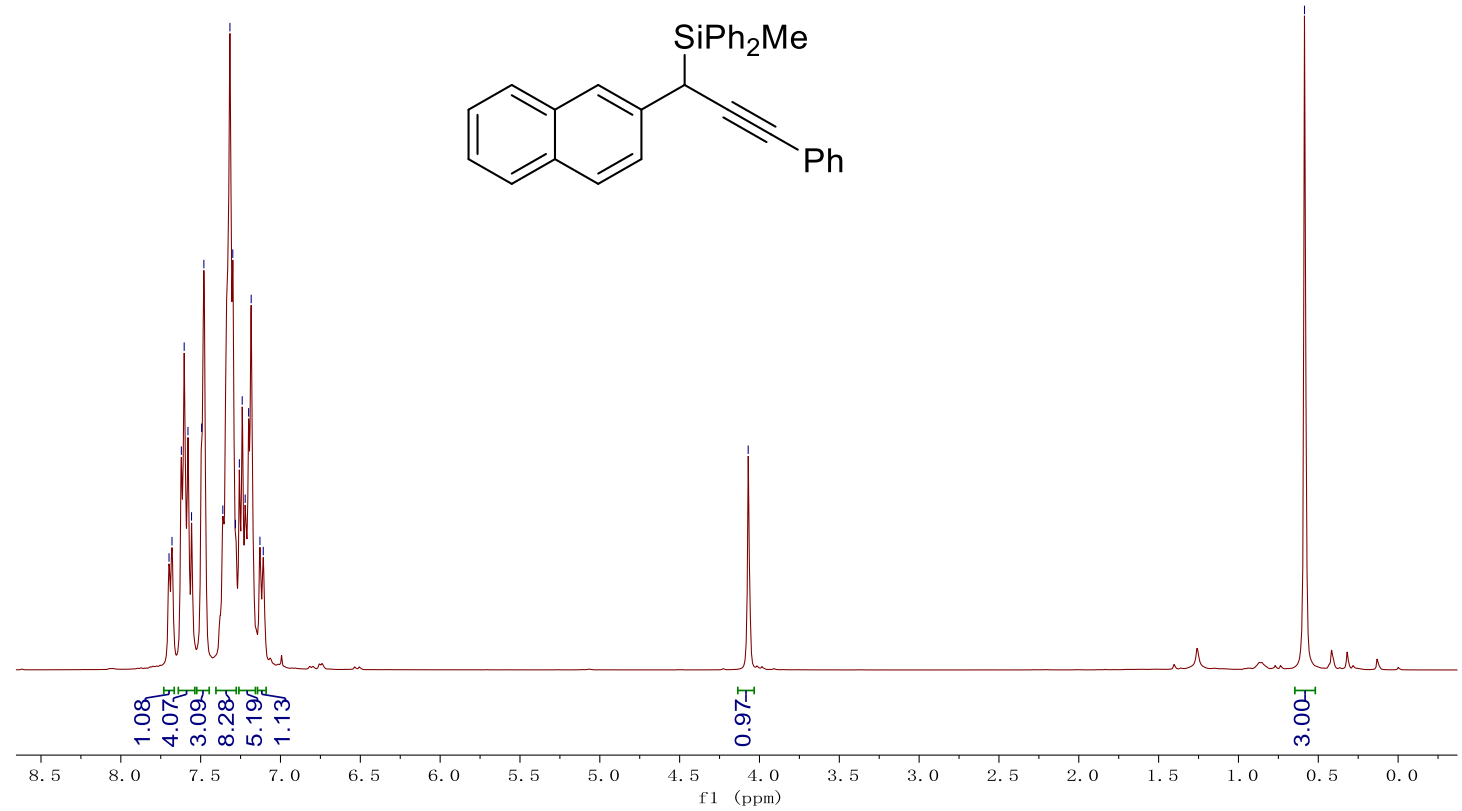

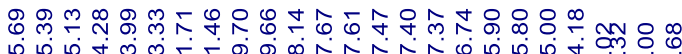

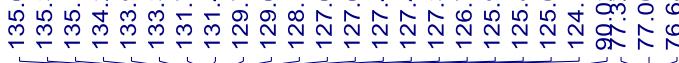
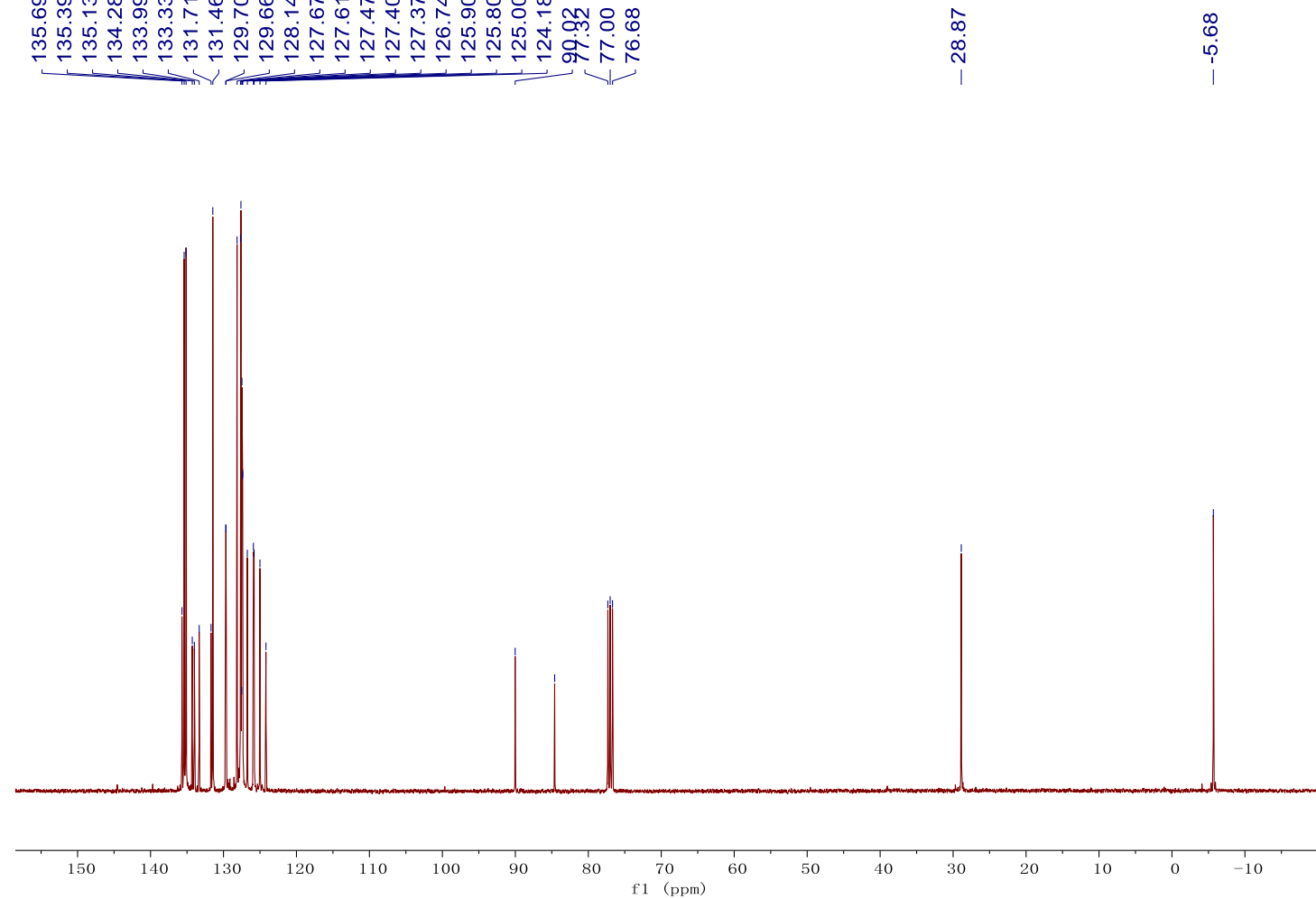
(-)-(1,3-diphenylprop-2-yn-1-yl)triethylsilane (3bb)<smiles>CCC(C#Cc1ccccc1)c1ccccc1</smiles>

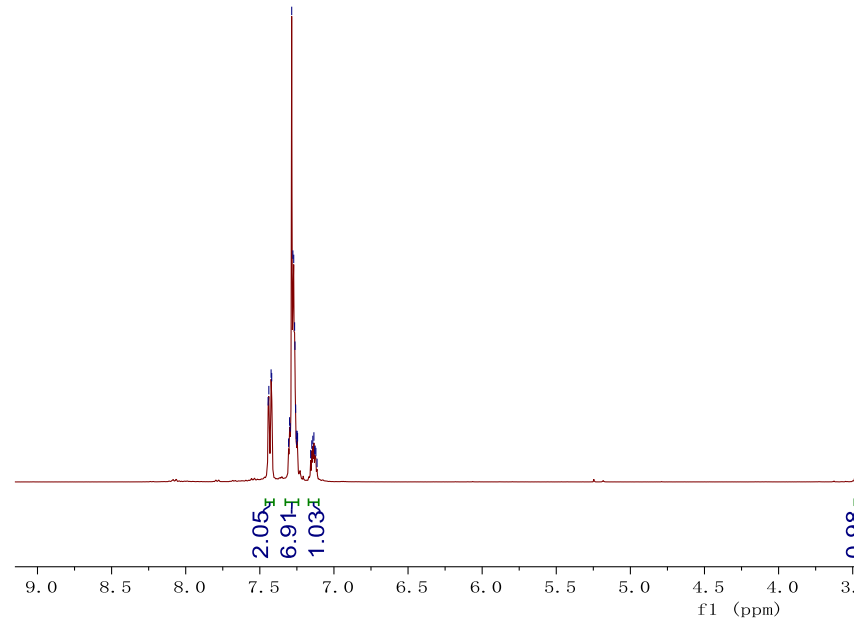

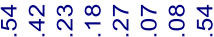

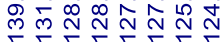

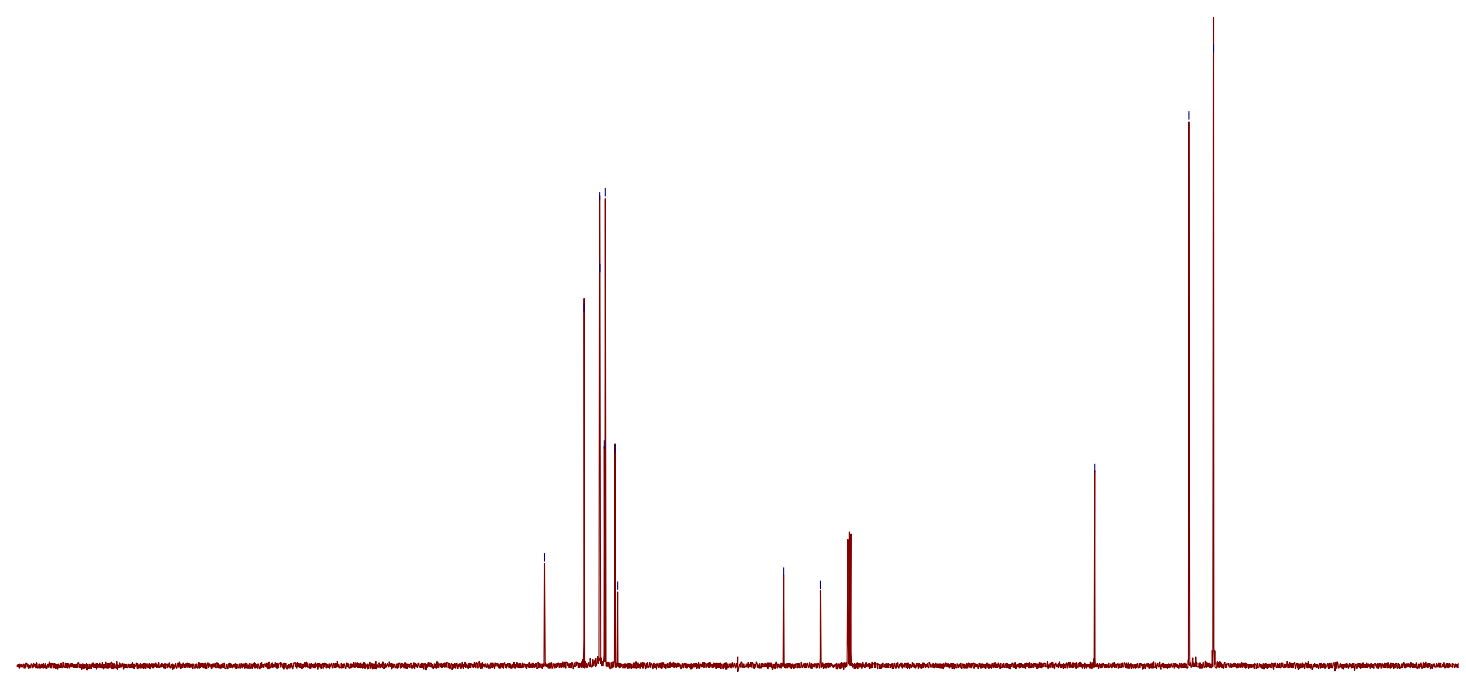

$\begin{array}{llllllllllllllll}240 & 230 & 220 & 210 & 200 & 190 & 180 & 170 & 160 & 150 & 140 & 130 & 120 & 110 & 100 & 90\end{array}$ 
(-)-(1-(2-chlorophenyl)-3-phenylprop-2-yn-1-yl)triethylsilane (3cb) ○

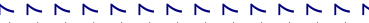<smiles>C[SiH2]C(C#CPc1ccccc1)c1ccccc1Cl</smiles>

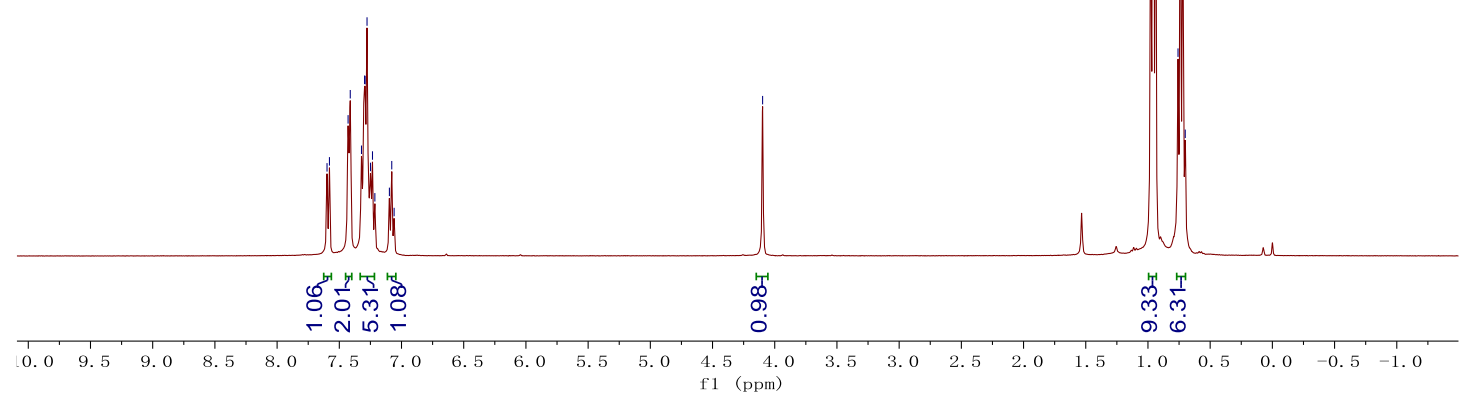

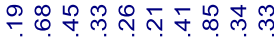

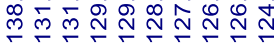

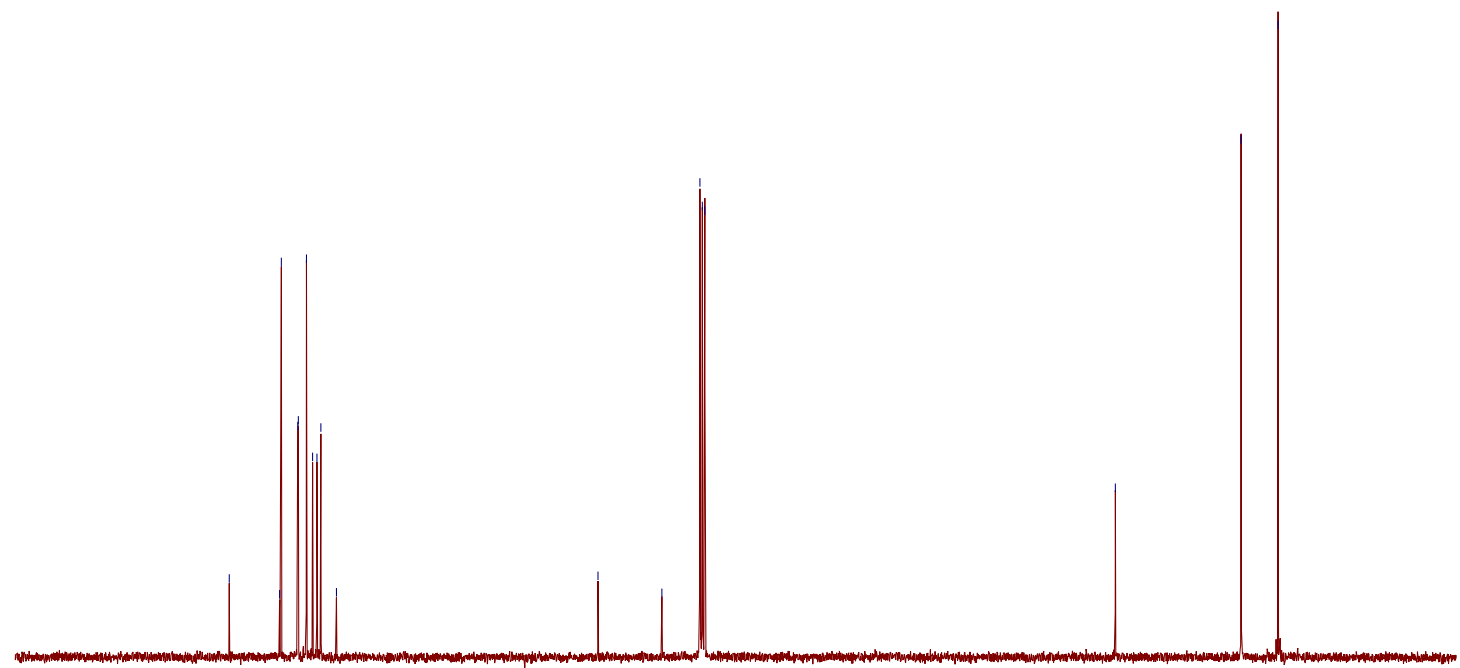


(-)-(1-(3-chlorophenyl)-3-phenylprop-2-yn-1-yl)triethylsilane (3db)

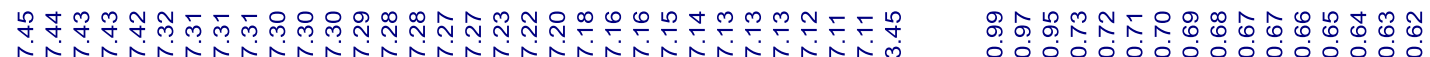

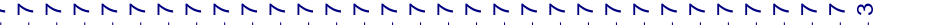<smiles>C[AsH2]C(C#Cc1ccccc1)c1cccc(Cl)c1</smiles>

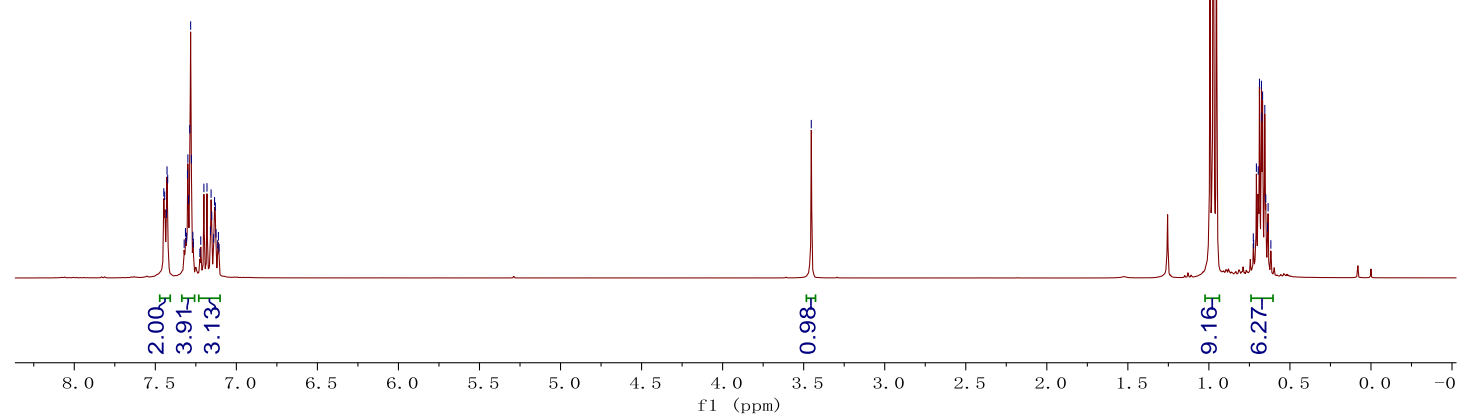

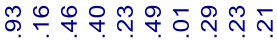

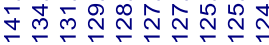

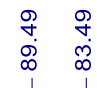
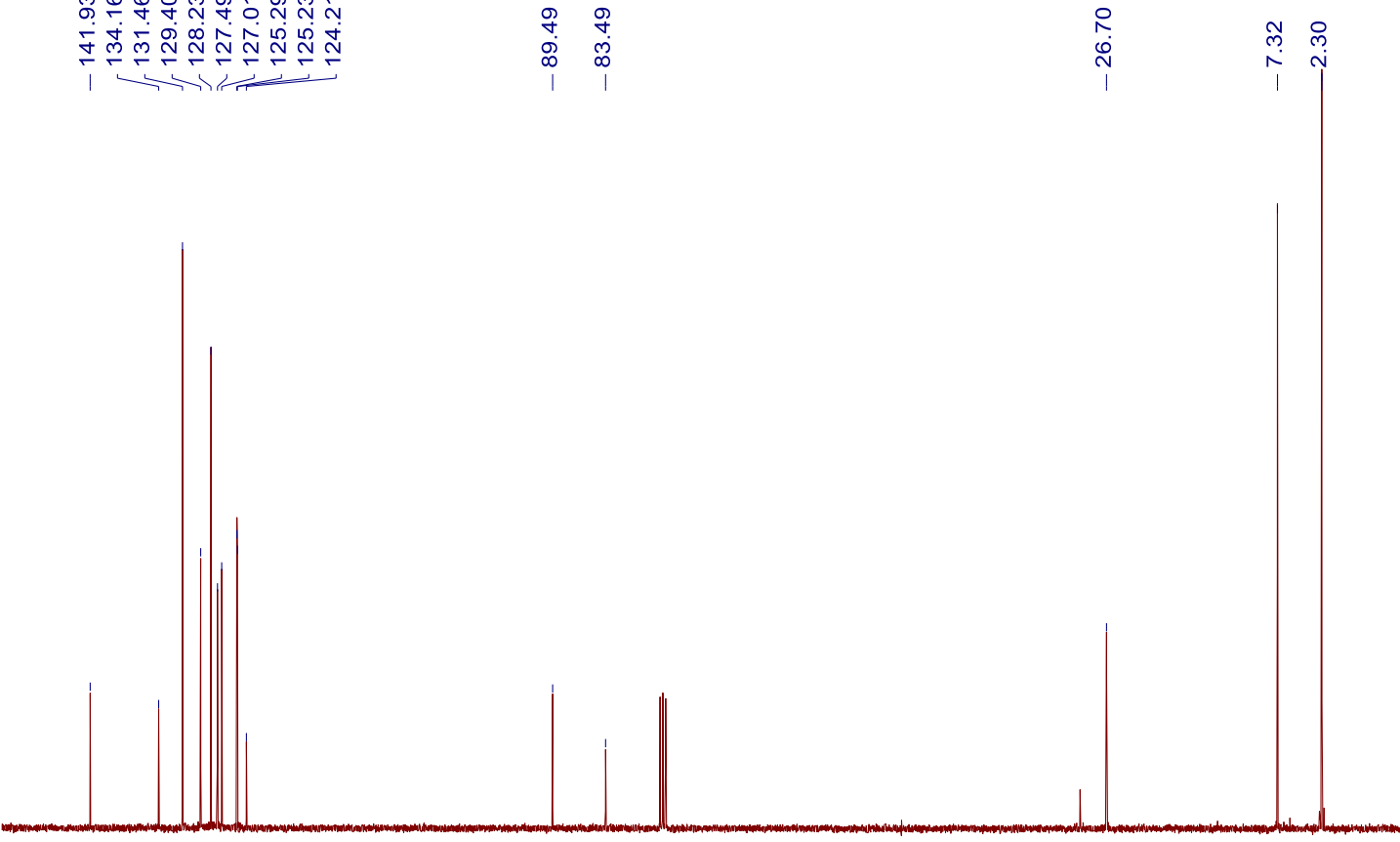
(-)-triethyl(1-(4-methoxyphenyl)-3-phenylprop-2-yn-1-yl)silane (3eb)

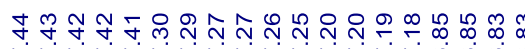

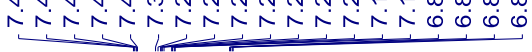

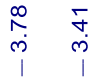

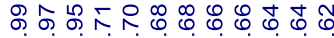

000000000000<smiles>CCC(C#Cc1ccccc1)c1ccc(OC)cc1</smiles>

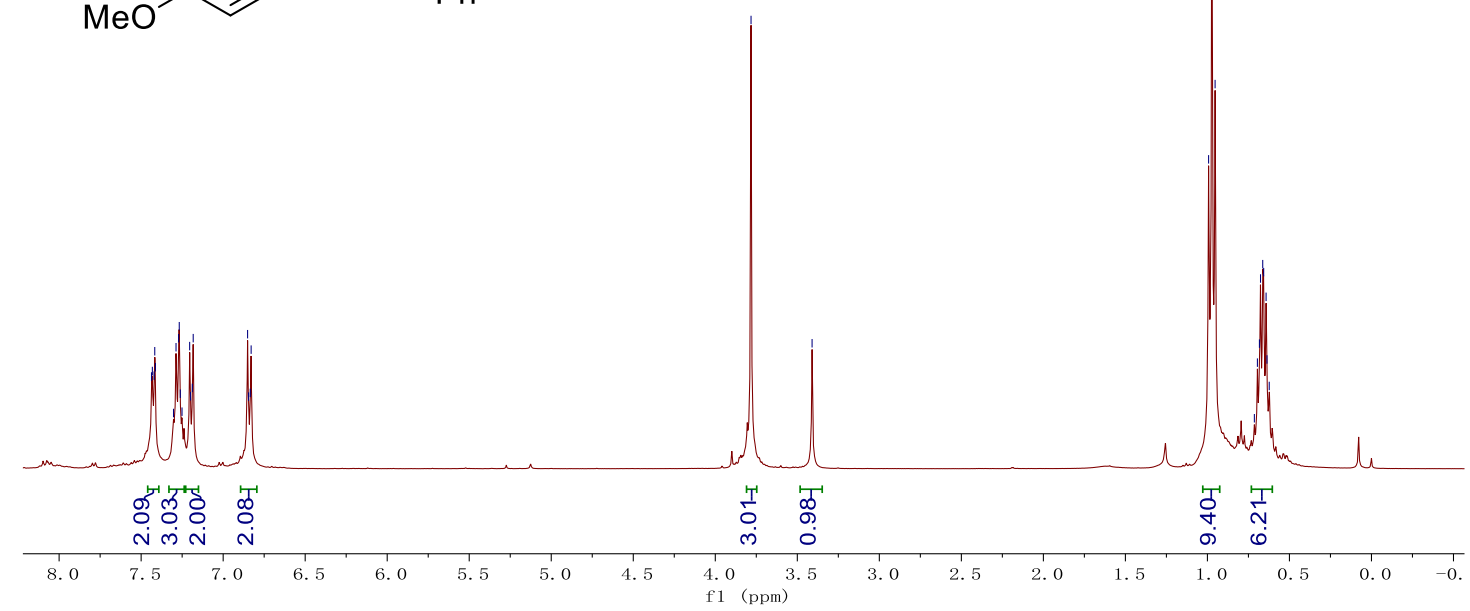

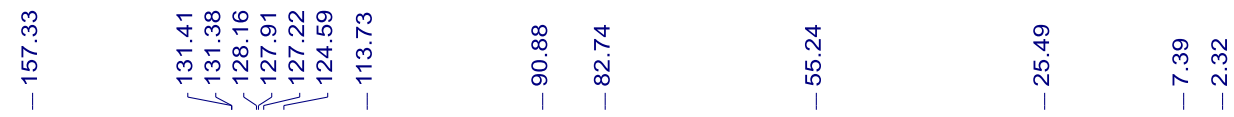

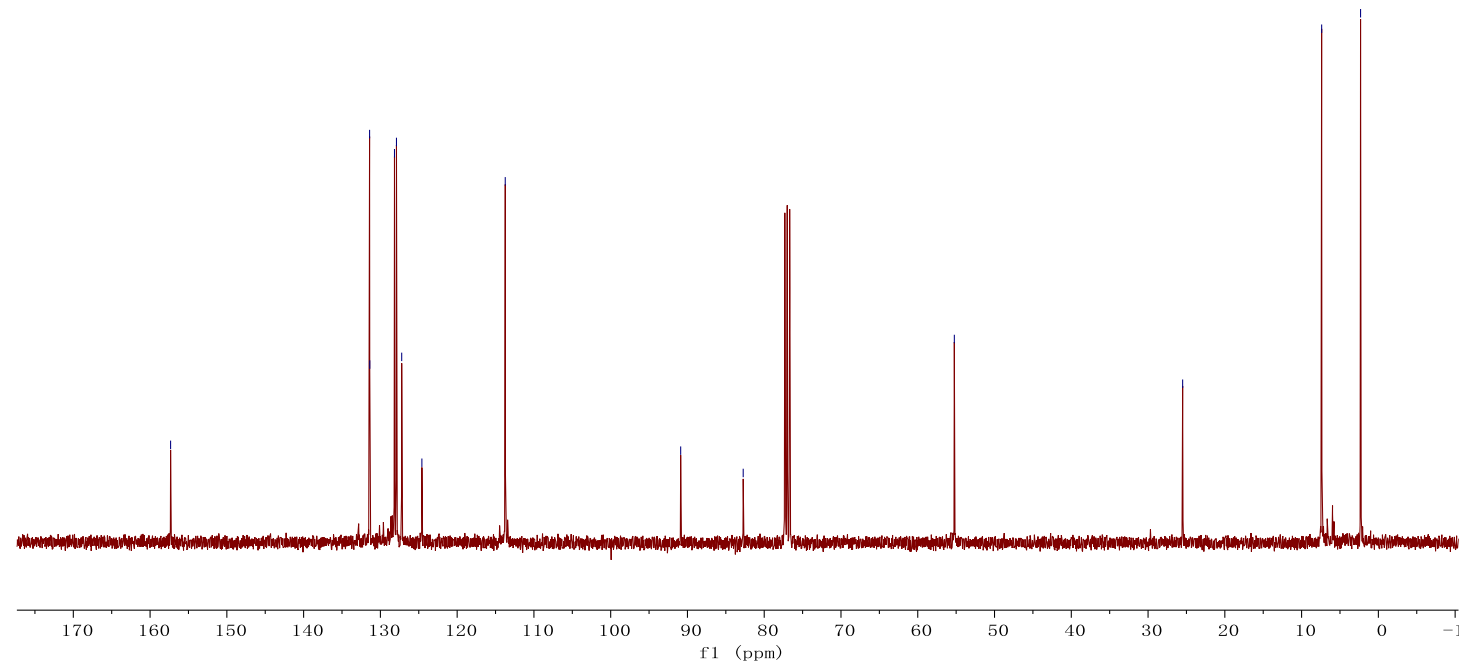


(-)-(1-(4-chlorophenyl)-3-phenylprop-2-yn-1-yl)triethylsilane (3fb)

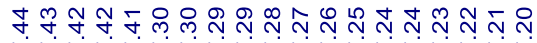

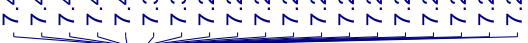

?

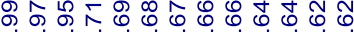

000000000000

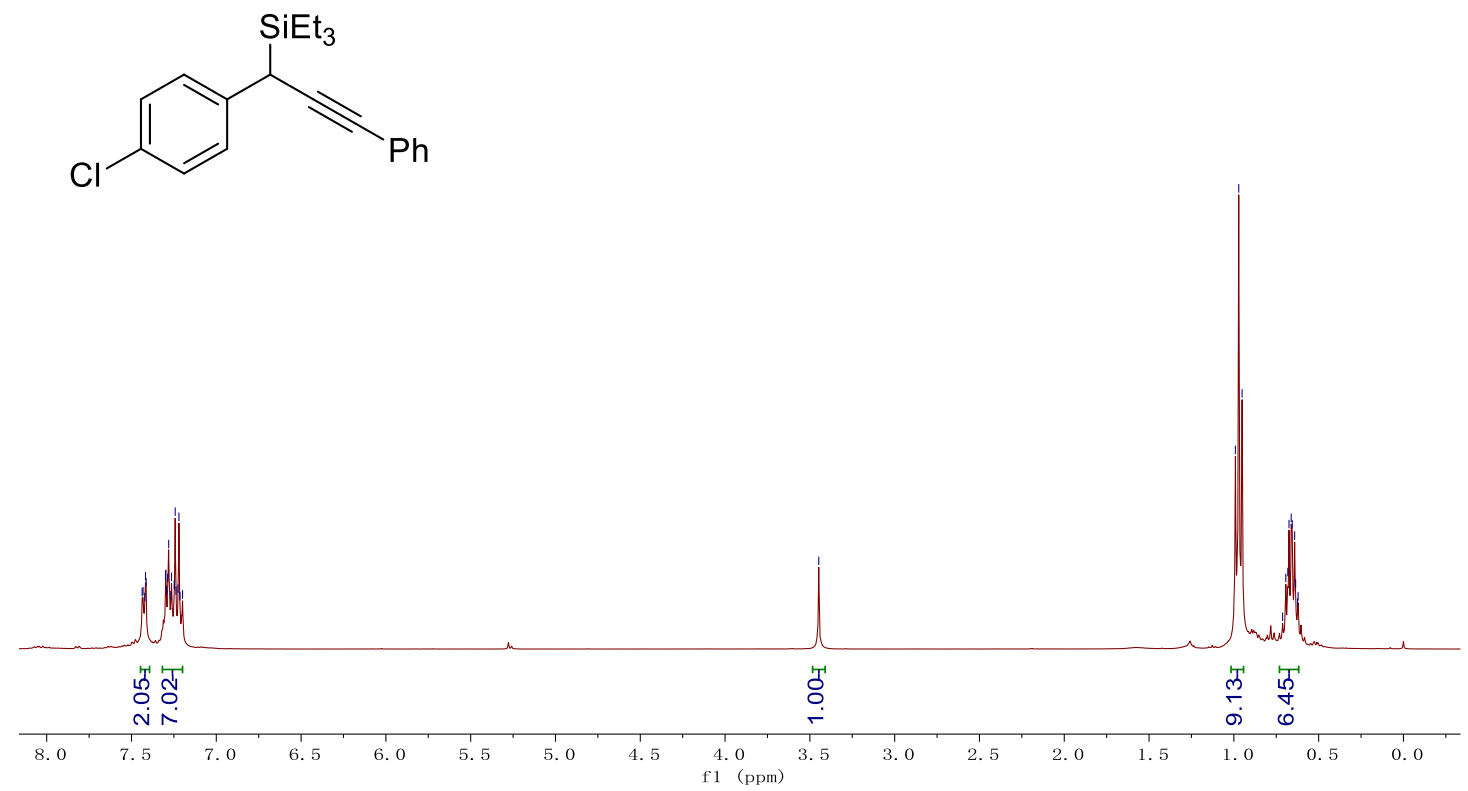

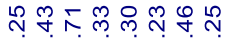

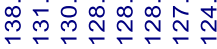

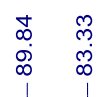

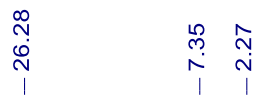

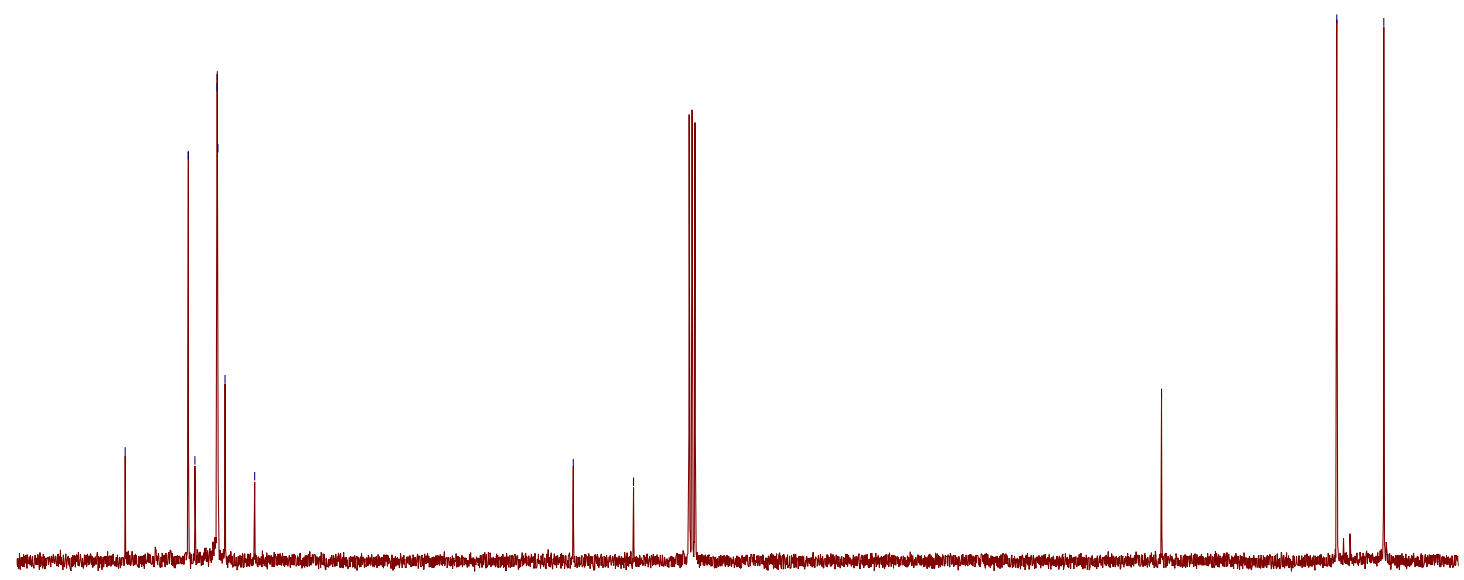

$\begin{array}{llllllllllllllllll}145 & 140 & 135 & 130 & 125 & 120 & 115 & 110 & 105 & 100 & 95 & 90 & 85 & 80 & 75 & 70 \\ & & & & & & & & & & & & \end{array}$ 
(-)-4-(3-phenyl-1-(triethylsilyl)prop-2-yn-1-yl)benzonitrile (3gb)

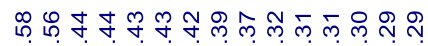

rinisinisinis

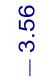

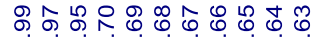

00000000000<smiles>CCC(C#CC#Cc1ccccc1)c1ccc(C#N)cc1</smiles>

$\mathrm{NC}$

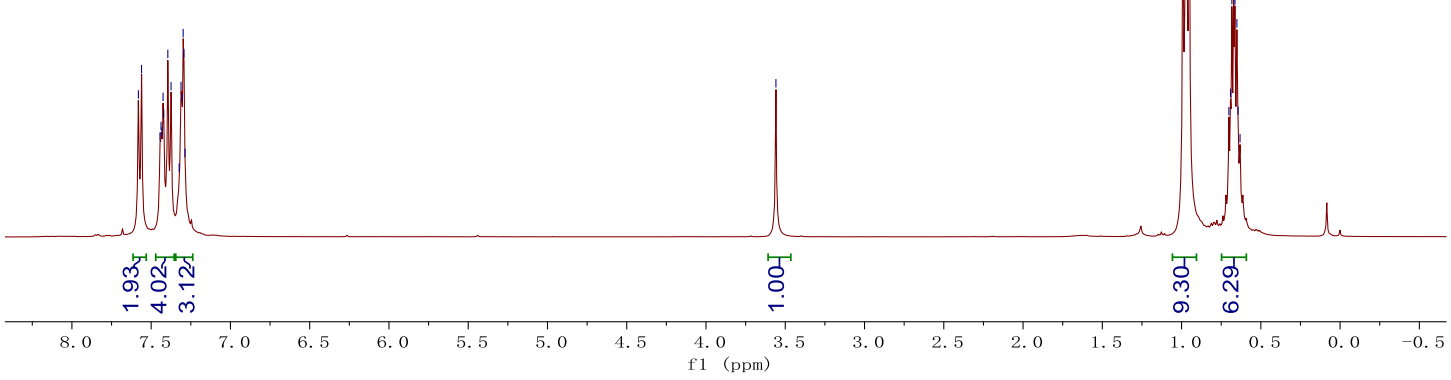

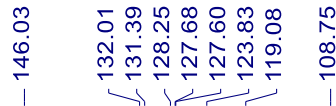

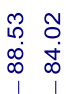

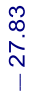
$\stackrel{m}{\sim} \stackrel{\sim}{N}$
î
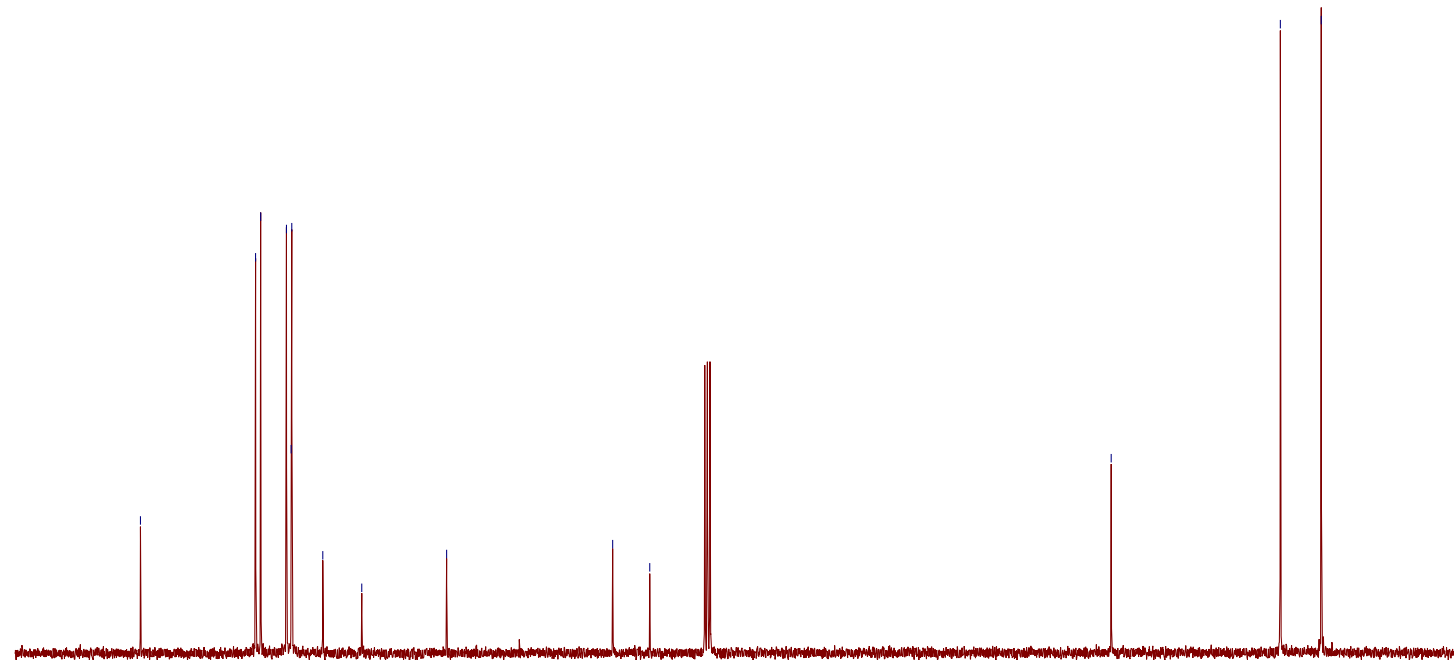

160

$\begin{array}{lllll}150-140 & 130-120 & 110 & 100-100\end{array}$

$80 \begin{array}{rr}1 & 0 \\ 81 & (\mathrm{ppm})\end{array}$

$60 \quad 50 \quad 40$

$30 \quad 20$

$0 \quad-10$ 
<smiles>CCCC(C#CC(C)(C)C)c1ccc2ccccc2c1</smiles>

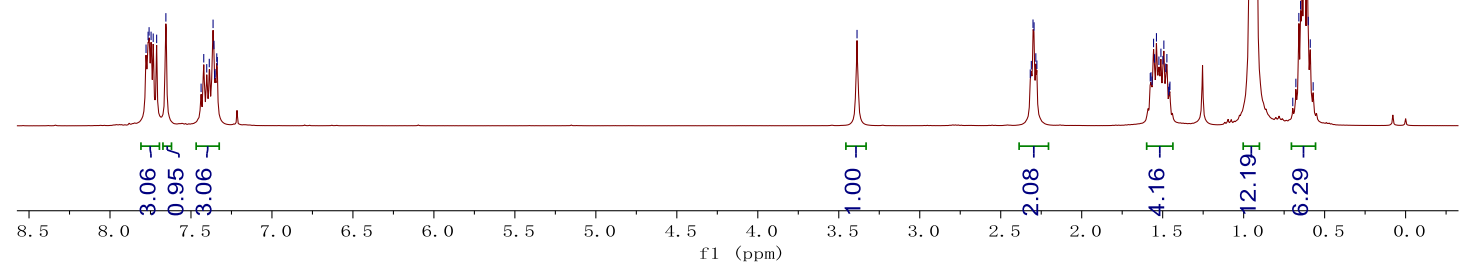

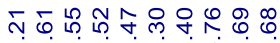

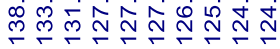

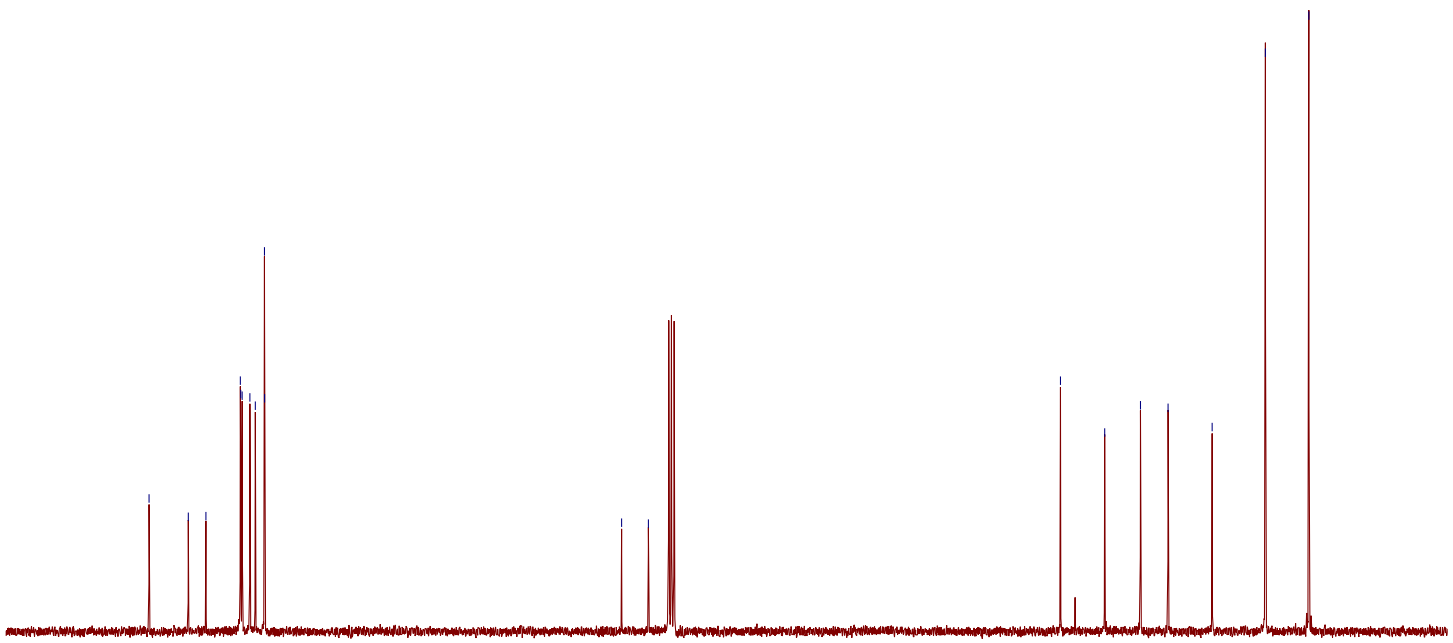

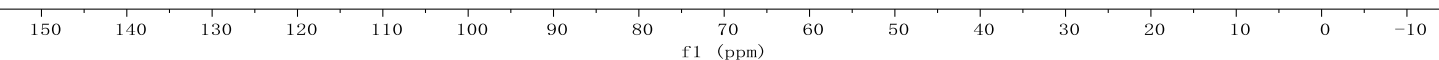


(-)-methyl(1-(naphthalen-2-yl)hept-2-yn-1-yl)diphenylsilane (3jc)

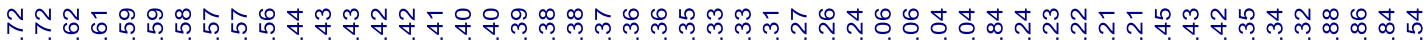<smiles>CCCCC(C#CC(C)(C)C)c1ccc2ccccc2c1</smiles>
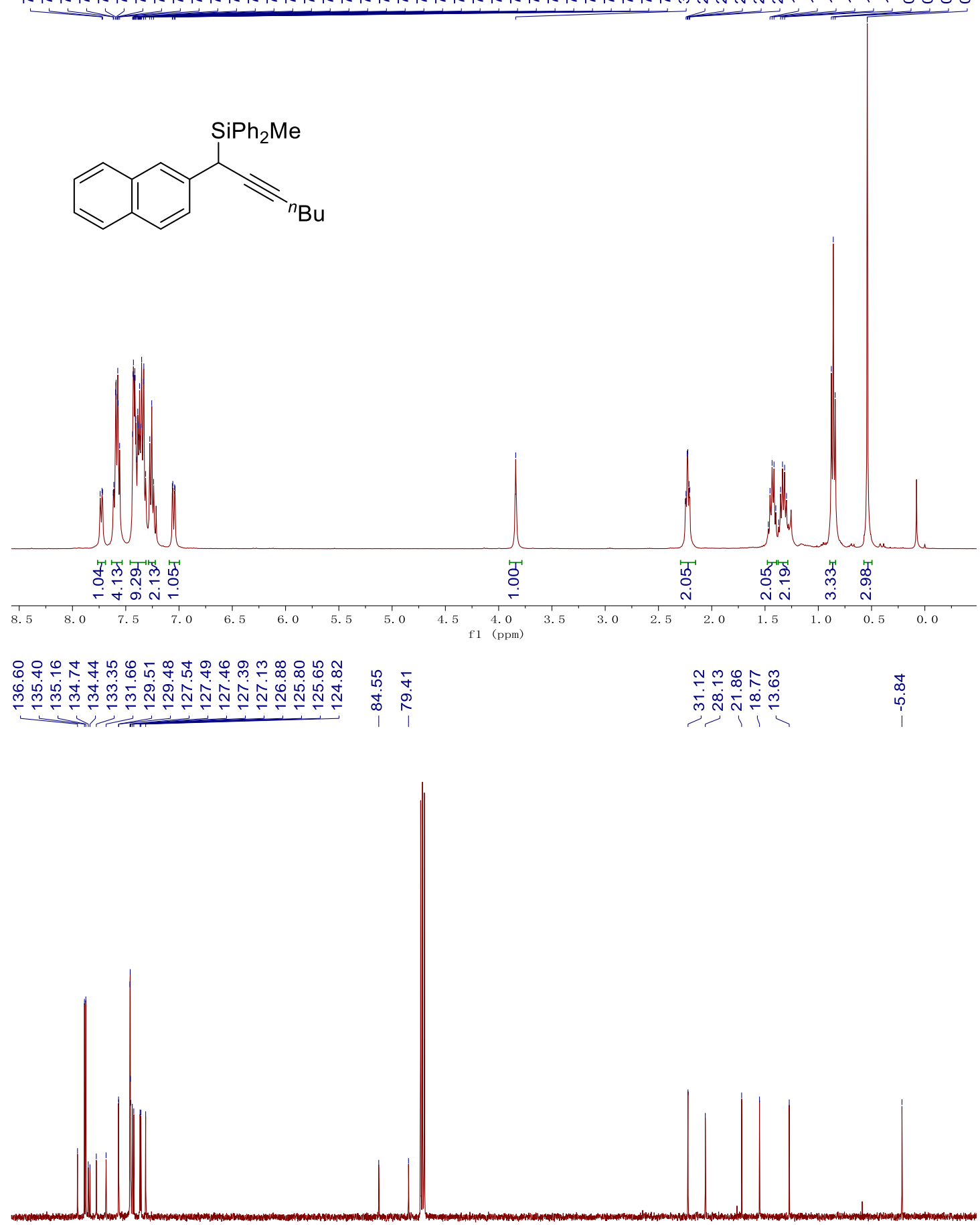

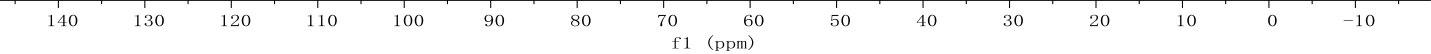


(-)-triethyl(1-(naphthalen-2-yl)but-2-yn-1-yl)silane (3kb)

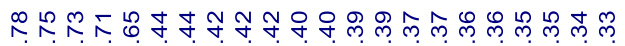

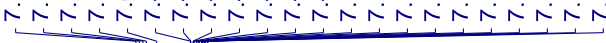

$\ddot{\infty}$

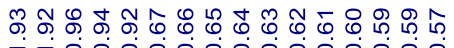

$\ln ^{\infty}$<smiles>CC#CC([SiH2]C)c1ccc2ccccc2c1</smiles>

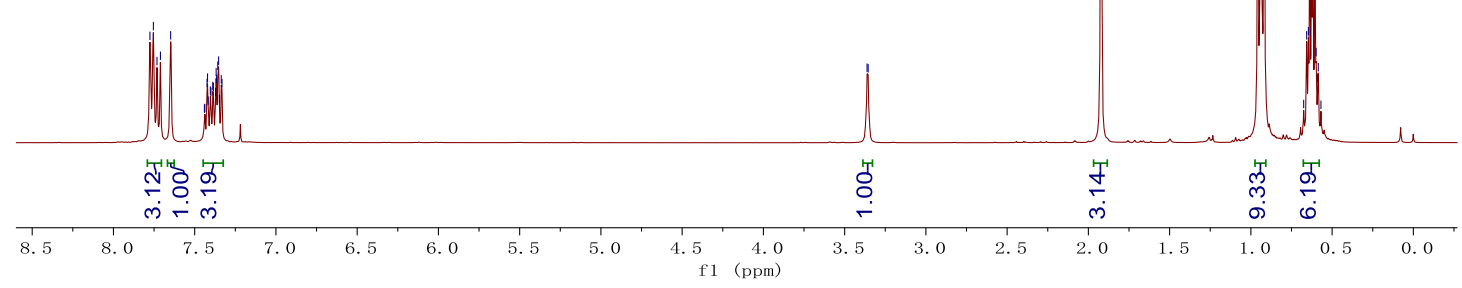

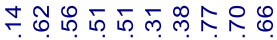

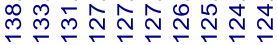

$\underset{\substack { \infty \\ \begin{subarray}{c}{\infty \\ i{ \infty \\ \begin{subarray} { c } { \infty \\ i } }\end{subarray}}{\substack{\infty \\ j}}$

$\stackrel{\stackrel{\sim}{N}}{\stackrel{\leftrightarrow}{N}}$

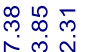

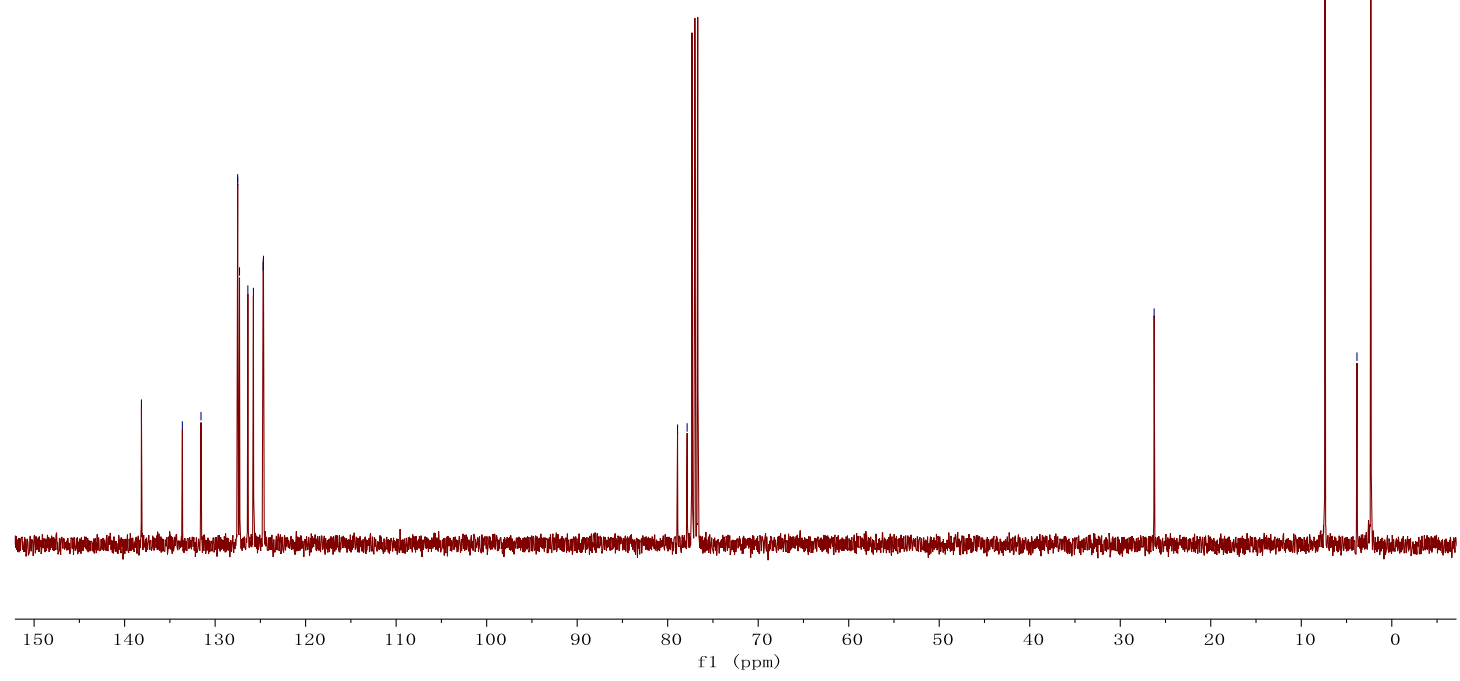



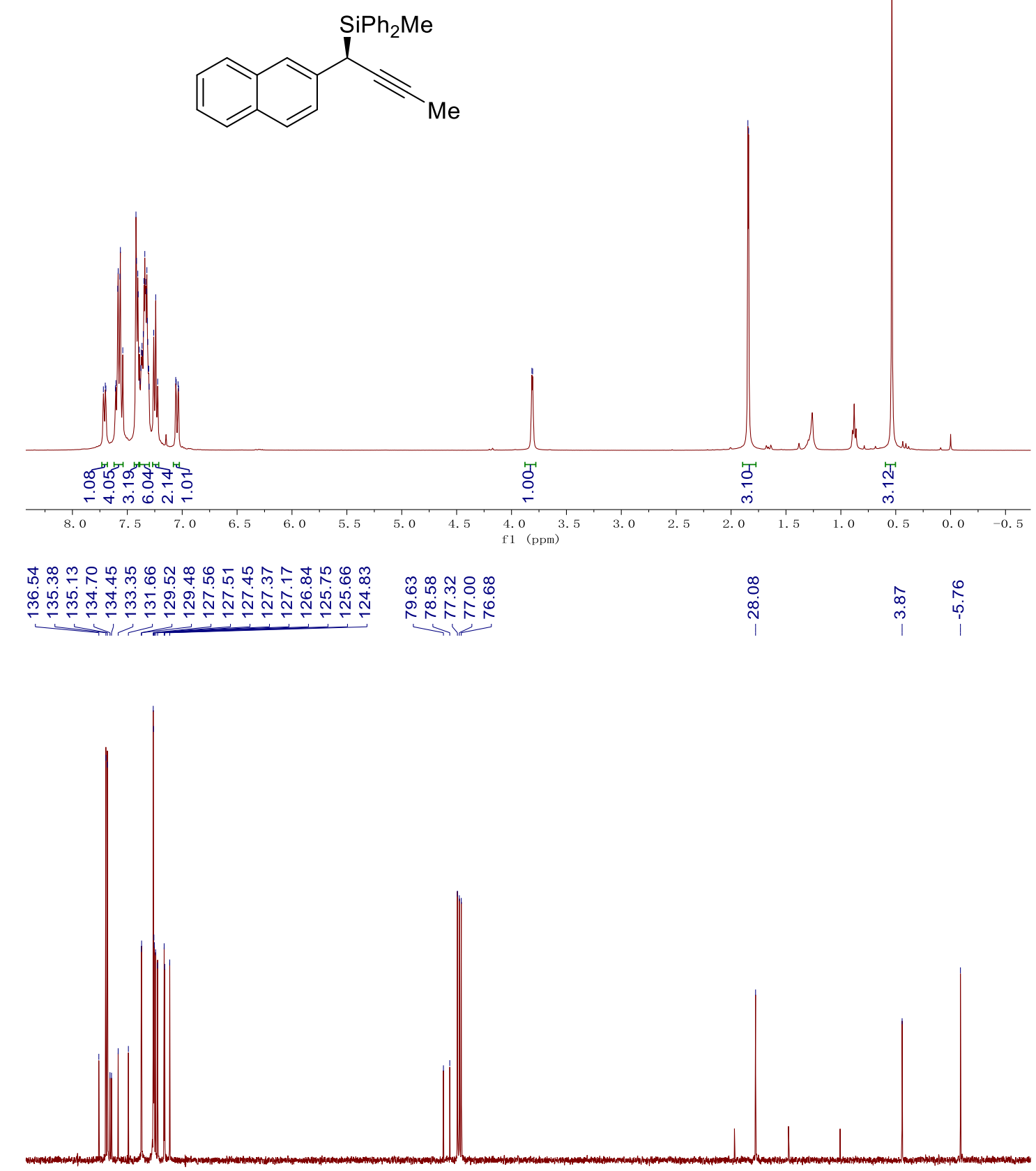
<smiles>CCCCCC#CC#CC(CCC)c1ccc2ccccc2c1</smiles>
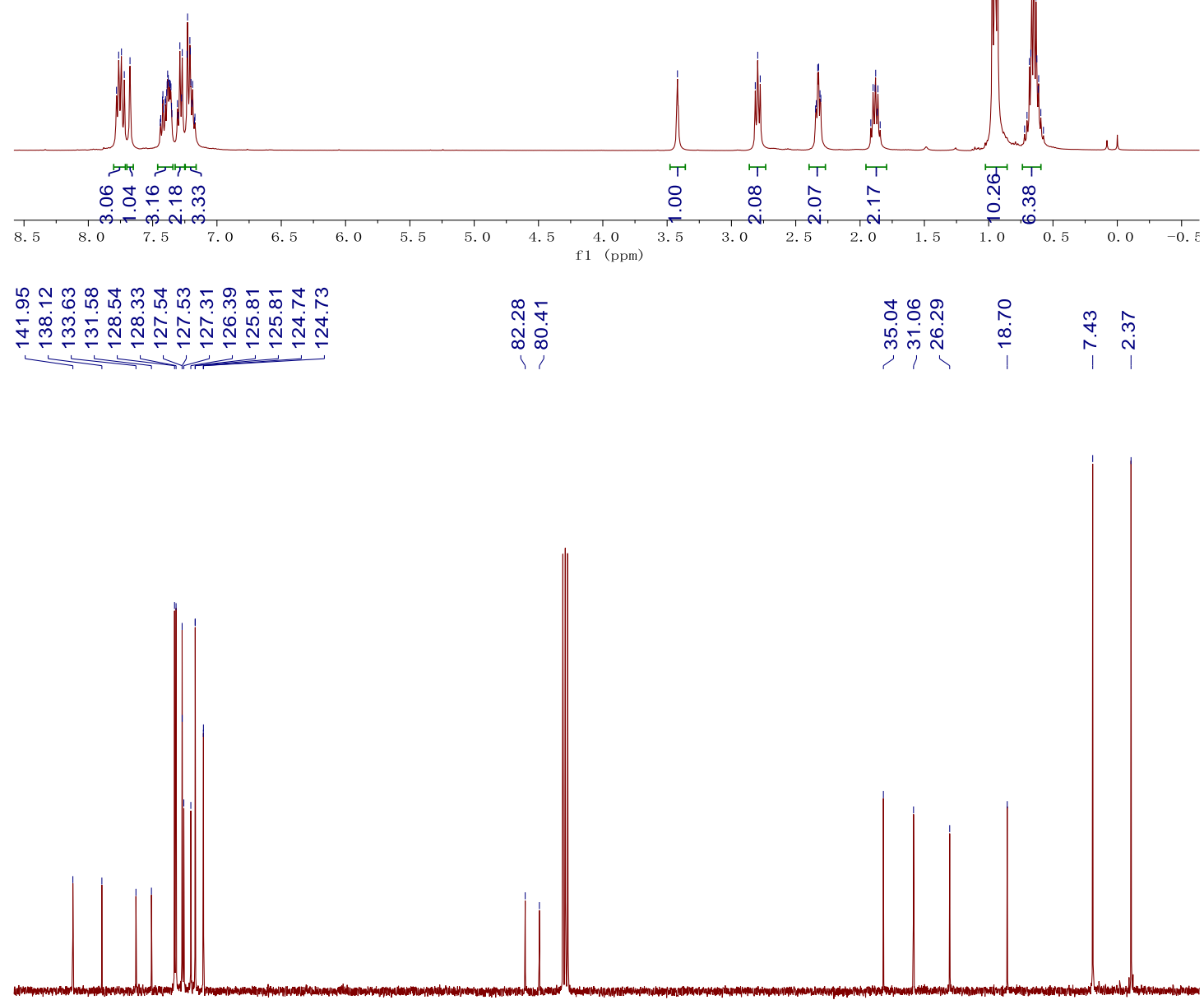
f1 $\stackrel{70}{(\mathrm{ppm})}$ 
<smiles>CC[SiH2]C(C#CC1CC1)c1ccc2ccccc2c1</smiles>

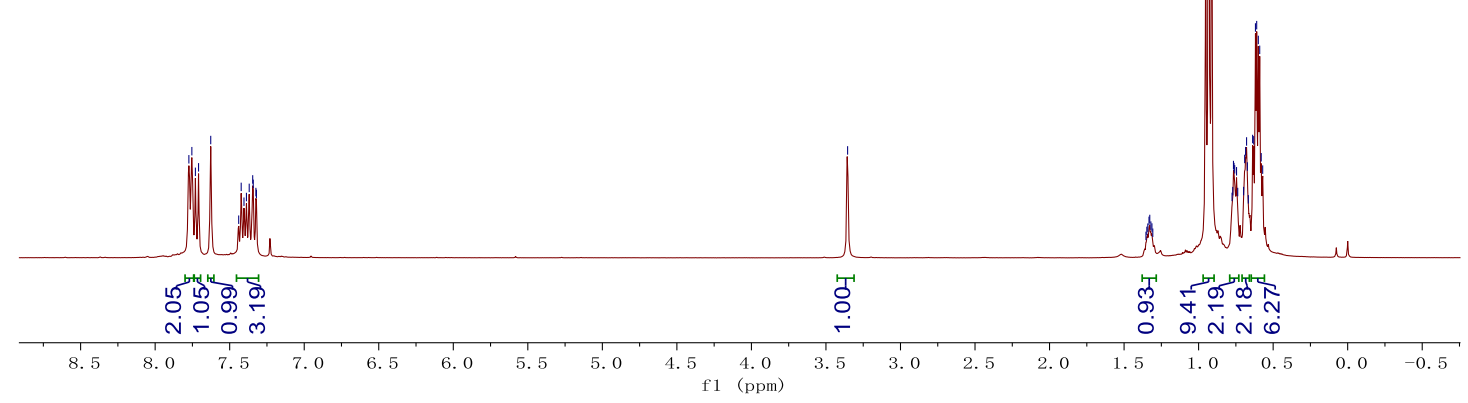

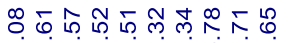

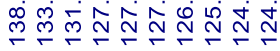

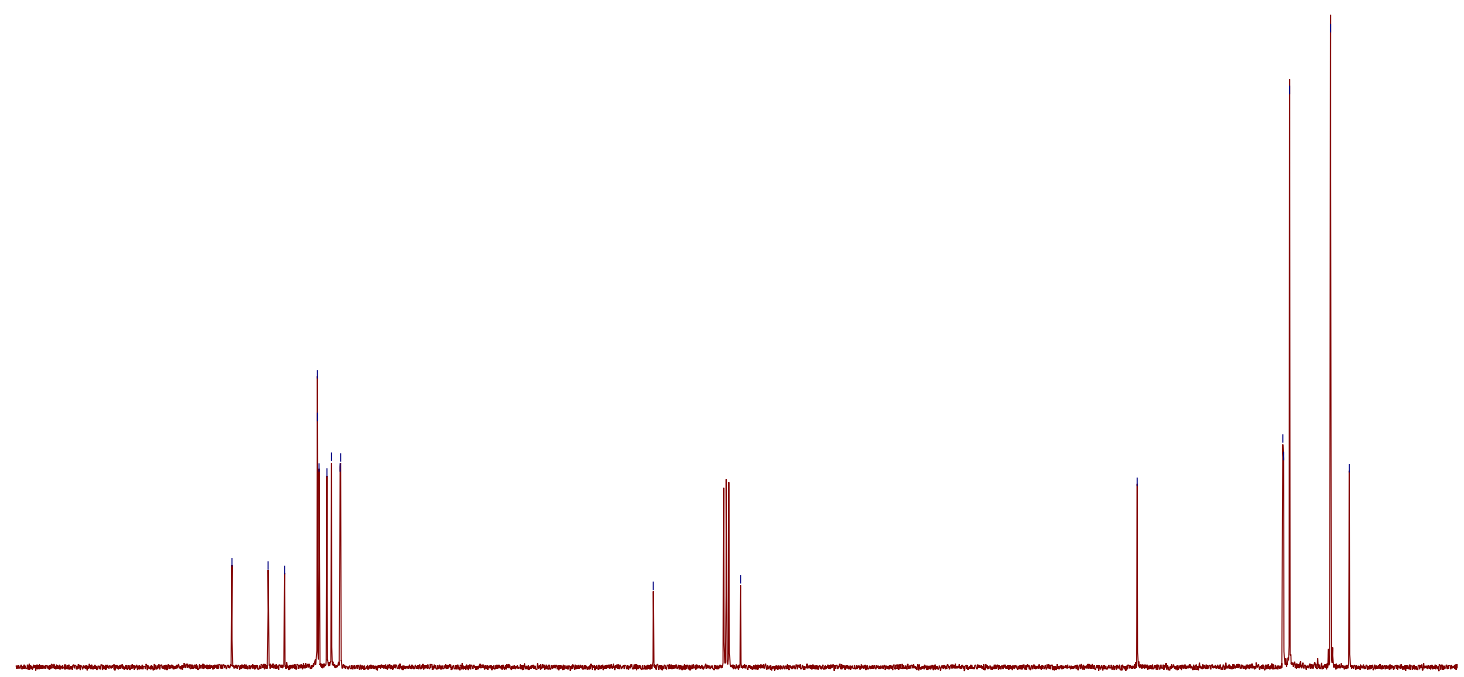

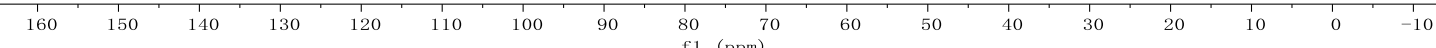


(-)-(3-cyclopropyl-1-(naphthalen-2-yl)prop-2-yn-1-yl)(methyl)diphenylsilane (3mc)

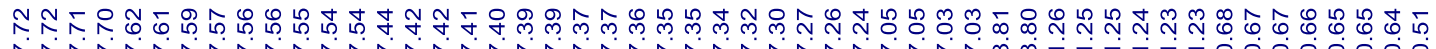

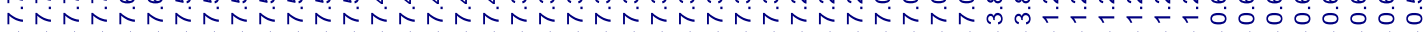<smiles>CCC[SnH2]C(C#CC1CC1)c1ccc2ccccc2c1</smiles>

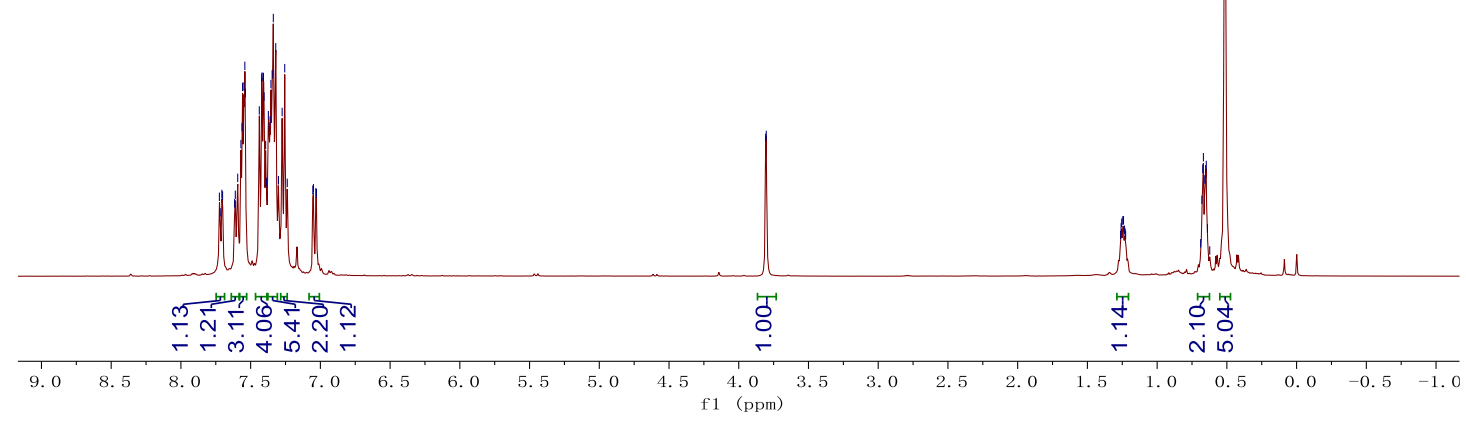

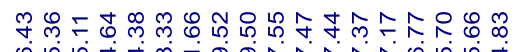

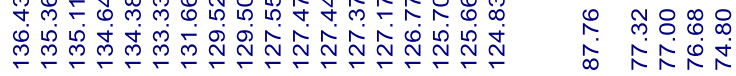

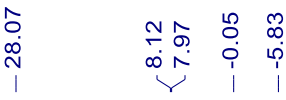

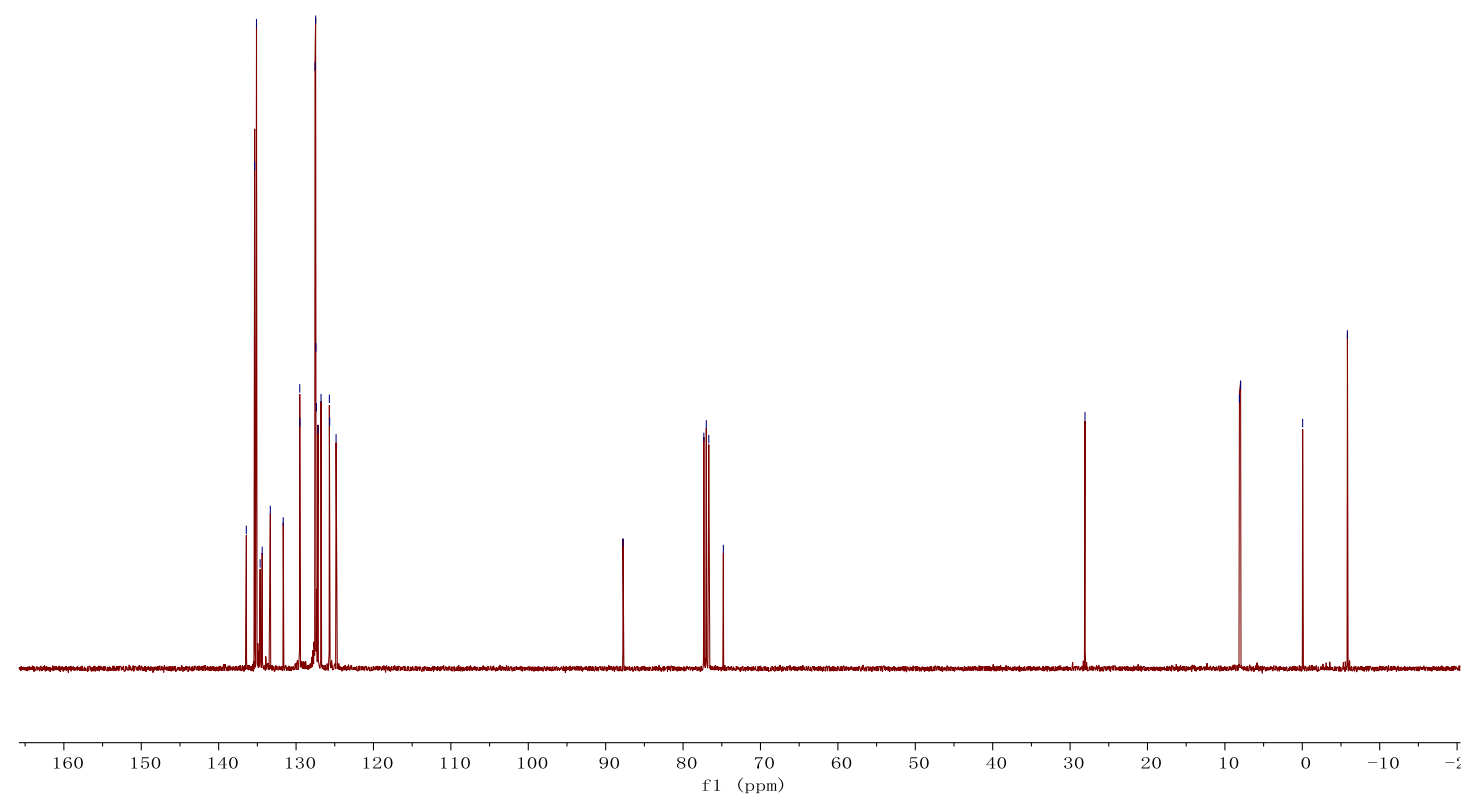




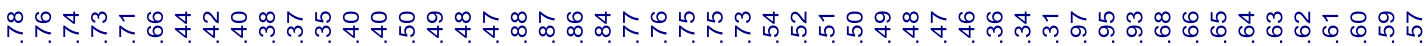

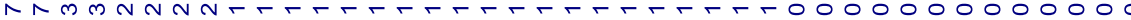<smiles>CCC(C#CC1CCCCC1)c1ccc2ccccc2c1</smiles>

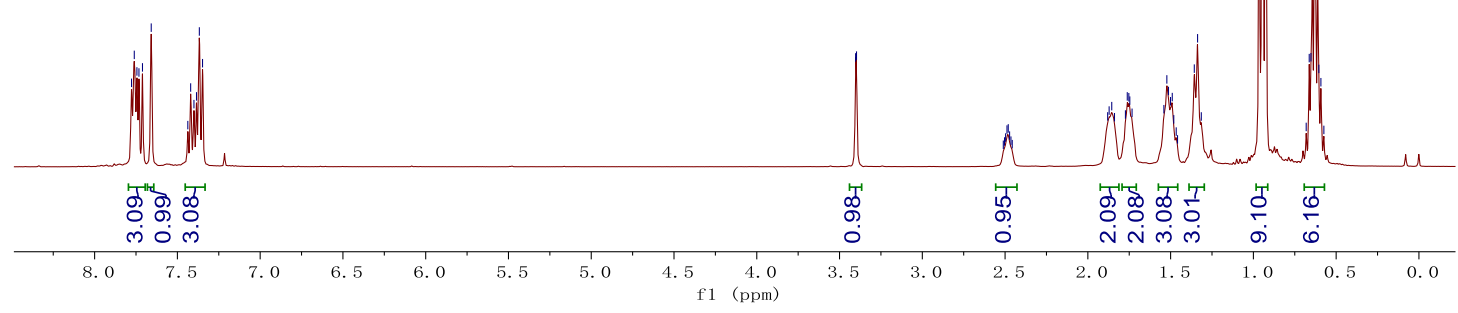

กำ

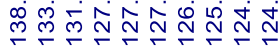

$\begin{array}{ll}\stackrel{\infty}{\infty} & \stackrel{\infty}{\infty} \\ \stackrel{0}{\infty} & \stackrel{0}{0} \\ 1 & 1\end{array}$

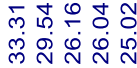

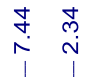

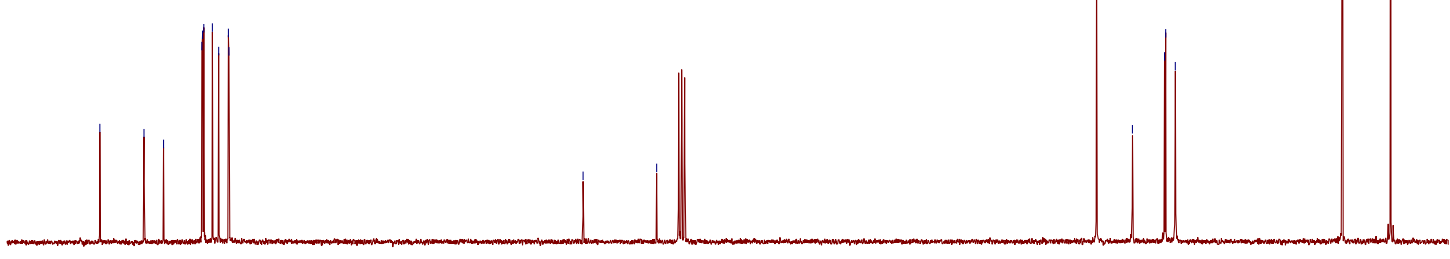

$\begin{array}{lllllllllllllllllllllllllllllll}145 & 140 & 135 & 130 & 125 & 120 & 115 & 110 & 105 & 100 & 95 & 90 & 85 & 80 & 75 & 70 & 65 & 60 & 55 & 50 & 45 & 40 & 35 & 30 & 25 & 20 & 15 & 10 & 5 & 0\end{array}$ 
(-)-triethyl(1-(naphthalen-2-yl)-3-(trimethylsilyl)prop-2-yn-1-yl)silane (3ob) œ risiriniñ

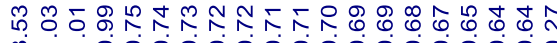
m- - 00000000000000000<smiles>CCC(C#CC#N)c1ccc2ccccc2c1</smiles>

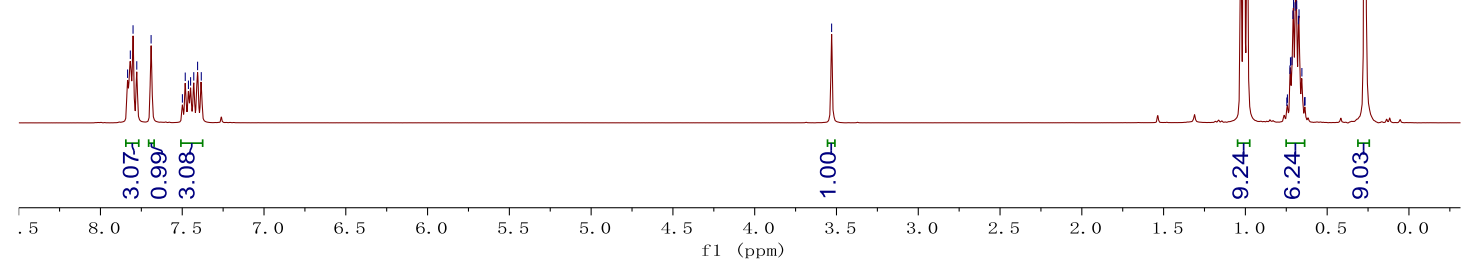

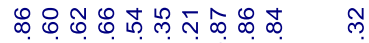

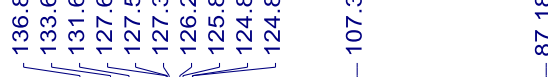
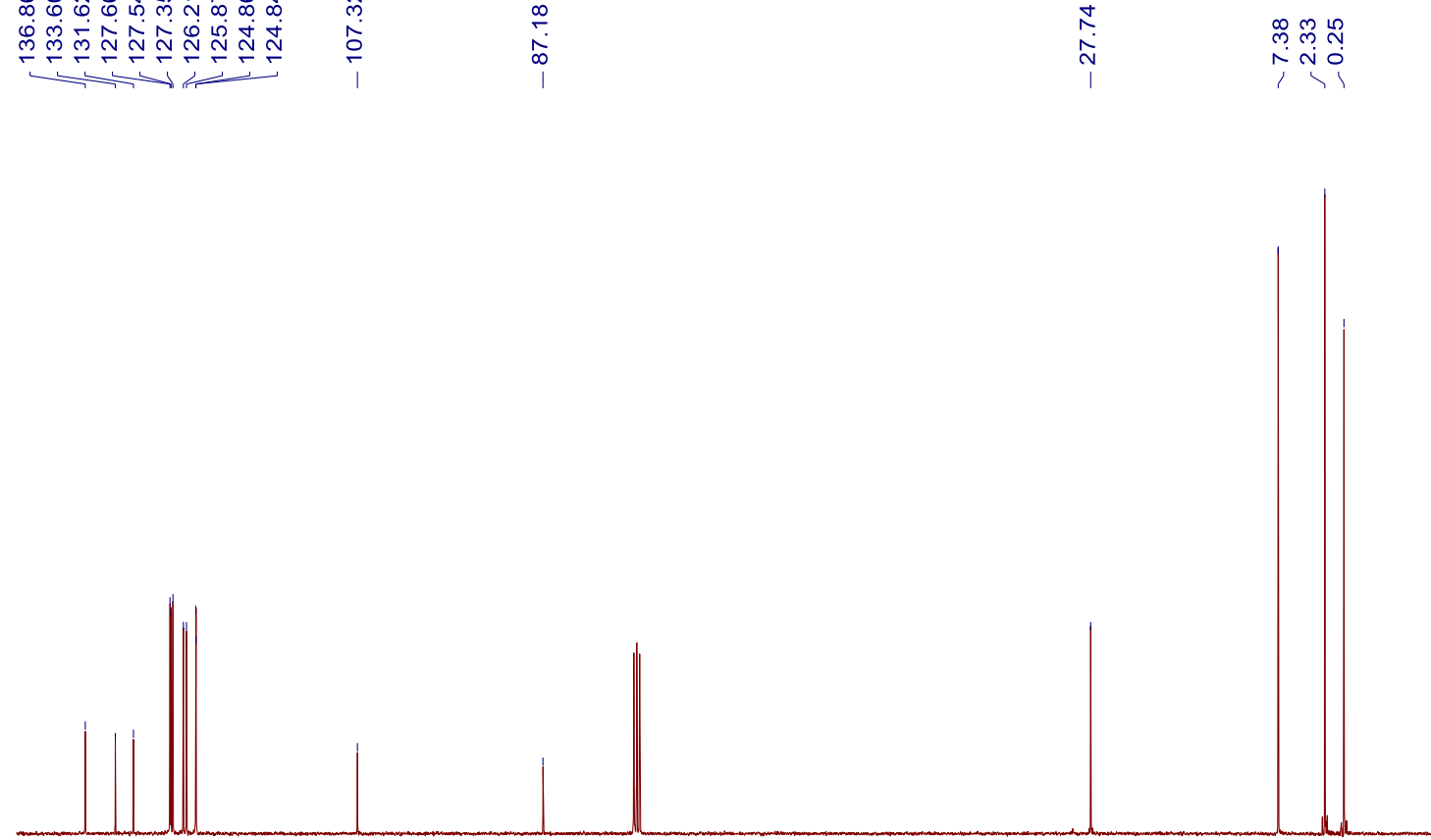

$\begin{array}{lllllllllllllllllllllllllllllllllllll}140 & 135 & 130 & 125 & 120 & 115 & 110 & 105 & 100 & 95 & 90 & 85 & 80 & 75 & 70 & 65 & 60 & 55 & 50 & 45 & 40 & 35 & 30 & 25 & 20 & 15 & 10 & 5 & 0 & -5\end{array}$ 
(-)-(3-(cyclohex-1-en-1-yl)-1-(naphthalen-2-yl)prop-2-yn-1-yl)triethylsilane (3pb)

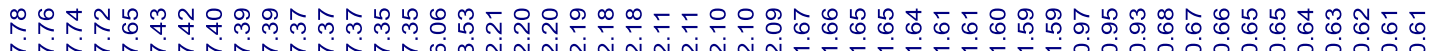

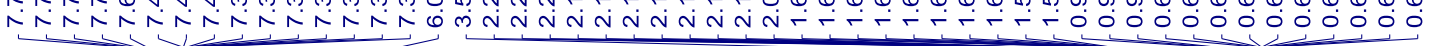<smiles>CCC(C#CC1=CCCCC1)c1ccc2ccccc2c1</smiles>

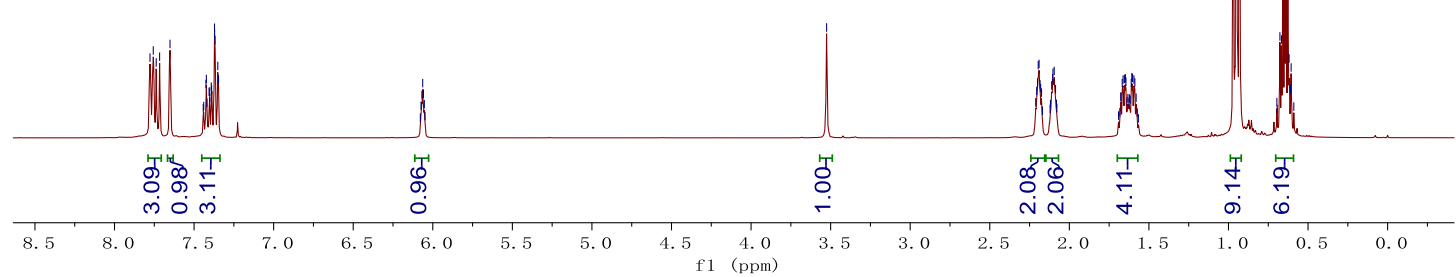

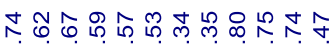

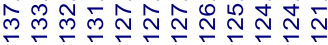

$ㅇ ㅜ ㅇ ㅛ$

宓空

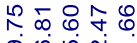

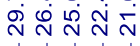

กั

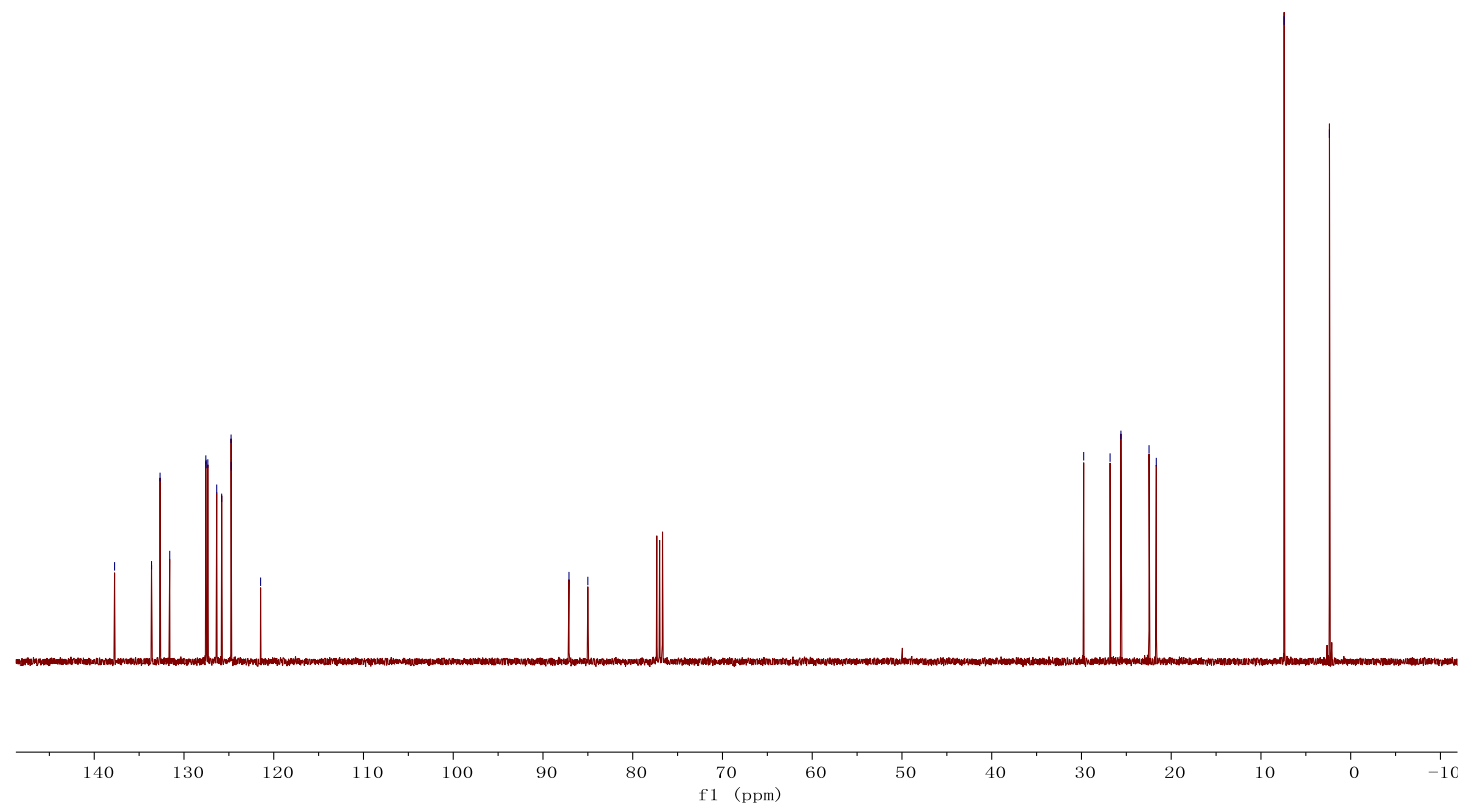


(-)-(3-(cyclohex-1-en-1-yl)-1-(naphthalen-2-yl)prop-2-yn-1yl)(methyl)diphenylsilane (3pc)

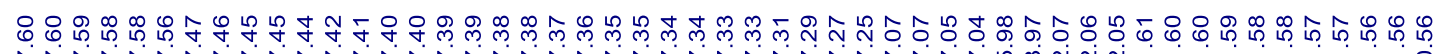

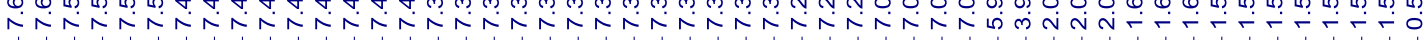<smiles>CCCCC(C#CC1=CCCCC1)c1ccc2ccccc2c1</smiles>

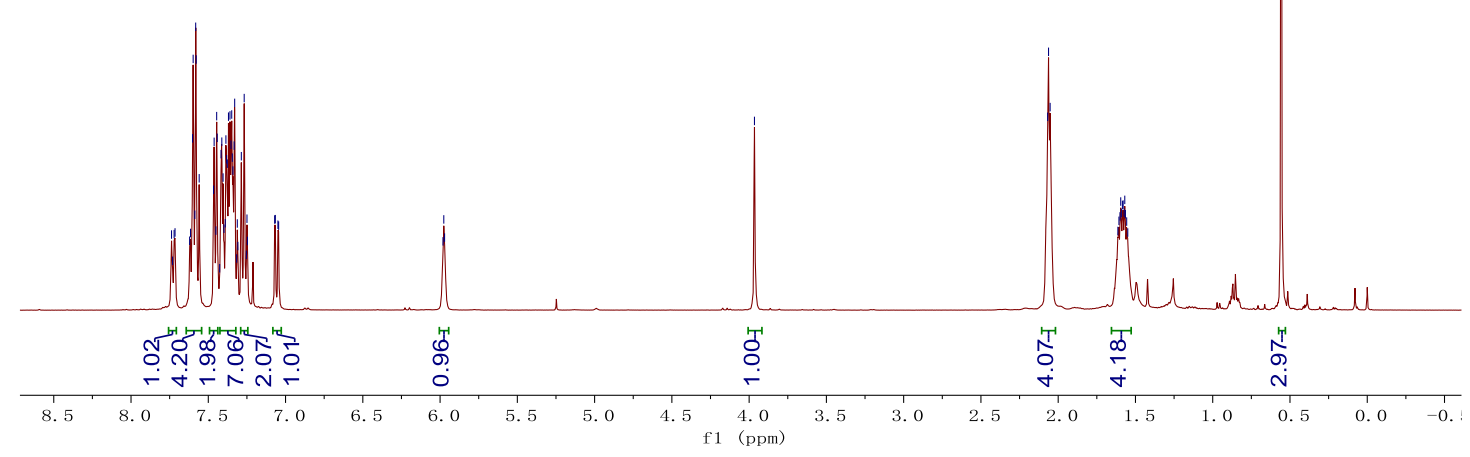

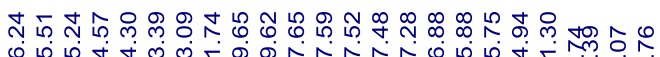

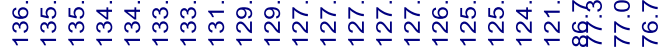

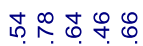

กิ

$\stackrel{\circ}{\circ}$

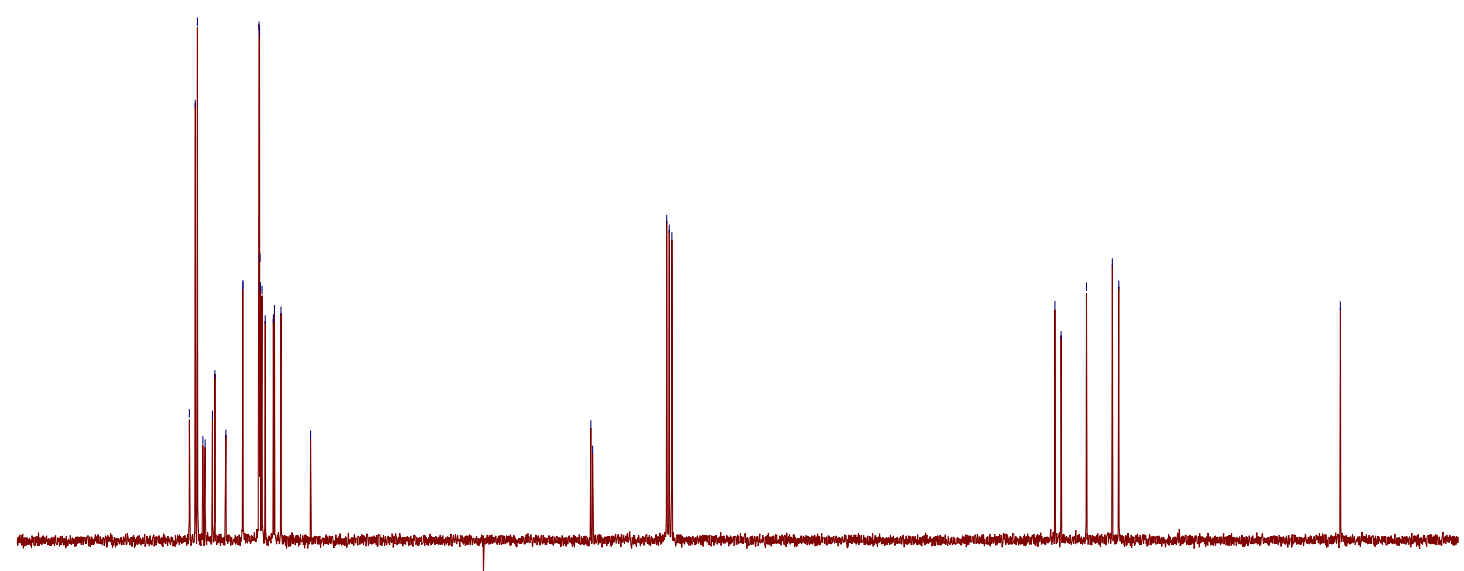


(-)-triethyl(1-(naphthalen-2-yl)undeca-2,4-diyn-1-yl)silane (3qb)

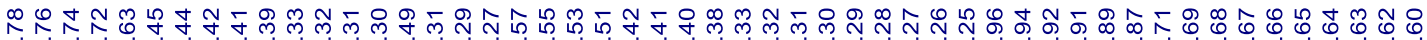

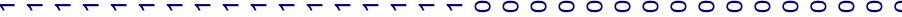<smiles>CCCCCCCC#CC#CC([SiH2]C)c1ccc2ccccc2c1</smiles>

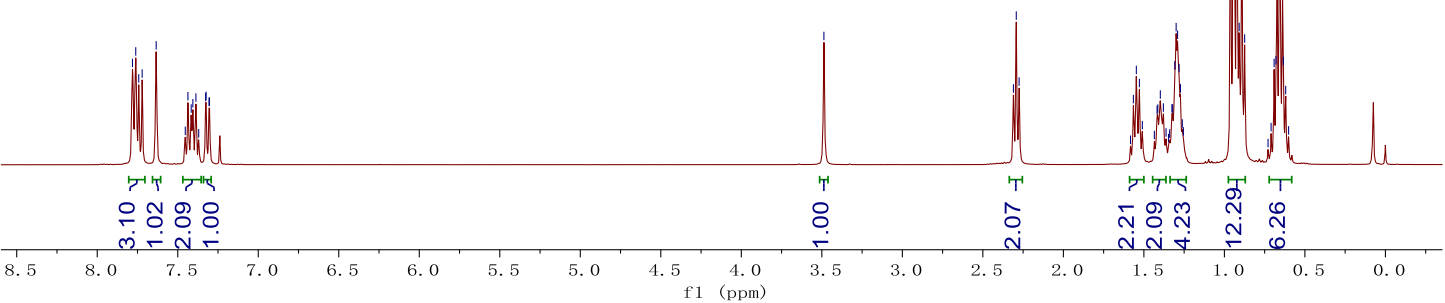

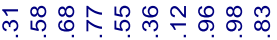

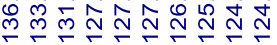


(-)-(6-chloro-1-(naphthalen-2-yl)hex-2-yn-1-yl)triethylsilane (3rb)

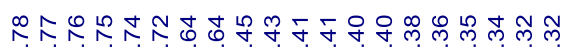

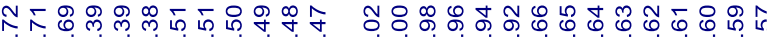

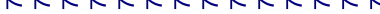

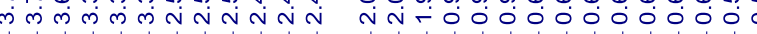<smiles>CCC(C#CC(Cl)Cl)c1ccc2ccccc2c1</smiles>

$\|$ U

n.

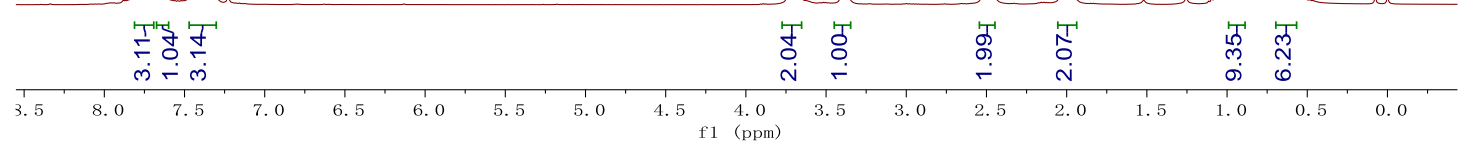

م⿻

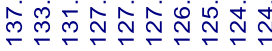

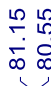

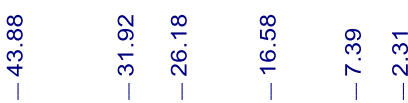

$\begin{array}{lllllllllllllllllllllllllllllllllllllllllll}145 & 140 & 135 & 130 & 125 & 120 & 115 & 110 & 105 & 100 & 95 & 90 & 85 & 80 & 75 & 70 & 65 & 60 & 55 & 50 & 45 & 40 & 35 & 30 & 25 & 20 & 15 & 10 & 5 & 0 & -5\end{array}$ 
<smiles>CCCC(C#CC(C)OCc1ccccc1)c1ccc2ccccc2c1</smiles>

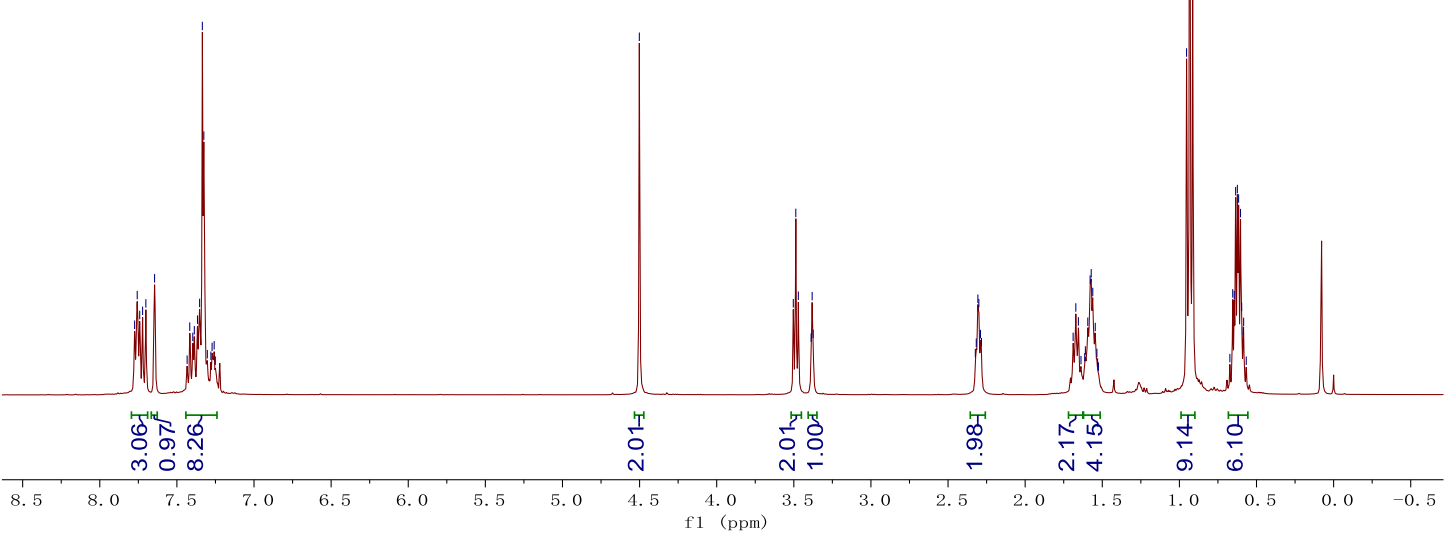

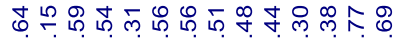

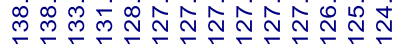

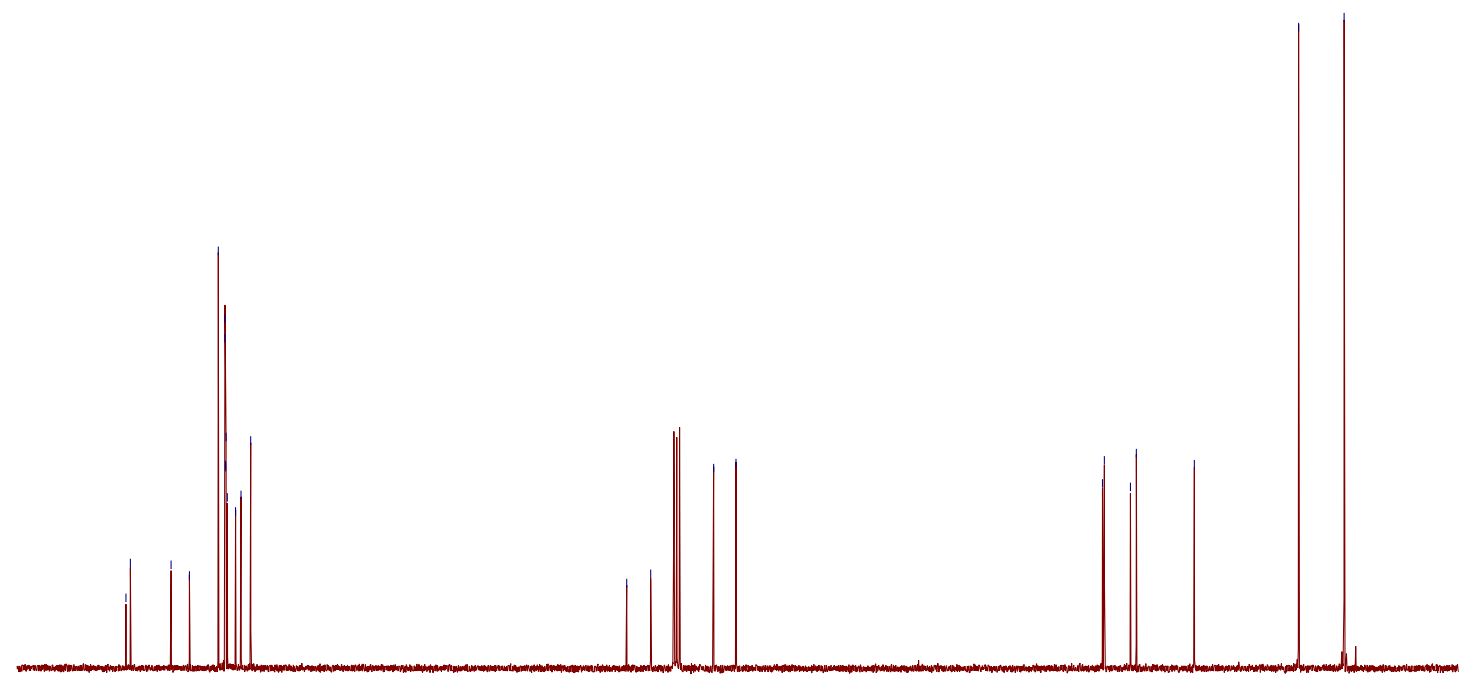

$\stackrel{T}{150}$
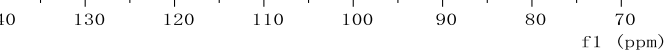

60 
<smiles>CCC(C#CC(C)n1ccc2ccccc21)c1ccc2ccccc2c1</smiles>
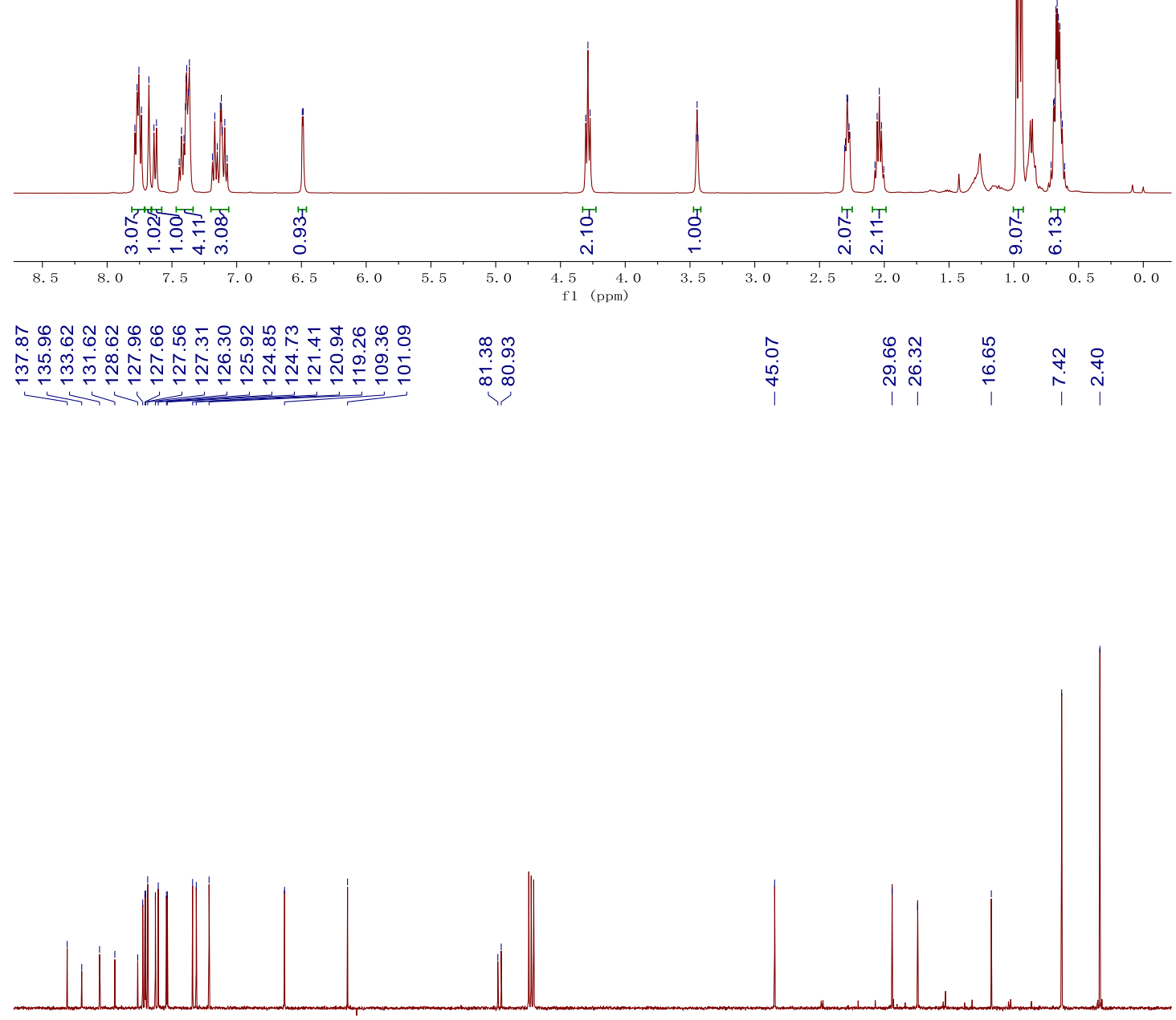

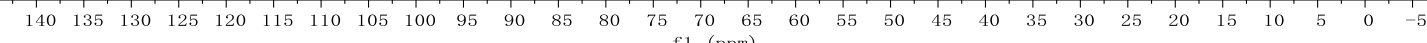


(-)-triethyl(1-(4-nitrophenyl)hept-2-yn-1-yl)silane (3ub)

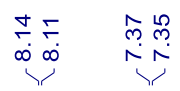

๙ิ

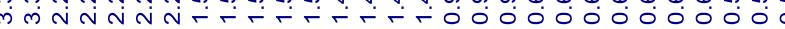<smiles>CCC(C#CC(Br)Br)c1ccc([N+](=O)[O-])cc1</smiles>

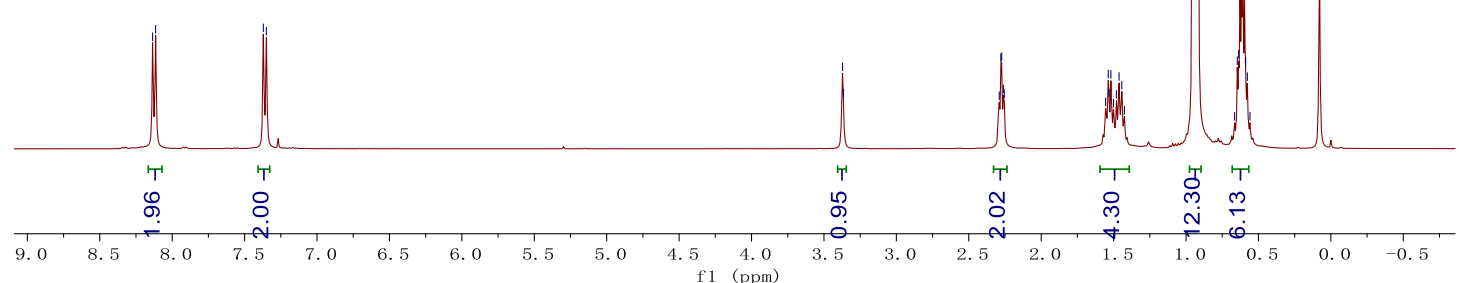

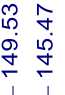

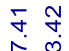

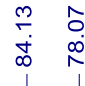

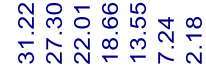

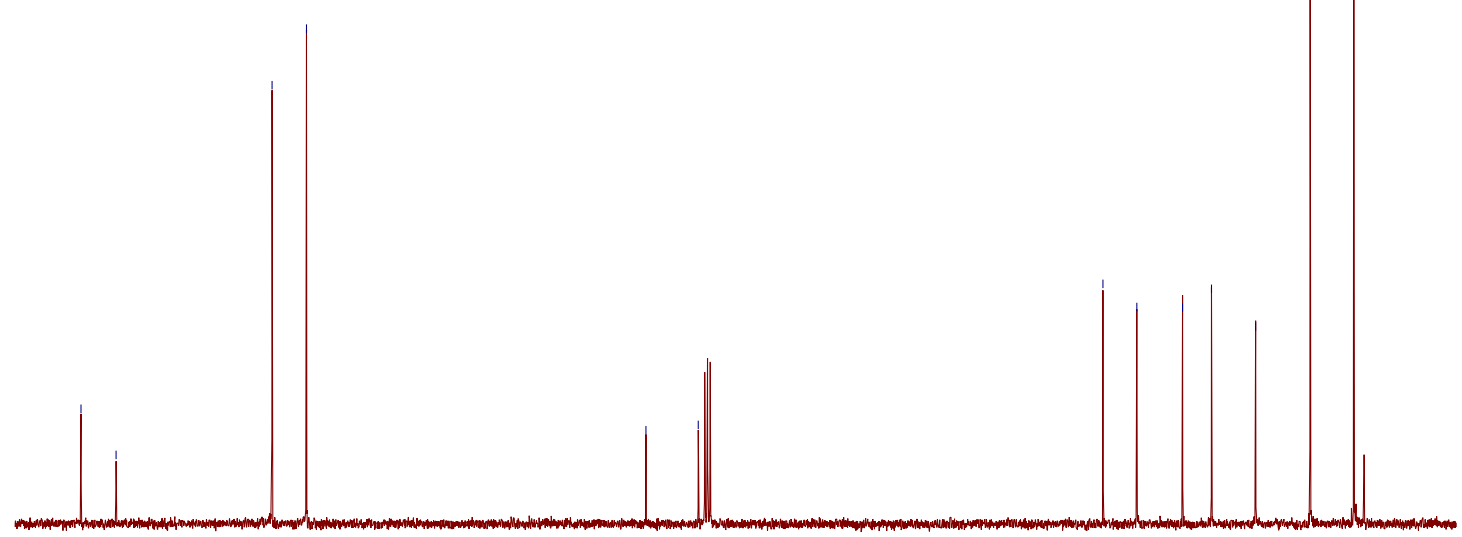

$\begin{array}{lllllllll}150 & 140 & 130 & 120 & 110 & 100 & 90 & 80 & 70 \\ & & & & & & & & \end{array}$ 
<smiles>CCC(C#CCBr)c1ccc(Cl)c(Cl)c1</smiles>

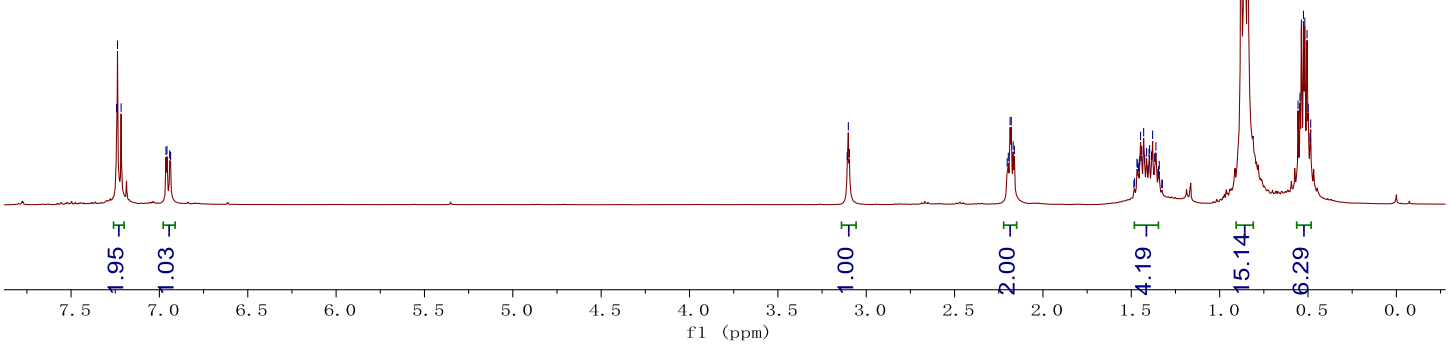

$\bar{c}$

咅

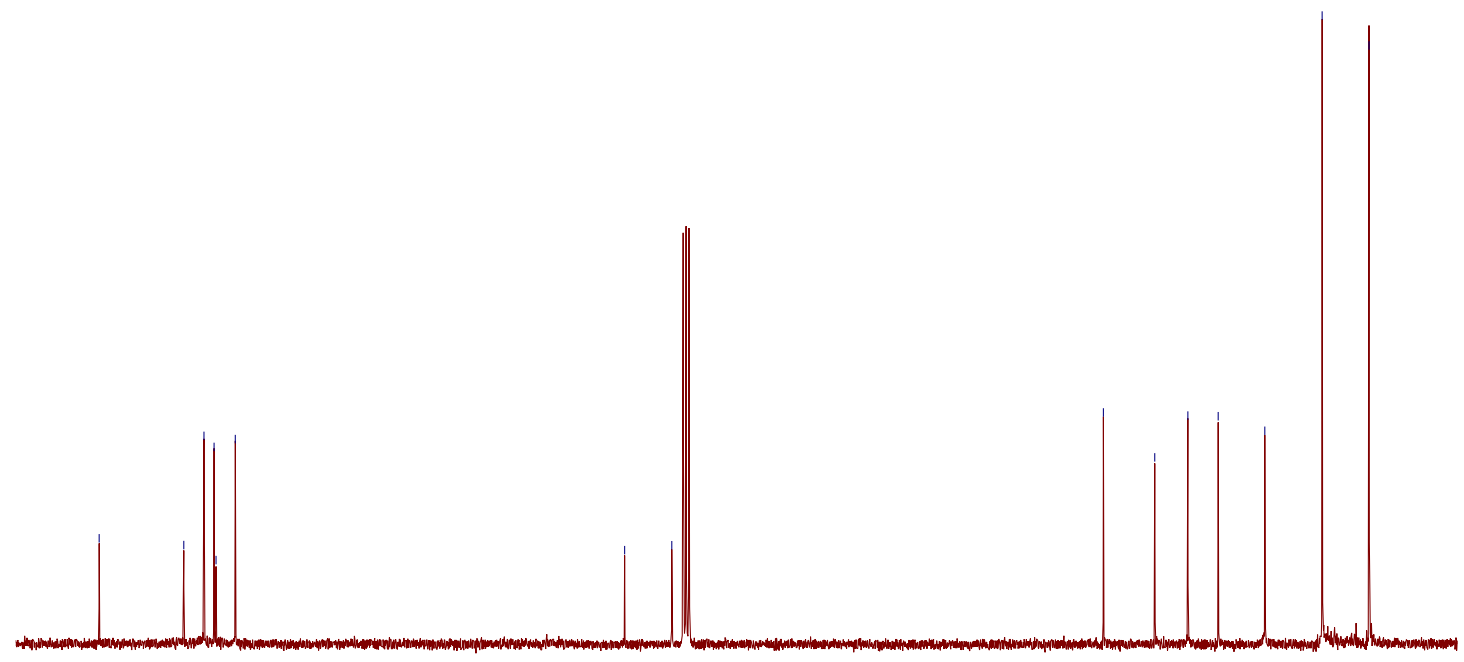

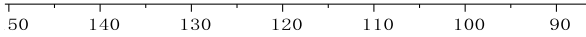
r1 70 
(-)-(1-(3,4-dichlorophenyl)hept-2-yn-1-yl)(methyl)diphenylsilane (3vc)

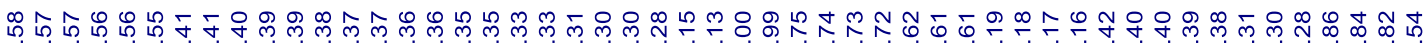

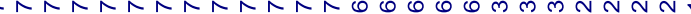<smiles>CCCCC(C#CC(C)(C)C)c1ccc(Cl)c(Cl)c1</smiles>
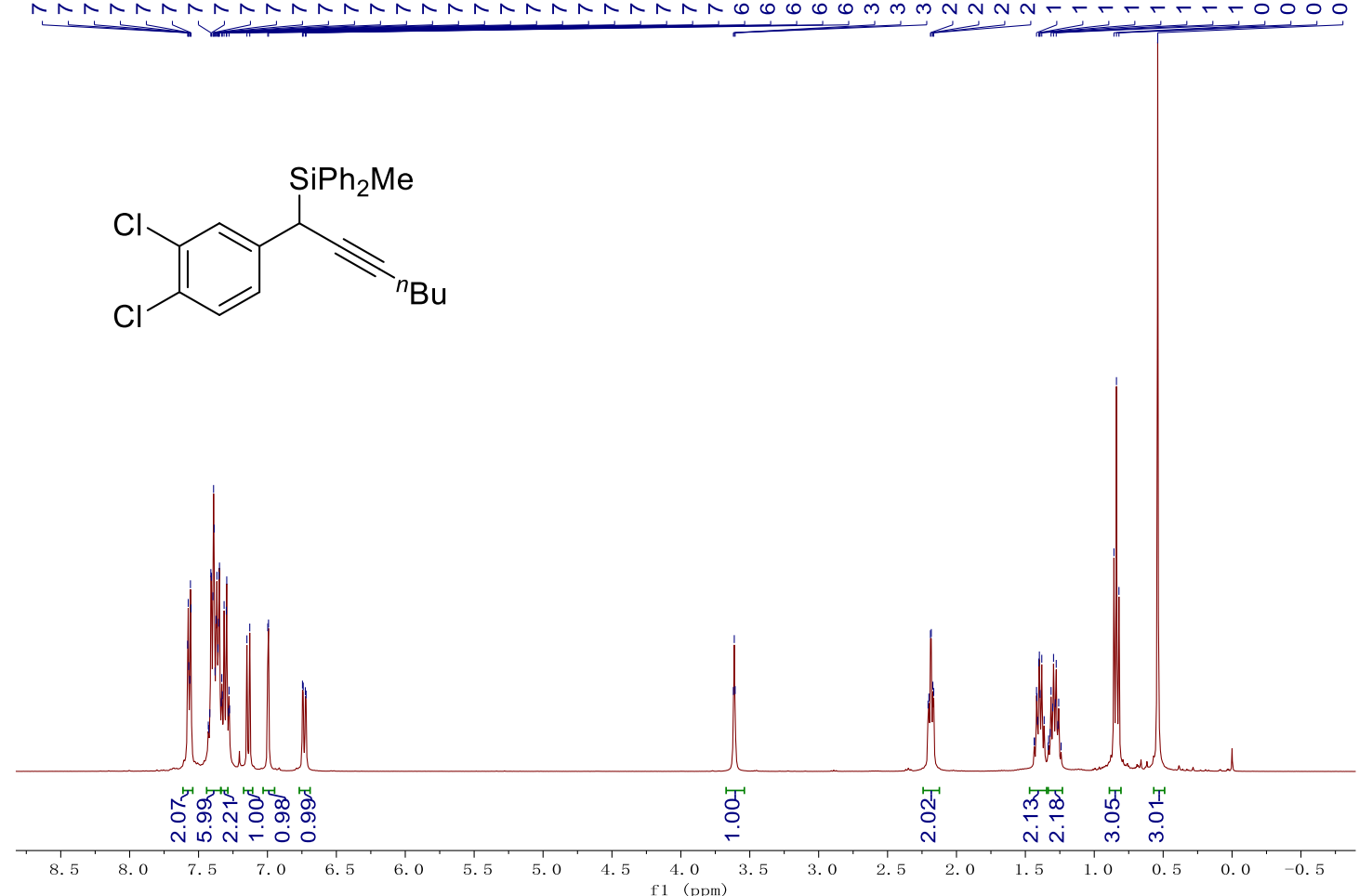

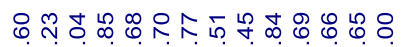

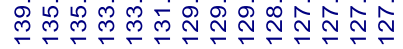

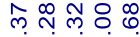

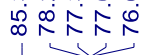

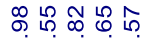

ì

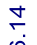

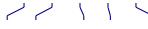
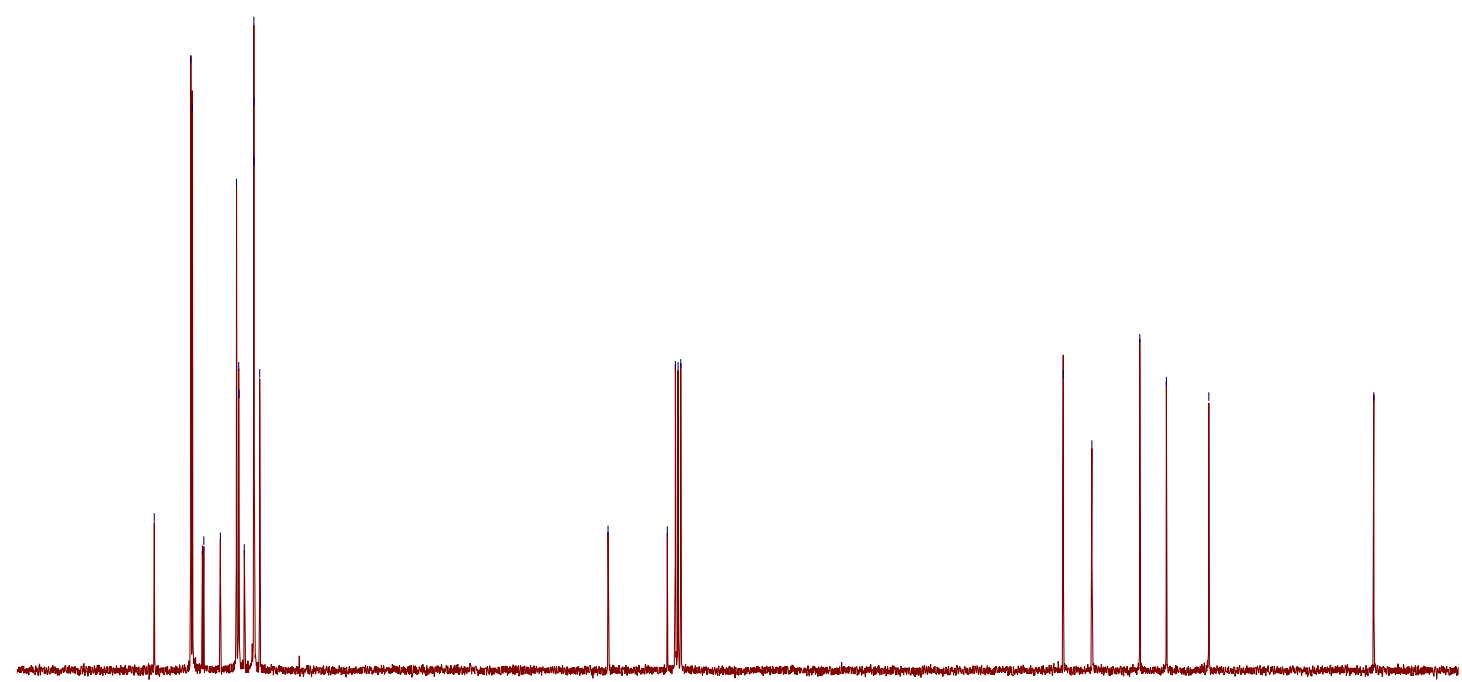

$150-140-130-120=110-100$

80

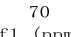

$60 \quad 50$

40

$30 \quad 20$

10

$-10$ 
(+)-(1-(3,4-dimethylphenyl)hept-2-yn-1-yl)(methyl)diphenylsilane (3wc)

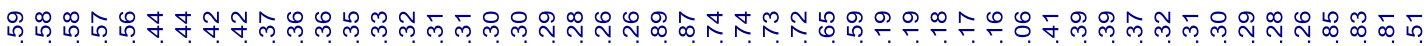

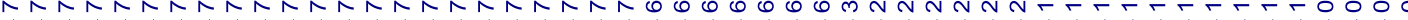

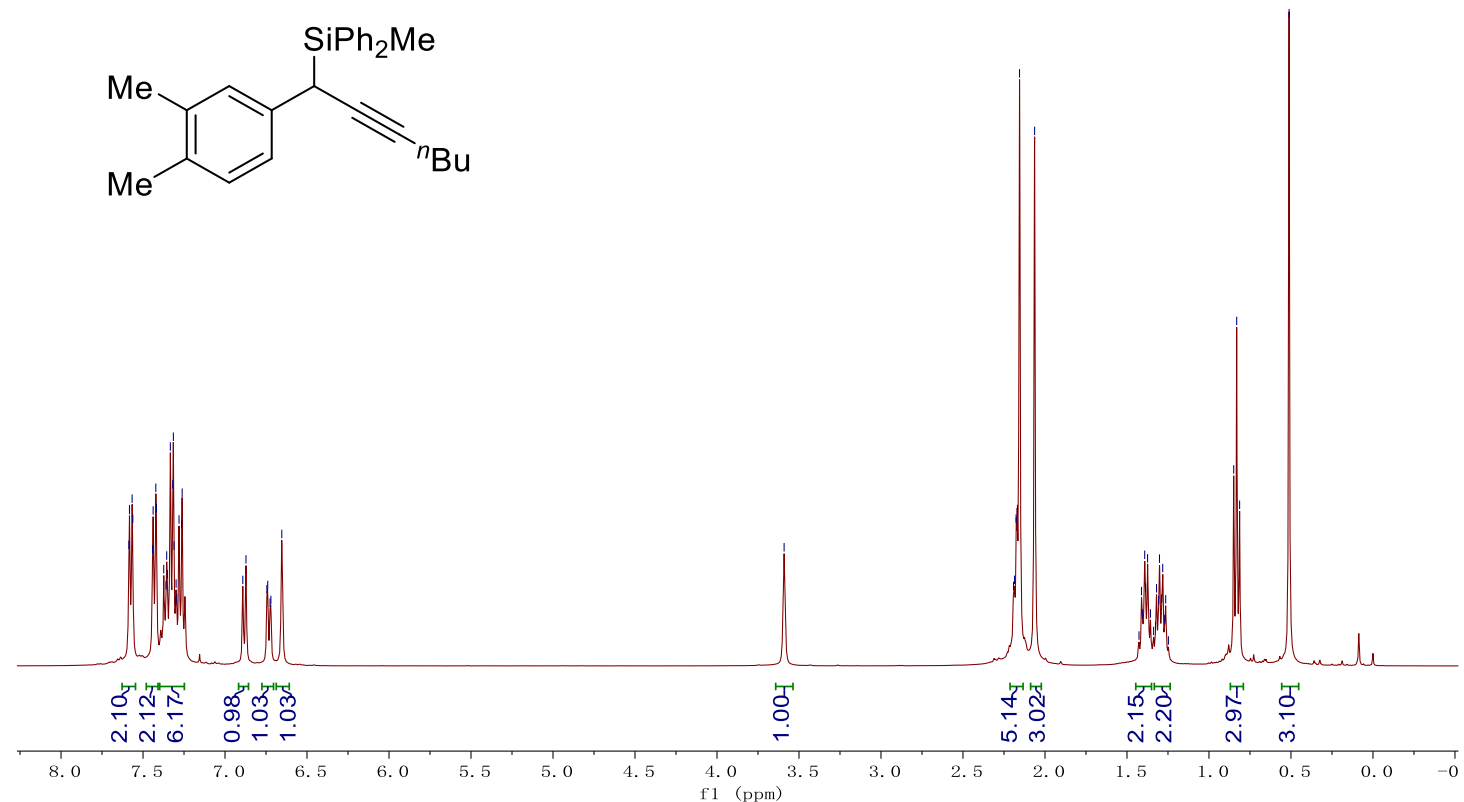

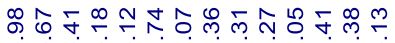

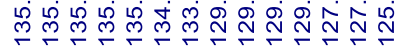

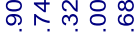

misisis

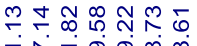

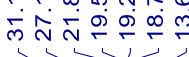

$\stackrel{\substack{\infty \\ \infty}}{\varphi}$

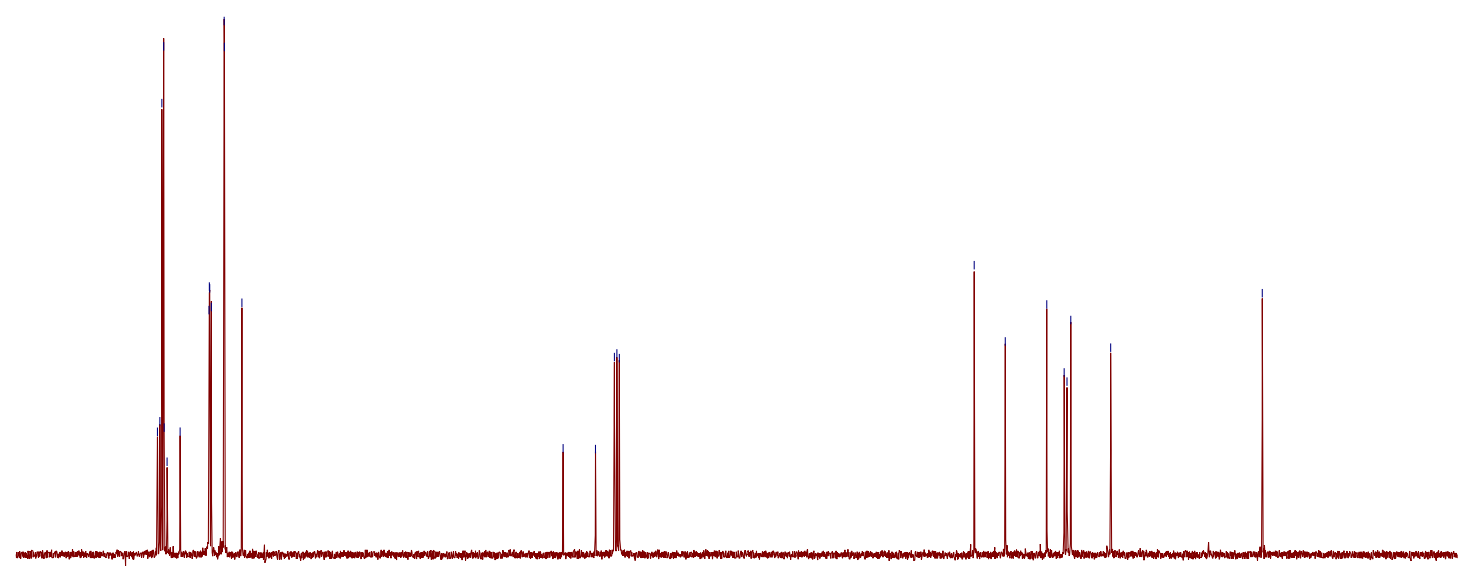

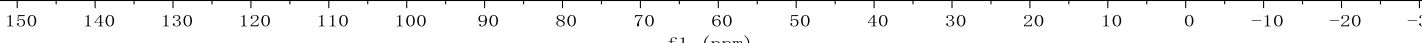


(-)-(1-(benzofuran-5-yl)hept-2-yn-1-yl)(methyl)diphenylsilane (3xc)

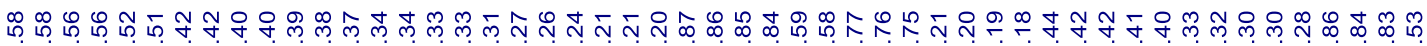

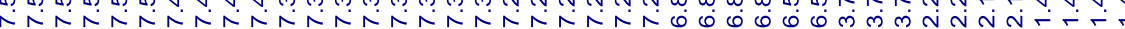<smiles>CCCCC(C#CC(C)(C)C)c1ccc2occc2c1</smiles>

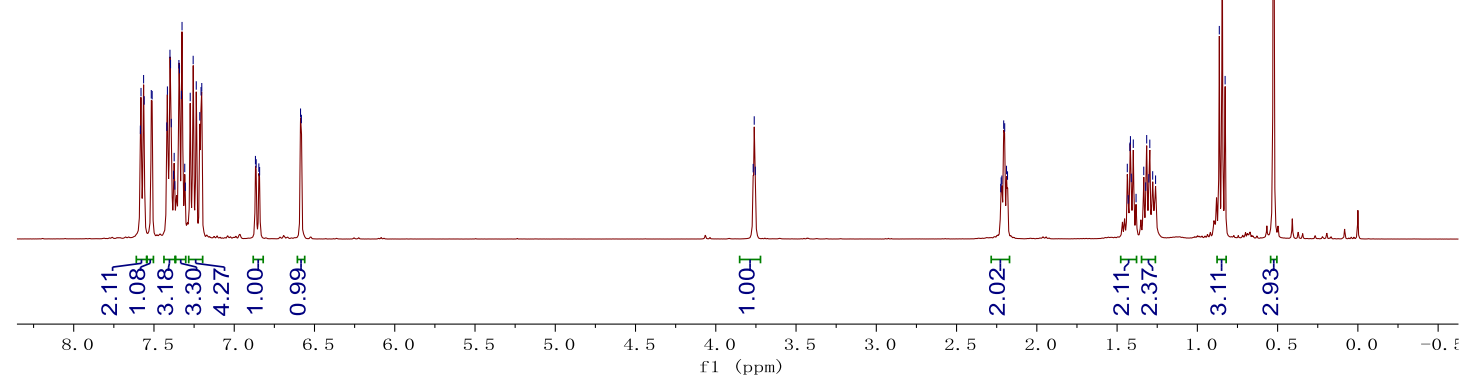

ำ

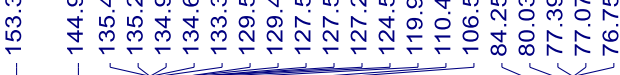

సุ

穴

$\stackrel{\substack{p \\ \dot{p}}}{i}$

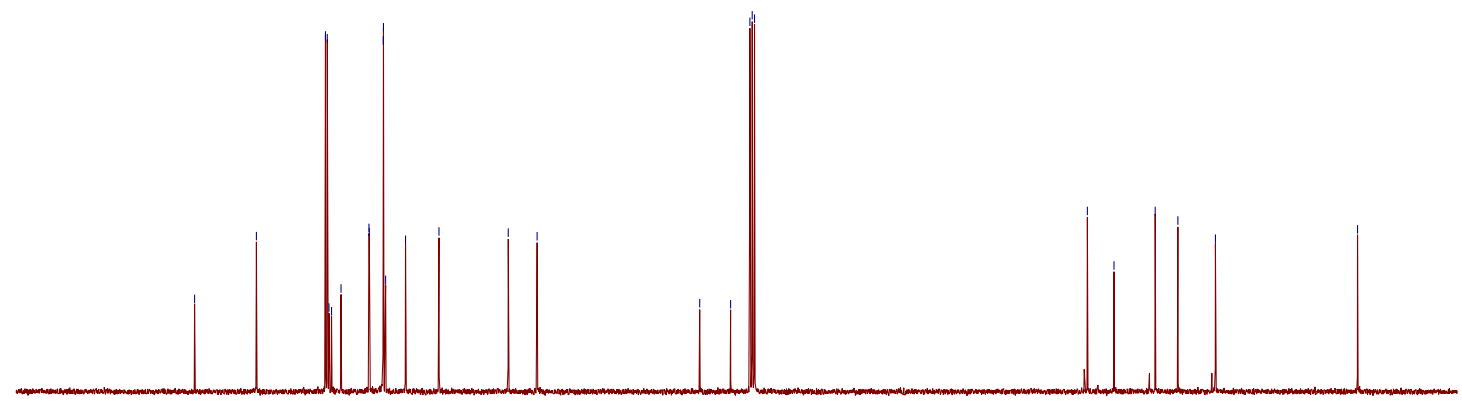

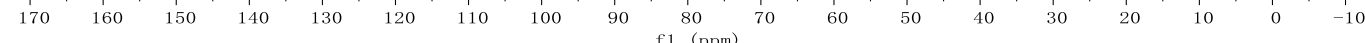


(+)-(Z)-triethyl(1-(naphthalen-2-yl)hept-2-en-1-yl)silane (4)

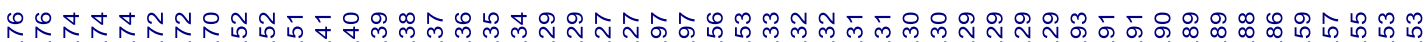

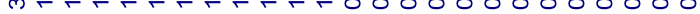<smiles>CCCCC(C)C=CC(CCCC)c1ccc2ccccc2c1</smiles>

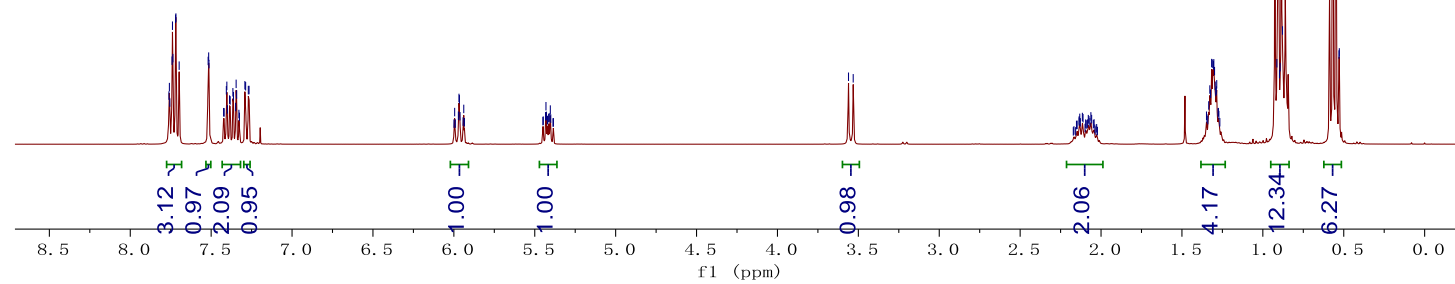

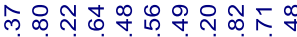

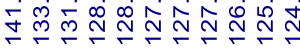

N.

走余

$\stackrel{\infty}{\infty} \stackrel{\infty}{\infty} \stackrel{\infty}{\infty}$

mั่

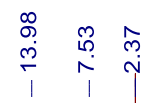

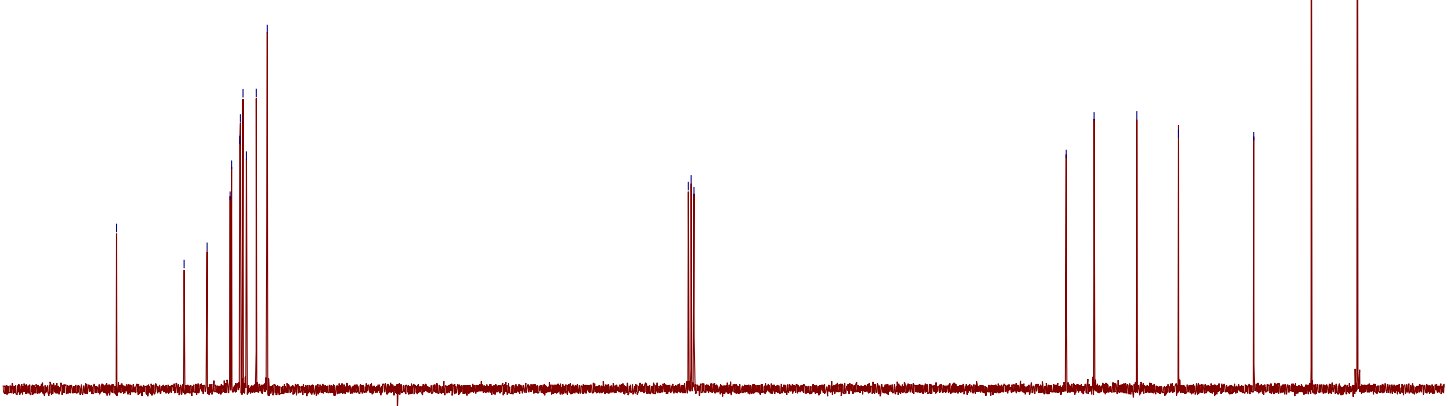

$80 \underset{\text { f } 12}{70}(\mathrm{ppm})$

60

$40 \quad 30$

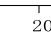


(+)-(Z)-methyl(1-(naphthalen-2-yl)-3-(4,4,5,5-tetramethyl-1,3,2-dioxaborolan-2yl)but-2-en-1-yl)diphenylsilane (5)

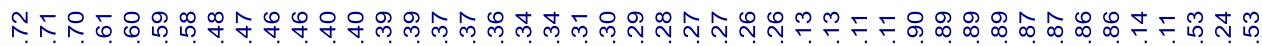

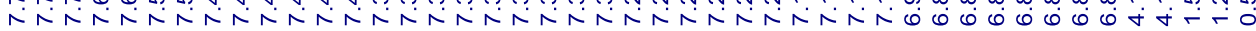<smiles>CCCCCCC(C)=CC([AlH2+])c1ccc2ccccc2c1</smiles>

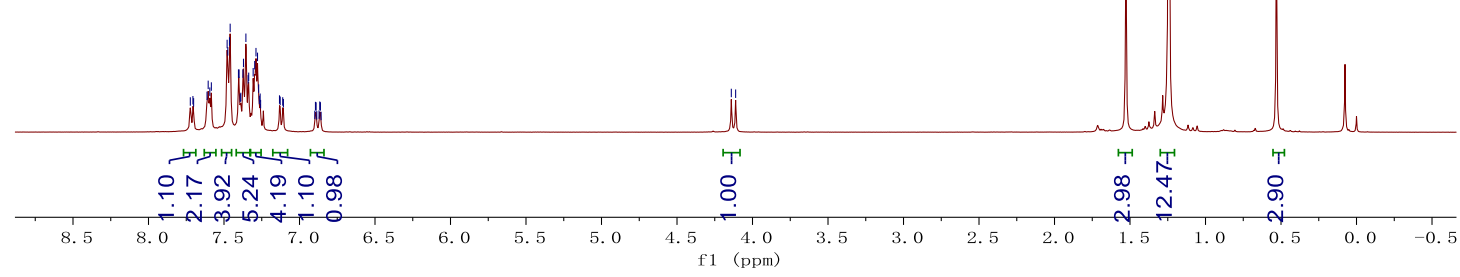

ㄸำ

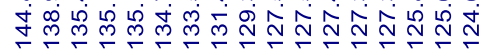

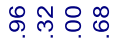

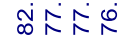
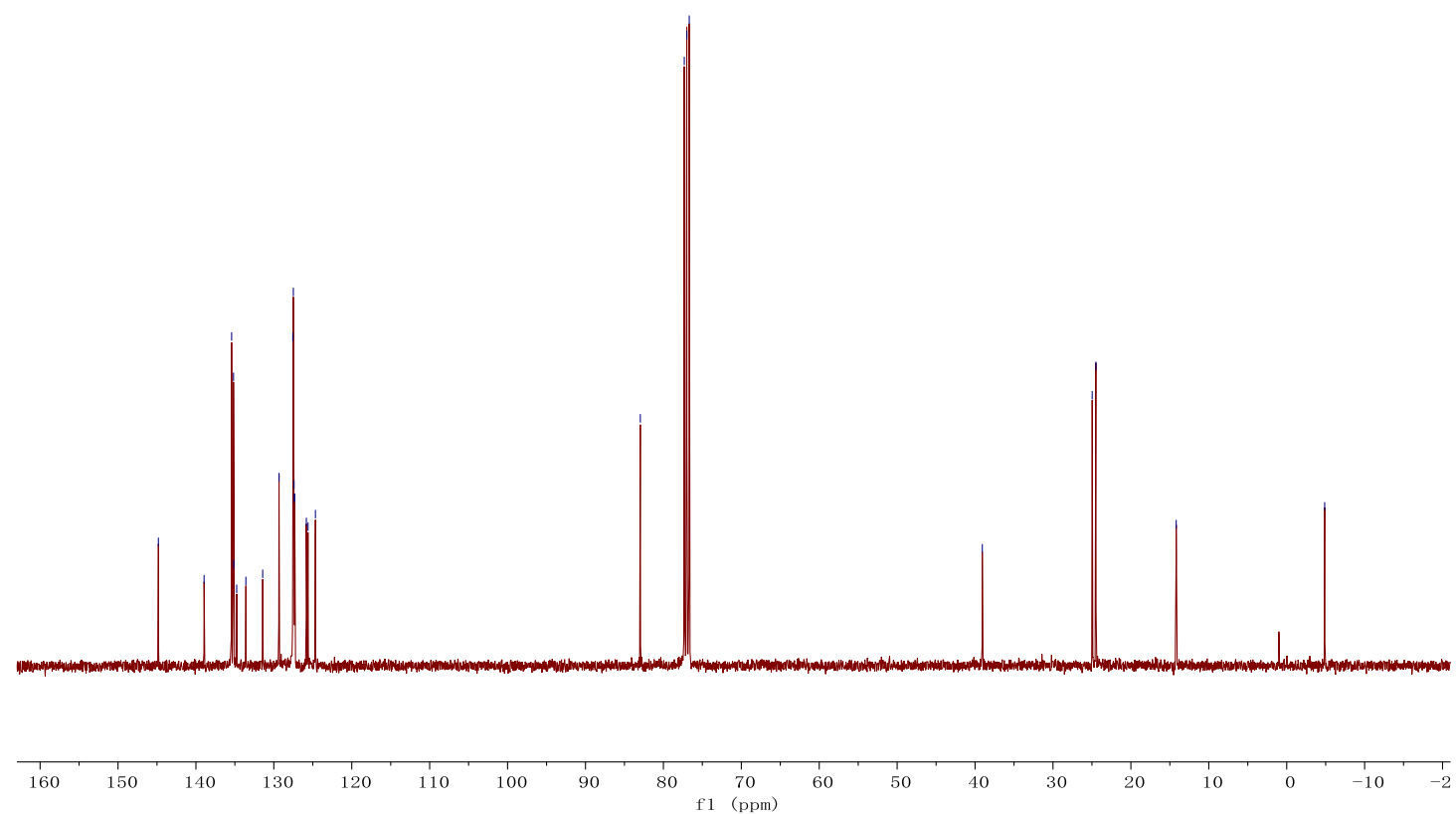


\section{(S)-dimethyl(1-(naphthalen-2-yl)-3-phenylpropyl)(phenyl)silane (6)}

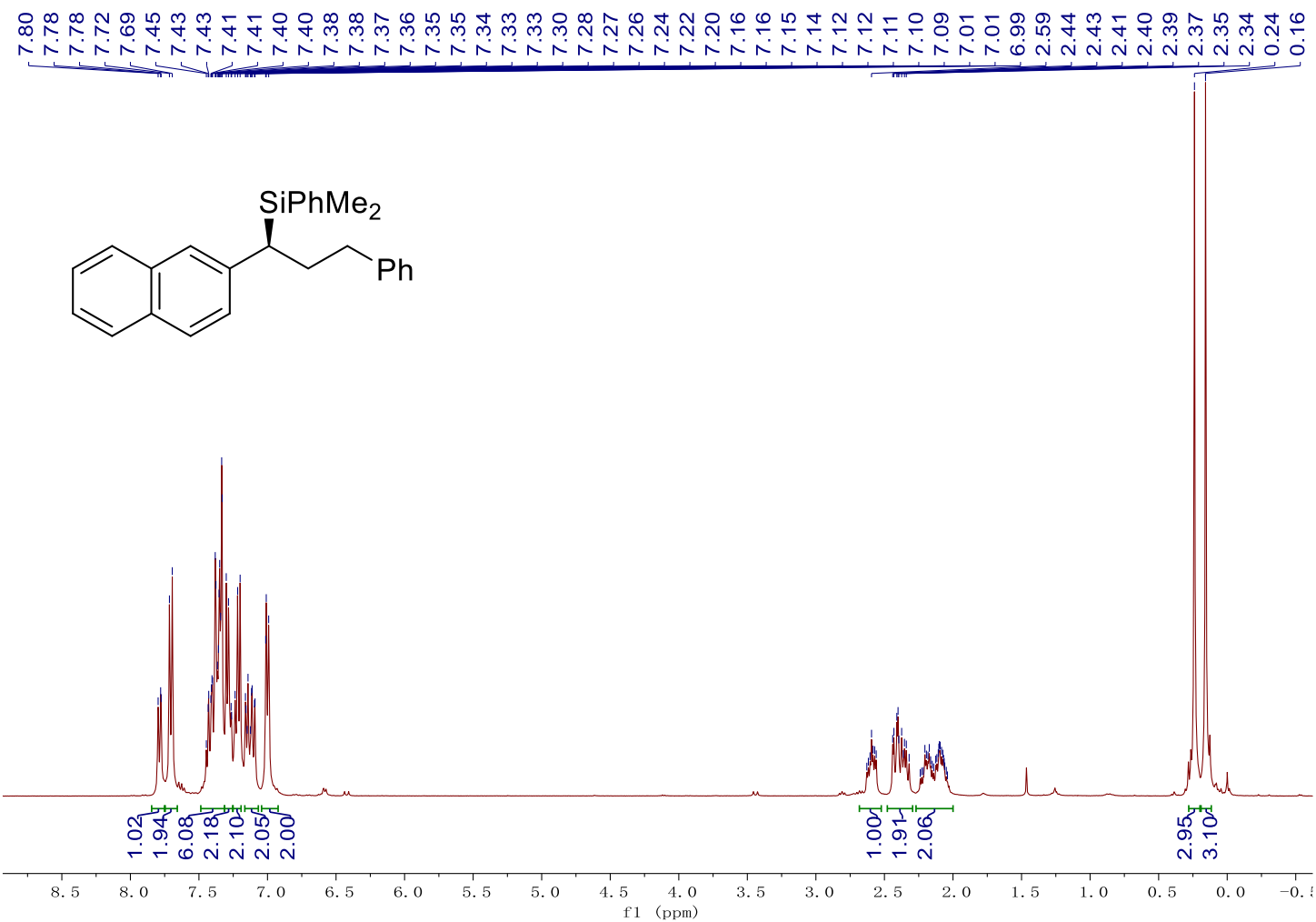

จำํำ

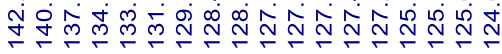

ํㅛ융

송

จุษ

लि

ㅇำ

mi

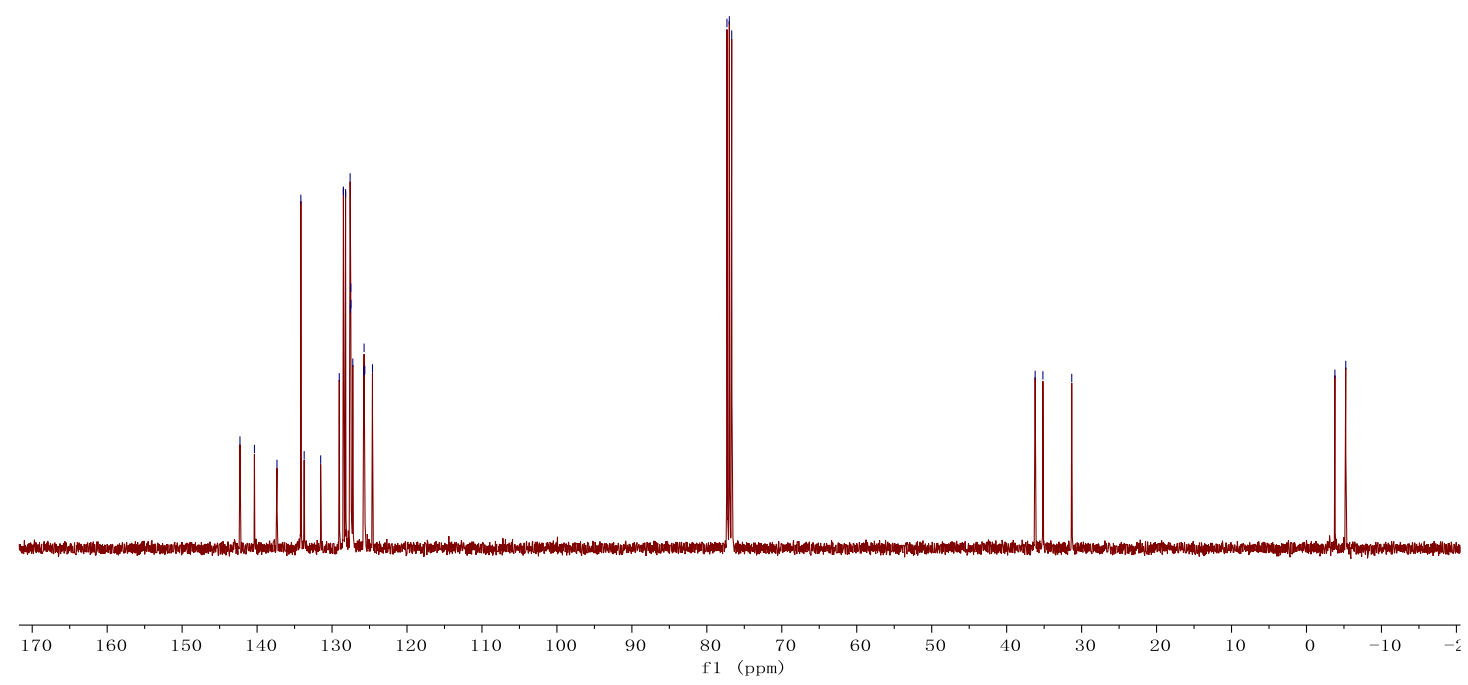




\section{(S)-methyl(1-(naphthalen-2-yl)butyl)diphenylsilane (3kc-H)}

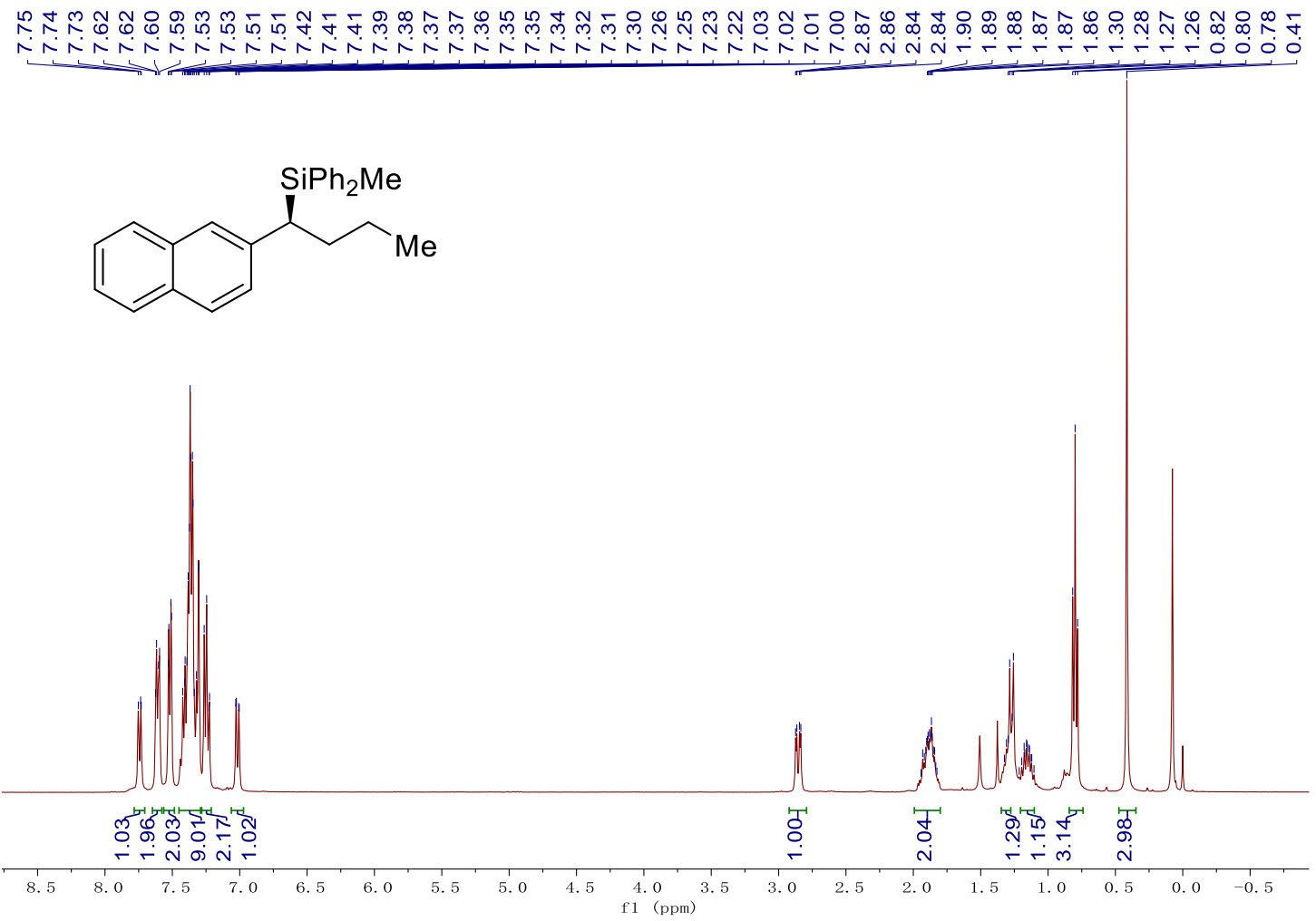

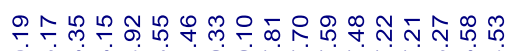

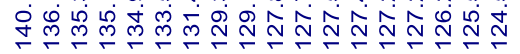

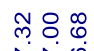

กิ̃

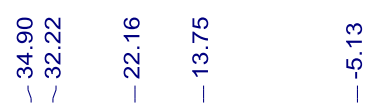

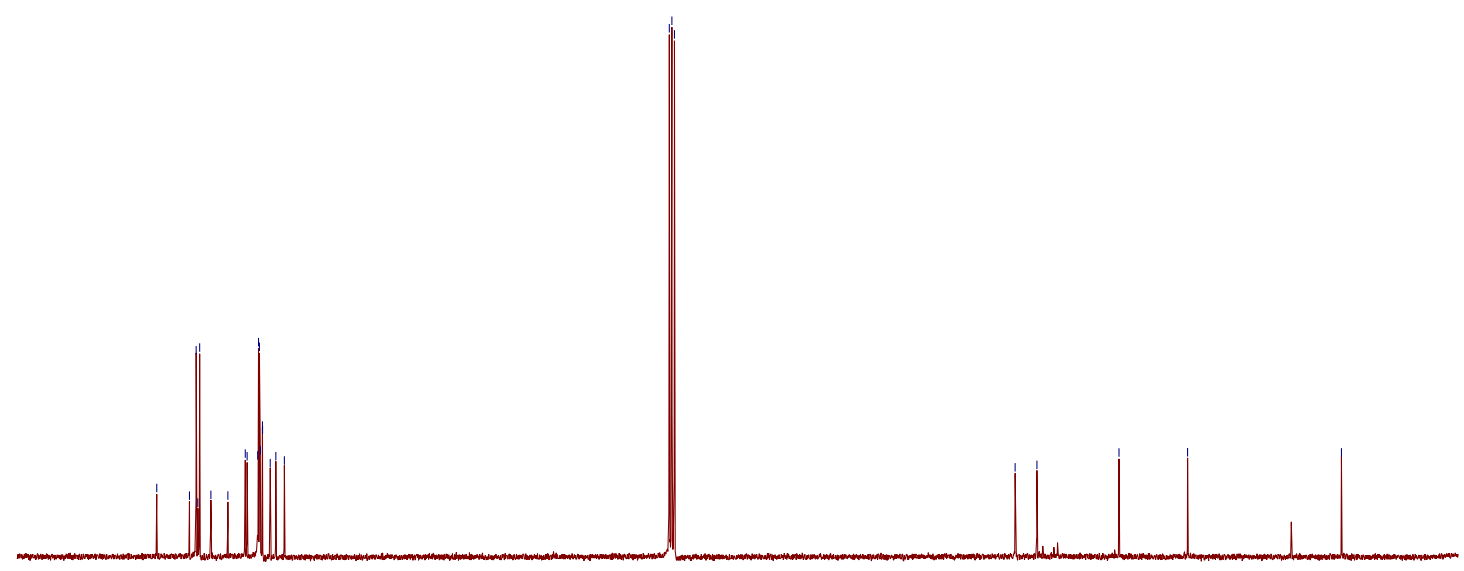

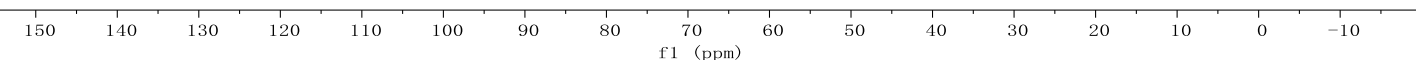




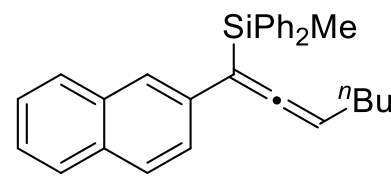

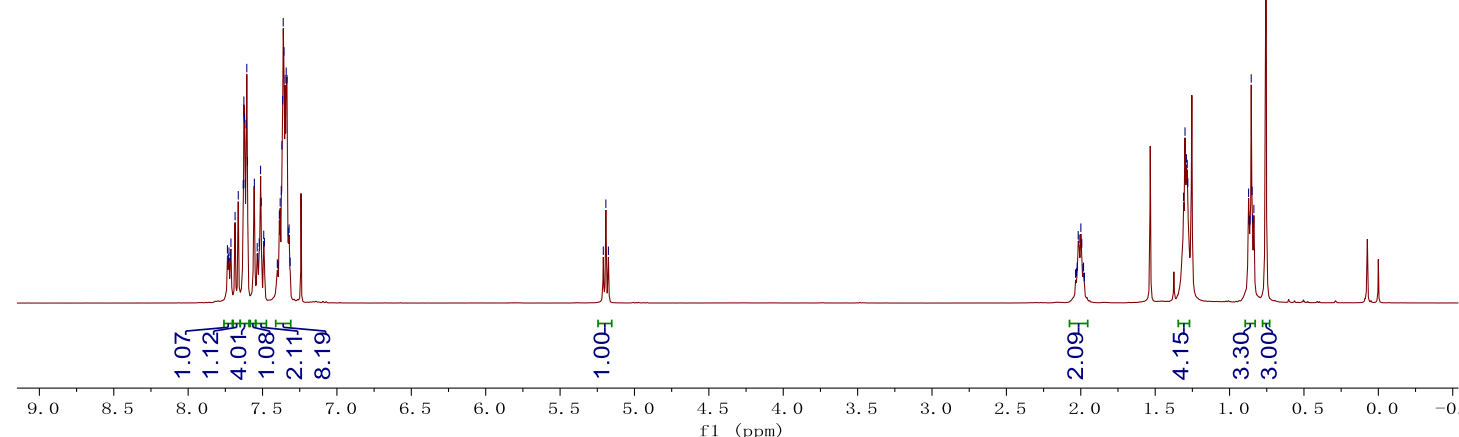

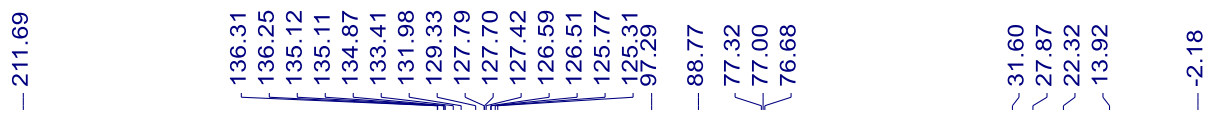

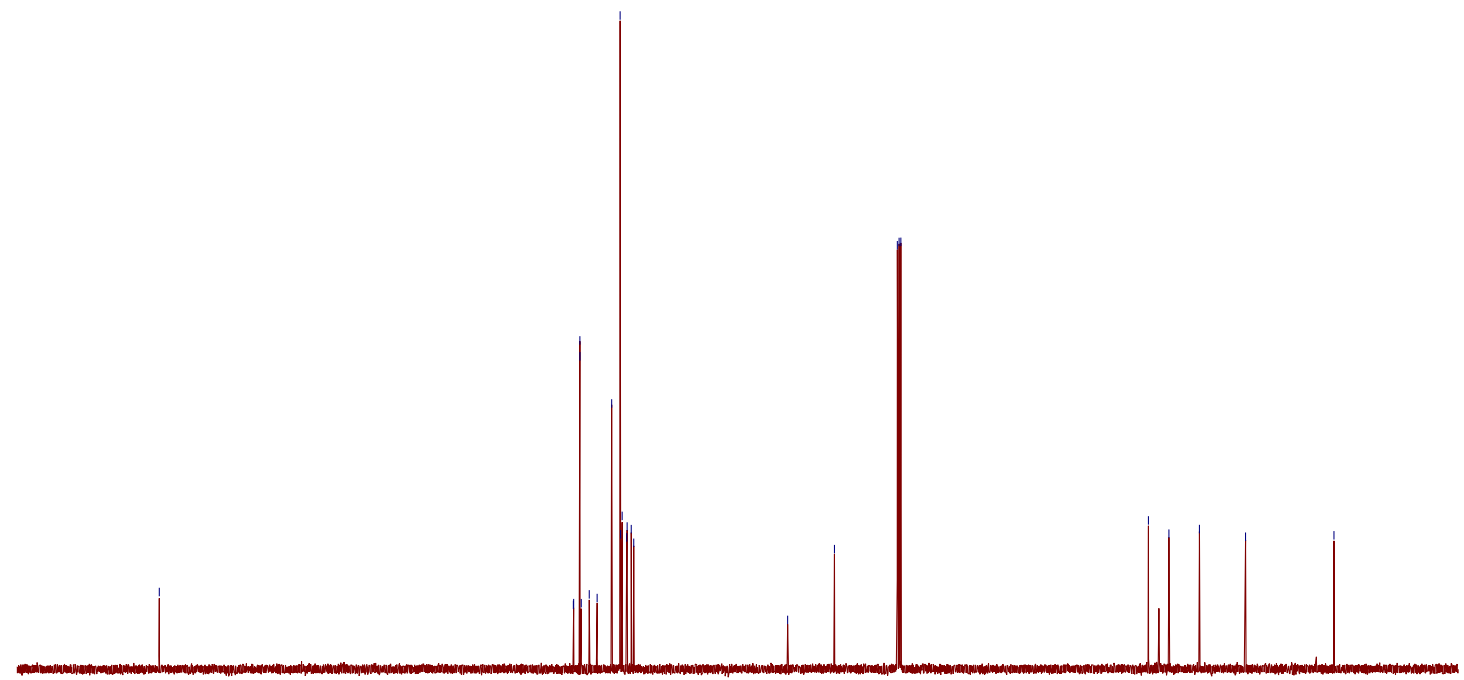

$\begin{array}{lllllllllllllllllllllllllllllllllll}230 & 220 & 210 & 200 & 190 & 180 & 170 & 160 & 150 & 140 & 130 & 120 & 110 & 100 & 90 & 80 & 70 & 60 & 50 & 40 & 30 & 20 & 10 & 0 & -10 & -20\end{array}$ 
<smiles>CC=C=C(CCCC)c1ccc2ccccc2c1</smiles>

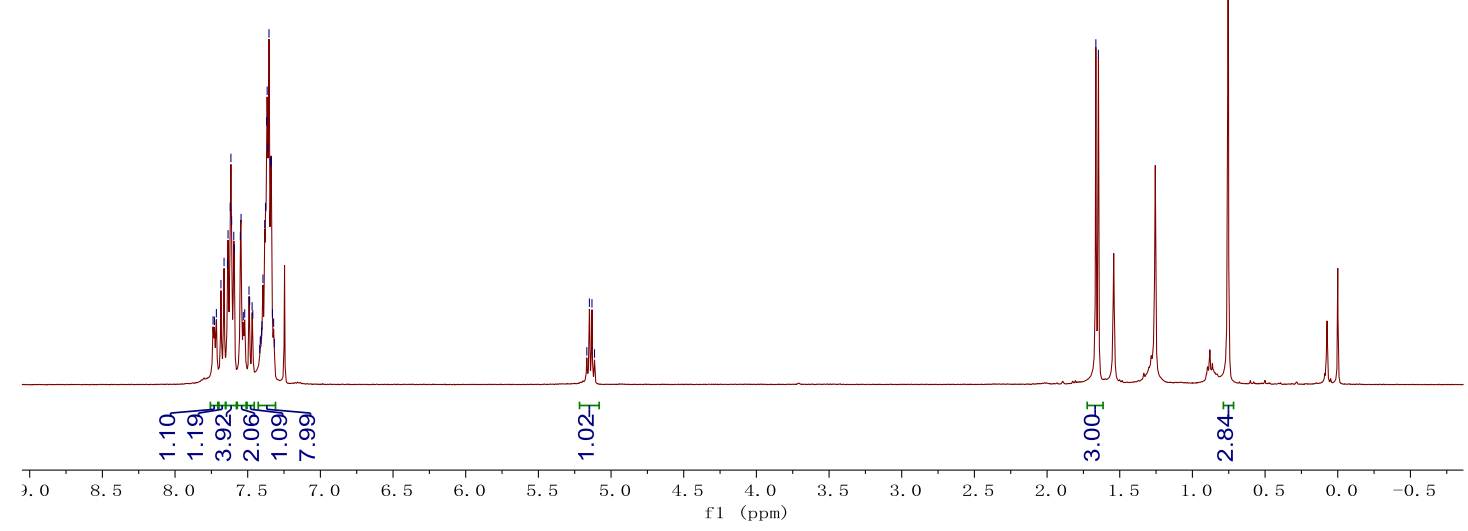

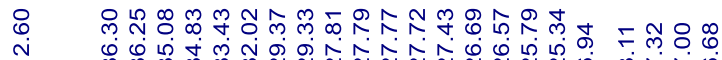

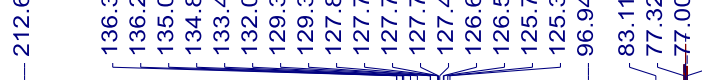
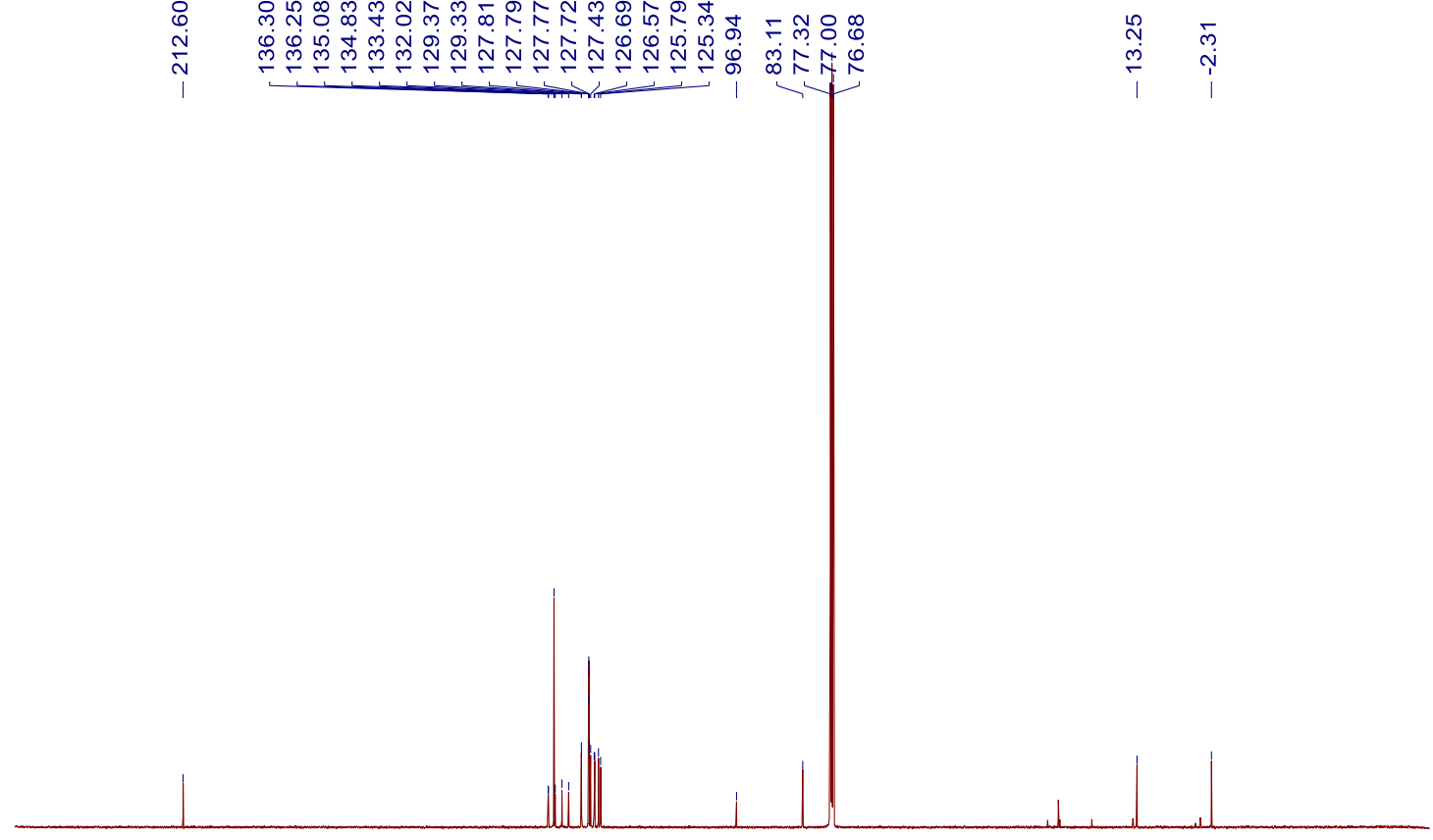

$\begin{array}{llllllllllllllll}240 & 230 & 220 & 210 & 200 & 190 & 180 & 170 & 160 & 150 & 140 & 130 & 120 & 110 & 100 & 90\end{array}$ 

(7c)

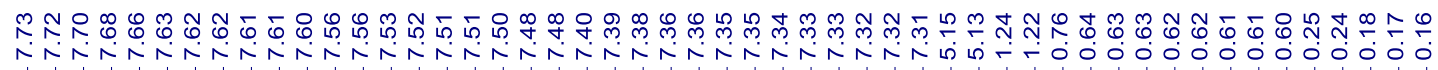

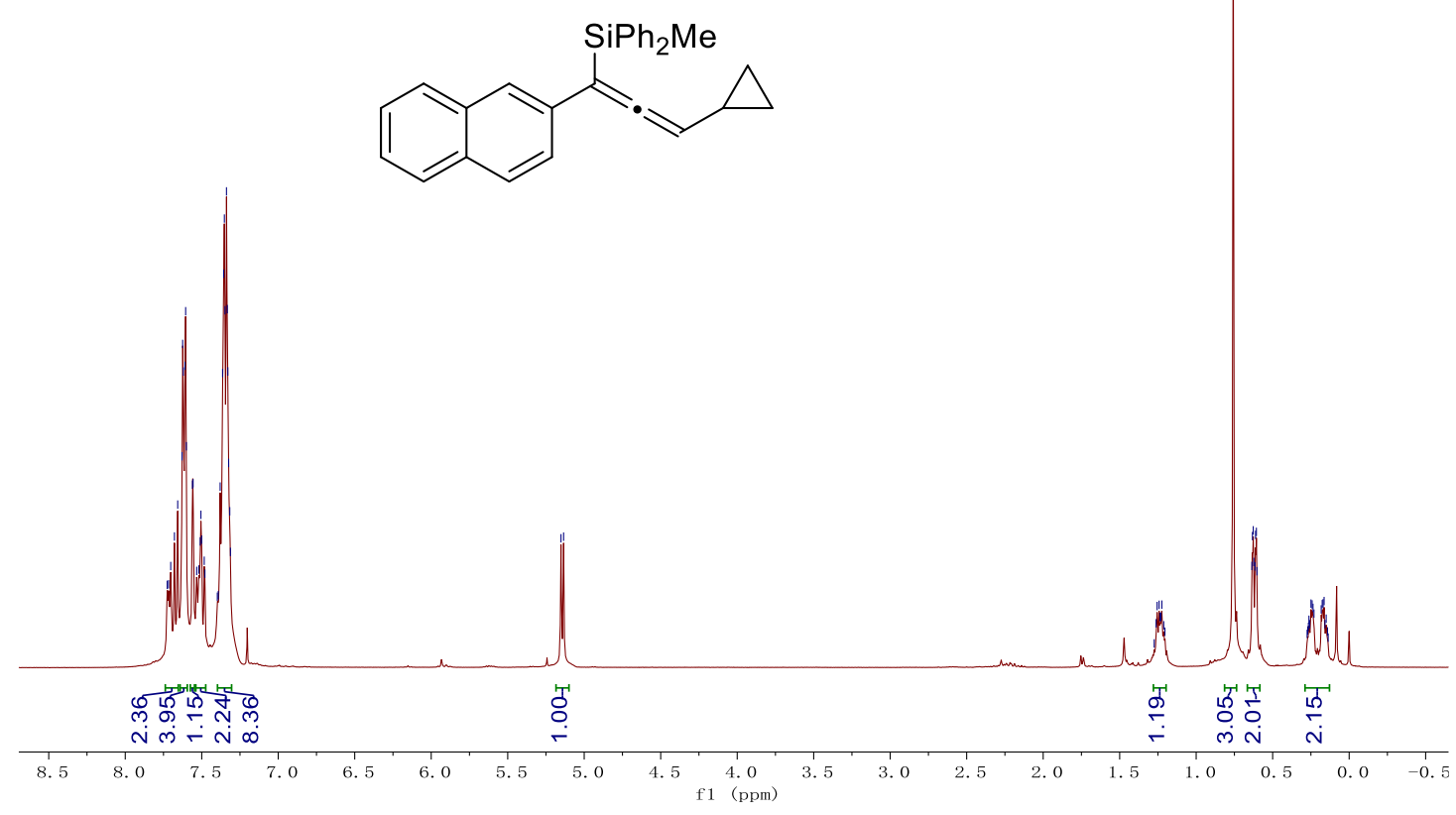

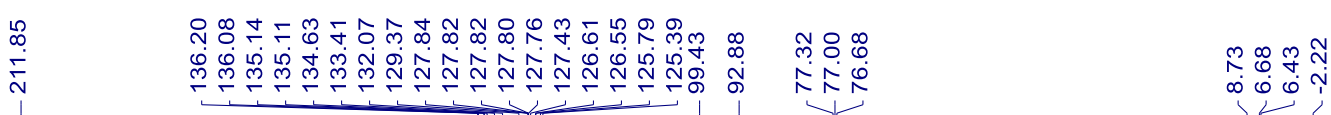

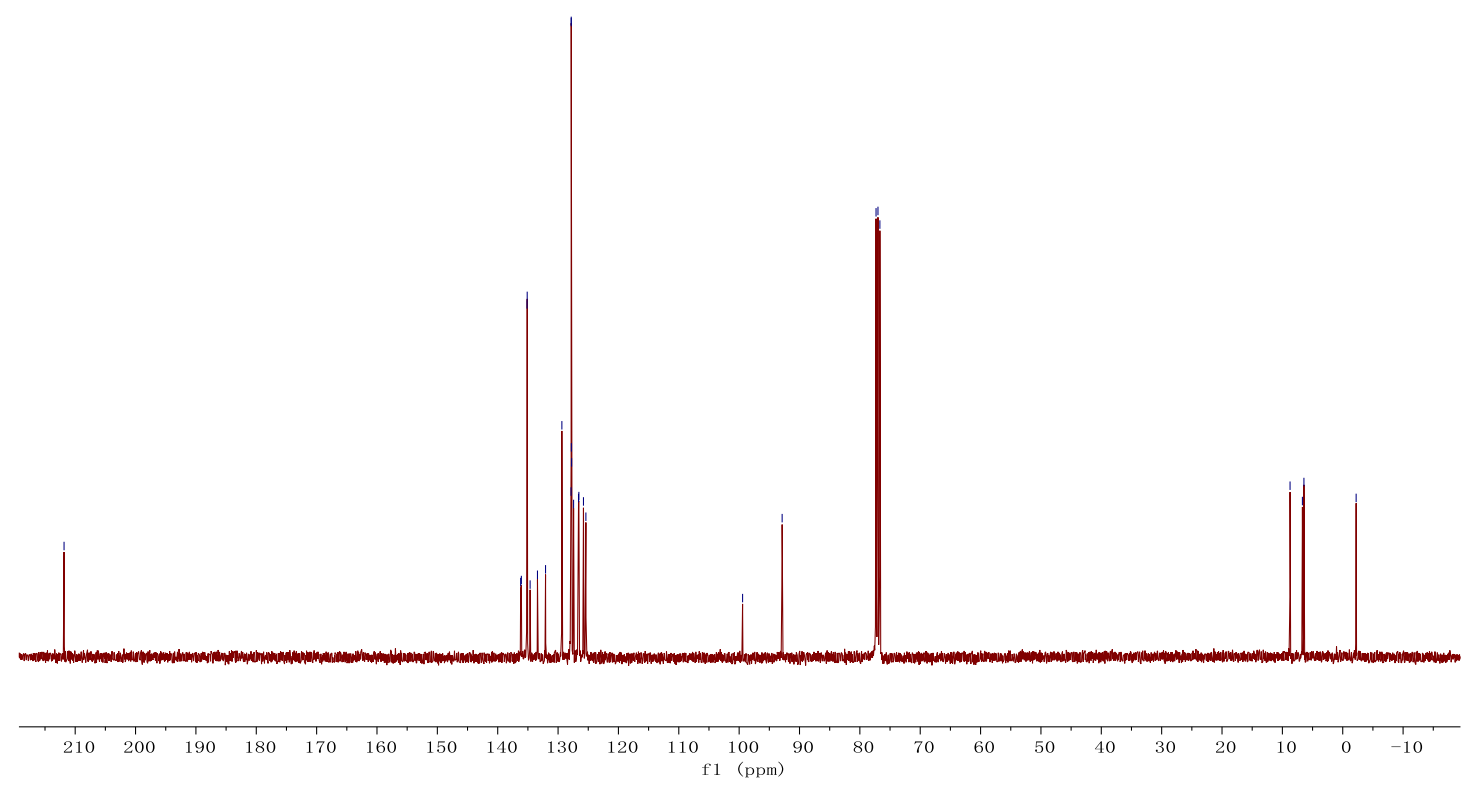




\section{(-)-(3-(cyclohex-1-en-1-yl)-1-(naphthalen-2-yl)propa-1,2-dien-1-}

\section{yl)(methyl)diphenylsilane (7d)}

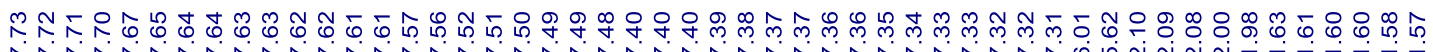

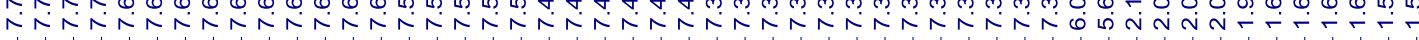<smiles>CCCCC(C=CC1=CCCCC1)c1ccc2ccccc2c1</smiles>

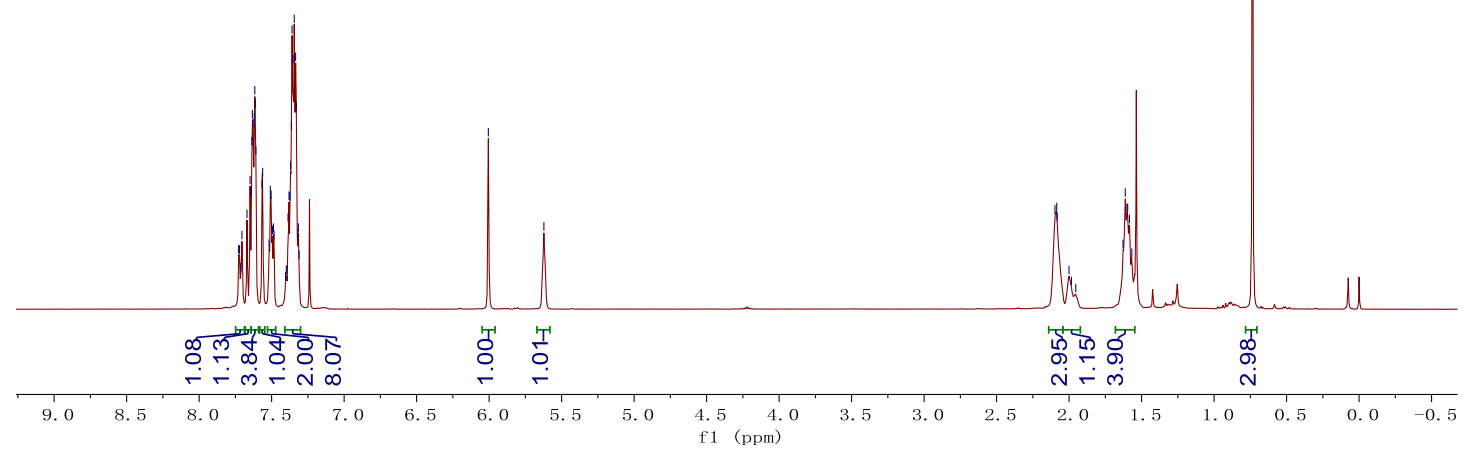

\begin{tabular}{|c|c|c|c|}
\hline $\begin{array}{l}\stackrel{N}{\infty} \\
\stackrel{N}{N}\end{array}$ & 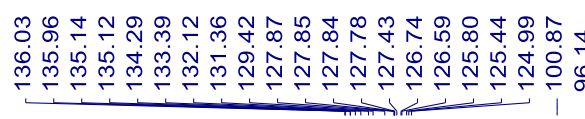 & 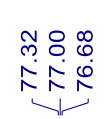 & 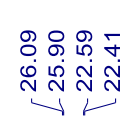 \\
\hline
\end{tabular}

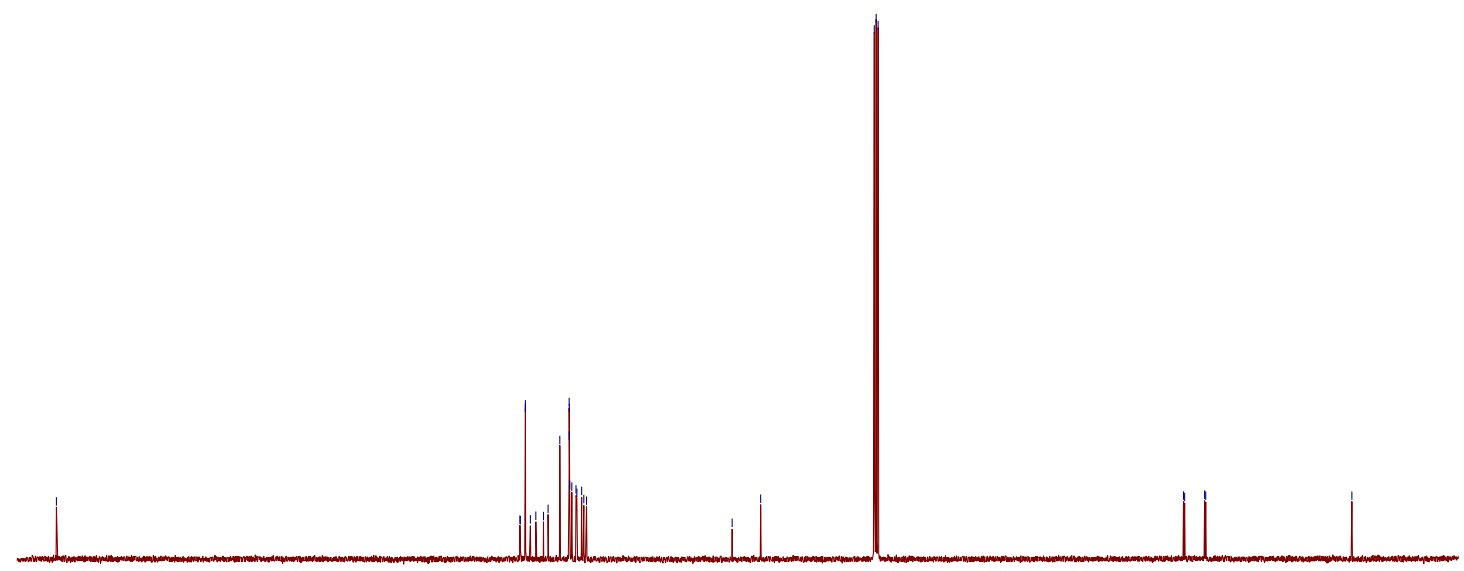

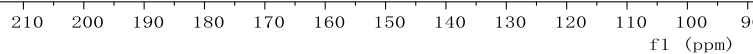


(-)-methyl(1-(naphthalen-2-yl)-3-phenylpropa-1,2-dien-1-yl)diphenylsilane (7e) 等

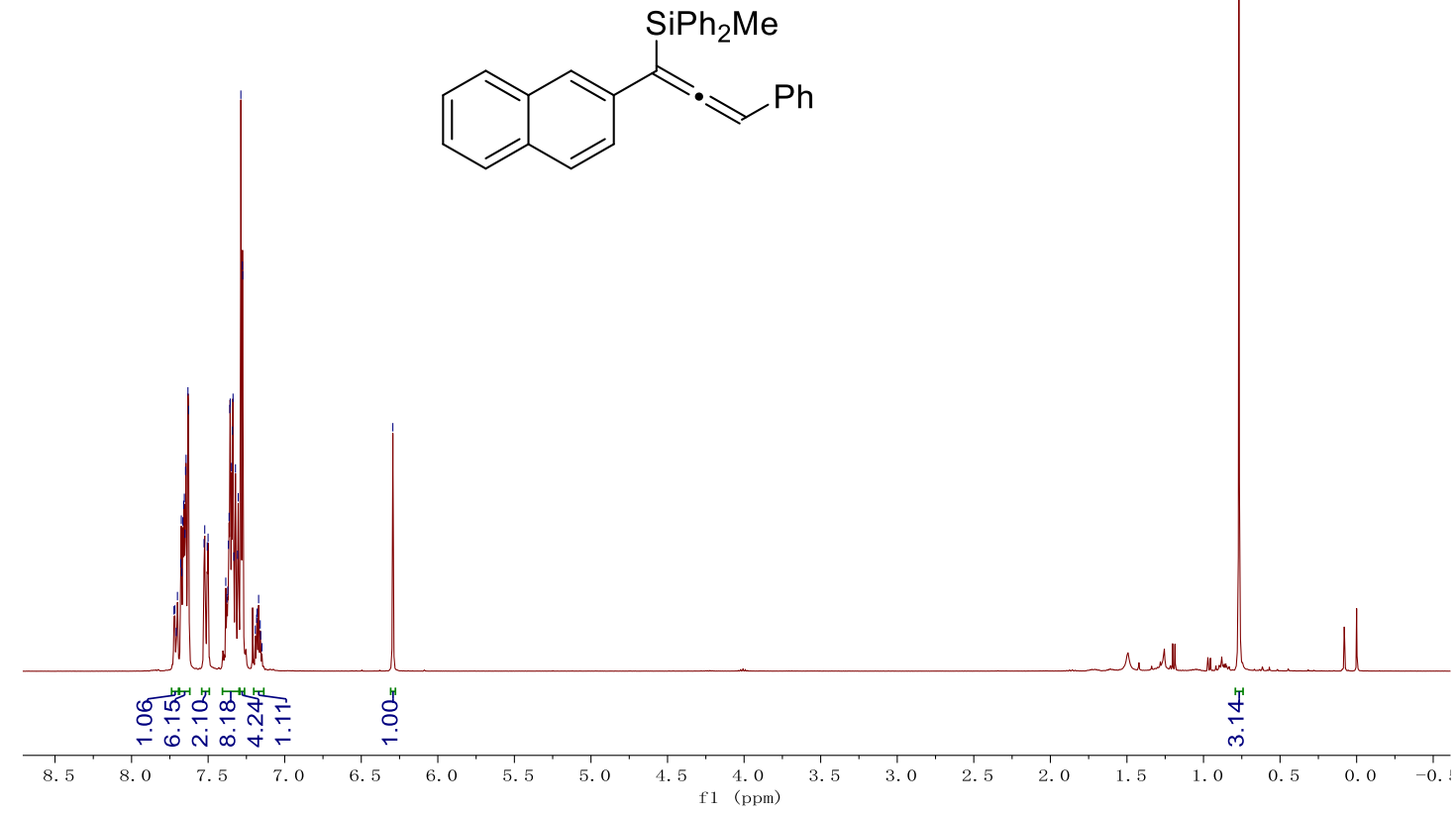

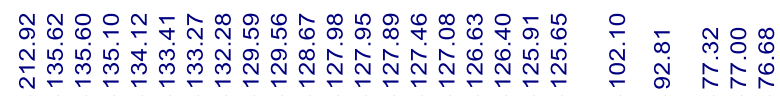

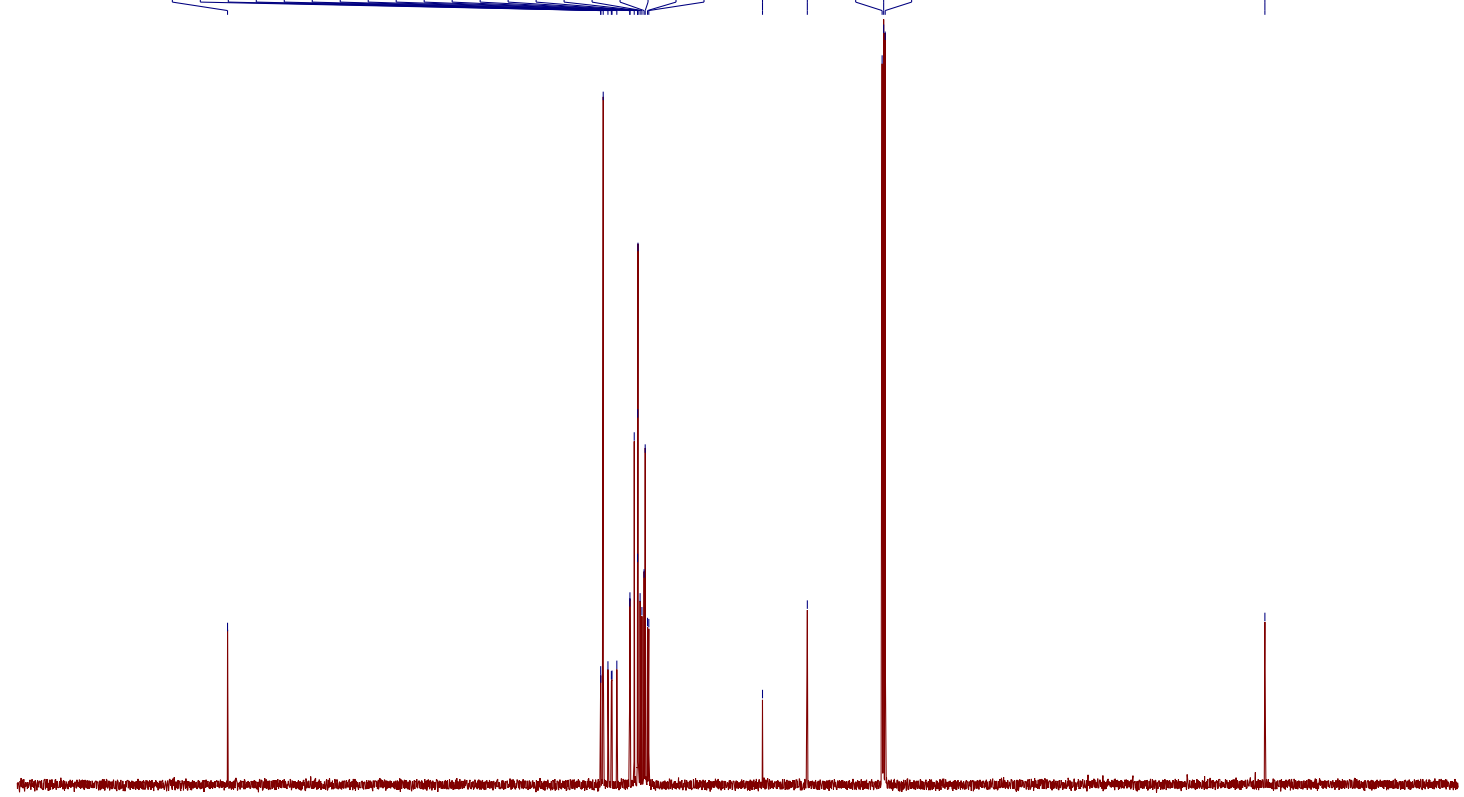

$\begin{array}{lllllllllllllllllllllllllllllllllllllll}1 & 250 & 240 & 230 & 220 & 210 & 200 & 190 & 180 & 170 & 160 & 150 & 140 & 130 & 120 & 110 & 100 & 90 & 80 & 70 & 60 & 50 & 40 & 30 & 20 & 10 & 0 & -10 & -20 & -30 & -4\end{array}$ f1 (ppm) 
<smiles>CCCCC(=CC=CC(C)(C)C)c1ccc(Cl)c(Cl)c1</smiles>

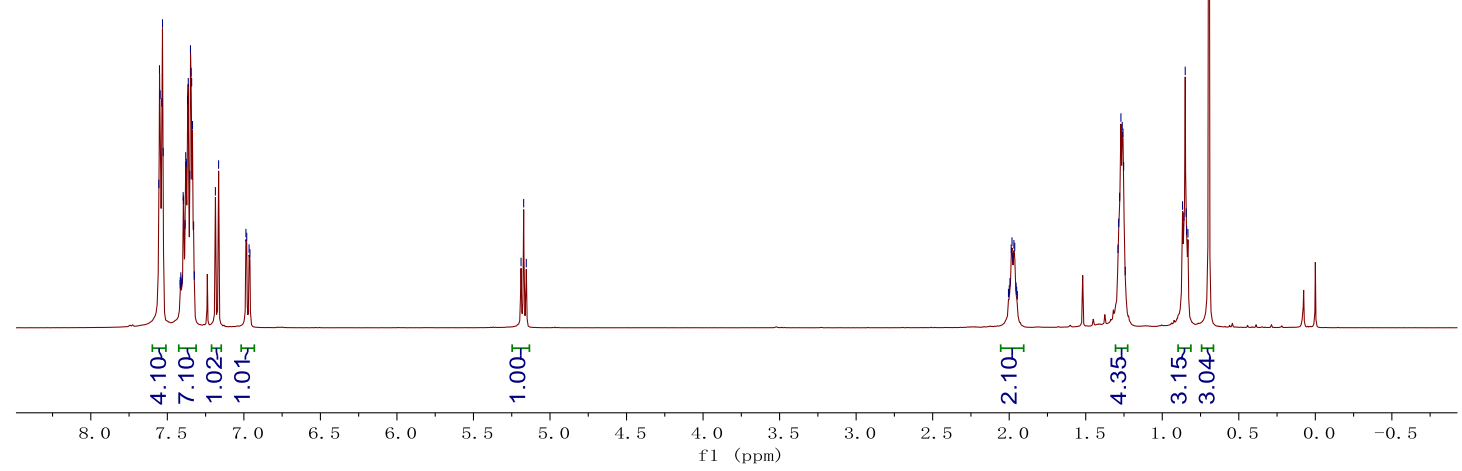

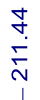

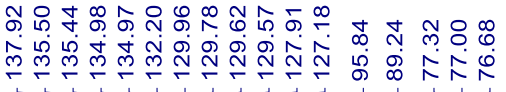

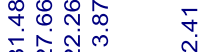

mก⿻

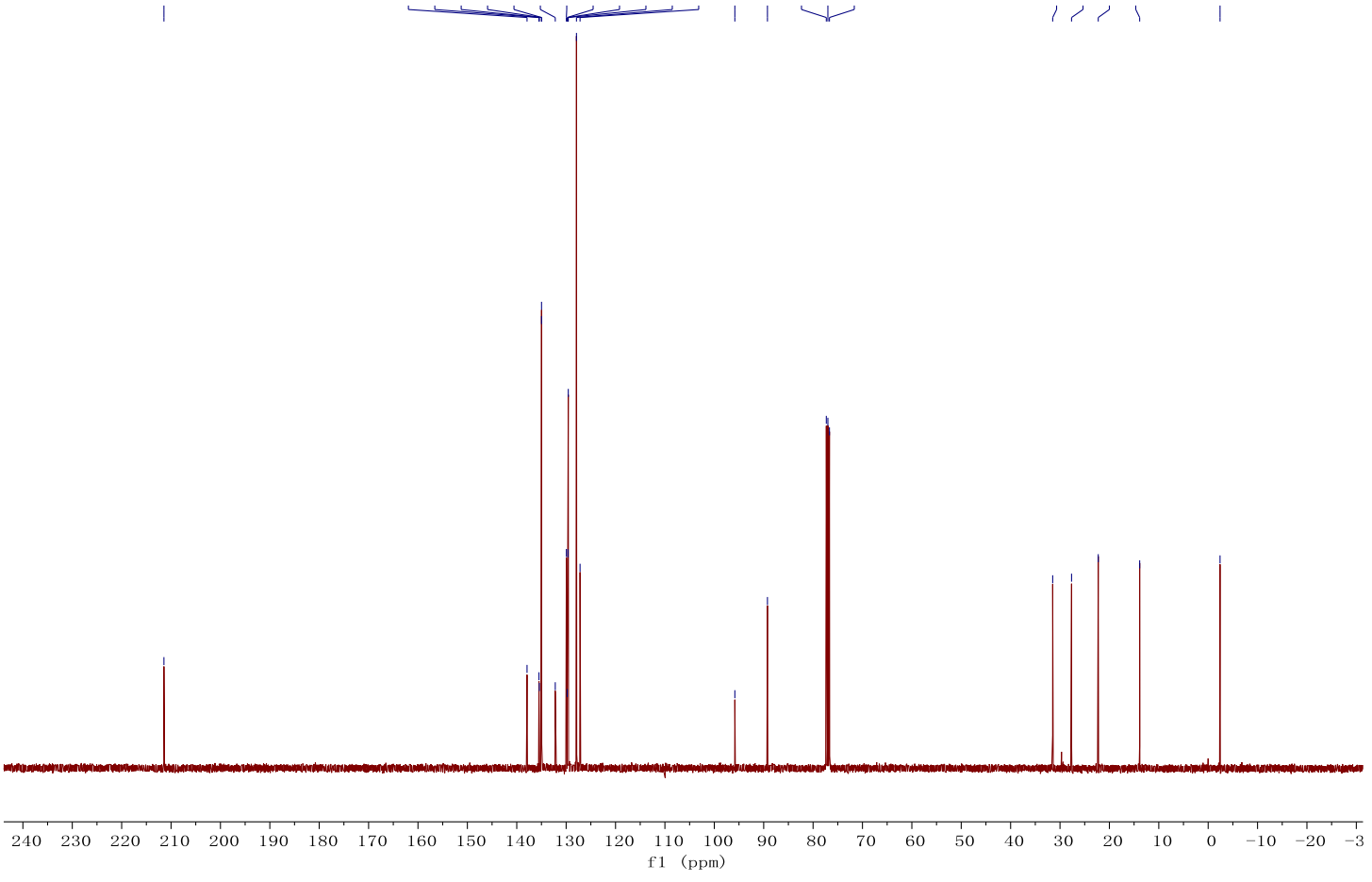


<smiles>Cc1ccc(C([SnH2])=CC(C)(C)C)cc1C</smiles>

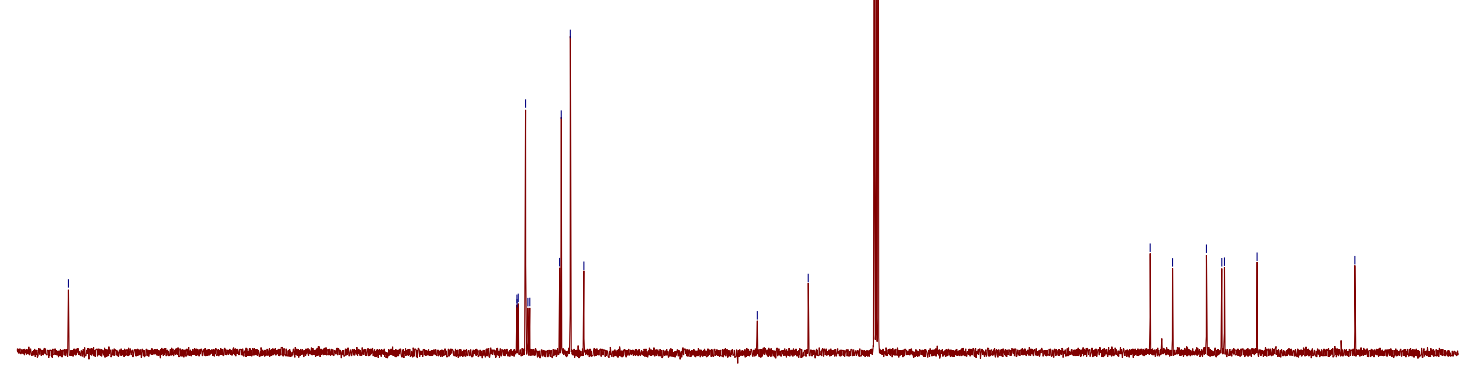




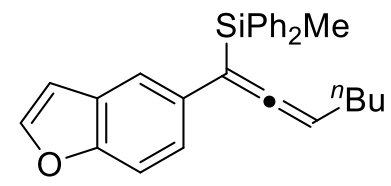

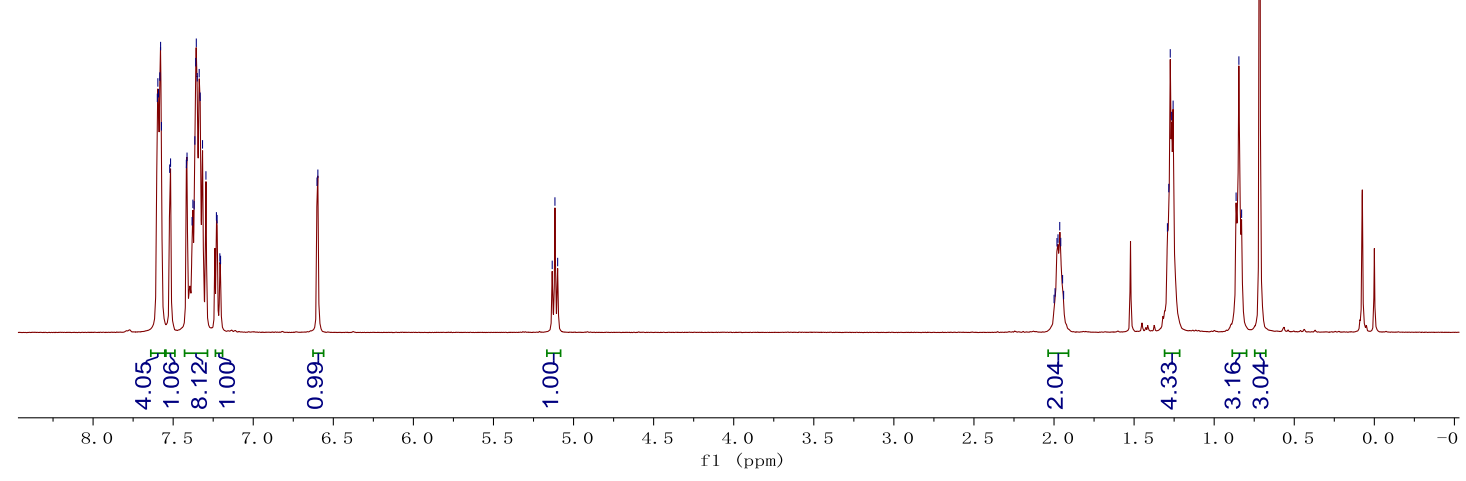

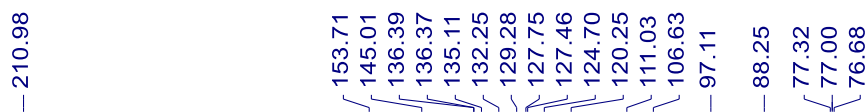

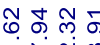

$\dot{m} \dot{\sim}$ ำ

$\min$

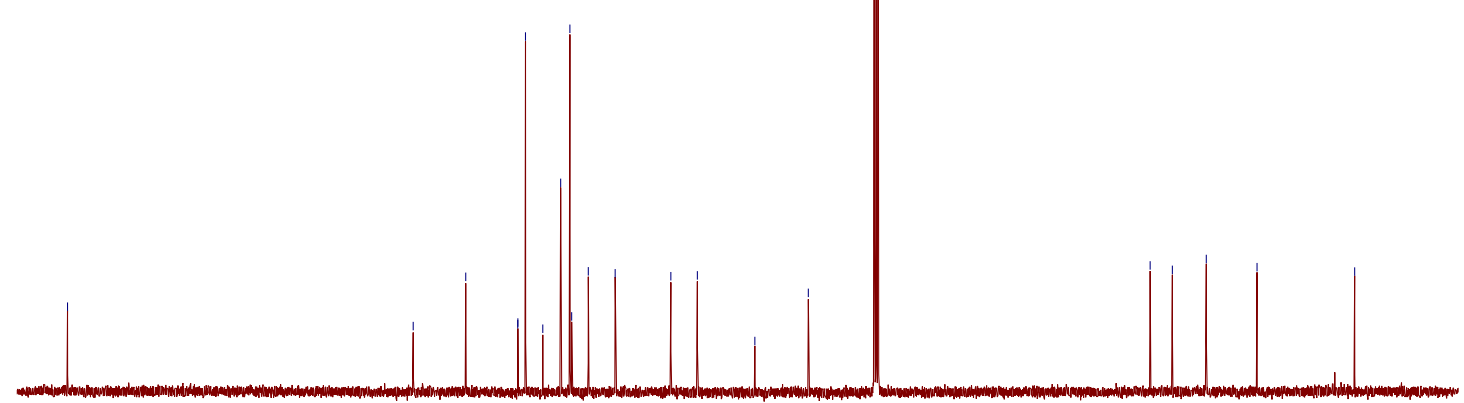

$\begin{array}{llllllllllll}210 & 200 & 190 & 180 & 170 & 160 & 150 & 140 & 130 & 120 & 110 & 100 \\ \mathrm{f} 1 & (\mathrm{ppm})\end{array}$ 
<smiles>CCCC=Cc1ccc2ccccc2c1</smiles>

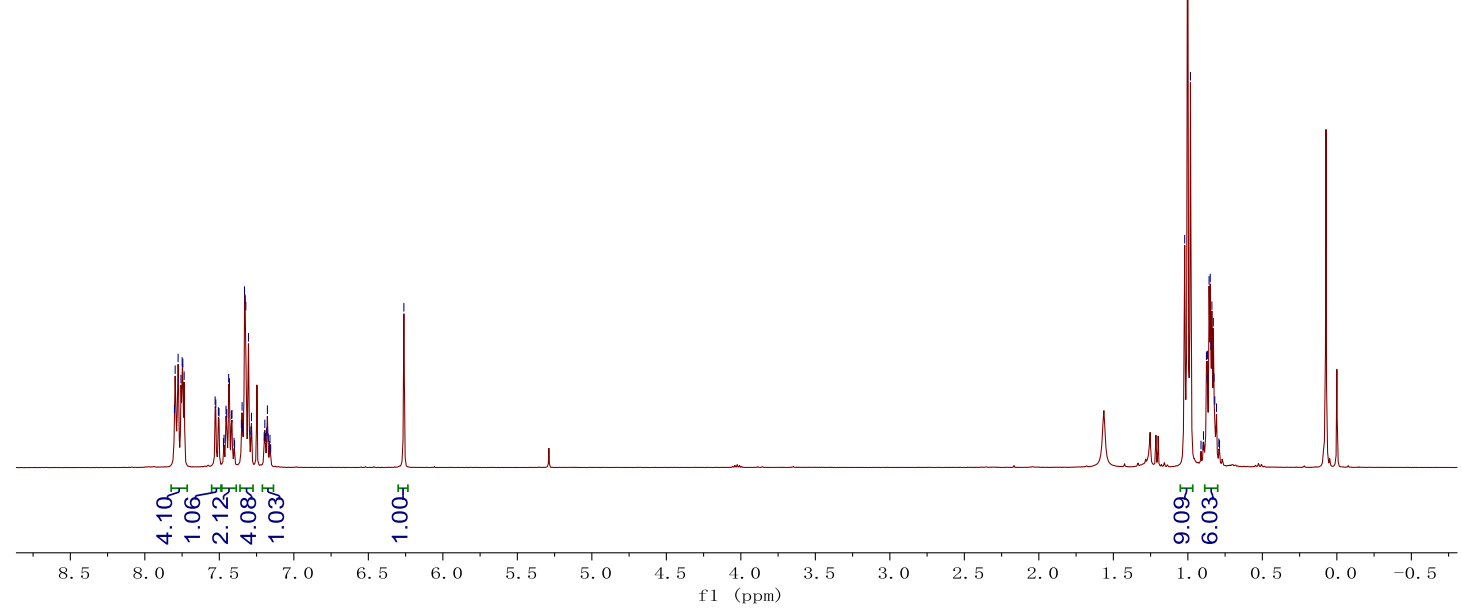

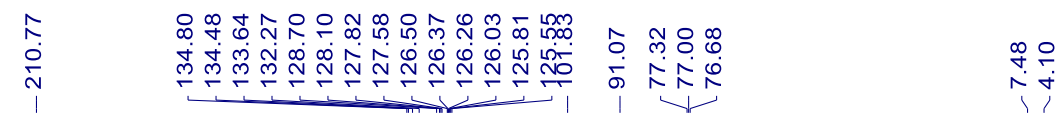

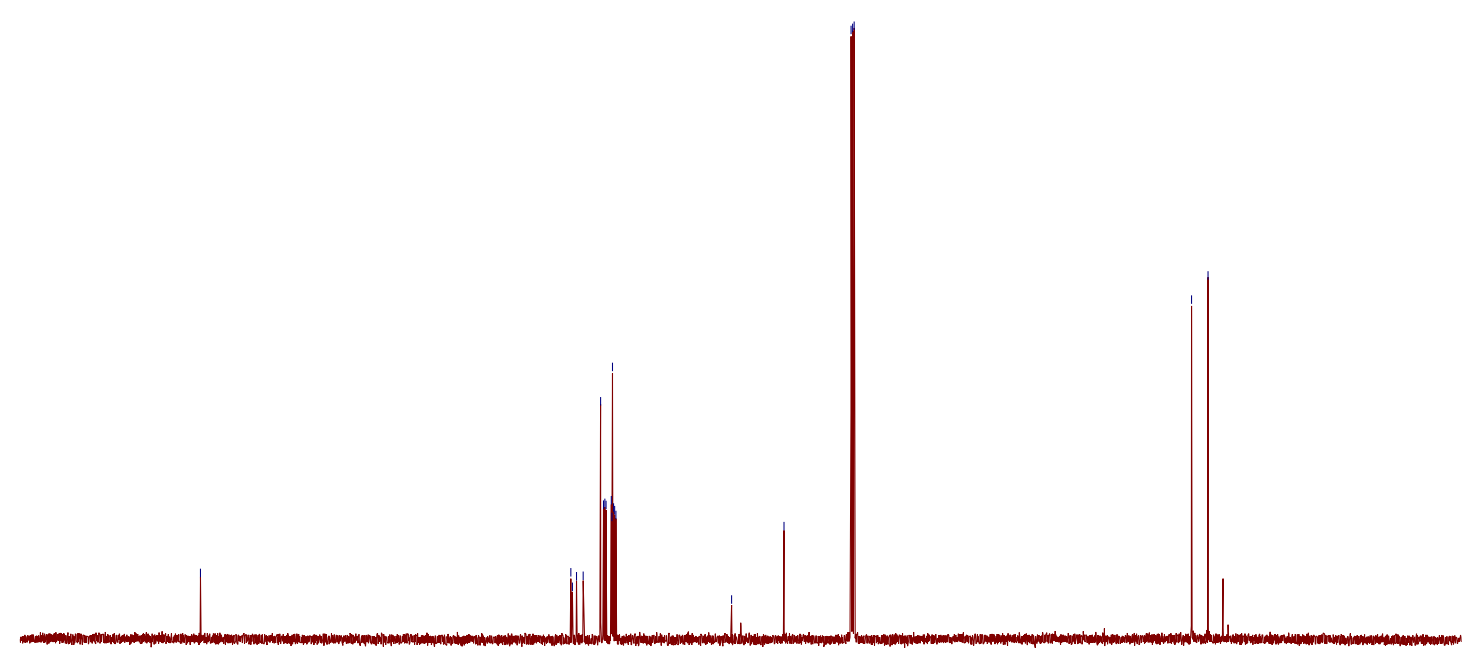
$\begin{array}{rlllllllllllllllllllllllllllllllll}240 & 230 & 220 & 210 & 200 & 190 & 180 & 170 & 160 & 150 & 140 & 130 & 120 & 110 & 100 & 90 & 80 & 70 & 60 & 50 & 40 & 30 & 20 & 10 & 0 & -10 & -20 & -30 & -40\end{array}$ 
<smiles>C[AsH2-]C(=CC=Cc1ccccc1)c1ccc2ccccc2c1</smiles>

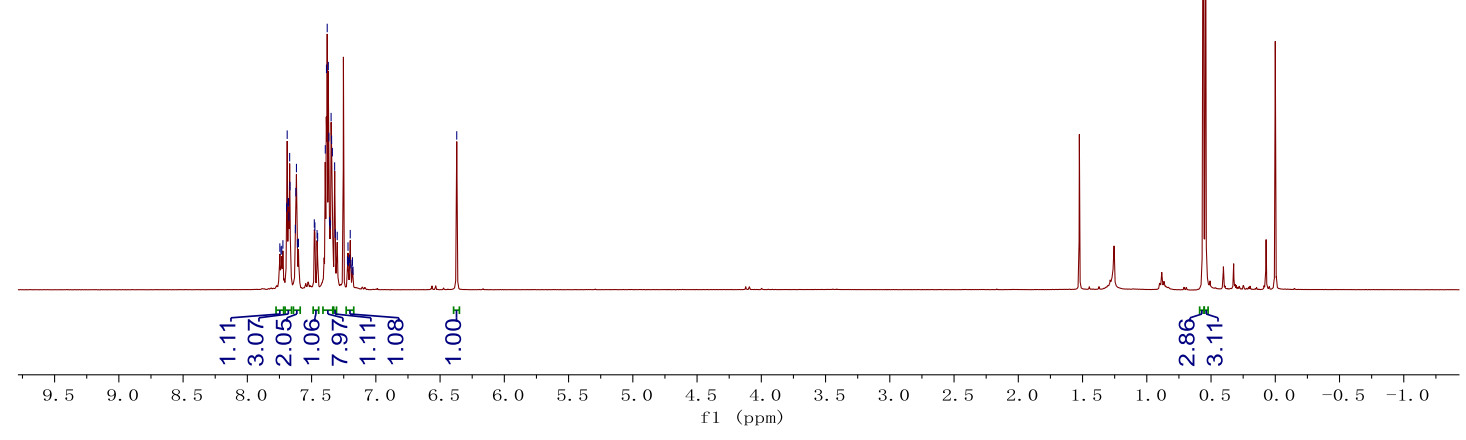

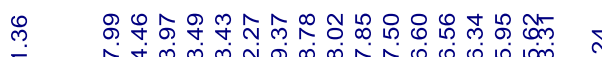

商

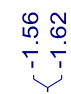




\section{HPLC Charts}

(R)-dimethyl(1-(naphthalen-2-yl)-3-phenylprop-2-yn-1-yl)(phenyl)silane (3aa)
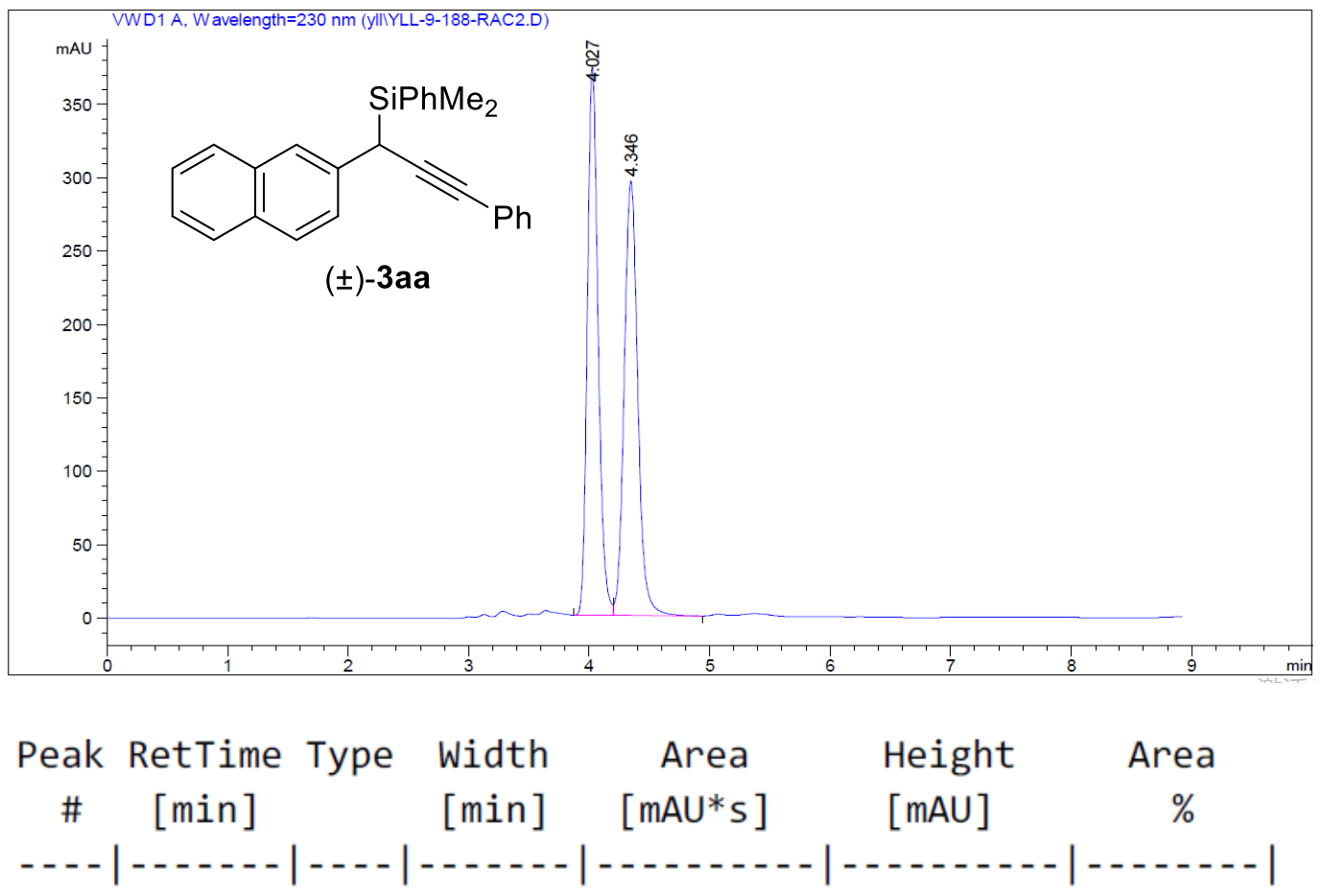
14.027 BV
$\begin{array}{llll}0.0951 & 2308.44067 & 373.57919 & 49.8844\end{array}$
$24.346 \mathrm{VB}$
$\begin{array}{llll}0.1224 & 2319.14404 & 296.15469 & 50.1156\end{array}$

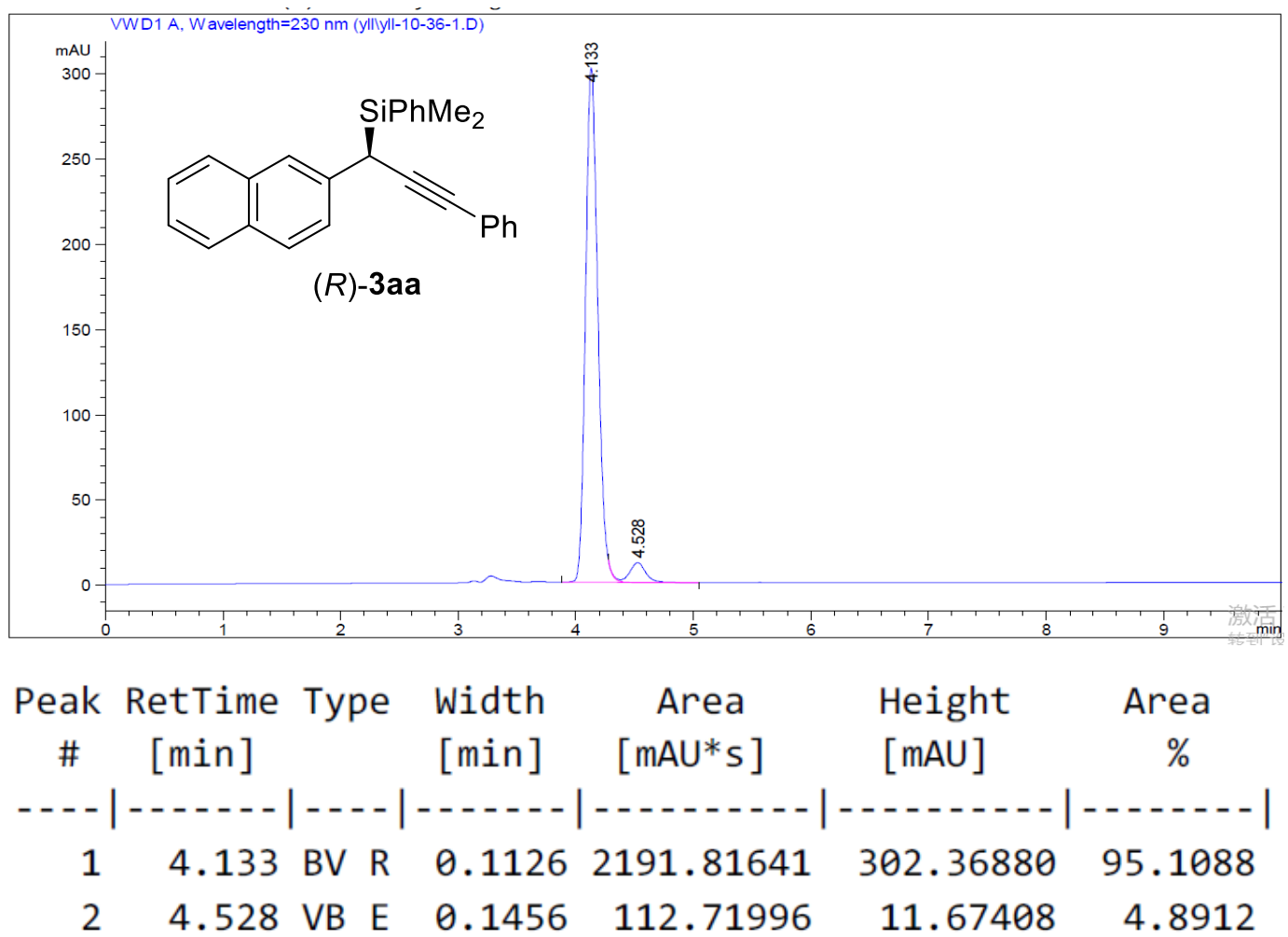


(-)-triethyl(1-(naphthalen-2-yl)-3-phenylprop-2-yn-1-yl)silane (3ab)
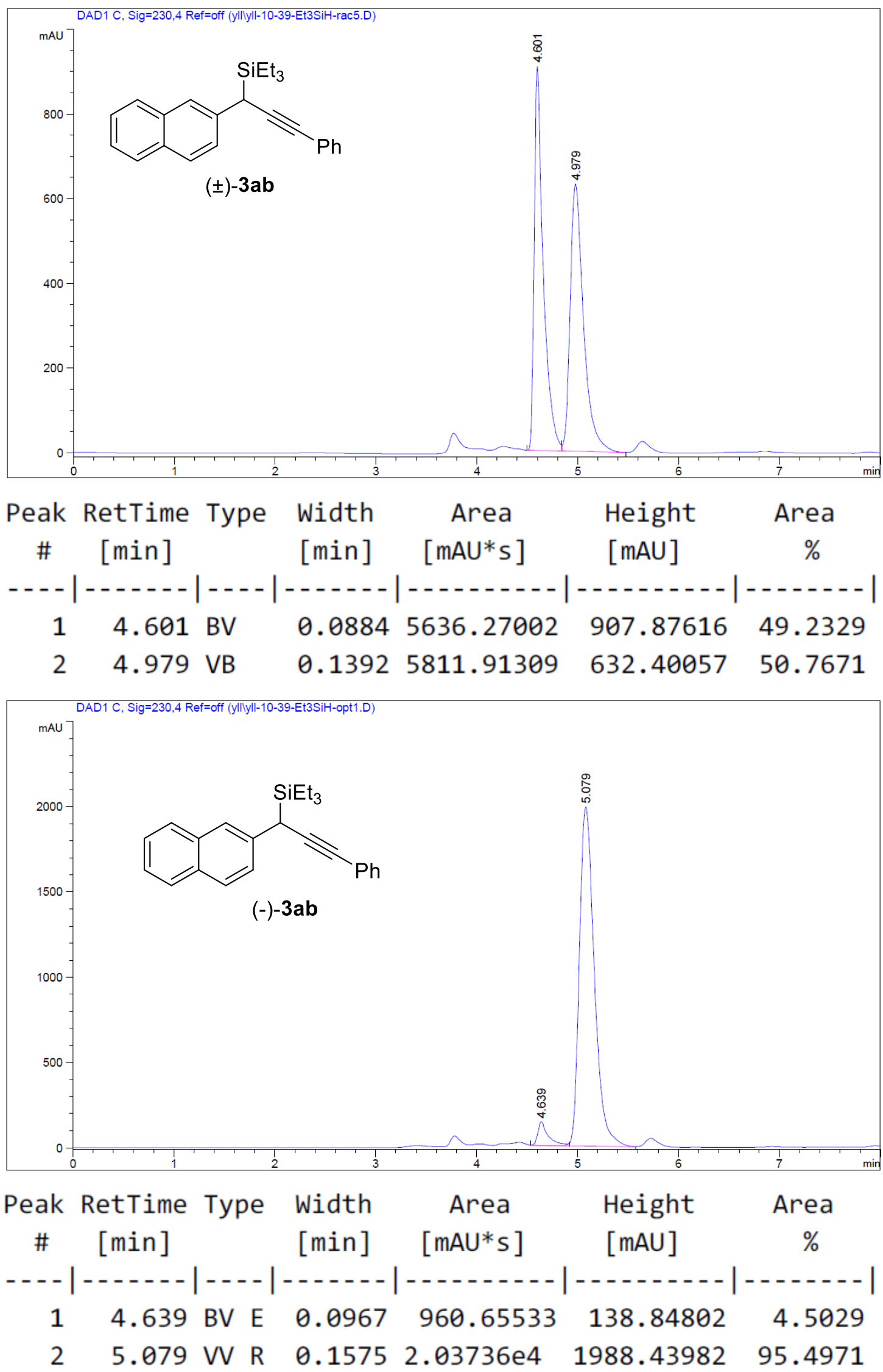
(-)-methyl(1-(naphthalen-2-yl)-3-phenylprop-2-yn-1-yl)diphenylsilane (3ac)

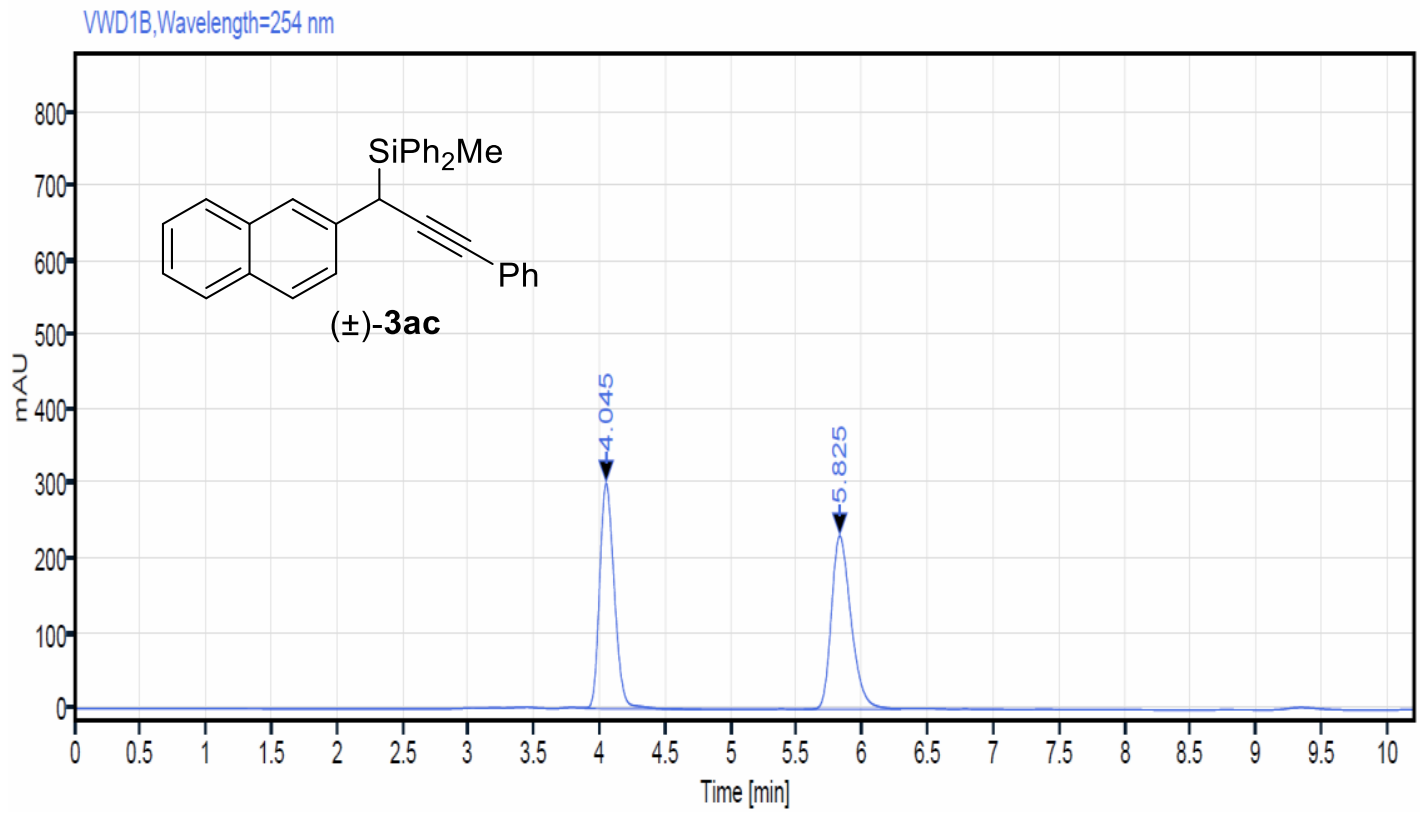

$\begin{array}{rrrrrr}\text { RT [min] } & \text { Type } & \text { Width [min] } & \text { Area } & \text { Height } & \text { Area\% } \\ 4.045 & \text { VB } & 0.89 & 2350.61 & 303.26 & 49.13 \\ 5.825 & \text { BV } & 0.74 & 2433.47 & 233.35 & 50.87\end{array}$

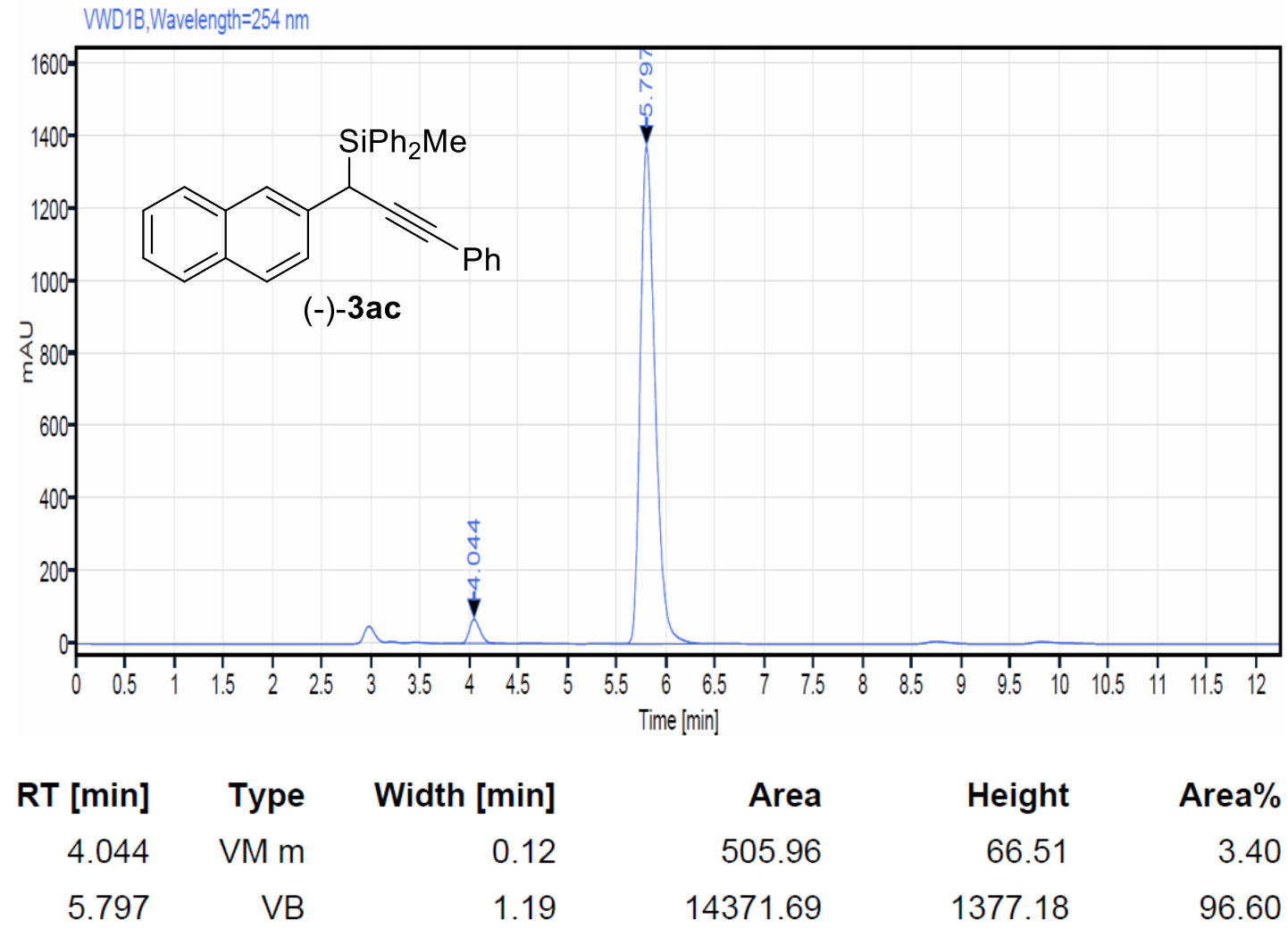


(-)-(1,3-diphenylprop-2-yn-1-yl)triethylsilane (3bb)
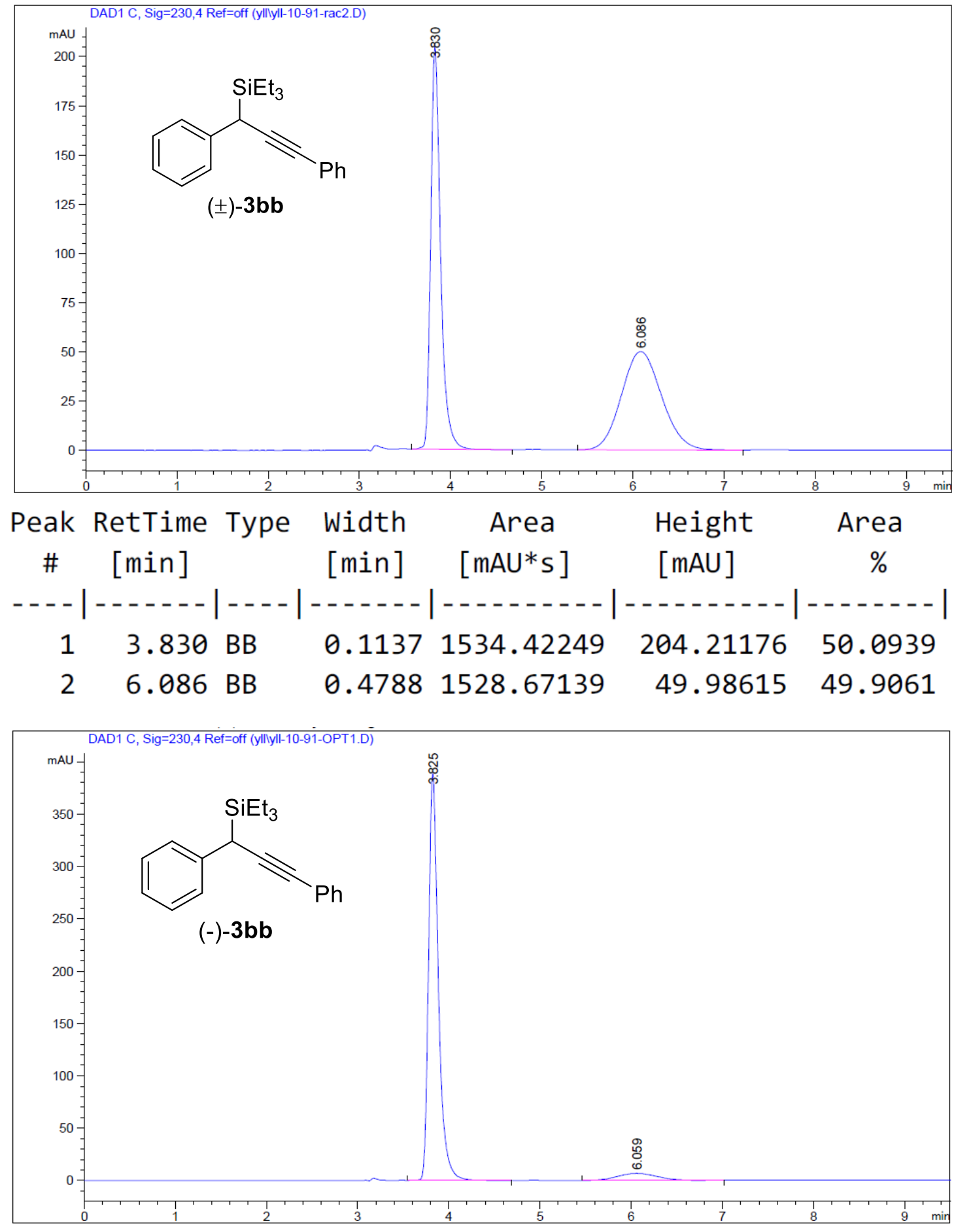

\begin{tabular}{|c|c|c|c|c|c|c|}
\hline $\begin{array}{c}\text { Peak } \\
\#\end{array}$ & $\begin{array}{c}\text { RetTime } \\
\text { [min] }\end{array}$ & Type & $\begin{array}{l}\text { Width } \\
\text { [min] }\end{array}$ & $\begin{array}{c}\text { Area } \\
{\left[\mathrm{mAU}^{*} \mathrm{~s}\right]}\end{array}$ & $\begin{array}{l}\text { Height } \\
{[\mathrm{mAU}]}\end{array}$ & $\begin{array}{c}\text { Area } \\
\%\end{array}$ \\
\hline & & & & 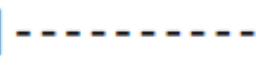 & & \\
\hline 1 & 3.825 & BB & .1129 & 2898.77881 & 389.10831 & 93.2921 \\
\hline 2 & 6.059 & BB & .4489 & 208.42972 & .81368 & 6.7079 \\
\hline
\end{tabular}


(-)-(1-(2-chlorophenyl)-3-phenylprop-2-yn-1-yl)triethylsilane (3cb)

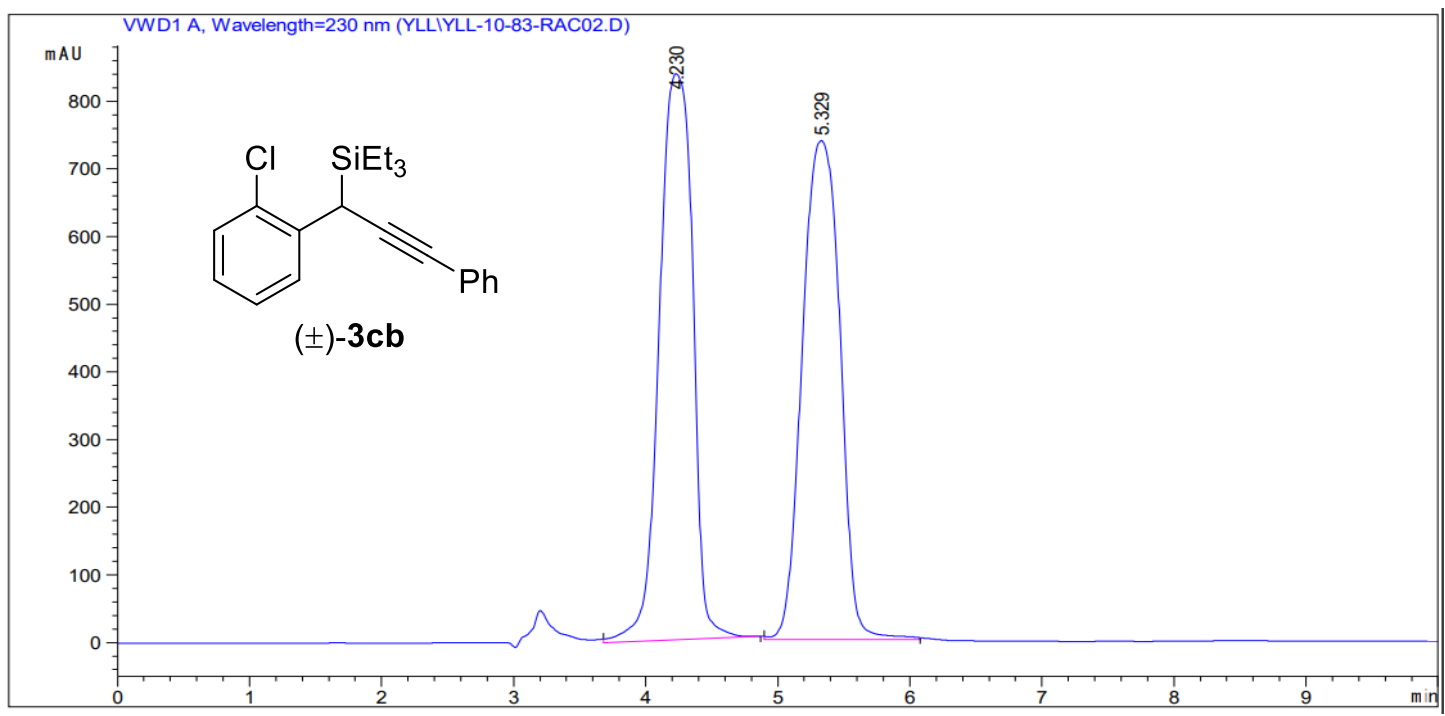

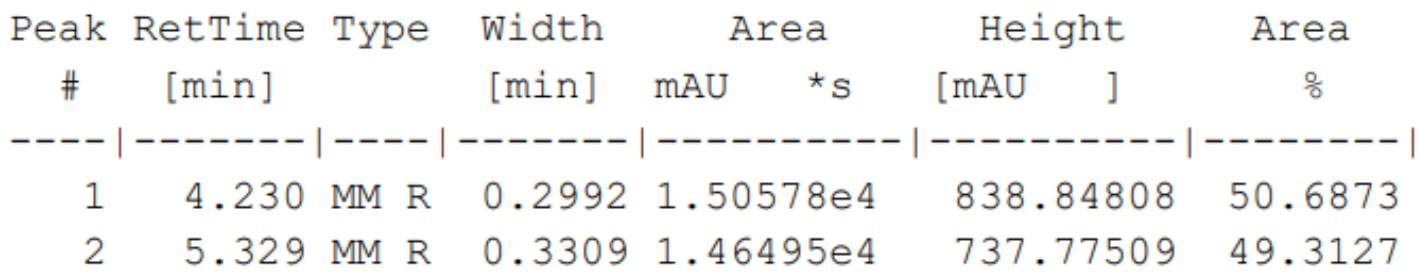

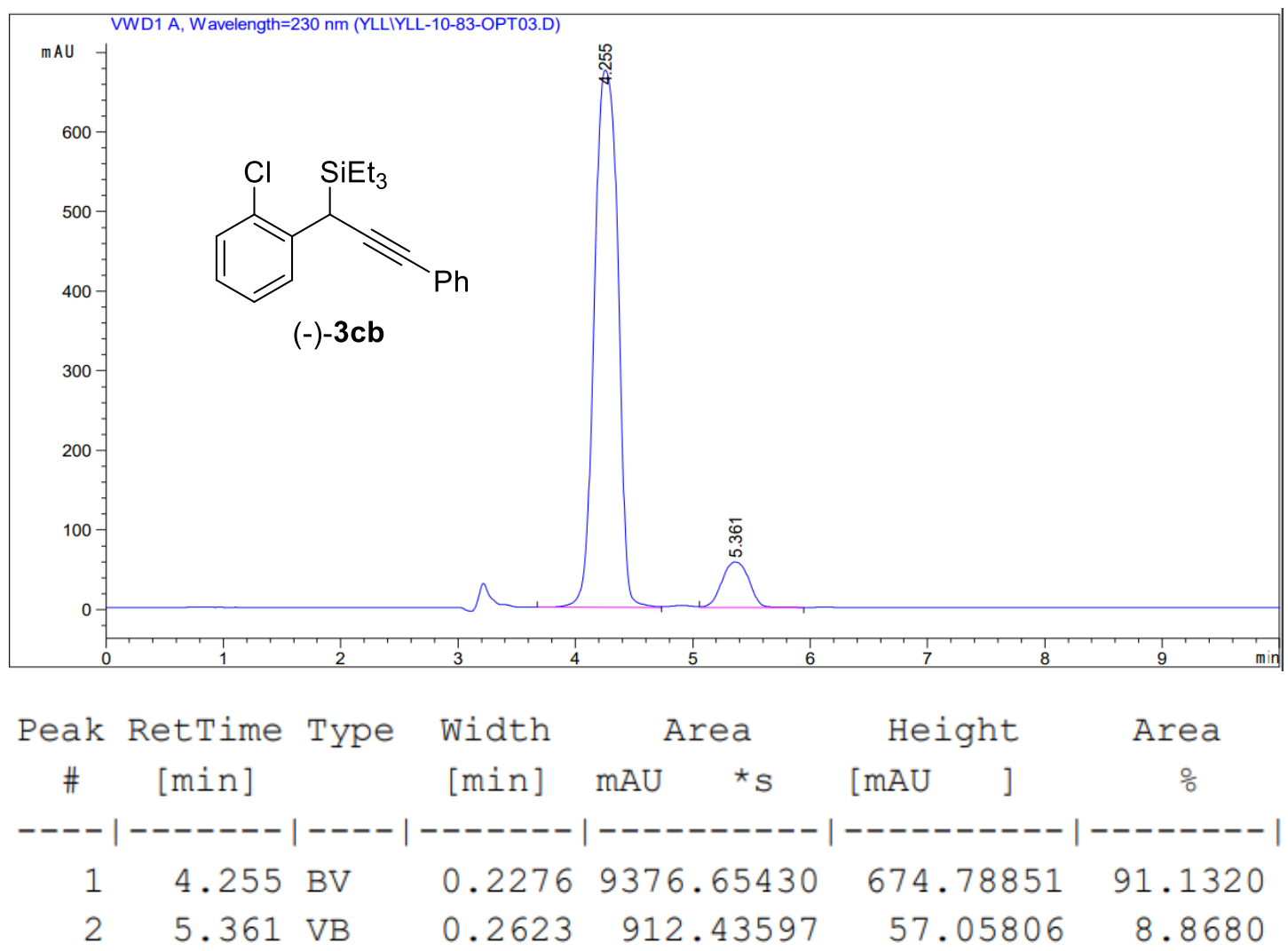


(-)-(1-(3-chlorophenyl)-3-phenylprop-2-yn-1-yl)triethylsilane (3db)

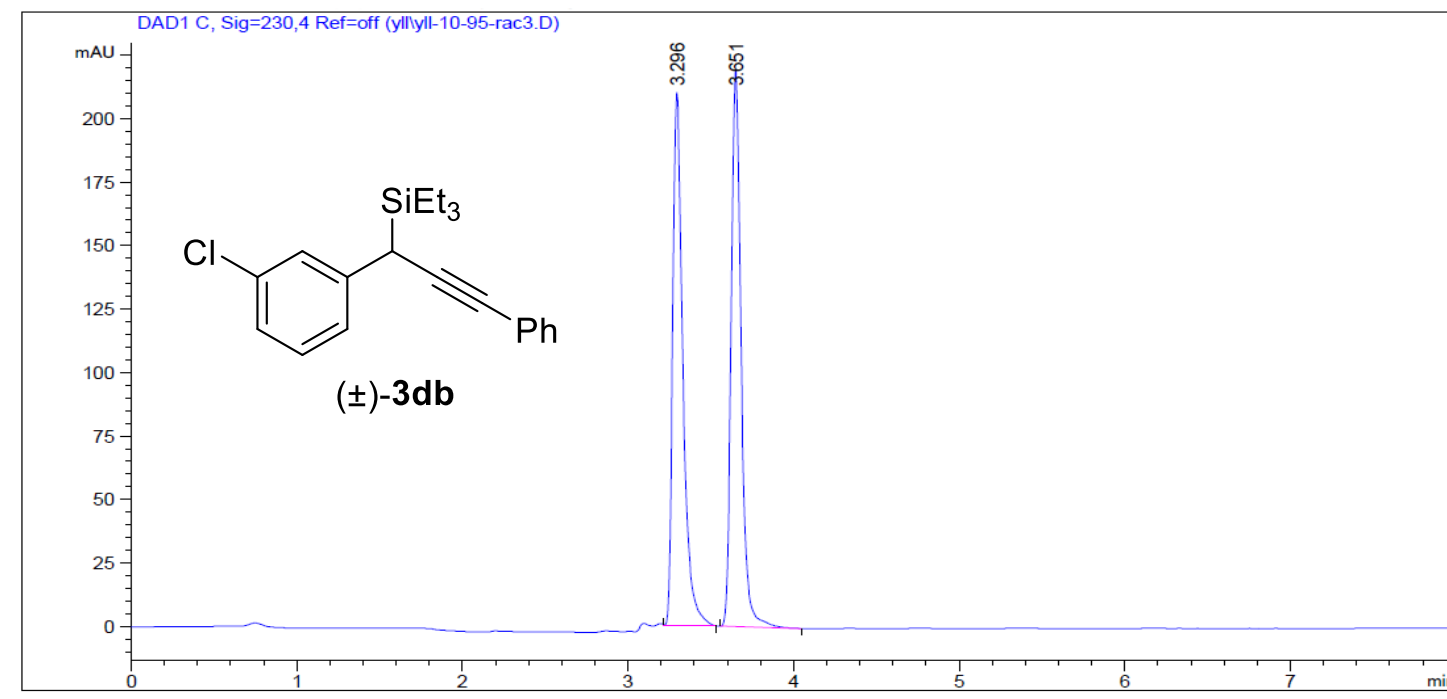

Peak RetTime Type Width Area Height Area

\# $[\mathrm{min}] \quad[\mathrm{min}] \quad[\mathrm{mAU} * \mathrm{~s}] \quad[\mathrm{mAU}] \quad \%$

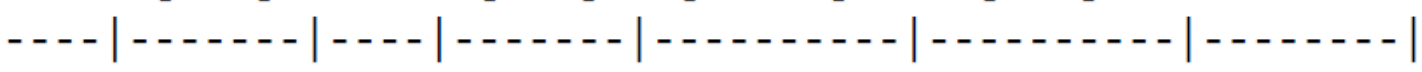

$\begin{array}{lllllll}1 & 3.296 & \text { MM R } & 0.0712 & 901.90845 & 211.09370 & 49.6656\end{array}$

$\begin{array}{lllllll}2 & 3.651 & \text { BB } & 0.0644 & 914.05469 & 220.26921 & 50.3344\end{array}$

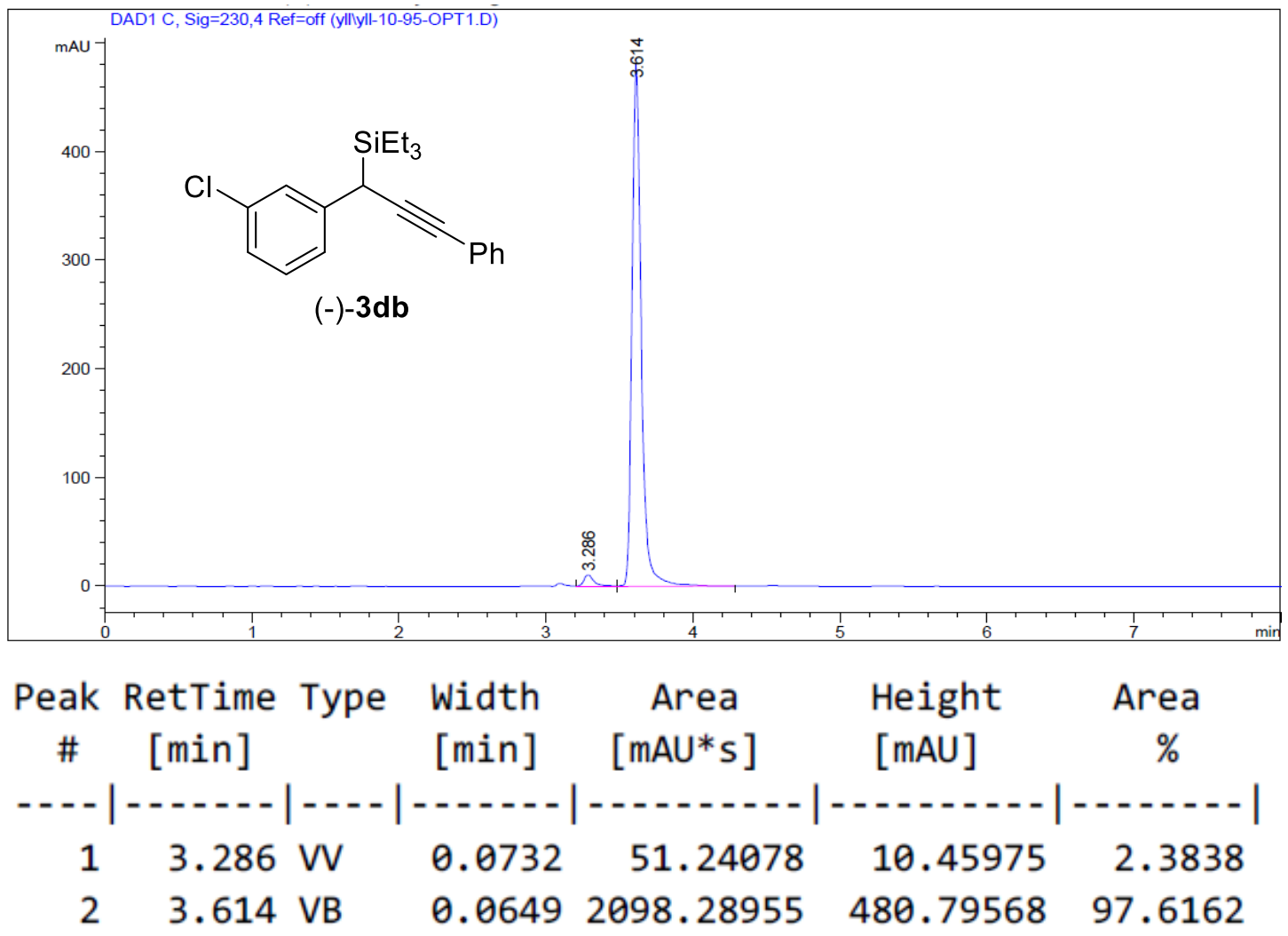


(-)-triethyl(1-(4-methoxyphenyl)-3-phenylprop-2-yn-1-yl)silane (3eb)

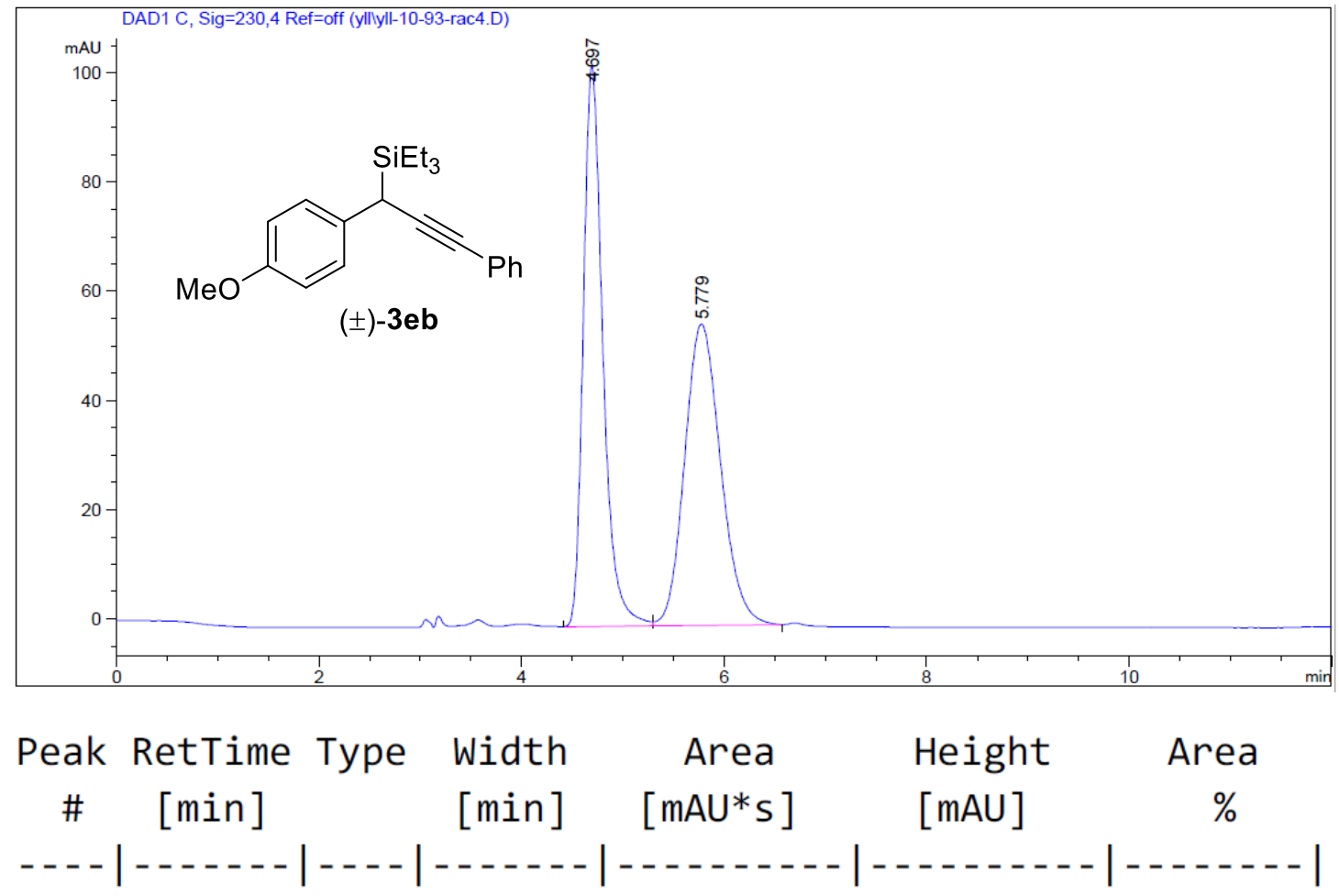

$\begin{array}{lllllll}1 & 4.697 & \text { BV } & 0.2064 & 1375.45471 & 102.61873 & 50.2174\end{array}$

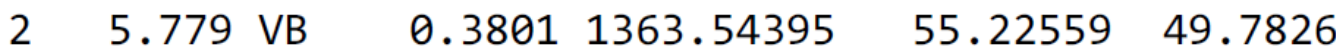

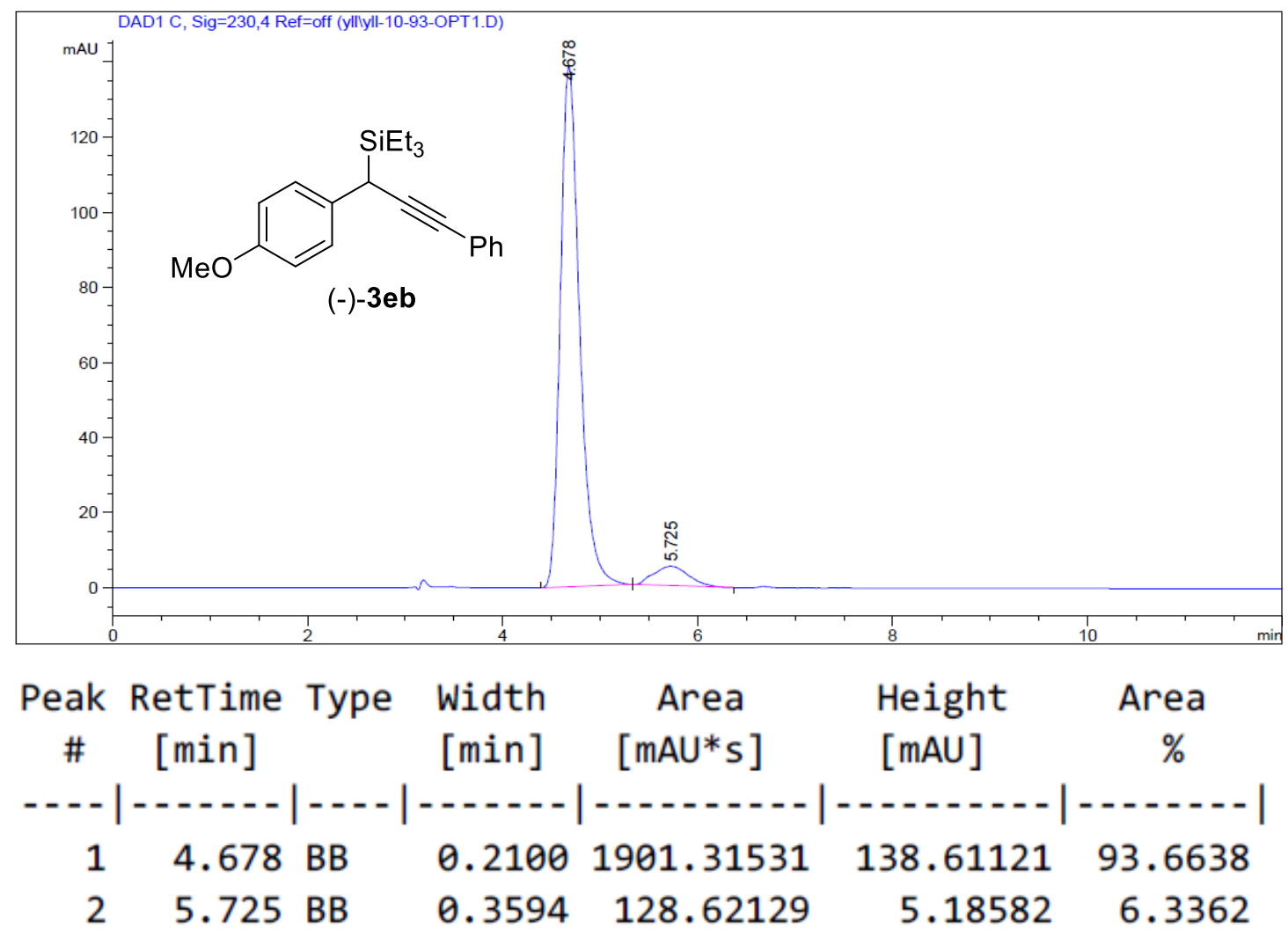


(-)-(1-(4-chlorophenyl)-3-phenyl-prop-2-yn-1-yl)triethylsilane (3fb)
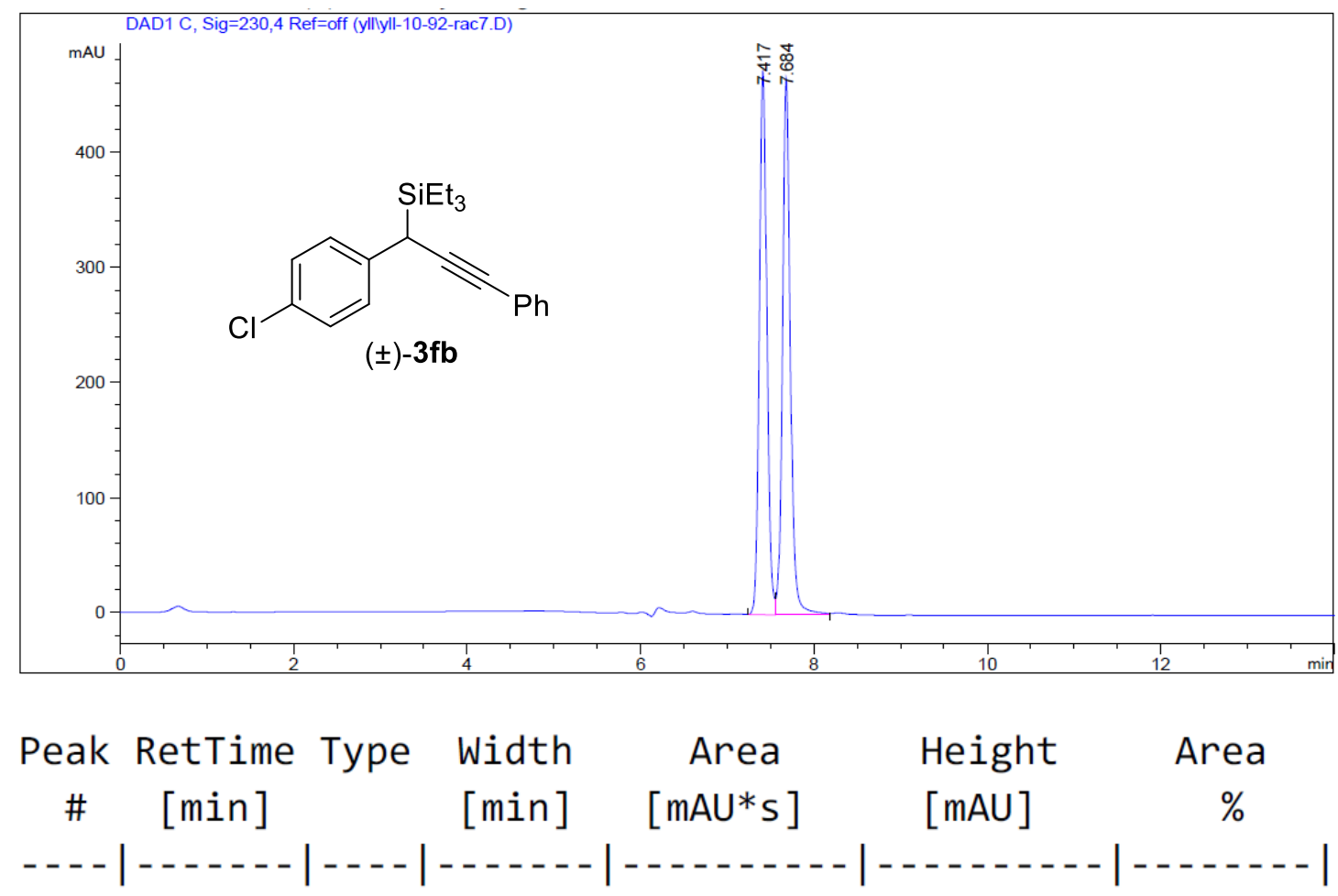

$\begin{array}{lllllll}1 & 7.417 & \text { BV } & 0.0928 & 2871.13330 & 473.10138 & 49.2116\end{array}$

$\begin{array}{lllllll}2 & 7.684 & \text { MM R } & 0.1056 & 2963.12354 & 467.57898 & 50.7884\end{array}$

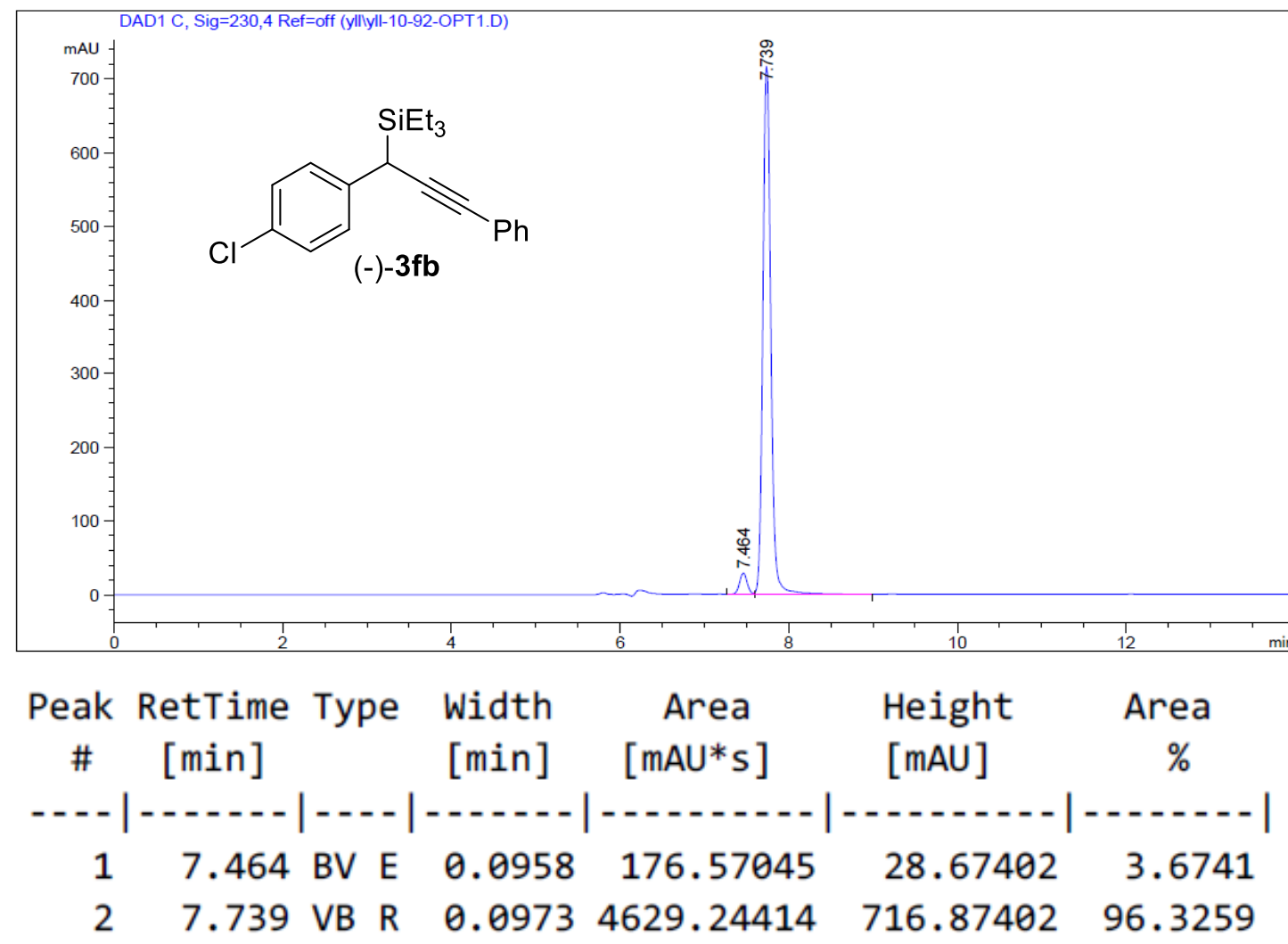


(-)-4-(3-phenyl-1-(triethylsilyl)prop-2-yn-1-yl)benzonitrile (3gb)

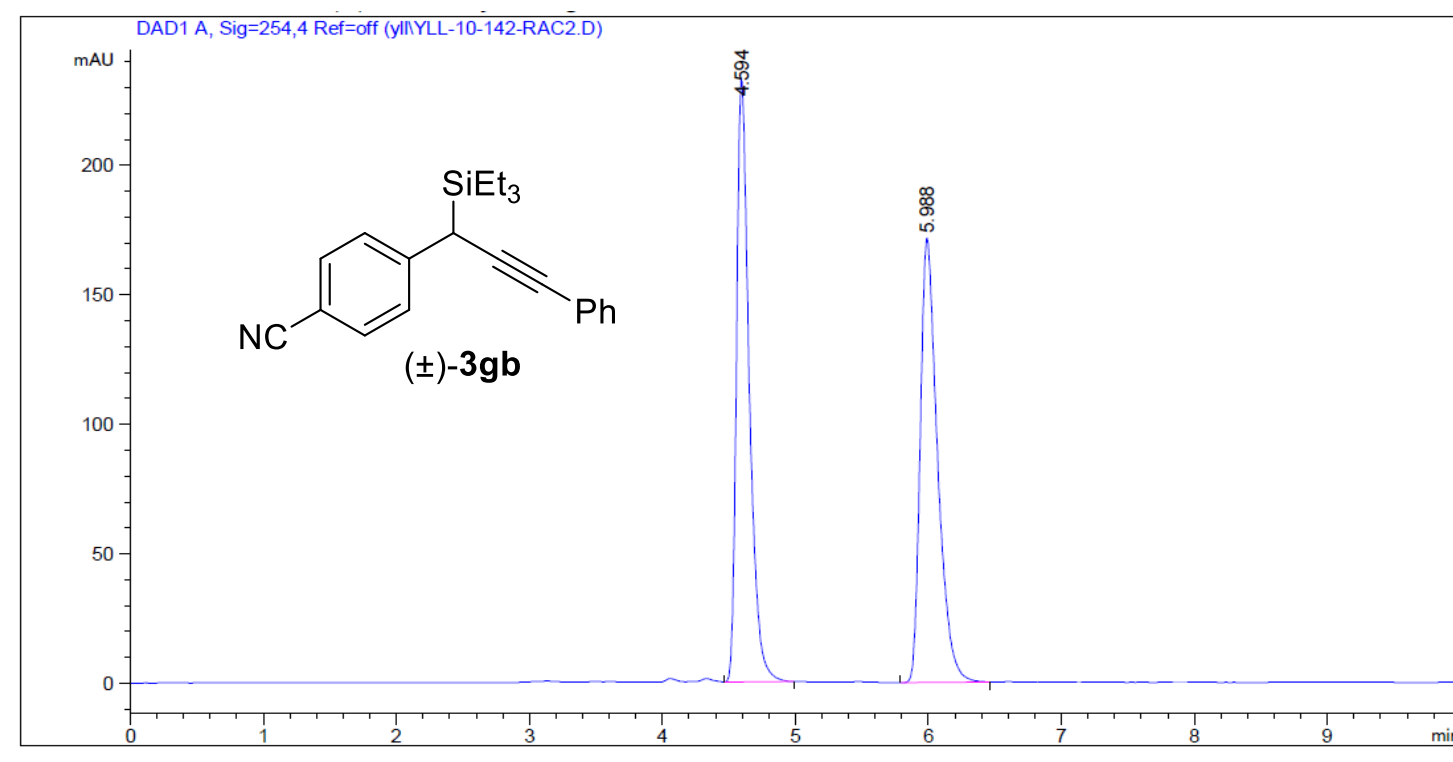

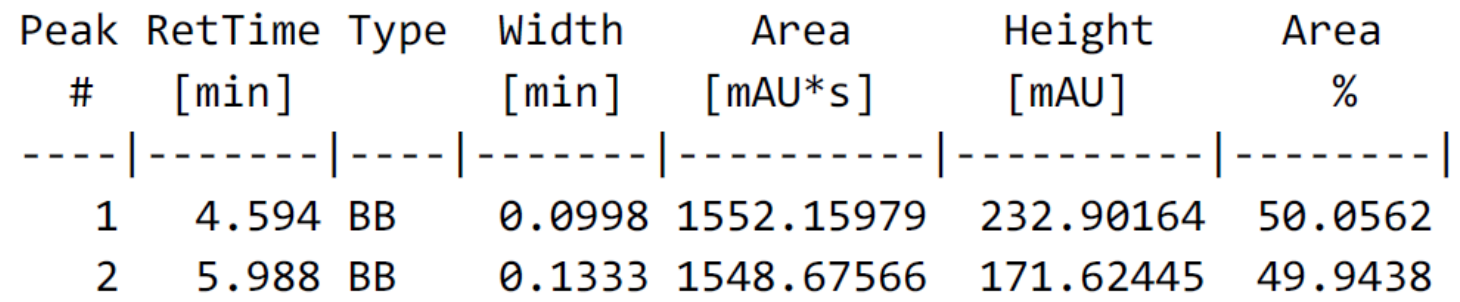

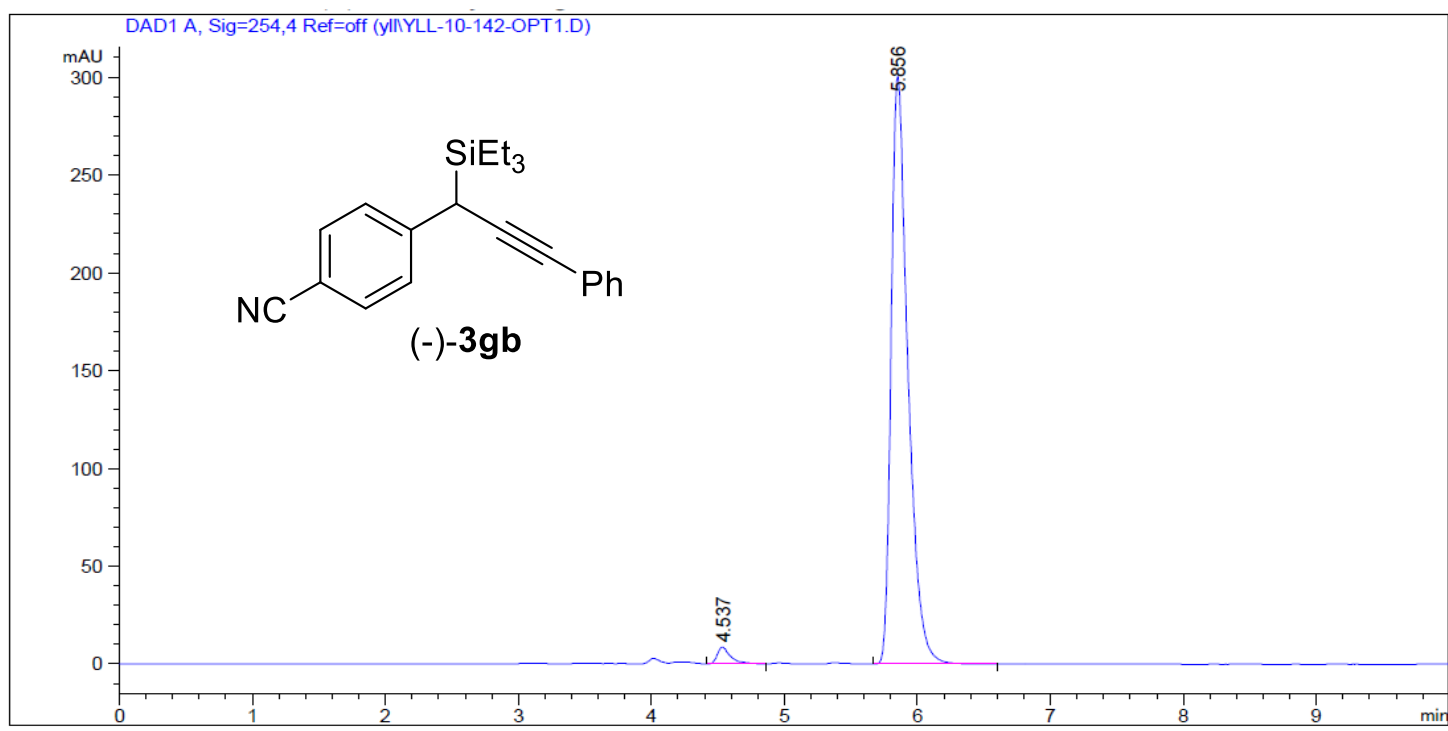

\begin{tabular}{cccccc}
$\begin{array}{c}\text { Peak RetTime Type } \\
\#\end{array}$ & $\begin{array}{c}\text { Width } \\
{[\text { min] }}\end{array}$ & $\begin{array}{c}\text { Area } \\
\text { [min] }\end{array}$ & $\begin{array}{c}\text { Height } \\
{[\text { mAU*s }]}\end{array}$ & $\begin{array}{c}\text { Area } \\
{[\text { mAU] }}\end{array}$ & $\%$ \\
\hline 1 & 4.537 BB & 0.1007 & 59.19971 & 8.55363 & 2.2006 \\
2 & 5.856 BB & 0.1301 & 2630.97290 & 300.76077 & 97.7994
\end{tabular}


(-)-4-(3-(naphthalen-2-yl)-3-(triethylsilyl)prop-1-yn-1-yl)benzonitrile (3hb)

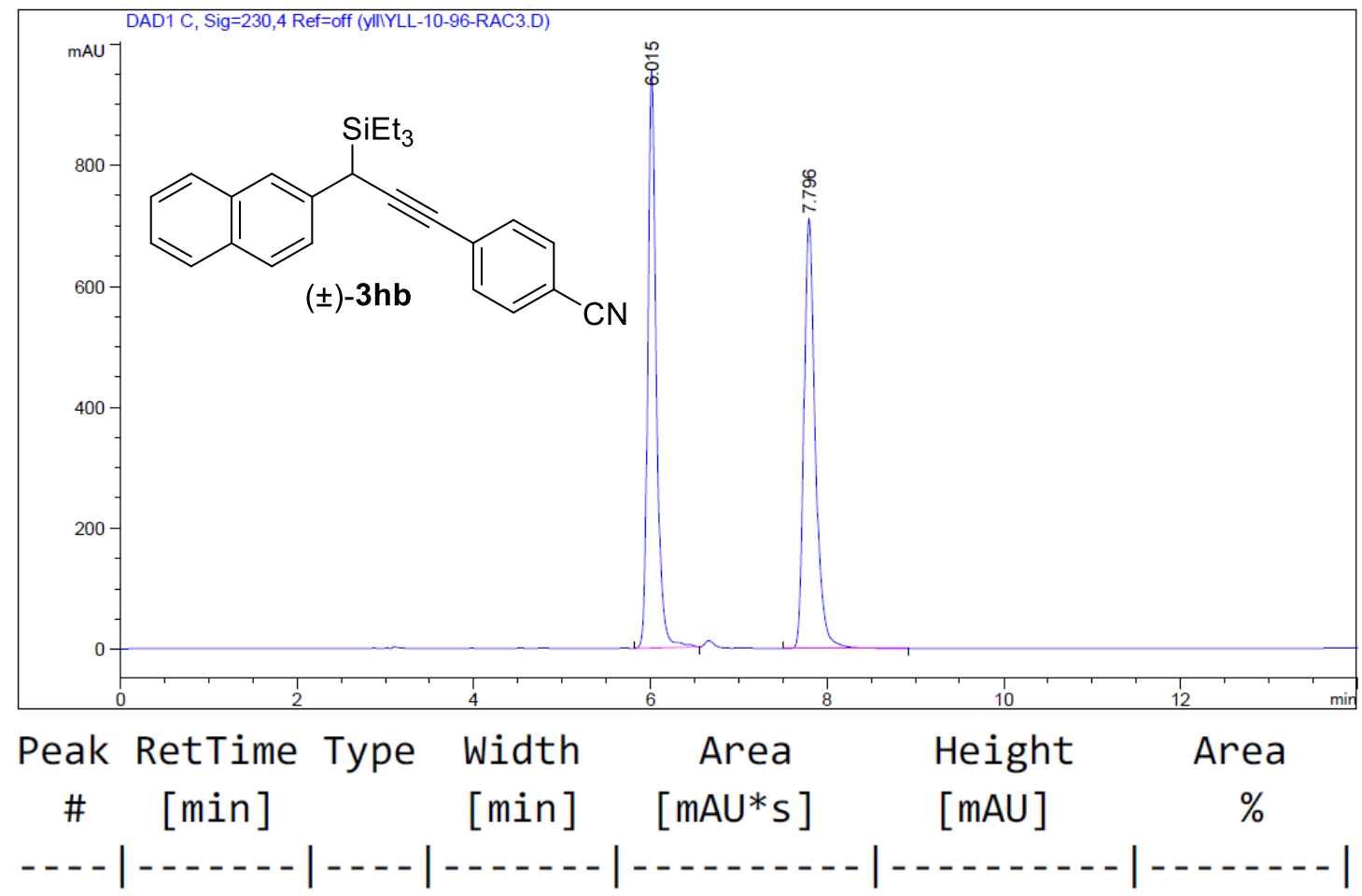

$\begin{array}{lllllll}1 & 6.015 & \text { BV R } & 0.1000 & 6424.51514 & 954.61127 & 50.1702\end{array}$

$\begin{array}{lllllll}2 & 7.796 & \text { BB } & 0.1349 & 6380.93506 & 709.86993 & 49.8298\end{array}$

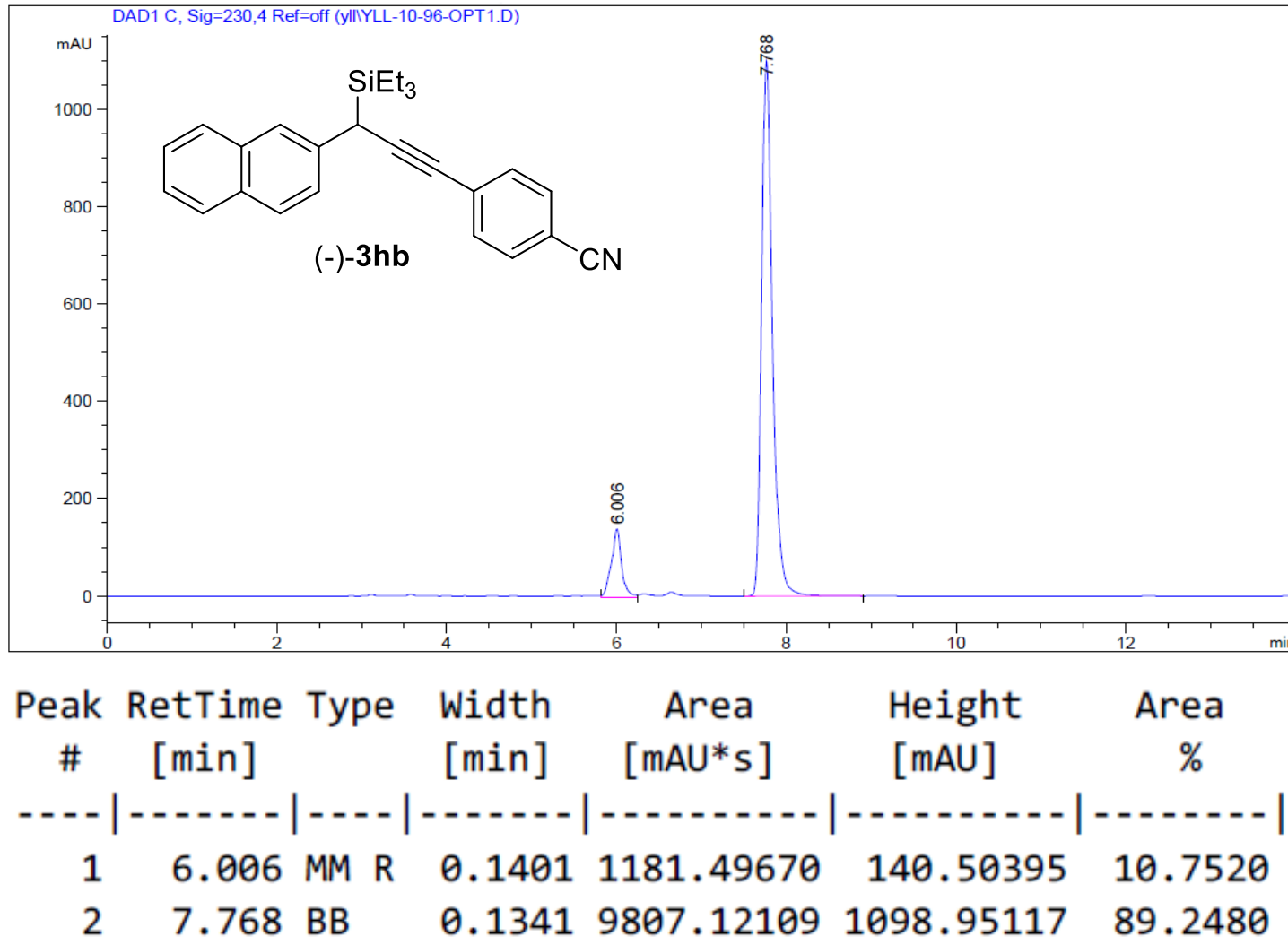


(-)-triethyl(1-(naphthalen-2-yl)-3-(thiophen-2-yl)prop-2-yn-1-yl)silane (3ib)
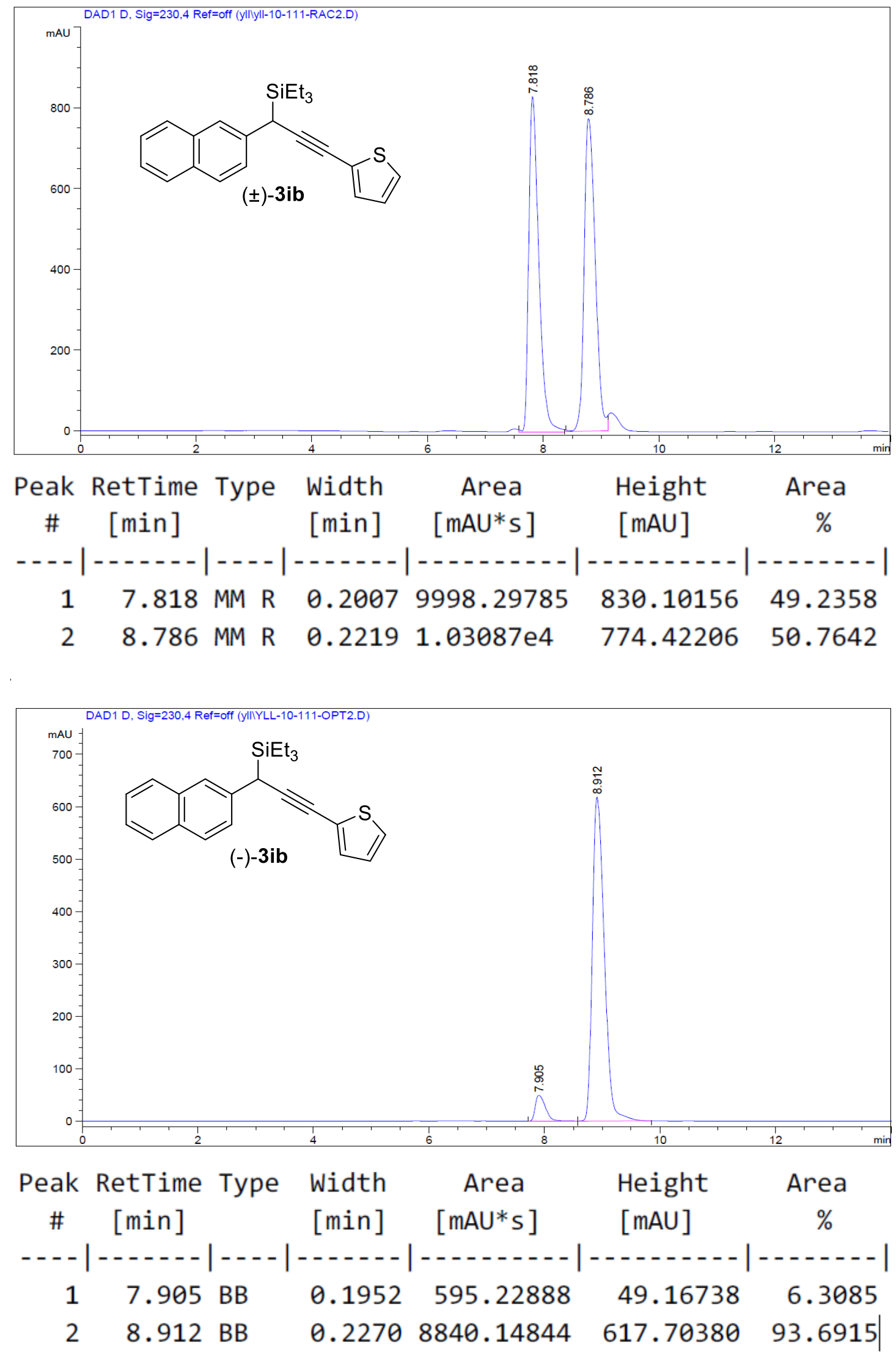
(-)-triethyl(1-(naphthalen-2-yl)hept-2-yn-1-yl)silane (3jb)

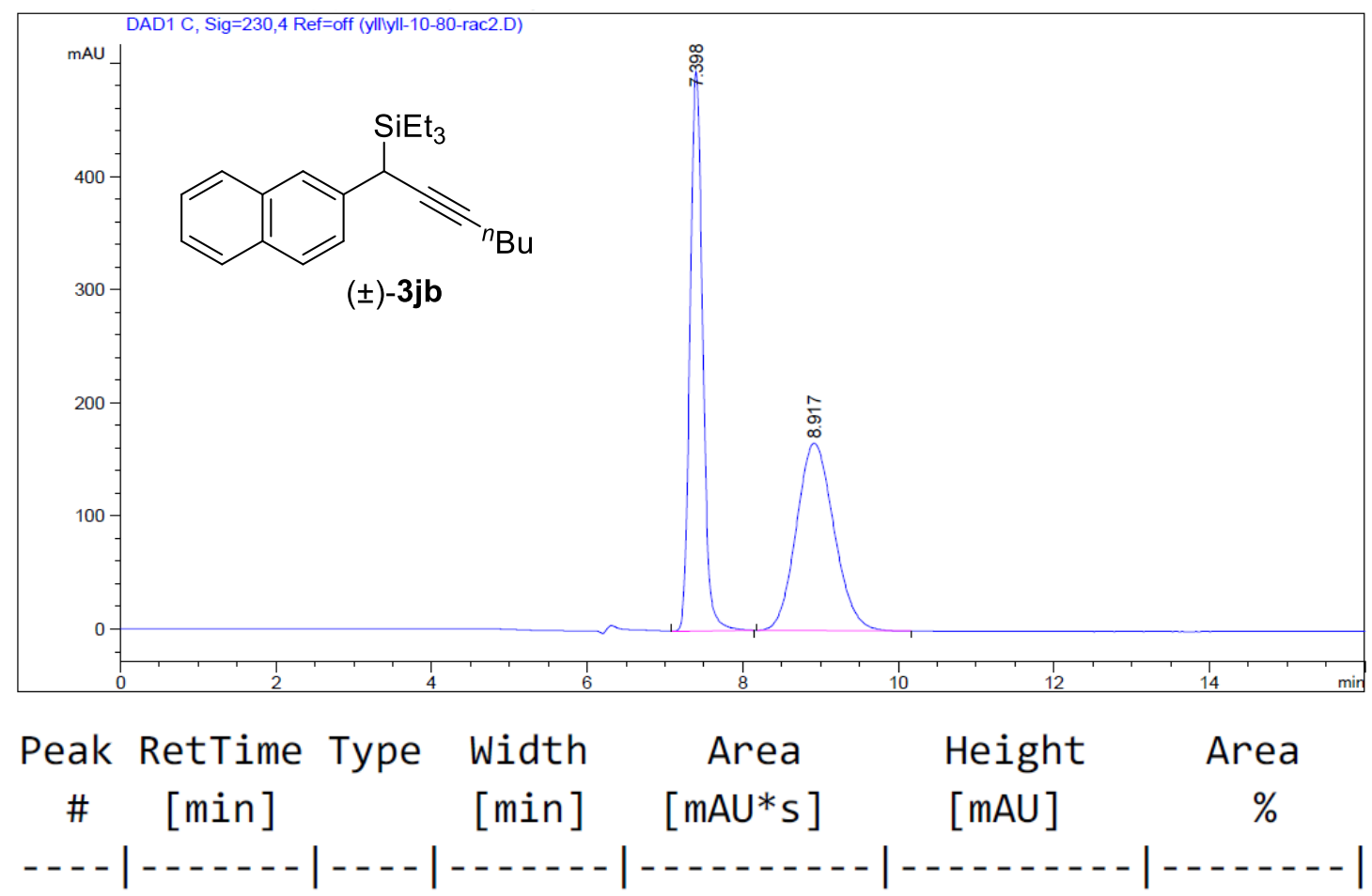

$\begin{array}{lllllll}1 & 7.398 & \text { MM R } & 0.1892 & 5613.01318 & 494.41409 & 50.2155\end{array}$

$\begin{array}{lllllll}2 & 8.917 & \text { BB } & 0.5198 & 5564.84424 & 165.71773 & 49.7845\end{array}$
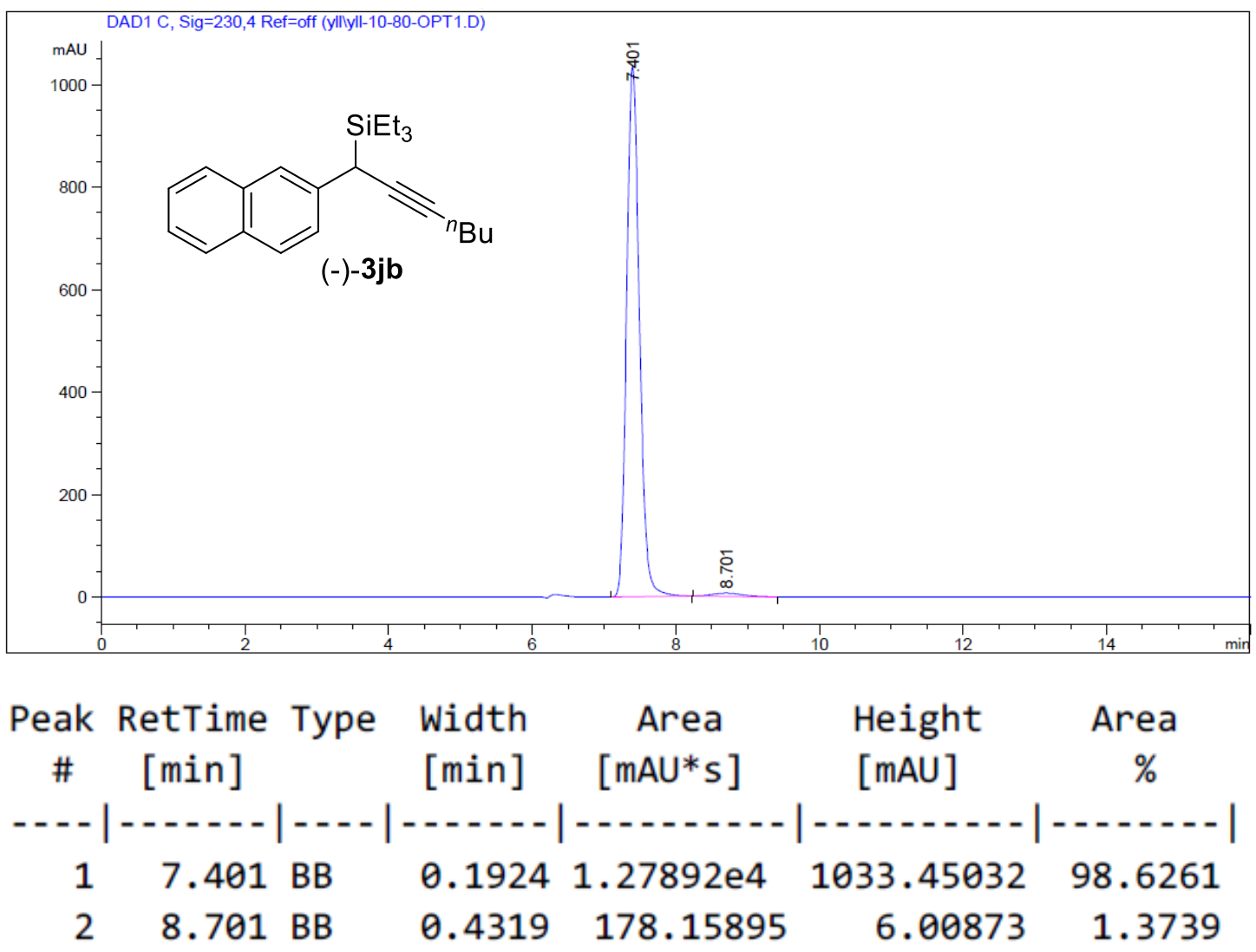
(-)-methyl(1-(naphthalen-2-yl)hept-2-yn-1-yl)diphenylsilane (3jc)

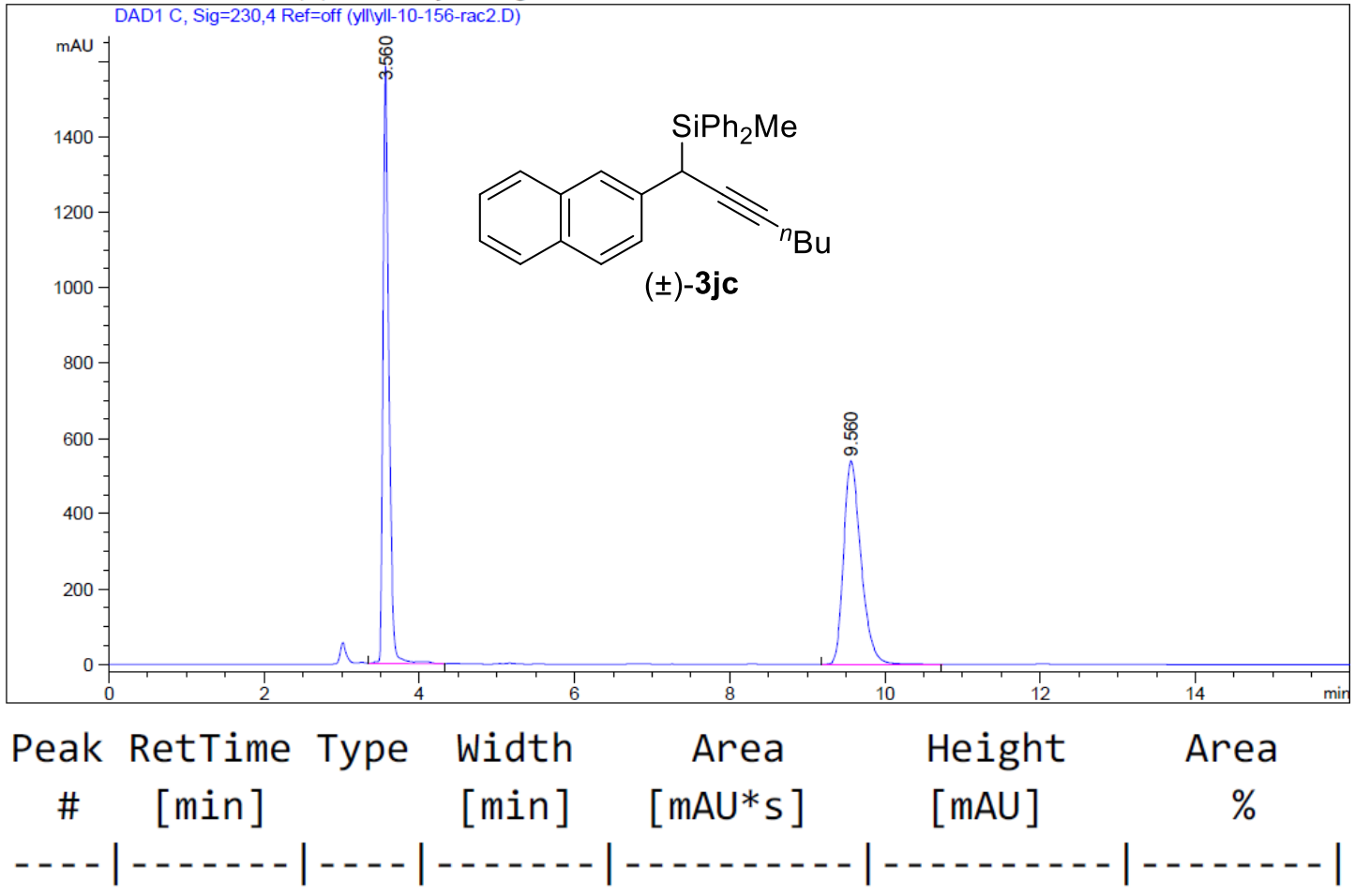

$\begin{array}{lllllll}1 & 3.560 \text { VV R } & 0.0788 & 8360.20996 & 1591.39587 & 49.8264\end{array}$

$\begin{array}{lllllll}2 & 9.560 & \text { BB } & 0.2377 & 8418.45508 & 540.43933 & 50.1736\end{array}$

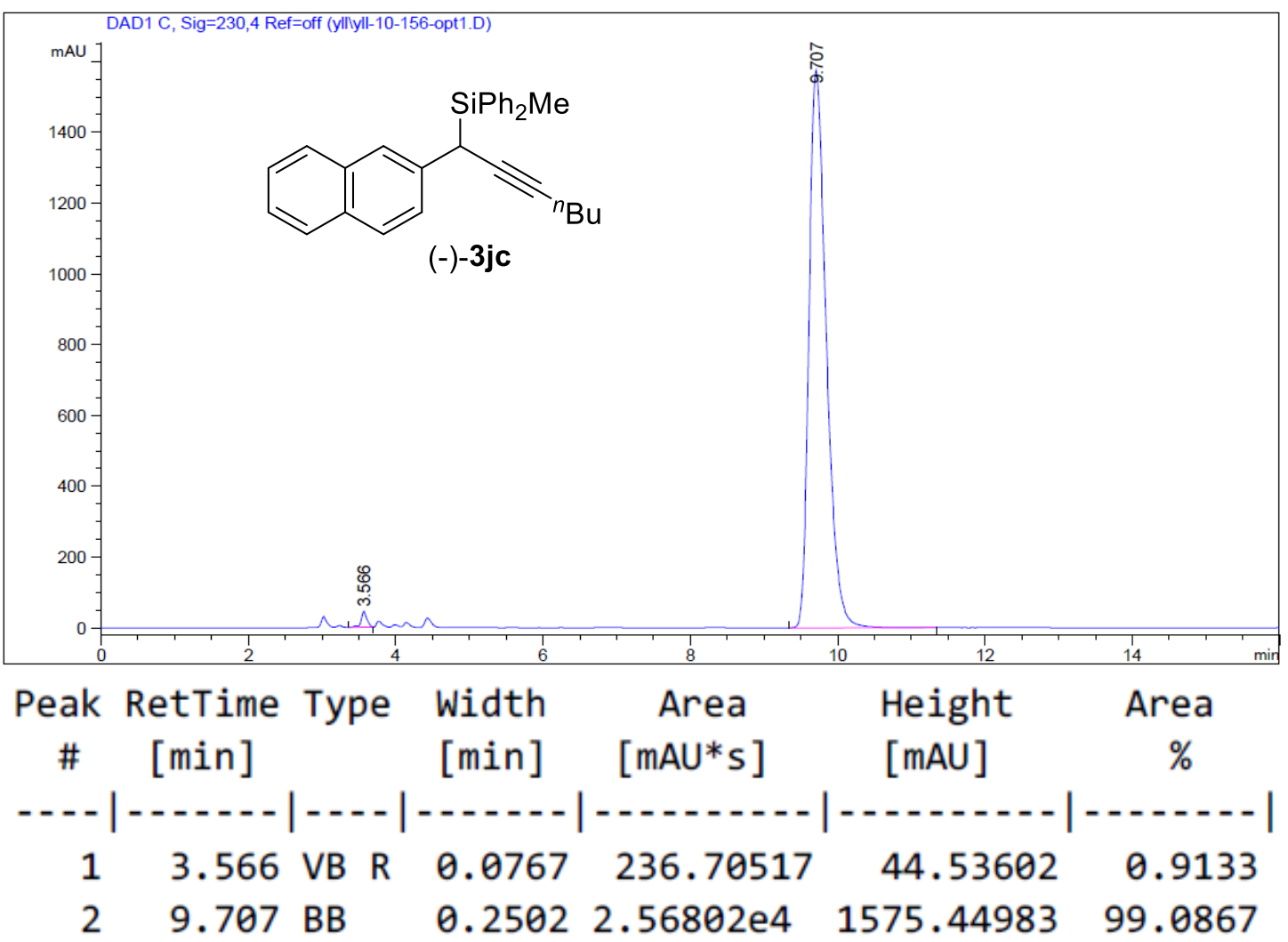


(-)-triethyl(1-(naphthalen-2-yl)but-2-yn-1-yl)silane (3kb)

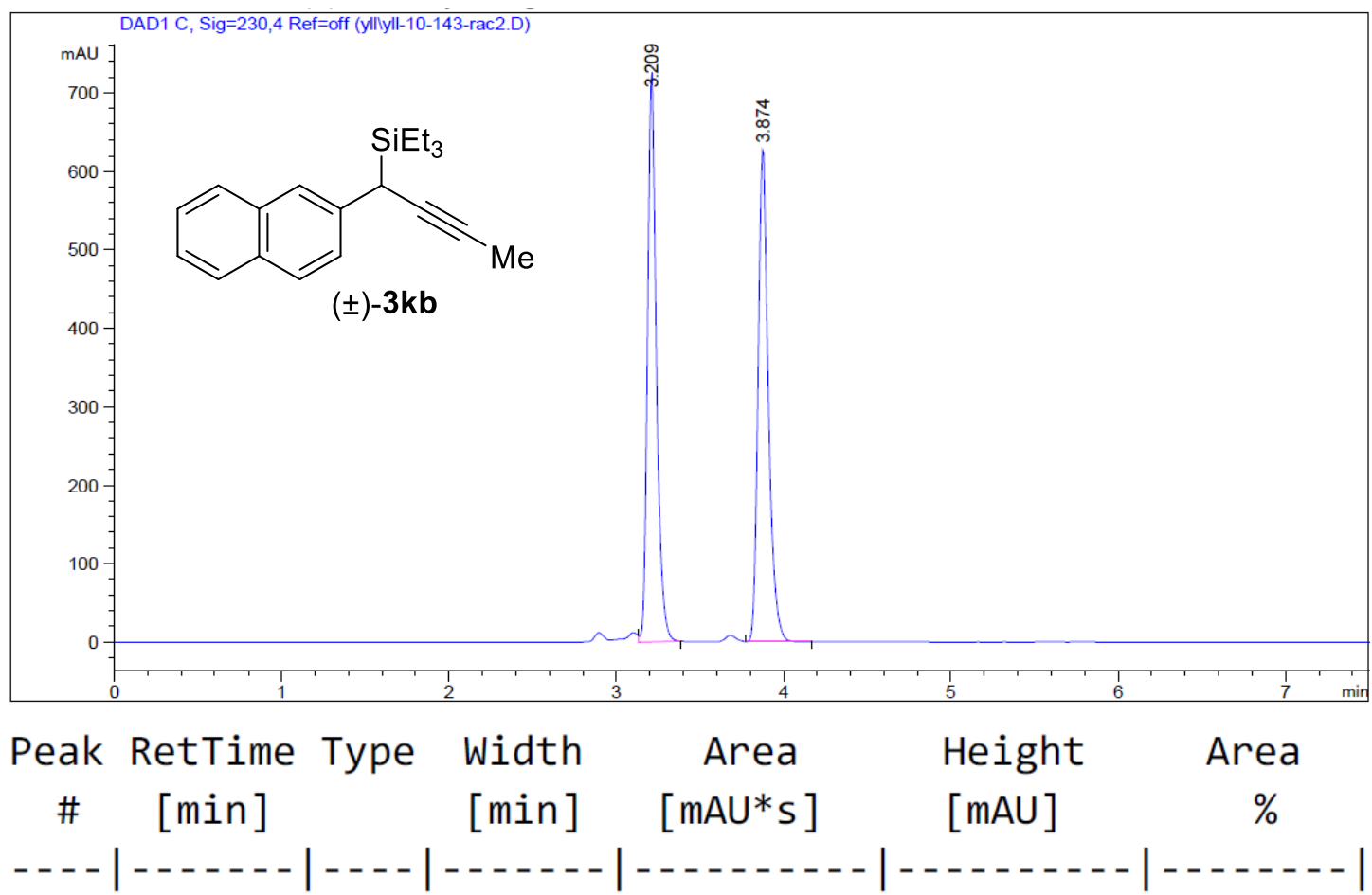

$\begin{array}{lllllll}1 & 3.209 & \text { MM R } & 0.0624 & 2736.43530 & 731.09265 & 50.0483\end{array}$

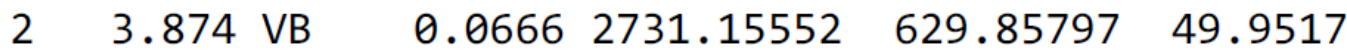

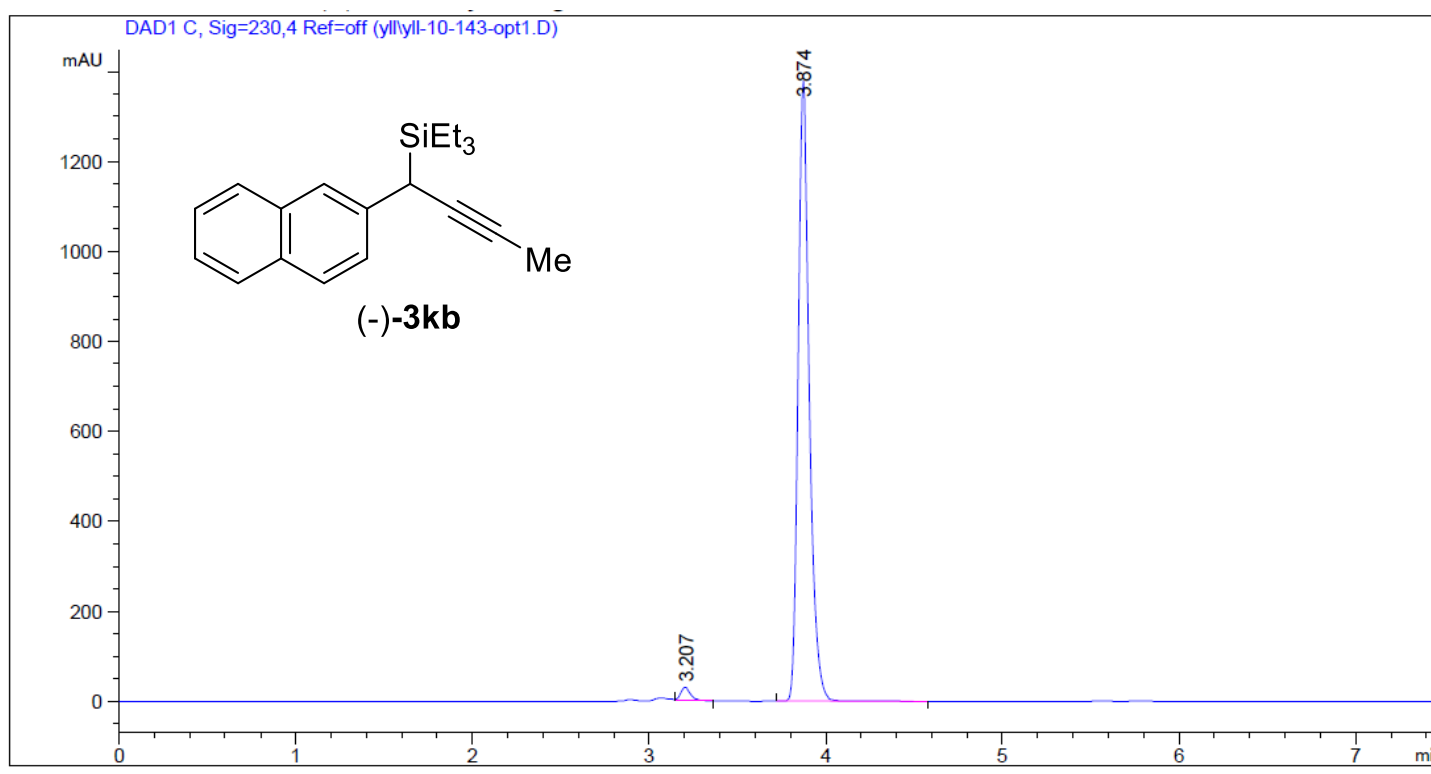

\begin{tabular}{|c|c|c|c|c|c|c|}
\hline $\begin{array}{c}\text { Peak } \\
\#\end{array}$ & $\begin{array}{c}\text { RetTime } \\
\text { [min] }\end{array}$ & Type & $\begin{array}{l}\text { Width } \\
\text { [min] }\end{array}$ & $\begin{array}{c}\text { Area } \\
{\left[\mathrm{mAU}^{*} \mathrm{~s}\right]}\end{array}$ & $\begin{array}{l}\text { Height } \\
{[\mathrm{mAU}]}\end{array}$ & $\begin{array}{c}\text { Area } \\
\%\end{array}$ \\
\hline & & & & -- & 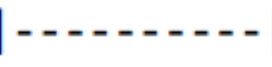 & \\
\hline 1 & 3.207 & VB & 515 & 124.53411 & 30.56877 & 1.9871 \\
\hline 2 & 3.874 & BV $R$ & .0679 & 6142.43848 & 1379.59985 & 98.0129 \\
\hline
\end{tabular}


(R)-methyl(1-(naphthalen-2-yl)but-2-yn-1-yl)diphenylsilane (3kc)

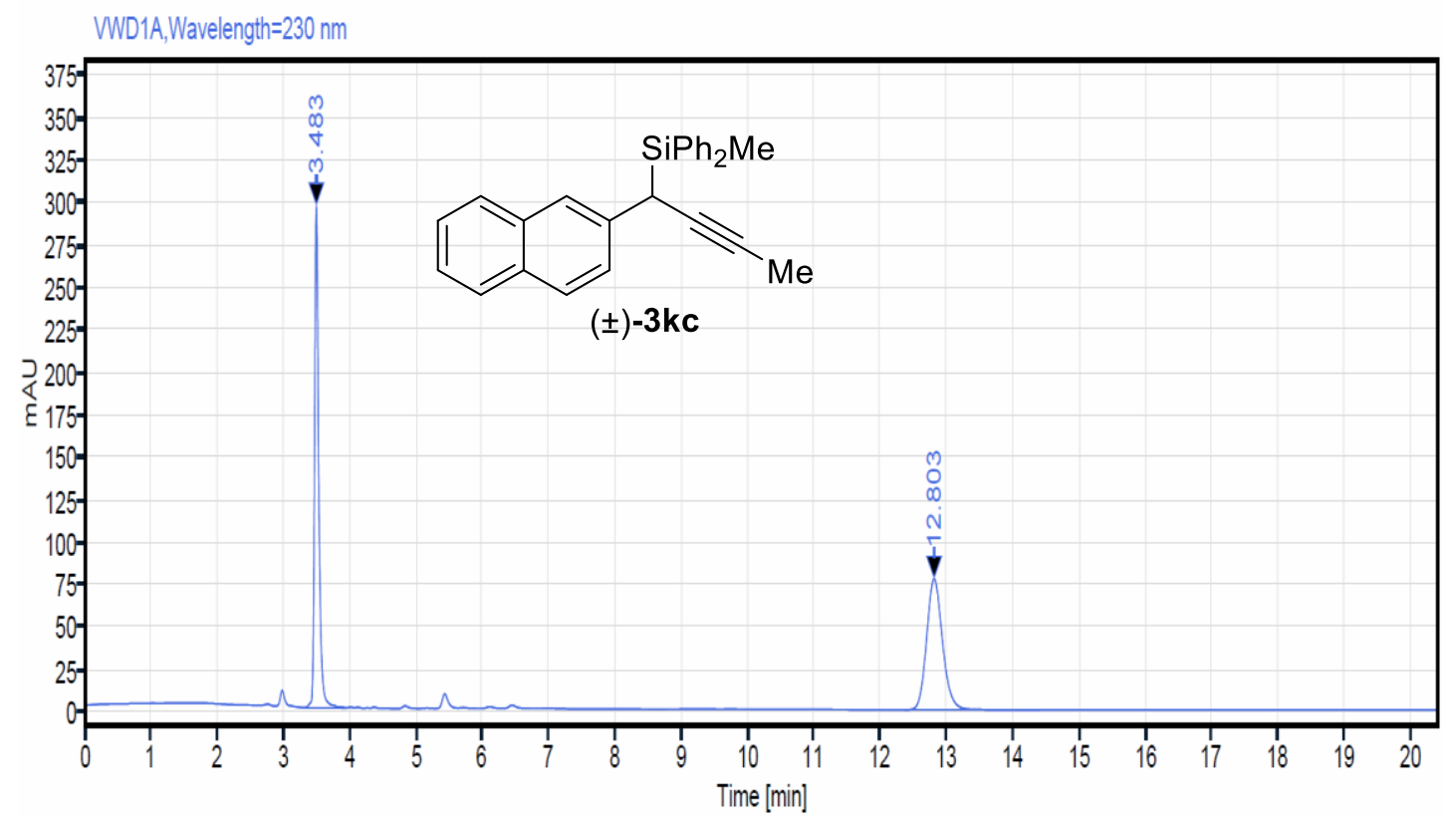

$\begin{array}{rrrrrr}\text { RT [min] } & \text { Type } & \text { Width [min] } & \text { Area } & \text { Height } & \text { Area\% } \\ 3.483 & \text { BV } & 0.63 & 1294.55 & 296.05 & 50.23 \\ 12.803 & \text { BB } & 2.06 & 1282.60 & 77.15 & 49.77\end{array}$

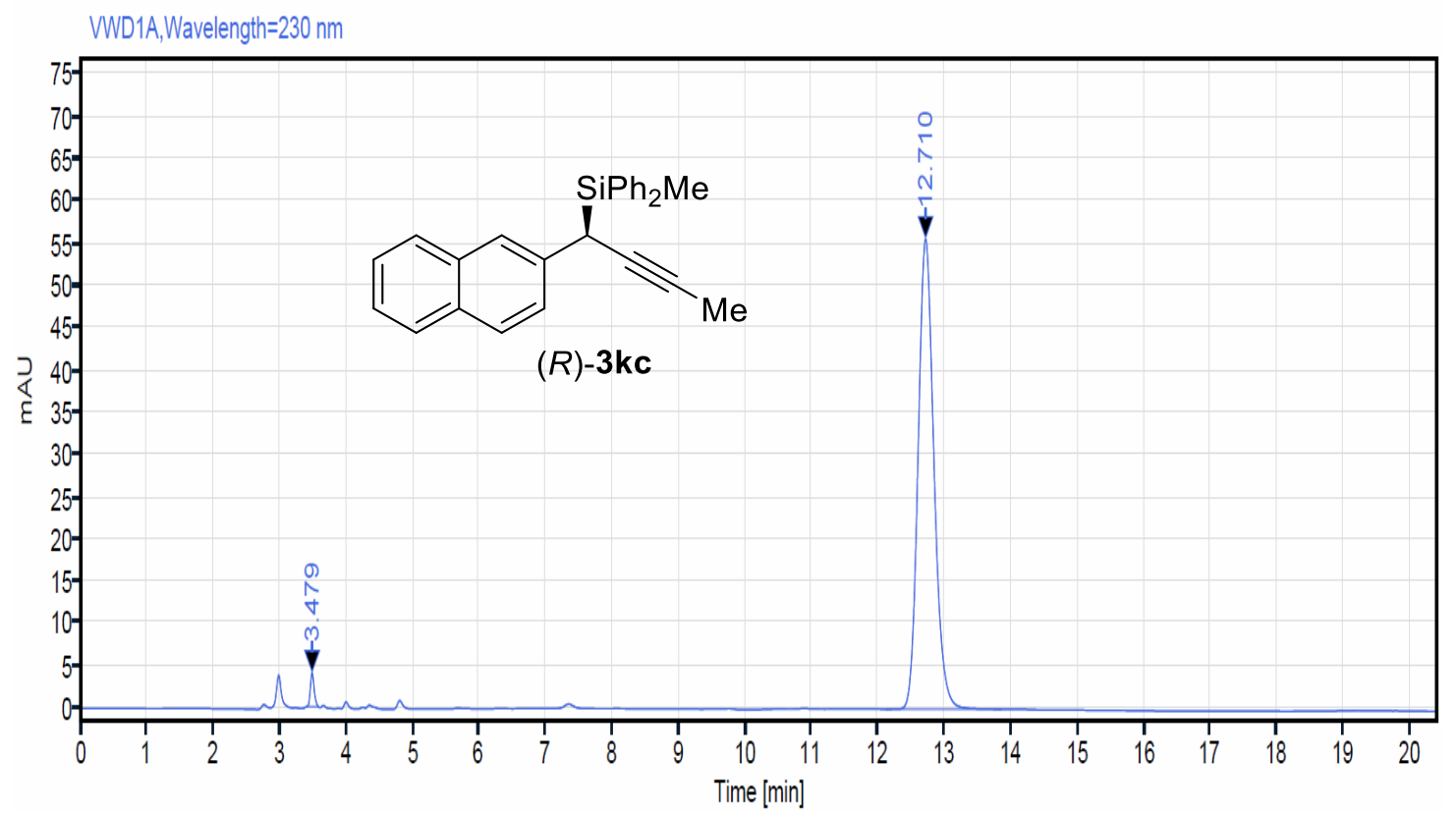

$\begin{array}{rrrrrr}\text { RT [min] } & \text { Type } & \text { Width [min] } & \text { Area } & \text { Height } & \text { Area\% } \\ 3.479 & \text { MM m } & 0.06 & 15.50 & 3.98 & 1.66 \\ 12.710 & \text { BB } & 1.74 & 920.51 & 55.68 & 98.34\end{array}$


(-)-triethyl(1-(naphthalen-2-yl)-6-phenylhex-2-yn-1-yl)silane (3lb)
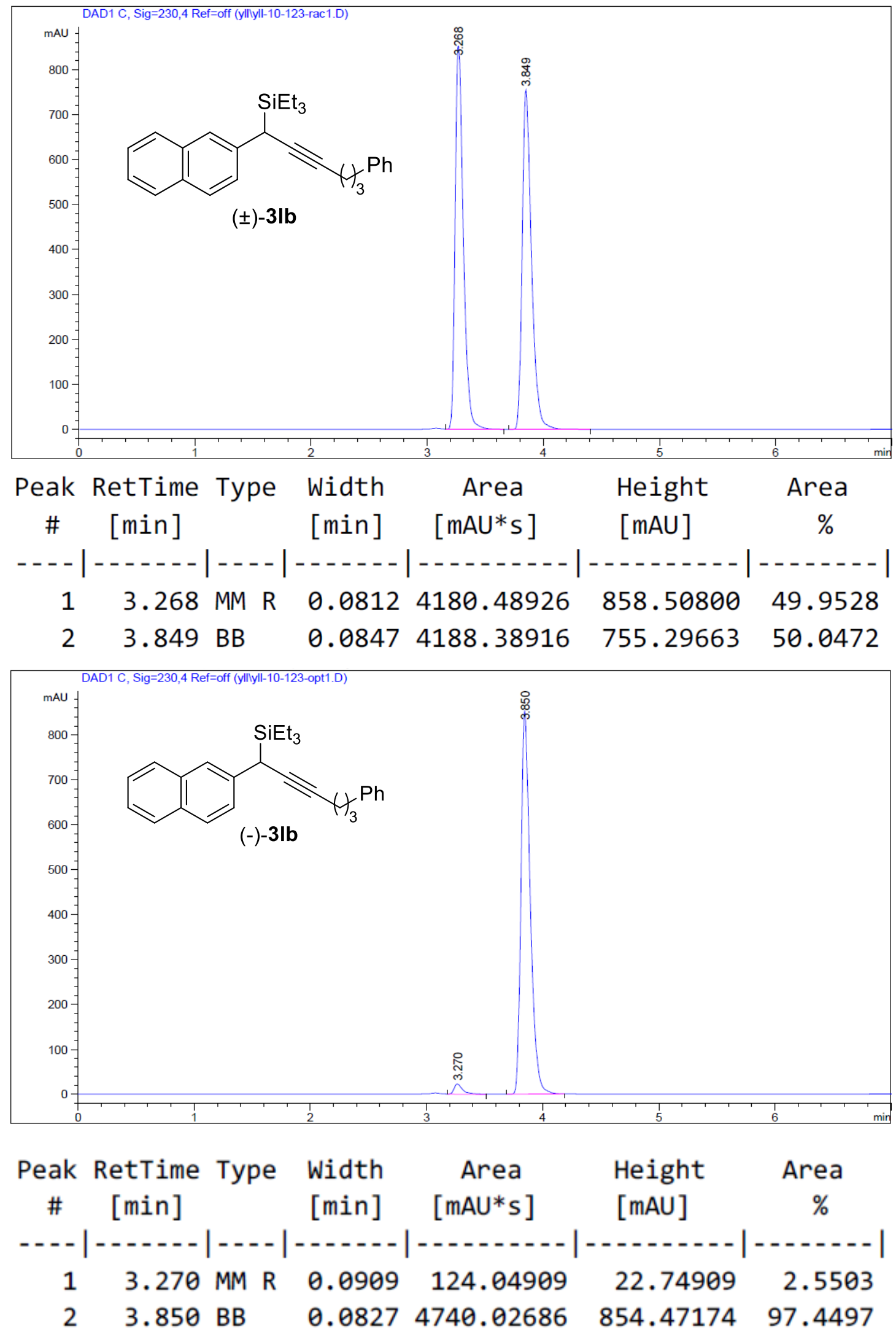
(-)-(3-cyclopropyl-1-(naphthalen-2-yl)prop-2-yn-1-yl)triethylsilane (3mb)

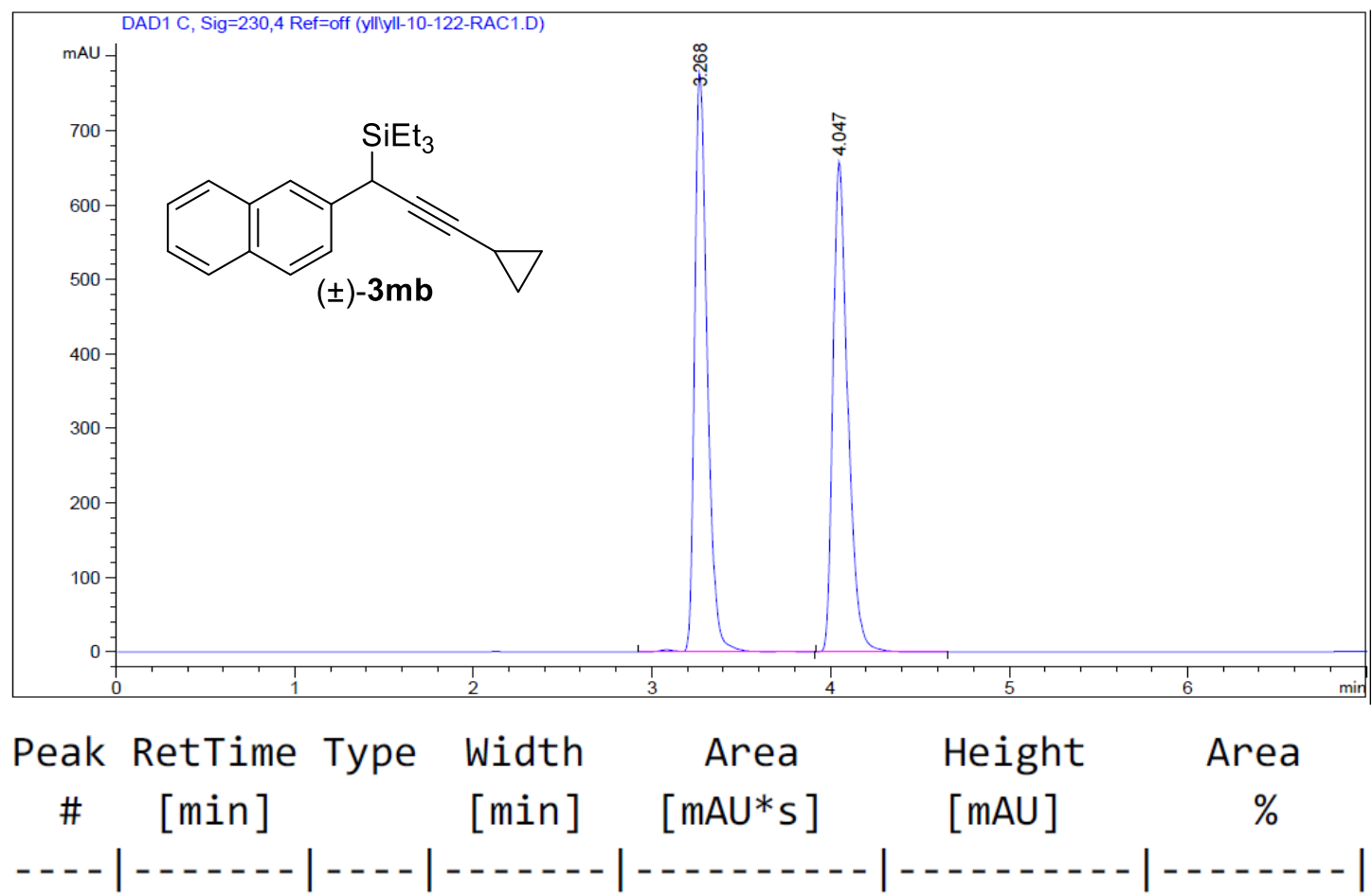

$\begin{array}{lllllll}1 & 3.268 \text { VV R } & 0.0747 & 3809.89160 & 780.26508 & 50.1348\end{array}$

$\begin{array}{lllllll}2 & 4.047 & \text { BB } & 0.0850 & 3789.40747 & 659.64691 & 49.8652\end{array}$

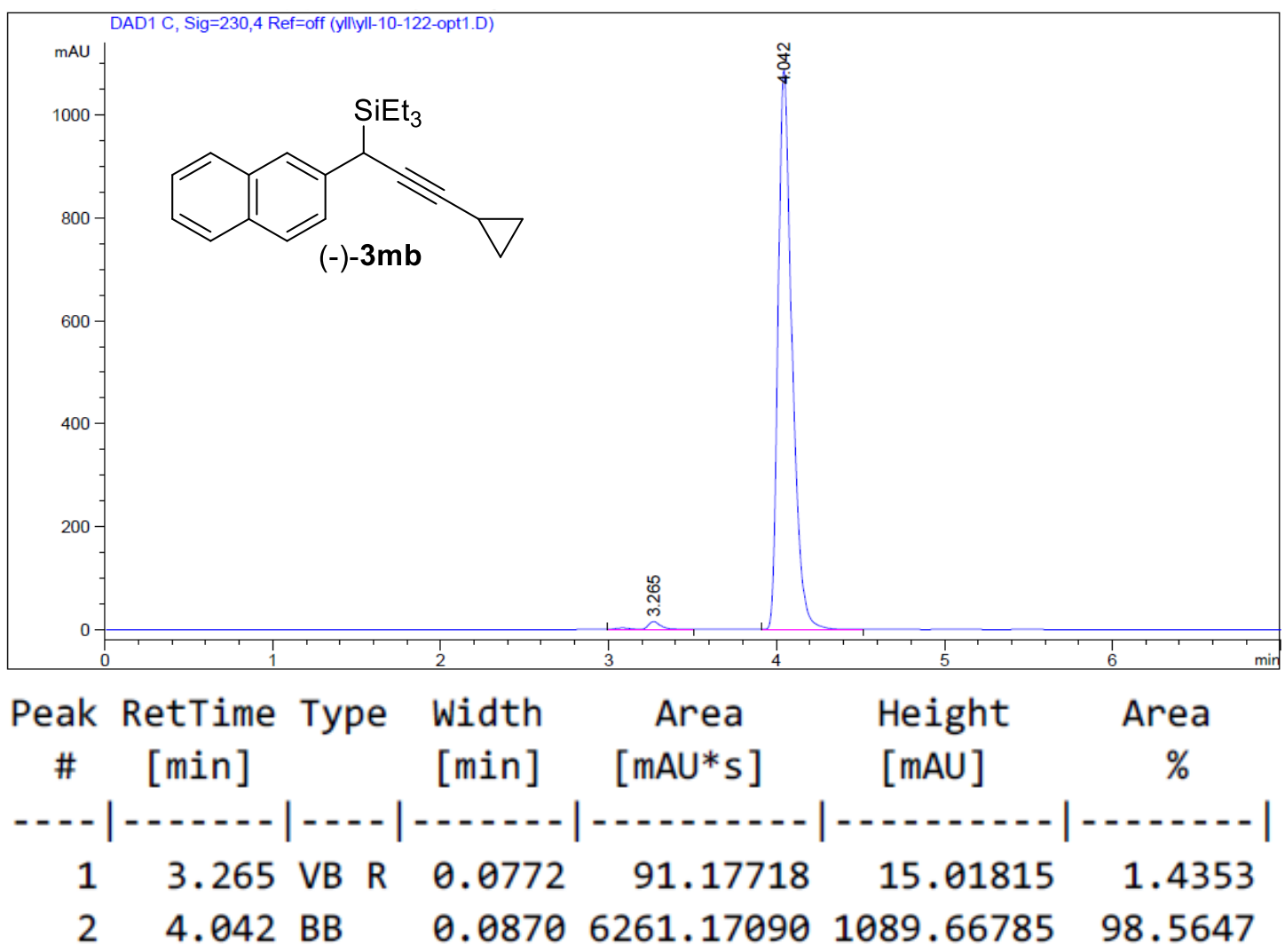


(-)-(3-cyclopropyl-1-(naphthalen-2-yl)prop-2-yn-1-yl)(methyl)diphenylsilane (3mc)

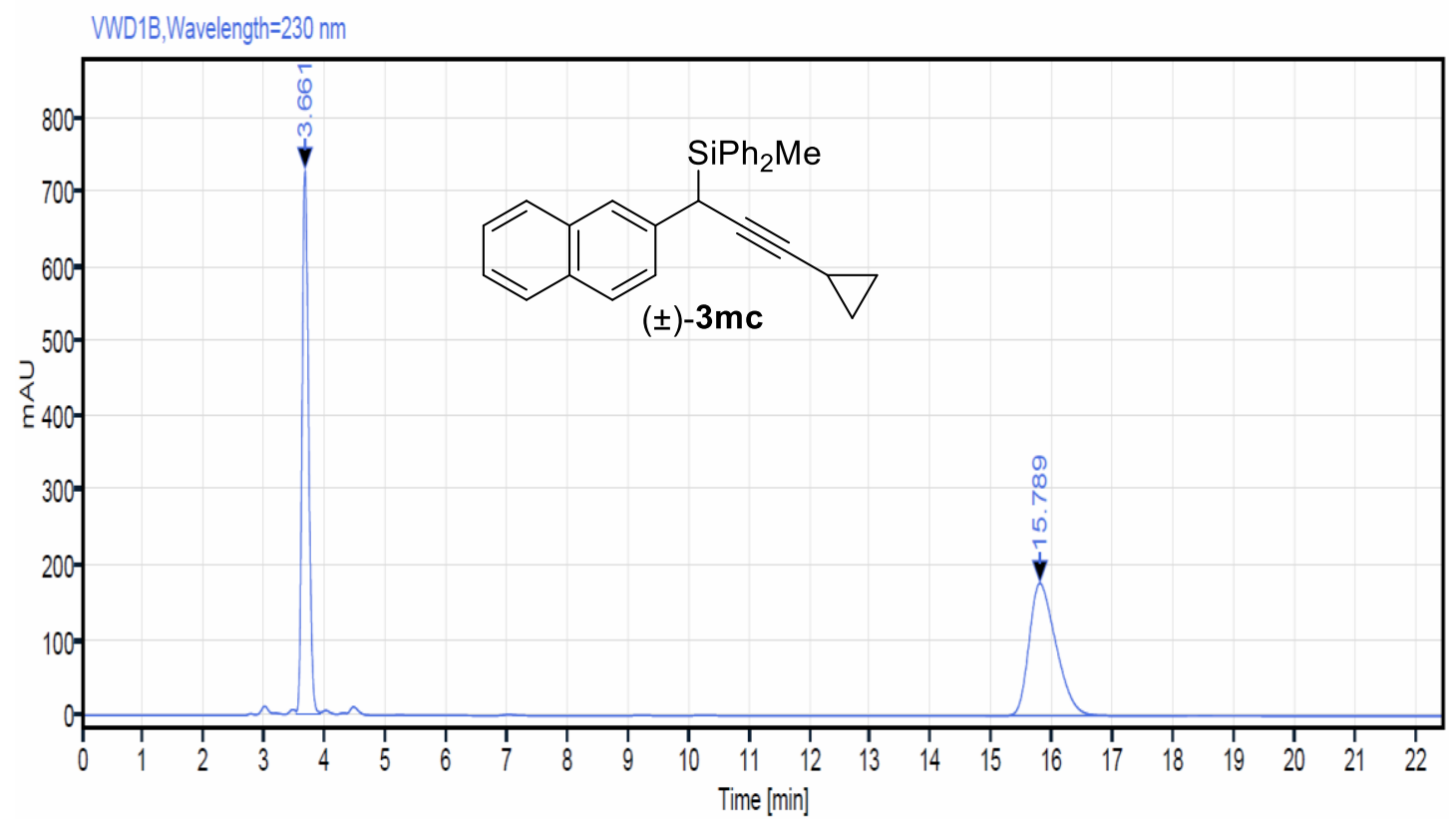

$\begin{array}{rrrrrr}\text { RT [min] } & \text { Type } & \text { Width [min] } & \text { Area } & \text { Height } & \text { Area\% } \\ 3.661 & \text { VV } & 0.40 & 5417.81 & 729.46 & 49.66 \\ 15.789 & \text { BB } & 3.33 & 5492.25 & 177.71 & 50.34\end{array}$

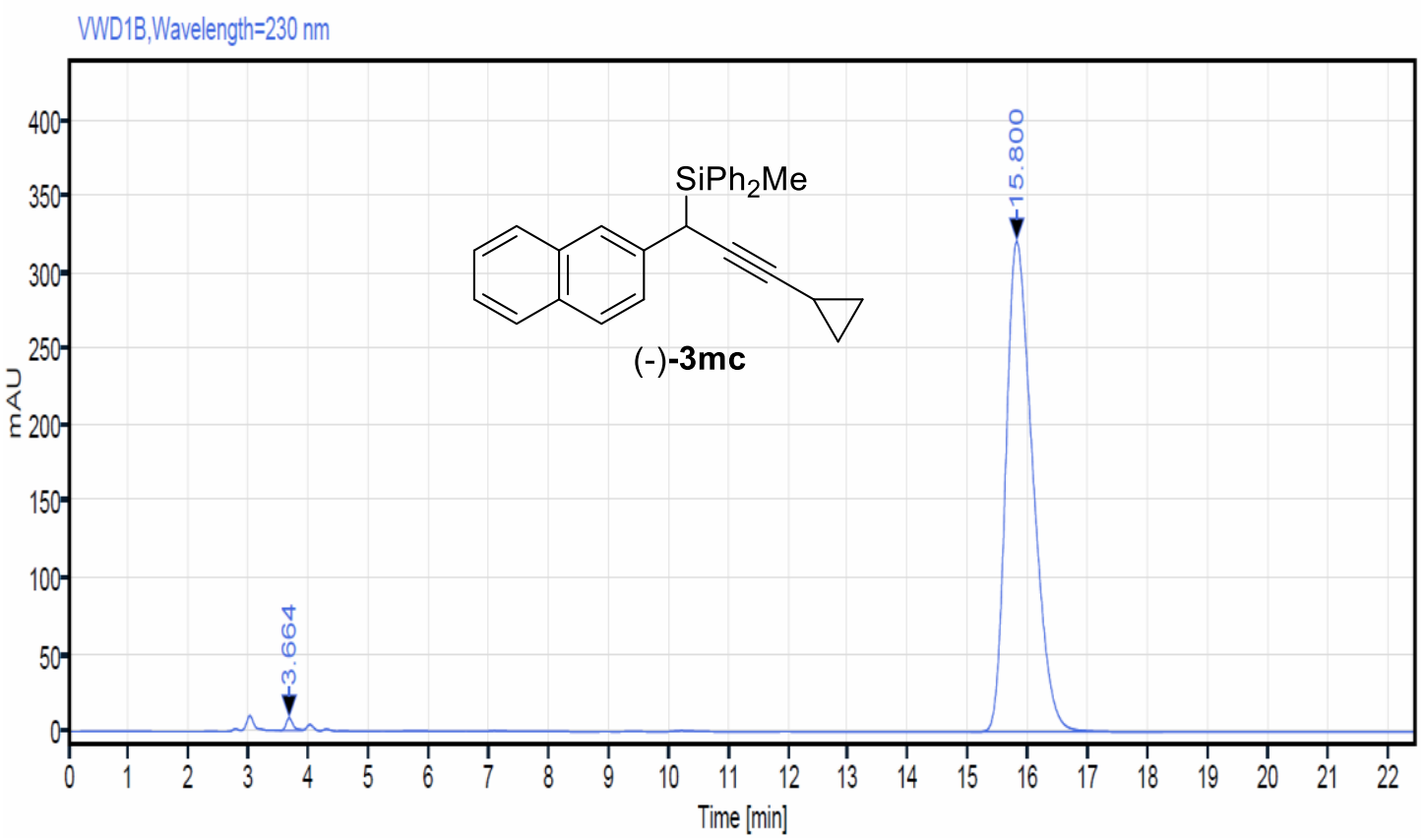

$\begin{array}{rrrrrr}\text { RT [min] } & \text { Type } & \text { Width [min] } & \text { Area } & \text { Height } & \text { Area\% } \\ 3.664 & \text { BV } & 0.47 & 67.93 & 8.23 & 0.68 \\ 15.800 & \text { BB } & 3.20 & 9948.26 & 321.59 & 99.32\end{array}$


(-)-(3-cyclohexyl-1-(naphthalen-2-yl)prop-2-yn-1-yl)triethylsilane (3nb)

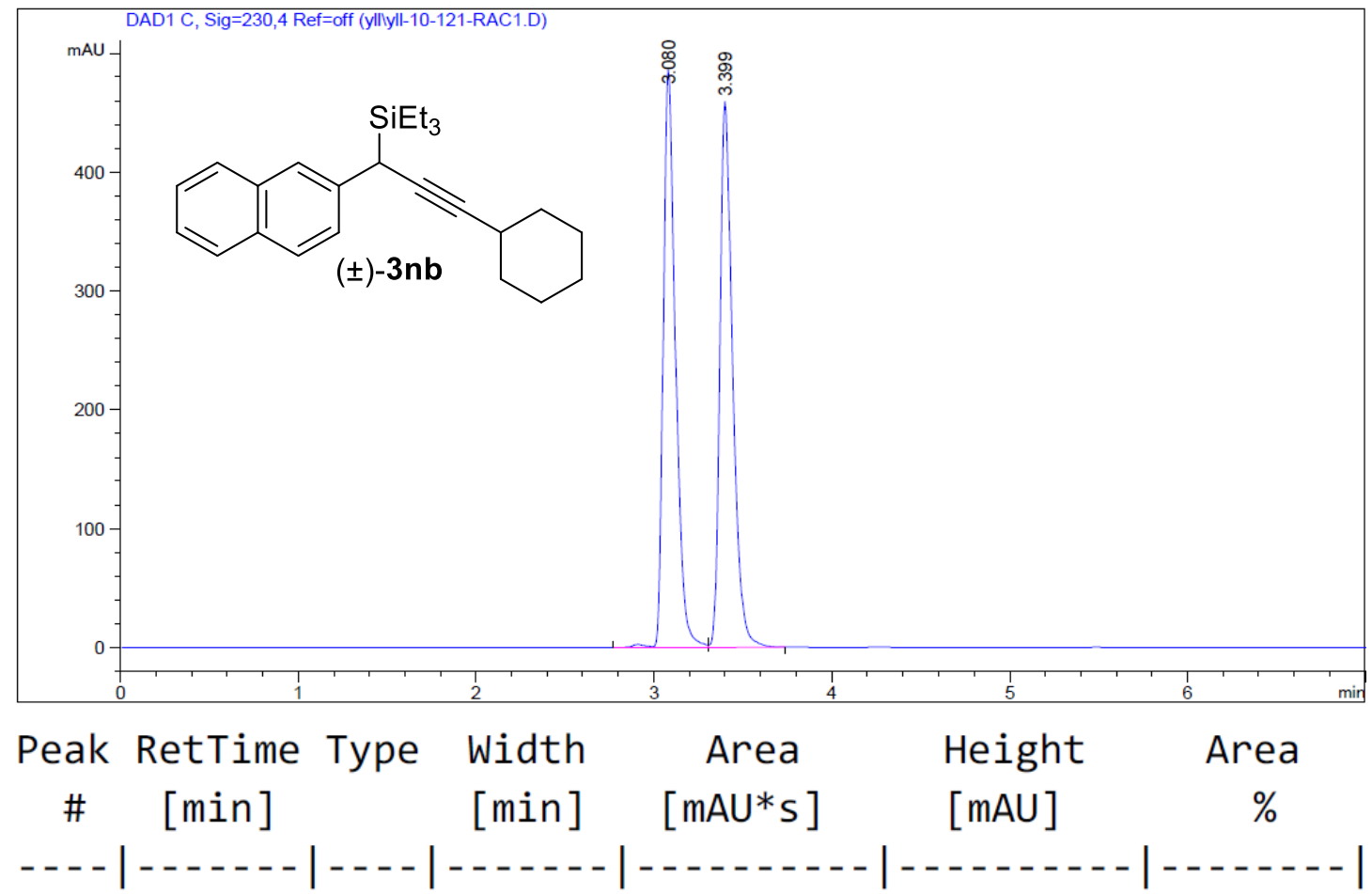

$\begin{array}{lllllll}1 & 3.080 & \text { V R R } & 0.0734 & 2327.90015 & 487.06311 & 50.2628\end{array}$

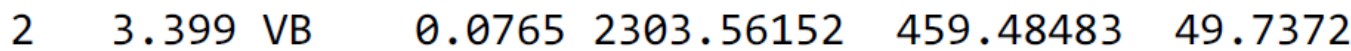

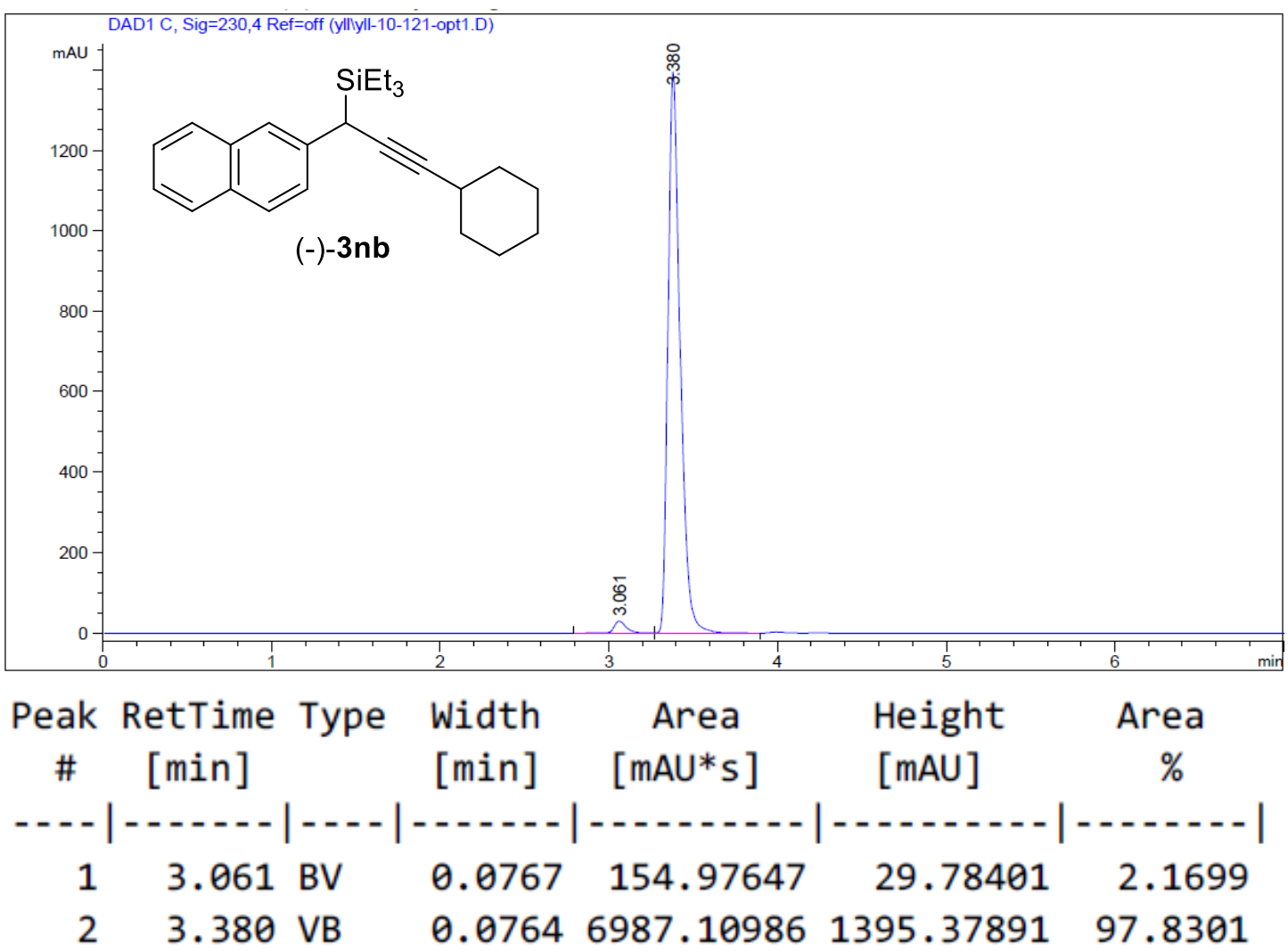


(-)-triethyl(1-(naphthalen-2-yl)-3-(trimethylsilyl)prop-2-yn-1-yl)silane (3ob)
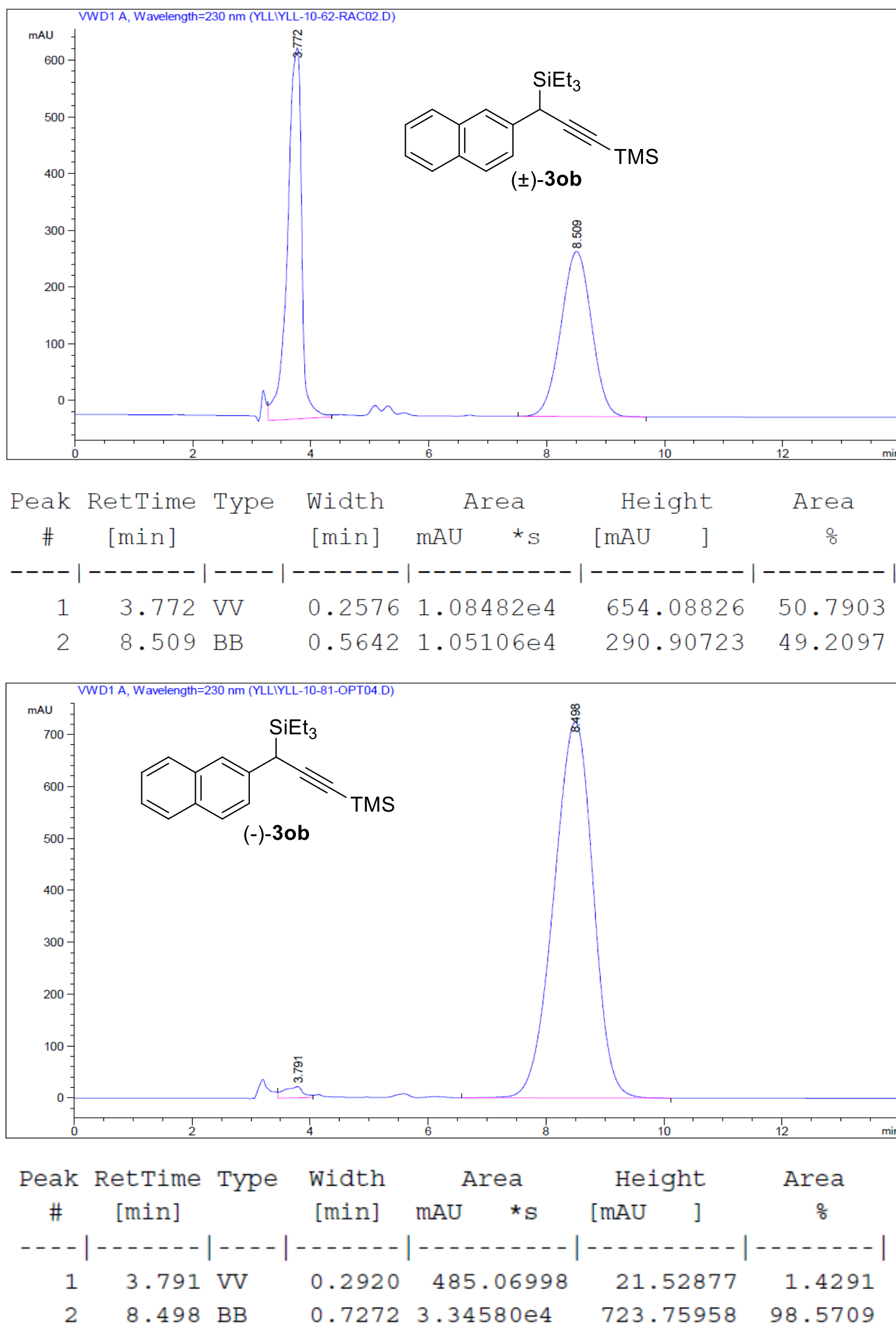
(-)-(3-(cyclohex-1-en-1-yl)-1-(naphthalen-2-yl)prop-2-yn-1-yl)triethylsilane (3pb)
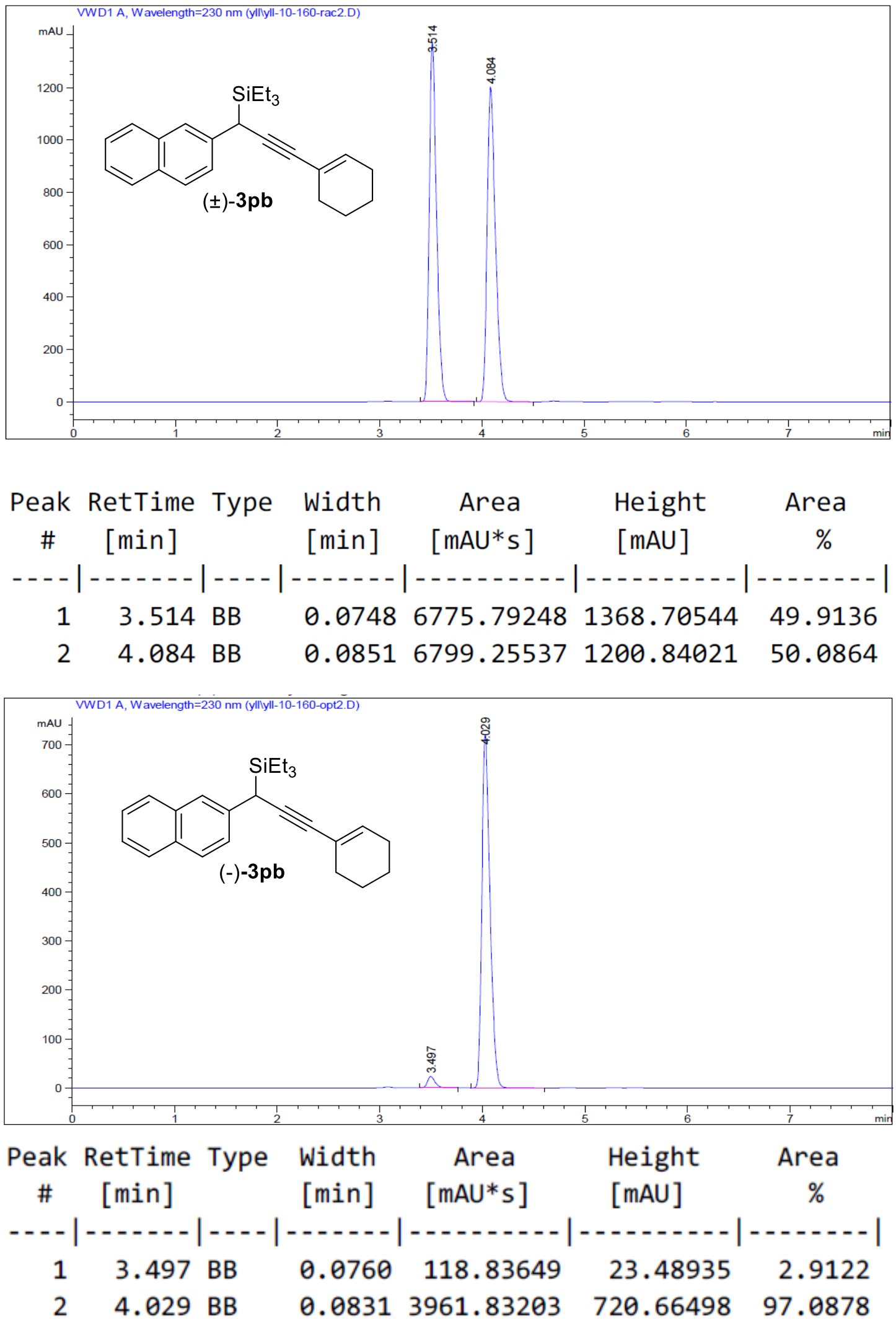
(-)-(3-(cyclohex-1-en-1-yl)-1-(naphthalen-2-yl)prop-2-yn-1yl)(methyl)diphenylsilane (3pc)
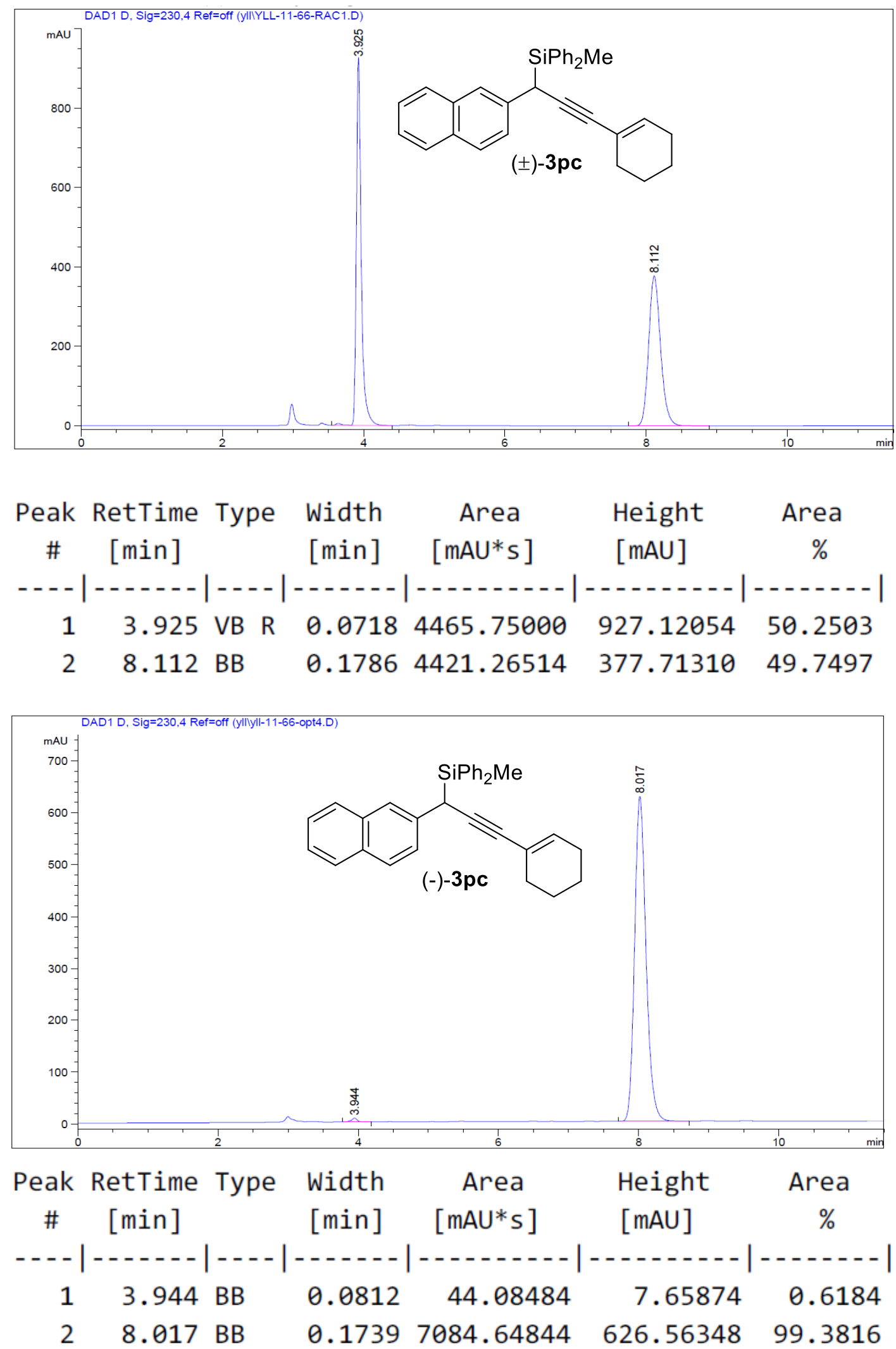


\section{(-)-triethyl(1-(naphthalen-2-yl)undeca-2,4-diyn-1-yl)silane (3qb)}

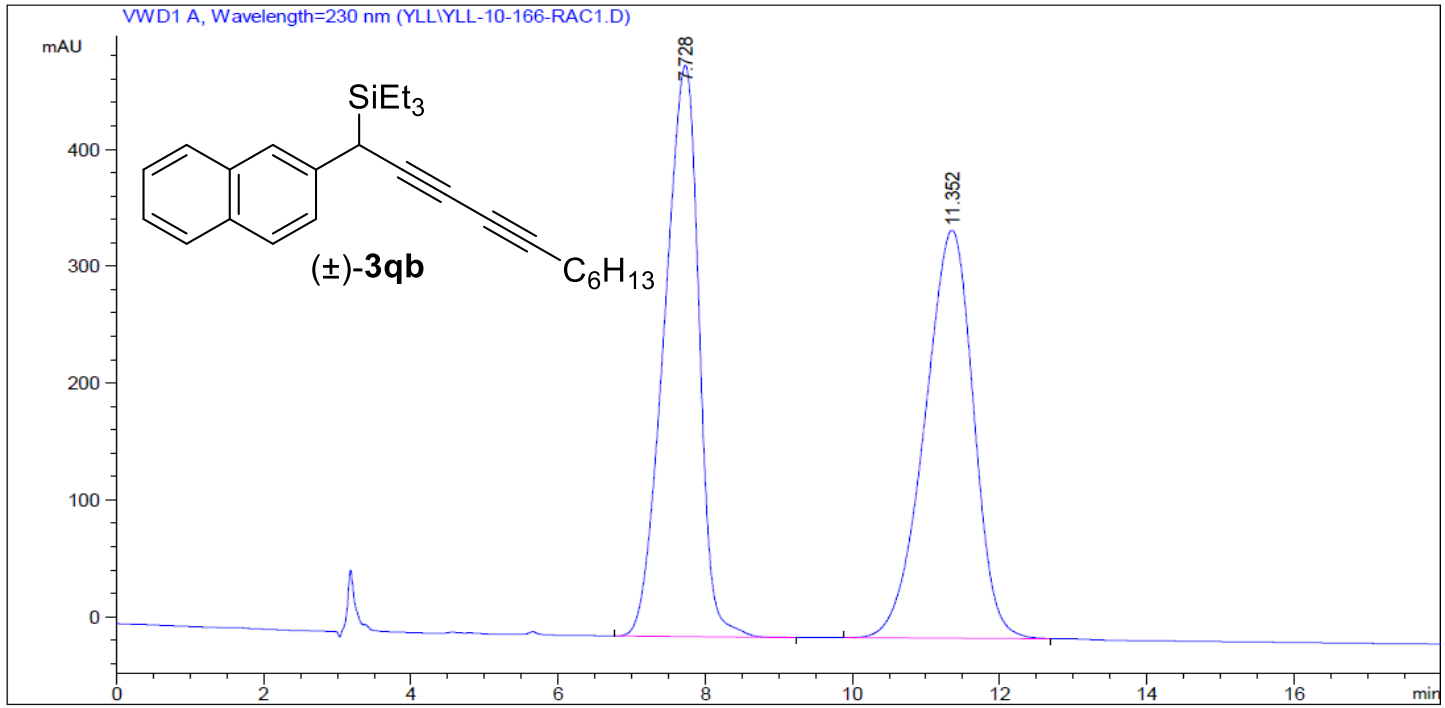

\begin{tabular}{|c|c|c|c|c|c|c|c|}
\hline \multirow{2}{*}{$\begin{array}{c}\text { Peak } \\
\#\end{array}$} & \multirow{2}{*}{$\begin{array}{c}\text { RetTime } \\
\text { [min] }\end{array}$} & \multirow[t]{2}{*}{ Type } & \multirow{2}{*}{$\begin{array}{l}\text { Width } \\
\text { [min] }\end{array}$} & \multicolumn{2}{|c|}{ Area } & Height & \multirow{2}{*}{$\begin{array}{c}\text { Area } \\
\frac{\circ}{0}\end{array}$} \\
\hline & & & & mAU & $\star_{\mathrm{S}}$ & {$[\mathrm{mAU}$} & \\
\hline & & & & & & & \\
\hline 1 & & & & 1 . & & 18 & \\
\hline 2 & 52 & E & 152 & 1.6 & $5 e 4$ & 349.03049 & 50 \\
\hline
\end{tabular}
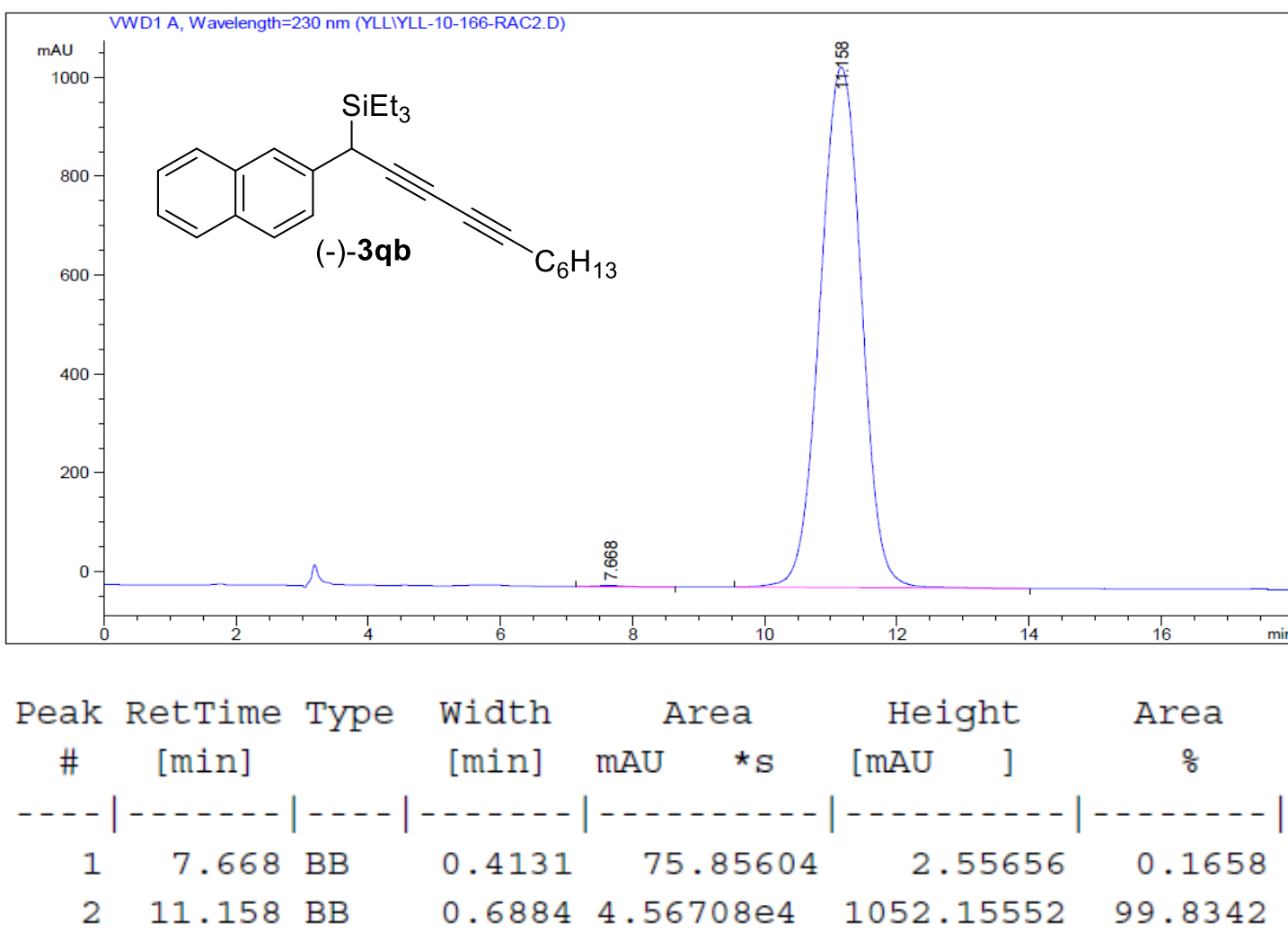
(-)-(6-chloro-1-(naphthalen-2-yl)hex-2-yn-1-yl)triethylsilane (3rb)
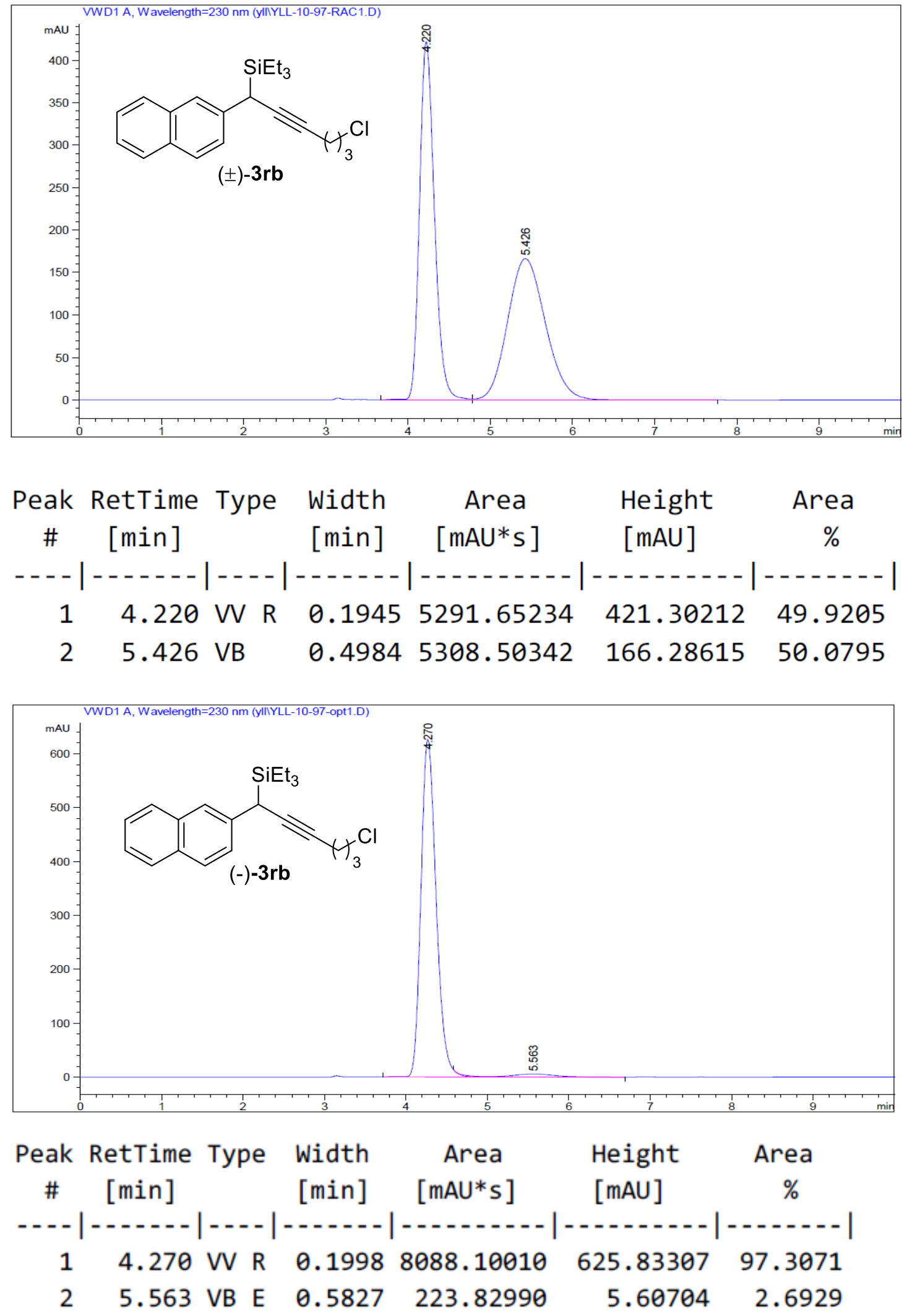
(-)-(8-(benzyloxy)-1-(naphthalen-2-yl)oct-2-yn-1-yl)triethylsilane (3sb)

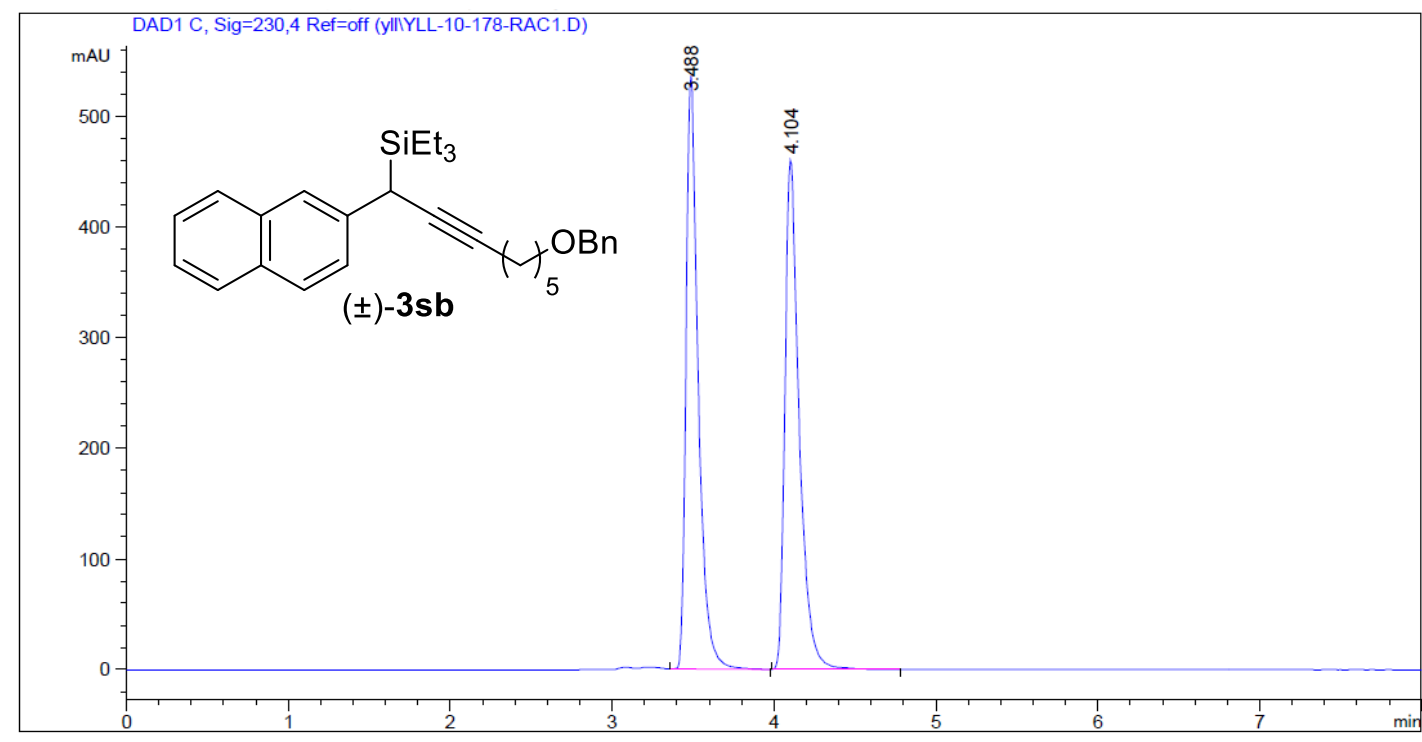

Peak RetTime Type width Area Height Area

\# [min] [min] [mAU*s $] \quad[\mathrm{mAU}] \quad \%$
- - - - | - - - - - | - - - - | - - - - - | - - - - - - - - | - - - - - - - | - - - - - - |

$\begin{array}{lllllll}1 & 3.488 & \text { BB } & 0.0767 & 2790.44116 & 536.34344 & 49.8428\end{array}$

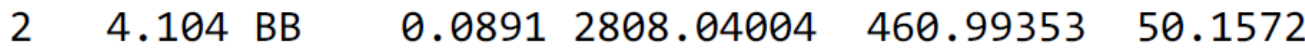

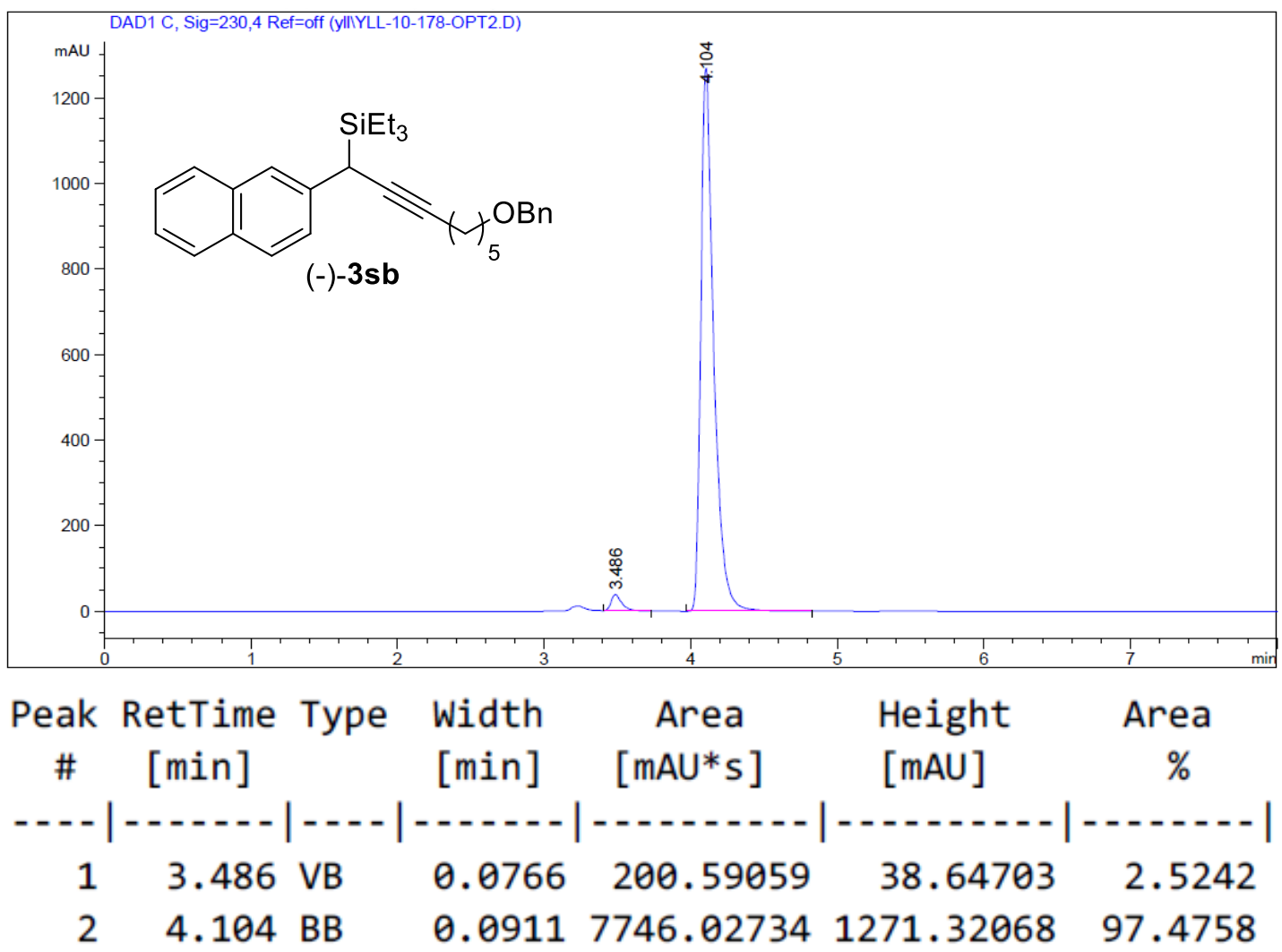


(-)-1-(6-(naphthalen-2-yl)-6-(triethylsilyl)hex-4-yn-1-yl)-1H-indole (3tb)
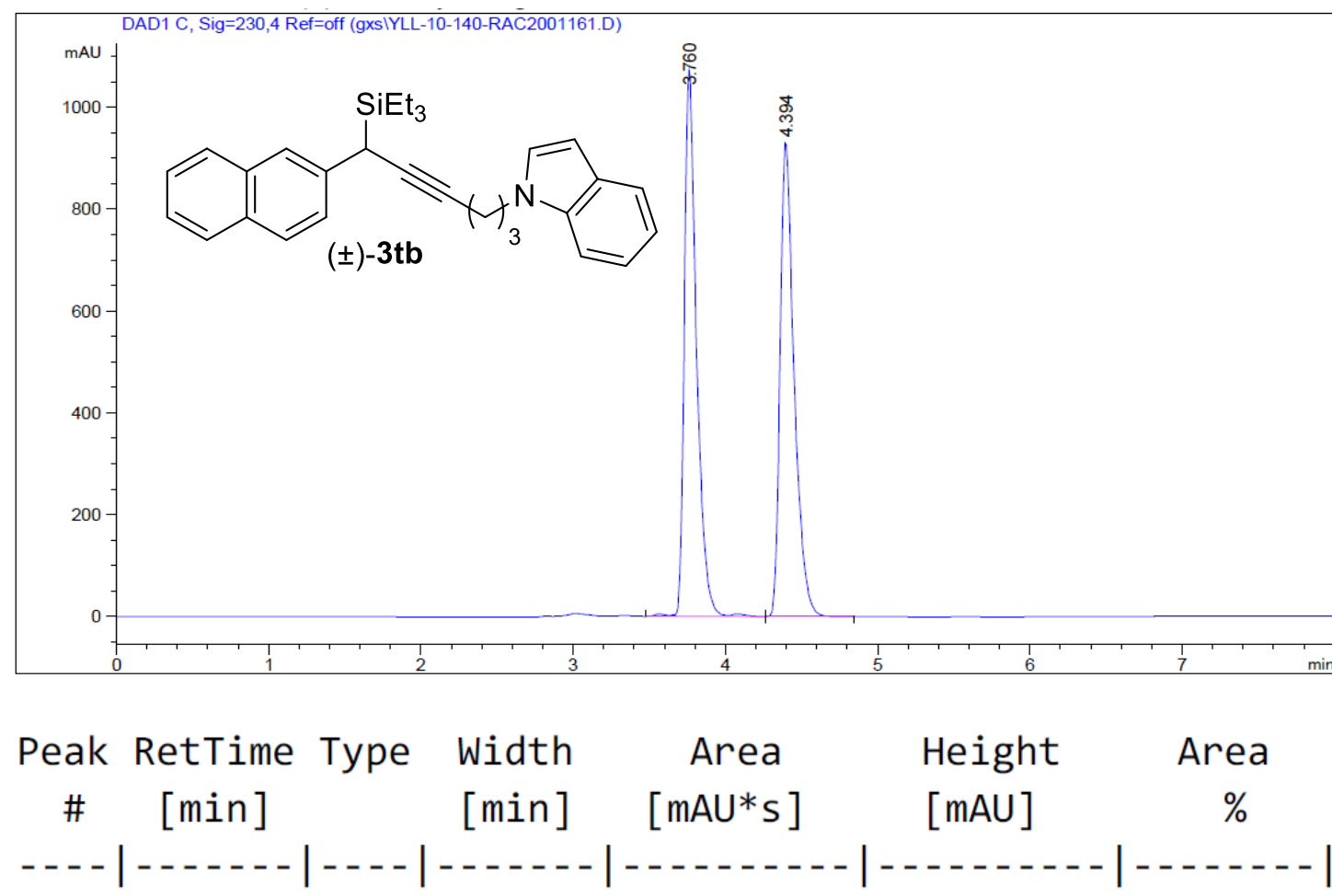

$\begin{array}{lllllll}1 & 3.760 & \text { V R R } & 0.0827 & 6013.06885 & 1073.73596 & 50.2157\end{array}$

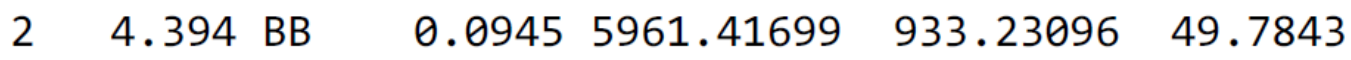

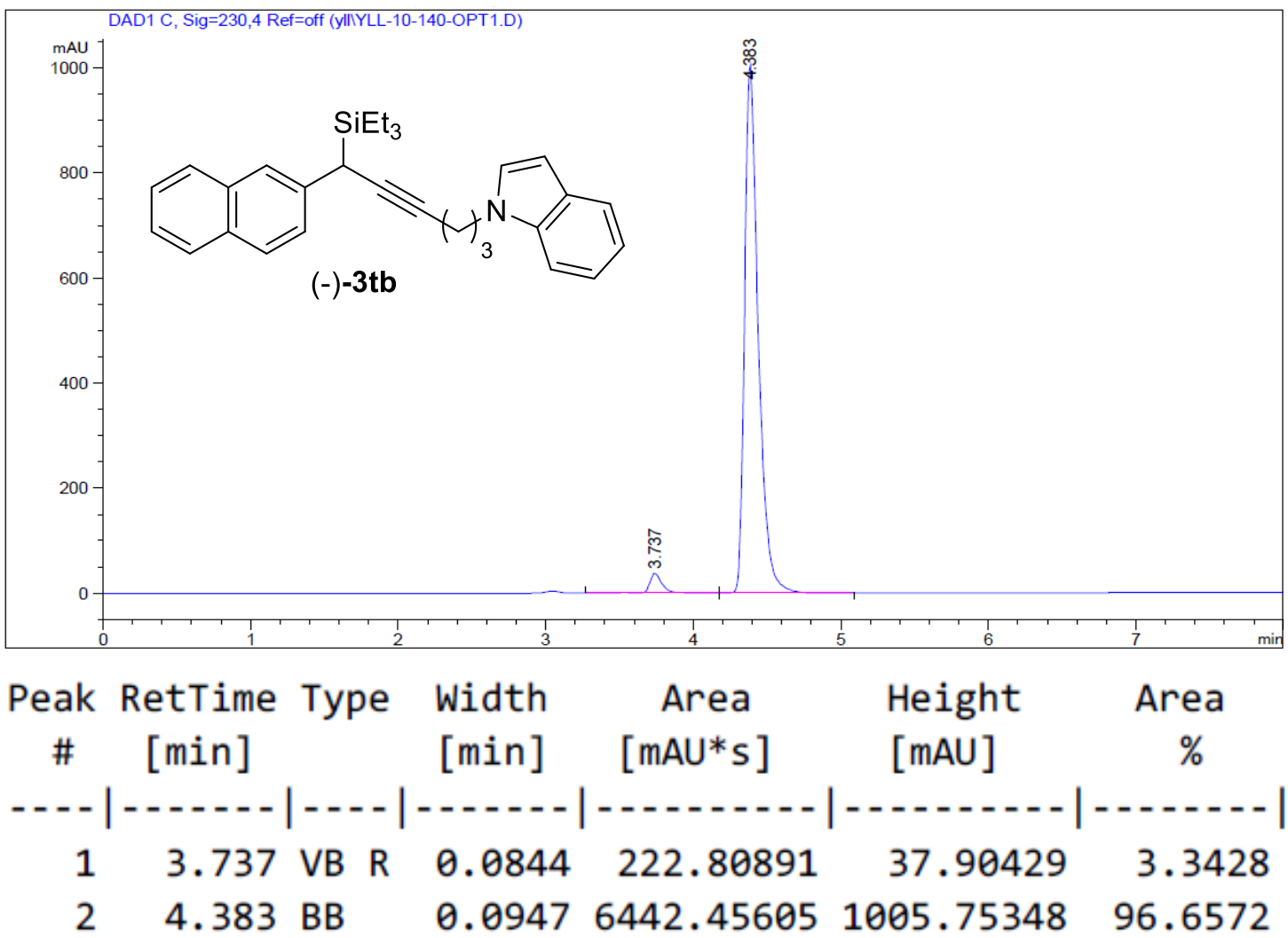


(-)-triethyl(1-(4-nitrophenyl)hept-2-yn-1-yl)silane (3ub)
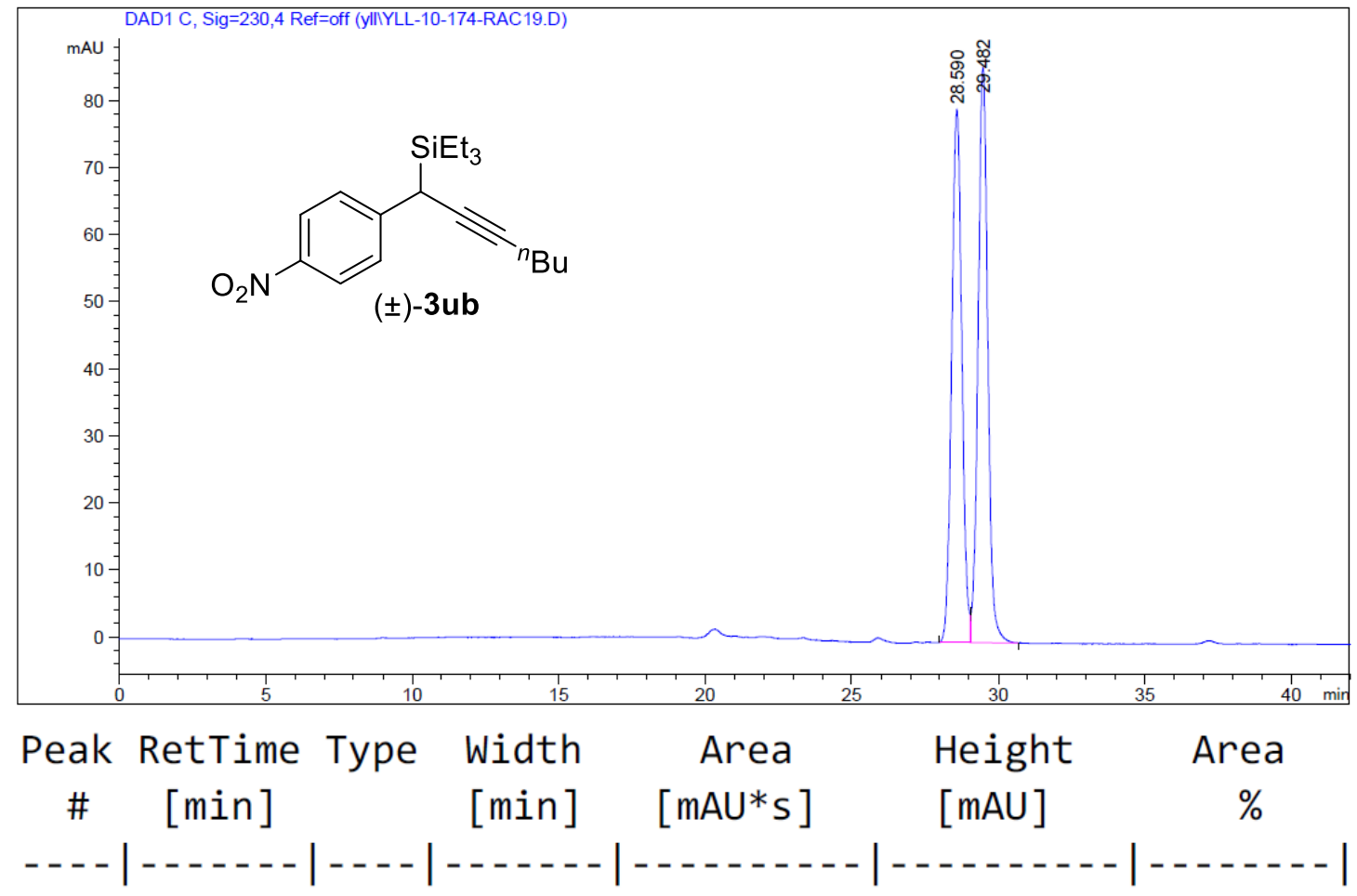

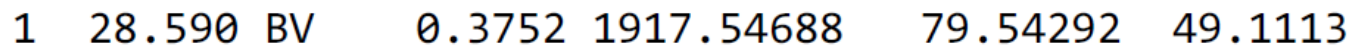

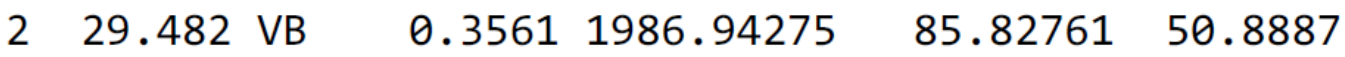

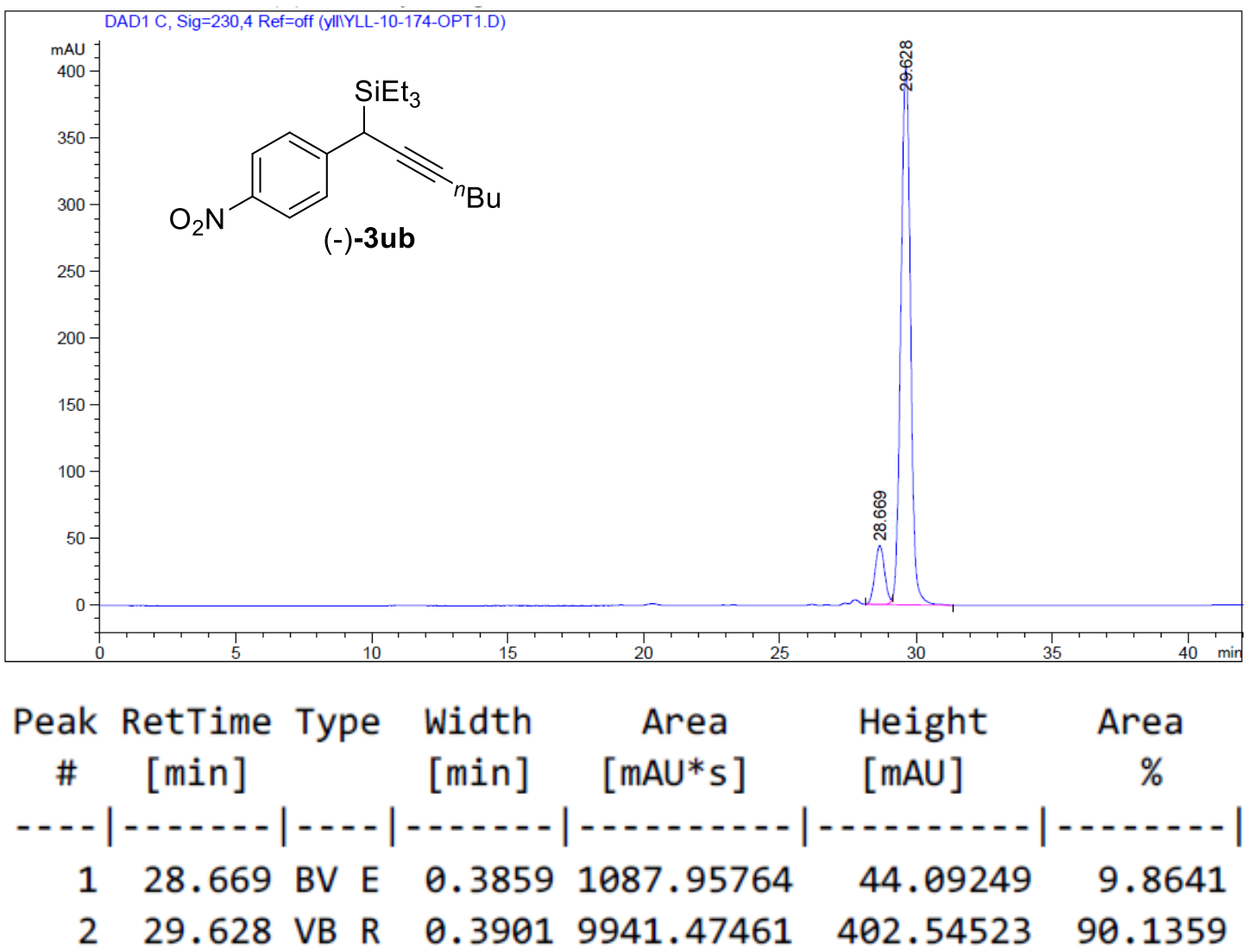


(-)-(1-(3,4-dichlorophenyl)hept-2-yn-1-yl)triethylsilane (3vb)

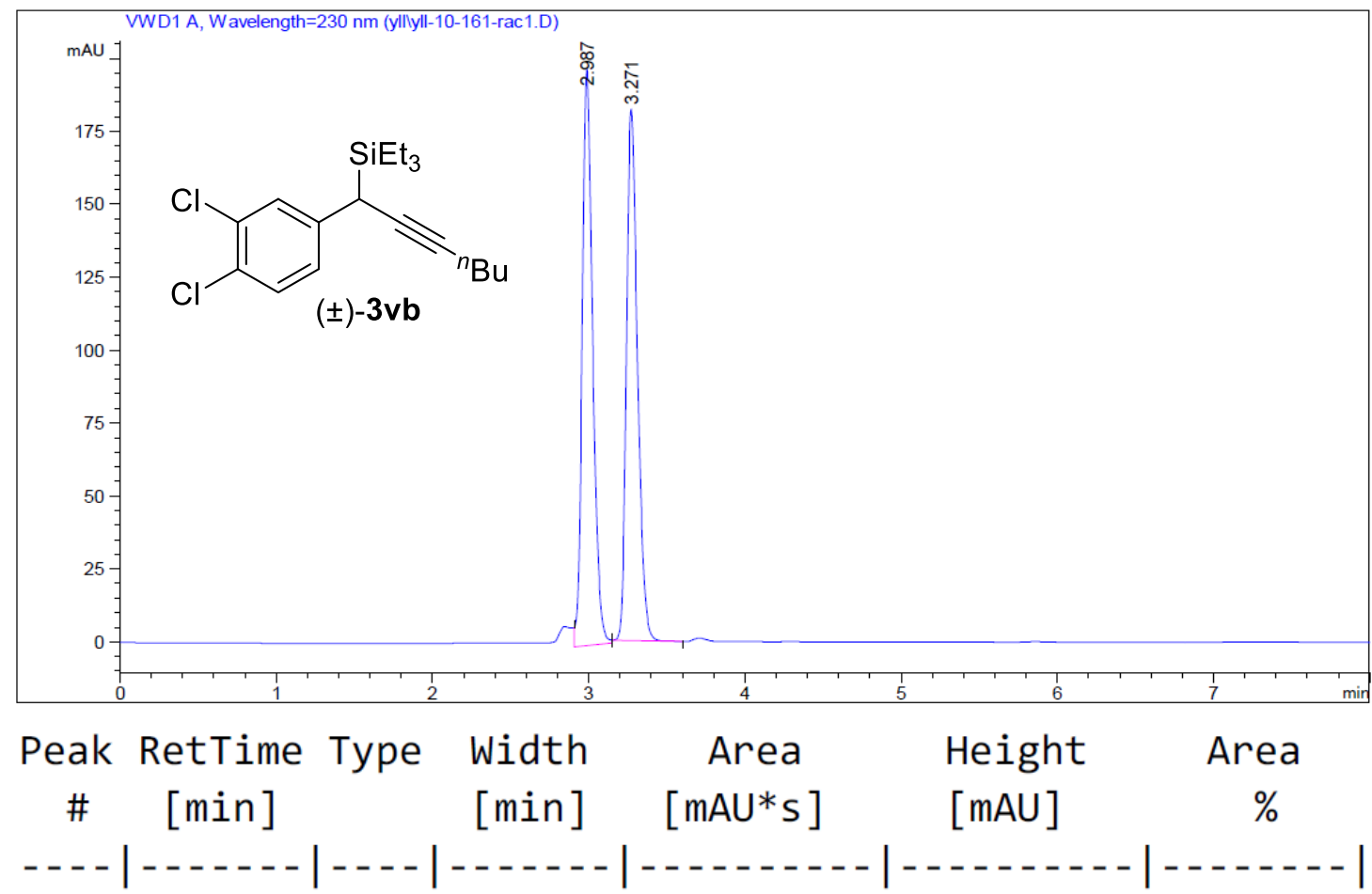

$\begin{array}{lllllll}1 & 2.987 \text { MM R } & 0.0775 & 919.69287 & 197.66266 & 50.8306\end{array}$

$\begin{array}{lllllll}2 & 3.271 & \text { BV R } & 0.0750 & 889.63623 & 182.14206 & 49.1694\end{array}$

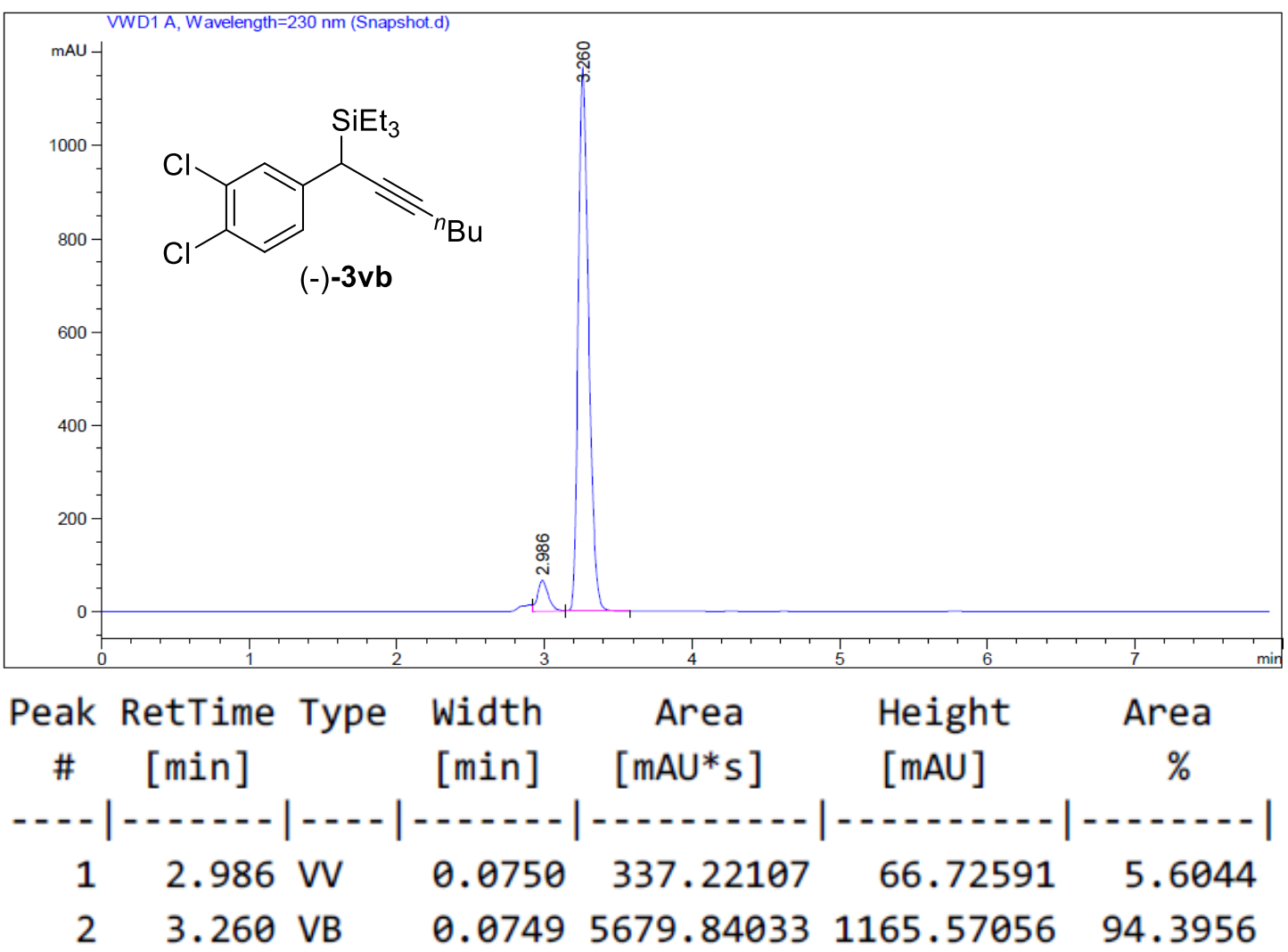


(-)-(1-(3,4-dichlorophenyl)hept-2-yn-1-yl)(methyl)diphenylsilane (3vc)

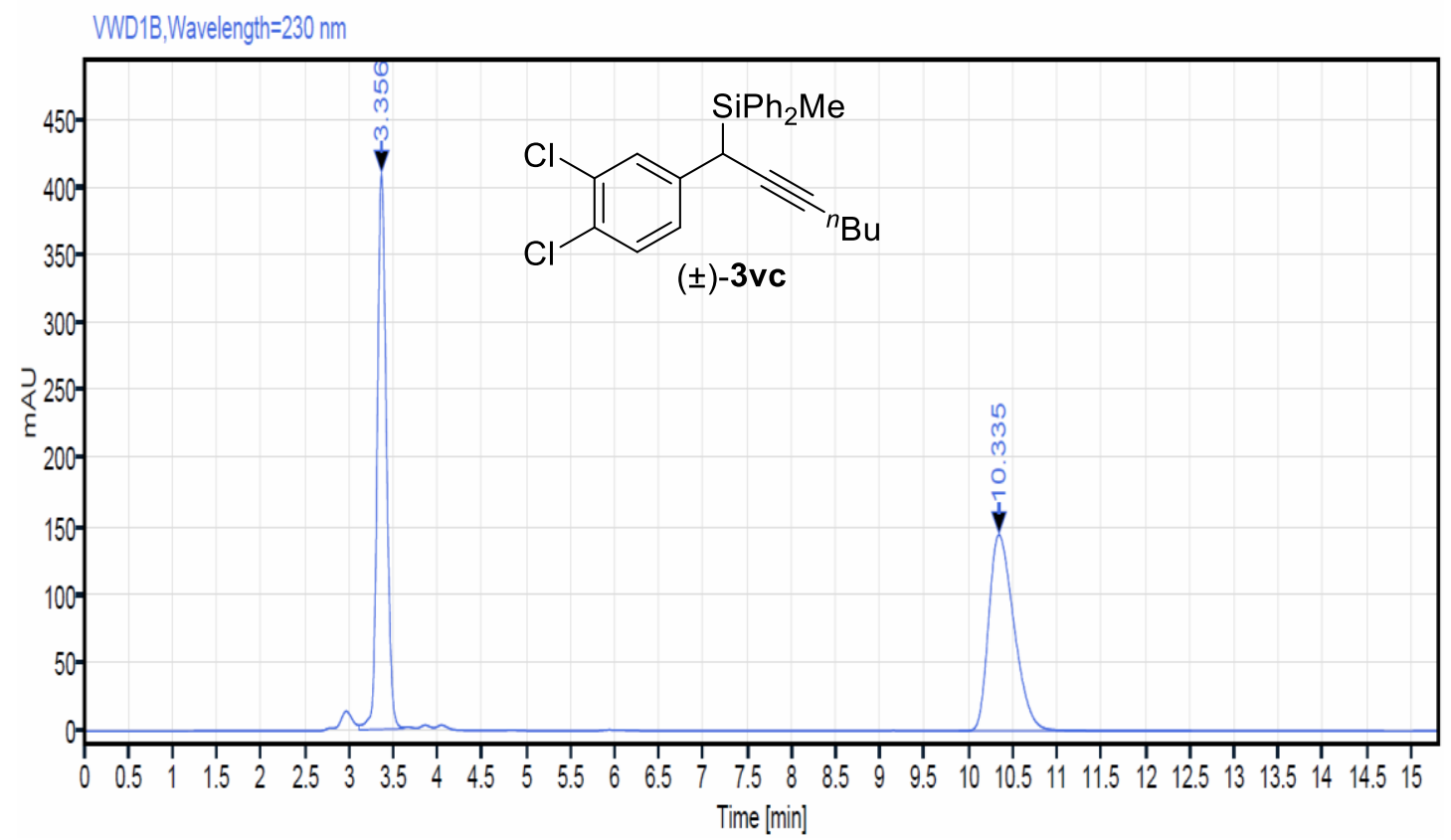

$\begin{array}{rrrrrr}\text { RT [min] } & \text { Type } & \text { Width [min] } & \text { Area } & \text { Height } & \text { Area\% } \\ 3.356 & \text { VV } & 0.50 & 2944.26 & 409.25 & 50.51 \\ 10.335 & \text { BB } & 2.63 & 2884.40 & 144.48 & 49.49\end{array}$

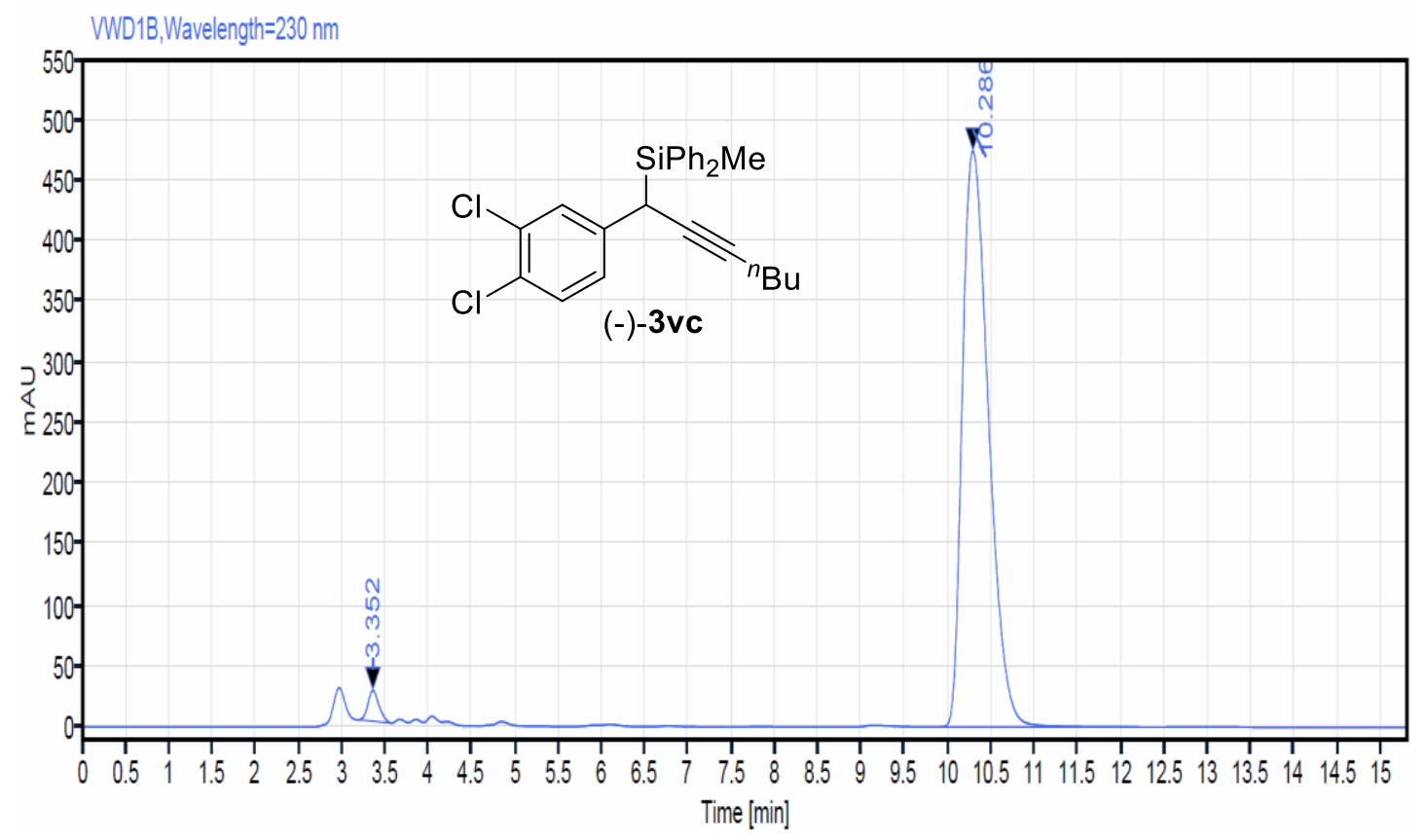

$\begin{array}{rrrrrr}\text { RT [min] } & \text { Type } & \text { Width [min] } & \text { Area } & \text { Height } & \text { Area\% } \\ 3.352 & \text { MM m } & 0.14 & 224.80 & 25.31 & 2.20 \\ 10.286 & \text { VB } & 2.45 & 9990.59 & 475.01 & 97.80\end{array}$


(+)-(1-(3,4-dimethylphenyl)hept-2-yn-1-yl)(methyl)diphenylsilane (3wc)

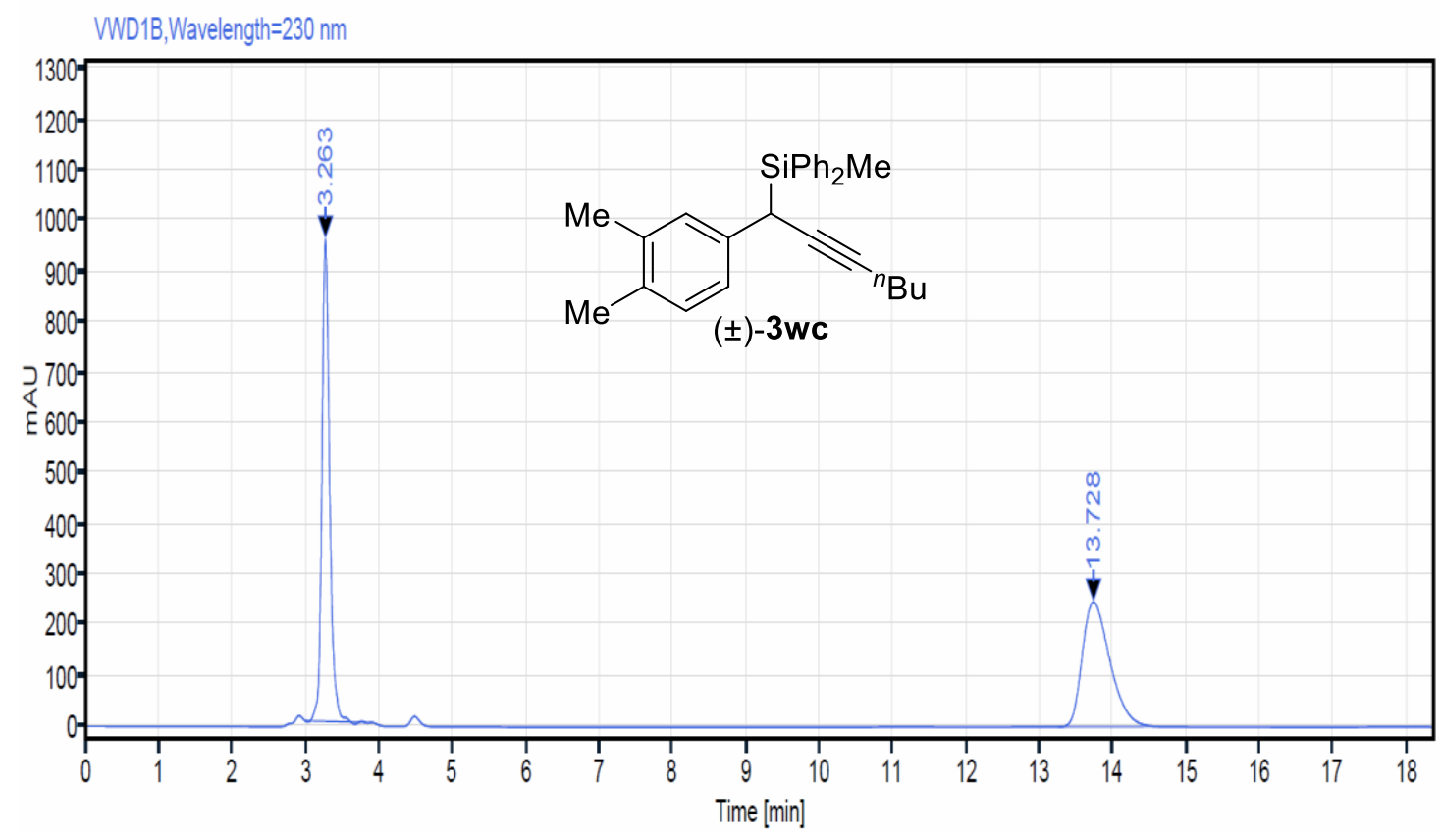

$\begin{array}{rrrrrr}\text { RT [min] } & \text { Type } & \text { Width [min] } & \text { Area } & \text { Height } & \text { Area\% } \\ 3.263 & \text { MM m } & 0.11 & 6954.41 & 956.68 & 51.78 \\ 13.728 & \text { MM m } & 0.41 & 6476.51 & 247.51 & 48.22\end{array}$

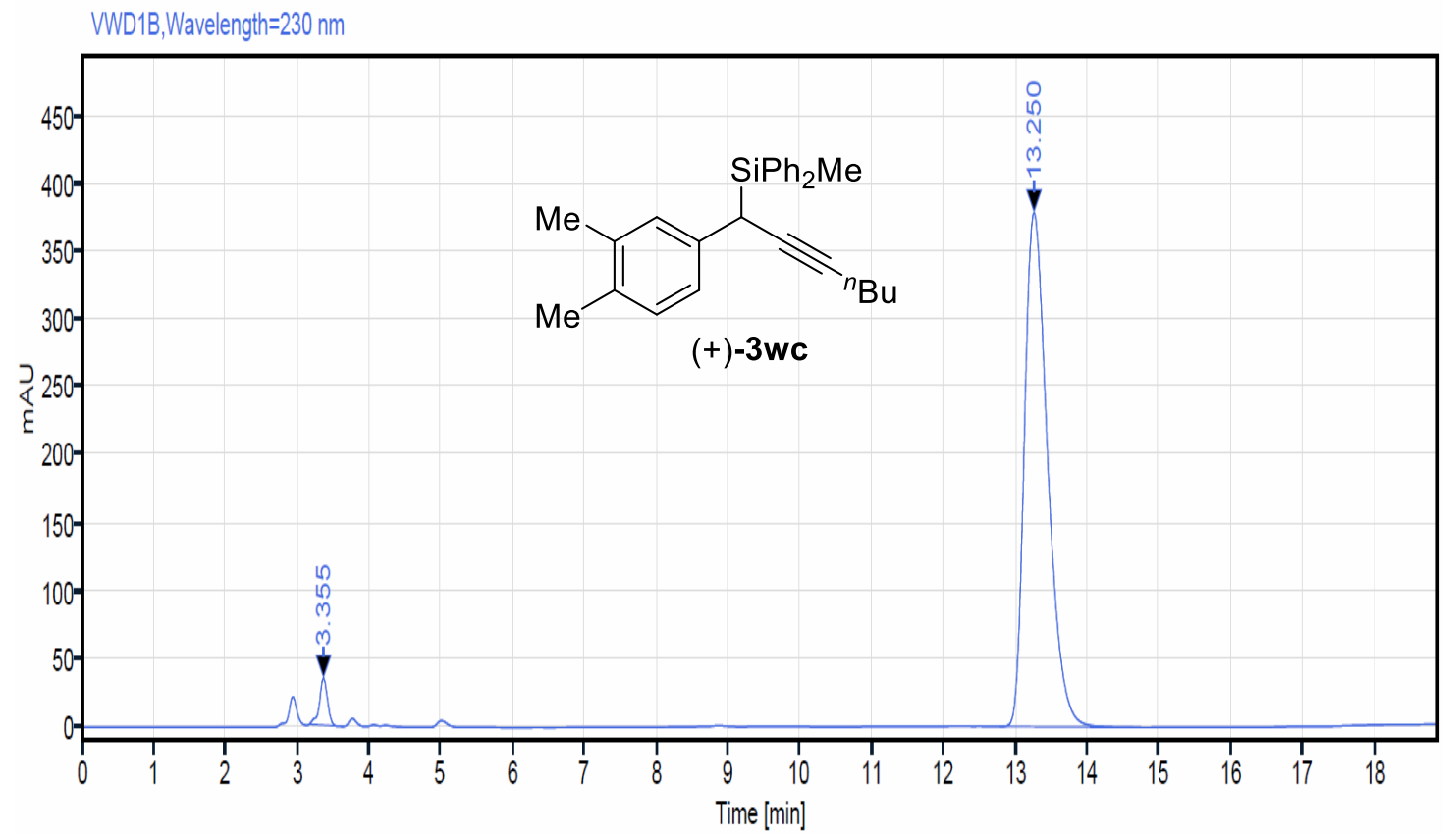

$\begin{array}{rrrrrr}\text { RT [min] } & \text { Type } & \text { Width [min] } & \text { Area } & \text { Height } & \text { Area\% } \\ 3.355 & \text { MB m } & 0.12 & 269.86 & 34.51 & 3.08 \\ 13.250 & \text { BB } & 1.85 & 8486.01 & 378.70 & 96.92\end{array}$


(-)-(1-(benzofuran-5-yl)hept-2-yn-1-yl)(methyl)diphenylsilane (3xc)

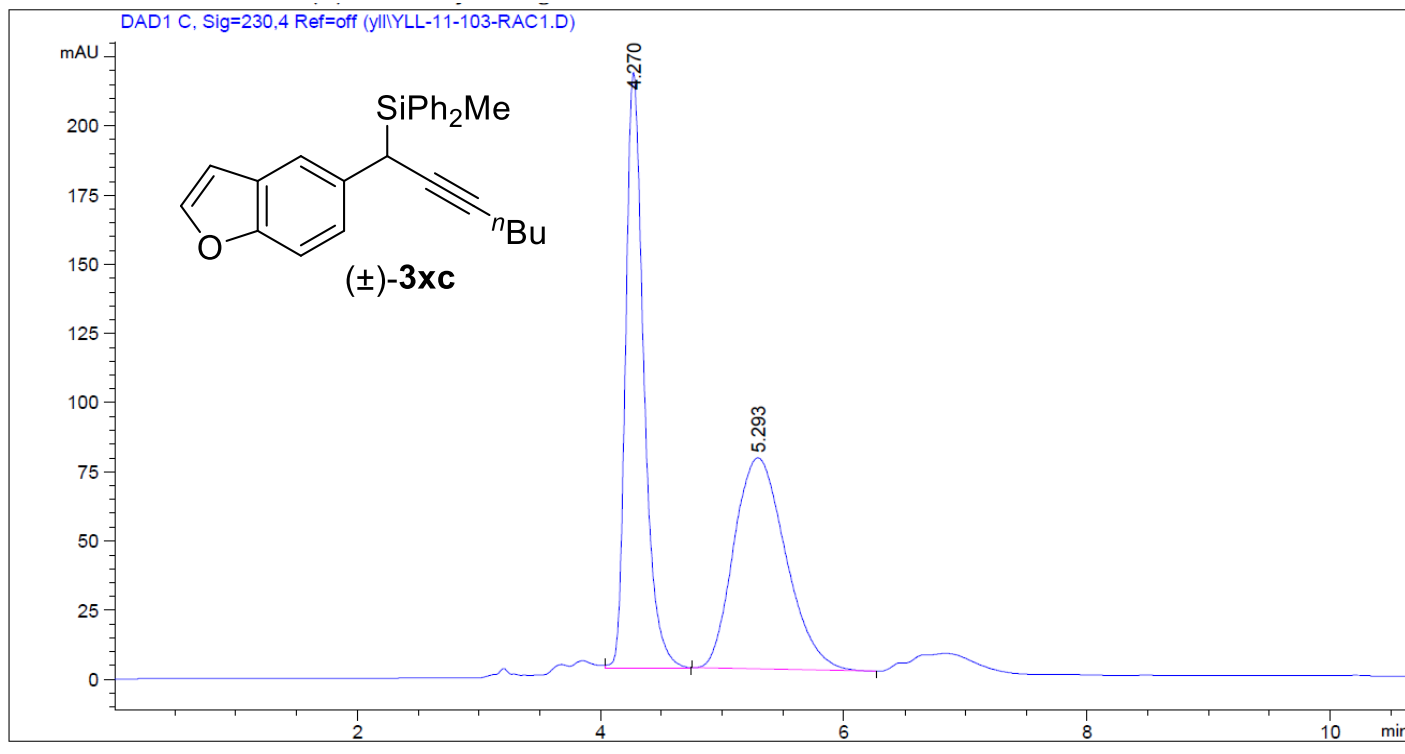

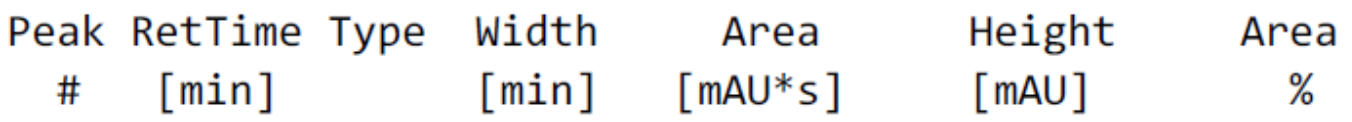
- - - | - - - - - - - | - - - - | - - - - - | - - - - - - - - - |

$\begin{array}{lllllll}1 & 4.270 \text { MM R } & 0.1735 & 2244.39111 & 215.60905 & 49.7473\end{array}$
25.293 BB
0.46442267 .19141
$76.36663 \quad 50.2527$

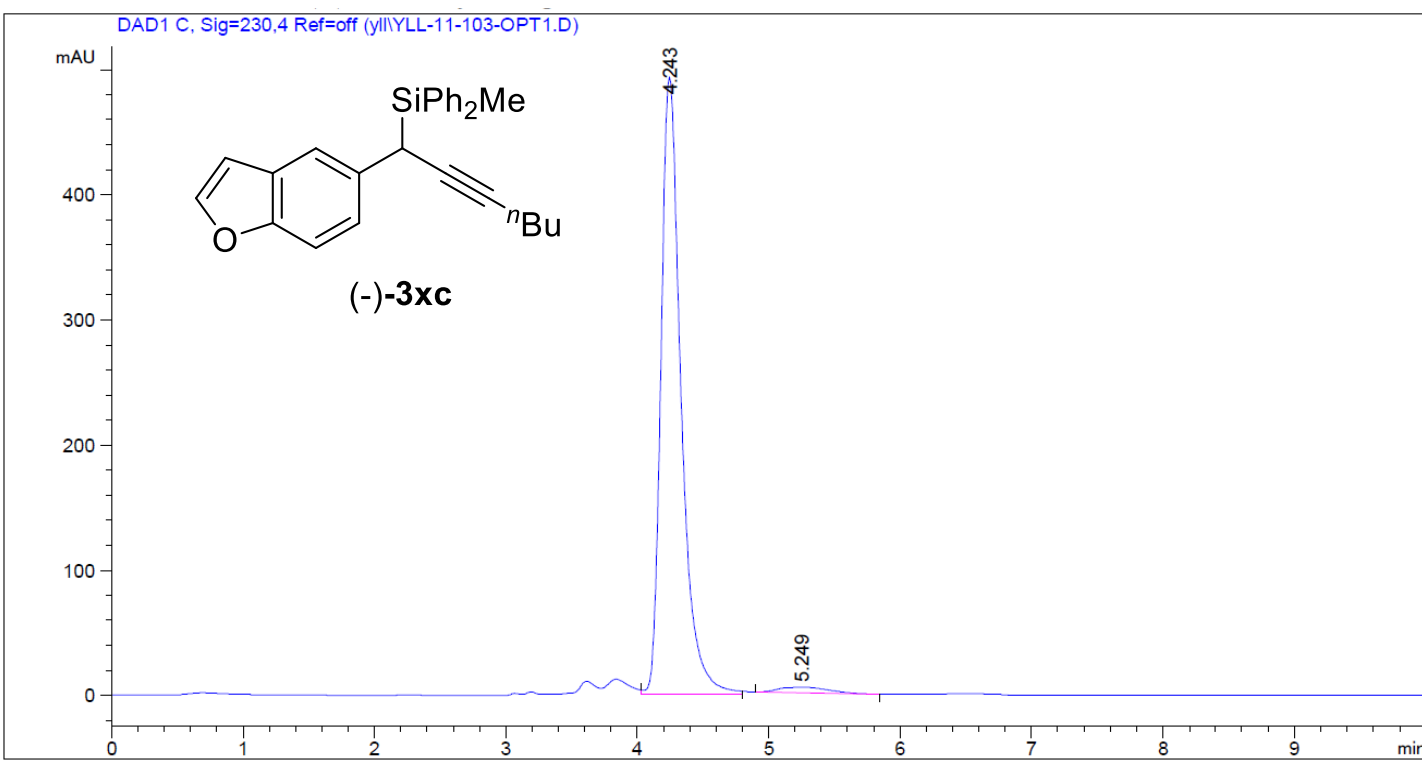

\begin{tabular}{|c|c|c|c|c|}
\hline $\begin{array}{c}\text { Peak } \\
\text { \# }\end{array}$ & $\begin{array}{l}\text { RetTime Type } \\
\text { [min] }\end{array}$ & $\begin{array}{l}\text { Width } \\
\text { [min] }\end{array}$ & $\begin{array}{c}\text { Area } \\
{\left[\mathrm{mAU}^{*} \mathrm{~s}\right]}\end{array}$ & $\begin{array}{l}\text { Height } \\
\text { [mAU] }\end{array}$ \\
\hline
\end{tabular}
- - - | - - - - - | - - - | - - - - | - - - - - - | - - - - - - | - - - - |

$\begin{array}{lllllll}1 & 4.243 & \text { MM R } & 0.1762 & 5209.85938 & 492.91833 & 97.7980\end{array}$
25.249 BB
$0.3799 \quad 117.30347$
$4.47390 \quad 2.2020$ 
(+)-(Z)-triethyl(1-(naphthalen-2-yl)hept-2-en-1-yl)silane (4)
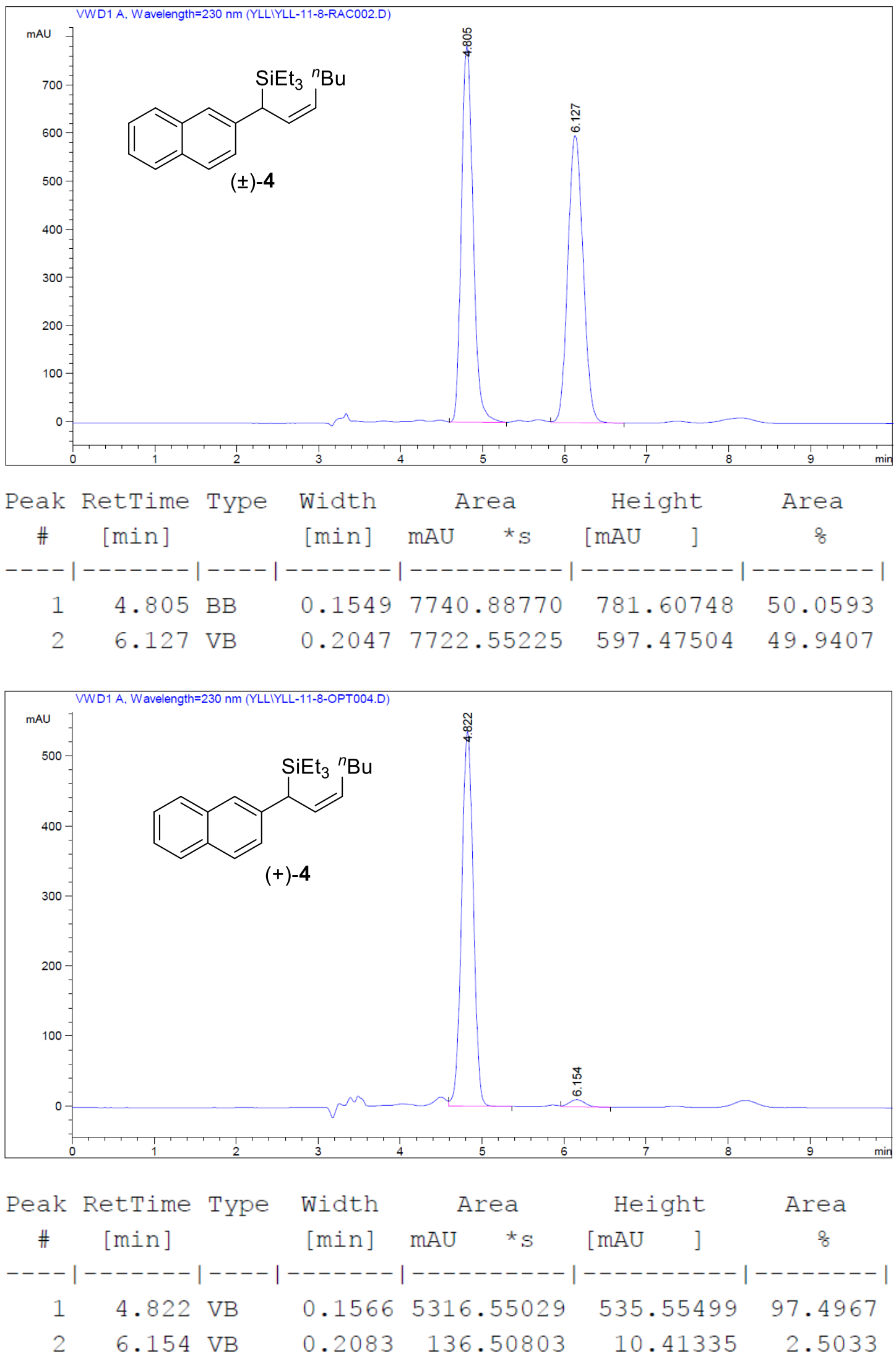
(+)-(Z)-methyl(1-(naphthalen-2-yl)-3-(4,4,5,5-tetramethyl-1,3,2-dioxaborolan-2yl)but-2-en-1-yl)diphenylsilane (5)
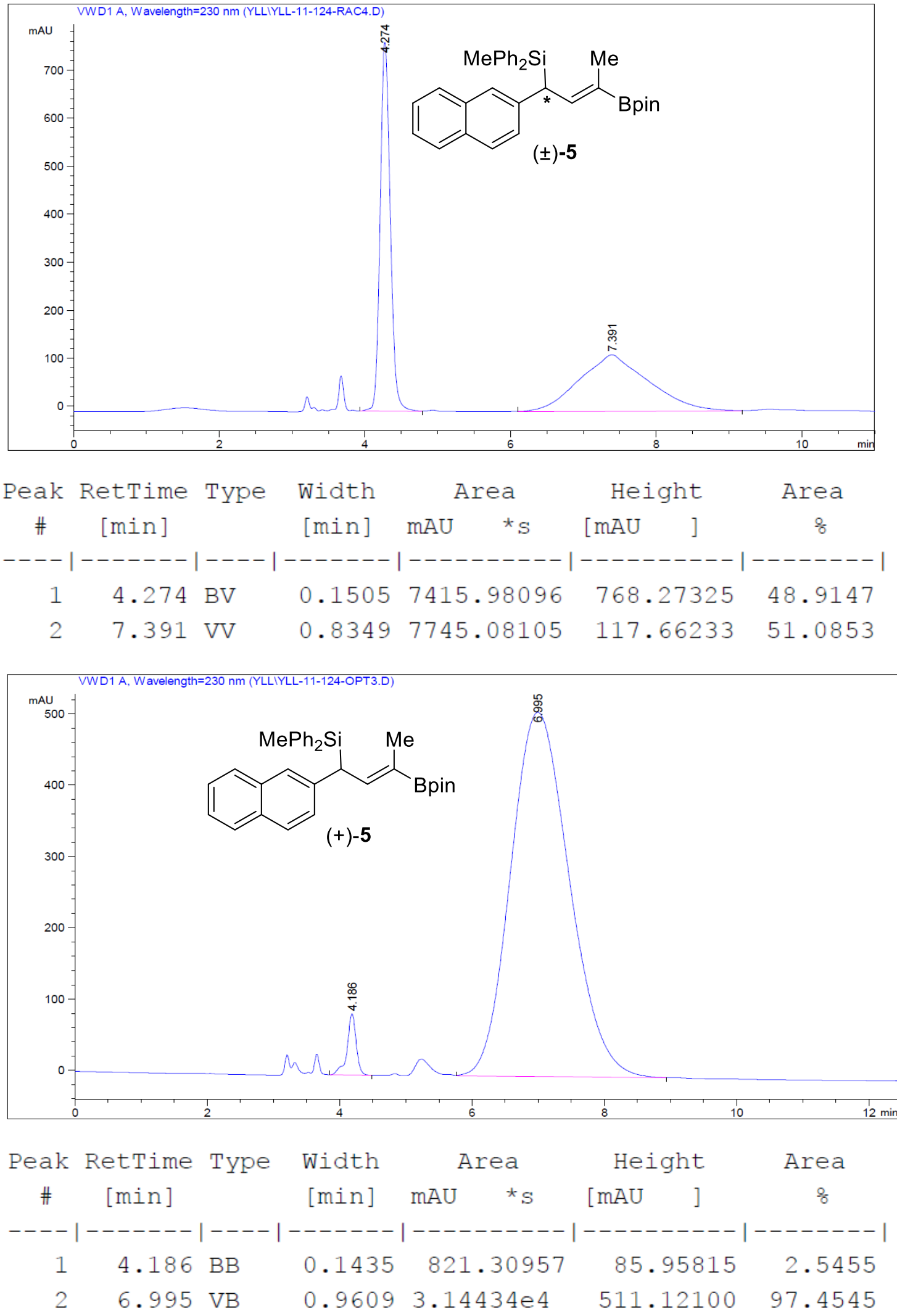
(S)-dimethyl(1-(naphthalen-2-yl)-3-phenylpropyl)(phenyl)silane (6)

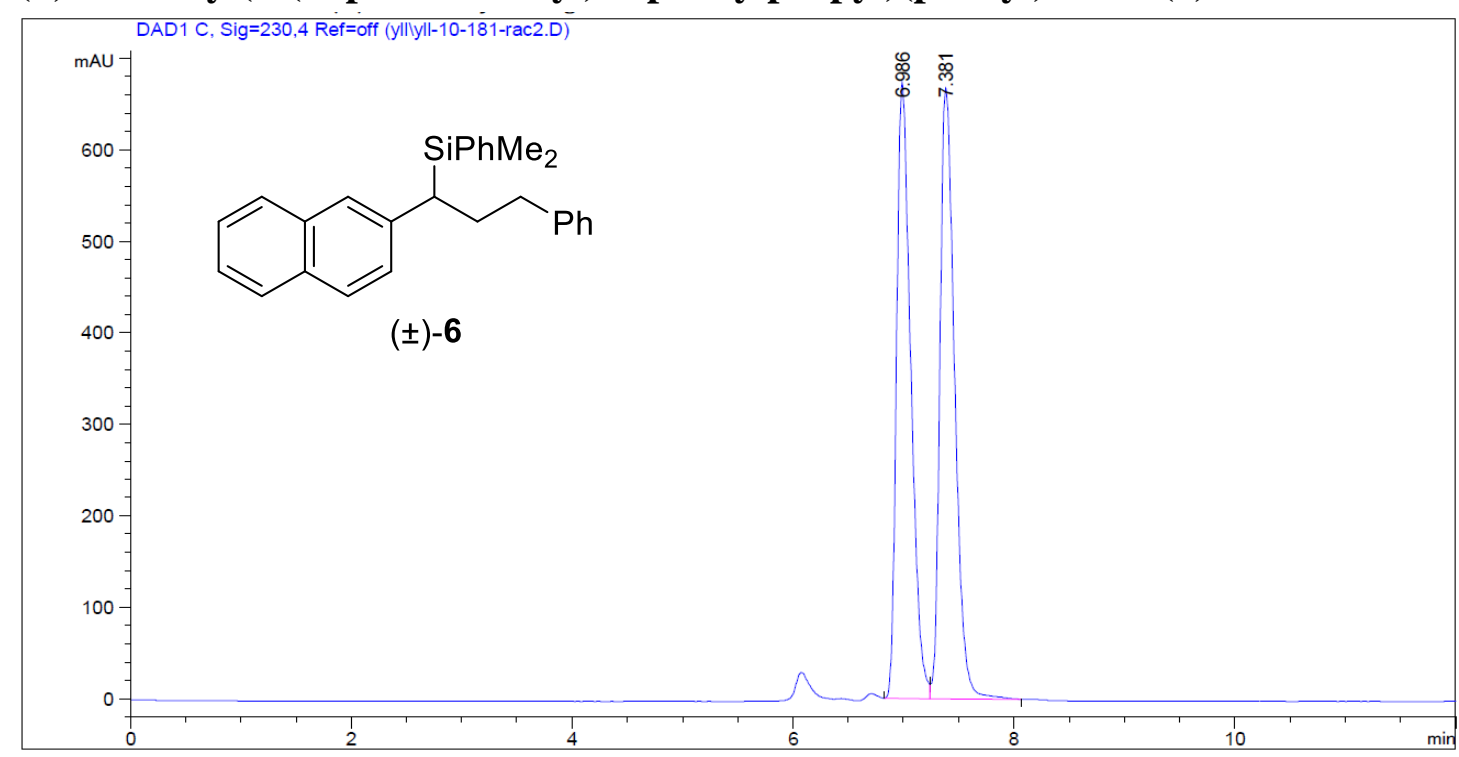

Peak RetTime Type Width Area Height Area

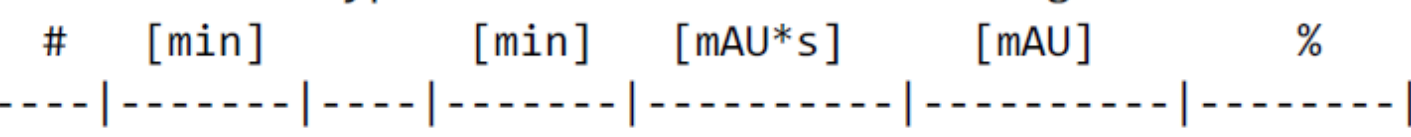
$16.986 \mathrm{BV}$
$0.1346 \quad 6042.64307 \quad 674.02802 \quad 49.3954$
27.381 VB
$0.14416190 .56299668 .20685 \quad 50.6046$

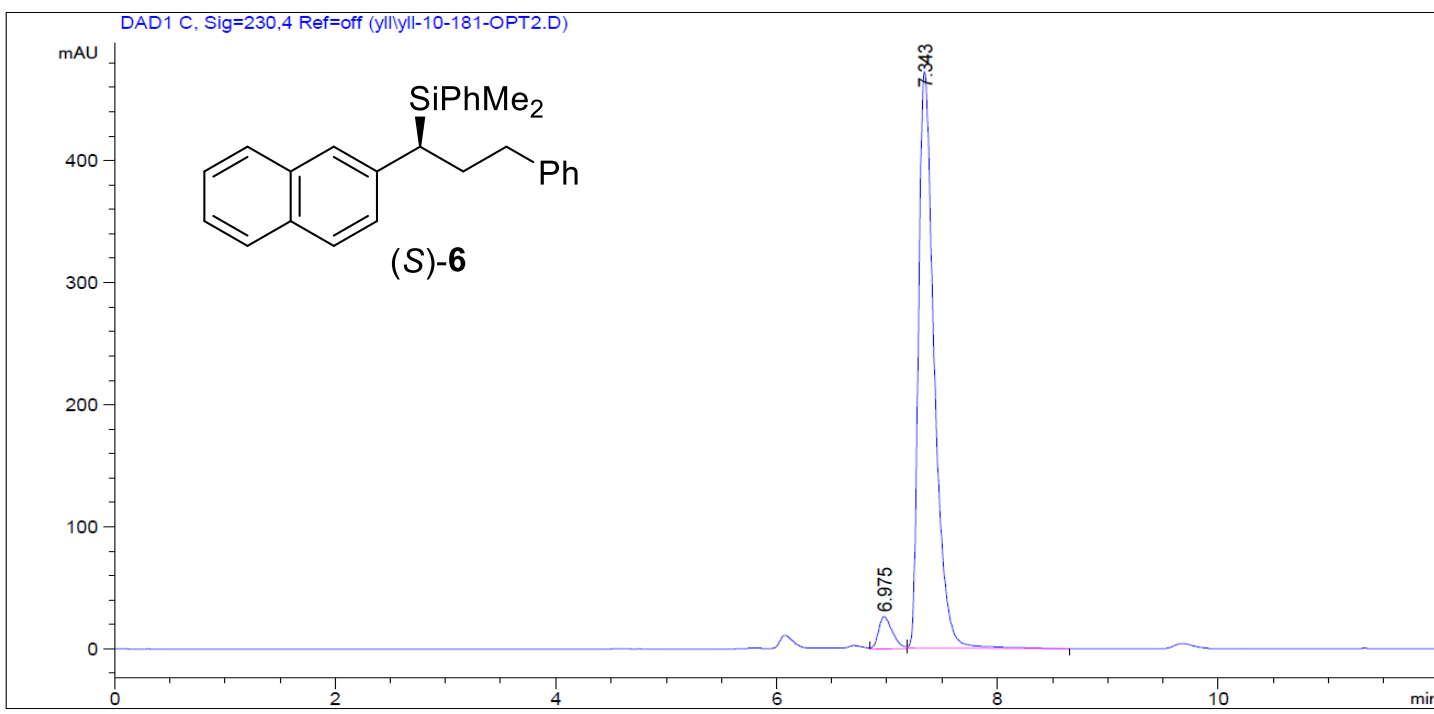

Peak RetTime Type Width Area Height Area

\# [min] [min $] \quad[\mathrm{mAU} * \mathrm{~s}] \quad[\mathrm{mAU}] \quad \%$
- - - | - - - - | - - | - - - - | - - - - - | - - - - - | - - - - |

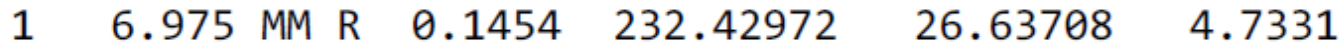

$\begin{array}{lllllll}2 & 7.343 & \text { VB } & 0.1496 & 4678.33838 & 472.10629 & 95.2669\end{array}$ 
(S)-1-(naphthalen-2-yl)-3-phenylpropan-1-ol (6ol)

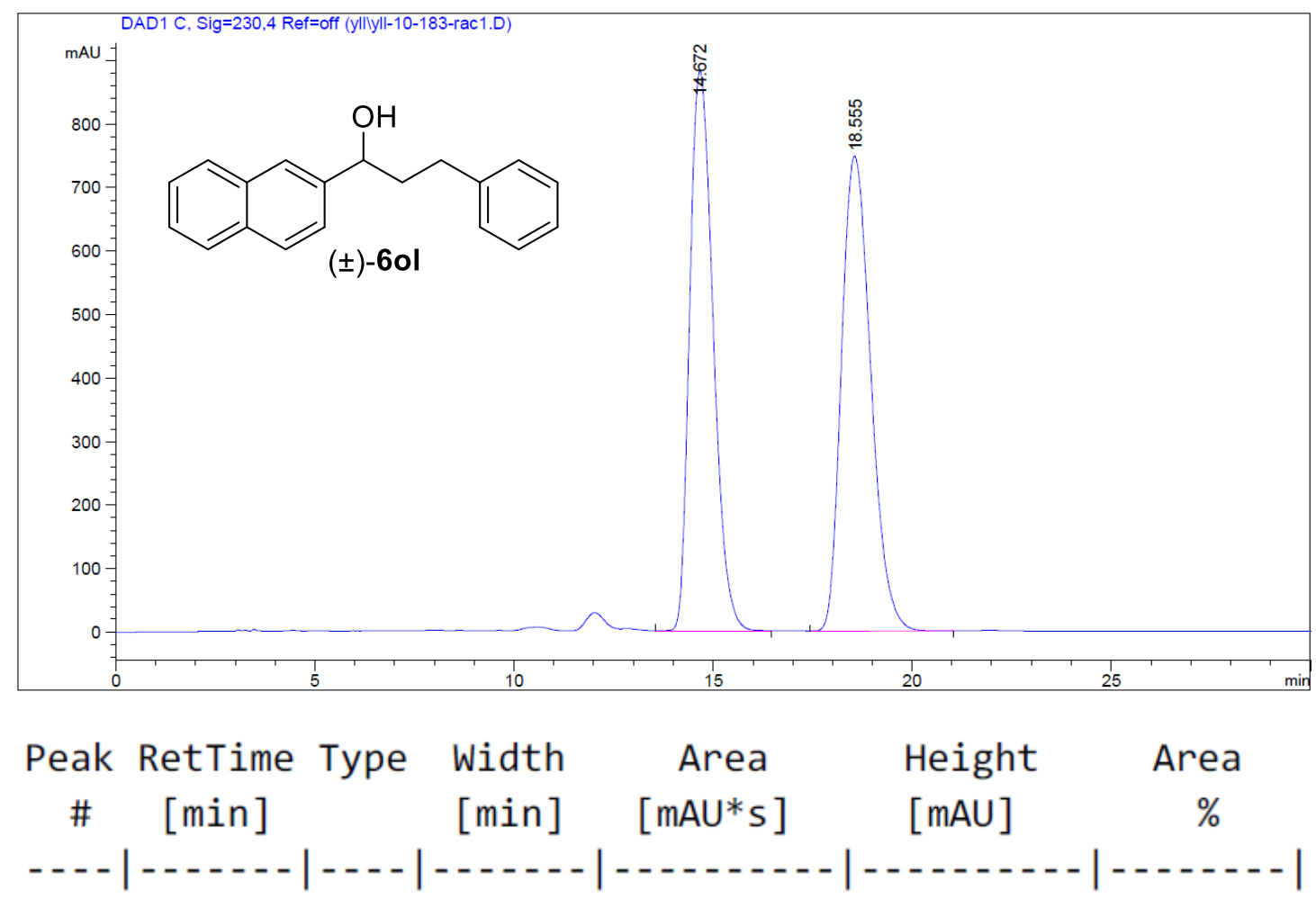

$\begin{array}{lllllll}1 & 14.672 & \text { BB } & 0.6511 & 3.60250 \mathrm{e} 4 & 881.01929 & 48.5072\end{array}$

$\begin{array}{lllllll}2 & 18.555 & \text { BB } & 0.8086 & 3.82424 \mathrm{e} 4 & 747.79346 & 51.4928\end{array}$

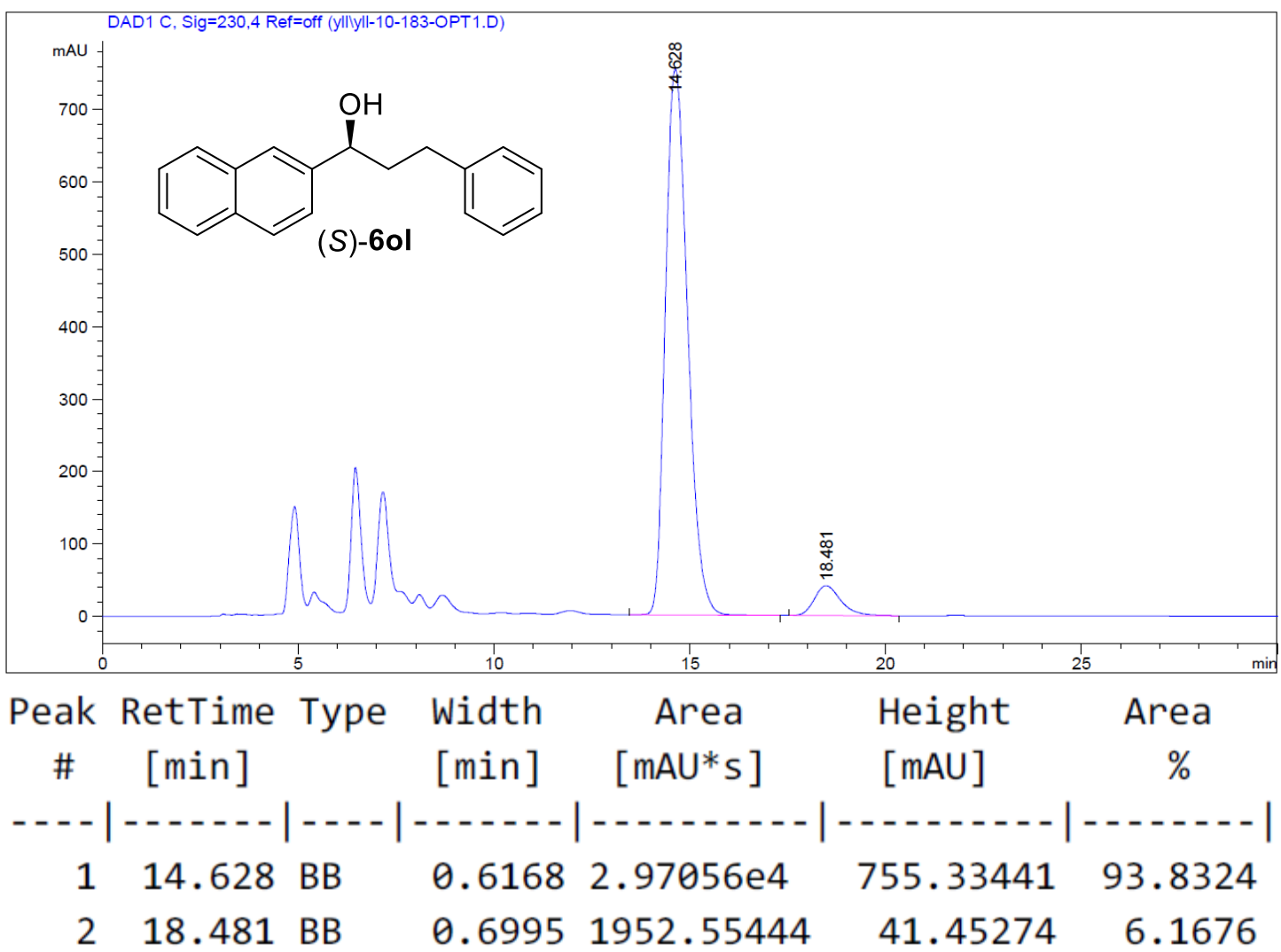




\section{(S)-methyl(1-(naphthalen-2-yl)butyl)diphenylsilane (3kc-H)}
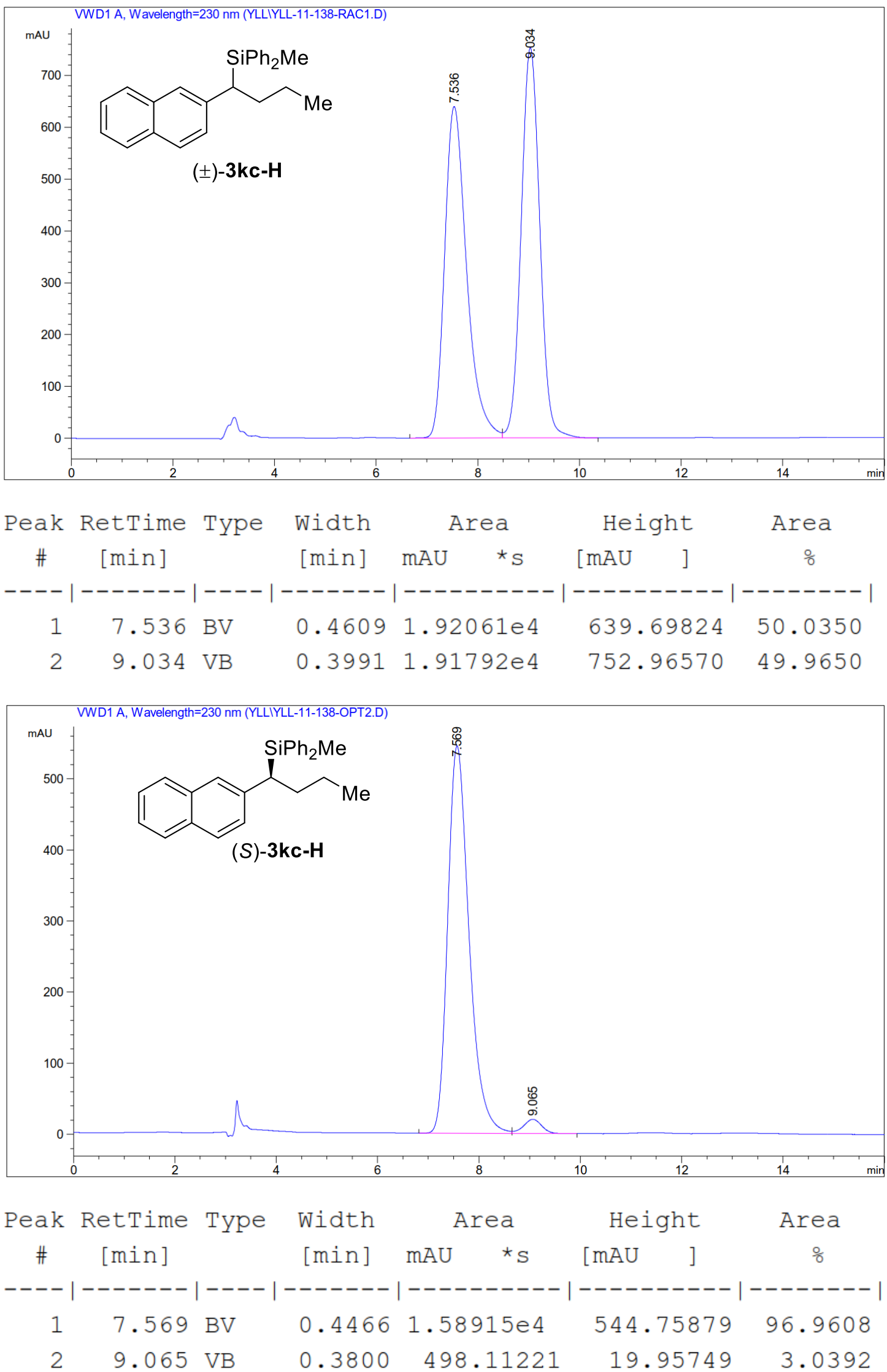
(S)-1-(naphthalen-2-yl)butan-1-ol (3kc-ol)
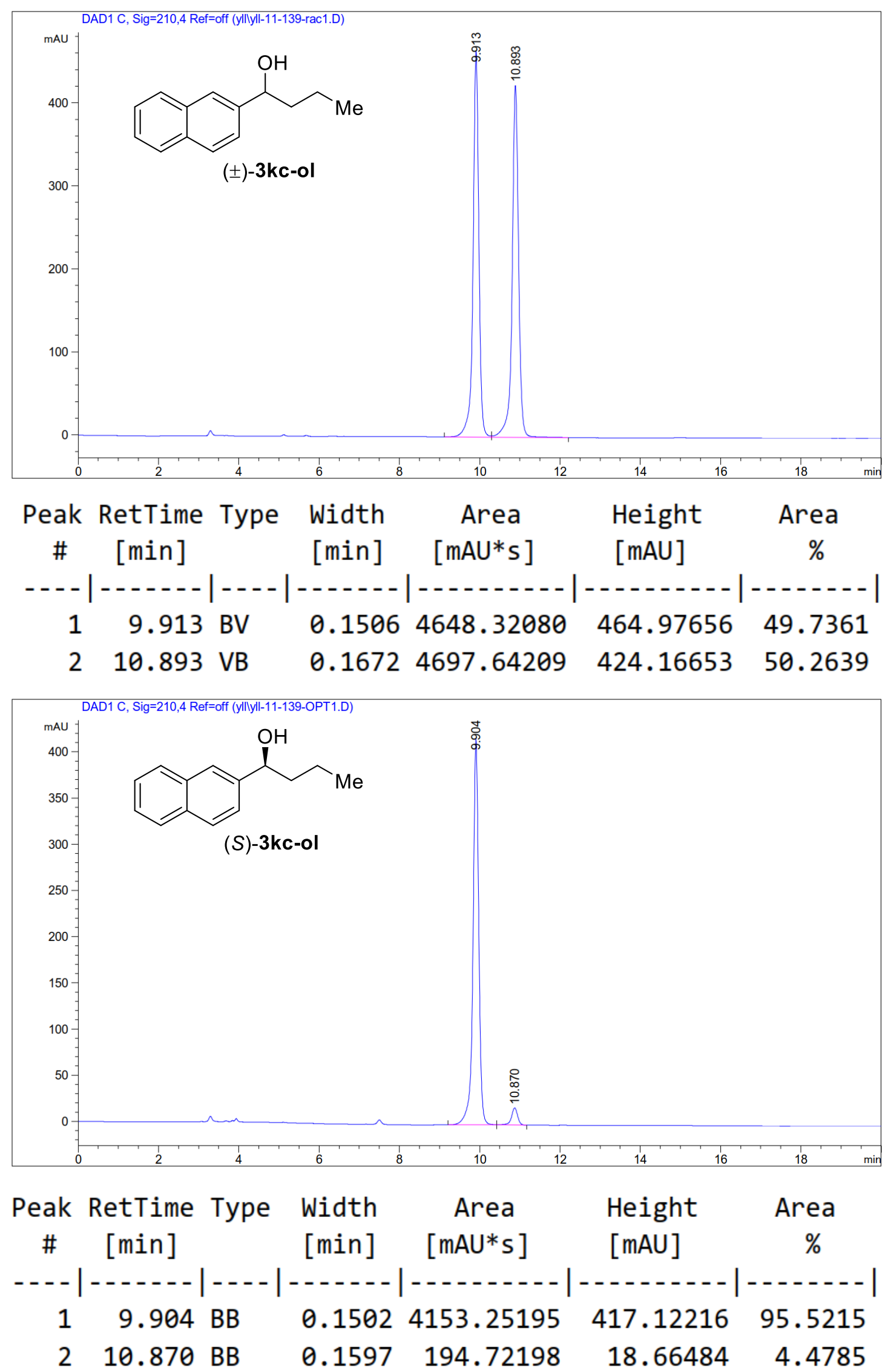
(-)-methyl(1-(naphthalen-2-yl)hepta-1,2-dien-1-yl)diphenylsilane (7a)

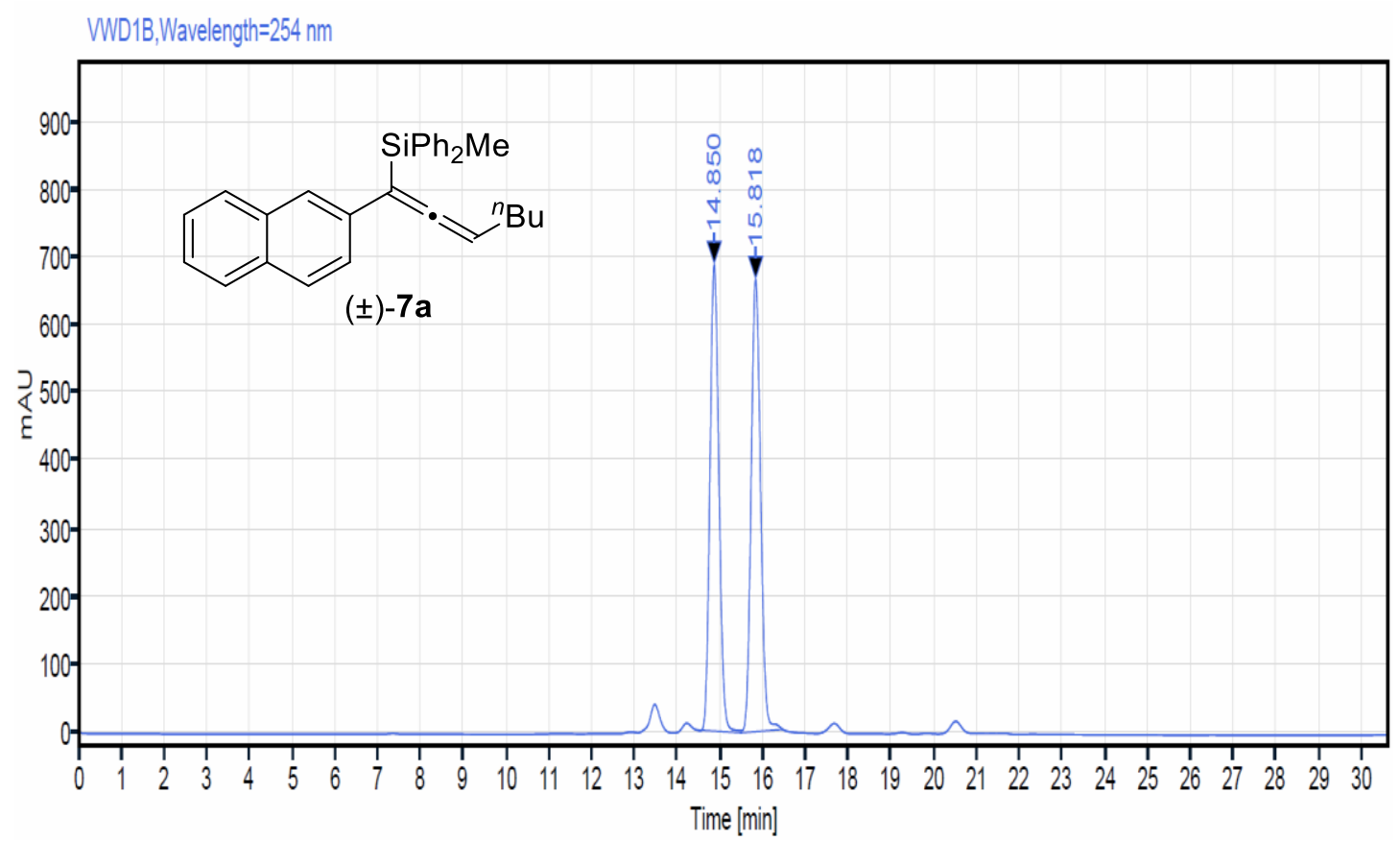

$\begin{array}{rrrrrr}\text { RT [min] } & \text { Type } & \text { Width [min] } & \text { Area } & \text { Height } & \text { Area\% } \\ 14.850 & \text { MM m } & 0.23 & 10024.13 & 688.04 & 49.54 \\ 15.818 & \text { VM m } & 0.24 & 10209.70 & 666.55 & 50.46\end{array}$

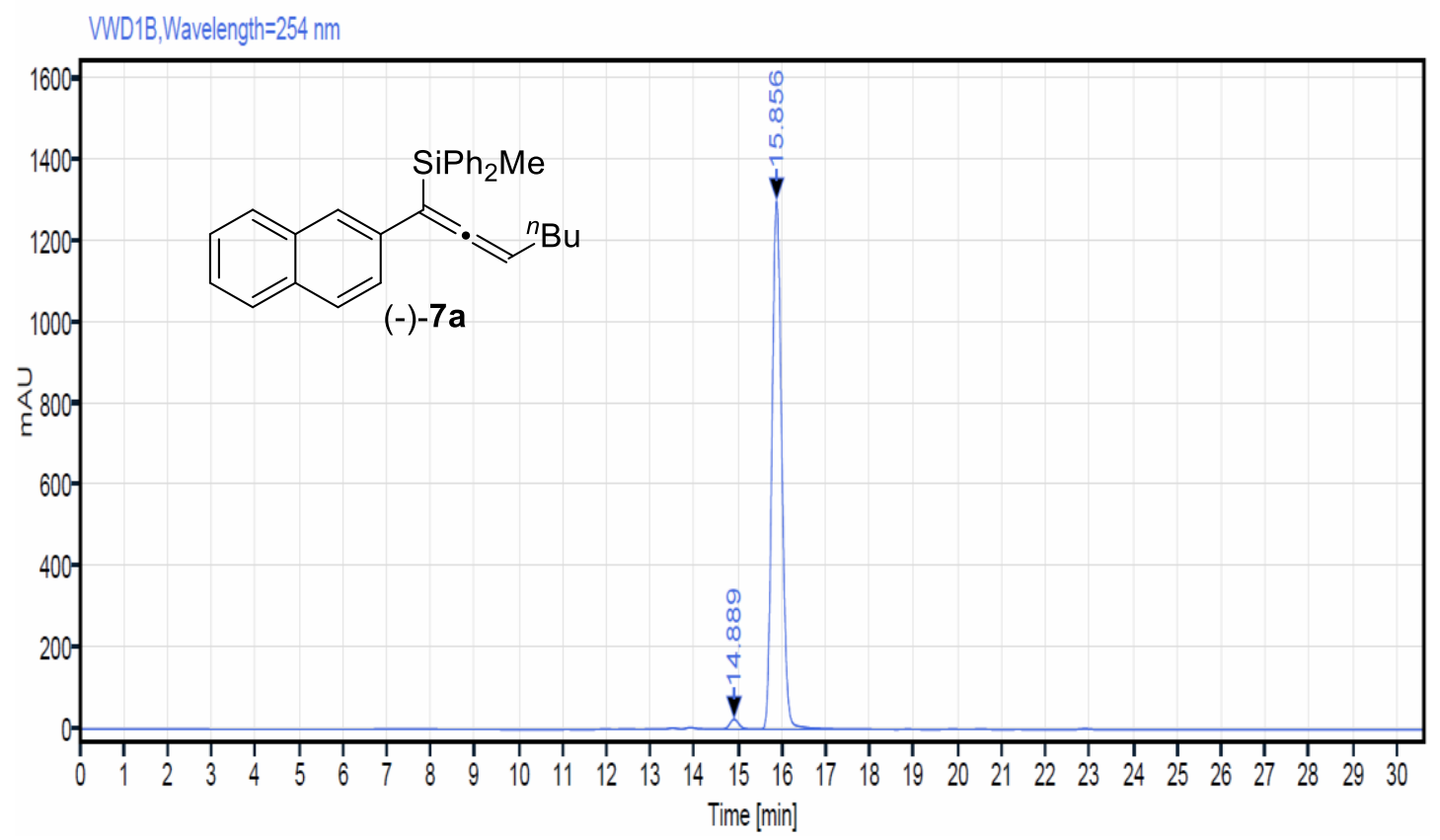

$\begin{array}{rrrrrr}\text { RT [min] } & \text { Type } & \text { Width [min] } & \text { Area } & \text { Height } & \text { Area\% } \\ 14.889 & \text { BB } & 1.05 & 354.58 & 24.46 & 1.74 \\ 15.856 & \text { BB } & 2.04 & 20071.13 & 1302.51 & 98.26\end{array}$


(S)-methyl(1-(naphthalen-2-yl)buta-1,2-dien-1-yl)diphenylsilane (7b)
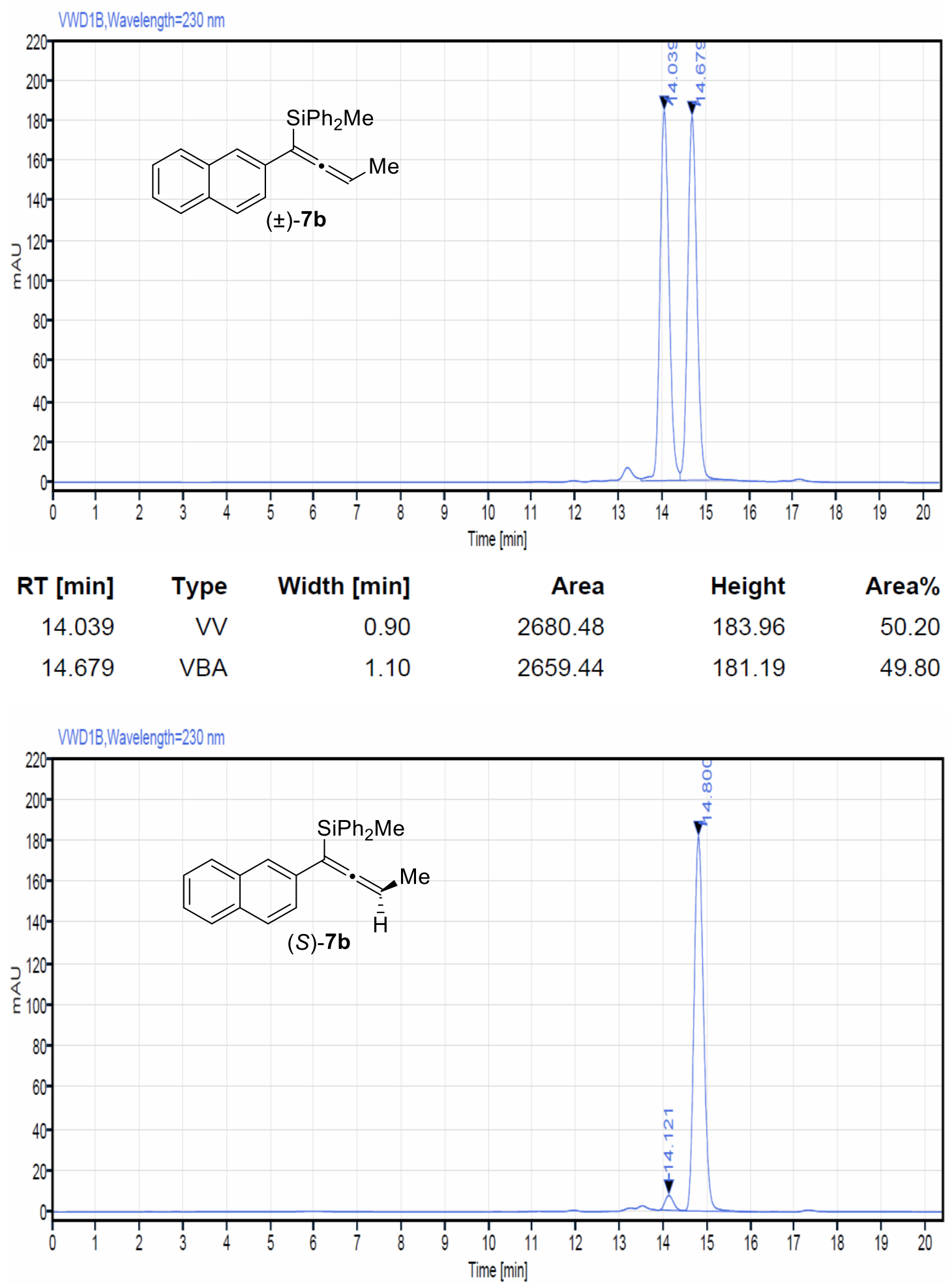

$\begin{array}{rrrrrr}\text { RT [min] } & \text { Type } & \text { Width [min] } & \text { Area } & \text { Height } & \text { Area\% } \\ 14.121 & \text { MB m } & 0.21 & 95.97 & 7.16 & 3.39 \\ 14.800 & \text { BB } & 1.55 & 2734.59 & 181.55 & 96.61\end{array}$


(-)-(3-cyclopropyl-1-(naphthalen-2-yl)propa-1,2-dien-1-yl)(methyl)diphenylsilane (7c)

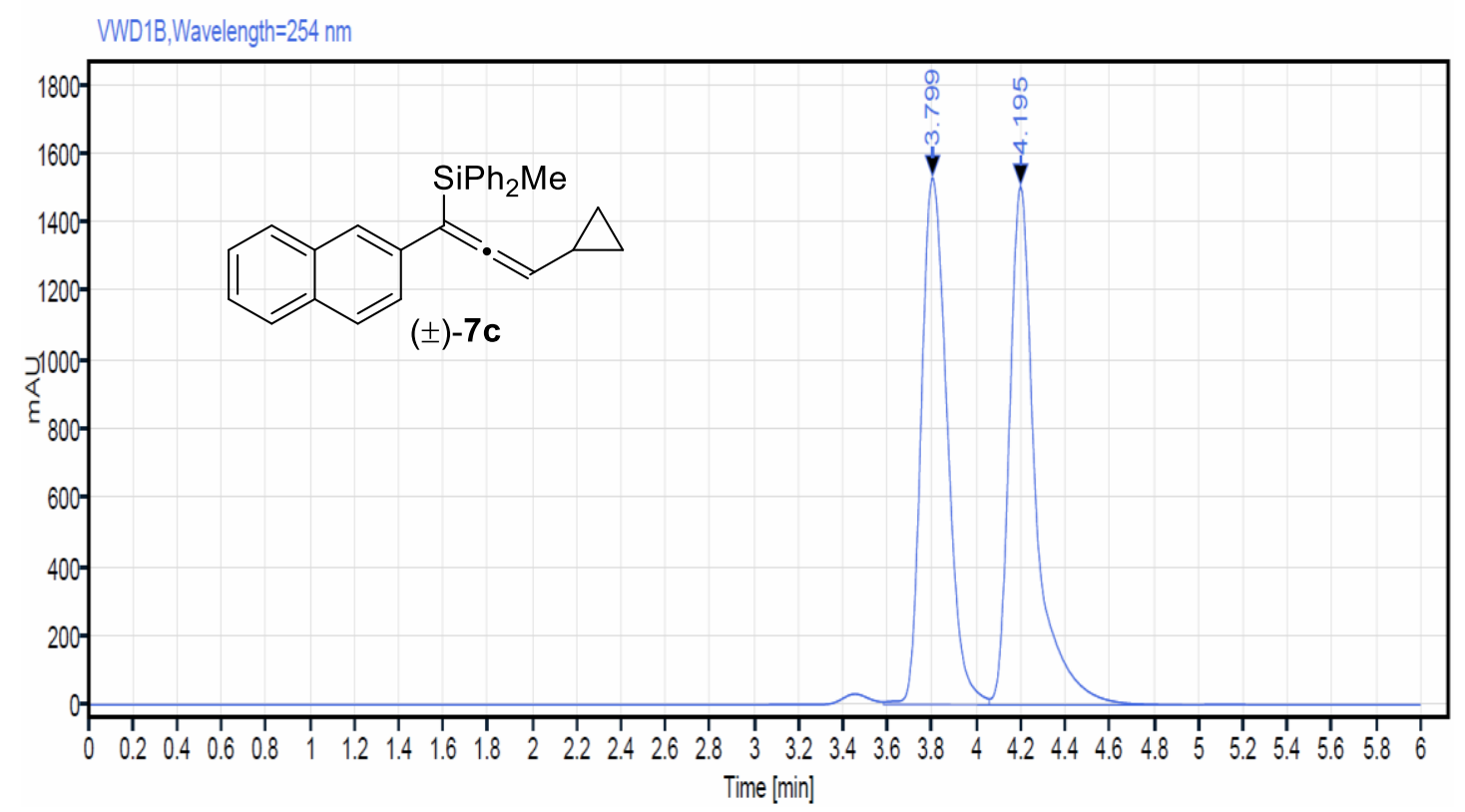

$\begin{array}{rrrrrr}\text { RT [min] } & \text { Type } & \text { Width [min] } & \text { Area } & \text { Height } & \text { Area\% } \\ 3.799 & \text { VV } & 0.48 & 12339.32 & 1531.62 & 50.04 \\ 4.195 & \text { VB } & 0.87 & 12319.63 & 1506.62 & 49.96\end{array}$

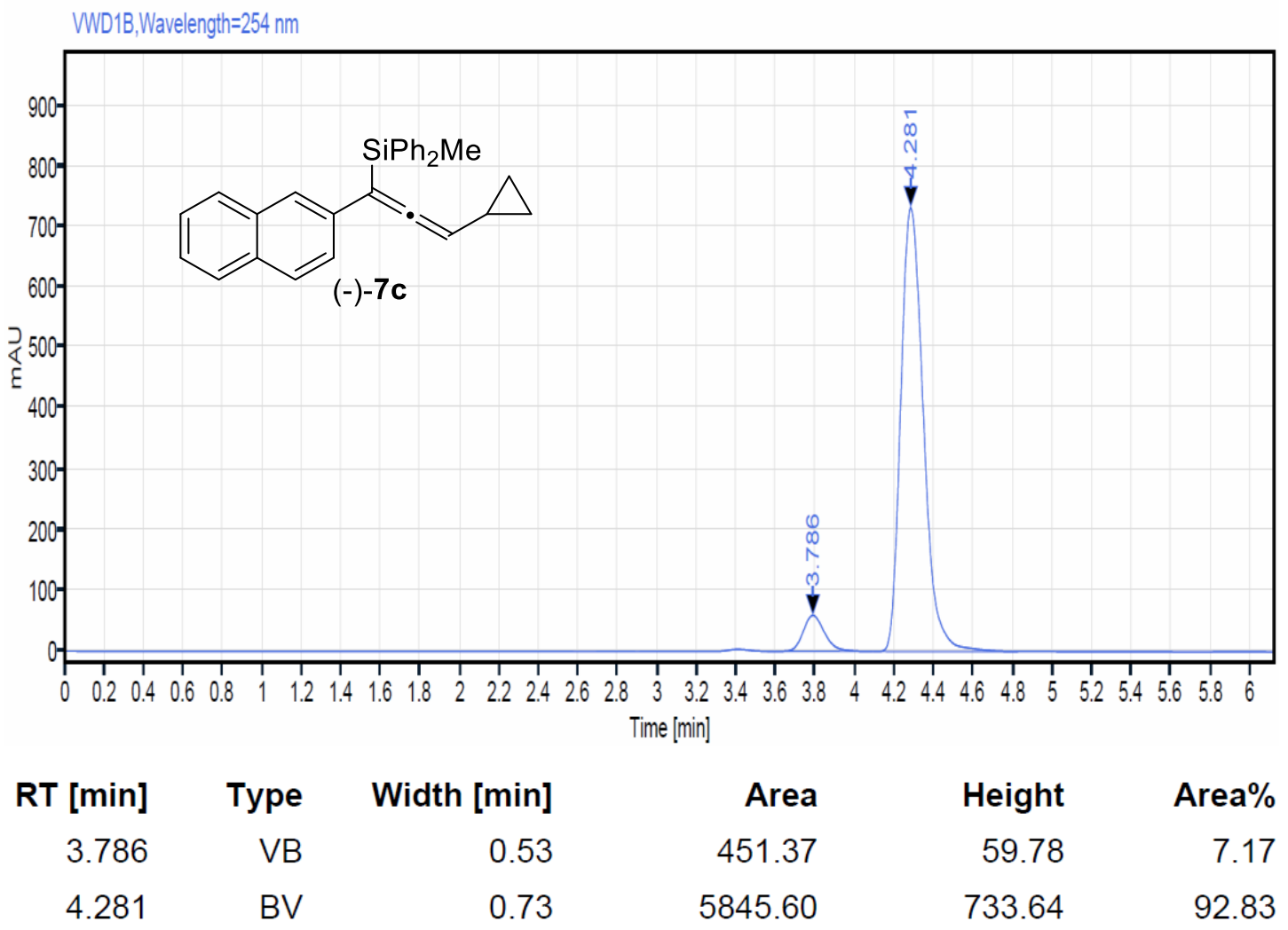


(-)-(3-(cyclohex-1-en-1-yl)-1-(naphthalen-2-yl)propa-1,2-dien-1-

yl)(methyl)diphenylsilane (7d)

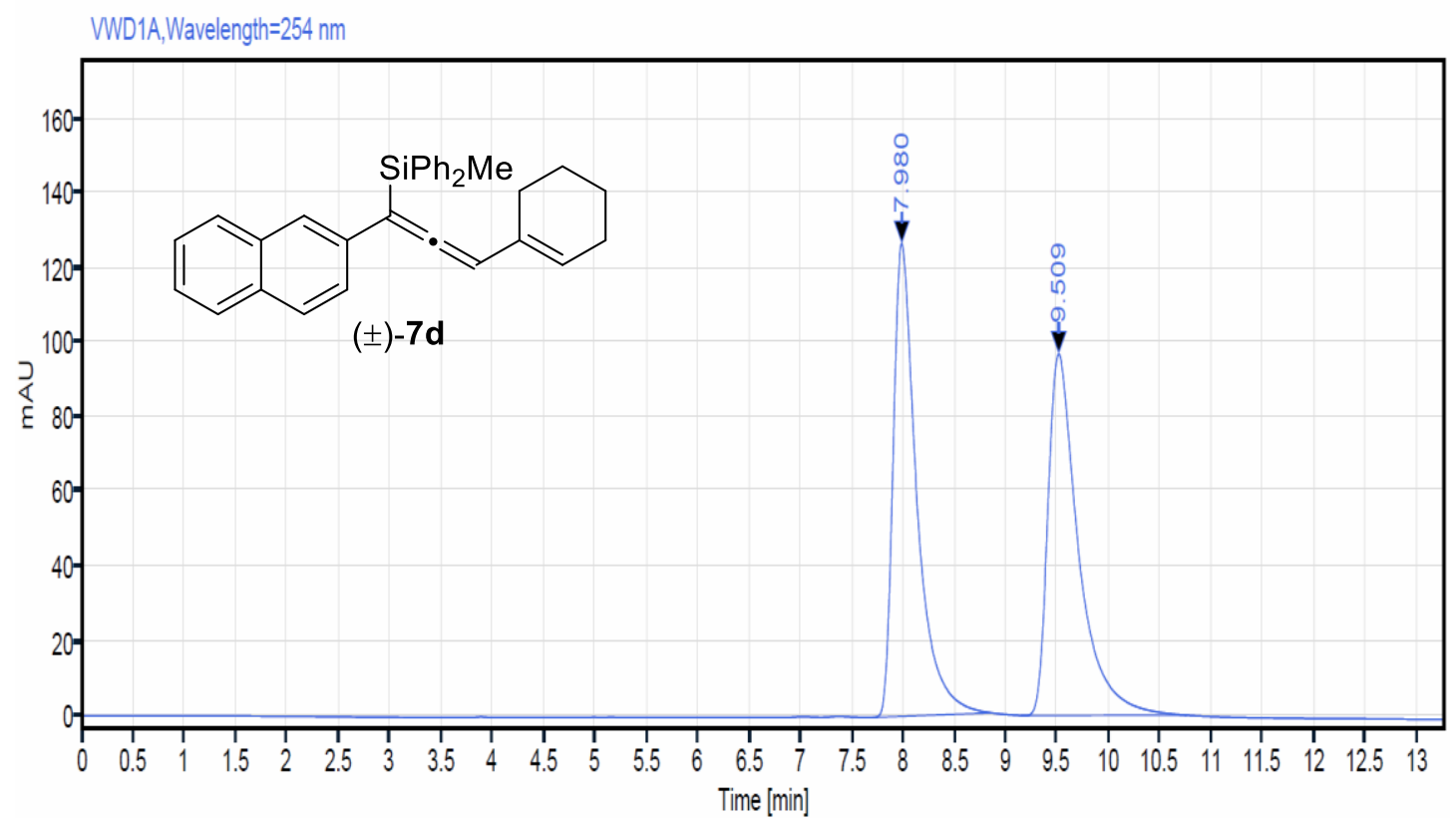

$\begin{array}{rrrrrr}\text { RT [min] } & \text { Type } & \text { Width [min] } & \text { Area } & \text { Height } & \text { Area\% } \\ 7.980 & \text { MM m } & 0.24 & 2027.26 & 126.80 & 50.24 \\ 9.509 & \text { MM m } & 0.31 & 2007.80 & 96.94 & 49.76\end{array}$

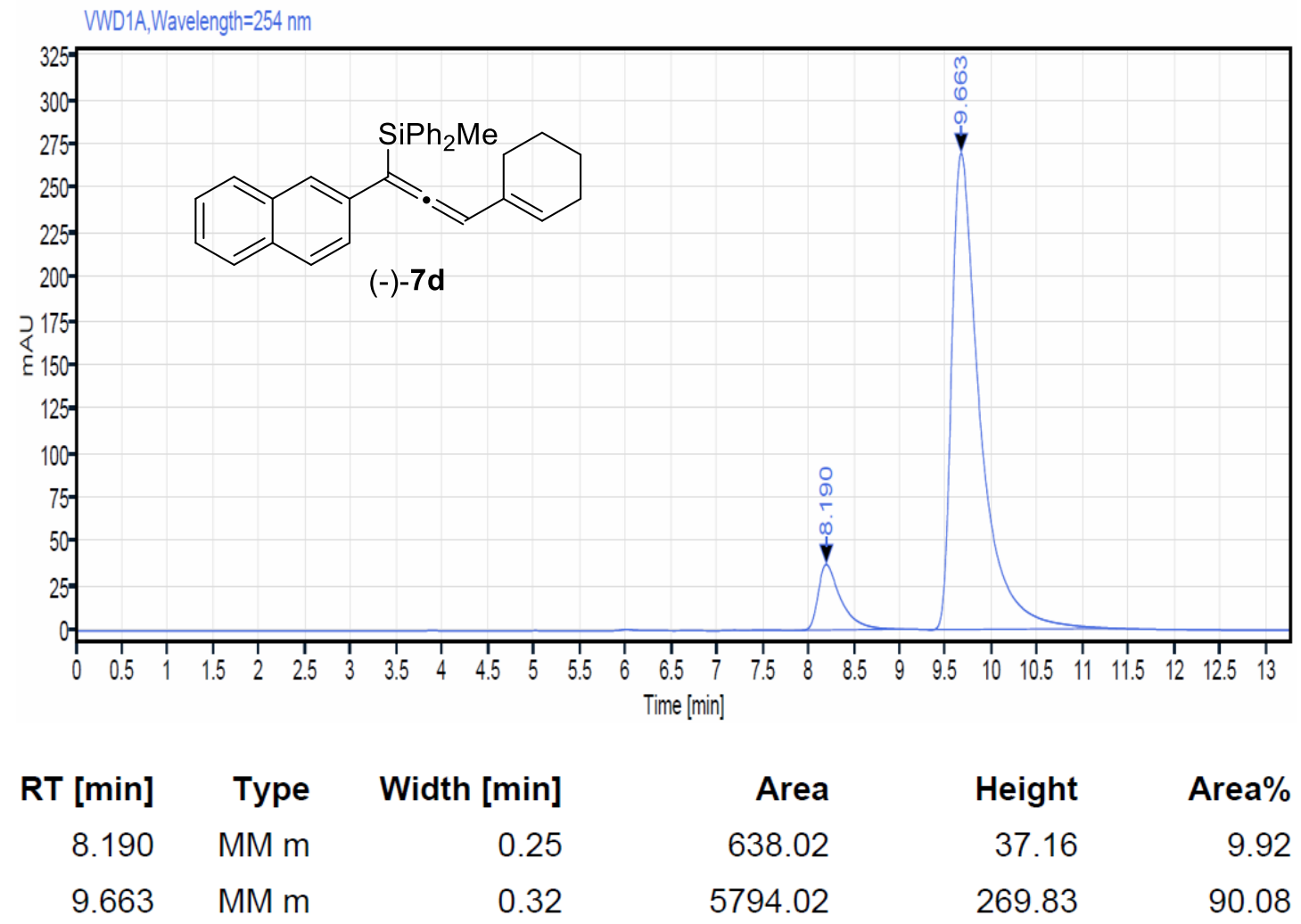


(-)-methyl(1-(naphthalen-2-yl)-3-phenylpropa-1,2-dien-1-yl)diphenylsilane (7e)

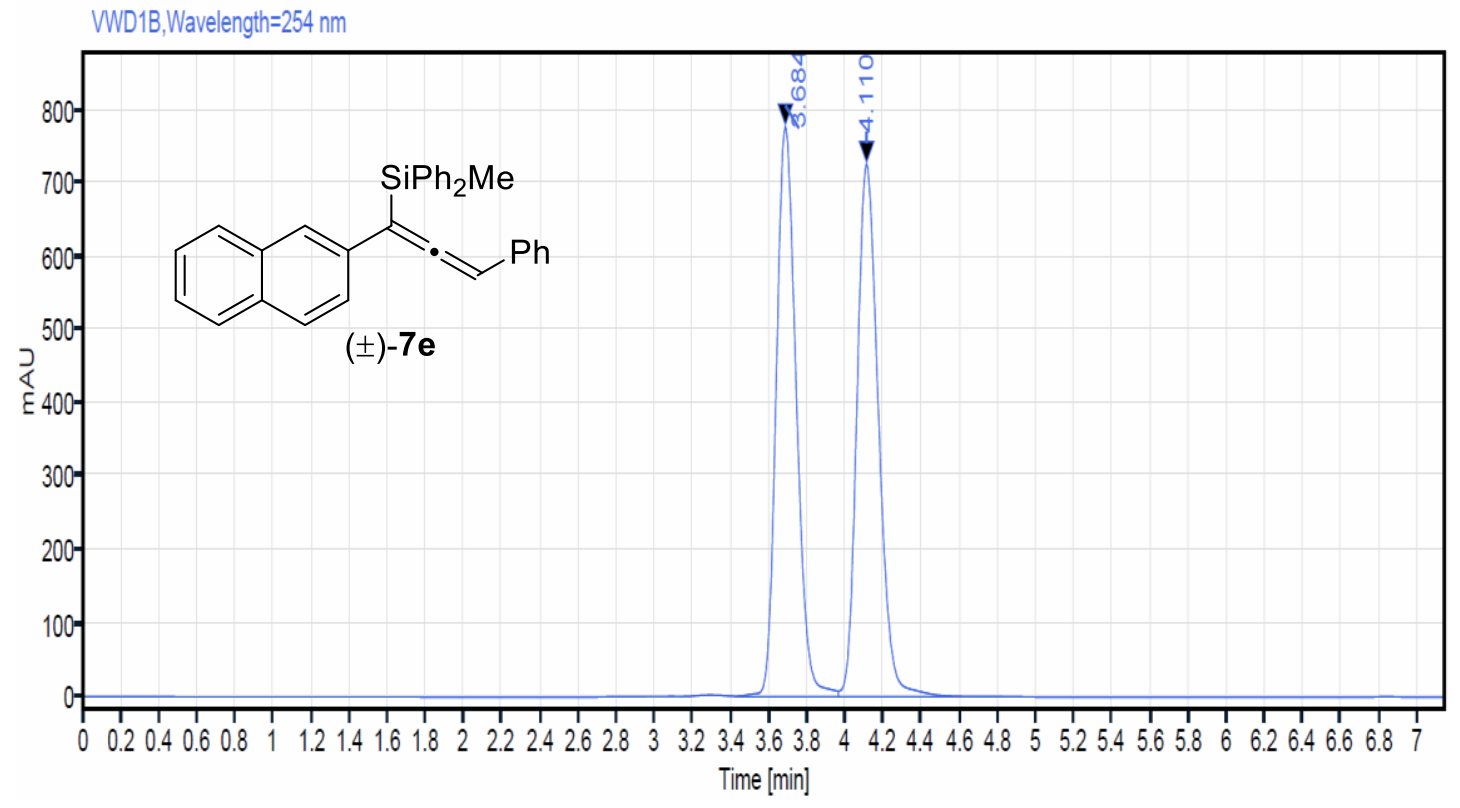

$\begin{array}{rrrrrr}\text { RT [min] } & \text { Type } & \text { Width [min] } & \text { Area } & \text { Height } & \text { Area\% } \\ 3.684 & \text { VV } & 0.54 & 5673.07 & 778.90 & 49.90 \\ 4.110 & \text { VB } & 0.86 & 5696.24 & 727.78 & 50.10\end{array}$

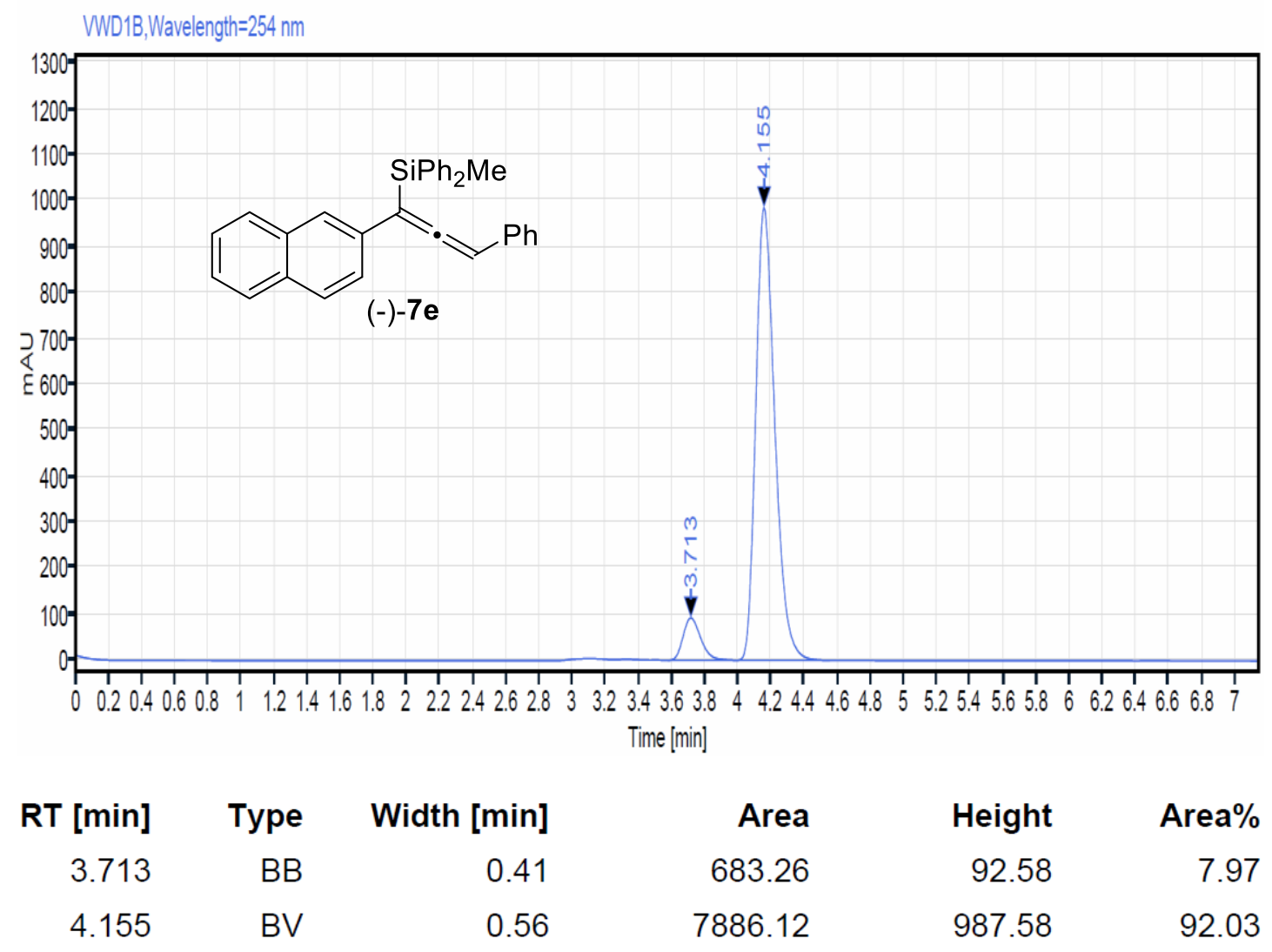



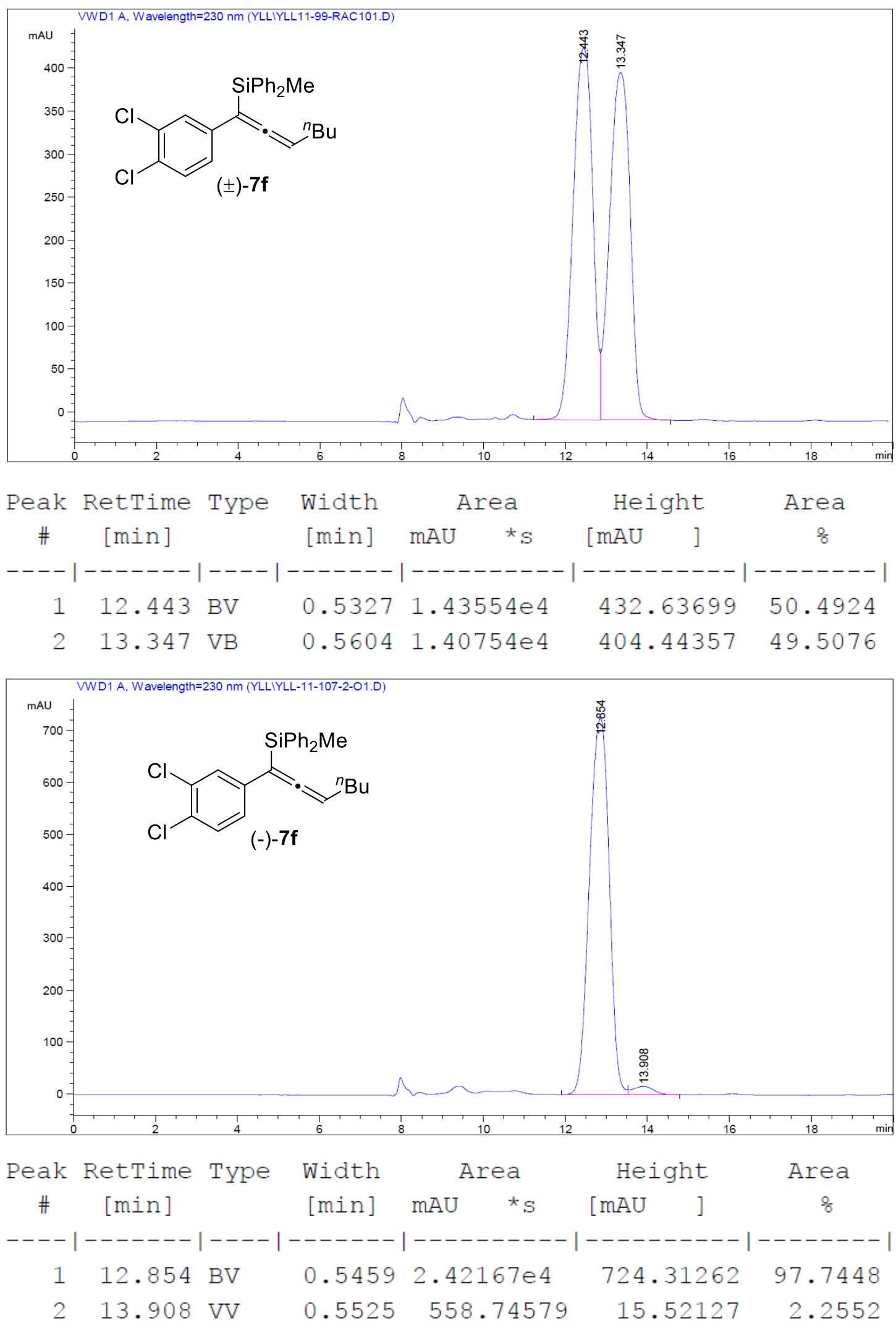
(-)-(1-(3,4-dimethylphenyl)hepta-1,2-dien-1-yl)(methyl)diphenylsilane (7g)

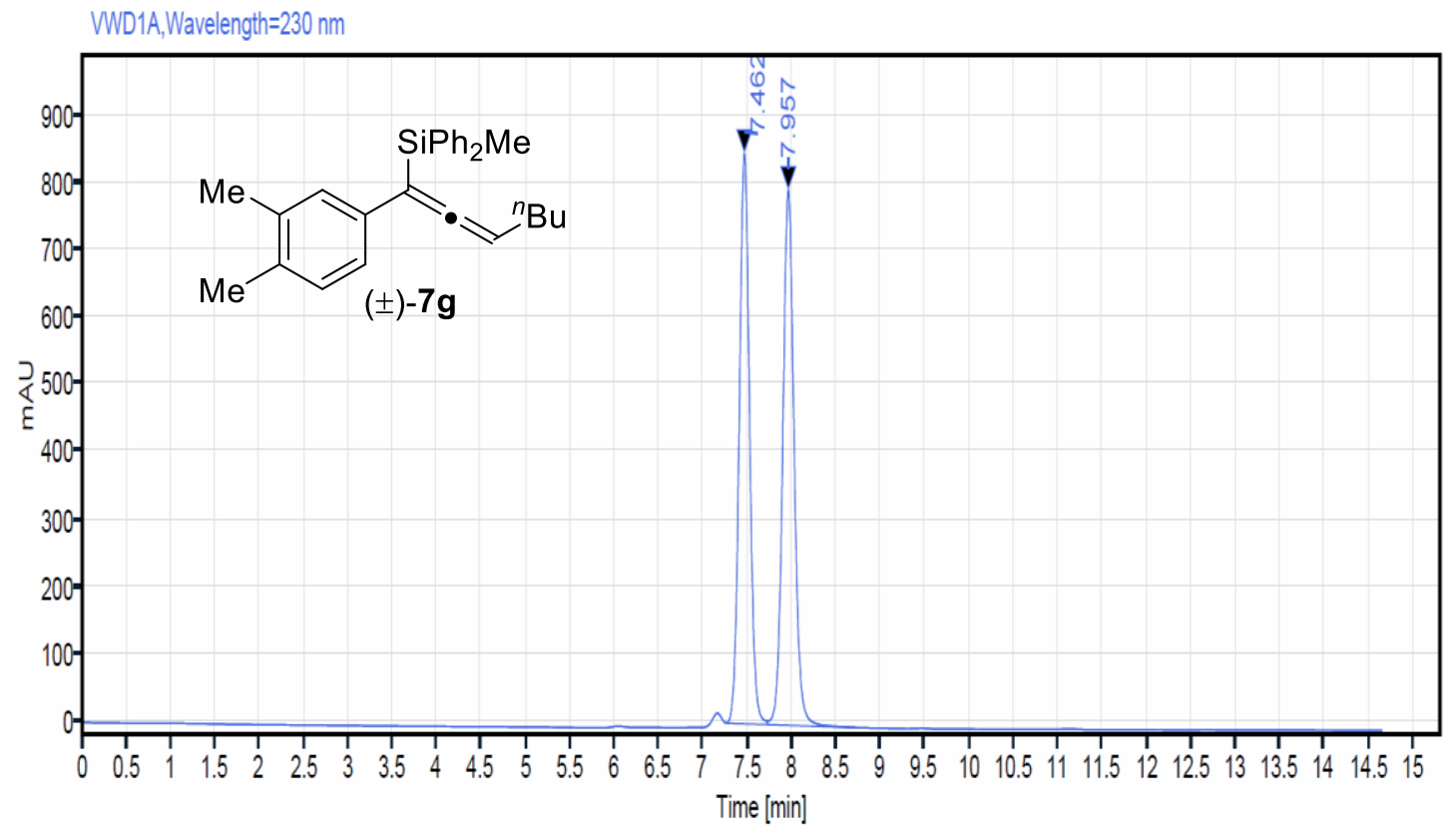

$\begin{array}{rrrrrr}\text { RT [min] } & \text { Type } & \text { Width [min] } & \text { Area } & \text { Height } & \text { Area\% } \\ 7.462 & \text { BV } & 0.46 & 6811.24 & 847.65 & 49.06 \\ 7.957 & \text { VBA } & 1.03 & 7071.16 & 795.22 & 50.94\end{array}$

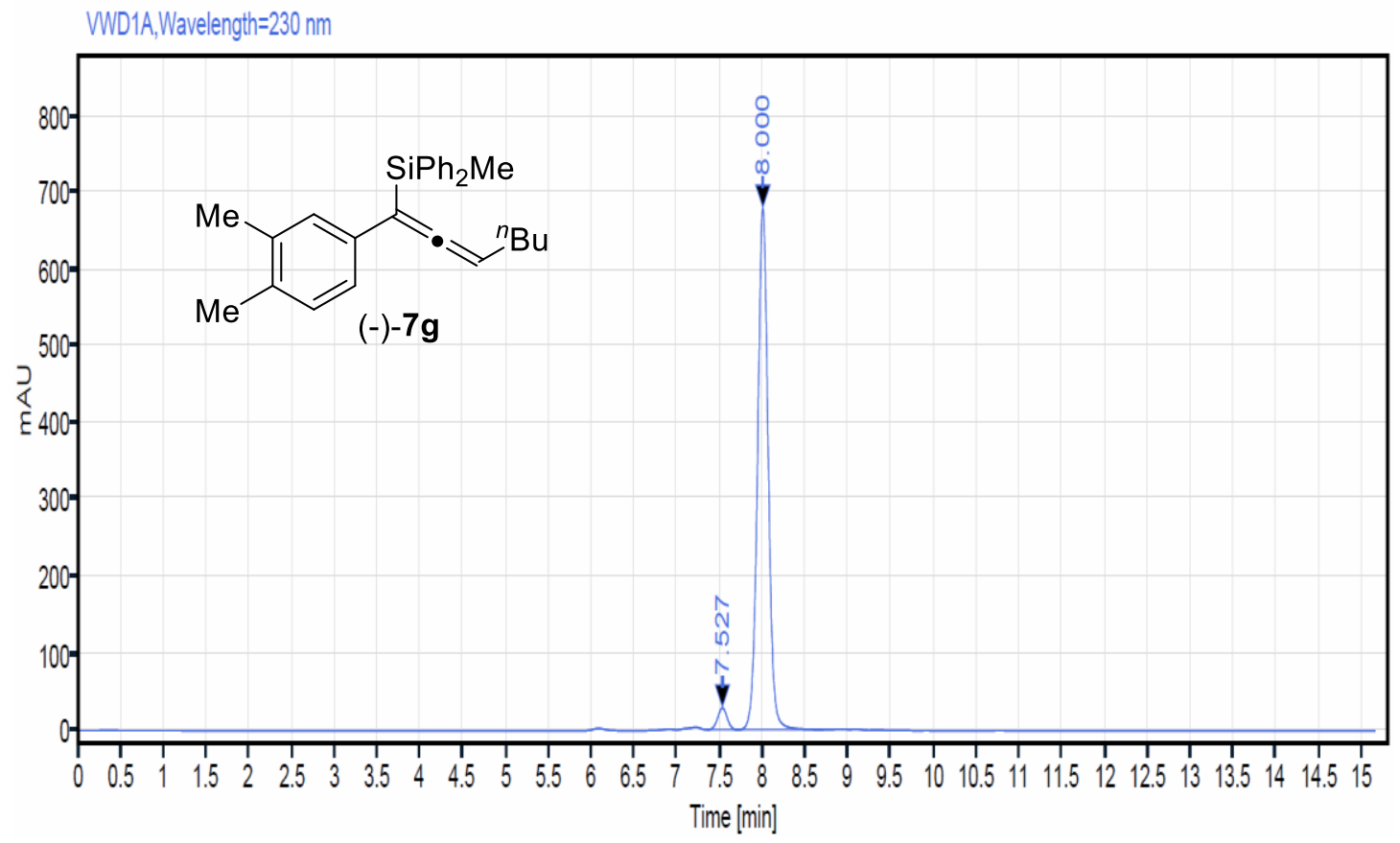

$\begin{array}{rrrrrr}\text { RT [min] } & \text { Type } & \text { Width [min] } & \text { Area } & \text { Height } & \text { Area\% } \\ 7.527 & \text { BB } & 0.36 & 218.91 & 28.43 & 3.60 \\ 8.000 & \text { BB } & 1.00 & 5864.30 & 679.87 & 96.40\end{array}$


(-)-(1-(benzofuran-5-yl)hepta-1,2-dien-1-yl)(methyl)diphenylsilane (7h)
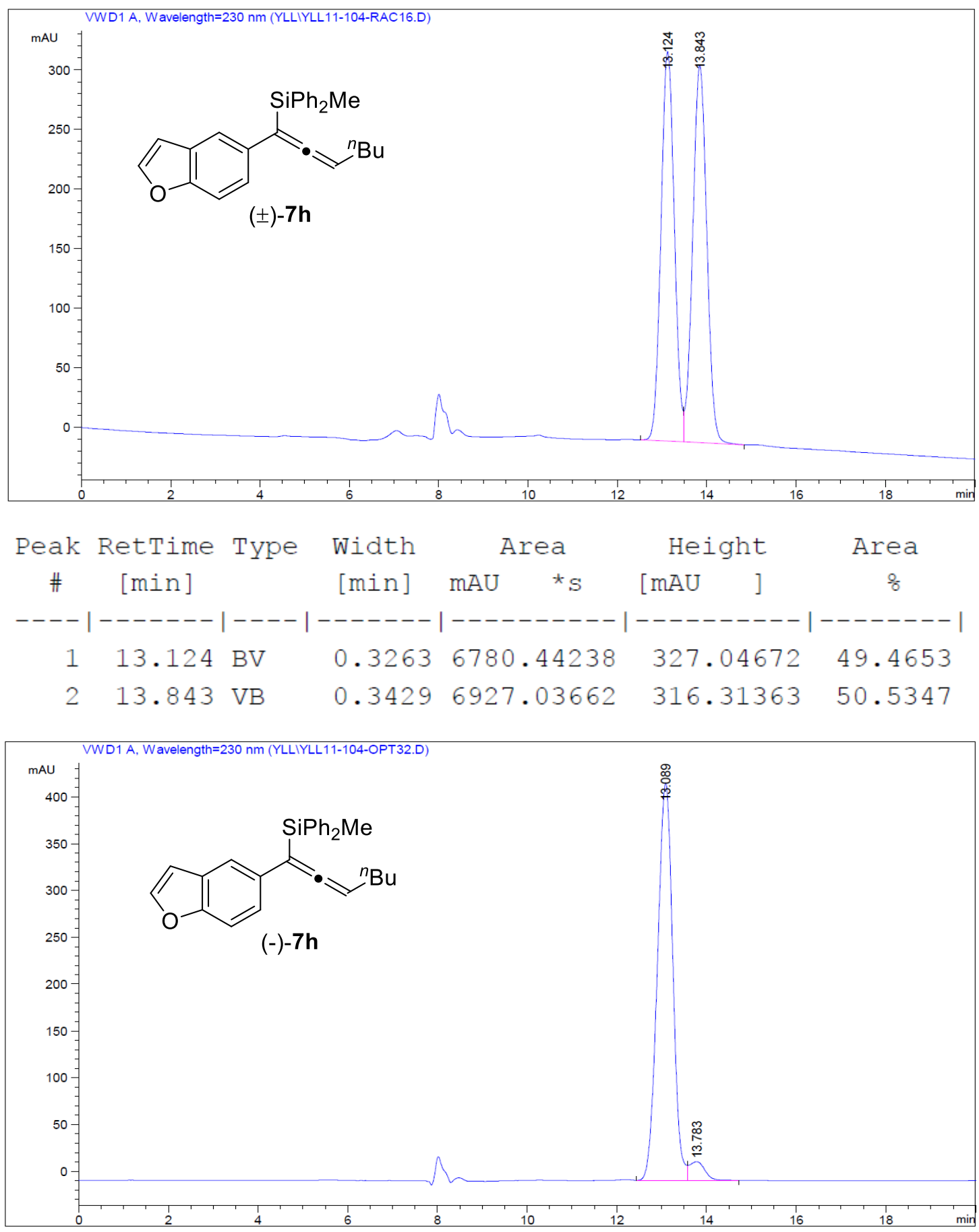

\begin{tabular}{|c|c|c|c|c|c|c|c|}
\hline eak & RetTime & Type & Width & & ea & Height & Area \\
\hline$\#$ & {$[\min ]$} & & [min $]$ & $\mathrm{mAU}$ & ${ }^{*} \mathrm{~S}$ & {$[\mathrm{mAU}$} & $\%$ \\
\hline & & & & |--- & . & ----- & \\
\hline 1 & 1 & 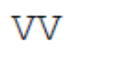 & 10 & 1.0 & e 4 & 424.3 & 540 \\
\hline 2 & 83 & B & 594 & 48 & 5994 & 20.09515 & 5460 \\
\hline
\end{tabular}



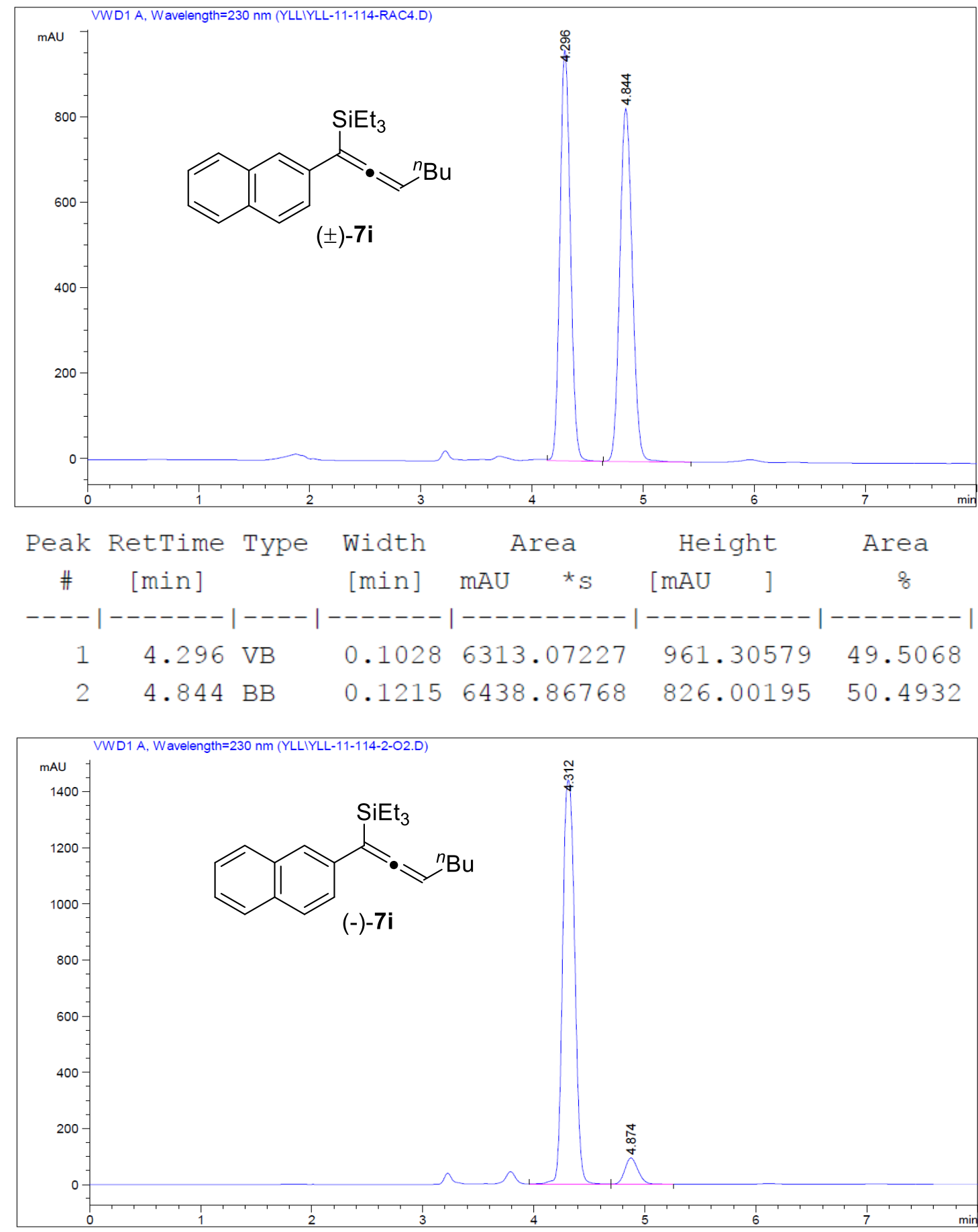

\begin{tabular}{|c|c|c|c|c|c|c|c|}
\hline eak & RetTime & Type & Width & Ar & ea & Height & Area \\
\hline \# & {$[\mathrm{min}]$} & & {$[\mathrm{min}]$} & $\mathrm{mAU}$ & ${ }^{*} \mathrm{~S}$ & {$[\mathrm{mAU}$} & 8 \\
\hline & & & & |--- & --- & $\mid-------$ & --- \\
\hline 1 & 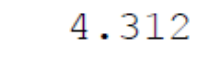 & & & 1.09 & $7 e 4$ & 1440.93 & 396 \\
\hline 2 & 874 & & 99 & 787 & 241 & 93.9 & 7104 \\
\hline
\end{tabular}


(-)-triethyl(1-(naphthalen-2-yl)-3-phenylpropa-1,2-dien-1-yl)silane (7j)

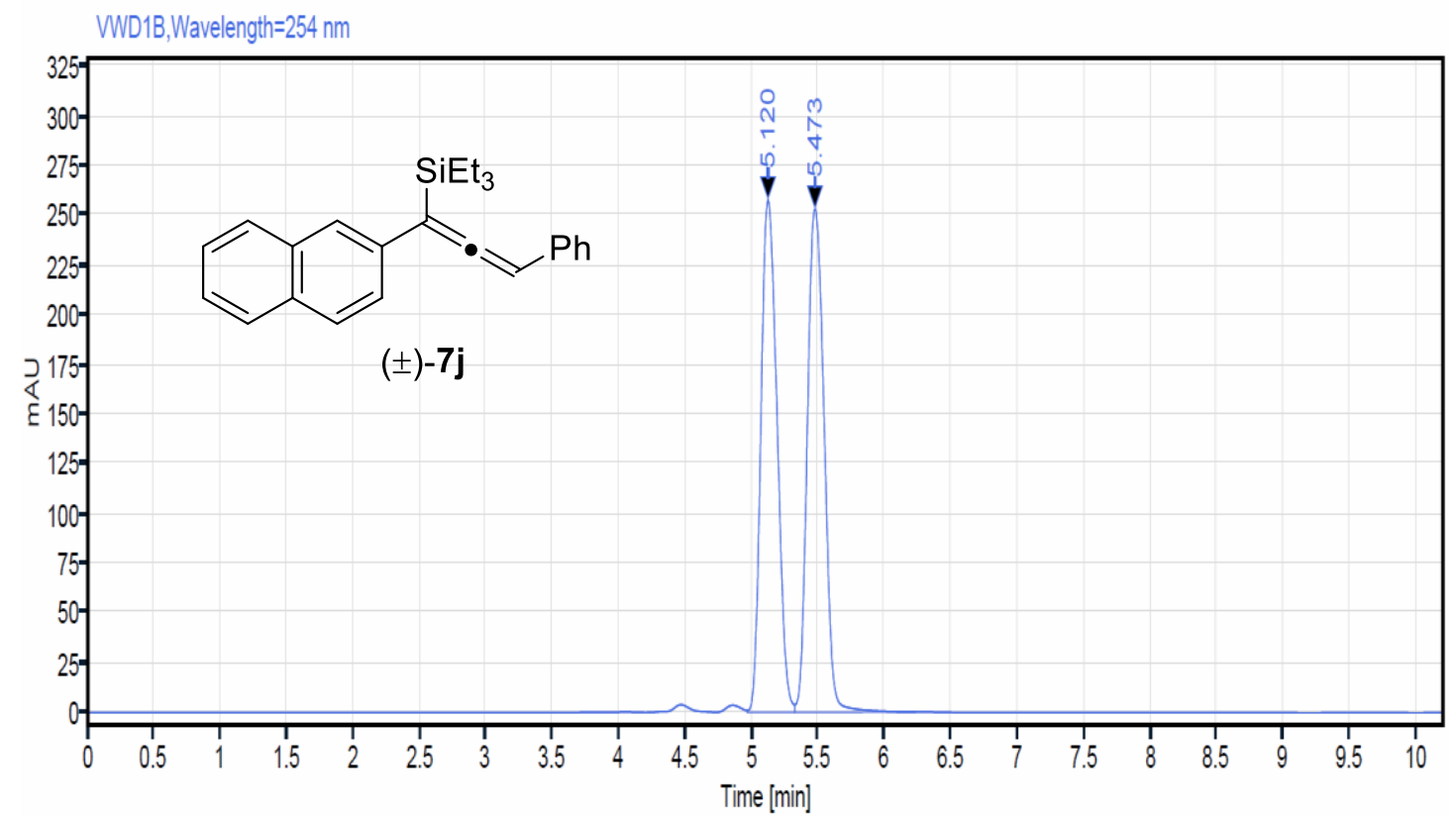

$\begin{array}{rrrrrr}\text { RT [min] } & \text { Type } & \text { Width [min] } & \text { Area } & \text { Height } & \text { Area\% } \\ 5.120 & \text { VV } & 0.36 & 2173.27 & 258.38 & 49.36 \\ 5.473 & \text { VB } & 0.91 & 2230.06 & 253.72 & 50.64\end{array}$

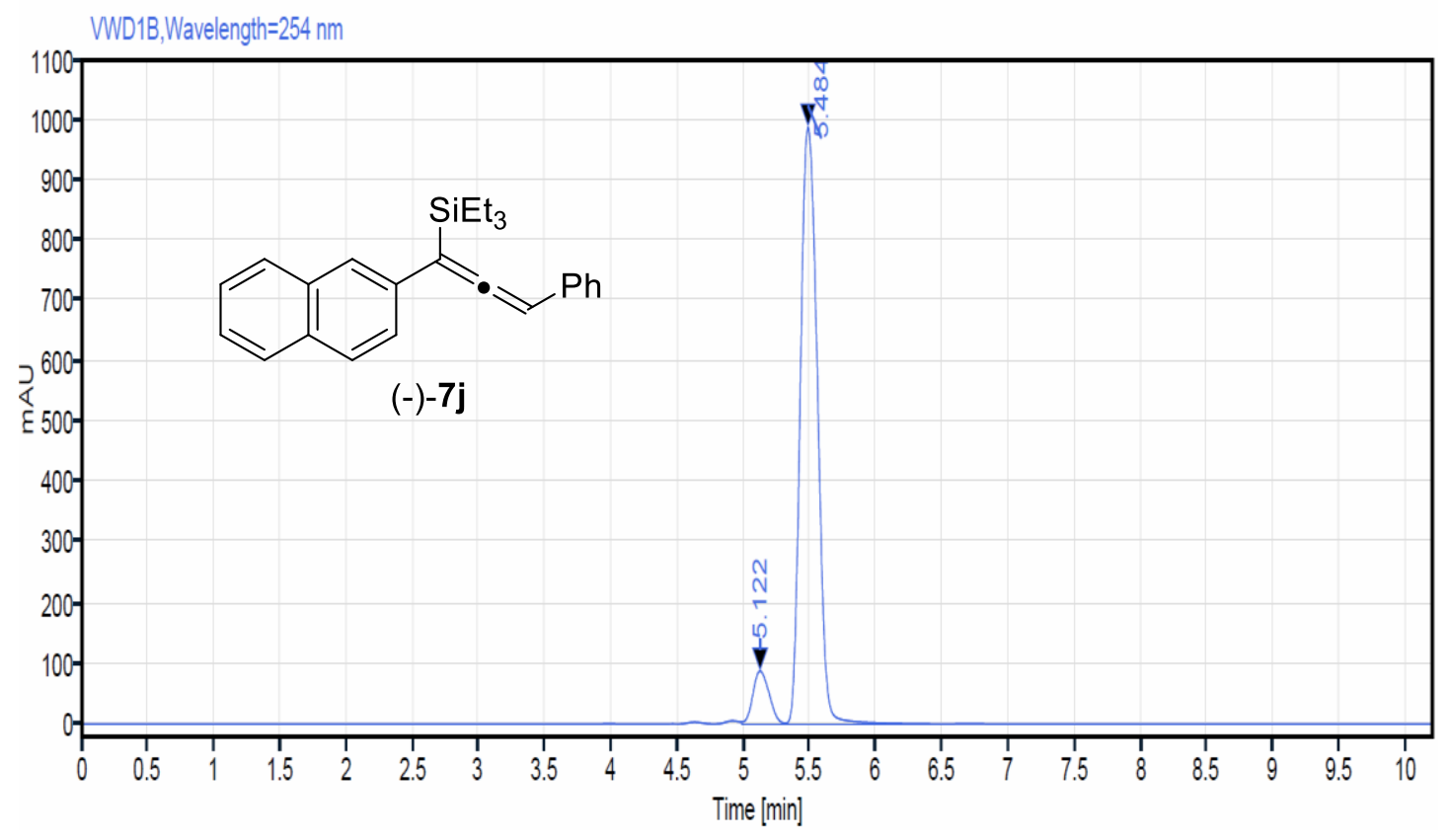

$\begin{array}{rrrrrr}\text { RT [min] } & \text { Type } & \text { Width [min] } & \text { Area } & \text { Height } & \text { Area\% } \\ 5.122 & \text { VV } & 0.33 & 750.08 & 87.93 & 7.84 \\ 5.484 & \text { VB } & 1.21 & 8815.54 & 989.00 & 92.16\end{array}$


(-)-dimethyl(1-(naphthalen-2-yl)-3-phenylpropa-1,2-dien-1-yl)(phenyl)silane (7k)

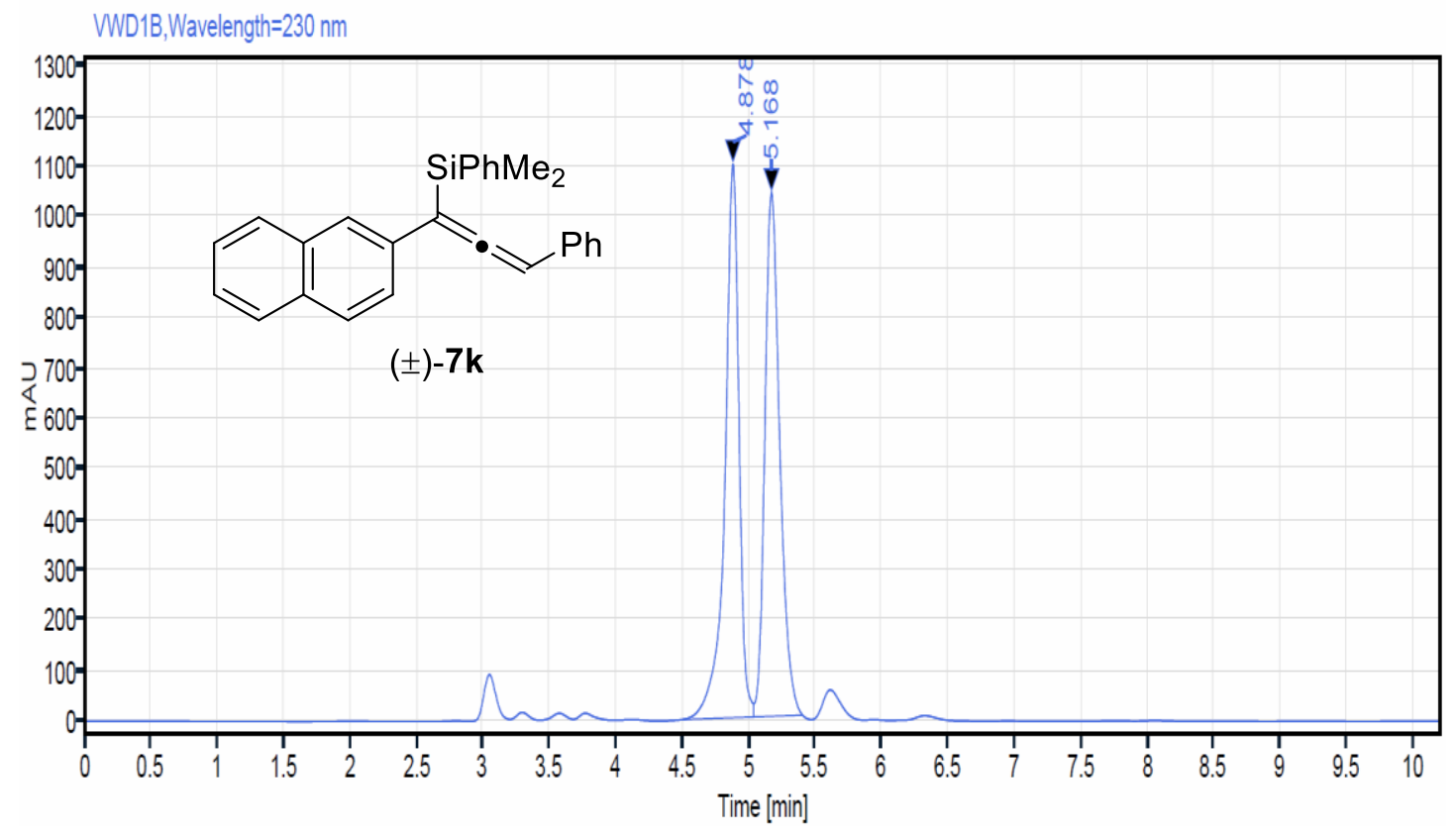

$\begin{array}{rrrrrr}\text { RT [min] } & \text { Type } & \text { Width [min] } & \text { Area } & \text { Height } & \text { Area\% } \\ 4.878 & \text { BV } & 0.53 & 8080.72 & 1102.42 & 49.78 \\ 5.168 & \text { VBA } & 0.37 & 8151.34 & 1042.28 & 50.22\end{array}$

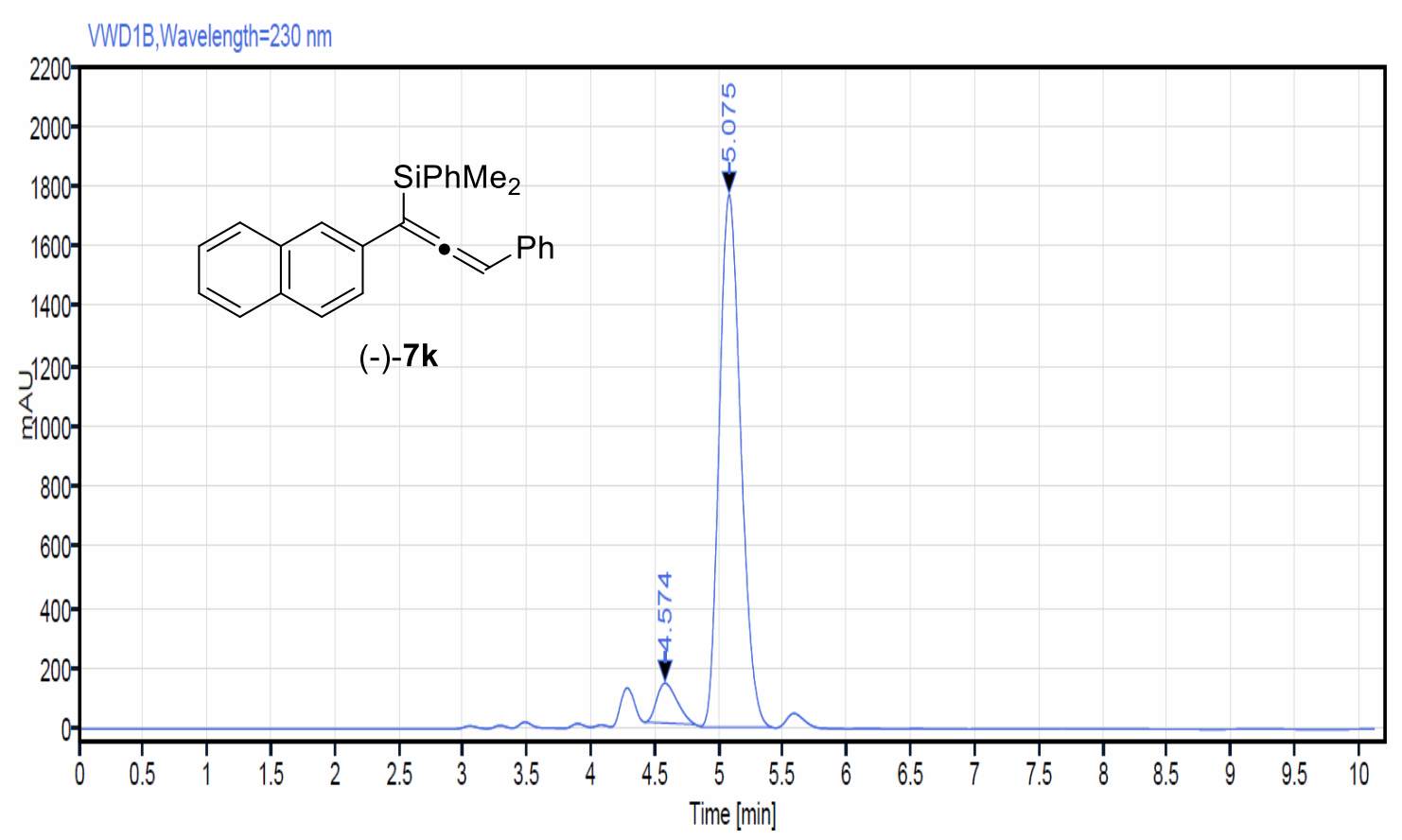

$\begin{array}{rrrrrr}\text { RT [min] } & \text { Type } & \text { Width [min] } & \text { Area } & \text { Height } & \text { Area\% } \\ 4.574 & \text { MB m } & 0.18 & 1463.43 & 132.23 & 6.60 \\ 5.075 & \text { VBA } & 0.56 & 20707.45 & 1768.95 & 93.40\end{array}$




\section{References}

${ }^{1}$ Armarego, W. L. F.; Chai, C. L. L. Purification of Laboratory Chemicals-Six Edition. Elsevier Inc.: London, 2009.

${ }^{2}$ Yang, L.-L.; Evans, D.; Xu, B.; Li, W.-T.; Li, M.-L.; Zhu, S.-F.; Houk, K. N.; Zhou, Q.-L. Enantioselective Diarylcarbene Insertion into $\mathrm{Si}-\mathrm{H}$ Bonds Induced by Electronic Properties of the Carbenes. J. Am. Chem. Soc. 2020, 142, 12394-12399.

3 DePinto, J. T.; deProphetis, W. A.; Menke, J. L.; McMahon, R. J. Triplet 1,3Diphenylpropynylidene (Ph-C-C-C-Ph). J. Am. Chem. Soc. 2007, 129, 2308-2315.

${ }^{4}$ Gandhamsetty, N.; Park, S.; Chang, S., Selective Silylative Reduction of Pyridines Leading to Structurally Diverse Azacyclic Compounds with the Formation of $\mathrm{sp}^{3} \mathrm{C}-\mathrm{Si}$ Bonds. J. Am. Chem. Soc. 2015, 137, 15176-15184.

5 Martin, R.; Martin-Montero, R.; Krolikowski, T.; Zarate, C.; Manzano, R., Stereospecific NickelCatalyzed Borylation of Secondary Benzyl Pivalates. Synlett 2017, 28, 2604-2608.

${ }^{6}$ Gonzalez, A. Z.; Soderquist, J. A. $\beta$-Silylated Homopropargylic Amines via the Asymmetric Allenylboration of Aldimines. Org. Lett. 2007, 9, 1081-1084.

${ }^{7}$ Larock, R. C.; Gupta, S. K.; Brown, H. C. Reaction of 2-Alkenyl-1,3,2-benzodioxaboroles with Mercuric Acetate. Convenient Stereospecific Procedure for the Conversion of Alkynes into Alkenylmercuric Salts. J. Am. Chem. Soc. 1972, 12, 4371-4373.

${ }^{8}$ Basch, C. H.; Cobb, K. M.; Watson, M. P. Nickel-Catalyzed Borylation of Benzylic Ammonium Salts: Stereospecific Synthesis of Enantioenriched Benzylic Boronates. Org. Lett. 2016, 18, 136139.

9 Nyman, C. J.; Wymore, C. E.; Wilkinson, G. Reactions of Tris(triphenylphosphine)platinum (0) and Tetrakis(triphenylphosphine)palladium (0) with Oxygen and Carbon Dioxide. J. Chem. Soc. A 1968, 561-563.

${ }^{10}$ Wilke, G.; Schott, H.; Heimbach, P. Oxygen Complexes of Zerovalent Nickel, Palladium, and Platinum. Angew. Chem. Int. Ed. 1967, 6, 92-93

${ }^{11}$ M. J. Frisch, G. W. Trucks, H. B. Schlegel, G. E. Scuseria, M. A. Robb, J. R. Cheeseman, G. Scalmani, V. Barone, G. A. Petersson, H. Nakatsuji, X. Li, M. Caricato, A. V. Marenich, J. Bloino, B. G. Janesko, R. Gomperts, B. Mennucci, H. P. Hratchian, J. V. Ortiz, A. F. Izmaylov, J. L. Sonnenberg, D. Williams-Young, F. Ding, F. Lipparini, F. Egidi, J. Goings, B. Peng, A. Petrone, T. Henderson, D. Ranasinghe, V. G. Zakrzewski, J. Gao, N. Rega, G. Zheng, W. Liang, M. Hada, M. Ehara, K. Toyota, R. Fukuda, J. Hasegawa, M. Ishida, T. Nakajima, Y. Honda, O. Kitao, H. Nakai, T. Vreven, K. Throssell, J. A. Montgomery, Jr., J. E. Peralta, F. Ogliaro, M. J. Bearpark, J. J. Heyd, E. N. Brothers, K. N. Kudin, V. N. Staroverov, T. A. Keith, R. Kobayashi, J. Normand, K. Raghavachari, A. P. Rendell, J. C. Burant, S. S. Iyengar, J. Tomasi, M. Cossi, J. M. Millam, M. Klene, C. Adamo, R. Cammi, J. W. Ochterski, R. L. Martin, K. Morokuma, O. Farkas, J. B. Foresman, and D. J. Fox, Gaussian 16, Revision A.03 (Gaussian, Inc., 2016).

12 Legault, C. Y. CYLview, 1.0b (Université de Sherbrooke, 2009); www.cylview.org. 